Christian Ricken

\title{
Determinanten \\ der Effektivität der Umweltpolitik
}




\section{Christian Ricken}

\section{Determinanten der Effektivität der Umweltpolitik}

Diegängigsten Variablen zurErklärungunterschiedlicherUmweltbelastungsniveaus sind die Höhe und das Wachstum der Wirtschaftsleistung. Dieses Buch möchte den Blickwinkel erweitern und untersucht die Relevanz des nationalen Politikstils für den Erfolg von Umweltpolitik. Sind Staaten mit konsensualem oder eher solche mit konfliktärem Politikstil auf dem Gebiet der Umweltpolitik erfolgreicher? Macht sich eine strategieorientierte Politik ökologisch bezahlt? Anhand einer quantitativen Analyse der OECD-Staaten wird der Effekt des nationalen Politikstils auf umweltökonomische Erfolgsgrößen untersucht.

Christian Ricken, geboren 1966, Studium der Betriebswirtschaftslehre in Hamburg, Promotion am Institut für Finanzwissenschaft. Von 1986 bis 1996 Offizierslaufbahn in der Bundeswehr. Seit 1996 ist er im Controlling der Konzernzentrale der Deutschen Bank tätig. Schwerpunkt: Operative Konzernplanung. 
Determinanten der Effektivität der Umweltpolitik 


\section{SCHRIFTEN ZUR \\ WIRTSCHAFTSTHEORIE UND \\ WIRTSCHAFTSPOLITIK}

Herausgegeben von

Rolf Hasse, Wolf Schäfer, Thomas Straubhaar und Klaus W. Zimmermann

Band 7

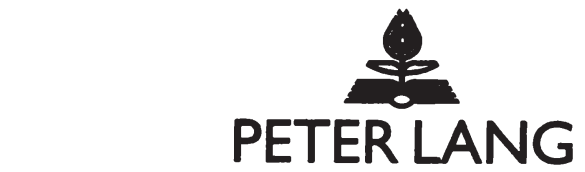

Frankfurt am Main - Berlin - Bern - New York · Paris - Wien 


\section{Christian Ricken}

\section{Determinanten der Effektivität der Umweltpolitik \\ Der nationale Politikstil im Spannungsfeld von Ökonomie, Politik und Kultur}

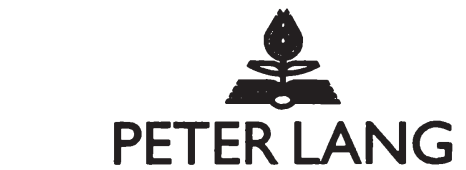

Europäischer Verlag der Wissenschaften 
Die Deutsche Bibliothek - CIP-Einheitsaufnahme

Ricken, Christian:

Determinanten der Effektivität der Umweltpolitik : der nationale Politikstil im Spannungsfeld von Ökonomie, Politik und Kultur / Christian Ricken. - Frankfurt am Main ; Berlin ; Bern ; New York ; Paris ; Wien : Lang, 1997

(Schriften zur Wirtschaftstheorie und Wirtschaftspolitik ; Bd. 7)

Zugl.: Hamburg, Univ. der Bundeswehr, Diss., 1997 ISBN 3-631-32071-X

Open Access: The online version of this publication is published on www.peterlang.com and www.econstor.eu under the international Creative Commons License CC-BY 4.0. Learn more on how you can use and share this work: http://creativecommons. org/licenses/by/4.0.

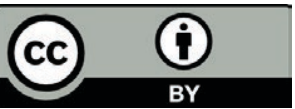

This book is available Open Access thanks to the kind support of ZBW - Leibniz-Informationszentrum Wirtschaft.

Gedruckt mit Unterstützung der Universităt der Bundeswehr Hamburg.

\author{
D 705 \\ ISSN 1433-1519 \\ ISBN 3-631-32071-X \\ ISBN 978-3-631-75118-3 (eBook) \\ (c) Peter Lang GmbH \\ Europäischer Verlag der Wissenschaften \\ Frankfurt am Main 1997 \\ Alle Rechte vorbehalten.
}

Das Werk einschließlich aller seiner Teile ist urheberrechtlich geschützt. Jede Verwertung außerhalb der engen Grenzen des

Urheberrechtsgesetzes ist ohne Zustimmung des Verlages unzulässig und strafbar. Das gilt insbesondere für

Vervielfältigungen, Übersetzungen, Mikroverfilmungen und die Einspeicherung und Verarbeitung in elektronischen Systemen.

Printed in Germany 123467 
Für Andrea

Christian Ricken - 978-3-631-75118-3

Downloaded from PubFactory at 01/11/2019 08:07:14AM

via free access 
Christian Ricken - 978-3-631-75118-3

Downloaded from PubFactory at 01/11/2019 08:07:14AM

via free access 


\section{Danksagung}

Die vorliegende Arbeit wäre ohne die Unterstützung und den Zuspruch zahlreicher Kollegen und Freunde nicht zustande gekommen.

Mein Dank gilt vor allem meinem Doktorvater, Herrn Prof. Dr. Klaus W. Zimmermann vom Institut für Finanzwissenschaft der Universität der Bundeswehr in Hamburg, der mich trotz der teilweise großen Entfernungen zwischen meinem Wohnort und der Universität als externen Doktoranden angenommen und über drei Jahre intensiv betreut hat. Seine Kritik war immer hart, aber auch konstruktiv. Ohne seine unermüdliche Unterstützung hätte ich weder seinen noch meinen Anforderungen an eine wissenschaftliche Arbeit gerecht werden können.

Ich danke auch Herrn Prof. Dr. Jürgen Hartmann, der als Zweitgutachter aus dem Bereich der Politikwissenschaft diese interdisziplinäre Arbeit erst ermöglicht hat. Auch Herrn Prof. Dr. Michel Domsch, der viele Jahre nachdem ich bei ihm studiert hatte, meine Bitte um Teilnahme am Prüfungsausschuß nicht ausgeschlagen hat, bin ich zu Dank verpflichtet.

Von den weiteren Lehr- und Fachkräften, die mir bei meiner Arbeit geholfen haben, möchte ich an dieser Stelle Herrn Eckehard Zirkmann und Frau Kruse besonders hervorheben. Beide haben dazu beigetragen, daß ich trotz der räumlichen Distanzen stets über alle administrativen und organisatorischen Punkte des Promotionsverfahres bestens informiert war.

Am meisten danken möchte ich jedoch meiner Frau. Während der drei Jahre meiner Promotion, die uns viel gemeinsame Zeit gekostet haben, hat sie mir mit ihrer Geduld und ihrem Verständnis durch manche Krise geholfen. 
Christian Ricken - 978-3-631-75118-3

Downloaded from PubFactory at 01/11/2019 08:07:14AM

via free access 


\section{Inhaltsverzeichnis}

Teil I: Elemente einer Theorie zur Erklärung des Einflusses des nationalen Politikstils auf die Effektivität von Umweltpolitik

1 Einführung 1

1.1 Problemstellung 1

1.2 Aufbau der Arbeit___ 8

1.3 Theoretischer und inhaltlicher Standort der Arbeit _ 15

1.3.1 Entdeckungszusammenhang _ 15

1.3.2 Begründungszusammenhang — 17

1.3.3 Verwendungszusammenhang__ 17

2 Erfolge nationaler Umweltpolitik _ 19

2.1 Systematik möglicher Erfolgskriterien__ 19

2.2 Kriterien zur Messung des Politikerfolges ___ 24

2.2.1 Effektivität —_ 24

2.2.2 Effizienz 25

2.2.3 Gerechtigkeit (Equity) _ 30

2.2.4 Diskussion und Auswahl __ 33

2.3 Effektivität von Umweltpolitik___ 35

2.3.1 Zielerreichungsgrad _ 35

2.3.2 Absolute Umweltbelastungen — 35

2.3.3 Relative Umweltbelastungen _ 37

2.3.4 Änderung der Umweltbelastungsindikatoren___ 38

2.4 Probleme der Zurechenbarkeit _ 39

2.4.1 Grenzüberschreitung von Schadstoffeinträgen __ 39

2.4.2 Gratiseffekte__ 40

3 Determinanten der Effektivität nationaler Umweltpolitik ___ 42

3.1 Überblick 42

3.2 Strukturelle Faktoren __ 43

3.2.1 Ökonomische Determinanten _ _ 43

3.2.2 Kulturelle Determinanten___ 44 
3.3 Situative Faktoren__ 48

4 Das Politikstil-Konzept im Kontext der Policy-Analyse _ 50

4.1 Policy-Analyse $\quad 50$

4.1.1 Gegenstände und Methoden der Policy-Analyse___ 50

4.1.2 Policy Networks___ 53

4.1.3 Policy Cycle _ 55

4.1.3.1 Problemdefinition 59

4.1.3.2 Agenda-Gestaltung 60

4.1.3.3 Politikformulierung __ 61

4.1.3.4 Politikimplementation _ 62

4.1.3.5 Evaluation 65

4.1.3.6 Politikterminierung bzw. Neuformulierung___ 66

4.2 Das Policy-Style-Konzept

4.2.1 Begriffsklärungen__ 69

4.2.2 Politikstil und Politische Kultur 79

4.2.3 Ansätze zur Erklärung und Darstellung nationaler Policy Styles _ 85

4.2.3.1 Das Konzept von Richardson (1982): Problemlösungsverhalten und Art der Beziehungen zwischen Regierung und Betroffenen als relevante Politikstildimensionen

4.2.3.2 Die Typologie von Kitschelt (1983): Offenheit und

Handlungskapazität als relevante Politikstildimensionen

4.2.3.3 Die Typologie von Bull (1992): Interessenaggregation nichtstaatlicher Akteure und staatliche Macht als relevante Politikstildimensionen

4.2.4 Schlußfolgerungen

5 Die Bedeutung des Politikstils als Determinante der Effektivität nationaler Umweltpolitik 96

5.1 Modellentwurf __ 96

5.2 Ökologisch relevante Politikstildimensionen___ 104

5.2.1 Strategieorientierung __ 104

5.2.2 Partizipationsgrad___ 111

5.3 Typologie nationaler Umweltpolitikstile __ 117

5.3.1 Schema _ 117

5.3.2 Planerischer Korporatismus___ 119

5.3.3 Reaktiver Korporatismus __ 120 
5.3.4 Innovativer Pluralismus 122

5.3.5 Laissez-faire 122

5.4 Zusammenfassende Bemerkungen 123

Teil II: Empirische Untersuchung

6 Vorhandene empirische Untersuchungen über die Bedeutung des Politikstils für den Erfolg von Umweltpolitik 125

6.1 Die Studien Jänickes 125

6.2 Weitere Studien 127

7 Politikstil und Effektivität von Umweltpolitik in OECD-Ländern:

Untersuchungsdesign 132

7.1 Die Festlegung der Fallzahl 132

7.2 Indikatorenauswahl und Index-Bildung 134

7.3 Strategie der Datenanalyse 145

8 Messung der Effektivität nationaler Umweltpolitik 148

8.1 Umweltbelastungsindikatoren (Basis: Einwohnerzahl) 148

8.1.1 Ressourcenverbrauch 148

8.1.2 Schadstoffemissionen 151

8.1.3 Gesamtbelastung 154

8.2 Umweltbelastungsindikatoren (Basis: BIP)

8.2.1 Ressourcenverbrauch 155

8.2.2 Schadstoffemissionen

8.2.3 Gesamtbelastung 155 157 159

9 Determinanten der Effektivităt nationaler Umweltpolitik 173

9.1 Ökonomische Determinanten 173

9.1.1 Ökonomisches Niveau

9.1.2 Sektorale Aufteilung der Volkswirtschaft 173

9.2 Politische Determinanten 185

10 Messung des nationalen Politikstils 189

10.1 Strategieorientierung 
10.1.1 (De-)Zentralisationsgrad _ـ 194

10.1.2 Technologische Kapazitäten _ـ 199

10.1.3 Administrative Kapazitäten 202

10.1.4 Gesamteinschätzung____ 205

10.2 Partizipationsgrad___ 205

10.2.1 Korporatismusgrad _ 205

10.2.2 Konfliktlösungsverhalten_ 208

10.2.3 Gesamteinschätzung der Staaten __ 211

10.3 Positionierung der Staaten __ 211

11 Politikstil als Determinante der Effektivität nationaler Umweltpolitik _ 218

11.1 Politikstildimensionen als Korrelate der Umweltbelastung ___ 218

11.1.1 Strategieorientierung __ 218

11.1.2 Partizipationsgrad____ 221

11.2 Multiple Regressionsanalysen aller Politikstilvariablen zur Erklärung der

Varianz der Umweltbelastung __ 223

11.3 Varianzanalysen der Politikstilcluster anhand der

Umweltbelastungsindizes___ 229

Teil III: Schlußfolgerungen

12 Zusammenfassung und Interpretation der empirischen

Untersuchungsergebnisse 237

13 Kritik an Methodik und Strategie der Datenanalyse 240

14 Literatur 242

15 Anhang 262 


\section{Abbildungsverzeichnis}

Abb. 1: Modell zur Erklärung des Einflusses des nationalen Politikstils auf die Effektivität von Umweltpolitik.................................................................. 7

Abb. 2: Input-Output-Modell des politischen Prozesses........................................ 16

Abb. 3: Systematik von Kriterien zur Messung des Erfolges von Umweltpolitik. 20

Abb. 4: Produktionsmöglichkeiten- und Indifferenzkurven ............................ 22

Abb. 5: Bestimmung des umweltökonomischen Optimums ............................... 27

Abb. 6: Systematik der Determinanten nationaler Umweltpolitik..................... 42

Abb. 7: Politikstil und Policy Cycle als Elemente des Input-Output-Modells .. 58

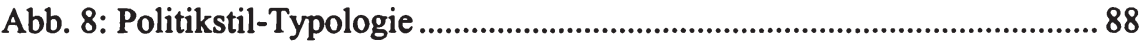

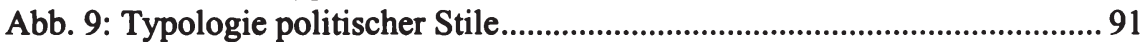

Abb. 10: Typologie möglicher Beziehungen von Staat und Interessen-

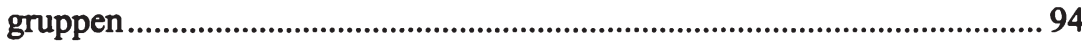

Abb. 11: Vergleich der vorgestellten Politikstil-Studien .....................................95

Abb. 12: Faktoren des Umweltpolitikzyklus................................................ 101

Abb. 13: Strategische Ansätze für eine präventive Umweltpolitik.................. 102

Abb. 14: Merkmalsdimensionen nationaler Umweltpolitik-Stile .................... 104

Abb. 15: Dezentralisierungstheorem ......................................................... 108

Abb. 16: Strategieoptionen im bilateralen Verhandlungsprozeß ..................... 113

Abb. 17: Umweltpolitikstil-Typologie .......................................................... 118

Abb. 18 : Zusammenhang zwischen Konsensorientierung staatlicher Politik und ökologischem Erfolg.................................................................... 126

Abb. 19: Ressourcenverbrauchsindex pro Einwohner 1990 ........................... 161

Abb. 20: Ressourcenverbrauchsindex pro Einwohner ausgewählter Staaten

1970 bis 1990

Abb. 21: Schadstoffindex pro Einwohner 1990 ............................................... 163

Abb. 22: Schadstoffindex pro Einwohner ausgewählter Staaten 1970 bis 1990

Abb. 23: Umweltbelastungsindex pro Einwohner 1990 .................................. 165

Abb. 24: Umweltbelastungsindex pro Einwohner ausgewählter Staaten

1970 bis $1990 \ldots \ldots$

Abb. 25: Ressourcenverbrauchsindex pro Einheit BIP 1990.......................... 167

Abb. 26: Ressourcenverbrauchsindex pro Einheit BIP ausgewählter Staaten

1970 bis $1990 \ldots \ldots$

Abb. 27: Schadstoffindex pro Einheit BIP 1990 .............................................. 169

Abb. 28: Schadstoffindex pro Einheit BIP ausgewählter Staaten 1970 bis 1990 
Abb. 29: Umweltbelastungsindex pro Einheit BIP

Abb. 30: Umweltbelastungsindex pro Einheit BIP ausgewählter Staaten

1970 bis 1990 ...

Abb. 31: Entwicklung der Umweltbelastungsindizes in Abhängigkeit vom elektoralen Erfolg grüner Parteien

Abb. 32: Positionierung der OECD-Staaten hinsichtlich ihres Politikstils ..... 212

Abb. 33: Positionierung der Clusterzentroide 217 


\section{Tabellenverzeichnis}

Tabelle 1: Korrelationsanalyse zwischen den Umweltbelastungsindikatoren pro Einwohner und der Wirtschaftsleistung . 175

Tabelle 2: Korrelationsanalyse zwischen den Umweltbelastungsindikatoren pro Einwohner und dem Wachstum der Wirtschaftsleistung.

Tabelle 3: Korrelationsanalyse zwischen dem Wachstum der

Umweltbelastungsindikatoren pro Einwohner und dem Wachstum der

Wirtschaftsleistung.

Tabelle 4: Korrelationsanalyse zwischen den Umweltbelastungsindikatoren pro Einheit BIP und der Wirtschaftsleistung. 180

Tabelle 5: Korrelationsanalyse zwischen den Umweltbelastungsindikatoren pro Einheit BIP und dem Wachstum der Wirtschaftsleistung.

Tabelle 6: Korrelationsanalyse zwischen dem Wachstum der

Umweltbelastungsindikatoren pro Einheit BIP und dem Wachstum der

Wirtschaftsleistung.

Tabelle 7: Korrelationen zwischen den Umweltbelastungsindizes und der

Wirtschaftsleistung von Staaten mit hohem bzw. niedrigem BIP pro

Einwohner

Tabelle 8: Einfache und partielle Korrelationsanalyse zwischen den

Umweltbelastungsindikatoren und der sektoralen Verteilung der

Volkswirtschaften

Tabelle 9: Elektoral erfolgreiche und erfolglose grüne Parteien in Westeuropa (1979-1989)

Tabelle 10: Föderalismusgrad der untersuchten Staaten................................ 198

Tabelle 11: Technologische Kapazitäten.............................................................. 200

Tabelle 12: Administrative Kapazitäten ........................................................204

Tabelle 13: Strategieorientierung der OECD-Staaten ..................................... 207

Tabelle 14: Partizipationsgrad der OECD-Staaten......................................... 210

Tabelle 15: Clusteranalyse der OECD-Staaten aufgrund ihrer

Politikstilmerkmale (Gruppenzentroide) ............................................. 214

Tabelle 16: Clustervarianzen und -zugehörigkeiten........................................ 214

Tabelle 17: Indikatoren der Strategieorientierung als Korrelate der

Umweltbelastung (Basis: Einwohnerzahl)

Tabelle 18: Indikatoren der Strategieorientierung als Korrelate der

Umweltbelastung (Basis: Einheit BIP)

Tabelle 19: Indikatoren des Partizipationsgrades als Korrelate der

Umweltbelastung (Basis: Einwohnerzahl) 
Tabelle 20: Indikatoren des Partizipationsgrades als Korrelate der Umweltbelastung (Basis: Einheit BIP).

Tabelle 21: Beta-Gewichte und t-Werte signifikanter Prädiktoren der Höhe des Ressourcenverbrauchsindexes pro Einwohner (multiple Regressionsanalyse)

Tabelle 22: Beta-Gewichte und t-Werte signifikanter Prädiktoren der Höhe des Schadstoffindexes pro Einwohner (multiple Regressionsanalyse).... 225

Tabelle 23: Beta-Gewichte und t-Werte signifikanter Prädiktoren der Höhe des Umweltbelastungsindexes pro Einwohner (multiple Regressionsanalyse)

Tabelle 24: Beta-Gewichte und t-Werte signifikanter Prädiktoren der Höhe des Ressourcenverbrauchsindexes pro Einheit BIP (multiple Regressionsanalyse)

Tabelle 25: Beta-Gewichte und t-Werte signifikanter Prädiktoren der Höhe des Schadstoffindexes pro Einheit BIP (multiple Regressionsanalyse)... 228

Tabelle 26: Beta-Gewichte und t-Werte signifikanter Prädiktoren der Höhe des Umweltbelastungsindexes pro Einheit BIP (multiple Regressionsanalyse)

Tabelle 27: Varianzanalyse der Staatengruppen anhand ihrer Umweltbelastungswerte (Basis: Einwohnerzahl)

Tabelle 28: Varianzanalyse der Staatengruppen anhand ihrer

Umweltbelastungswerte (Basis: Einheit BIP)

Tabelle 29: Varianzanalyse der Staatengruppen anhand ihrer

Umweltbelastungswerte (Basis: Einwohnerzahl).

Tabelle 30:Varianzanalyse der Staatengruppen anhand ihrer

Umweltbelastungswerte (Basis: Einheit BIP) 


\section{Verzeichnis der Tabellen im Anhang}

Tab. 1: Wasserverbrauch

Tab. 2: $\quad$ Landwirtschaftsfläche

Tab. 3: Primärenergieverbrauch

Tab. 4: Energiebedingte $\mathrm{CO}_{2}$-Emissionen

Tab. 5: Abfallaufkommen

Tab. 6: Handelsdüngerabsatz

Tab. 7: Veränderung des Wasserverbrauches

Tab. 8: Veränderung der Landwirtschaftsfläche

Tab. 9: Veränderung des Primärenergieverbrauches

Tab. 10: Veränderung der energiebedingten $\mathrm{CO}_{2}$-Emissionen

Tab. 11: Veränderung des Abfallaufkommens

Tab. 12: Veränderung des Handelsdüngerabsatzes

Tab. 13: Wasserverbrauch pro Einwohner

Tab. 14: Landwirtschaftsfläche pro Einwohner

Tab. 15: Primärenergieverbrauch pro Einwohner

Tab. 16: Energiebedingte $\mathrm{CO}_{2}$-Emissionen pro Einwohner

Tab. 17: Abfallaufkommen pro Einwohner

Tab. 18: Handelsdüngerabsatz pro Einwohner

Tab. 19: Ressourcenverbrauchsindex (Basis: Einwohnerzahl)

Tab. 20: Schadstoffindex (Basis: Einwohnerzahl)

Tab. 21: Umweltbelastungsindex (Basis: Einwohnerzahl)

Tab. 22: Veränderung des Wasserverbrauches pro Einwohner

Tab. 23: Veränderung der Landwirtschaftsfläche pro Einwohner

Tab. 24: Veränderung des Primärenergieverbrauches pro Einwohner

Tab. 25: Veränderung der energiebedingten $\mathrm{CO}_{2}$-Emissionen pro Einwohner

Tab. 26: Veränderung des Abfallaufkommens pro Einwohner

Tab. 27: Veränderung des Handelsdüngerabsatzes pro Einwohner

Tab. 28: Veränderung des Ressourcenverbrauchsindexes (Basis:

Einwohnerzahl)

Tab. 29: Veränderung des Schadstoffindexes (Basis: Einwohnerzahl)

Tab. 30: Veränderung des Umweltbelastungsindexes (Basis: Einwohnerzahl)

Tab. 31: Wasserverbrauch pro Einheit BIP

Tab. 32: Landwirtschaftsfläche pro Einheit BIP

Tab. 33: Primärenergieverbrauch pro Einheit BIP 
Tab. 34: Energiebedingte $\mathrm{CO}_{2}$-Emissionen pro Einheit BIP

Tab. 35: Abfallaufkommen pro Einheit BIP

Tab. 36: Handelsdüngerabsatz pro Einheit BIP

Tab. 37: Ressourcenverbrauchsindex (Basis: BIP)

Tab. 38: Schadstoffindex (Basis: BIP)

Tab. 39: Umweltbelastungsindex (Basis: BIP)

Tab. 40: Veränderung des Wasserverbrauches pro Einheit BIP

Tab. 41: Veränderung der Landwirtschaftsflăche pro Einheit BIP

Tab. 42: Veränderung des Primärenergieverbrauches pro Einheit BIP

Tab. 43: Veränderung der energiebedingten $\mathrm{CO}_{2}$-Emissionen pro Einheit BIP

Tab. 44: Veränderung des Abfallaufkommens pro Einheit BIP

Tab. 45: Veränderung des Handelsdüngerabsatzes pro Einheit BIP

Tab. 46: Veränderung des Ressourcenverbrauchsindexes (Basis: BIP)

Tab. 47: Veränderung des Schadstoffindexes (Basis: BIP)

Tab. 48: Veränderung des Umweltbelastungsindexes (Basis: BIP)

Tab. 49: Einwohnerzahl

Tab. 50: Bruttoinlandsprodukt

Tab. 51: Wachstum des Bruttoinlandsproduktes

Tab. 52: Bruttoinlandsprodukt pro Einwohner

Tab. 53: Wachstum des BIP pro Einwohner

Tab. 54: Sektorale Aufteilung der Volkswirtschaft 1990

Tab. 55: Regressionsgleichungen der multiplen Regressionsanalysen 


\section{Abkürzungs- und Variablenverzeichnis}

ADM01

ADM02

BIP

C

$\mathrm{CO} 2$

d.f.

ENER

ENVIR

EU

FERT

F\&E01
Variablenkurzbezeichnung für Ausgaben zur Durchführung öffentliche Aufgaben

Variablenkurzbezeichnung für den Anteil der mit der Erfüllung öffentlicher Aufgaben Beschäftigter

Bruttoinlandsprodukt (Variablenkurzbezeichnung)

Einwohnerzahl

Variablenkurzbezeichnung des Umweltbelastungsindikators Energiebedingte $\mathrm{CO}_{2-}$ Emissionen

degrees of freedom (Freiheitsgrade)

Variablenkurzbezeichnung des Umweltbelastungsindikators Primärenergieverbrauch

Variablenkurzbezeichnung des Umweltbelastungsindexes

Europäische Union

Variablenkurzbezeichnung des Umweltbelastungsindikators Handelsdüngerabsatz

Variablenkurzbezeichnung für die inländischen Bruttoausgaben für Forschung und Entwicklung als Prozentsatz des Bruttoinlandsproduktes (in Kaufkraftparitäten) 
F\&E02

F\&E03

FCKW

FÖDERAL

F\&E

$\mathrm{H} 2 \mathrm{O}$

KORPORAT

LAND

M

MITI

MTOE

$\mathrm{N}$

n.s.

OECD

$\mathrm{p}$
Variablenkurzbezeichnung für den Quotienten aus Export und Import von Gütern aus F\&Eintensiven Industriesektoren

Variablenkurzbezeichnung für die öffentlichen Bildungsausgaben zur F\&E-Spitzenförderung als Prozentsatz des Bruttoinlandsproduktes

Fluorchlorkohlenwasserstoff

Variablenkurzbezeichnung des Föderalismusgrades

Forschung und Entwicklung

Variablenkurzbezeichnung des Umweltbelastungsindikators Wasserverbrauch

Variablenkurzbezeichnung des Korporatimusgrades

Variablenkurzbezeichnung des Umweltbelastungsindikators Landwirtschaftsfläche

Mittelwert

Japanisches Ministerium für Technologie und Außenhandel

Millionen Tonnen Rohöläquivalent

Fallzahl

statistisch nicht signifikant

Organisation For Economic Co-Operation And Development

probability (Irrtumswahrscheinlichkeit) 
PARTIZIP

POPUL

PRESS

r

RESOU

SD

TG

Vi

$\mathrm{Vz}$

WASTE
Variablenkurzbezeichnung des Partizipationsgrades

Einwohnerzahl (Variablenkurzbezeichnung)

Variablenkurzbezeichnung des Schadstoffindexes

Korrelationskoeffizient

Variablenkurzbezeichnung des Ressourcenverbrauchsindexes

standard deviation (Standardabweichung)

Testgröße

Varianz innerhalb der Gruppen

Varianz zwischen den Gruppen

Variablenkurzbezeichnung des Umweltbelastungsindikators Abfallaufkommen 
Christian Ricken - 978-3-631-75118-3

Downloaded from PubFactory at 01/11/2019 08:07:14AM

via free access 
Teil I: Elemente einer Theorie zur Erklärung des Einflusses des
nationalen Politikstils auf die Effektivităt von Umweltpolitik

\section{Einführung}

\subsection{Problemstellung}

Ausgehend von Analyseresultaten der Politikwissenschaft, welche positive Auswirkungen korporatistischer, d.h. kooperativer, den Konsens betonender Strukturen im Interaktionsverhältnis von Kapital und Arbeit belegen ${ }^{1}$, ziehen einige Autoren einen Analogieschluß, durch welchen der Politikstil als Erfolgsbedingung politischen Handelns aus dem ökonomischen auch in den ökologischen Politikbereich übertragen werden soll.

„Ein aktiver, kooperativer Politikstil bekommt der Ökonomie wie der Ökologie besser als die Philosophie des Laissez-faire", formuliert beispielsweise Jänicke ${ }^{2}$. Empirisch belegt wird diese These durch die Feststellung, daß diejenigen Staaten zu den „umweltpolitischen Erfolgsfällen" gehören, in welchen konsensbetonte Aushandlungsprozesse die normale Form der Auseinandersetzung zwischen den politischen Akteuren sind, während Staaten mit einer eher schwachen umweltpolitischen Performanz meist einen konfliktbetonten Politikstil praktizieren ${ }^{3}$.

$\mathrm{Zu}$ einem ähnlichen Ergebnis gelangen Kern und Bratzel im Rahmen einer Sekundäranalyse umweltpolitischer Studien: „Für die Politikstil-Forschung in der Umweltpolitikanalyse läßt sich ein weitgehender Konsens dahingehend feststellen, daß sich kooperative Problemverarbeitungs- und Interakti-

\footnotetext{
${ }^{1}$ Vgl. Pryor, Frederic L. (1988): Corporatism as an Economic System: A Review Essay, in: Journal of Comparative Economics, 12, S. $334 \mathrm{ff}$.; sehr differenziert und kritisch: Crepaz, Markus M. L. (1992): Corporatism in Decline? An Empirical Analysis of the Impact of Corporatism on Macroeconomic Performance and Industrial Disputes in 18 Industrialized Democracies, in: Comparative Political Sciences, 2, S. 148 ff.; Cusack, Thomas R. (1994): Economic Problems in the OECD Countries during the Period 1950 through 1990, unveröffentlichter Entwurf, Berlin, S. 54 ff.

${ }^{2}$ Vgl. Jänicke, Martin (1990): Erfolgsbedingungen von Umweltpolitik im internationalen Vergleich, in: Zeitschrift für Umweltpolitik und Umweltrecht, 3, S. 224.

${ }^{3}$ Edenda, S. 223.
} 
onsmuster sowohl unter Effektivitäts-als auch unter Effizienzgesichtspunkten positiv auf den Erfolg von Umweltpolitik auswirken "4. Empirische Evidenz für diese Behauptung liefern vor allem Arbeiten, in welchen die Interaktionsformen in umweltpolitischen Prozessen in den USA einerseits und den Demokratien Westeuropas und Japans andererseits anhand eines dichotomen Schemas in „konfliktär“" bzw. „kooperativ“" unterschieden werden". Während die US-amerikanische Betonung individualistischer Selbstentfaltungswerte die Zusammenarbeit der Akteure behindere, würden die Standardverfahren der Politikformulierung und -implementation in Westeuropa, Skandinavien und Japan aufgrund historischer und kultureller Faktoren eher auf einen Konsens zwischen den Vertretern konfligierender Interessen abzie$\operatorname{len}^{6}$.

Die Operationalisierung und Instrumentalisierung des Konzeptes „Politikstil“" als erklärende Variable unterschiedlicher Erfolgsniveaus nationaler Umweltpolitiken setzt drei Grundannahmen voraus:

1. Das Konzept beschreibt die Realitäten des politischen Prozesses und läßt sich ausreichend exakt definieren.

2. Es läßt sich für jeden Staat ein nationaler Politikstil identifizieren.

3. Trotz der Vielzahl der Fälle lassen sich anhand von Ähnlichkeiten und Übereinstimmungen einzelner Politikstilmerkmale Staatengruppen mit einer heuristisch praktikablen Anzahl von Elementen bilden.

Richardson definiert den nationalen Politikstil eines Staates als „main characteristics of the policy process “7 oder ",standard operating procedures

${ }^{4}$ Kern, Kristine, und Bratzel, Stefan (1994): Erfolgskriterien und Erfolgsbedingungen von (Umwelt-)Politik im internationalen Vergleich. Eine Literaturstudie, FFU-report 94-3, Berlin, S. 40, sowie: Dies. (1995): Umweltpolitischer Erfolg im internationalen Vergleich, unveröffentlichtes Manuskript, Berlin, S. 9 ff.

${ }^{5}$ Vgl. u.a.: Vogel, David (1986): National Styles of Regulation. Environmental Policy in Great Britain and the United States, Ithaca und London; Kelman, Steven (1981): Regulating America, Regulating Sweden: A Comparative Study of Occupational Safety and Health Policy, Cambridge; Badaracco, Joseph L. (1985): Loading the Dice. A Five-Country Study of Vinyl Chloride Regulation, Boston.

${ }^{6}$ Vgl. Kelman, Steven (1981): Regulating America ...., a.a.O., S. 39; Weidner, Helmut (1989): Japanese Environmental Policy in an International Perspective: Lessons for a Preventive Approach, In: Tsuru, Shigeto, und Weidner, Helmut (Hg.): Environmental Policy in Japan, Berlin, S. $499 \mathrm{ff}$.

${ }^{7}$ Richardson, Jeremy (1982): Convergent Policy Styles in Europe?, In: Richardson, Jeremy, et al. (Hg.): Policy Styles in Western Europe, Boston und Sydney, S. 197. 
which are usually used to formulate and implement public policies ". ${ }^{\circ}$ Durch diese Definition werden die Politikinhalte, die Policies, zur abhängigen, vom Politikstil determinierten Größe erklärt. Ähnlich formuliert es Freeman in seiner zusammenfassenden Darstellung einiger komparatistischer Analysen der „national decisionmaking styles": ,[...] historical, cultural, or structural characteristics of nations [...] are thought to have caused the differences in policy, which is then treated as a dependent variable ". ${ }^{10}$

Diese definitorischen Überlegungen werden in Kapitel 4 der vorliegenden Studie noch weiter vertieft, jedoch ist bereits an dieser Stelle festzuhalten, daß Policy Styles die nationalen Policies beeinflussen, und daß ein vergleichender politikwissenschaftlicher Ansatz dieses Konzept somit in seine Theorie des Policymaking einbeziehen muß: "If national styles are a cause of policy differences in their own right, they have to be taken in account by those who seek to develop models or theories of comparative policymaking. "11

Auch wenn einige Autoren hinsichtlich der tatsächlichen Ausprägung nationaler Politikstile in Westeuropa eine gewisse Konvergenz zu erkennen glauben ${ }^{12}$, scheint es dennoch ohne größere Probleme möglich zu sein, unterschiedliche nationale Politikstile zu identifizieren und anhand eindeutiger Merkmale zu beschreiben ${ }^{13}$. Diese einzelnen nationalen Policy Styles lassen sich dann im Rahmen einer Typologie, welche dem Kriterium der Prakti-

\footnotetext{
${ }^{8}$ Ebenda.
}

9 Zur Definition des Policy-Begriffes vgl. grundlegend: Jann, Werner (1987): Policy, In: Nohlen, Dieter, und Schultze, Rainer-Olaf (Hg.): Politikwissenschaft. Theorien - Methoden Begriffe, München, S. 702.

${ }^{10}$ Freeman, Gary P. (1985): National Styles and Policy Sectors: Explaining Structured Variation, in: Journal of Public Policy, 5, S. 470.

${ }^{11}$ Ebenda, S. 472.

12 Vgl. Hartmann, Jürgen (1995): Vergleichende Politikwissenschaft, Frankfurt/Main und New York, S. 101; Richardson, Jeremy, et al. (1982) (Hg.): Policy Styles in Western Europe, Boston und Sydney; Eine konvergente Einwicklung der politischen Kultur westlicher Demokratien hin zu einer sogenannten civic culture beschreiben: Almond, Gabriel A., und Verba, Sidney (1963): The Civic Culture, Boston. Die Begriffe Politische Kultur und Politikstil werden in Kapitel 4 des ersten Teils dieser Untersuchung noch ausfuhrlich voneinander abgegrenzt.

${ }^{13}$ Vgl. Hartmann, Jürgen (1995): Vergleichende . . . , a.a.O., S. 101 ff.; Freeman, Gary P. (1985): National Styles ..., a.a.O., S. 475 f. 
kabilität entspricht und ausreichend flexibel für komparatistische Zwecke ist, zu einigen wenigen Grundtypen zusammenfassen ${ }^{14}$.

$\mathrm{Da}$ also das Politikstil-Konzept ein brauchbares Instrument zur komparatistischen Analyse und Erklärung unterschiedlicher nationaler Policies darstellt, und außerdem ein Effekt des jeweiligen Policy Styles auf die Inhalte und Resultate nationaler Umweltpolitik anzunehmen ist, wird in der vorliegenden Untersuchung der Frage nachgegangen, ob der nationale Politikstil eines jeweiligen Staates eine wichtige Determinante für die Resultate seiner umweltpolitischen Anstrengungen ist. Oder anders formuliert: Ist die kulturell und historisch geprägte Art und Weise, in welcher die politischen Eliten eines Landes politisch relevante Entscheidungen treffen und diese umsetzen, für die Umweltqualität in eben diesem Staat von signifikanter Bedeutung? Oder geht dieser Faktor in einer Vielzahl von weitaus wichtigeren, vor allem ökonomischen und politischen Determinanten unter?

Es gibt zahlreiche, auch empirisch fundierte Studien, welche z.B. den $\mathrm{Zu}$ sammenhang von ökonomischem Entwicklungsstand oder struktureller Verteilung der Wirtschaftstätigkeit einerseits und dem Grad der Umweltbelastung andererseits belegen ${ }^{15}$. Ebenfalls bereits häufig thematisiert wurde der

${ }^{14}$ Vgl. Richardson, Jeremy; Gustafsson, Gunnel, und Jordan, Grant (1982): The Concept of Policy Style, In: Richardson, Jeremy, et al. (Hg.): Policy Styles in Western Europe, Boston und Sydney, S. $10 \mathrm{ff}$.

${ }^{15}$ Vgl. Knoepfel, Peter, und Weidner, Helmut (1985): Luftreinhaltepolitik (stationäre Quellen) im internationalen Vergleich, Berlin; Jänicke, Martin, und Mönch, Harald (1988): Ökologischer und wirtschaftlicher Wandel im Industrieländervergleich, in: Schmidt, Manfred G. (Hg.): Staatstätigkeit. International und historisch vergleichende Analysen, Politische Vierteljahresschrift, Sonderheft 19, Opladen, S. 391 ff.; Ringquist , Evan J. (1993): Environmental Protection at the State Level. Politics and Progress in Controlling Pollution, Armonk, London; Jänicke, Martin; Mönch, Harald; Binder, Manfred, et al. (1993): Umweltentlastung durch industriellen Strukturwandel? Eine explorative Studie über 32 Industrieländer (1970 bis 1990), 2. Aufl., Berlin.

Für die Luftreinhaltungspolitik verschiedener europäischer Staaten attestieren Knoepfel und Weidner den ökonomischen Variablen einen größeren Effekt als den unterschiedlichen Implementations-Regimen.

Jänicke und Mönch stellen fest, daß Veränderungen diverser Umweltqualitätsindikatoren primär vom wirtschaftlichen Wohlstand eines Staates abhängen.

Auch Ringquist bezeichnet als Resultat eines intranationalen Vergleiches des Erfolges der Luft- und Gewässerreinhaltungspolitiken der 50 Bundesstaaten der USA die ökonomische Stärke als signifikantesten Erklärungsfaktor.

Die großangelegte Vergleichsstudie Jänickes et al. stellt für verschiedene ökologische Belastungsfaktoren eine unterschiedliche Entwicklung in Abhängigkeit von der industriellen Wertschöpfung fest. 
Zusammenhang zwischen politischen Faktoren, etwa Entstehen und Erfolg "grüner" Parteien ${ }^{16}$ oder Spezifika der Staatsorganisation ${ }^{17}$, sowie der Ausprägung bestimmter Umweltindikatoren.

Alle diese Studien sind jedoch trotz ihrer z.T. hohen inhaltlichen und methodischen Qualität mit Mängeln behaftet, denn sie messen und analysieren entweder nur den zeitlichen Ablauf der Entwicklung der politischen bzw. ökonomischen Faktoren und der durch diese beinflußten Indikatoren zur Bestimmung der Umweltqualität ${ }^{18}$ oder die konkreten regionalen oder nationalen Auswirkungen einzelner politischer Maßnahmen ${ }^{19}$.

Im ersten Fall kommen sie zu Aussagen wie: Mit dem Ansteigen des ProKopf-Einkommens und dem Fortschreiten des Strukturwandels von der gewerblichen Wirtschaft hin zum Dienstleistungsbereich kann für die entwikkelten Länder ein Sinken der Umweltbelastung festgestellt werden. Oder: Staaten mit einer landwirtschaftlich geprägten Wirtschaft erhöhen mit dem Grad ihrer Industrialisierung zunächst auch die absolute und Pro-KopfUmweltbelastung.

In den meisten dieser Studien wird also die Gesamtheit der untersuchten Staaten nicht weiter differenziert, sondern die entdeckten, allgemeinen $\mathrm{Zu}$ sammenhänge werden auf alle Staaten übertragen. Man unterstellt somit, daß der Grad der Umweltbelastung eines Staates einem bestimmten, idealtypischen zeitlichen Muster folgt, jeweils abhängig vom ökonomischen ${ }^{20}$ Entwicklungsstand. Ausgehend von diesem Ansatz sind dann Unterschiede im Ausprägungsgrad von Umweltqualitätsindikatoren zwischen einzelnen Staaten primär nicht durch unterschiedliche Kulturen, Wertesysteme und nationale Spezifika der Entscheidungsfindung zu erklären, sondern lediglich durch einen aufholbaren Vorsprung bzw. Rückstand auf der ökonomischpolitischen Zeitachse.

${ }^{16}$ Vgl. Jănicke, Martin (1990): Erfolgsbedingungen . . ., a.a.O., S. 215.

17 Vgl. vor allem die diversen Literaturhinweise bei Kem, Kristine, und Bratzel, Stefan (1994): Erfolgskriterien . ., a.a.O., S. 59 ff.

${ }^{18}$ Siehe Jănicke, Martin; Mönch, Harald; Binder, Manfred, et al. (1993): Umweltentlastung . . . , a.a.O.

${ }^{19}$ Siehe Ringquist, Evan J. (1993): Environmental Protection . . . , a.a.O.; Knoepfel, Peter, und Weidner, Helmut (1985): Luftreinhaltepolitik . . , a.a.O.

${ }^{20}$ Vgl. Jänicke, Martin, und Mönch, Harald (1988): Ökologischer . . . , a.a.O., S. 391. 
Die zweite Kategorie von Untersuchungen - hier handelt es sich vornehmlich um (bi-)nationale Ländervergleiche oder Einzelfallstudien - kommt als Fazit ihrer Analyse meist zu Schlußfolgerungen dieser Art: Die im Jahre X durch den Staat $Y$ beschlossenen Richtlinien zur Luft-reinhaltung konnten aufgrund institutionell günstiger Implementationsbedingungen schon nach 2 Jahren eine signifikante Reduktion der „Treibhausgas"-Emissionen bewir$\mathrm{ken}^{21}$.

Diese Aussagen sind dann meist von einer sehr hohen Validität, weisen jedoch einen eher geringen Theoriegehalt auf.

In dieser Studie soll deshalb anders gefragt werden. Wie kann es sein, daß Staaten mit einem ähnlich hohen ökonomischen Entwicklungsstand und einer nahezu parallel verlaufenden Verschiebung der sektoralen Wirtschaftsanteile zu völlig unterschiedlichen Ergebnissen ihrer Umweltpolitik gelangen $^{22}$ ? Oder: Wieso geht der parlamentarische Erfolg ökologisch orientierter Parteien in einigen Staaten einher mit einer deutlichen Verbesserung der Umweltqualität, während dieser Effekt in anderen Ländern nicht in diesem Ausmaß feststellbar ist ${ }^{23}$ ?

${ }^{21}$ Als Beispiele für den Zusammenhang von Luftreinhaltepolitk und politisch-institutionellen Arrangements vgl. Lundquist, Lennart J. (1980): The Hare and the Tortoise: Clean Air Policies in the United States and Sweden, Ann Arbor; Ringquist, Evan, J. (1993): Environmental Protection . . , a.a.O.; Knoepfel, Peter und Weidner, Helmut (1985): Luftreinhaltepolitik . .. , a.a.O.

${ }^{22}$ Als Beispiel sind die Daten von Kanada und Schweden anzuführen. Beide Staaten halbierten im Zeitraum 1974-90 den Agraranteil an der strukturellen Zusammensetzung des BIP (CAN: $4.6 \%$ in 1974 bzw. $2.4 \%$ in 1990; SWE: 4.5 bzw. $2.6 \%$ ), haben einen ähnlich verlaufenden stetigen Rückgang des sekundären Sektors zu verzeichnen (CAN: 33.1 bzw. 28.9\%; SWE: 37.6 bzw. 29.6\%) und steigerten den ohnehin hohen Anteil des Dienstleistungssektors noch weiter (CAN: 62.3 bzw. 67.3\%; SWE: 57.9 bzw. 67.9\%). Die sich damit für 1990 ergebende strukturelle Aufteilung des BIP ist nahezu identisch. Dennoch sind die Umweltbelastungwerte beider Staaten völlig unterschiedlich. Während Kanada 1990 für einen zahlreiche Indikatoren erfassenden Index gemessen an der Einwohnerzahl den zweithöchsten und gemessen an der Höhe des Bruttoinlandsproduktes den vierthöchsten Wert unter allen OECDStaaten aufweist (siehe Tabellen A21 und A39), zählt Schweden zu den umweltpolitischen Erfolgsfällen (Rangplätze 14 und 16; siehe ebenda).

23

So zählt zum Beispiel Müller-Rommel Finnland und Irland $\mathrm{zu}$ den Staaten mit „erfolgreichen“ grünen Parteien (Ders. (1992): Erfolgsbedingungen Grüner Parteien in Westeuropa, in: Politische Vierteljahresschrift, 2, S. 189-218.). Dennoch weisen beide Staaten OECD-weit überdurchschnittlich hohe Umweltbelastungswerte auf (siehe Tabellen A21 und A39; FIN: Rangplätze 4 und 8, IRL: Rangplätze: 6 und 5), während Schweden (siehe FN 22), Österreich (Rangplätze 19 und 21) und die Schweiz (jeweils der beste Rangplatz: 22), zu de- 


\section{Abb. 1: Modell zur Erklärung des Einflusses des nationalen Politikstils auf die Effektivität von Umweltpolitik}

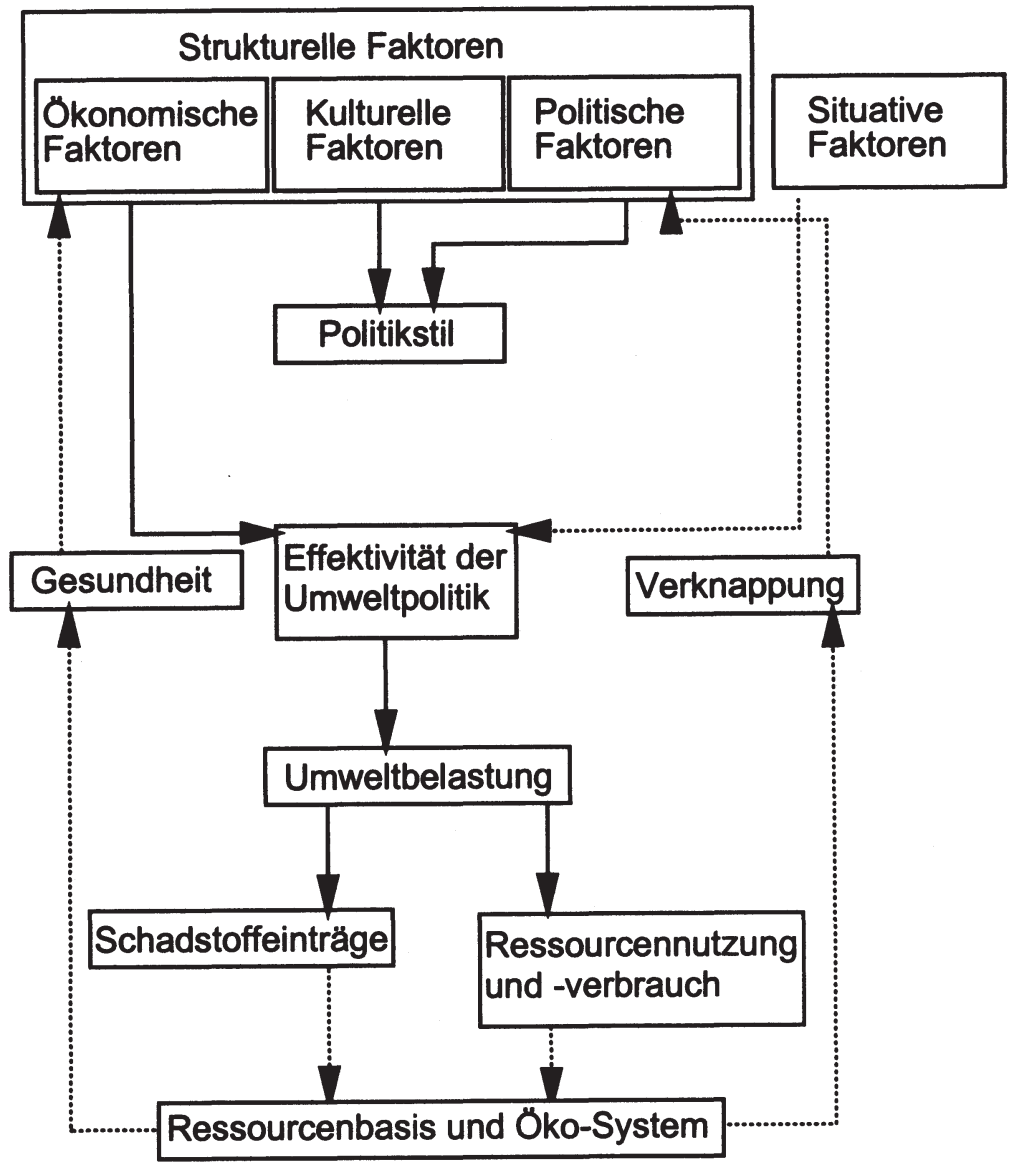

Quelle: Eigene Darstellung in Anlehnung an Bossel (1990, S. 8 ff.) sowie Kern und Bratzel (1994).

Bemerkung: Die mit durchgezogenen Linien dargestellten Beziehungen werden im Rahmen dieser Untersuchung thematisiert.

ren Parteienspektrum ebenfalls erfolgreiche grüne Parteien gehören, umweltpolitisch ungleich effektiver sind. 
Es geht also zum einen um eine möglichst breite Datenbasis, durch welche das Erklärungspotential der gefundenen Zusammenhänge erhöht werden soll, und zum anderen um die Aufdeckung unterschiedlicher, von der Position auf der ökonomischen und politischen Zeitachse unabhängiger ökologischer Belastungsmuster verschiedener Staaten und Staatengruppen.

Ein wichtiges Mittel zur Beantwortung dieser Fragen wird es also sein, nicht nur die zeitliche Entwicklung der Umweltbelastungsindikatoren $\mathrm{zu}$ verfolgen, sondern auch die relative Ausprägung dieser Variablen, d.h. es ist beispielsweise $\mathrm{zu}$ untersuchen, warum diese Indikatoren in den USA zwar in den vergangenen 15 Jahren $z . T$. deutlich zurückgegangen sind ${ }^{24}$ (als Folge des strukturellen und technologischen Wandels sowie der Umweltgesetzgebung), jedoch noch immer sowohl absolut als auch pro Kopf und pro BIPEinheit weit über den Werten Japans oder Deutschlands liegen ${ }^{25}$. Hier vermutet die vorliegende Studie ein hohes Erklärungspotential des Faktors Politikstil.

In Abbildung 1 ist der Grundgedanke dieser Untersuchung schaubildhaft dargestellt. Von den zahlreichen strukturellen und situativen Faktoren, welche auf die Umweltpolitik und deren Outcomes, d.h. die tatsächlichen, intendierten und nicht-intendierten Wirkungen staatlichen Umwelthandelns, einwirken, wird der nationale Politikstil als Ausfluß kultureller und politischer Determinanten isoliert und in seiner Wirkung auf die Politikergebnisse im Bereich der Ökologie analysiert.

Am Ende dieser Untersuchung soll dann eine Aussage darüber stehen, ob es hinreichende empirische Evidenz für diese Vermutung gibt oder nicht. Wie vorausgesetzt, der Politikstil erweist sich tatsächlich als in diesem Zusammenhang relevant - diese Erkenntnis zum Zwecke einer Beeinflussung der umweltpolitischen Aktivitäten der Industrieländer praktisch zu nutzen wäre, wird nicht weiter thematisiert.

\subsection{Aufbau der Arbeit}

Die vorliegende Untersuchung gliedert sich in einen theoretischen und in einen empirischen Teil.

Während sich der erste Teil mit begrifflichen Klärungen, der Einordnung der Arbeit in den umweltökonomischen und politikwissenschaftlichen Kontext

${ }^{24}$ Vgl. Tabellen A22 bis A30 sowie A40 bis A48.

${ }^{25}$ Vgl. Tabellen A19, A20, A21, A37, A38 und A39. 
sowie dem Entwurf eines Erklärungsmodells beschäftigt, erfolgt im analog gegliederten zweiten Teil die empirisch-quantitative Überprüfung der aufgestellten Hypothesen mittels bi- und multivariater Analyseverfahren.

Im einzelnen ist die Arbeit wie folgt gegliedert:

\begin{tabular}{|l|l|}
\hline Theorie (Teil I) & Empirie (Teil II) \\
\hline Problemstellung (Kap. 1) & $\begin{array}{l}\text { Vorhandene empirische Untersu- } \\
\text { chungen (Kap. 6) }\end{array}$ \\
\hline $\begin{array}{l}\text { Erfolge nationaler Umweltpolitik } \\
\text { (Kap. 2) }\end{array}$ & $\begin{array}{l}\text { Messung der Effektivität nationa- } \\
\text { ler Unweltpolitik (Kap. 8) }\end{array}$ \\
\hline $\begin{array}{l}\text { Derterminanten der Effektivität } \\
\text { nationaler Umweltpolitik (Kap. 3) }\end{array}$ & $\begin{array}{l}\text { Derterminanten der Effektivität } \\
\text { nationaler Umweltpolitik (Kap. 9) }\end{array}$ \\
\hline $\begin{array}{l}\text { Das Politikstil-Konzept im Kon- } \\
\text { text der Policy-Analyse (Kap. 4) }\end{array}$ & $\begin{array}{l}\text { Messung des nationalen Poli- } \\
\text { tikstils (Kap. 10) }\end{array}$ \\
\hline $\begin{array}{l}\text { Politikstil als Determinante der } \\
\text { Effektivität nationaler Umwelt- } \\
\text { politik (Kap. 5) }\end{array}$ & $\begin{array}{l}\text { Politikstil als Determinante der } \\
\text { Effektivität nationaler Umwelt- } \\
\text { politik (Kap. 11) }\end{array}$ \\
\hline
\end{tabular}

Im Anschluß an dieses einleitende Kapitel wird zunächst die abhängige Variable, nämlich der Erfolg nationaler Umweltpolitik thematisiert. Dabei ist insbesondere zu klären, ob das Anstellen mikroökonomischer Überlegungen im Sinne einer gesamtstaatlichen ökologischen Nettonutzenmaximierung unter Berücksichtigung individueller Zahlungsbereitschaften eine geeignete Methode zur Messung umweltpolitischen Erfolges ist, oder ob statt Effizienz- und Optimalitätsgesichtspunkten das weniger anspruchsvolle Kriterium der Effektivität für die Zwecke dieser Untersuchung praktikabler ist. Da durch die exakte Beschreibung des Erfolgskriteriums der Gehalt der im Laufe der Überlegungen gewonnenen Erkenntnisse entscheidend bestimmt wird, sind außer Plausibilitätsüberlegungen also auch solche über die Eignung des Kriteriums zu einer quantitativen Operationalisierung bedeutsam.

Ein weiteres mögliches Kriterium des Erfolges von Umweltpolitik ist die Verteilungsgerechtigkeit (Equity), also das jeweilige $\mathrm{Maß}$, in welchem sich Umweltbelastungen innerhalb eines Territoriums regional und personal unterscheiden. Dabei sind zwei wichtige Aspekte zu unterscheiden: 
1. Sind die durch die Umweltpolitik veranlaßten Veränderungen von Umweltnutzen (bzw. -schäden) über alle Einkommensklassen sowie auf die regionalen Einheiten gerecht verteilt?

2. Ist die Veränderung der monetären Verteilung innerhalb eines Staates durch die Kosten umweltpolitischer Maßnahmen insofern gerecht, als daß sich die Kostenbelastungen der einzelnen Wirtschaftssubjekte (Konsumenten und Unternehmen) am Maß der durch sie im Rahmen des Produktions- und Konsumtionsprozesses verursachten Umweltschäden orientieren?

Nach der Auswahl des Kriteriums Effektivität als Zielgröße für diese Untersuchung und dessen Präzisierung erfolgen Überlegungen bezüglich der $\mathrm{Zu}-$ rechenbarkeitsproblematik, da grenzüberschreitende Wirkungen und Gratiseffekte, d.h. ursprünglich nicht intendierte positive Effekte einer Maßnahme oder Entwicklung auf die Umwelt, zu einer Veränderung der Umweltlast innerhalb eines staatlichen Territoriums beitragen können.

So führt der grenzüberschreitende Import von Schäden zu einem Anstieg der inländischen Umweltbelastungen ${ }^{26}$, während andererseits der Import von Nutzen einen Gratiseffekt im Sinne einer sinkenden Umweltbelastung bedeutet. Es sind auch inländische Gratiseffekte möglich, z.B. durch einen Nachfragerückgang (die dann gedrosselte Produktion hat geringere Emissionen zur Folge [siehe beispielsweise den Zusammenbruch der Industrieproduktion in der ehemaligen DDR]), eine technologische Innovation oder eine Änderung der volkswirtschaftlichen Struktur.

Da neben der in dieser Untersuchung angenommenen Bedeutung des Faktors Politikstil auch zahlreiche andere Variablen für die Umweltbelastung eines Staates verantwortlich sind, also im empirischen Teil zur Vermeidung von Kollinearitäten eine weitgehende Kontrolle dieser anderen Determinanten von besonderer Bedeutung ist, werden diese in Kapitel 3 ausführlich behandelt.

Dabei ist zunächst zwischen strukturellen und situativen Faktoren zu unterscheiden. Während es sich bei letzteren um nicht-vorhersehbare Ereignisse wie Katastrophen, Embargos oder singuläre Geschehnisse wie z.B. den politischen und ökonomischen Zusammenbruch der ehemaligen DDR handelt, sind mit dem Begriff der strukturellen Faktoren vor allem solche Determinanten gemeint, welche den langfristigen politischen, sozio-ökonomischen

${ }^{26}$ So sind z.B. die durch atmosphärische Transporte von Großbritannien nach Deutschland importierten Mengen an oxidierten Schwefel- und Stickstoffverbindungen etwa um ein Vierfaches größer als die von Deutschland nach Großbritannien exportierten Mengen (vgl. Umweltbundesamt (1995): Umweltdaten Deutschland 1995, Berlin, S. 22). 
und historisch-kulturellen Hintergrund des jeweiligen nationalen umweltpolitischen Handelns bilden. So ist etwa anzunehmen, daß die Staatsorganisation, die makroökonomische Entwicklung oder das gesellschaftliche Wertesystem eines Landes den Erfolg seiner umweltpolitischen Bemühungen signifikant beeinflussen.

In Kapitel 4 wird dann schließlich die unabhängige Variable dieser Studie, also der nationale Politikstil thematisiert.

Zunächst erfolgt eine kontextuale Einordnung des Policy-Style-Begriffes in das übergreifende politikwissenschaftliche Konzept der Policy-Analyse als einer Methode des internationalen Vergleiches der Art und Weise staatlicher Problemlösungsaktivitäten. In diesem Zusammenhang ist es auch notwendig, den Policy-Begriff, der primär auf die inhaltliche Dimension der Politik abhebt, von den verwandten Begriffen der Politics und der Polities, welche für die prozessualen und institutionellen Aspekte staatlichen Handelns stehen, definitorisch abzugrenzen.

Für das Verständnis des politischen Entscheidungsprozesses, also der Politikformulierung und der Implementation, sind die Beziehungen der am Problemlösungsprozeß Beteiligten von besonderer Wichtigkeit. Die konkrete Ausgestaltung dieser Interaktionen wird im Konzept der Policy Networks beschrieben.

Wichtige Instrumente der Policy-Analyse sind außerdem das Input-OutputModell der Politik sowie das darin eingebettete Policy-Cycle-Konzept. Danach wirken zahlreiche sozioökonomische, politische und kulturelle Faktoren, zu denen im Rahmen dieser Studie natürlich auch der Politikstil zählt, als Input auf das Policymaking-System, in welchem die Entscheidungen getroffen und implementiert werden, ein. Dieser Prozeß der Generierung einer politischen Entscheidung wiederum läßt sich am besten in Form eines Politikzyklus beschreiben, innerhalb dessen ein politische Problem die Phasen der Problemdefinition, des Gelangens auf die politische Agenda, der Formulierung und schließlich der Implementation in Form von konkreten Programmen (Policies) durchläuft. Diese sind der politische Output, welcher im Idealfall als einer weiteren Phase des Policy Cycles eine Evaluation durchläuft, die dann zu einer Bestätigung der Policy, deren Neuformulierung oder Terminierung führen kann.

Nachdem auf diese Weise der theoretische Kontext beschrieben ist, in welchen das Politikstil-Konzept eingebettet ist, wird dieses im weiteren Verlauf des Kapitels dann ausführlich behandelt.

Der nationale Politikstil wird dabei als die länderspezifische Art und Weise der Politikformulierung und -implementation durch die staatlichen Entscheidungsträger bezeichnet. 
Neben einer eindeutigen Definition des Policy-Style-Begriffes ist auch eine Abgrenzung vom verwandten Ansatz Politischer Kultur notwendig. Der Kulturbegriff kann danach als zeitlich stabile Hintergrundvariable gelten, welche die Einstellungen und Werte der handelnden administrativen Eliten umfaßt, und somit auch den Politikstil als der länderspezifischen Art und Weise, in der diese Eliten den politischen Prozeß gestalten, beeinflußt.

Kapitel 4 schließt mit einer Darstellung von drei Politikstil-Konzepten, welche anhand bestimmter Merkmalsdimensionen jeweils eine Typologie staatlichen Handelns entwerfen.

Im dem den theoretischen Teil beschließenden fünften Kapitel geht es um den Versuch, ein Modell für die Bedeutung des Politikstils als Determinante der Effektivität nationaler Umweltpolitik zu entwerfen, d.h. nach der bis dahin isolierten Betrachtung von abhängiger und unabhängiger Variable wird nun der (angenommene) kausale Zusammenhang zwischen beiden GröBen analysiert.

Dazu ist zunächst die Frage nach den für den Erfolg von Umweltpolitik relevanten Merkmalsdimensionen des Politikstils zu beantworten, wobei von den besonderen Eigenschaften der im umweltpolitischen Politikfeld auftretenden Probleme und zu treffenden Maßnahmen auszugehen ist; diese sind Wirkungstiefe, Irreversibilität sowie Komplexität. Außerdem handelt es sich bei Fragestellungen der Umweltpolitik häufig um besonders kontroverse und emotional belastete Themen. Vor diesem Hintergrund erscheinen die Merkmalsdimensionen der Strategieorientierung sowie der Partizipationsgrad der von umweltpolitischen Entscheidungen Betroffenen als besonders relevant. Eine strategisch ausgerichtete Umweltpolitik, die außerdem die wichtigen nicht-staatlichen Akteure in den politischen Prozeß einbezieht, verspricht den positivsten Effekt im Sinne einer ökologischen Schadensminderung.

Nach der Bestimmung der zwei grundlegenden Dimensionen des Politikstils ist dann eine Typologisierung möglich. Dabei werden durch Kombination der beiden relevanten Dimensionen vier unterschiedliche UmweltpolitikIdealtypen dargestellt.

Der dann folgende Schritt ist der Entscheidende: anhand theoretischer Überlegungen wird der Einfluß der vier unterschiedlichen Politiktypen auf die Umweltbelastung der den jeweiligen Politikstil praktizierenden Staaten prognostiziert. Dabei ergibt sich ein idealtypischer Politikstil, nämlich der planerische Korporatismus, der unter der Vorausssetzung der Konstanz aller anderen Faktoren den positivsten Effekt auf die durch die Volkswirtschaft eines Staates verursachten Umweltbelastungen hat. Dieser Politikstil vereint sowohl eine hohe Strategiefähigkeit durch die Antizipation von künftigen Entwicklungen und die Inrechnungstellung der langfristigen Folgen des ei- 
genen Handelns als auch ein überdurchschnittliches Partizipationsmaß der durch die umweltpolitischen Maßnahmen Betroffenen.

Diese zentrale These der vorliegenden Untersuchung wird im zweiten Teil der Studie anhand eines empirischen Industrieländervergleiches überprüft. Im ersten Kapitel werden die bereits vorhandene empirische Untersuchungen über die Bedeutung des Politikstils für den Erfolg von Umweltpolitik detaillierter vorgestellt. Diese unterscheiden die Staaten meist anhand eines eindimensionalen Kontinuums in solche mit eher kosensualem bzw. konfliktärem Politikstil und attestieren, daß eine konsensorientierte Politik zu den besseren umweltpolitischen Resultaten führt. Neben zahlreicher wichtiger Erkenntnisse, welche diese Arbeiten liefern, weisen sie jedoch die bereits erwähnten Defizite mangelnder Stichprobengröße und Quantifizierung der behandelten Variablen auf.

Für die eigene Untersuchung ist zunächst die Fallzahl festzulegen, wobei zwischen den konkurrierenden Zielen möglichst großer Reichweite sowie methodischer Verläßlichkeit abzuwägen ist. Grundgesamtheit der vorliegenden Studie sind deshalb die OECD-Staaten, welche zum einen eine ökonomisch und politisch relativ homogene Gruppe bilden, und zum anderen eine große Fülle an Datenmaterial bieten. Nach der Festlegung der Fallzahl gilt es, die Problematik der Operationalisierung des Erfolgskriteriums, also der relativen Effektivität nationaler Umweltpolitik zu lösen. Zu diesem Zweck werden zahlreiche Einzelindikatoren zur Messung der Emisssionen und des Ressourcenverbrauches nationaler Volkswirtschaften genutzt, auf die Einwohnerzahl und das Bruttoinlandsprodukt des jeweiligen Staates bezogen und zu Indexgrößen aggregiert (Kapitel 7).

Kapitel 8 ist der Darstellung der so gemessenen umweltpolitischen Performance der untersuchten Staaten gewidmet.

In Kapitel 9 geht es dann um die ökonomischen und politischen Determinanten, von denen ein besonders signifikanter Einfluß auf die Effektivität der Umweltpolitik angenommen werden kann. Diesen gilt es zu messen und im weiteren Verlauf der Untersuchung zu kontrollieren. Die zu diesem Zweck in ihrer Wirkung auf die ökologischen Indikatoren analysierten Faktoren sind das ökonomische Niveau, die sektorale Aufteilung der Volkswirtschaft sowie der elektorale Erfolg grüner Parteien. Dabei wird vermutet, daß sich ein hohes Wohlstandsniveau, ein eher hoher Anteil des Dienstleistungssektors an der Wirtschaftsstruktur sowie Wahlerfolge und parlamentarische Präsenz grüner Parteien positiv auf die umweltpolitische Effektivität von Staaten auswirken. Während die Thesen hinsichtlich der sektoralen Aufteilung der Volkswirtschaft sowie des elektoralen Erfolgs grüner Parteien durch die statistische Prüfung nicht gestützt werden, ist im Hinblick auf das ökonomische Niveau ein deutlicher Effekt meßbar. Während die Umweltbelastung 
mit der Wirtschaftsleistung zunächst anwächst, ist ab einem bestimmten ökonomischen Niveau eine Umkehrung des Kausalzusammenhanges feststellbar, d.h. je höher das Bruttoinlandsprodukt, desto geringer sind die Werte für die Indikatoren zur Messung des Ressourcenverbrauchs sowie der Schadstoffemissionen.

Nach der Behandlung der abhängigen Variable und möglicher Determinanten derselben wird dann in Kapitel 10 die Operationalisierung der erklärenden Variable vorgenommen. Als Indikatoren für ein hohes Maß an Strategieorientierung sind dabei die technologisch-administrativen Kapazitäten sowie der (De-)Zentralisierungsgrad eines Staates wichtig.

Während die Bedeutung ausreichender technologischer und administrativer Ressourcen für die Konzeption und Durchführung einer langfristig orientierten Politik unmittelbar einleuchtet, ist dieser Zusammenhang für den Grad, in welchem die Staatsorganisation in zentralen oder dezentralen Mustern erfolgt, nicht so klar. Ein eher dezentraler, föderalistischer Staatsaufbau wird deshalb als Einzelindikator für die Strategieorientierung eines Staates angenommen, da zum einen nur in diesem Fall eine ausreichende regionale Implementations- und Überwachungsbürokratie für umweltpolitisch verfügte Maßnahmen vorhanden ist, welche sogar über die zentral verfügten Standards hinausgehen kann, und zum anderen die subnationalen Einheiten als „Frühwarnsysteme“ fungieren können, welche krisen- oder chancenhafte Entwicklungen rechtzeitig weitergeben und somit vorausschauendes Handeln der Zentralmacht ermöglichen.

Zur Messung des Partizipationsgrades wird u.a. auf die in den zahlreichen empirischen Studien zum Thema Korporatismus - einer wichtigen Ausformung innerstaatlicher Kooperationsbereitschaft - verwendeten Indikatoren zurückgegriffen. Außerdem werden die Staaten hinsichtlich ihres Konfliktlösungsverhaltens in solche mit eher konsensualem bzw. eher konfliktärem Problemlösungsverhalten unterschieden.

Anhand der so gewonnenen Meßergebnisse werden die Staaten der untersuchten Grundgesamtheit in einem zweidimensionalen, durch die Merkmalsdimensionen Strategieorientierung und Patizipationsgrad aufgespannten Koordinatensystem positioniert und mittels der multivariaten StatistikMethode der Clusteranalyse zu aufgrund ihres Datenmusters homogenen Gruppen zusammengefaßt.

Im letzten Kapitel des zweiten Teiles geht es dann um den eigentlichen Falsifizierungsversuch ${ }^{27}$ der Ausgangsthese. Zunächst werden beide PolitikstilDimensionen einer einfachen sowie einer multiplen Regeressionsanalyse mit

${ }^{27}$ Vgl. Popper, K. R. (1976): Logik der Forschung, 6. Aufl.,Tübingen, S. 8. 
den Effektivitätsindikatoren unterzogen, anschließend erfolgt der varianzanalytische Versuch, die in der Clusteranalyse anhand ihres Politikstils generierten Ländergruppen auch anhand ihrer Umweltbelastungsdaten im statistisch signifikanten Sinne zu trennen. Sollte dies gelingen, so wäre der erste Baustein einer Theorie zur Bedeutung des nationalen Politikstils als Determinante einer effektiven Umweltpolitik gelegt, und die Lücke im Bereich der empirischen Studien auf dem Feld der Umweltpolitik ${ }^{28}$ etwas verkleinert. Teil III faßt dann noch einmal die wichtigsten Erkenntnisse dieser Untersuchung zusammen und zieht einige politisch-strategische Schlüsse - in aller Vorsicht hinsichtlich der Grenzen, die jede theoretische und empirische Analyse dieser Art auferlegt.

\subsection{Theoretischer und inhaltlicher Standort der Arbeit}

Um dem Leser schon eingangs eine Einordnung dieser Arbeit zu erleichtern, sollen nachfolgend kurz der wissenschaftstheoretische Kontext sowie der inhaltliche und methodische Standort der vorliegenden Studie dargelegt werden. Da Aussagen zur Umweltpolitik - insbesondere im Zusammenhang mit dem Erfolg oder der Effektivität selbiger - in der Perzeption durch unterschiedliche gesellschaftliche Gruppen durch interessenbezogene Werturteile und nichteinheitliche, teilweise ideologisierte Sprachregelungen überlagert werden, wird im folgenden zunächst das Verständnis des Verfassers im Hinblick auf den Entdeckungs-, Begründungs- und Verwendungszusammenhang der Arbeit erläutert.

\subsubsection{Entdeckungszusammenhang}

In dieser Studie steht der Zusammenhang von Politikstil und der Effektivität nationaler Umweltpolitik im Zentrum des Interesses. Die Beschränkung auf diese beiden Faktoren als unabhängige bzw. abhängige Variablen bedeutet eine Ausblendung der Problematik der Politikinhalte. Von besonderer Bedeutung ist also die Frage (siehe dazu Abbildung 2): Wie muß die Policy eines Staates beschaffen sein, damit die Outcomes seiner Politik ökologisch effektiv sind?, und nicht: was soll der jeweilige administrative Output der

\footnotetext{
${ }^{28}$ Kern, Kristine, und Bratzel, Stefan (1994): Erfolgskriterien . . , , a.a.O., S. 58.
} 
Politik sein? An einem Beispiel erläutert bedeutet diese Sichtweise: wir fragen nicht nach dem Zusammenhang zwischen dem Erlaß einer staatlichen Luftreinhaltungsverordnung (Output) und der Entwicklung der durch die betroffene Volkswirtschaft verursachten Emissionen (Outcome), sondern danach, ob ein autoritärer oder ein partizipativer Stil (Policy Style) in der Umweltpolitik zu eher niedrigen Werten der Umweltbelastungsindikatoren (Outcome) führt ${ }^{29}$.

\section{Abb. 2: Input-Output-Modell des politischen Prozesses}

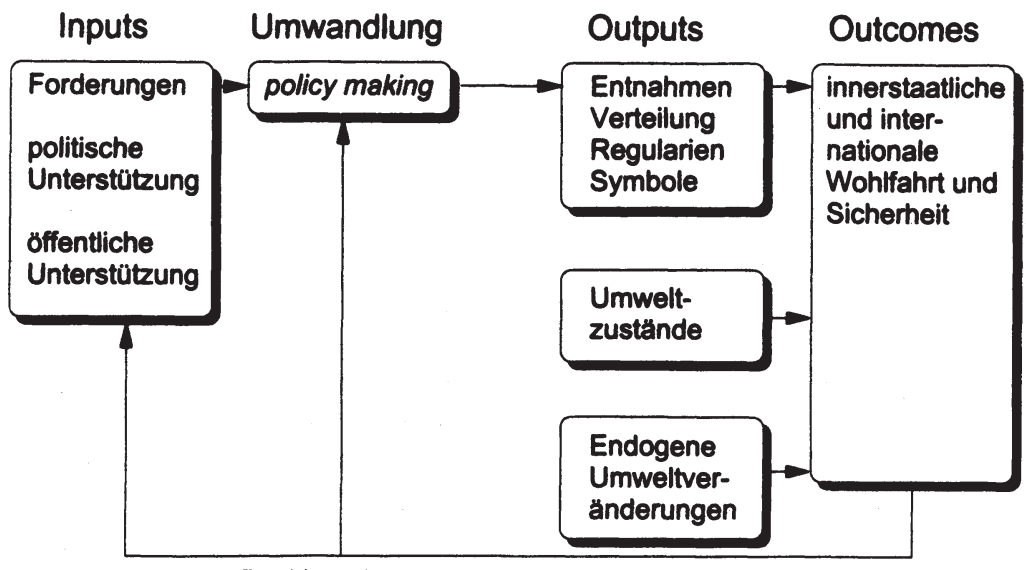

Rückkopplungsprozesse

Quelle: Almond und Powell (1992, S.10); Eigene Übersetzung.

Natürlich sind letztlich auch die Outputs verantwortlich für die Qualität der umweltpolitischen Outcomes, und Staaten, die zu den ökologischen „Erfolgsfällen“ zählen, dürften neben einem ähnlichen Politikstil auch ähnliche umweltpolitische Instrumente nutzen, doch aus methodischen Gründen und auch solchen der thematischen Beschränkung bleiben letztere in der vorliegenden Studie weitgehend unbeachtet.

${ }^{29}$ Zur Unterscheidung von Output und Outcome vgl. ebenda, S. 3; eine ausfürliche Darstellung dieser Konzepte erfolgt außerdem in Kapitel 4. 


\subsubsection{Begründungszusammenhang}

Im Sinne des Kritischen Rationalismus ${ }^{30}$ soll in der vorliegenden Arbeit versucht werden, Hypothesen zu gewinnen, welche quantitativen, empirischen Falsifizierungsversuchen standhalten, ohne daß ihr Informationsgehalt (ihre Allgemeinheit) zu sehr gemindert wird. Diesen Hypothesen soll jedoch kein Gesetzes- oder Theoriecharakter zugesprochen werden, sondern sie sollen weniger anspruchsvoll als raum-zeitlich relativierte Quasi-Theorien gelten, die weiteren Falsifizierungsbemühungen auszusetzen sind. Die Relativierung der später empirisch abgestützten Ergebnisse ist auch bedingt durch die mangelnde ökonomische und politische Repräsentativität der untersuchten Grundgesamtheit.

\subsubsection{Verwendungszusammenhang}

Eine politische Instrumentalisierung der im Rahmen dieser Studie ermittelten Zusammenhänge ist nur sehr begrenzt sinnvoll.

Erstens können aus der Tatsache, daß ein bestimmter Politikstil zu eher günstigen Folgen für das Öko-System führt, zunächst keine Folgerungen für die Ausgestaltung der praktischen Politik von Staaten gezogen werden, denn der Politikstil ist als Resultat u.a. der kulturell-religiösen und historischen Eigenarten eines Staates eine nur sehr langfristig änderbare Größe. Insbesondere eine Empfehlung an die Staaten Asiens oder Afrikas, ihren Politikstil an bestimmten Vorbildern aus der westlichen Hemisphäre zu orientieren, dürfte auf Ablehnung stoßen.

Zweitens ist die empirisch untersuchte Staatengruppe, nämlich die marktwirtschaftlich und auf die repräsentative Demokratie hin orientierten OECDStaaten Nordamerikas, Europas sowie Japan, - wie schon erwähnt - nicht heterogen genug, als daß aus den statistischen Signifikanzen der empirischen Untersuchung Handlungsempfehlungen für Staaten mit anderer Wirtschaftsverfassung und anderem politischem System abgeleitet werden dürften.

Der inhaltliche Standort dieser Arbeit ist zunächst im interdisziplinären Grenzbereich zwischen vergleichenden Politik-, Wirtschafts- und Umwelt-

${ }^{30}$ Vgl. Popper, K. R. (1976): Logik, a.a.O., S. XV; Spinner, Helmut F. (1995): Kritischer Rationalismus bzw. Pluralismus, In: Nohlen, Dieter (Hg.): Wörterbuch Staat und Politik, Bonn, S. 377-382. 
wissenschaften zu finden. Die Komplexität der ökologischen Problematik und ihre Interdependenz mit zahlreichen insbesondere ökonomischen und politischen Faktoren macht diese übergreifende Vorgehensweise notwen$\mathrm{dig}^{31}$. Neben der Verifizierung der Grundthese soll diese Arbeit inhaltlich außerdem noch zweierlei leisten:

1. Das bisher von den Politikfeldern relativ unabhängige Konzept der Policy Styles soll explizit auf das Feld der Umweltpolitik angewendet und modifiziert werden ${ }^{32}$;

2. Es soll eine auch für weitere Studien brauchbare Systematik von Indikatoren zur Messung der Effektivität von Umweltpolitik entwickelt werden.

Methodisch soll die Studie sowohl einen - allerdings im Anspruch begrenzten - Beitrag zur Theoriebildung auf dem Gebiet des Zusammenhanges von Politikstil und Umweltbelastung leisten, als auch diese Ansätze durch geeignete Indikatorauswahl und multivariate Datenanalyse empirisch verifizieren. Dabei zählt es zu einem der primären Interessen dieser Arbeit, das "ausgeprägte Defizit an quantitativen Studien" auf dem Gebiet der "international vergleichenden Umweltpolitikanalyse“ zu verringern ${ }^{33}$.

${ }^{31}$ Vgl. Bossel, Hartmut (1990): Umweltwissen, Berlin, S. 3.

${ }^{32}$ Zur bisherigen Bedeutung des Konzeptes der Policy Styles für den Bereich der Umweltpolitik siehe: Knoepfel, Peter; Lundquist, Lennart; Prud'homme, Rémy, und Wagner, Peter (1987): Comparing Environmental Policies: Different Styles, Similar Content, In: Dierkes, Meinolf, et al. (Hg.): Comparative Policy Research, Aldershot, S. 174 f.

${ }^{33}$ Kern, Kristine, und Bratzel, Stefan (1994): Erfolgskriterien ..., a.a.O., S. 58. 


\section{Erfolge nationaler Umweltpolitik}

\subsection{Systematik möglicher Erfolgskriterien}

„Es gibt bisher keinen Erfolgsfall der Umweltpolitik. "34 „[...] truly successful environmental policy exists in no country. "35 Diese Einschätzung Jänickes und Weidners wird sicherlich von vielen Beobachtern und Analytikern der umweltpolitschen Aktivitäten der Industrieländer geteilt. Trotz vielfältiger Entsorgungsbemühungen am Ende des Produktions- und Konsumtionsprozesses und trotz der zahlreicher gewordenen Versuche einiger Unternehmen zu ökologisch angepaßten Produktionsweisen bleiben auf der negativen Seite der Bilanz zwei Fakten unverändert bestehen:

1. Die Daten für die absolute und Pro-Kopf- bzw. Pro-Einheit-BIPBelastung an Emissionsmengen und Ressourcenverbräuchen haben sich in den vergangenen zwei Jahrzehnten kaum signifikant verringert ${ }^{36}$, und

2. es ist bisher in keinem Industrieland der ernsthafte Versuch einer strukturellen Wirtschafts- und Umweltpolitik gemacht worden, welche nicht versucht, bei Vorgabe des ökonomischen Wachstumszieles lediglich eine Begrenzung der dadurch induzierten Umweltbelastungen $\mathrm{zu}$ erreichen, sondern welche eine langfristige und nachhaltige Verbesserung des "state of the environment" zu einer verbindlichen Nebenbedingung des volkswirtschaftlichen Prozesses macht ${ }^{37}$, oder sogar die Bewahrung der natürlichen Umwelt in einer definierten Qualität zur politischen Priorität erklärt, an welcher sich die Art und das Ausmaß der ökonomischen Aktivitäten zu orientieren haben.

Trotz dieser Defizite räumt jedoch auch Jänicke ein, daß in der umweltpolitischen (Miß-)Erfolgsbilanz der Industrieländer interessante relative

${ }^{34}$ Jänicke, Martin (1990): Erfolgsbedingungen . . . , a.a.O., S. 213.

35 Jänicke, Martin, und Weidner, Helmut (1995): Successful Environmental Policy: An Introduction, In: Jänicke, Martin, und Weidner, Helmut (Hg.): Successful Environmental Policy. A Critical Evaluation of 24 Cases, Berlin, S.10.

${ }^{36} \mathrm{Vgl}$. Tabellen A1 bis A48.

37 Zum Thema der strukturellen Ökologisierung vgl. insbesondere Prittwitz, Volker von (1990): Das Katastrophenparadox - Elemente einer Theorie der Umweltpolitik, Opladen, S. 84 ff. 
Unterschiede bestehen ${ }^{38}$. Doch welche Daten sollten in der Erfolgsbilanz der Umweltpolitik erscheinen, oder anders gefragt: wie sollte der umweltpolitische Erfolg eines Staates definiert und gemessen werden ${ }^{39}$ ? $\mathrm{Zu}$ diesem Zweck ist eine Systematisierung möglicher Erfolgsbegriffe politischen Handelns notwendig.

\section{Abb. 3: Systematik von Kriterien zur Messung des Erfolges von Umwelt- politik}

\begin{tabular}{|c|c|c|c|}
\hline \multicolumn{3}{|c|}{ Performanz } & \multirow{2}{*}{$\begin{array}{c}\text { ProzeB- } \\
\text { erfolg }\end{array}$} \\
\hline Effektivität & Effizienz & Gerechtigkeit & \\
\hline $\begin{array}{l}\text { Erreichung } \\
\text { der Pro- } \\
\text { grammziele } \\
\text { Objektiver } \\
\text { Zielerreich- } \\
\text { ungsgrad }\end{array}$ & $\begin{array}{l}\text { - Ökologisch } \\
\text { - Ökonomisch }\end{array}$ & $\begin{array}{l}\text { - Räumlich } \\
\text { - Sozial }\end{array}$ & $\begin{array}{l}\text { - Partizipation } \\
\text { - Berechenbar- } \\
\text { keit } \\
\text { - Verfahrens- } \\
\text { gerechtigkeit }\end{array}$ \\
\hline
\end{tabular}

Eine erste wichtige Unterscheidung ist diejenige zwischen Ergebnis- und Prozeßzielen ${ }^{40}$. Letztere sind im Zusammenhang mit unserer Thematik jedoch vorerst nicht relevant, denn sie umfassen Konzepte wie Partizipation und Verfahrensgerechtigkeit, also Kriterien, die eventuell einen ethischen Wert besitzen, aus umweltpolitischer Sicht jedoch kein Selbstzweck sein können, denn es geht hier nicht um möglichst gerechte Politikverfahren, sondern um eine niedrige Belastung des Öko-Systems als

38 Jänicke, Martin (1990): Erfolgsbedingungen . . , , S. 213.

39 Jänicke et al. messen als Erfolgsindikator die über mehrere Jahre durchschnittliche Veränderung der Produktion umweltbelastender Wirtschaftsbereiche (jeweils bezogen auf die Einwohnerzahl der untersuchten Staaten) (Jänicke, Martin, et al. (1993): Umweltentlastung . . . , a.a.O.). Ähnlich gehen Jänicke sowie Jänicke und Mönch vor, indem sie als Kriterium die langfristige Änderung diverser Belastungsfaktoren auswählen (Jänicke, Martin (1990): Erfolgsbedingungen . . . , a.a.O.; Jänicke, Martin, und Mönch, Harald (1988): Ökologischer . . . , a.a.O.).

Weitere Einzelindikatoren finden sich in den Übersichten bei Kern, Kristine, und Bratzel, Stefan (1994): Erfolgskriterien . . . , a.a.O., S. 50 f.; Knoepfel, Peter, et al. (1987): Comparing environmental ..., a.a.O., S. $167 \mathrm{ff}$.

${ }^{40}$ Vgl. Nagel, Stuart S. (1991): Public Policy. Goals, Means, and Methods, Lanham. 
Resultat eben dieser Verfahren. Überspitzt ließe sich auch sagen: sollte am Ende dieser Analyse die Erkenntnis stehen, daß eher undemokratische und verfahrenstechnisch ungerechte Politiksysteme zu einer tatsächlichen Verringerung der Umweltlasten führen, dann wäre dies der ,, ideale“ Politikstil im Kontext unserer Thematik. Es wird sich aber später erweisen, $\mathrm{da} ß$ eine bestimmte Ausgestaltung einiger Prozeßziele positive Effekte auf die Ergebnisziele ausübt, somit also kein anzustrebendes Ziel umweltpolitischen Handelns ist, sondern eine notwendige Voraussetzung für den Erfolg desselben.

Festzuhalten ist also an dieser Stelle, daß zunächst eine Konzentration auf mögliche Ergebnisziele der Umweltpolitik zu erfolgen hat, und diese sind vor allem Effektivität und Effizienz ${ }^{41}$. Nachfolgend soll unter Effektivität ein im Sinne eines zuvor definierten Zieles wirksamer Einsatz von Ressourcen verstanden werden, während der Begriff Effizienz auf solche Faktorkombinationen zutrifft, in denen die Zielerreichung unter Einsatz möglichst geringer Faktormengen gelingt. Somit handelt es sich beim Effektivitätskriterium um eine Ergebnis- oder Wirkungsgröße, und beim Effizienzkriterium um eine Beurteilung des Verhältnisses von Input und Output.

Aus Ökonomischer Sicht sind die in Abbildung 4 dargestellten Sachverhalte für die Diskussion des Effizienz- und des Effektivitätsbegriffes wichtig $^{42}$. Die konkav verlaufende Verbindung zwischen den Punkten A und $B$ ist die volkswirtschaftliche Transformationskurve für die beiden Güter $\mathrm{X}_{1}$ und $\mathrm{X}_{2}$. Jeder Punkt auf dieser auch als Produktionsmöglichkeitenkurve bezeichneten Kurve repräsentiert eine im Sinne gesamtwirtschaftlicher Allokation effiziente Kombination der produzierten Mengen der Güter $X_{1}$ und $X_{2}$. Die drei weiteren in der Abbildung dargestellten Kurven sind Indifferenzkurven verschiedener Volkswirtschaften, in denen sich jeweils die Präferenzfunktion der Bevölkerungen hinsichtlich des Nutzens der beiden Güter ausdrücken. Alle Gütermengenkombinationen, die auf der Indifferenzkurve liegen, haben für die Bevölkerung den gleichen Nutzen, sind also im Sinne der Bedürfnisbefriedigung effektiv. Die mittlere Indifferenzkurve repräsentiert eine ungefähre Gleichgewichtung

${ }^{41}$ Vgl. Kern, Kristine, und Bratzel, Stefan (1994): Erfolgskriterien . . , a.a.O., S. 7 ff.

${ }^{42}$ Vgl. grundlegend: Schumann, Jochen (1987): Grundzüge der mikroökonomischen Theorie, 5. Aufl., Berlin; Heidelberg; New York, S 232 ff.; Zimmermann, Klaus W., und Kahlenborn, Walter (1994): Umweltföderalismus. Einheit und Einheitlichkeit in Deutschland und Europa, Berlin, S. $98 \mathrm{ff}$. 
den beiden Güter, da bei der Aufgabe einer bestimmten Menge des Gutes $\mathrm{X}_{1}$ eine Erhöhung der Produktion von $\mathrm{X}_{2}$ um etwa die gleiche Menge zu demselben Nutzen führt, die Grenzrate der Substitution des Gutes $X_{1}$ durch das Gut $\mathrm{X}_{2}$ also ungefähr Eins beträgt. Die obere Indifferenzkurve ist diejenige einer Volkswirtschaft, welche dem Gut $X_{1}$ einen höheren Nutzen zuschreibt als dem Gut $X_{2}$, was zu einer Grenzrate der Substitution des Gutes $\mathrm{X}_{1}$ durch Gut $\mathrm{X}_{2}$ von deutlich kleiner als 1 führt, während die Präferenzfunktion der Volkswirtschaft mit der unteren Indifferenzkurve eine höhere Gewichtung des Gutes $\mathrm{X}_{2}$ zum Ausdruck bringt.

\section{Abb. 4: Produktionsmöglichkeiten- und Indifferenzkurven}

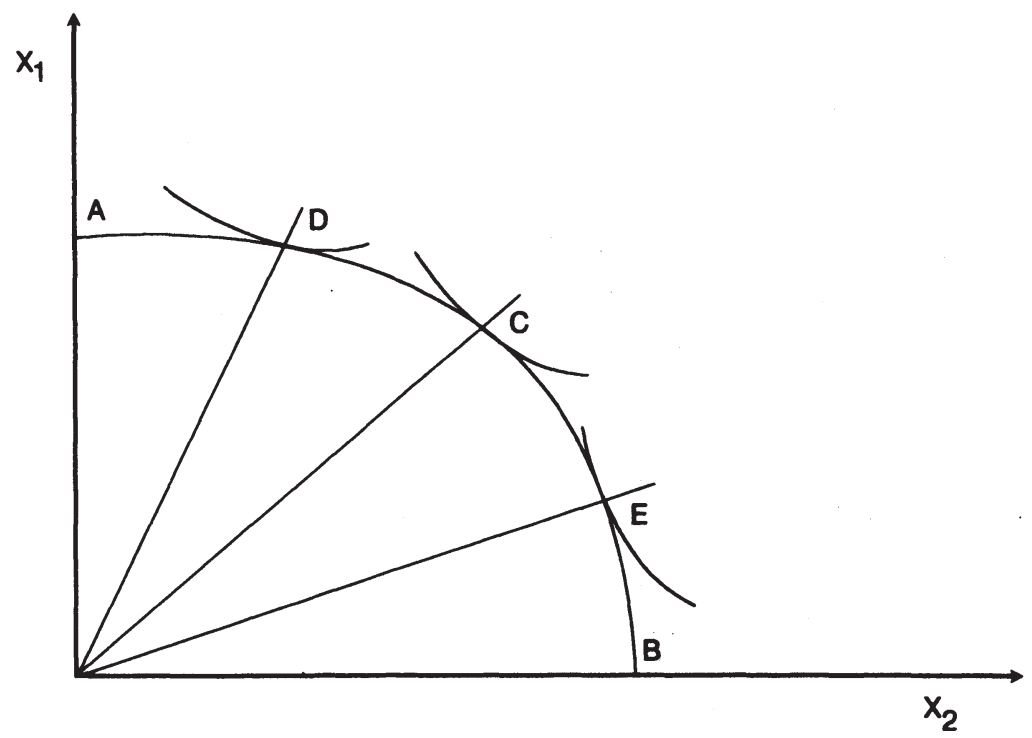

Für alle drei Volkswirtschaften gilt - vorausgesetzt, die Produktionsmöglichkeitenkurven sind identisch -, daß das wohlfahrtstheoretische Optimum dann vorliegt, wenn die Grenzraten der Transformation und der Substitution identisch sind. In diesen Punkten (C, D und E) tangieren die Indifferenzkurven die Produktionsmöglichkeitenkurve, und es liegt eine sowohl effiziente als auch effektive Kombination der Güter $\mathrm{X}_{1}$ und $\mathrm{X}_{2}$ 
vor. Entsprechend der unterschiedlichen Präferenzfunktionen der drei Volkswirtschaften ergeben sich unterschiedliche optimale Gütermengenkombinationen.

Der Inhalt dieses mikroökonomischen Basiswissens läßt sich auch auf die unweltökonomische Problematik übertragen ${ }^{43}$. Zu diesem Zweck ist das Gut $\mathrm{X}_{1}$ als normales Konsumgut und das Gut $\mathrm{X}_{2}$ als Umweltgut zu definieren. Analog zu den obigen Überlegungen drücken die Indifferenzkurven somit die Präferenzfunktionen hinsichtlich der beiden Güter aus. Gesellschaften, die aufgrund des Mangels an Konsumgütern eher materialistisch ausgerichtet sind, kommen im volkswirtschaftlichen Optimum (Punkt D) zu einer Gütermengenkombination, in welcher die Menge des Konsumgutes diejenige des Umweltgutes deutlich überwiegt. Im Falle einer aus dem Überfluß von Konsumgütern entspringenden eher postmaterialistischen Orientierung des gesellschaftlichen Wertesystems ${ }^{44}$ ergibt sich die in Punkt E vorliegende Gütermengenkombination, wobei entsprechend der Präferenzfunktion die Menge des im volkswirtschaftlichen Transformationsprozeß erzeugten Umweltgutes zu Lasten des Konsumgutes deutlich größer ist.

Diese Überlegungen zeigen also, daß eine Bestimmung des wohlfahrtsökonomischen Optimums zumindest theoretisch möglich ist. Es ist jedoch die Problematik zu lösen, ob für die Bestimmung des wohlfahrtstheoretischen Optimums das subjektive Nutzenempfinden der Bevölkerung (was die Menschen tatsächlich wollen) oder ein objektiver Nutzenbegriff (was die Menschen zur Bewahrung der natürlichen Lebensgrundlagen wollen müßten) zugrundegelegt werden sollte. Diese Frage wird in Abschnitt 2.2.2. im Zusammenhang mit der Problematik individueller Zahlungsbereitschaften noch weiter thematisiert.

${ }^{43} \mathrm{Vgl}$. Ebenda.

44 Inglehart, Ronald (1977): The Silent Revolution, Princeton; Zur Problematik einer Erklärung unterschiedlicher Präferenzfunktionen mittels unterschiedlicher Niveaus der ökonomischen Bedürfnisbefriedigung siehe: Zimmermann, Klaus W., und Kahlenborn, Walter (1994): Umweltföderalismus, a.a.O., S. 99. 


\subsubsection{Effektivität}

Effektivität kann - wie bereits im obigen Kapitel ausgeführt - ganz allgemein als der Grad bezeichnet werden, mit dem eine intendierte Wirkung des damit verbundenen Handelns bzw. Ressourceneinsatzes eintritt. Diese Definition läßt also zwei verschiedene Effektivitätsvarianten zu:

1. Eine „subjektive“ Effektivität, welche nur darauf abhebt, ob die explizit formulierten Programmziele erreicht wurden, und

2. Eine „objektive“ Effektivität, welche die Politikergebnisse zu den tatsächlichen Problemen in Beziehung setzt und dabei von allgemeingültigen, von der jeweiligen subjektiven Zielformulierung unabhängigen Politikzielen ausgeht ${ }^{45}$.

Während also die erste Effektivitätsvariante zu dem Resultat führen könnte, daß wir die Umweltpolitik eines Staates deshalb als effektiv bezeichnen müßten, weil er seine Programmziele äußerst bescheiden formuliert hat, erlaubt der zweite Begriff einen sinnvollen Vergleich und eine Bewertung der Umweltpolitik unterschiedlicher Staaten. In diesem Sinne einer objektive Effektivität definieren auch Jänicke und Weidner den Erfolg umweltpolitischer Bemühungen ${ }^{46}$.

Zur Definition des von ihnen verwendeten Erfolgsbegriffes nennen sie drei Hauptkriterien:

1. Es muß sich um eine relative Bestleistung handeln, d.h. erfolgreiche Fälle umweltpolitischen Handelns sind die mit den im Vergleich zu den Bemühungen anderer Staaten, Regionen oder Unternehmen positivsten Resultate.

2. Es wurde eine deutliche Verringerung eines speziellen Umweltverschmutzungstypus erreicht. Diese Reduktion bedeutet im besten Fall einen absoluten

${ }^{45}$ In diesem Kontext ist anzumerken, daß eine tatsächliche Objektivität in der Behandlung politischer Probleme auch für wissenschaftliche Experten nicht möglich ist, es sich bei einer Aussage über „objektive“ Notwendigkeiten auf dem Gebiet der Umweltpolitik also immer nur um eine unter Zugrundelegung vernünftiger, transparenter und nachvollziehbarer Maßstäbe quasi-objektive Annäherung an die „tatsächlichen“ Probleme handeln kann. Außerdem ist zu beachten, daß auch die ökologische Forschungsperspektive niemals ausschließlich gemeinwohlorientiert sein kann, sondern ebenfalls interessegeleitet, also am self interest orientiert bleibt.

${ }^{46}$ Jänicke, Martin, und Weidner, Helmut (1995): Successful . ., a.a.O., S. 14 f. 
Rückgang der Belastungsdaten, doch auch ein Rückgang relativ zum ökonomischen Wachstum gilt als ökologischer Erfolg.

3. Erfolgreich ist das umweltpolitische Handeln dann, wenn es im Vergleich zum Nichthandeln eine Verbesserung bedeutet. Wenn es also aufgrund von Gratiseffekten zu einer ökologischen Entlastung kommt, so ist dies kein umweltpolitischer Erfolg, sondern dieser kann erst dann behauptet werden, wenn die Reduzierung durch umweltpolitisches Handeln diejenige des Gratiseffektes noch übersteigt.

Diese drei Kriterien sind jedoch nicht hinreichend. So ist es beispielsweise denkbar, daß bestimmte Erfolge der Schadstoffreduktion vor allem durch eine kurzfristige ökonomische Rezessionstendenz bedingt sind, oder daß die Eliminierung eines umweltbelastenden Schadstoffes aus dem Produktionsprozeß das Resultat einer Substitution durch eine andere, ebenso schädliche Substanz ist. Aufgrund dieser Komplexität möglicher Ursachen für positive ökologische Effekte sind noch weitere Kriterien zu berücksichtigen:

- Die zeitliche Dimension der Reduzierung ist zu hinterfragen. Hierbei sind solche Lösungen, welche für eine endgültige oder langfristige Umweltentlastung sorgen, von nur kurz- oder mittelfristigen Erfolgen zu unterscheiden. Es ist also von Bedeutung, ob die Verbesserungen lediglich durch end-ofpipe-Verfahren erreicht wurden, oder ob grundsätzliche technologische und organisatorische Innovationen zu tatsächlich umweltschonenden und sauberen Produktionsverfahren geführt haben.

- Ein weiteres Kriterium für umweltpolitischen Erfolg ist die Vermeidung einer Verlagerung der Umweltbelastung durch Transfer in andere Regionen oder die Nutzung anderer Umweltmedien.

- Schließlich kann auch nach dem ökonomischen Nutzen der umweltpolitischen Maßnahme für die jeweilige Volkswirtschaft (z.B. durch Materialeinsparungen, positive Beschäftigungseffekte, geringere Kontrollkosten usw.), Region oder das Unternehmen gefragt werden.

\subsubsection{Effizienz}

Während das Erfolgskriterium der Effektivität allein den Nutzen (Wirkung) politischen Agierens mißt, werden zur Messung der Effizienz diesem Nutzen die mit den durchgeführten Maßnahmen verbundenen Kosten gegenübergestellt. Effizienz mißt also die Nutzen-Kosten-Relation umweltpolitischen Handelns. Die wichtigste Systematisierung in diesem Kontext ist die Unterscheidung zwischen ökologischer und ökologischökonomischer Effizienz. 
Die ökologische Effizienz stellt dem Nutzen einer staatlichen Umweltpolitik, also ihren positiven Auswirkungen auf die Indikatoren zur Messung der Umweltbelastung und des Ressourcenverbrauches, die ökologischen Kosten, d.h. die negativen Auswirkungen auf eben diese Indikatoren, gegenüber ${ }^{47}$. So können zum Beispiel Recyclingverfahren insofern das Erfolgskriterium der Effektivität erfüllen, als daß sie zur Reduzierung des Ressourcenverbrauches beitragen, jedoch gleichzeitig ökologisch ineffizient sein, weil die Umweltbeeinträchtigungen durch die im Recyclingproze $ß$ freigesetzten Emissionen den Umweltnutzen übersteigen.

Problematisch am ökologischen Effizienzkriterium sind die folgenden Punkte:

- Die methodische Problematik der Verrechnung unterschiedlicher Meßdimensionen (siehe obiges Beispiel) scheint kaum lösbar ${ }^{48}$.

- Das Effizienzkriterium mißt stets eine Relation, ergibt also einen Faktor, der keine Aussage über die absoluten Auswirkungen auf die Umweltqualität mehr zuläßt. So kann etwa eine Maßnahme mit einem verschwindend geringen Nutzen und noch geringeren Kosten effizienter sein als ein umfangreiches Programm, das neben absolut gemessen hohen positiven Umweltwirkungen auch ökologische Kosten verursacht.

Diese Kritikpunkte treffen in ähnlicher Weise auch für die ökologischökonomische Effizienz $\mathrm{zu}^{49}$. Dieses Kriterium stellt die erzielte Verbesserung der Umweltqualität in eine Relation zum dazu notwendigen volkswirtschaftlichen Aufwand. Die Problematik des Vergleiches von Variablen mit unterschiedlichen Meßdimensionen tritt hier noch verschärfter zutage. Die Frage, wieviel die Reduktion der durch eine Volkswirtschaft verursachten Emissionen um eine Maßeinheit kosten darf, damit die ökologisch-ökonomische Effizienz gewahrt ist, öffnet subjektiver und weltanschaulicher Beliebigkeit Tür und Tor.

Zur Lösung dieser Problematik führen einige Autoren das Konzept der individuellen Zahlungsbereitschaften an $^{50}$.

${ }^{47}$ Vgl. Prittwitz, Volker von (1990): Das Katastrophenparadox . . , a.a.O., S. $67 \mathrm{ff}$.

${ }^{48}$ Prittwitz schlägt eine Lösung dieses Problems durch Entscheidungsfragen vor, aus denen qualitative Relationen der unterschiedlichen Umweltwirkungen abgeleitet werden können. Dieses Vorgehen ist wegen des hohen Maßes an Subjektivität, mit denen die Beantwortung der Entscheidungsfragen behaftet sein dürfte, fragwürdig (Ebenda, S. 68).

${ }^{49}$ Vgl. Wicke, Lutz (1991): Umweltökonomie. Eine praxisorientierte Einführung, München, S. 401 . 


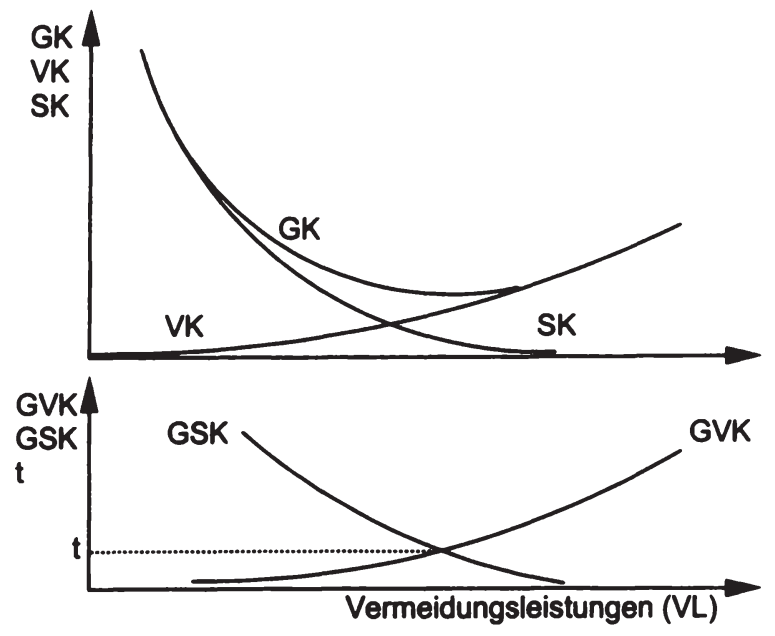

Quelle: Zimmermann und Kahlenborn (1994); S. 114

Eine die gesamtstaatliche Wohlfahrt optimierende Umweltpolitik liegt dann vor, wenn die Gesamtkosten der Vermeidung von Umweltschäden (z.B. für Reinigungsmaßnahmen oder die Umstellung von Produktionsverfahren) und der Umweltbelastung (z.B. Schädigung von Menschen und sonstigen Lebewesen, Gesundheitsschäden, Nutzenminderung öffentlicher Güter) - kurz: GK - ihr Minimum erreichen (Abbildung 5) ${ }^{5}$.

Die Vermeidungskosten steigen mit zunehmenden Vermeidungsleistungen steil an. Während im Falle eines völligen Verzichts auf Maßnahmen der Vermeidung von Umweltschäden keine Minderung der Umweltbelastung eintritt, steigen die Schadensvermeidungskosten in der Nähe des Punktes maximaler Umweltverbesserung überproportional an, d.h. die

${ }^{50}$ Vgl. Schulz, Wemer (1985): Der monetăre Wert besserer Luft. Eine empirische Analyse individueller Zahlungsbereitschaften und ihrer Determinanten auf der Basis von Repräsentativumfragen, Frankfurt/Main; Bern; New York; Zimmermann, Klaus W., und Kahlenborn, Walter (1994): Umweltfoderalismus, a.a.O., S. 109 ff.

${ }^{51}$ Siehe fur die folgenden Überlegungen auch: Schulz, Werner (1985): Der monetäre Wert ..., a.a.O., S. 10 ff.; Zimmermann, Klaus W., und Kahlenborn, Walter (1994): Umweltfoderalismus, a.a.O., S. 113 ff.; Wicke, Lutz (1991): Umweltðkonomie, a.a.O., S. 20 ff. 
Grenzvermeidungskosten (GVK) erreichen extrem hohe Werte. Die Vermeidung der Umweltbelastung um eine Einheit ist rechts des Minimums von GK nur noch unter Inkaufnahme rasch ansteigender Kosten zu erreichen. Für die Kosten der Umweltbelastung kann ein gegensätzlicher Kurvenverlauf angenommen werden. Während also im Falle minimaler Vermeidungsleistungen die Schadenskosten hoch sind, sinken diese Kosten mit zunehmenden Einsatz von Vermeidungstechnologie. Die zunächst deutlich negative Steigung der Kurve wird jedoch im weiteren Verlauf immer flacher, d.h. der Grenznutzen der Umweltentlastung nimmt entsprechend des 1 . Gossenschen Gesetzes $a b^{52}$. Aufgrund des gegensätzlichen Verlaufes der beiden Kostenfunktionen weist die Gesamtkostenkurve ein Minimum auf. Dieses Umweltkostenoptimum liegt dann vor, wenn die Grenzkosten des Umweltschutzes gleich den Grenzkosten der Umweltbelastung (bzw. dem Grenznutzen der Umweltverbesserung) sind.

Aus gesamtstaatlicher Sicht ist die optimale Allokation also nicht identisch mit dem Punkt minimaler Umweltbelastungskosten. Das Verhältnis der hier extrem hohen Grenzvermeidungskosten zum sehr geringen Grenznutzen der Umweltverbesserung ist so disproportional, daß eine kostenintensive Bindung von Produktionsmitteln zum Zwecke einer weiteren Verminderung der Umweltbelastung eine aus wohlfahrtstheoretischer Sicht unsinnige Reduktion anderer Ausgaben (z.B im Sozialbereich) bedeuten würde ${ }^{53}$. Oder anders formuliert: es gibt ein volkswirtschaftliches Optimum der Umweltbelastung. Die tatsächliche Bestimmung dieses Optimums ist jedoch problematisch. Mit noch tolerablen Fehlermöglichkeiten behaftet ist die Ermittlung der Vermeidungskosten. Zwar ist eine exakte Bestimmung der volkswirtschaftlichen Opportunitätskosten, d.h. des Nutzenentgangs, der durch eine Ressourcenbindung zugunsten ökologischer und zu Lasten ökonomischer Ziele entsteht, nur schwer möglich, doch die reinen monetären Kosten von Umweltschutzmaßnahmen (z.B. die Anschaffung einer industriellen Entschwefelungsanlage) sind relativ exakt $\mathrm{zu}$ bestimmen ${ }^{54}$. Viel schwieriger ist jedoch die Ermittlung

\footnotetext{
${ }^{52}$ Schumann, Jochen (1987): Grundzüge . . ., a.a.O., S. 14 f.

${ }^{53}$ Vgl. Schulz, Werner (1985): Der monetäre Wert . ., a.a.O., S. 11 f.
}

${ }^{54}$ Wenn beispielsweise durch Aufklärungskampagnen und Verpackungsverordnungen Bevölkerung und Unternehmen zu einer Verringerung der Verpackungsmengen angehalten werden, wie sind dann die volkswirtschaftlichen Kosten zu quantifizieren? Kampagne und Gesetzgebungsverfahren verursachen Kosten, in der Verpackungsindustrie und in der Müllbeseitigung fallen eventuell Arbeitsplătze weg usw. Diese 
des Verlaufes der Umweltbelastungskurve, da hier neben nur schwer quanitifizierbaren monetären Schäden vor allem der soziale Nutzenentgang der Bevölkerung zu berücksichtigen ist. Es stellt sich also beispielsweise die Frage, wie die Tatsache monetär zu bewerten ist, daß die Bevölkerung einer dichtbesiedelten Industriestadt einer durchschnittlich weit höheren Luftverschmutzung ausgesetzt ist als die Bewohner eines Ferienortes mit nahezu unbelasteter Luftqualität. Zur Lösung dieser Problematik schlägt u.a. Schulz das Konzept der individuellen Zahlungsbereitschaft der Wirtschaftssubjekte vor ${ }^{55}$. Zur Erfassung der marginalen Konsumentenrente werden repräsentativ ausgewählte Individuen befragt, welche Summe sie bereit wären, für eine bestimmte Verbesserung der Luftqualität zu zahlen. Um den Gesamtnutzen einer solchen Umweltqualitätsverbesserung zu bestimmen, erfolgt dann eine vertikale Aggregation der individuellen Nachfragekurven. Diese Gesamtkurve gibt dann den ökonomischen Wert unterschiedlicher Luftreinhaltungsgrade an. Im Schnittpunkt der daraus ableitbaren Grenznutzenfunktion mit der Grenzkostenfunktion des Umweltschutzes liegt dann das volkswirtschaftliche Optimum.

Gegen diese Vorgehensweise einer Nutzung individueller Präferenzen zur Bestimmung eines gesellschaftlichen Allokations-Solls gibt es vor allem die folgenden Argumente ${ }^{56}$ :

1. Informationsdefizit: die mangelnde Information der Wirtschaftssubjekte in einer so schwierigen Materie wie der Umweltbelastung muß zu einer Fehleinschätzung des monetären Wertes besserer Umweltgüte führen.

2. Irrationalität: die Irrationalität der Individuen hat meist einen zu hohen Diskontsatz für öffentliche Güter zur Folge, d.h. zukünftiger Konsum wird unterbewertet.

3. Minderschätzung der Bedürfnisse zukünftiger Generationen: eine Ermittlung aktueller individueller Zahlungsbereitschaften erfaßt nur die Präferenzen gegenwärtiger Generationen, die Bedürfnisse zukünftiger Generationen bleiben jedoch unberücksichtigt bzw. unterrepräsentiert.

\footnotetext{
Kosten genau zu bestimmen und dann sinnvoll zu aggregieren dürfte ebenfalls zu einem immensen Aufwand und großen methodischen Schwierigkeiten führen.

${ }^{55}$ Vgl. Schulz, Werner (1985): Der monetäre Wert . . ., a.a.O., S. 52; Wicke, Lutz (1991): Umweltőkonomie, a.a.O., S. 72 ff.

${ }^{56}$ Schulz, Werner (1985): Der monetäre Wert ..., a.a.O., S. 58 ff.
} 
Als Gegenargumentation ist anzuführen, daß Individuen aufgrund mangelnder Information mögliche Umweltrisiken eher über- als unterschätzen werden. Außerdem gibt es keine demokratische Legitimation, wegen eines angenommenen Informationsdefizites und einer unterstellten Irrationalität in die Präferenzen der Bürger einzugreifen ${ }^{57}$. Die Minderschätzung der Bedürfnisse zukünftiger Generationen schließlich ist eher ein Fehler des politischen, an die Dauer von Legislaturperioden orientierten Prozesses als der Bürger.

Eine Diskussion der hier nur skizzierten Einwände und Gegenargumente soll an dieser Stelle nicht erfolgen, eine wichtige Schlußfolgerung ist jedoch möglich: die Tatsache, daß die Bürger im Rahmen von Befragungen durchgängig äußern, daß sie bereit sind, eine gewisse, egal ob objektiv gerechtfertigte oder unterschätzte Summe zu zahlen, beweist, daß die gegenwärtige Allokation ökonomischer und ökologischer Güter in den Industriestaaten nicht optimal bzw. effizient ist.

\subsubsection{Gerechtigkeit (Equity)}

Dieses Kriterium berührt primär die ethischen Dimensionen des umweltpolitischen Entscheidungsprozesses, und zwar im Hinblick auf die Ergebnisverteilung und die Verfahren. Der Verteilungsaspekt kann dabei sowohl räumlich als auch sozial verstanden werden.

Die räumliche Dimension der Equity ist so zu verstehen, daß von zentralstaatlichen umweltpolitischen Entscheidungen die einzelnen subnationalen Einheiten in einem langfristig gleichen Maße betroffen sein müssen. Als Beispiel sei der Betrieb von Truppenübungsplätzen durch die Streitkräfte eines Staates aufgeführt. Da der Übungsbetrieb durch Lärm- und Emissionsentwicklungen $\mathrm{zu}$ Umweltbelastungen der jeweiligen Region führt, ist das Equity-Kriterium für ein Übungsplatzkonzept in einem dichtbesiedelten Staat, der über keine größeren, menschenleeren Flächen verfügt, nur dann erfüllt, wenn sich die genutzten Gelände annähernd gleichmäßig über das Staatsgebiet verteilen ${ }^{58}$. Eine räumlich sinnvolle Verteilung von Umweltschäden kann jedoch auch deren Konzentration an

${ }^{57}$ Ebenda.

58 Vgl. Jarre, Jan (1976): Die verteilungspolitische Bedeutung von Umweltschäden, Göttingen, S. 161 . 
einem oder wenigen Orten sein (z.B. Sondermülldeponien für hochtoxische Abfallstoffe).

Im sozialen Sinne ist das Equity-Kriterium dann erfüllt, wenn alle Bevölkerungsgruppen von den Umweltbe- und -entlastungen sowie den Kosten für Umweltverbesserungsmaßnahmen in dem Maß betroffen sind, in dem sie durch ihre Nachfrage zur Umweltbelastung beitragen. Die Tatsache, daß sozial Schwache in stark umweltbelasteten Innenstadtbezirken wohnen müssen, während es der Mittel- und Oberklasse möglich ist, in den grünen und ruhigen suburbs der Städte den negativen Folgen des volkswirtschaftlichen und privaten Wohlstandes teilweise zu entgehen, verletzt also dieses Erfolgskriterium.

Die gesellschaftspolitische Relevanz mangelnder Verteilungsgerechtigkeit konnte von Pearce für die USA empirisch belegt werden ${ }^{59}$. Danach sind u.a. die folgenden Ergebnisse gesichert: ${ }^{60}$

"[...]

- Die Verteilung der Umweltschäden auf die Einkommensgruppen ist stark regressiv.

- Die Verteilung der Umweltnutzung wandelt sich von Proportionalität zu Progressivität, je weiter der Umweltbegriff gefaßt (Erholung, Naturparknutzung usw.) und je einkommenselastischer damit die Umweltnutzung wird.

- Rückwirkungen einer durch Umweltpolitik verbesserten Umweltqualität können zu Preisveränderungen (Bodenwerte/Mieten) führen, welche die regressive Struktur der Umweltschäden verstärken.

- Die monetären Kosten der Umweltpolitik sind signifikant regressiv verteilt."

Eine Beseitigung dieses als verbesserungsbedürftig anzusehenden Status quo ist jedoch keine reine Allokationsproblematik, denn der Zustand einer ungerechten Verteilung der Nutzen und Kosten des Kollektivgutes Umwelt ist auch im Falle volkswirtschaftlicher Pareto-Optimalität (im Sinne des im vorausgegangenen Abschnitt erläuterten Effizienzbegriffes)

59 Pearce, D.W. (1982): The Distribution of the Costs and Benefits of Environmental Policy, Environment Committee OECD, ENV/ECO, 82.3, vervielf. Manuskript, Paris; Zitiert nach: Zimmermann, Klaus W. (1983): Ansatzpunkte einer verteilungsorientierten Umweltpolitik, in: Kyklos, 3, S. 421.

${ }^{60}$ Zimmermann, Klaus W. (1983): Ansatzpunkte ..., a.a.O., S. 421; Vgl. auch: Decker, Frank (1994): Ökologie und Verteilung. Eine Analyse der sozialen Folgen des Umweltschutzes, in: Aus Politik und Zeitgeschichte, 49, Bonn, S. 23 f. 
denkbar, oder anders formuliert: umweltökonomische Optimierung ist nicht identisch mit Verteilungsgerechtigkeit. Zur Schaffung der letzteren ist vielmehr eine umfassende Berücksichtigung von Verteilungskriterien in allen Phasen des politischen Prozesses notwendig ${ }^{61}$. Das Ziel einer solchen verteilungsorientierten Umweltpolitik wäre es dann, „die Umweltqualität [...] im Aktionsbereich der besonders betroffenen Bevölkerungsgruppen überdurchschnittlich [zu verbessern] "62. Die Problematik einer so orientierten Politik läge jedoch darin, daß eine ausschließliche Equity-Orientierung zu umweltpolitischen Effizienzverlusten führen, und der volkswirtschaftliche "Kuchen " somit kleiner werden könnte. ${ }^{63}$

Equity im Sinne von Verfahrensgerechtigkeit ist dann gegeben, wenn zum einen die direkt Betroffenen einer Entscheidung und zum anderen die gesamte interessierte Öffentlichkeit am Entscheidungverfahren partizipieren kann, etwa durch Anhörungen und Einspruchsrechte. Der Gebrauch bundesrechtlicher Weisungen durch den Bundesumweltminister etwa im Streit zwischen der Bundesregierung und dem Land Niedersachsen über die Inbetriebnahme des atomaren Endlagers bei Salzgitter berührt also aus Sicht des betroffenen Landes die Verfahrensgerechtigkeit. Über die Relevanz dieses Kriteriums wurde schon in Abschnitt 2.1. geurteilt, so daß lediglich zur Verteilungsgerechtigkeit Stellung genommen werden muß.

Sicherlich ist es aus Sicht der Betroffenen nicht unwichtig, ob die Umweltschäden und die Kosten zur Beseitigung dieser Schäden innerhalb eines Staates sozial und geographisch gerecht verteilt sind, und eine gerechte Verteilung steigender Umweltlasten kann sicherlich als Erfolg einer Politik interpretiert werden. Letztlich bleibt Umweltpolitik jedoch primär Allokationspolitik, d.h. es geht vor allem um den politisch gewollten Einsatz von Ressourcen, deren Quantität und Kombination, und erst in nachgeordneter Hinsicht um Gerechtigkeitsaspekte. Denn nur wenn tatsächlich Effekte im Sinne einer absoluten Umweltentlastung und bestenfalls sogar einer strukturellen Ökologisierung der Volkswirtschaft zu attestieren sind, kann das Equity-Kriterium als Nebenbedingung dieses Prozesses Sinn machen.

${ }^{61}$ Zimmermann; Klaus W. (1983): Ansatzpunkte . . ., a.a.O., S. 425 ff.

${ }^{62}$ Jarre, Jan (1976): Die verteilungspolitische Bedeutung . . ., a.a.O., S. 161.

${ }^{63} \mathrm{Vgl}$. Zimmermann, Klaus W. (1985): Umweltpolitik und Verteilung. Eine Analyse der Verteilungswirkungen des öffentlichen Gutes Umwelt, Bonn, S. 279. 


\subsubsection{Diskussion und Auswahl}

Da das Equity-Kriterium aufgrund der zuletzt angestellten Überlegungen als Erfolgsgröße für diese Studie nicht brauchbar ist, soll nachfolgend abgewogen werden, ob wir im weiteren Verlauf die Effizienz oder die Effektivität nationaler Umweltpolitik als die abhängige Variable, sprich: die Zielgröße, ansehen wollen.

Aus ökonomischer Sicht ist die Antwort auf diese Fragestellung eigentlich klar: der Punkt des umweltökonomischen Optimums, in welchem Grenzschadenskosten und Grenzvermeidungskosten der Umweltbelastung identisch sind, muß das eigentliche Kriterium zur Messung der Politikqualität sein. Weichen die Leistungen zur Vermeidung von Umweltschäden von diesem Punkt ab, so liegt eine Fehlallokation und somit ein Politikversagen vor. Dies bedeutet andersherum formuliert: je näher die Vermeidungsleistungen eines Landes an diesem Punkt effizienter Allokation liegen, desto erfolgreicher ist seine Umweltpolitik. Diese Festlegung auf das umweltökonomische Effizienzkriterium wäre jedoch aus mehreren Gründen problematisch:

- Zur Feststellung der Gesamtzahlungsbereitschaft für eine Reduzierung der Umweltbelastungen müßten außer dem bisher abgedeckten Bereich der Luftqualität ${ }^{64}$ auch alle anderen Teilbereiche der Umweltpolitik erfaßt (Gewässerqualität, Recyclingquote, Düngemitteleinsatz usw.) und zu einer Gesamtgröße aggregiert werden. Die Komplexität der umweltökonomischen Zusammenhänge würde also $\mathrm{zu}$ einem immensen empirischen Aufwand führen.

- Es ist anzunehmen, daß die einzelnen Staaten unterschiedliche Zahlungsbereitschaften ihrer Bevölkerungen für eine Vermeidung von Umweltbelastungen aufweisen ${ }^{65}$. Daraus resultieren national unterschiedliche Optima ökologischer Vermeidungsleistungen. Die sich somit ergebende Frage ist dann, wie eine vergleichende Bewertung der umweltpolitischen Erfolge der einzelnen Staaten durchgeführt werden kann. Soll als Erfolgskriterium der (möglichst kleine) absolute Abstand

\footnotetext{
64 Vgl. Schulz, Werner (1985): Der monetäre Wert . . ., a.a.O.; Wicke, Lutz (1991): Umweltökonomie, a.a.O., S. 72 ff.

${ }^{65}$ Empirisch belegt ist beispielsweise, daß die Zahlungsbereitschaft der Bürger der ehemaligen DDR deutlich geringer ausfallt als diejenige der Einwohner der alten Bundesrepublik (Zimmermann, Klaus W., und Kahlenborn, Walter (1994): Umweltfoderalismus, a.a.O., S. 109).
} 
zwischen den optimalen und den tatsächlichen Vermeidungsleistungen gelten oder die Relation der beiden Größen? Außerdem stellt sich die Frage, ob ein Staat mit einer aufgrund von Ignoranz gegenüber ökologischen Belastungen nur sehr geringen Zahlungsbereitschaft und im umweltökonomischen Optimum dementsprechend geringen Vermeidungsleistungen als der umweltpolitisch erfolgreichere Staat gelten kann, wenn seine Vermeidungsleistungen diesem Optimum näher sind als diejenigen eines anderen Staates, dessen weit höheren Vermeidungsleistungen wegen einer sehr hohen Zahlungsbereitschaft seiner eher post-materialistisch orientierten Bevölkerung noch weit vom Niveau einer umweltökonomischen Optimierung entfernt sind?

Eine Lösung dieser Problematik ist aus Sicht des Verfassers jedoch nicht notwendig. Wenn man die Hypothese akzeptiert, da $B$ in allen Industriestaaten innerhalb der Bevölkerung die Bereitschaft vorhanden ist, über die gegenwärtige Abgabenlast hinaus Zahlungen für eine Verbesserung der Umweltqualität zu leisten ${ }^{66}$, dann kann daraus abgeleitet werden, daß in allen diesen Staaten eine Fehlallokation hinsichtlich der Produktion des Kollektivgutes Umweltqualität vorliegt. Mit anderen Worten: die derzeitigen Vermeidungsleistungen in den westlichen Industriestaaten liegen mehr oder weniger weit vom Niveau entfernt, welches die Optimalitätsbedingung der Gleichheit von Grenzschadens- und Grenzvermeidungskosten erfüllt. In dieser Situation bedeutet umweltpolitischer Erfolg also eine Erhöhung der Vermeidungsleistungen bzw. eine Senkung der Kosten durch Umweltbelastungen. Ablesen läßt sich dieses Bemühen um eine Annäherung an das umweltökonomische Optimum am Ausmaß der Umweltqualität. Der so gefundene praktikable Erfolgsbegriff ist also das Effektivitätskriterium einer möglichst geringen Umweltbelastung. Es sei in diesem Kontext noch einmal an Abbildung 5 erinnert. Theoretisch ist die Forderung nach einer Minimierung der Umweltbelastungen unsinnig, da im Extremfall der Grenznutzen der zusätzlichen Vermeidungsleistungen gegen Null geht, und die Grenzkosten extrem ansteigen. Doch solange wir annehmen dürfen - und vieles spricht dafür -, daß sich die gegenwärtigen Vermeidungsleistungen der untersuchten Staaten mehr oder weniger weit links vom dem Punkt befinden, in dem sich die Kurven von Grenzschadens- und Grenzvermeidungskosten schneiden, ist dieses Kriterium sinnvoll. Also: unter der Annahme, daß die volkswirtschaftlichen Vermeidungsleistungen der westlichen Industriestaaten zur Reduktion von

${ }^{66}$ Allein in der Alt-BRD beträgt die Zahlungsbereitschaft für eine Verbesserung der Umweltqualität z.Zt. ca. 40 Milliarden DM (ebenda, S. 110). 
Umweltbelastungen unterhalb des optimalen Niveaus liegen, ist eine nationale Umweltpolitik aus ökologischer Sicht dann erfolgreich, wenn sie möglichst geringe Werte der Umweltbelastungsindikatoren erreicht. Somit ist das Effektivitätskriterium der im weiteren Verlauf dieser Studie angewandte Erfolgsbegriff.

\subsection{Effektivität von Umweltpolitik}

\subsubsection{Zielerreichungsgrad}

Wie oben ausgeführt soll das Erfolgskriterium dieser Studie also die „objektive“"Effektivität sein, d.h. das Maß, in welchem allgemeingültige, der Problemstellung inhärente Politikziele erreicht werden. Da in Kapitel 2.2.4. außerdem dargelegt wurde, daß das Niveau der volkswirtschaftlichen Vermeidungsleistungen in den westlichen Industriestaaten noch weit vom Punkt des Optimums entfernt sein dürfte, kann nachfolgend eine Umweltpolitik dann als effektiv gelten, wenn die auf die Umweltqualität (state of the environment) einwirkenden Belastungen (pressures on the environment $)^{67}$ möglichst günstige Indikatorwerte aufweisen. Die Forderung des „möglichst günstig“ ist jedoch recht unpräzise und läßt zahlreiche Variationen zu. Zunächst sei festgehalten, daß die subjektiven Politikziele der einzelnen Staaten hier nicht durch willkürlich selbstgesetzte Zielmarken ersetzt werden sollen. Ein Vergleich der gemessenen Indikatorwerte mit "Idealwerten" wird also nicht vorgenommen. Es erfolgt vielmehr eine mehrfache Relativierung der gefundenen Werte, zunächst durch den Vergleich mit den anderen Staaten dieser Untersuchung, dann durch die Einbeziehung von Bevölkerungszahl und Wirtschaftskraft des jeweiligen Staates.

\subsubsection{Absolute Umweltbelastungen}

Vergleichen wir z.B. die absoluten Indikatorwerte zur Messung der Umweltbelastung der USA und Dänemarks zur Feststellung des Erfolges ihrer jeweiligen Umweltpolitik miteinander, so müßte das Resultat lauten: die Umweltpolitik der Vereinigten Staaten ist im Vergleich zu derjenigen des kleinen Königreiches katastrophal schlecht. So setzt der Energiever-

${ }^{67}$ Vgl. zu diesen Termini jeweils OECD (1994): Environmental Indicators, Paris, S. 10 ff. 
brauch der USA jährlich mehr als fünf Milliarden Tonnen $\mathrm{CO}_{2}$ frei, derjenige Dänemarks nur etwa 56 Millionen Tonnen. Dies entspricht einem Verhältnis von $90 \mathrm{zu} 1^{68}$. Bei der Menge des produzierten Hausmülls ist das Verhältnis ähnlich disproportional ${ }^{69}$. Dennoch ist unmittelbar einsichtig, daß diese Vergleichsmethode unsinnig ist, da verschiedene Faktoren unberücksichtigt bleiben:

- Die Einwohnerzahl der USA ist ungefähr $45 \mathrm{mal}$ so groß wie diejenige Dänemarks. Unter der Voraussetzung eines ähnlichen Produktions- und Konsumtionsverhaltens der beiden Bevölkerungen ist damit notwendigerweise auch eine höhere absolute Umweltbelastung duch die USamerikanische Volkswirtschaft gegeben.

- Das Bruttoinlandsprodukt der USA ist tatsächlich auch mehr als 45 mal so groß wie das Dänemarks ${ }^{70}$. Innerhalb einer relativ homogenen Gruppe, wie sie die OECD-Staaten als weitgehend entwickelte Industriestaaten darstellen, schlagen sich große Unterschiede in der Einwohnerzahl auch auf die Proportionen der jeweiligen Volkswirtschaften nieder.

Somit ist festzuhalten, daß ein bloßer Vergleich der absoluten Umweltbelastungsdaten verschiedener Staaten ohne Berücksichtigung der Population und der Wertschöpfung kaum einen Sinn macht.

Es ist jedoch in diesem Zusammenhang auch festzuhalten, daß die Zentralregierungen großer Staaten aufgrund der absoluten Größe der durch ihre Produktions- und Konsumtionsverfahren hervorgerufenen pressures on the environment eine besondere Verantwortung tragen, da wegen der teilweise globalen Auswirkungen von Umweltbelastungen auch zahlreiche andere Staaten von der Qualität ihrer Entscheidungs- und Implementationsprozesse betroffen sind. Außerdem könnte es für die Resultate dieser Studie von besonderer Bedeutung sein, sollte sich herausstellen, daß der Politikstil sich zum einen tatsächlich signifikant auf die Effektivität der Umweltpolitik auswirkt und er zum anderen durch die Größe des jeweiligen Staates determiniert ist, also große Staaten nur eine bestimmte Art von Umweltpolitik praktizieren können, da die Staatsgröße auf spezifische Weise die wichtigen Dimensionen der politischen Entscheidungsfindung präformiert.

${ }^{68} \mathrm{Vgl}$. Tabelle A4.

${ }^{69} \mathrm{Vgl}$. Tabelle A5.

${ }^{70} \mathrm{Vgl}$. OECD (1995): OECD in Figures. Statistics of the member countries, Paris, S. $28 \mathrm{f}$. 


\subsubsection{Relative Umweltbelastungen}

Wie oben festgestellt, ist es also zur Durchführung einer sinnvollen vergleichenden Studie notwendig, die Umweltbelastungsindikatoren auf Merkmale der Staats- und Wirtschaftsgröße zu beziehen. Neben den Faktoren Einwohnerzahl und Bruttoinlandsprodukt sind noch weitere Bezugsgrößen denkbar. Die naheliegensten wären: Staatsfläche und Wirtschaftswachstum.

Staatsfläche: Ein mögliches Maß für den Grad der Ausbeutung der Natur in einem Staat könnte die Relation von Umweltbelastung und Fläche sein. Die geographischen Ausmaße eines Staates dürften keinen wichtigen Einfluß auf die absoluten Umweltbelastungen haben. Zwar könnte in großen, teilweise kontinentalen Flächenstaaten das Vorhandensein fossiler Brennstoffe in großen und leicht verfügbaren Mengen den verschwenderischen Umgang mit selbigen fördern, doch zeigen die Erfahrungen der Exploration fossiler Brennstoffe, daß zwischen der Staatsfläche und der Menge der Öl- oder Gasvorkommen kein unbedingter Zusammenhang besteht. Auch das Argument, in einem großflächigen Staat bestehe eher die Neigung zu bedenkenloser Emissionsfreisetzung, da dies ja in relativ unbewohntem Gebiet geschehen könne und somit eine große Assimilationskapazität bestehe, trifft nicht zu, da enge Korrelationen zwischen dem Ausmaß von Industrieansiedlungen und der Bevölkerungsdichte bestehen, Emissionen also auch in diesen Staaten eher dort freigesetzt werden, wo der größte Teil der Bevölkerung lebt ${ }^{71}$.

Wirtschaftswachstum: Da es sich beim BIP-Wachstum um eine Änderungsrate handelt, bei den Indikatoren zur Messung der pressures on the environment jedoch um zu einem Zeitpunkt gemessene Größen, wäre eine Relation dieser beiden Größen nicht sinnvoll.

Diese Überlegungen zeigen also, daß zur Berücksichtigung der Größenunterschiede zwischen den untersuchten Staaten eine Verwendung der Faktoren Bevölkerungszahl und Bruttoinlandsprodukt zu präferieren ist.

${ }^{71} \mathrm{Vgl}$. dazu die zahlreichen Luft- und Wasserqualitätsindikatoren von Großstadtregionen in OECD (1994): Environmental Indicators, a.a.O. 


\subsection{4 Änderung der Umweltbelastungsindikatoren}

Viele Autoren wählen zur Messung des Erfolges nationaler Umweltpolitik die Veränderungsraten der Umweltbelastungsindikatoren ${ }^{72}$. Hinter dieser Vorstellung steht der Grundgedanke, daß sich eine konkrete Maßnahme zur Emissionsreduktion oder zur Verringerung des Ressourcenverbrauches auch in meßbaren Veränderungen eben dieser Indikatoren niederschlagen sollte bzw. das strukturelle Wandlungsmuster der umweltbelastenden Industriebereiche auch einen direkten Einfluß auf die Umweltqualität ausübt. Gegen die Anwendung dieses Erfolgskriteriums im Rahmen der vorliegenden Untersuchung sprechen jedoch einige inhaltliche und methodische Überlegungen:

- Es ist oft äußerst schwierig, Änderungen der Indikatorwerte bestimmten umweltpolitischen Maßnahmen zuzuordnen. Überlagerungen verschiedener Maßnahmen sowie die Möglichkeit von time lags erschweren diesen Vorgang noch zusätzlich.

- Änderungen der Indikatorwerte könne oft auch von anderen Faktoren als der Umweltpolitik des jeweiligen Staates determiniert sein. So schlägt sich ein konjunkturell bedingter Rückgang der Industrieproduktion natürlich auch in zwischenzeitlich günstigeren Umweltdaten nieder.

- Es gibt inzwischen hinreichende empirische Evidenz für die Annahme, $\mathrm{da} ß$ die Umweltbelastungskurve eines Staates von seinem ökonomischen Entwicklungsstand sowie der sektoralen Aufteilung seiner Wirtschaftstätigkeit determiniert wird. Nimmt man außerdem an, daß alle Industriestaaten mit gewissen zeitlichen Verschiebungen eine ähnliche Entwicklung ihrer Wirtschaftsdaten sowie einen ähnlich gestalteten Strukturwandel vollziehen, dann sind Unterschiede der Änderungsraten der Umweltbelastungsindikatoren möglicherweise auch dadurch gegeben, daß sich diese Staaten auf einem entlang der Zeitachse unterschiedlichen Punkt ihrer ansonsten ähnlich verlaufenen Belastungskurve befinden.

- Diese oben beschriebene Gesetzmäßigkeit vorausgesetzt, geht es in dieser Studie also nicht darum, Änderungen im Verlauf der Umweltbelastungskurve, also zeitliche relative Unterschiede, zu erklären, sondern darum, die absoluten Unterschiede im Verlauf dieser Kurve zu klären, also die Frage, warum diese Kurve bei einigen Staaten flacher und bei anderen steiler als beim Durchschnitt verläuft.

${ }^{72}$ Vgl. hierzu sämtliche Studien Jänickes. 
Das Resultat der Überlegungen zur Auswahl der verwendeten Erfolgskriterien lautet also: wir wählen zur Beschreibung des Erfolges das Kriterium einer objektiven Effektivität, die sich nicht an den nationalstaatlich definierten Zielen, sondern übergeordneten, an das Problem angepaßten Zielen orientiert. Zur Wahrung der Vergleichbarkeit der Daten und zur Berücksichtigung des Faktors Staatsgröße sind die Indikatoren zur Zielmessung jedoch auf die jeweilige Einwohnerzahl und Wirtschaftskraft der untersuchten Staaten zu beziehen.

\subsection{Probleme der Zurechenbarkeit}

Obwohl die Auswahl des Effektivitätskriteriums unter Berücksichtigung vielfältiger möglicher inhaltlicher und methodischer Probleme erfolgte, ist die Verwendung dieser Erfolgsgröße dennoch mit weiteren Schwierigkeiten behaftet, die es im folgenden zu analysieren gilt.

\subsubsection{Grenzüberschreitung von Schadstoffeinträgen}

Bei der noch erfolgenden Festlegung der konkreten Meßgrößen für den Grad der Umweltbelastungen eines Staates werden auch Indikatoren zur Messung der Emissionsbelastung innerhalb eines Staates gebildet, u.a. auch des $\mathrm{CO}_{2}$-Ausstoßes, einem der Hauptverursacher des Treibhauseffektes. Dieser Treibhauseffekt ist jedoch von globaler Wirkung, d.h. ein hoher Indikatorwert eines Staates für diese Emissionsart hat Bedeutung auch über die nationalen Grenzen hinaus ${ }^{73}$.

Wenn also das Konstrukt „Umweltqualität eines Staates“ anhand der originär durch diesen Staat verursachten Umweltlasten quantifiziert wird, dann berücksichtigt dieses Vorgehen nicht die Effekte grenzüberschreitender und interdependenter Umweltprobleme ${ }^{74}$. Es ist also denkbar, daß

${ }^{73}$ Vgl. Bossel, Hartmut (1990): Umweltwissen, Berlin, S. 130.

${ }^{74}$ Zur Interdependenz von Umweltproblemen vgl. Henn, Heike, et al. (1993): Weltökologie, In: Hauchler, Ingomar (Hg.): Globale Trends 93/94. Daten zur Weltentwicklung, Frankfurt am Main, $280 \mathrm{ff}$. Die Konsequenzen der grenzüberschreitenden Wirkung von Umweltbelastungen werden insbesondere von Prittwitz thematisiert: Prittwitz, Volker von (1986): Drei Ansätze 
ein Staat aufgrund einer vorbildlichen Umweltpolitik die relativ niedrigsten Umweltbelastungwerte erzielt, durch die geographische Nachbarschaft zu weniger „ökologischen“ Staaten jedoch einen eher hohen Grad der Luft-, Gewässer- und Bodenverschmutzung aufweist ${ }^{75}$.

Diese Tatsache der Interdependenz der Umweltproblematik und der eventuell notwendigen Inter- oder gar Supranationalität umweltpolitischen Handelns ist für die Aussagekraft der im Rahmen dieser Studie gefundenen Resultate jedoch eher zu vernachlässigen. Denn es geht hier primär um die Frage, wie sich der nationale Politikstil eines Staates auf die durch diesen Staat verursachten Umweltbelastungen auswirkt, d.h. es werden Emissionen und nicht Imissionen betrachtet ${ }^{76}$. Daß der State of the Environment eines Staates auch von der Qualität der Umweltpolitik anderer Staaten abhängt, ist eindeutig, aber ein anderes Problem. Die Fähigkeiten der Exekutive, diese grenzüberschreitenden Effekte mangelhafter Umweltpolitik anderer internationaler Akteure durch politische Maßnahmen zu minimieren, wird in dieser Studie also nicht weiter berücksichtigt ${ }^{77}$.

\subsubsection{Gratiseffekte}

Als Gratiseffekte sollen solche Effekte gelten, welche eine nichtintendierte positive Wirkung auf den Zielerreichungsgrad der Umweltpolitik ausüben ${ }^{78}$ :

- Die Verbesserung der technologischen Kapazitäten kann die wirtschaftlich rentable Substitution fossiler Brennstoffe durch weniger umweltbelastende Energiequellen gestatten.

zur Analyse internationaler Umweltpolitik, In: Kohler-Koch, Beate (Hg.): Technik und internationale Politik, Baden-Baden, S. 389-405.

${ }^{75}$ Zum Thema Schadstoffimport/-export vgl. das Beispiel Schwefel bei Bossel (1990): Umweltwissen, Berlin, S. 127.

${ }^{76}$ Zum Unterschied vgl. ebenda.

${ }^{77}$ Dies wäre das Metier einer Umweltaußenpolitik (Prittwitz, Volker von (1986): Drei Ansätze

. . , a.a.O., S. 494 ff.), als der ,Vertretung (nationaler) Umweltinteressen gegenüber anderen Grundeinheiten der internationalen Politik" (Ebenda, S. 495).

${ }^{78}$ Vgl. Kern, Kristine, und Bratzel, Stefan (1994): Erfolgskriterien . . , , a.a.O., S. 8. 
- Ein struktureller Wandel der sektoralen Aufteilung einer Volkswirtschaft in Richtung Dienstleistung führt automatisch zu einer Reduktion der durch industrielle Produktionsverfahren verursachten Umweltbelastungen.

- Temporäre rezessive Tendenzen schlagen sich aufgrund nachlassender industrieller Produktion und verminderter Konsumtion sofort in einem Rückgang des Ressourcenverbrauches und der Emissionen nieder.

- Bemühungen anderer Politikbereiche (z.B. der Verkehrspolitik) haben oft unbeabsichtigte, aber nachhaltige Wirkung auf umweltpolitischem Gebiet $^{79}$.

- Singuläre Ereignisse wie der Zusammenbruch der industriellen Strukturen in der ehemaligen DDR können ebenfalls positive Wirkungen im Sinne eines deutlichen Rückganges der Umweltbelastung haben ${ }^{80}$.

Diese Beispiele zeigen, daß der Wert bestimmter Umweltqualitätsindikatoren das Resultat multikausaler Zusammenhänge ist, es im Rahmen der empirischen Untersuchung also notwendig sein wird, den Wirkungsanteil der anderen Faktoren zumindest teilweise zu identifizieren und - soweit möglich - herauszurechnen.

${ }^{79}$ Zum Querschnitt-Charakter der Umweltpolitik vgl. Bungarten, Harald H. (1978): Umweltpolitik in Westeuropa, Bonn, S. 87.

${ }^{80} \mathrm{Vgl}$. Zimmermann, Klaus W. (1992): Umweltpolitische Perspektiven in den neuen Ländern, in: Aus Politik und Zeitgeschichte, 39-40, S. 8. 


\section{Determinanten der Effektivität nationaler Umweltpolitik}

\section{1 Überblick}

In ihrer umfangreichen Literaturstudie der international vergleichenden Umweltpolitikanalyse unterscheiden Kern und Bratzel zwischen strukturellen und situativen Faktoren, welche den Erfolg von Umweltpolitik determinieren $^{81}$. Abbildung 6 gibt einen Überblick über die einzelnen Variablen und Variablengruppen dieser Systematik.

Abb. 6: Systematik der Determinanten nationaler Umweltpolitik

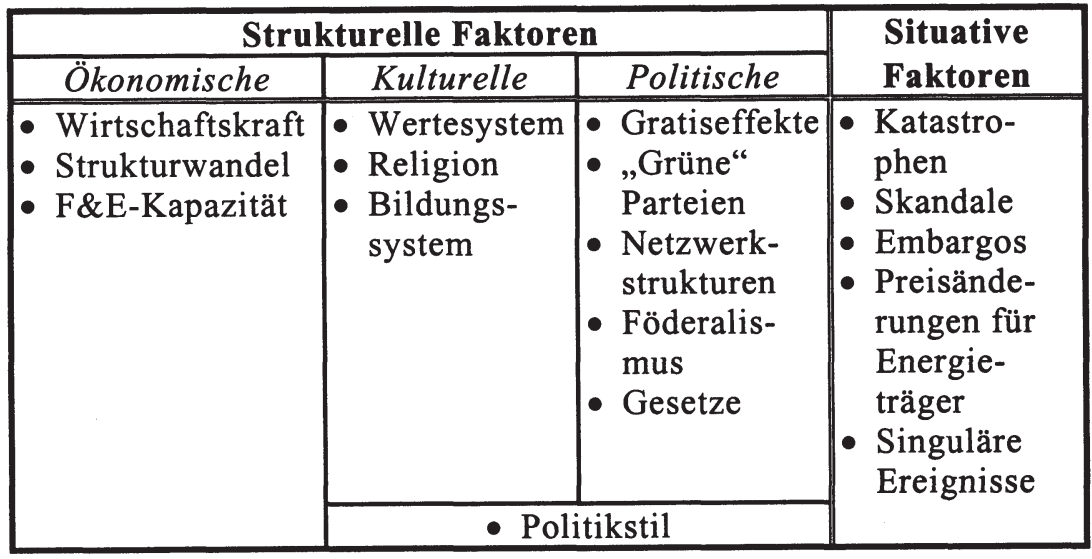

Quelle: Eigene Darstellung in Anlehnung an Kern und Bratzel (1994)

Während es sich bei den strukturellen Faktoren ${ }^{82}$ um Variablen handelt, welche eher langfristigen Wandlungen unterliegen und somit im ta-

${ }^{81}$ Vgl. Kern, Kristine, und Bratzel, Stefan (1994): Erfolgskriterien . . , a.a.O., S. 15 ff.

${ }^{82}$ Es ist zu beachten, daß sich der politische Struktur-Begriff vom ökonomischen deutlich unterscheidet. Die ökonomische Struktur gibt das Verhältnis von primärem (Landwirtschaft), sekundärem (Verarbeitendes Gewerbe) und tertiärem Sektor (Dienstleistungen, Transport, Baugewerbe) innerhalb einer Volkswirtschaft wieder. 
gespolitischen Agieren als konstante Nebenbedingungen des Handelns gelten können, handelt es sich bei den situativen Faktoren um meist kurzfristig auftretende, unmittelbar wirkende Veränderungen der politischökonomischen Rahmenbedingungen. Diese kurzfristigen Effekte können allerdings auch strukturelle Folgen haben, indem etwa eine Umweltkatastrophe die gesellschaftliche Werteskale modifiziert.

\subsection{Strukturelle Faktoren}

\subsection{1 Ökonomische Determinanten}

Wie im Abschnitt über die Umweltqualität beeinflussenden Gratiseffekte bereits ausgeführt, ist eine signifikante Korrelation zwischen den Indikatoren zur Messung der Umweltqualität sowie ökonomischen Variablen wie dem Bruttoinlandsprodukt-pro-Einwohner oder der Industrieproduktion zu erwarten. Diese These wird auch von zahlreichen empirischen Studien belegt. Einige Wissenschaftler sehen im ökonomischen Faktor sogar die wichtigste Determinante der Umweltqualität ${ }^{83}$. Der vereinfachenden These: „Mit der Zunahme der Wirtschaftstätigkeit wachsen auch Ressourcenverbrauch und Schadstoffeinträge", ist jedoch nicht zuzustimmen. Zahlreiche Argumente widerlegen die simplifizierende Annahme einer alleinigen Dominanz des ökonomischen Faktors:

- Einige vorliegende empirische Studien belegen entweder eine ab einem bestimmten Niveau der Wirtschaftsleistung erfolgende Entkopplung von Bruttoinlandsprodukt und Umweltbelastung oder liefern sogar Evidenz für die Annahme, daß durch Wandel von Struktur und technologischer Kapazität ab einem bestimmten Wohlstandsniveau Pro-KopfEinkommen und Umweltbelastungsindikatoren eines Staates negativ korrelieren $^{84}$.

- Die Muster der Umweltbelastungsdaten von Staaten mit nahezu identischem Verlauf der ökonomischen Entwicklung weisen teilweise erhebliche Unterschiede auf. Hier ist auf die bereits oben erfolgten Überle-

\footnotetext{
${ }^{83}$ Vgl. Ringquist, Evan J. (1993): Environmental Protection . . , a.a.O.

${ }^{84} \mathrm{Vgl}$. die Studien Jänickes.
} 
gungen zu verweisen, daß die Kurven der Umweltbelastung in Abhängigkeit vom Bruttoinlandsprodukt für unterschiedliche Staaten zwar meist einen ähnlichen Verlauf aufweisen, die wichtigsten Werte zur Beschreibung dieser Kurven (Steigung, Wendepunkte, Maxima) jedoch teilweise deutlich voneinander abweichen.

Die Wirtschaftsstruktur hat insofern einen Einfluß auf die Umweltqualität, als daß davon ausgegangen werden kann, daß hohe Anteile von Industrie- und Agrarproduktion tendenziell eher umweltbelastend wirken (Rohstoffverbrauch, Emissionen, Düngemitteleinsatz etc.), und im Falle einer Expansion des tertiären Sektors mit umweltentlastenden Effekten zu rechnen ist ${ }^{85}$.

Auch strukturelle Veränderungen innerhalb des sekundären Sektors können umweltentlastend wirken, wenn z.B. die Anteile stark umweltunverträglicher Industrien sinken, und weniger rohstoff- und energieintensive Branchen ihre gesamtwirtschaftliche Bedeutung erhöhen können.

Eng verknüpft mit dieser Thematik ist das Vorhandensein ausreichender F\&E-Kapazitäten eines Staates, denn nur in diesem Fall sind strukturelle Veränderungen der gesamtwirtschaftlichen Produktion, z.B. durch den auch ökonomisch rentablen Ersatz gegenwärtiger Energiequellen mit ökologisch verträglichen Ressourcen, denkbar.

Durch technologisches Know-How kann jedoch auch die bereits bestehende Produktionsstruktur ökologisch entlastet werden, etwa durch ,endof-pipe"-Systeme, Recyclingtechnologien oder energie- und materialsparende Fertigungsverfahren.

\subsubsection{Kulturelle Determinanten}

Unter dem Konstrukt der „kulturellen Determinanten“ sind solche Faktoren subsumiert, welche aus den historischen, religiösen und gesellschaftlichen Spezifika der einzelnen Staaten hervorgehen, z.B. das Wertesystem einer Gesellschaft und die Positionierung des Gutes „Bewahrung der natürlichen Umwelt" innerhalb desselben.

Eine hohe Wertschätzung der umweltpolitischen Ziele innerhalb des nationalen Wertekanons kann sich sowohl im Verhalten der Konsumenten (Vermeidung von Verpackungsmüll, Mülltrennung, Kauf umweltgerech-

${ }^{85}$ Falls die Zunahme des Individual- und Güterverkehrs diesen Effekt nicht überkompensiert (siehe z.B. Bossel, Hartmut (1990): Umweltwissen, a.a.O., S. 126). 
ter Produkte etc.) als auch in dem von Industrie (Emissionsvermeidung, hohe Recyclingquote) und Politik (Gesetz-gebung, Kontrollen) indirekt zugunsten der ökologischen Qualität auswirken ${ }^{86}$.

In diesem Kontext ist allerdings auch zu fragen, warum sich diese hohe Priorität, die dem öffentlichen Gut „Umwelt“ in nahezu allen OECDStaaten eingeräumt wird ${ }^{87}$, letztendlich nur sehr unzureichend und zögerlich im tatsächlichen Verhalten der Bürger, seien sie Konsumenten oder Produzenten, niederschlägt. Die Zurschaustellung eines „ökolo-gischen Gewissens" durch Verbraucher in Umfragen sowie die Betonung von corporate responsibility oder business ethics durch Unternehmen scheinen gemessen am praktischen Handeln doch nur oberflächliche Imagepflege zu sein.

Mit dem jeweiligen gesellschaftlichen Wertesystem interdependent verknüpft sind zwei weitere kulturelle Faktoren: die Bedeutung der Religion sowie das Bildungssystem eines Staates.

Wenn die Bewahrung der Umwelt in einem Staat mit eher niedrigem Säkularisationsniveau einen wichtigen Bestandteil der dominierenden Religion darstellt, dürfte dies nicht ohne positive Wirkungen auf den Umgang mit dem Öko-System bleiben. Auch die Frage, ob die Religion eher „diesseitig“ orientiert ist oder aber jenseitige, nicht-irdische Paradiese verspricht, dürfte einen Einfluß auf die Anstrengen zum Schutze der gegenwärtigen materiellen Grundlagen haben ${ }^{88}$.

Im Hinblick auf die Bedeutung der Bildung ist anzunehmen, daß mit einer Zunahme des Bildungsniveaus zum einen die zur Umweltbelastung führenden wirtschaftlichen und gesellschaftlichen Prozesse und zum anderen die Tragweite der ökologischen Krise für das eigene Wohlbefinden und das der kommenden Generationen besser erkannt werden. Außerdem ist die wichtige Rolle der Lehrer als Vermittler ökologischer Werte zu beachten.

\footnotetext{
${ }^{86}$ Zum Zusammenhang zwischen „,neuen Wertorientierungen“ und dem Erfolg grüner Parteien vgl. Müller-Rommel, Ferdinand (1992): Erfolgsbedingungen Grüner Parteien in Westeuropa, in: Politische Vierteljahresschrift, 2, S. $207 \mathrm{ff}$.

${ }^{87}$ Siehe die Umfrageergebnisse zur Umweltschutz-Thematik in: OECD (1993): OECD Environmental Data, Paris, S. $287 \mathrm{ff}$.

${ }^{88}$ Vgl. Kern, Kristine, und Bratzel, Stefan (1994): Erfolgskriterien . . . a.a.O., S. 16.
} 
Als weiterer wichtiger kultureller Faktor sind die Medien der modernen Massenkommunikation (vor allem Presse und TV) anzuführen. Diese Medien sind nicht nur die Berichterstatter und Kommentatoren von Ereignissen der Umweltpolitik, sondern sie sind auch Instrumente und Akteure in der politischen Arena. Die nur 30 Sekunden währende AntiAtomtest-Aktion von Greenpeace im August 1995 in Peking hat ihre politische Wirkung ausschließlich der Medienberichterstattung zu verdanken. Im Fall der geplanten Versenkung einer Ölverlade-Plattform des Shell-Konzerns brachte erst eine durch die Medien aktiv geführte Boykott-Kampagne das Unternehmen und die britische Regierung zum Einlenken.

\subsubsection{Politische Determinanten}

Neben den bereits in Abschnitt 2.4.2. erwähnten Gratiseffekten durch nicht-intendierte Auswirkungen anderer Politikbereiche auf die Umweltproblematik sind noch weitere wichtige politische Determinanten der Effektivität nationaler Umweltpolitik denkbar.

So könnten sich Existenz und parlamentarischer Erfolg „grüner Parteien“, also solcher politischen Interessenformationen, die primär oder sogar alleine aus ökologischen Motivationen gebildet wurden und die Erreichung umweltpolitischer Ziele zur Hauptsache ihrer politischen Einflußnahme auf die Gesellschaft machen, positiv auf das Maß der ökologischen Ausrichtung der Politik auswirken. Dabei sind zwei Mechanismen der Einflußnahme möglich:

1. Existenz und Erfolg ökologischer Parteien auf der einen sowie das Umweltbewußtsein der Bevölkerung auf der anderen Seite scheinen in einer sich gegenseitig verstärkenden, interdependenten Beziehung zu stehen. Ist innerhalb der Bevölkerung aufgrund einer wertemäßigen ohnehin vorhandenen Prädisposition oder aufgrund eines Wertewandels eine Abwendung von ökonomischen Wachstumszielen hin zu einer stärkeren Betonung der Lebens- und Umweltqualität zu beobachten, dann schlägt sich dies auch im politischen Aufstieg postmateriell orientierter politischer Gruppierungen nieder. Andererseits erhöht der politische Erfolg die Attraktivität und somit auch den Einfluß auf die Formulierung der gesellschaftlich relevanten Probleme ${ }^{89}$.

${ }^{89}$ Müller-Rommel mißt zwischen dem Erfolg grüner Parteien sowie dem Ausmaß des Postmaterialismus einer Gesellschaft einen Korrelationskoeffizienten von 0.57 (ohne Angabe des 
2. Der Erfolg „grüner Parteien“ führt trotz anfänglicher Abwehrreflexe der bis dahin etablierten Parteien mittel- und langfristig zu einer Übernahme offensichtlich populärer Ziele in den Fundus der eigenen Programmatik. Dieser Prozeß der „Ökologisierung“ führt somit zu einer zunehmenden Koalitionsfähigkeit „grüner“ Parteien, und damit wiederum ist eine stärkerer Berücksichtigung umweltentlastender Argumente im politischen Entscheidungs- und Implementationsprozeß verbunden.

Auch von der wichtigsten Strukturvariable zur Beschreibung der Staatsorganisation, dem Zentralisationsgrad, sind signifikante Effekte auf die Umweltpolitik anzunehmen ${ }^{90}$. So dürfte sich die Verlagerung von Entscheidungskompetenz auf subnationale Einheiten auf die Effizienz und Effektivität des umweltpolitischen Prozesses eher positiv auswirken. Die Frage der umweltpolitischen Bedeutung einer föderalistischen Staatsorganisation wird in Kapitel 5 noch ausführlich thematisiert.

Auch die umweltpolitische Gesetzgebung gehört in die Reihe der politischen Determinanten. Erst auf der Grundlage eines nationalen und internationalen Systems von rechtlichen Prinzipien können die verschiedenen umweltpolitischen Instrumente zur Anwendung kommen.

Von besonderer Bedeutung sind in diesem Zusammenhang auch die Zeitpunkte einer rechtlichen Institutionalisierung des Umweltschutzes durch die Schaffung eines Umweltrahmengesetzes und die Aufnahme des Schutzes von Natur und Umwelt in die nationale Verfassung ${ }^{91}$.

Eine weitere wichtige Determinante der Umweltpolitik aus dem Bereich der politischen Faktoren ist die Gestaltung der nationalen Politiknetzwerke, also die Struktur derjenigen politischen Gremien, welche Entscheidungen vorbereiten, treffen oder implementieren ${ }^{92}$. Unter den zahlreichen Dimensionen zur Beschreibung von Netzwerkstrukturen seien an dieser

Signifikanzniveaus) (Müller-Rommel, Ferdinand (1992): Erfolgsbedingungen . . ., a.a.O., S. 213).

${ }^{90}$ Vgl. Zimmermann, Klaus W., und Kahlenborn, Walter (1994): Umweltföderalismus, a.a.O.

${ }^{91}$ Vgl. hierzu: Jänicke, Martin (1990): Erfolgsbedingungen . . , a.a.O., S. 215 f.

${ }^{92}$ Zur Theorie der policy networks siehe Waarden, Frans van (1992): Dimensions and Types of Policy Networks, in: European Journal of Political Research, 21, S. 29-52, sowie die Ausführungen in Kapitel 4. 
Stelle Art und Anzahl der Netzwerkteilnehmer, die Machtverteilung innerhalb des Netzwerkes sowie die Phase des politischen Prozesses, in welcher das Netzwerk agiert, genannt. Wie die bereits oben erwähnten kulturellen Prädispositonen der am Entscheidungsprozeß Beteiligten, so steht auch die spezifische Ausprägung der nationalen Politiknetzwerke in einem engen Kontext mit der Ausgangsthese dieser Arbeit. Der Politikstil der Entscheidungsträger wird nicht nur durch kulturelle Faktoren bestimmt, sondern er ergibt sich oft auch aus der besonderen Ausgestaltung der den politischen Proze $B$ dominierenden Entscheidungsgremien. Wenn also beispielsweise strukturell ein machtpolitisches Übergewicht einer Seite innerhalb der Netzwerke besteht, dann wird sich dies sicherlich auch auf die Art und Weise niederschlagen, in welcher dieser mächtigere Akteur mit den anderen umgeht. Andererseits ist aber auch zu vermuten, daß sich kulturell determinierte Prädispositonen der Entscheidungsträger bezüglich der Art und Weise, wie Entscheidungen $\mathrm{zu}$ treffen seien, auf die Gestaltung der Netzwerkstrukturen auswirken. Wenn also das Konsensprinzip innerhalb eines Staates wertemäßig stark verankert ist, so wird sich dies sicherlich auch insofern in der Gestaltung der nationalen Politiknetzwerke niederschlagen, als daß die Machtverteilung unter den Betroffenen eher gleichmäßig erfolgt.

Diese kurzen Überlegungen verdeutlichen die Interdependenz des Faktors Struktur der nationalen Politiknetzwerke sowie des Konstrukts Politikstil. Die Abgrenzung dieser Faktoren sowie die Berücksichtigung ihrer Wechselwirkungen erfolgt ausführlich in Kapitel 4 dieser Untersuchung.

\subsection{Situative Faktoren}

Als situative Faktoren subsumieren wir solche Ereignisse, welche erstens außerhalb der direkten Beeinflußbarkeit durch die Regierung des betroffenen Staates liegen, und zweitens zu kurz- oder zumindest mittelfristigen und unmittelbaren Änderungen der Werte der Umweltbelastungsindikatoren führen ${ }^{93}$.

$\mathrm{Zu}$ dieser Kategorie der Determinanten gehören katastrophale oder skandalöse Ereignisse wie zum Beispiel Unfälle oder Fälle von Umweltkriminalität. Ihre Wirkung entfalten diese Ereignisse zum einen durch sofortige Maßnahmen der Exekutive und zum anderen durch eine Sensibilisierung der gesellschaftlichen Perzeption der Umweltproblematik. So können die-

${ }^{93}$ Vgl. Kern, Kristine, und Bratzel, Stefan (1994): Erfolgskriterien . . ., a.a.O., S. 21 f. 
se situativen Geschehnisse durch die mediale Vermittlung auf die politische Agenda gelangen und durch ein Ingangsetzen des politischen Prozesses und einen entsprechenden Output zu durchaus langfristigen Veränderungen der umweltpolitischen Realität einer Gesellschaft beitragen. Ein gutes Beispiel für diesen Mechanismus ist der Unfall im ukrainischen Atomkraftwerk Tschernobyl, welcher der entscheidende Faktor zur zumindest längerfristigen Abkehr von dieser Art der Energieerzeugung in Deutschland war.

$\mathrm{Zu}$ den situativen Faktoren sind außerdem Ereignisse wie Embargos oder starke und plötzliche Preisänderungen für Energieträger zu zählen. Diese Geschehnisse wirken vor allem durch eine Änderung des künftig genutzten Energiemixes sowie durch Umstellungen des industriellen Produktionsprozesses. Das Öl-Embargo und der Preisschock der 70er Jahre haben somit möglicherweise mehr zu einer Senkung der Energieintensität in den westlichen Industrieländern beigetragen als der Einsatz sämtlicher umweltpolitischer Instrumentarien.

Auch singuläre Ereignisse wie die deutsche Einheit und die mit ihr verbundene Umweltunion können einen wichtigen situativen Faktor der Umweltpolitik darstellen. So wurde die Umweltpolitik in den neuen Ländern nach der Vereinigung primär durch die ökologische Katastrophe der ehemaligen DDR bestimmt. Statt auf langfristiger Strukturpolitik lag der Schwerpunkt staatlicher Maßnahmen deshalb zunächst auf Sofortmaßnahmen der Stillegung und Gefahrenabwehr für die Gesundheit der Bevölkerung ${ }^{94}$.

${ }^{94}$ Zimmermann, Klaus W. (1992): Umweltpolitische Perspektiven . . ., a.a.O., S. 3-13. 


\section{Das Politikstil-Konzept im Kontext der Policy-Analyse}

\subsection{Policy-Analyse}

Die thematische Beschäftigung mit dem Begriff des Politikstils (Policy Styles) setzt eine exakte definitorische Bestimmung dieses politikwissenschaftlichen Konzeptes, sowie dessen zutreffende kontextuale Einordnung in übergreifende oder verwandte Analysekonzepte voraus.

$\mathrm{Zu}$ diesem Zweck erfolgt zunächst eine Darstellung der Policy-Analyse als einer Methode, welche ,die Art und Weise staatlicher Aktivitäten, die Problembewältigung und ihre Instrumente ${ }^{\text {،95 }}$ u.a. auch zum Zwecke des internationalen Vergleiches ${ }^{96}$ untersucht.

Die zum Verständnis des politischen Gestaltungsprozesses besonders wichtigen Beziehungen der an der Problemlösung beteiligten Akteure sowie deren konkrete strukturelle Ausgestaltung werden im PolicyNetwork-Konzept thematisiert.

Zum Zwecke einer zeitlichen Differenzierung und um dem Input-OutputCharakter politischer Prozesse zu entsprechen, bedient sich die PolicyAnalyse eines Phasen-Modells, auch als Policy-Zyklus (Policy Cycle) bezeichnet, welches die Geschichte einer Policy anhand einzelner Entwicklungsstadien nachzeichnet ${ }^{97}$. Der Darstellung des Policy-Cycle-Konzeptes ist der letzte Abschnitt dieses Kapitels gewidmet.

\subsubsection{Gegenstände und Methoden der Policy-Analyse}

Aus der Ablehnung des begrifflich eindimensionalen und inhaltlich vieldeutigen deutschen „Politik"-Begriffes hat sich in der Politikwissenschaft eine aus dem angelsächsischen Sprachraum entlehnte Differenzierung von Politik in die unterschiedlichen Dimensionen Polity, Politics und Policy durchgesetzt ${ }^{98}$.

95 Jann, Werner (1995): Politikfeldanalyse, In: Nohlen, Dieter (Hg.): Wörterbuch Staat und Politik, Bonn, S. 551.

${ }^{96}$ Vgl. Schubert, Klaus (1991): Politikfeldanalyse, Opladen.

97 Berg-Schlosser, Dirk, und Stammen, Theo (1992): Einführung in die Politikwissenschaft, München, S. 217. 
Der Begriff Polity beschreibt den institutionellen Aspekt ${ }^{99}$ des politischen Systems, also den konkreten strukturellen und formalen Rahmen, in dem die staatlichen Entscheidungsträger agieren. Basis dieser institutionellen Dimension ist die Verfassungsordnung eines Staates ${ }^{100}$. Ihren sichtbaren Ausdruck findet sie in den Organen der politischen Willensbildung und Administration. Die in der Politikwissenschaft am häufigsten thematisierten Institutionen sind dabei die Parlamente, Regierungsbürokratien und Parteien $^{101}$.

Die prozessuale Dimension von Politik wird im angelsächsischen Sprachraum mit dem Begriff Politics bezeichnet ${ }^{102}$. Analysegegenstände sind dabei die aus Interessenkonkurrenz entspringenden Konflikte, die Mechanismen zu deren Regelung sowie die Frage, wer seine Interessen, Ziele oder Inhalte letztlich durchsetzt. Zentrale Begriffe der Betrachtung von Politics sind deshalb auch Macht, Konsens und Legitimität ${ }^{103}$.

Drittes Element einer umfassenden Betrachtung von Politik ist die auch als Policy bezeichnete inhaltliche Dimension. In der Policy-Forschung geht es also um die "Art und Weise staatlicher Aktivitäten", d.h. um die Frage ,wie und mit welchen Instrumenten der Staat die gesellschaftlichen Probleme bearbeitet "104. Somit sind also Entscheidungsinhalte öffentli-

${ }^{98}$ Vgl. Jann, Werner (1987): Policy, In: Nohlen, Dieter, und Schultze, Rainer-Olaf (Hg.): Politikwissenschaft, a.a.O., S. 702; Windhoff-Héritier, Adrienne (1987): Policy-Analyse. Eine Einführung, Frankfurt; New York, S. 17; Berg-Schlosser, Dirk, und Stammen, Theo (1992): Einführung ..., a.a.O., S. 33.

${ }^{99}$ Vgl. Pihan, Andreas (1995): Der Policy Cycle der Pflegeversicherung - Zur Bedeutung von Politiknetzwerken, Dissertation, Hamburg, S. 47.

${ }^{100}$ Vgl. Alemann, Ulrich von (1995): Politikbegriffe, In: Nohlen, Dieter (Hg.): Wörterbuch Staat und Politik, Bonn, S. 544.

${ }^{101}$ Vgl. Hartmann, Jürgen (1995): Vergleichende . ., a.a.O., S. 45.

${ }^{102}$ Vgl. ebd; Berg-Schlosser, Dirk, und Stammen, Theo (1992): Einführung . ., a.a.O., S. 33; Jann, Werner (1987): Politics, In: Nohlen, Dieter, und Schultze, Rainer-Olaf (Hg.): Politikwissenschaft, a.a.O., S. 704.

${ }^{103}$ Vgl. ebd.

104 Jann, Werner (1987): Policy, In: Nohlen, Dieter, und Schultze, Rainer-Olaf (Hg.): Politikwissenschaft, a.a.O., S. 702. 
cher Organisationen gemeint ${ }^{105}$. Diese Beschränkung auf Public Policy (,whatever governments choose to do or not do "106), die in den Definitionen der beiden Policy-Pioniere Laswell und Dye angelegt ist, muß laut Windhoff-Héritier dann aufgehoben, und private Entscheidungen in die Analyse einbezogen werden, „wenn es um die Durchführung dieser Entscheidungen geht und gesellschaftliche Organisationen, Gruppen und Individuen beteiligt sind. "107 In Anlehnung an Blankenburg/Schmid/Treiber definiert sie Policy als "politische[n] Entwurf, Plan oder Programm und Einzelentscheidung zur Gestaltung gesellschaftlicher Verhältnisse, als Strategie und konkretes Handeln ".

Die Policy-Analyse befaßt sich mit der konkreten inhaltlichen Ausgestaltung staatlicher Policies in verschiedenen Politikbereichen, mit den Folgen und Wirkungen eben dieser Policies sowie mit den Faktoren, welche den Unwandlungsproze $\beta$ von politischen Programmen (Output) in tatsächliche, auch nicht-intendierte Wirkungen (Outcomes) beeinflussen ${ }^{109}$. Da zu den dabei von der Policy-Analyse betrachteten Faktoren außer den unterschiedlichen parteipolitischen Zusammensetzungen der Regierungen auch unterschiedliche nationale Regimetypen zählen ${ }^{910}$, ist eine der Methoden dieses Teilgebietes der Politikwissenschaft der internationale Politikvergleich.

Der historische Ursprung der Policy-Analyse liegt in den Arbeiten der Policy Sciences in den USA der 50er Jahre. Die Pioniere dieser For-

${ }^{105}$ Lasswell, Harold D. (1971): A Pre-View of Policy Sciences, New York, S. 1.

106 Dye, Thomas R. (1976): Policy Analysis: What Governments Do, Why They Do it, and What Difference it Makes, Alabama, S. 1.

${ }^{107}$ Windhoff-Héritier, Adrienne (1987): Policy-Analyse, a.a.O., S. 18.

${ }^{108}$ Ebd. in Anlehnung an Blankenburg, Erhard; Schmid, Günther, und Treiber, Hubert (1974): Von der reaktiven zur aktiven Politik? Darstellung und Kritik des Policy Sciences-Ansatzes, in: Grottian, Peter, und Murswieck, Axel (Hg.): Handlungsspielräume der Staatsadministration, Hamburg, S. 35-51.

${ }^{109}$ Vgl. Jann, Werner (1995): Politikfeldanalyse, a.a.O., S. 551.

${ }^{110}$ Vgl. Berg-Schlosser, Dirk, und Stammen, Theo (1992): Einführung . . ., a.a.O., S. 216. 
schungsrichtung, Lerner, Laswell, Dye sowie Dror, verfolgten stets zwei Absichten. Neben der analytisch-beschreibenden Funktion von Entscheidungsprozessen der Politik ging es im Rahmen einer eher präskriptivnormativen Orientierung auch um wissenschaftliche Politikberatung, welche durch die Evaluation der Implementation staatlicher Politik den politischen Entscheidern für zukünftige Politikgestaltung die relevanten Informationen bereitstellt ${ }^{111}$. Die deutsche Policy-Forschung definiert die Policy-Analyse heute nahezu ausschließlich als deskriptiv-analytische Forschung zur Erstellung von Entscheidungs- und Wirkungsanalysen ${ }^{112}$.

\subsubsection{Policy Networks}

Ein weiteres wichtiges Konzept der Policy-Analyse, dessen Kenntnis zum Verständnis politischer Gestaltungsprozesse notwendig ist, ist das der Policy Networks (Politiknetzwerke).

Ein Policy Network ist definiert als die institutionalisierte, protoorganisatorische Gesamtheit der Beziehungen zwischen den konkreten, an einem politischen Problemlösungsprozeß beteiligten Akteuren, also meist dem Staat und nicht-staatlichen Interessenvertretern ${ }^{113}$. Institutionalisierung bedeutet, daß die Beziehungen nicht auf zufälligem Zusammentreffen beruhen, sondern daß es gewisse (eventuell auch informelle) Regeln bezüglich der Art und Weise der Kooperation gibt. Dennoch sind diese Netzwerke keine Organisationen, da ihr Grad an Formalisierung und Koordination dieser Kategorisierung nicht entspricht. Wichtige Dimensionen zur Beschreibung von Policy Networks sind u.a. Funktion, Konventionen der Interaktion, Machtverteilung sowie die Strategie der staatlichen Netzwerkteilnehmer.

${ }^{111}$ Vgl. Lerner, Daniel, und Lasswell, Harold D. (1951) (Hg.): The Policy Sciences. Recent Development in Scope and Method, Stanford; Lasswell, Harold D. (1971): A Pre-View . . ., a.a.O.; Dror, Yehezkel (1971): Ventures in Policy Sciences: Concept and Applications, New York. Siehe außerdem die weiteren Hinweise bei Pihan, Andreas (1995): Der Policy Cycle . . , a.a.O., S. 45 f., sowie Windhoff-Héritier, Adrienne (1987): Policy-Analyse, a.a.O., S. 18 f.

112 Vgl. Hartwich, Hans-Hermann (1985) (Hg.): Policy-Forschung in der Bundesrepublik Deutschland. Ihr Selbstverstăndnis und ihr Verhăltnis zu den Grundfragen der Politikwissenschaft.

${ }^{113}$ Berg-Schlosser, Dirk, und Stammen, Theo (1992): Einfulhrung . . ., a.a.O., S. 217; sowie Waarden, Frans van (1992): Dimensions ..., a.a.O., S. 1. 
Von großer Bedeutung ist die Funktion des Netzwerkes im Hinblick auf die Beziehung des Staates zu den Interaktionspartnern. Es besteht dabei ein Kontinuum des Beteiligungsausmaßes der nicht-staatlichen Akteure. So kann das Netzwerk lediglich als Instrument zur Konsultation der Betroffenen dienen, es sind jedoch auch Verhandlungs-, Koordinierungsoder Kooperationsfunktionen denkbar.

Alle diese Netzwerkfunktionen spiegeln sich in der Art der Kommunikation zwischen dem Staat und den anderen Teilnehmern wider. Mit zunehmender Einbeziehung der nicht-staatlichen Akteure verändert sich durch einen höheren Anteil von bottom-up-Informationen auch die Richtung der Einflußnahme im Netzwerk.

Für die Art der Einflußnahme durch Interessenvertreter sind dabei zwei Grundtypen zu beobachten: der Lobbyismus und die Konzertierung ${ }^{114}$. Der Lobbyismus versucht durch eher unidirektionales Einwirken auf Entscheidungsträger und Administration Resultate in seinem Sinne zu erwirken. Im Falle der Konzertierung verläuft zwar ebenfalls ein Teil der Kommunikation von den Interessenvertretern in Richtung Staat, insgesamt ist die Netzwerkstruktur jedoch eher interdependent und ausbalanciert, und die Informationsstränge und Wege der Einflußnahme verlaufen bi- oder multidirektional.

Im Hinblick auf die Interaktionsformen innerhalb des Netzwerkes ist relevant, ob politische Gegnerschaften mit dem Ziel der individuellen Interessenverwirklichung ausgetragen werden (Adversarialism), oder ob die Zusammenkünfte der Konsenssuche dienen. Eng verknüpft mit dieser Thematik ist die Frage, ob die Treffen der Netzwerkakteure das öffentliche Wohl mehren sollen (serving public interest) oder nicht (particularistic self-interest) $^{115}$.

Als wichtigste Dimension zur Beschreibung des Netzwerktypus muß die Machtverteilung gelten. Hier sind vier verschiedene Typen der distribution of power zu unterscheiden ${ }^{116}$ :

- staatliche Autonomie gegenüber den gesellschaftlichen Interessen;

\footnotetext{
${ }^{114}$ Waarden, Frans van (1992): Dimensions ..., S. 34.

115 Ebenda, S. 36.

${ }^{116}$ Ebenda.
} 
- Instrumentalisierung privater oder gesellschaftlicher Interessen durch den Staat;

- Dominanz gesellschaftlicher Interessengruppen gegenüber dem Staat (z.B. durch Lobbyismus oder Klientelismus);

- eine Machtbalance beider Seiten im Rahmen einer intensiven und konsensualen Zusammenarbeit.

Als letzte Dimension der Politiknetzwerke ist die „Strategie der staatlichen Netzwerkteilnehmer" anzuführen. Hier ist ein Kontinuum denkbar, das bei Strategien beginnt, welchen es um die Ausschaltung spezieller Interessenformationen geht, über solche, welche lediglich die staatliche Zugänglichkeit für Argumente aus dem öffentlichen Bereich demonstrieren sollen, bis hin zu Strategien mit dem Ziel der Übertragung (delegation) staatlicher Autorität.

Anhand der hier geschilderten Netzwerkdimensionen lassen sich die Interaktionen der an politischen Problemlösungsprozessen Beteiligten detailliert beschreiben, und in eine durch die Kombination der Dimensionen erzeugte Typologie einordnen. Auf diese Weise können für einzelne Staaten bzw. Staatengruppen typische Netzwerkkonfigurationen identifiziert werden.

\subsubsection{Policy Cycle}

Als wichtiges Analyseinstrument der Policy-Forschung hat sich das Konzept des Policy Cycles (oder auch: Politikzyklus) fest etabliert ${ }^{117}$. Dabei wird dem prozessualen Charakter der Politikgestaltung dadurch Rechnung getragen, daß die Entstehung einer Policy in einzelne, zeitlich differenzierte Sequenzen unterteilt wird. Diese temporale Sichtweise von Politik bietet wichtige Vorteile:

- Auch wenn sie als theoretisches Konstrukt keine Abbildung der realen Abläufe leisten kann, so wird sie doch der politischen Wirklichkeit mit im Zeitablauf veränderlichen Merkmalen (Akteursbeziehungen, Metho-

${ }^{117}$ Vgl. Jann, Werner (1995): Politikfeldanalyse, a.a.O., S. 552 f.; Windhoff-Héritier, Adrienne (1987): Policy-Analyse, a.a.O., S. 18 sowie S. 64 ff.; Almond, Gabriel A., und Powell, Jr., Bingham G. (1992): Comparative ..., a.a.O., S. 14 f.; Pihan, Andreas (1995): Der Policy Cycle ..., a.a.O., S. 51; Brewer, Garry D., und deLeon, Peter (1983): The Foundations of Policy Analysis, Homewood, S. 17 ff.; Kern, Kristine, und Bratzel, Stefan (1995): Umweltpolitischer Erfolg im internationalen Vergleich, unveröffentlichtes Manuskript, Berlin, S. 4 ff. 
den der Machtausübung, Konfliktlösungsstrategien etc.) gerechter als statische Sichtweisen.

- „Der policy cycle setzt sich [...] über die auf einzelne Institutionen fixierte Betrachtung aus der Akteursperspektive hinweg. "118

- Die Veränderung von Politikinhalten kann nur dann adäquat untersucht werden, wenn die Policy-Analyse „eine dynamische, prozeßorientierte Sicht von Policies einnimmt.

Theoretisch eingebettet ist das Policy-Cycle-Konzept in ein umfassenderes Input-Output-Modell ${ }^{120}$ (Abbildung 7). Danach wirken soziokulturelle und ökonomische Umweltbedingungen sowie das politisch-administrative System $^{121}$ als Input-Faktoren in Form von Forderungen und Unterstützungen auf das noch enger definierte Policymaking-System ${ }^{122}$, welches nur noch den staatlichen Entscheidungsapparat umfaßt, und in dem dieser Input in verbindliche Entscheidungen umgewandelt wird (Conversion), ein. Die durch das Policymaking-System getroffenen Entscheidungen wirken in Gestalt von Politikinhalten und Programmen als Policy Output steuernd auf das soziokulturelle und ökonomische Umfeld zurück. Die Wirkungen des Policy Outputs auf den jeweiligen Adressatenkreis sind die sogenannten Policy Impacts.

Die umfassenderen, auch die nicht beabsichtigten Auswirkungen staatlicher Aktivitäten auf alle Betroffenen werden schließlich als Policy Outcome bezeichnet ${ }^{123}$. Als Feedback haben die Impacts und Outcomes von

${ }^{118}$ Brewer, Garry D., und deLeon, Peter (1983): The Foundations . . ., a.a.O., S. 18 ff.

${ }^{119}$ Windhoff-Héritier, Adrienne (1987): Policy-Analyse, a.a.O, S. 64.

${ }^{120}$ Easton, David (1965): A Framework for Political Analysis, Englewood Cliffs.

${ }^{121}$ Das politisch-administrative System umfaßt alle relevanten politischen Akteure (Exekutive, Parlament, Gerichte, Parteien, Interessengruppen etc.) sowie deren Strukturen, Prozesse, Einstellungen und Beziehungen untereinander (vgl. vor allem Jann, Werner (1981): Kategorien der Policy-Forschung, Speyer, S. 18).

122 Jann definiert das Policymaking-System wie folgt: „Unter Policymaking-System werden [...] sämtliche öffentliche Institutionen und Akteure subsumiert, die mit der Entwicklung und Durchführung staatlicher Politiken beschäftigt sind. “(Ebd., S. 19)

${ }^{123}$ Zur Unterscheidung von Output, Impact und Outcome vgl. insbesondere: Jann, Werner (1981): Kategorien der Policy-Forschung, Speyer, S. 26 f. 
Politik wiederum einen Effekt auf die soziokulturellen und ökonomischen Umweltbedingungen ${ }^{124}$.

Zur Beschreibung des Konversionsprozesses, durch welchen die gesellschaftlichen Inputs in konkrete Policy Outputs umgewandelt werden, bedient sich die Policy-Analyse des Policy-Cycle-Konzeptes. Dabei unterscheidet sie meist sechs Phasen des Policy Cycles ${ }^{125}$ :

- Problemdefinition,

- Agenda-Gestaltung,

- Politikformulierung,

- Politikimplementation,

- Evaluation und

- Politik-Terminierung bzw. Neuformulierung.

Da sich das Policy-Style-Konzept ausdrücklich auf das politische Phasenmodell bezieht (diese Zusammenhänge werden im folgenden Kapitel noch ausführlich dargestellt), ein Verständnis der Bedeutung unterschiedlicher Politikstile für die Outcomes nationaler Umweltpolitik ohne dieses heuristische Analyseinstrument also nicht möglich ist, sollen die einzelnen Sequenzen der Politikgestaltung nachfolgend im einzelnen erläutert werden.

${ }^{124}$ Vgl. zum Input-Output-Modell auch jeweils: Windhoff-Héritier, Adrienne (1987): PolicyAnalyse, a.a.O, S. 18 f.; Almond, Gabriel A., und Powell, Jr., Bingham G. (1992): Comparative ..., a.a.O., S. 9 ff.; Jann, Werner (1995): Politikfeldanalyse, a.a.O., S. 553.

${ }^{125}$ Die folgende Darstellung lehnt sich terminologisch und inhaltlich weitgehend an Windhoff-Héritier, Adrienne (1987): Policy-Analyse, a.a.O, S. 64 ff., an. Anzahl, Bezeichnung und inhaltliche Festlegung der einzelnen Policy-Phasen hängen in der politikwissenschaftlichen Forschung oft vom Forschungsinteresse und -gegenstand ab, und so gibt es auch zahlreiche andere Unterteilungen. Vgl. etwa die Vier-Phasen-Modelle, die bei Jann, Werner (1995): Politikfeldanalyse, a.a.O., S. 552 f., und Almond, Gabriel A., und Powell, Jr., Bingham G. (1992): Comparative . . ., a.a.O., S. 14 f., dargestellt sind; siehe auch die bei Pihan, Andreas (1995): Der Policy Cycle . . ., a.a.O., S. 52 f., und Brewer, Garry D., und deLeon, Peter (1983): The Foundations . .., a.a.O., S. 17, Fußnote 55, angefuhrten weiteren Phasenmodelle (vor allem die von Anderson, James E. (1979): Public Policymaking, Chicago; San Franzisko, sowie Lasswell, Harold D. (1956): The Decision Process, Maryland). 
Abb. 7: Politikstil und Policy Cycle als Elemente des Input-Output-Modells

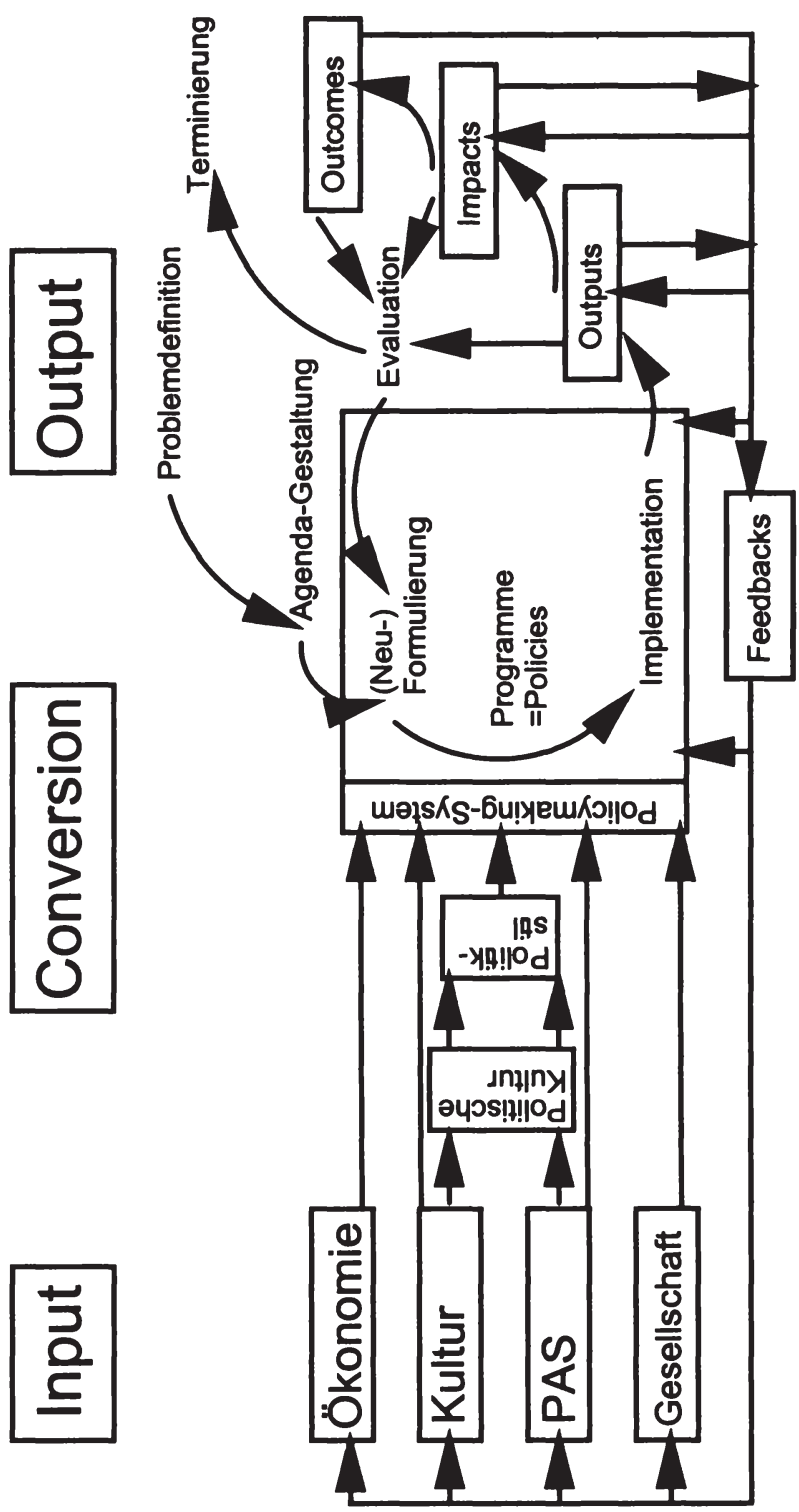




\subsubsection{Problemdefinition}

Ein Policy-Problem entsteht aus der „Überzeugung, daß ein gesellschaftliches Problem politisch-administrativ zu bewältigen " ${ }^{26}$ ist. Zuständig für die Problemdefinition oder -wahrnehmung sind keine institutionellen Strukturen, sondern es sind vielmehr die politischen Forderungen unterschiedlicher gesellschaftlicher Gruppen, die eine Thematik als handlungsrelevant erscheinen lassen oder nicht. Ob eine solche Thematik dann tatsächlich als Policy-Problem definiert wird, bestimmen normgeleitete Auswahlprozesse, in denen die Interessengruppen mit dem größten politischen Durchsetzungsvermögen auch über den größten Einfluß bei der Auswahl verfügen. Motive der Akteure in der Phase der Problemdefinition, also der Phase, in welcher die handlungsrelevanten Aspekte des politischen Prozesses festgelegt werden, können sein: „Verteilungskämpfe“ zwischen Gruppen, das persönliche Nutzenkalkül von Politikern, Gemeinwohlorientierung, Anstöße durch Medienberichterstattung oder Konzentration auf ein einziges Thema durch eine „single issue group “127. Für die Wahrnehmung umweltpolitischer Probleme scheint es vor allem zwei begünstigende Faktoren zu geben ${ }^{128}$ : die Struktur der Problematik ${ }^{129}$ sowie die Problemdeutungs- und -vermittlungsfähigkeiten von ökologischen Interessengruppen.

${ }^{126}$ Windhoff-Héritier, Adrienne (1987): Policy-Analyse, a.a.O, S. 68.

${ }^{127}$ Ebd., S 68 f.

${ }^{128}$ Kern, Kristine, und Bratzel, Stefan (1995): Umweltpolitischer Erfolg . ., a.a.O., S. 4 ff.

${ }^{129}$ Ein ökologisches Problem wird insbesondere dann als handlungsrelevant wahrgenommen, wenn es (1) meßbar, (2) durch spektakuläre Einzelfälle illustrierbar und (3) darüber hinaus zeitlich und räumlich generalisierbar ist (Vgl. Solesbury, William (1976): Issues and Innovation in Environmental Policy in Britain, West Germany, and California, in: Policy Analysis, 2, S. 14ff., sowie Enloe; Cynthia H. (1975): The Politics of Pollution in a Comparative Perspective. Ecology and Power in Four Nations, New York, S. 321). 


\subsubsection{Agenda-Gestaltung}

Die Agenda-Gestaltung (auch als Agenda setting bezeichnet) ist eine Art Filter, in dem die wahrgenommenen Probleme selektiert und der formal verbindlichen Entscheidungsfindung durch die politisch administrativen Organe, also der Politikformulierung, zugeführt werden.

Eine gebräuchliche Definition des Agenda-Begriffes liefern $\mathrm{Cobb}$ und $\mathrm{El}$ der als ,diejenigen Fragen, die für die politischen Entscheider zur aktiven und ernsthaften Behandlung anstehen. " 130

Bei den Voraussetzungen für ein erfolgreiches Agenda setting ist zwischen einem instrumentellen und einem normativen Aspekt zu unterscheiden.

So braucht es zum einen ein notwendiges $\mathrm{Maß}$ an politischer Macht zur Gewinnung von Schlüsselakteuren und Behörden (instrumenteller Aspekt) und zum anderen eine weitgehende Deckung des PolicyAñliegens mit dem normativen Konsensus des politischen Systems (kulturell-normativer Aspekt), um eine Idee auf die Agenda zu setzen. Als wichtigste mögliche Hindernisse bei der Einreihung eines Problems in den Kreis der entscheidungswürdigen Fragen sind gemäß Downs zu nennen ${ }^{131}$ :

- geringes Problembewußtsein der breiten Bevölkerung,

- das Tangieren der Interessen einflußreicher Gruppen sowie

- das Nachlassen des Medieninteresses.

Gerade für die Wahrnehmung und Bearbeitung von Umweltthemen können sich diese Faktoren als hinderlich erweisen. Andererseits ist die Wahrscheinlichkeit einer aktiven und ernsthaften Bearbeitung von ökologischen Problemen durch das politisch-administrative System im Falle einer thematischen und institutionellen Offenheit der politischen Strukturen besonders hoch ${ }^{132}$. D.h. wenn zum einen die politischen Werte und Einstellungen der relevanten Akteure gegenüber Umweltthemen positiv sind und zum anderen für Umweltgruppen und -parteien institutionalisier-

${ }^{130}$ Cobb, Roger W., und Elder, Charles D. (1983): Participation in American Politics: The Dynamics of Agenda-Building, Boston, S. 86.

131 Downs, Anthony (1972): Up and Down with Ecology - The Issue-Attention-Cycle, in: Public Interest, 28, S. $42 \mathrm{f}$.

${ }^{132} \mathrm{Vgl}$. Solesbury, William (1976): Issues . ., a.a.O. 
te Wege des Zugangs zur politischen Arena bestehen, sind die Chancen für eine Wahrnehmung von Umweltproblemen groß.

\subsubsection{Politikformulierung}

Als Ergebnis der Informationsgewinnung und -verarbeitung und der Prozesse von Konfliktregelung und Konsensbildung ${ }^{133}$ wird schließlich eine konkrete Politik formuliert, d.h.: „Eine Policy wird beschlossen.

Dieses konkrete Programm dient dann als Handlungsauftrag für die nachfolgende Phase der Implementation.

Das Ergebnis dieser Phase des Policy Cycles kann auch die Vermeidung einer Entscheidung sein. Diese non-decisions sind insbesondere im Fall inhaltlich komplexer, risikobehafteter und langfristig irreversibler Issues möglich ${ }^{135}$.

Gibt es jedoch eine Entscheidung, dann sind grundsätzlich zwei PolicyTypen zu unterscheiden: rule of law bzw. policy without law ${ }^{136}$.

Kennzeichen des ersten Typus ist eine genaue, meist mit Sanktionsandrohungen verbundene Festlegung von Ausführungsstandards in allen materiellen Details.

Im Falle einer policy without law überläßt die Legislative die materielle Ausgestaltung von Rahmenentscheidungen den Verwaltungen. Die Vorteile solcher Lösungen, die im umweltpolitischen Kontext oft mit Begriffen wie "nach dem Stand der Technik" verbunden sind, liegen für die Gesetzgeber darin, daß sie den Zorn von eventuellen Verlierern der politischen Auseinandersetzung nicht fürchten müssen, und sich gleichzeitig den Anschein reformorientierter, aktiver Politik geben können. Aus demokratietheoretischer Sicht mag diese Verschiebung der Gestaltungsmacht von der Legislative hin zur Verwaltung zwar problematisch sein, doch für die praktische Ausgestaltung der so gefundenen Policies sind auch positive Effekte denkbar. Gerade für Umweltthemen dürfte nämlich die folgende Feststellung Windhoff-Héritiers Gültigkeit besitzen ${ }^{137}$ : „In

${ }^{133}$ Vgl. Jann, Wemer (1995): Politikfeldanalyse, a.a.O., S. 554.

${ }^{134}$ Windhoff-Héritier, Adrienne (1987): Policy-Analyse, a.a.O, S. 74.

${ }^{135}$ Vgl. Brewer, Garry D., und deLeon, Peter (1983): The Foundations . . ., a.a.O., S. 19.

${ }^{136}$ Vgl. Lowi, Theodore J. (1969): The End of Liberalism, New York, S. 125.

${ }^{137}$ Windhoff-Héritier, Adrienne (1987): Policy-Analyse, a.a.O, S. 84. 
der nicht unbegründeten Hoffnung, daß problemgerechte Lösungen eher in enger Zusammenarbeit mit den betroffenen Bürgern als aus der Ferne der Zentralregierung zu [finden] sind, werden nachgeordneten Verwaltungsinstitutionen Gestaltungskompetenzen eingeräumt." In diese Richtung deuten auch die Schlußfolgerungen Knoepfels und Weidners in ihrer internationalen Vergleichsstudie über die Luftreinhaltepolitik ${ }^{138}$ : „Clean air policies tend to be more successful if they leave to the regional implementation agencies a consideration leeway of political and administrative or technical discretion."

Diese Ausführungen verweisen bereits auf die in der Praxis von der Politikformulierung nicht klar zu trennende Phase der Politikimplementation.

\subsubsection{Politikimplementation}

„Implementation is the execution of the selected option ... "139 Eine präzisere Definition der ,Umsetzung politischer Programme in die Wirklichkeit "140 findet sich wiederum bei Windhoff-Héritier ${ }^{141}$ : „Als Implementation [...] wird in der Politikwissenschaft die Durchführung von rechtsverbindlichen Entscheidungen bezeichnet, die durch politische und administrative Organe beschlossen wurden [und], in vager und präziser Weise formuliert, [...] unter Beteiligung verschiedener öffentlicher und privater Organisationen, von Gruppen und Einzelpersonen mit durchaus divergierenden Zielen, konflikthaft oder konsensual, schematisch oder frei gestaltend in die Praxis umgesetzt [werden]. "

Begünstigt wird der Implementationsverlauf laut empirischen Implementationsstudien vor allem von zwei Faktoren:

- Je weniger Entscheidungsstellen ein Programm durchläuft, desto höher sind die Durchführungschancen ${ }^{142}$.

${ }^{138}$ Knoepfel, Peter, und Weidner, Helmut (1986): Explaining Differences in the Performance of Clean Air Policies: An International and Interregional Comparative Study, in: Policy and Politics, 14, S. 85 f.

${ }^{139}$ Brewer, Garry D., und deLeon, Peter (1983): The Foundations . ., a.a.O., S. 19.

${ }^{140}$ Vgl. Jann, Werner (1995): Politikfeldanalyse, a.a.O., S. 553.

${ }^{141}$ Windhoff-Héritier, Adrienne (1987): Policy-Analyse, a.a.O, S. 86.

${ }^{142}$ Vgl. Pressman, Jeffrey, und Wildavsky, Aaron B. (1973): Implementation, Berkeley. 
- Je höher die durch die Durchführungsorganisation investierten Ressourcen, desto größer der Programmerfolg ${ }^{143}$.

Die besondere Problematik der Implementationsphase liegt darin, daß das konkrete politische Handeln durch die in der Formulierungsphase festgelegten Programme und Zielvorgaben meist nicht endgültig festgelegt ist und ,daher in dieser Phase politische Programme und deren Intentionen verzögert, verändert oder sogar vereitelt werden können. “144

Insbesondere Konflikte, die in der Phase der Politikformulierung nicht endgültig ausgetragen wurden, treten in der Implementationphase wieder auf. Oder anders formuliert: werden politische Programme präzise formuliert, so ist in der Implementationsphase mit weniger Auseinandersetzungen zu rechnen.

$\mathrm{Da}$ im Implementationsprozeß die tatsächliche Kosten-Nutzen-Verteilung erfolgt, sind die Versuche politischer Einflußnahme in dieser Phase besonders intensiv. Die konfliktär oder konsensual ausgeprägten Akteursbeziehungen, die letztlich den Erfolg dieser Interventionsversuche bestimmen, werden im struktur- bzw. akteurorientierten Ansatz der Implementationsforschung mit dem Instrument der Netzwerkananlyse untersucht $^{145}$.

Die wichtigsten Netzwerktypen sind in diesem Kontext die pluralistische und die korporatistische Variante ${ }^{146}$.

Der im folgenden verwendete Pluralismusbegriff meint nicht den normativen Ansatz einer inhaltlich und strukturell vielfältigen (,pluralistischen") Demokratie als Gegenkonzeption zu totalitär-diktatorischen Systementwürfen ${ }^{147}$, sondern den primär im angelsächsischen Sprachraum akzeptierten Ansatz einer deskriptiven Berücksichtigung der innerhalb des politischen Systems „vorhandenen, mit ungleicher Durchsetzungsfä-

${ }^{143}$ Vgl. Meter, Donald S. van, und Horn, Carl E. van (1975): The Policy Implementation Process. A Conceptual Framework, in: Administration and Society, S. 445-488.

${ }^{144}$ Vgl. Jann, Werner (1995): Politikfeldanalyse, a.a.O., S. 553.

${ }^{145}$ Windhoff-Héritier, Adrienne (1987): Policy-Analyse, a.a.O, S. 92 ff.

${ }^{146} \mathrm{Vgl}$. ausführlich $\mathrm{zu}$ diesen beiden sowie weiteren Varianten die Typologie bei Waarden, Frans van (1992): Dimensions ..., a.a.O.

147 Vgl. Eisfeld, Rainer (1995): Pluralismus/Pluralismustheorie, In: Nohlen, Dieter (Hg.): Wörterbuch ..., a.a.O., S. 540. 
higkeit (Macht) ausgestatteten Meinungs-, Interessen- und Organisations-(Gruppen- bzw. Verbands-)Vielfalt. " ${ }^{488}$ Kennzeichen pluralistischer Netzwerkstrukturen sind ein relativ geringer Grad selbständiger Interessenkonzentration der Betroffenen sowie ein zurückhaltend agierender, eher „schwacher" Staat ${ }^{149}$. Somit ist der politische Einfluß abhängig vom Ausmaß der individuellen oder Pressure-Group-Macht, und als Resultat des politischen Prozesses werden einzel- oder gruppenspezifische Ziele realisiert.

Als Korporatismus bezeichnet man „die Beteiligung von Interessengruppen an der Politik, und zwar durch wechselseitige Organisationsbeziehungen zwischen Regierung und politischer Verwaltung einerseits und starken, zentralisierten Verbänden andererseits. "150

Ein besonderes Merkmal korporatistischer Netzwerkstrukturen liegt darin, daß den nicht-staatlichen Akteuren auch Befugnisse im Bereich der Politik-Implementation zugestanden werden, d.h. der Staat berücksichtigt nicht nur die Interessen einer bestimmten Klientel bei seinem Handeln, sondern er macht diese Klientel zum kooperativen Partner des politischen Prozesses. Dadurch werden zum einen die durch die Implementation einer Politik eventuell entstehenden Konfliktkosten minimiert und zum anderen der Teilnehmer des korporatistischen Netzwerkes in die Verantwortung für das Politikergebnis einbezogen. Weitere Strukturmerkmale sind: Stabilität, Institutionaliserung, Zentralität und Konsensorientierung. Als allgemeines Charakteristikum korporatistischer Netzwerkstrukturen ist die Mäßigung interessenpolitischer Forderungen durch kooperative Konfliktregulierung zu bezeichnen.

148 Ebd. S. 537; vgl. auch Waarden, Frans van (1992): Dimensions . . ., a.a.O, S. 44, sowie Pihan, Andreas (1995): Der Policy Cycle . . ., a.a.O., S. 65.

${ }^{149}$ Vgl. Bull, Martin J. (1992): The Corporatist Ideal-Type and Political Exchange, in: Political Studies. XL, S. 265.

${ }^{150}$ Streeck, W., und Schmitter, P.C. (1991): From National Corporatism to Transnational Pluralism: Organized Interests in the Single European Market, in: Politics and Society, 19, S. 135, zit. nach Czada, Roland (1995): Korporatismus/Neo-Korporatismus, In: Nohlen, Dieter (Hg.): Wörterbuch . . ., a.a.O., S. 365. Zum Korporatismus siehe grundlegend auch: Lehmbruch, Gerhard (1977): Liberal Corporatism and Party Government, in: Comparative Political Studies, 10, S. 91-126; Pryor, Frederic, L. (1988): Corporatism . . . a.a.O.; Czada, Roland (1983): Korporatismus, In: Schmidt, Manfred G. (Hg.): Westliche Industriegesellschaften. Wirtschaft - Gesellschaft - Politik, München, S. 209-217. 
In Anlehnung an das Korporatismuskonzept kommt auch die international vergleichende Umweltpolitikanalyse zu dem Ergebnis, daß nicht pluralistische, sondern kooperative Netzwerkstrukturen den Politikerfolg erhöhen ${ }^{151}$. So betont Vogel in seiner Studie über die Styles of Regulation in Großbritannien und den USA, daß die britische Regierung Regulationsstandards auf einem ähnlichen Niveau wie die USA, aber mit erheblich geringeren Kosten erreicht hat, indem sie kooperative governmentindustry relations praktizierte ${ }^{152}$, während die Netzwerkbeziehungen in der pluralistischen amerikanischen Gesellschaft extrem konfliktären Charakter haben.

Knoepfel bezieht auch die Umweltorganisationen in die Netzwerkanalyse mit ein, und führt als eine der Bedingungen für die wirksame Umsetzung umweltpolitischer Programme in Westeuropa die Kooperation eines iron triangle aus Regierung, Umweltverschmutzern und Umweltschutzgruppen $a^{153}$. Laut Jänicke und Weidner kann dieses Netzwerk manchmal sogar auf die Betrachtung der Beziehung zwischen nur zwei Partnern reduziert werden: ,environmentalists and polluters. “154

\subsubsection{Evaluation}

Die Evaluation von Policies ist primär ein Ex-post-Vergleich von erwarteten bzw. angestrebten Policy-Wirkungen mit den tatsächlich eingetretenen Resultaten ${ }^{155}$. Die Maßgröße für diesen Bewertungsvorgang kann entweder ein eher allgemein definiertes Ziel oder ein spezifisches, meßbares Kriterium wie z.B. Effektivität, Effizienz oder Verteilungsgerechtigkeit sein.

${ }^{151}$ Kern, Kristine, und Bratzel, Stefan (1995): Umweltpolitischer Erfolg . . ., a.a.O., S. 17 ff.

${ }^{152}$ Vogel, David (1986): National Styles ..., a.a.O., S. $21 \mathrm{ff}$.

${ }^{153}$ Knoepfel, Peter (1993): Bedingungen einer wirksamen Umsetzung umweltpolitischer Programme - Erfahrungen aus Westeuropäischen Staaten, Lausanne.

${ }^{154}$ Jänicke, Martin, und Weidner, Helmut (1995): Successful . . ., a.a.O., S. 21.

${ }^{155}$ Vgl. Brewer, Garry D., und deLeon, Peter (1983): The Foundations . . ., a.a.O., S. 20 bzw. $319 \mathrm{ff}$. 
Ein weiteres Thema der Evaluation ist die Verantwortlichkeit im Falle von Abweichungen zwischen den gewollten und den realen PolicyWirkungen.

Neben dem Evaluationstyp der formellen wissenschaftlichen Untersuchung ${ }^{156}$ gibt es auch die informelle Evaluation. Diese ist die praktisch wohl am meisten verbreitete Form der Policy-Bewertung und gibt oft erst den Ansto $ß \mathrm{zu}$ weiteren, formellen Evaluationsanstrengungen:

". . . if evaluation is thought of very generally as mere information about ongoing activities to responsible officials, then the innumerable informal evaluations of this type plainly dominate: they are the most common, the least expensive, the least disruptive (under normal circumstances), and in toto probably the most effective type.

Zwar gibt es keine empirischen Studien, welche Faktoren aufzeigen, die sich in der Evaluationsphase umweltpolitischer Policies günstig auswirken, doch ist zu vermuten, daß es aufgrund der komplexen und wissenschaftlich anspruchsvollen Thematik ökologischer Probleme ähnliche Faktoren wie in den Phasen der Problemdefinition und der AgendaGestaltung sind, welche informelle, und daran anschließend schließlich auch formelle Evaluationsbemühungen in Gang setzen, nämlich die Problemdeutungs- und -vermittlungsfähigkeiten der ökologischen Interessengruppen sowie die thematische und institutionelle Offenheit des Systems.

\subsubsection{Politikterminierung bzw. Neuformulierung}

Als letzte Sequenz des Policy Cycles folgt auf die Evaluation in zahlreichen Fällen die Beendigung einer Policy. Die Politikterminierung als Reaktion auf die Tatsache, da $\beta$ die mit dem Programm intendierte Problemlösung erreicht wurde, der Policy-Zweck also erfüllt ist, stellt eher die Ausnahme dar ${ }^{158}$.

${ }^{156}$ Windhoff-Héritier, Adrienne (1987): Policy-Analyse, a.a.O, S. 65.

${ }^{157}$ Brewer, Garry D., und deLeon, Peter (1983): The Foundations . . ., a.a.O., S. 20 bzw. S. 345.

${ }^{158}$ Vgl. Windhoff-Héritier, Adrienne (1987): Policy-Analyse, a.a.O, S. 107. 
In den meisten Fällen sind finanzielle, politisch-ideologische und effektivitätsorientierte Motive der Anlaß zur Programmbeendigung ${ }^{159}$. Ähnlich formulieren Brewer und deLeon ${ }^{160}$ : „Termination generally refers to the adjustment of policies and programs that have become dysfunctional, redundant, outmoded, unnecessary, or even counterproductive [...] a sense of finality is easily seen in the concept. " Der hier zusätzlich angeführte Aspekt der Finalität von Politik-Terminierung ist allerdings zu relativieren, da eine komplette, ersatzlose Einstellung einer Policy, und nur dies würde das tatsächliche Ende des Policy Cycles bedeuten, meist nicht erfolgt. Vielmehr werden oft nur Änderungen an einzelnen Programmelementen vorgenommen, und mit dieser partiellen Politikneuformulierung der Politikzyklus neu in Gang gesetzt ${ }^{16}:$, [...] termination is frequently only the replacement of one set of practices with another: Termination signals a beginning of the policy process as much as it does its end." Von Terminierungsabsichten besonders betroffen sind Policies mit geringer politischer und administrativer Unterstützung. Deren Merkmale sind häufig:

- Mangelnde Exklusivität der Policy-Leistungen.

- Wahrnehmung der Problemdauer als eher kurzfristig.

- Mangelnde normative oder soziale Akzeptanz des Problems oder der Betroffenen.

- Policy-Alter ${ }^{162}$.

Aus umweltpolitischer Sicht können vor allem der erste und der letzte Punkt problematisch sein. Der Querschnittcharakter ökologischer Probleme findet sich so auch in den jeweiligen Policies und Programmen wieder, d.h. die aus subjektiver Sicht positiven Wirkungen (z.B. Reduzie-

${ }^{159} \mathrm{Zu}$ politisch-ideologischen Motiven vgl. deLeon. Peter (1983): Policy Evaluation and Program Termination, In: May, Judith V., und Wildavsky, Aaron B. (Hg.): The Policy Cycle, London u.a., S. 634; zit. nach Windhoff-Héritier, Adrienne (1987): Policy-Analyse, a.a.O, ebd.

${ }^{160}$ Brewer, Garry D., und deLeon, Peter (1983): The Foundations ..., a.a.O., S. 385.

${ }^{161}$ Ebd.; siehe auch Brewer, Garry D. (1978): Termination: Hard Choices - Harder Questions, in: Public Administration Review, 4, S. 338-344.

${ }^{162}$ Im Falle finanzieller Engpäße der öffentlichen Kassen oder politisch-ideologischer Akzentverschiebungen aufgrund von Regierungswechseln, sind eher junge Programme gefährdet, da diese noch keine traditionelle und einflußreiche Klientel von Leistungsempfängern schaffen konnten. 
rung von Emissionen) umweltpolitischer Maßnahmen verteilen sich oft gleichmäßig und kaum wahrnehmbar auf die gesamte Bevölkerung. Entsprechend gering sind die Widerstände im Falle der Programmbeendigung $^{163}$.

\subsection{Das Policy-Style-Konzept}

Seit Anfang der 80er Jahre spielt in der wissenschaftlichen Diskussion der international vergleichenden Policy-Analyse unter der Grundannahme: "Nations matter" das Policy Style oder Politikstil-Konzept eine wichtige Rolle zur Erklärung national unterschiedlicher Policies, Implementationsbemühungen und Politikwirkungen (Outcomes) ${ }^{164}$.

In diesem Sinne führt Freeman aus ${ }^{165}:$,A rigorous tradition in comparative politics argues that national policymakers develop characteristic and durable methods for dealing with public issues, that these can be linked to policy outcomes, and that they can be systematically compared."

Bevor im nachfolgenden Kapitel 5 der Wirkungszusammenhang zwischen dem Politikstil als erklärender Variable einerseits und der Effektivität nationaler Environmental Policy als zu erklärender Variable andererseits genauer thematisiert wird, sollen in diesem Kapitel die begrifflichen und konzeptionellen Grundlagen gelegt werden.

Dabei geht es zunächst um die verbindliche Definition des Begriffes Politikstil. Insbesondere eine Abgrenzung vom Policy-Begriff sowie eine kontextuale Einordnung des Konzeptes in das Policy Cycle-Modell werden vorgenommen.

${ }^{163}$ Andererseits sind die von solchen Policies negativ Betroffenen (z.B. Unternehmen, die gesetzlich zu Umweltschutzinvestitionen gezwungen sind) oft gut organisiert und durchsetzungsstark, wodurch eine Besiegelung des Programmschicksals ebenfalls begünstigt wird.

${ }^{164}$ Vgl. Richardson, Jeremy, et al. (1982): Policy Styles . ., a.a.O.; Freeman, Gary P. (1985): National Styles . . ., a.a.O., S. 467-496; Feick, Jürgen, und Jann, Werner (1988): „Nations matter" - Vom Elektizismus zur Integration in der vergleichenden Policy-Forschung?, In: Schmidt, Manfred G. (Hg.): Staatstătigkeit: International und historisch vergleichende Analysen, Politische Vierteljahresschrift, Sonderheft 19, S. 196-220; Vogel, David (1986): National Styles ..., a.a.O.

${ }^{165}$ Freeman, Gary P. (1985): National Styles ..., a.a.O., S. 467. 
Im folgenden wird dann die politisch-kulturelle ${ }^{166}$ und institutionellstrukturelle ${ }^{167}$ Bedingheit der national unterschiedlichen Art und Weise, in der das politische System in die Gesellschaft interveniert, skizziert.

Den Abschluß dieses Kapitels bilden verschiedene Ansätze der PolicyForschung zur Erklärung und Darstellung nationaler Policy Styles sowie der in diesem Zusammenhang angebotenen Typologien länderspezifischer Formulierung bzw. Implementation von Policies. Die dabei verwendeten Politikstilmerkmale bilden den Anknüpfungspunkt für das nachfolgende Kapitel 5, in dem es zunächst darum gehen wird, von den möglichen Variablen zur Beschreibung von Policy Styles die umweltpolitisch relevanten auszuwählen.

\subsubsection{Begriffsklärungen}

Einer des Pioniere des Policy-Style-Konzeptes, Jeremy Richardson, definiert gemeinsam mit Gustafsson und Jordan Policy Style als

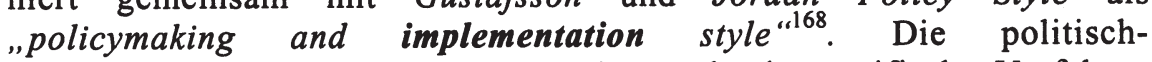
administrativen Entscheidungsträger hätten länderspezifische Verfahren (,standard operating procedures") zur politischen Problemlösung und Implementation entwickelt, d.h. es gibt , [...] main characteristics of the way in which a given society formulates and implements its public policies."

${ }^{166}$ Vgl. Mayntz, R., et al. (1982): Regulative Politik und politisch-administrative Kultur. Ein Vergleich von fünf Ländern und vier Interventionsprogrammen (Projektbericht des Instituts für angewandte Sozialforschung), Köln; Prokop, H. (1987): Koordinierung und Abschottung. Muster staatlichen Problemlösungsverhaltens in Frankreich und Italien, Dissertation, Speyer; Sturm, R. (1985): Die Politikstilanalyse. Zur Konkretisierung des Konzeptes der Politischen Kultur in der Policy-Analyse, in: Hartwich, H.-H. (Hg.): Policy-Forschung . . ., a.a.O., S. 111116.

${ }^{167}$ Vgl. Feick, Jürgen, und Jann, Werner (1988): „Nations matter“ . ., a.a.O., S. 207 ff.; Kitschelt, Herbert (1983): Politik und Energie: Energie-Technologiepolitiken in den USA, der Bundesrepublik Deutschland, Frankreich und Schweden, Frankfurt/Main; New York, S. 70 ff.

${ }^{168}$ Richardson, Jeremy; Gustafsson, Gunnel, und Jordan, Grant (1982): The Concept of Policy Style, In: Richardson, Jeremy, et al. (Hg): Policy Styles ..., a.a.O., S. 2 f. (auch Quelle der beiden folgenden Zitate; Hervorhebungen wie im Original) 
In seiner bereits oben erwähnten Definition fügt Freeman dieser Beschreibung noch den Aspekt der Dauerhaftigkeit (,durable ") hinzu ${ }^{169}$. Das Verhältnis von Politikstil einerseits und Politikinhalten (Policies) andererseits wird schließlich von Feick und Jann verdeutlicht ${ }^{170}$ : „, Wenn hier der Begriff Stil verwendet wird, geht es um „Policymaking “, d.h. um Interaktionen und Verhaltensweisen, die regelmäßig bei der Formulierung und Durchsetzung von Politikinhalten eine Rolle spielen."

Diese Definitionsversuche treffen zahlreiche explizite und implizite Grundannahmen, die im folgenden problematisiert werden sollen:

1. Bei den Policy Styles Praktizierenden handelt es sich um die politischadministrativen Eliten.

2. Policy Styles beziehen sich (vor allem) auf die Formulierungs- und Implementationsphase des Politikzyklus und sind in diesen beiden Phasen identisch.

3. Es gibt tatsächlich divergierende nationale Policy Styles.

4. Diese Policy Styles sind im Zeitablauf relativ stabil.

5. Der Policy Style-Begriff bezieht sich nicht direkt auf Politikinhalte, sondern vielmehr auf die länderspezifische Art und Weise, in der diese Policies formuliert und umgesetzt werden.

$\mathrm{Zu} 1 .:$ Die Tatsache, daß sich das Politikstil-Konzept auf den staatlichen Entscheidungsapparat, also das Policymaking-System, bezieht, wirft die Frage auf, ob der Politikstil-Begriff subjektiv-normativ oder objektivdeskriptiv verstanden werden soll. Oder anders formuliert: Ist der nationale Politikstil aus den Handlungsintentionen und -präferenzen der Eliten (wie aus Sicht der Decisionmakers gehandelt werden sollte) oder aus dem tatsächlichen praktischen Tun der am politischen Prozeß Beteiligten (wie gehandelt wird) ableitbar.

Die Relevanz dieser Fragestellung zeigt sich etwa in Haywards Darstellung des französischen Politikstils ${ }^{171}$. Der aus Sicht der staatlichen Eliten präferierte Politikstil ist aktiv und vorausschauend, rational und langfristig angelegt. Das tatsächliche Handeln der Entscheidungsträger ist jedoch als eine eher reaktive, kurzfristige und stückweise Anpassung an reale Notwendigkeiten $\mathrm{zu}$ beschreiben. D.h. die durch Erziehung und so-

${ }^{169}$ Vgl. Fußnote 179.

${ }^{170}$ Feick, Jürgen, und Jann, Werner (1988): „Nations matter“ . ., a.a.O., S. 201.

${ }^{171}$ Vgl. Hayward, Jack (1982): Mobilizing Private Interests in the Service of Public Ambitions: The Salient Element in the Dual French Policy Style?, In: Richardson, Jeremy, et al. (Hg.): Policy Styles ..., a.a.O., S. 112 ff. 
ziale Abstammung geprägte Einstellung der politischen Klasse gegenüber der Art und Weise politischer Problemlösung, ein Aspekt der Politischen Kultur $^{172}$, widerspricht dem tatsächlichen Policymaking Style. In der politischen Realität gibt es also nicht, wie von Hayward behauptet, eine Dualität widerstreitender Ansätze von Policymaking, sondern lediglich den Zustand, daß die Entscheidungsträger nicht so handeln, wie sie es selbst für richtig halten.

$\mathrm{Da}$ es in dieser Studie um den Zusammenhang von Politikstil und dessen realen Wirkungen geht, ist im folgenden auch der objektiv praktizierte, wie auch immer „meßbare“ Politikstil gemeint. Auch wenn im Sinne dieser Festlegung die subjektiven Vorstellungen der politischen Eliten über einen wünschenswerten Policymaking Style nicht mehr relevant zu seien scheinen, ist jedoch zu berücksichtigen, daß im Falle einer Konvergenz beider Stile eine höhere Effektivität des politischen Prozesses zu erwarten ist, da die Entscheidungsträger nicht ihren eigentlichen Intentionen widerstrebend handeln müssen. Diesen Zustand der weitgehenden Deckung von subjektivem und objektivem Policy Style sieht beispielsweise Anton in Schweden, dessen Politikstil er als "deliberative, rationalistic, open, consensual " beschreibt, erreicht: "... this pattern [...] represents a national style, which is itself a reflection of Swedish political culture.

Im Hinblick auf den Gegenstand dieser Studie ist im Zusammenhang mit der Frage, wer Policy Styles praktiziert, somit folgendes festzuhalten: der Environmental Policy Style beschreibt das Verhalten der staatlichen Entscheidungsträger im umweltpolitischen Bereich, also vor allem das der Regierung, des zuständigen Ministeriums, der parlamentarischen Gremien, sowie das der mit der administrativen Umsetzung von umweltpolitischen Entscheidungen betrauten Institutionen.

Zu 2.: Das wichtigste Problem im Zusammenhang mit der Einbettung des Policy-Style-Konzeptes in den Politikzyklus ist die Frage, ob der Politikstil während der gesamten Zeitdauer, in welcher die Policy bearbeitet wird, konstant bleibt. Da es sich nämlich sowohl bei der Politikformulierungsphase als auch bei der Implementationsphase um Prozesse der Konfliktaustragung und Konsensfindung handelt, auf die das Stilkonzept anwendbar ist, könnte laut Feick und Jann die Annahme sinnvoll sein,

${ }^{172}$ Auf diesen Begriff wird in Abschnitt 4.2.2. noch genauer eingegangen.

${ }^{173}$ Anton, Thomas J. (1969): Policymaking and Political Culture in Sweden, in: Scandinavian Political Studies, Heft 4, S. 94, zit. nach Freeman, Gary P. (1985): National Styles ..., a.a.O., S. 475. 
„daß Policy-Formulierung und -Durchführung weitgehend voneinander isolierte Prozesse mit jeweils spezifischen Charakteristika sind "174, und es somit in den einzelnen Zyklusphasen unterschiedliche Politikstile gibt. Diese Einschätzung widerspricht jedoch der in der Policy-Analyse allgemein akzeptierten Auffassung einer engen Verflechtung von Formulierungs- und Implementationsphase ${ }^{175}$.

Für diese Annahme einer Verflechtung spricht unter anderem die Überlegung, daß diejenigen Entscheidungsinstanzen, die eine Politik formulieren, mit der Absicht einer intentionsgemäßen Umsetzung in die Implementationsbemühungen intervenieren werden. Außerdem gibt es Versuche der mit der Implementation betrauten administrativen Stellen der Staatsbürokratie, die Politikformulierung zu beeinflussen. In diesem Sinne formuliert Windhoff-Héritier ${ }^{176}$ : „Politikformulierung [...] und Implementation streng als logische und chronologische Sequenz zu verstehen, würde somit eine unzulässige Vereinfachung der komplexen Wirklichkeit bedeuten, die insbesondere die Bedeutung von hohen Verwaltungsbeamten in der Programmkonzipierung verkennt." Diese personelle und inhaltliche Verflechtung von Politikformulierung und Implementation läßt eine strikt getrennte Betrachtung beider Phasen außer zu Zwecken der Modellbildung nicht sinnvoll erscheinen ${ }^{177}$.

Für das Policy-Style-Konzept folgt aus diesen Überlegungen, daß sich der Politikstil beim Übergang von der Politikformulierungs- in die Implementationsphase nicht wesentlich ändern dürfte und somit die selben $\mathrm{Be}$ schreibungsmerkmale verwendet werden können. Die im Rahmen dieser Studie aufgrund von konkreten Merkmalsausprägungen noch $\mathrm{zu}$ beschreibenden länderspezifischen Environmental Policy Styles beziehen sich somit auf beide zentrale Phasen des Policymakings.

${ }^{174}$ Feick, Jürgen, und Jann, Werner (1988): „Nations matter“ . ., a.a.O., S. 214.

${ }^{175} \mathrm{Vgl}$. die Abschnitte 4.1.3.3. und 4.1.3.4. sowie insbesondere Windhoff-Héritier, Adrienne (1987): Policy-Analyse, a.a.O, S. 74 ff., und Jann, Werner (1995): Politikfeldanalyse, a.a.O., S. $552 \mathrm{ff}$.

${ }^{176}$ Windhoff-Héritier, Adrienne (1987): Policy-Analyse, a.a.O, S. 89.

${ }^{177}$ Vgl. Brewer, Garry D., und deLeon, Peter (1983): The Foundations . . ., a.a.O., S. 255. 
$\mathrm{Zu}$ 3.: Für die Vermutung, daß es gerade in der Gruppe der entwickelten Industrieländer gar keine deutlich voneinander abgrenzbaren, divergierenden Politikstile gibt, sprechen einige Argumente ${ }^{198}$ :

- Die internationale Problemangleichung in den OECD-Staaten und die sich daraus ergebende Ähnlichkeit der Agendas und Programme könnten sich auf den Politikstil im Sinne einer langfristigen Angleichung auswirken.

- Die weltweiten Tendenzen ökonomischer, infrastruktureller und informationeller Integration und Interdependenz und die damit zunehmende Notwendigkeit von Kooperation und gemeinsamen Problemlösungsstrategien düften ebenfalls eine Konvergenz der nationalen Policy Styles begünstigen.

- Es ist außerdem denkbar, daß die politische Elite eines Landes die erfolgreichen Policies anderer Staaten imitieren, und dabei außer den konkreten Politikinhalten auch Elemente der Art und Weise des Policymakings übernehmen könnte. So wird Schweden oft als politische Vorbildnation für die anderen skandinavischen Staaten angeführt ${ }^{179}$.

- Schließlich wird von den Vertretern der Konvergenzhypothese behauptet, der vor allem durch Inglehart beschriebene Wertewandel von materialistischen hin zu postmaterialistischen Werten habe in allen westlichen Industriestaaten zu einer Erweiterung der Partizipationsrechte von Interessengruppen geführt ${ }^{180}$. Dadurch seien die Akteurs-interaktionen, vor allem diejenigen zwischen dem Staat und den durch die jeweilige Policy Betroffenen, in den Staaten Westeuropas tendenziell konsensual geprägt, und somit in einer wichtigen Merkmalsdimension zur Beschreibung von Policy Styles keine wesentliche Varianz zwischen den untersuchten Ländern feststellbar.

Das im folgenden trotzdem von länderspezifischen, hinreichend unterschiedlichen Policy Styles ausgegangen wird, hat vor allem zwei Gründe: Zum einen lehrt die politische Realität, daß die hochindustrialisierten

${ }^{178} \mathrm{Vgl}$. auch im folgenden Feick, Jürgen, und Jann, Werner (1988): „Nations matter“ . . , a.a.O., S. 196, Freeman, Gary P. (1985): National Styles . .., a.a.O., S. 478 ff., Richardson, Jeremy (1982): Convergent Policy Styles in Europe?, In: Richardson, Jeremy (Hg.): Policy Styles ..., a.a.O., S. 197-209.

${ }^{179}$ Vgl. etwa für den Gesundheitssektor: Heidenheimer, Arnold J., und Johansen, Lars (1985): Organized Medicine and Scandinavian Professional Unionism: Hospital Policies and Exit Options in Denmark and Sweden, in: Journal of Health Politics, Policy, and Law, 2, S. 347370, hier: S. 368 f.; zit. nach Freeman, Gary P. (1985): National Styles . ., a.a.O., S. 481.

Inglehart, R. (1977): The Silent Revolution ..., a.a.O. 
westlichen Länder und Japan obwohl sie „in vielen Bereichen vor ähnlichen Problemen stehen und ähnliche technische Lösungspotentiale besitzen "181 zu divergierenden Policy-Reaktionen und -Outcomes gelangen. Der Grund für diese Unterschiede der Problembewältigungsbemühungen und -erfolge kann zumindest teilweise in den Charakteristika nationaler politischer Kultur, institutionellen Besonderheiten und spezifischen Merkmalsausprägungen des jeweiligen Policy Styles liegen ${ }^{182}$. Zum anderen ist die Annahme von Konvergenz meist nur eine Folge des zu geringen Diskriminanzpotentials der gewählten Merkmalsausprägungen bzw. der diese operationalisierenden Variablen. In diesem Sinne führt Freeman mit Blick auf die Studien Richardsons aus ${ }^{183}$ : „One of the deficencies of the typology employed in the volume by Richardson and his associates is that it does not really differentiate the cases, which all tend toward the upper right-hand 'reactive-consensual' quadrant.[...] If our interest is in elucidating pecularities among the set of Western nations, we may need a finer-grained framework. [...] Despite the fact that each state would be classified as predominantly reactive-consensual, the individual chapters are filled with richly documented descriptions of variety and idiosyncracy of policymaking in particular national settings. Some more discriminating typology ought to capture these differences."

Die vorliegende Studie bejaht also die Existenz hinreichend verschiedener nationaler Politikstile. Im folgenden Kapitel 5 wird es darum gehen, diejenigen Merkmalsdimensionen auszuwählen und entsprechend zu operationalisieren, welche über ein so großes Diskriminanzpotential verfügen, daß die länderspezifischen Eigenheiten des Policymakings verortet werden können.

$\mathrm{Zu}$ 4.: Nachdem in Punkt 2. die langfristige Konstanz des Politikstils in den einzelnen Phasen des Policy Cycles bejaht wurde, soll es nun um die Frage einer langfristigen Stabilität gehen.

Die Annahme eines über einen längeren Zeitraum stabilen, dominierenden Policy Styles, eine wichtige Voraussetzung, um reliable Aussagen über den Kausalzusammenhang zwischen Politikstil und Policy-Wirkungen

${ }^{181}$ Feick, Jürgen, und Jann, Werner (1988): „Nations matter“ . ., a.a.O., S. 196.

${ }^{182}$ Vgl. Scharpf, F.W. (1987): Sozialdemokratische Krisenpolitik in Europa, Fankfurt; New York, S. 26-27; zit. nach Feick, Jürgen, und Jann, Werner (1988): „Nations matter“ . . ., a.a.O., ebd.

${ }^{183}$ Freeman, Gary P. (1985): National Styles . ., a.a.O., S. 476. 
machen zu können, wird in der Literatur der Policy-Forschung zumindest teilweise in Frage gestellt.

Laut Freeman sind vor allem die folgenden Ursachen für eine zeitliche Varianz des nationalen Policy Styles denkbar ${ }^{184}$ :

- Regierungswechsel,

- Systemwechsel ${ }^{185}$ sowie

- konjunkturelle und zyklische Änderungen der sozio-ökonomischen Rahmenbedingungen.

Entsprechend dem eigenen Anspruch könnte von einer sozialdemokratisch dominierten Regierung ein eher aktiver und konsensorientierter Politikstil erwartet werden. Im Gegensatz dazu wäre für eine konservative Regierung aufgrund ihrer weniger reformorientierten inhaltlichen Prioritäten (im ökonomischen Bereich z.B. Preisstabilität und Ausgleich der Zahlungsbilanz) und ihrer ideologischen Präferenz für einen durchsetzungsstarken Staat ein eher reaktives und konfliktäres Problemlösungsverhalten typisch. ${ }^{186}$ Für die praktische Politik würde dieser Unterschied im Politikstil bedeuten, daß sich im Falle eines Regierungswechsels nicht nur die politische Agenda und die Policies, sondern auch der Policy Style kurzfristig ändern würden.

In noch krasserer Form könnte dieser Effekt auftreten, wenn sich nicht nur die politisch-ideologische Couleur ändert, sondern wenn ein komplettes politisches System inklusive seiner Institutionen und Akteure durch ein neues ersetzt wird. Als Beispiel kann der Übergang Frankreichs zur V. Republik angeführt werden, mit seinen Begleiterscheinungen einer Zentralisation und Verlagerung des Policymakings vom Parlament zur Exekutive, der Schaffung von institutionellen Kapazitäten zur industriellen Langfrist-Planung (planification) sowie dem Anspruch einer aktiven Politikgestaltung. Noch deutlichere Systemwechsel waren der Übergang (West-)Deutschlands vom nationalsozialistischen Regime zur parlamen-

${ }^{184}$ Vgl. ebd., S. 477 ff.

${ }^{185}$ Der Begriff „politisches System“ ist im Sinne der Systemtheorie als „dasjenige gesellschaftliche Teilsystem, das für die Produktion kollektiv verbindlicher Entscheidungen zuständig ist“" (Nohlen, Dieter, und Thibaut, Bernhard (1995): Politisches System, In: Nohlen, Dieter (Hg.): Wörterbuch Staat und Politik, Bonn; S. 610-613) weiter gefaßt als derjenige der Regierung.

${ }^{186}$ Hinsichtlich der parteipolitisch unterschiedlichen wirtschaftspolitischen Zielsetzungen vgl. etwa: Cusack, Thomas R. (1994): Economic Problems in the OECD Countries during the Period 1950 through 1990, unveröffentlichter Entwurf, Berlin, S. 2 ff. 
tarischen Demokratie der Bundesrepublik Deutschland oder derjenige Ostdeutschlands vom totalitären Sozialismus der DDR zum demokratischen gesamtdeutschen Staat. Der Unterschied in allen Aspekten des politischen Systems könnte kaum größer sein als in diesen beiden Fällen, und es leuchtet unmittelbar ein, daß damit auch eine Transformation der Art und Weise des Policymakings einhergeht.

Schließlich ist denkbar, daß Schwankungen insbesondere der volkswirtschaftlichen Lage zu einer Änderung des „eigentlichen“ nationalen Politikstils führen. So dürften die Chancen für eine aktive, reformorientierte Politik, die außerdem auf einen weitgehenden Konsensus der von der jeweiligen Entscheidung Betroffenen abzielt, in Zeiten von Rezession und öffentlicher Sparpolitik eher gering sein. Vielmehr ist unter diesen Rahmenbedingungen ein eher reaktives Krisenmanagement mit konfliktträchtigen Einschnitten in die Besitzstände von Interessengruppen zu erwarten.

Zusammenfassend läßt sich aus diesen Ausführungen schlußfolgern: Es spricht einiges für die zeitliche Instabilität des in einem Land dominanten Politikstils. Insbesondere ein Systemwechsel führt mit hoher Wahrscheinlichkeit zu einer Modifikation des praktischen Policy Styles. Doch auch Regierungswechsel zwischen ideologisch divergierenden politischen Lagern sowie konjunkturellen Zyklen und Einbrüchen könnten die nationalen „Spielregeln“ der Problemlösung und Konfliktaustragung in der politischen Arena signifikant beeinflussen.

$\mathrm{Da}$ im folgenden trotz der hohen Plausibilität dieser Überlegungen von einer Stabilität des länderspezifischen Policy Styles ausgegangen werden soll, hat die folgenden Gründe:

- Die Zahl der Systemwechsel in den im Rahmen dieser Studie relevanten Industriestaaten Europas, Nordamerikas und Asiens war in den fünf Jahrzehnten seit Ende des Zweiten Weltkriegs äußerst gering ${ }^{187}$.

- Da es sich bei Regierungswechseln und konjunkturellen Zyklen hingegen um recht häufige Phänomene handelt, sind die anderen Argumente gegen die Annahme einer zeitlichen Stabilität der nationalen Policy Styles ernster zu nehmen. Vor allem ein Blick auf einen so krassen Fall wie den Regierungswechsel in Großbritannien im Jahre 1979, der die Ablösung einer linken Labour-Regierung durch das extrem marktwirt-

\footnotetext{
${ }^{187}$ Außer der Transformation Frankreichs von der Dritten zur Vierten und schließlich zur Fünften Republik sind nur noch die Re-Demokratisierungsprozesse in Südeuropa (Portugal, Spanien und Griechenland) sowie der Beitritt der Ex-DDR zur Bundesrepublik Deutschland zu nennen.
} 
schaftlich-individualistisch ausgerichtete Kabinett Thatcher bedeutete, scheint die auch kurzfristige Beeinflußbarkeit der Art und Weise des Policymakings zu belegen. Doch so sehr sich durch diesen Machtwechsel das innenpolitische Koordinatensystem Großbritanniens auch verschoben hat, und so sehr sich die ideologischen und inhaltlichen Prioritäten im Vergleich zur Vor-Thatcher-Ära auch geändert haben, so ist dennoch das Fortdauern eines typisch-britischen Politikstils feststellbar, d.h. die radikale Umgestaltung der politischen Agenda und der praktizierten Policies kann die im Vergleich zu anderen Staaten weiterhin divergierenden und somit auch konstanten Merkmale britischer Verfahren der Politikformulierung und Implementation - nämlich vor allem der kompromißlosen Durchsetzung einer einmal majoritär festgelegten Policy - nicht überdecken. Eine Änderung des nationalen Politikstils aufgrund von Regierungswechseln ist also durchaus wahrnehmbar, doch diese interne Varianz der durch einen Staat praktizierten Politikstilvarianten scheint geringer als die externe Varianz, also die Unterschiede zwischen den dominanten Politikstilvarianten der einzelnen Staaten. Unabhängig vom politisch-ideologischen Standort gelte deshalb laut Rose das folgende Zitat eines Labour-Premierministers als typisch für den Policymaking Style im Vereinigten Königreich ${ }^{188}$ : „, The essential thing in a democracy is a general election in which a government is elected with power to do any damned thing it likes and if the people don't like it they have the right to chuck it out. "Der Einfluß der im Zeitablauf nur sehr langfristig änderbaren Grundmuster Politischer Kultur ${ }^{189}$ hat auf den nationalen Politikstil also einen stablisierenden Effekt.

- Ähnlich läßt sich auch gegen eine zeitliche Instabilität des Politikstils aufgrund von konjunkturellen Zyklen argumentieren. Sicherlich ist anzunehmen, daß beispielsweise in Zeiten hoher Wachstumsraten und ausreichender öffentlicher Mittel, die Neigung zu einvernehmlichen, konsensualen Problemlösungsverfahren zunimmt, und andererseits im Falle einer tiefen Rezession und entsprechender Defizite der öffentli-

${ }^{188}$ Putnam, Robert D. (1973): The Beliefs of Politicians, New Haven, S. 172; zit. nach: Rose, Richard (1992): Politics in England, In: Almond, Gabriel A., und Powell Jr., Bingham (Hg.): Comparative ..., a.a.O., S. 154.

${ }^{189}$ Vgl. etwa Inglehart, Ronald (1988): Politische Kultur und stabile Demokratie, In: Politische Vierteljahresschrift, 3, S. 370. Eine ausfuhrliche Darstellung des Konzeptes der Politischen Kultur und des Zusammenhanges zwischen Kultur und Stil-Begriff erfolgt im folgenden Abschnitt 4.2.2. 
chen Haushalte Politikformulierung und Implementation verstärkt konfliktäre und reaktive Züge aufweisen. Dennoch geht die vorliegende Untersuchung davon aus, daß die kulturell bedingten länderspezifischen Charakteristika des Policymakings auch die konjunkturell bedingten, nuancenhaften Verschiebungen der Politikstilmerkmale überdecken.

Aus den Überlegungen zur Zeitstabilität nationaler Politikstile kann also abschließend gefolgert werden: Policy Styles sind zeitlich nicht unveränderbar, doch solange extreme politische Systemwechsel ausbleiben, ist von einer relativen Stabilität auszugehen.

Zu 5.: Der Begriff Policy Style könnte nahelegen, daß damit bestimmte typische Merkmalskombinationen zur Beschreibung von Policies (Politikinhalten) bezeichnet werden sollen. Der in der Policy-Forschung dominierende Ansatz bezieht den typologisierenden Stil-Begriff jedoch eher auf die prozessuale Dimension der Politik ${ }^{190}$. D.h. es sollen hier nicht länderspezifische Gemeinsamkeiten von Politkinhalten, sondern spezifische Verhaltens- und Handlungsweisen zur Formulierung und Implementation eben dieser Policies interessieren. Die meist nur in Einzelfallstudien zu erschließenden Politikinhalte, also der eigentliche PolicyOutput in Form von politischen Programmen, Gesetzen, Verordnungen etc., werden innerhalb der angenommenen Kausalkette PolitikstilPolitikinhalte-Politikergebnisse als Black Box ${ }^{191}$ betrachtet. Wenn nämlich von einem signifikanten Einfluß des Politikstils auf die Politikinhalte ausgegangen werden kann, und die Outcomes letztlich das kausale Ergebnisse der Policy-Auswirkungen auf das sozio-ökonomische und gesellschaftliche System sind, dann kann unter weitgehender Nichtbetrachtung der Politikinhalte der Effekt unterschiedlicher Politikstile auf die Policy Outcomes analysiert werden.

Unter Berücksichtigung aller Konkretisierungen und Relativierungen soll im weiteren Verlauf die folgende Definition gelten:

Ein nationaler Politikstil ist die Gesamtheit der länderspezifischen und zeitlich relativ stabilen Methoden und Verhaltensweisen, mit welchen die staatlichen Entscheidungsträger politische

${ }^{190}$ Vgl. vor allem Feick, Jürgen, und Jann, Werner (1988): „Nations matter“ . . ., a.a.O., S. 201.

${ }^{191}$ Vgl. das Input-Output-Modell Eastons (Easton, David (1967): A Systems Analysis of Political Life, New York). 
Problemlösungen in Form von Policies formulieren und implementieren.

Die Grundthese läßt sich unter Berücksichtigung dieser Definition und der weiteren, in Abbildung 7 zusammenfassend dargestellten Zusammenhänge wie folgt umformulieren:

Die länderspezifische Art und Weise der Policy-Formulierung und -implementation ist eine Determinante der PolicyWirkungen (Impacts) und schließlich auch der PolicyErgebnisse (Outcomes).

Für den Bereich der Umweltpolitik bedeutet dies:

Die Effektivität nationaler Umweltpolitik - gemessen durch das anhand der Einwohnerzahl und das Bruttoinlandsprodukt relativierte allgemeine Niveau der Umweltbelastung - wird als Policy Outcome durch den Environmental Policy Style zumindest teilweise determiniert.

Bevor einzelne konkrete Politikstilkonzepte und -typologien genauer dargestellt werden, soll im folgenden Abschnitt zunächst der Zusammenhang zwischen Politikstil und dem umfassenderen Konzept der Politischen Kultur dargestellt werden.

\subsubsection{Politikstil und Politische Kultur}

Richardson et al. interpretieren die nationalen Politikstile der westeuropäischen Staaten u.a. auch als Reflektion von „often deep-rooted values in society "192. Feick und Jann führen aus, „nationale Schablonen“ politischen Problemlösungsverhaltens müßten auch im Zusammenhang mit der Kontextvariable ,[...] nationaler Kultur im Sinne vorherrschender kognitiver und normativer Orientierungen [ ...] gesehen werden. "Diese sei ein Faktor, der ",[...] die konkreten Orientierungen und Verhaltensweisen von individuellen wie kollektiven Akteuren im Policy-Proze $\beta^{\prime 193}$ beeinflusse. Beide Zitate verweisen auf die historisch-kulturellen Prädispositonen der politischen Eliten als Ursache national unterschiedlicher Politikstile.

192 Richardson, Jeremy; Gustafsson, Gunnel, und Jordan, Grant (1982): The Concept . . ., a.a.O., S. 2.

${ }^{193}$ Feick, Jürgen, und Jann, Werner (1988): „Nations matter“ . ., a.a.O., S. 201. 
Dieser letztlich subjektive Faktor, welcher die Meinungen, Einstellungen und Werte der politisch Handelnden umfaßt, wird in der Politikwissenschaft im Konzept der „Politischen Kultur“ thematisiert und als ursächlich für nationenspezifische Ausprägungen des politischen Systems beschrieben ${ }^{194}$. Die Bedeutung der kulturellen Bedingheit politischer Prozesse zu ignorieren, wäre deshalb eine problematische Verkürzung der Realität ${ }^{195}$. Diese Tatsache wird besonders deutlich von Joppke formuliert: „Political preferences are not merely reflections of external opportunities but are culturally determined patterns of perception that select suitable objects and appropriate lines of action. A political process theory that treats preferences and interests as external givens and only considers their ex post facto incorporation into the polity is incomplete.

Bevor der Einfluß kultureller Faktoren auf das Policymaking genauer analysiert und eine inhaltliche Abgrenzung zum Politikstil-Begriff vorgenommen werden kann, ist eine exakte Begriffsbestimmung des Konzeptes politischer Kultur notwendig.

Almond und Powell definieren political culture als: "[...] a particular distribution of political attitudes, values, feelings, information, and skills. "197

Eine ähnliche Definition findet sich bei Berg-Schlosser und Stammen, welche politische Kultur als die "[...] Gesamtheit aller Meinungen (,,beliefs"), Einstellungen (,, attitudes") und Werte (,values"), die in einer Gesellschaft zu einem gegebenen Zeitpunkt anzutreffen sind "198, beschreiben.

Zur Konkretisierung dieser noch recht unpräzisen Konzepte bedarf es einer Festlegung konkreter Beschreibungsmerkmale. Zu diesem Zweck führen die beiden Autoren insgesamt vier Variablengruppen an ${ }^{199}$ :

194 Vgl. grundlegend: Berg-Schlosser, Dirk (1987): Politische Kultur. In: Nohlen, Dieter, und Schultze, Rainer-Olaf (Hg.): Politikwissenschaft ..., a.a.O., S. 746-751.

${ }^{195}$ Vgl. Inglehart, Ronald (1988): Politische Kultur . . ., a.a.O., S. 370.

196 Joppke, Christian (1993): Mobilizing Against Nuclear Energy: A Comparison of Germany and the United States, Berkeley; Los Angeles, S. $13 \mathrm{f}$.

${ }^{197}$ Almond, Gabriel A., und Powell, Jr., Bingham G. (1992) (Hg.): Comparative . ., a.a.O., S. 39.

${ }^{198}$ Berg-Schlosser, Dirk, und Stammen, Theo (1992): Einfuhrung . . ., a.a.O., S. 181. 
- Variablen sozialer Identifikation, welche den Grad der affektiven und kognitiven Übereinstimmung mit dem politischen System bestimmen;

- Psychische Prädispositonen im Hinblick auf die bevorzugte Art der politischen Konfliktaustragung;

- sozio-ökonomische und religiöse Orientierung im Hinblick auf die gesellschaftliche Entscheidungsfindung;

- politische Einstellungen im Hinblick auf Partizipation am politischen System sowie auf die Legitimität desselben.

Diesem Operationalisierungsschema entspricht in weiten Teilen ein Vorschlag Kaases mit den folgenden Elementen politischer Kultur ${ }^{200}$ :

„1. Orientierungen gegenüber dem politischen System insgesamt. Also die 'diffuse' Zustimmung und Identifikation mit der Nation, den politischen Institutionen und den politischen Eliten, kurz, das Legitimationseinverständnis [...].

2. Orientierungen gegenüber den Leistungen (output) des politischen Systems; die Zuschreibung und Bewertung spezifischer Gratifikationen von bestimmten Akteuren und Institutionen.

3. Orientierungen gegenüber den Willensbildungs-(input)strukturen politischer Organisationen und Ideologien.

4. Orientierungen gegenüber dem eigenen Selbst, die Kenntnis von Rechten und Pflichten und das Gefühl politischer Kompetenz."

Eine Mangel der bisher vorgestellten Systematiken zur Beschreibung von politischer Kultur liegt darin, daß nicht zwischen Gesellschaft und politischen Akteuren unterschieden wird. Da es im Kontext der vorliegenden Arbeit jedoch um den Zusammenhang zwischen Kultur-Begriff und der Art und Weise des Handelns der politisch-administrativen Elite geht, soll nachfolgend stärker auf die kulturell-subjektiven Orientierungen der Mitglieder des Policymaking-Systems und deren Effekt auf das konkrete Handeln eben dieser Akteure abgehoben werden.

${ }^{199}$ Vgl. ebd., S. 184.

${ }^{200}$ Kaase, M. (1983): Sinn und Unsinn des Konzepts politischer Kultur für die vergleichende Politikforschung, In: Kasse, M., und Klingemann (Hg.): Wahlen und politisches System, Opladen, S. 144-172, zit. nach Fenner, Christian (1995): Politische Kultur, In: Nohlen, Dieter: (Hg.): Wörterbuch . . ., a.a.O., S. 568. Vgl. auch die Unterscheidung Almonds und Verbas zwischen einem gegenüber dem politischen System indifferenten, sogenannten ,parochialen“ Kulturtyp, einem Untertanentyp und einer Beteiligungskultur (Almond, Gabriel A., und Verba, Sidney (1963): The Civic Culture . . ., a.a.O.); Vgl im folgenden auch: Berg-Schlosser, Dirk, und Stammen, Theo (1992): Vergleichende . . ., a.a.O., S. 182 ff., sowie Fenner, Christian (1995): Politische Kultur, In: Nohlen, Dieter: (Hg.): Wörterbuch . ., a.a.O., S. 568. 
Wichtig im Zusammenhang mit dem Politikstil-Konzept sind also vor allem diejenigen Kulturmerkmale, welche auf die staatlichen Eliten abzielen. Diese Merkmale können wie folgt zusammengefaßt werden: Es gibt eine länderspezifische politische Kultur der staatlichen Eliten, welche sich vor allem in deren Einstellung zu Art und Ausmaß der politischer Konfliktlösung manifestiert.

Diese subjektiven Dimensionen politischer Systeme stellen "Prädispositionen zu Handlungen "201 dar. Eine solche Verbindung zwischen Kultur-Begriff und politischem Agieren beschreibt auch Fenner, indem er politische Kultur als ,die geschriebenen und ungeschriebenen Ideen und Wertecodes, die politisches Handeln der Gesellschaftsmitglieder regulieren “202, definiert. Die Pioniere des politischen KulturKonzeptes, Almond und Powell, gehen ebenfalls von einer Kausalität zwischen politischer Kultur und dem Handeln der politischen Akteure aus: "As people's attitudes affect what they will do, a nation's political culture affects the conduct of its citizens and leaders throughout the political system. " ${ }^{203}$ Schließlich seien in diesem Kontext noch Feick und Jann zitiert: „Es bestehen kaum Zweifel, daß Meinungen, Einstellungen, Werte, kurzum: kognitive, normative und evaluative Orientierungen, Einflu $\beta$ auf die Ausgestaltung politischer Institutionen und die in und zwischen ihnen ablaufenden Prozesse haben. “204

Diese Sichtweise der politikwissenschaftlichen Kultur-Forschung betont also die Relevanz kultureller Grundlagen für die konkrete Ausgestaltung nationaler politischer Systeme, oder wie es Inglehart formuliert: „,...] politische Kultur besteht in einem Konsensus über verschiedene Spielregeln oder konstitutionelle Vorgehensweisen [...].

Somit ist von einem kausalen Nexus zwischen kulturellen Mustern einerseits und dem politischen Prozeß, als einem der neben den Polities grundlegenden Elemente des politischen Systems, andererseits auszuge-

${ }^{201}$ Berg-Schlosser, Dirk, und Stammen, Theo (1992): Einführung . ., a.a.O., S. 181.

${ }^{202}$ Fenner, Christian (1995): Politische Kultur, In: Nohlen, Dieter: (Hg.): Wörterbuch . . ., a.a.O., S. 565 .

${ }^{203}$ Almond, Gabriel A., und Powell, Jr., Bingham G. (1992) (Hg.): Comparative ..., a.a.O., S. 39.

${ }^{204}$ Feick, Jürgen, und Jann, Werner (1988): „Nations matter“ . ., a.a.O., S. 210.

${ }^{205}$ Inglehart, Ronald (1988): Politische Kultur ..., a.a.O., S. 371 f. 
hen. D.h. die politische Kultur eines Staates ist ein ursächlicher Faktor für die konkrete, länderspezifische Art und Weise, in welcher die Eliten dieses Staates den politischen Prozeß gestalten.

In diesem Sinne beschreibt Hartmann die Realität der politischen Institutionen (Polities) und Prozesse (Politics) in einigen beispielhaft aufgeführten westlichen Industriestaaten sowie in Japan als Resultat kultureller Prädispositionen ${ }^{206}$ :

- Hauptkennzeichen skandinavischer politischer Kultur ist das Bestreben zur Minimierung politischer Konflikte durch eine weitgehende Egalisierung der ökonomischen und sozialen Verhältnisse. Diese Politikvariante wird in korporatistischen Strukturen, in denen die Forderungen der Bürger an den Staat durch repräsentative Verbände mediatisiert werden, vollzogen.

- In den USA hingegen spielt der Staat eine weniger wichtige Rolle. Vielmehr bleibt es jedem selbst überlassen, seine Interessen zu vertreten und durchzusetzen. Die christlich-protestantisch geprägten Hauptmerkmale des amerikanischen politischen und ökonomischen Systems, nämlich ein starker Individualismus und eine ebenso ausgeprägte Leistungs- und Wettbewerbsorientierung, liefern die Grundlage für den extrem konfliktären Charakter der Interessenaustragung innerhalb der politischen Arena.

- In Japan bilden die religiös-philosophischen Wurzeln von Buddhismus, Shintoismus und Neo-Konfuzianismus die Grundlagen der politischen Kultur $^{207}$. Als Grundregel des politischen Prozesses und der Austragung von Interessengegensätzen gilt dabei das Streben nach harmonischen, konfliktvermeidenden Lösungen, die allen Akteuren die Chance zur Gesichtswahrung lassen. Der wichtigste Unterschied im Vergleich zur europäisch-skandinavischen Konsensvariante des Korporatismus liegt in dem wohl einmaligen, sich nicht behindernden Zusammentreffen von Klientelismus ${ }^{208}$ und strategischer Industriepolitik.

${ }^{206}$ Hartmann, Jürgen (1995): Vergleichende . ., a.a.O., S. 106 f.

${ }^{207}$ Vgl. Kreiner, Josef (1994): Religionen heute, In: Mayer, Hans Jürgen, und Pohl, Manfred (Hg.): Länderbericht Japan, Bonn, S. 505 ff.

${ }^{208}$ Klientelismus läßt sich definieren als das Vorhandensein einer personalen Beziehung zwischen Akteuren oder Gruppen von Akteuren, der zum beiderseitigen Vorteil vorgenommene, allenfalls halblegale Austausch in der Regel ungleicher Ressourcen und damit verbunden das Bestehen eines faktischen Dependenzverhältnisses (in Anlehnung an Ziemer, Klaus (1995): Klientelismus, In. Nohlen, Dieter (Hg.): Wörterbuch . . ., a.a.O., S. 316). 
Wenn wir als Ergebnis dieser Überlegungen den nationalen policy style also als Resultat kultureller Bestimmungsfaktoren ansehen können, stellt sich im Kontext einer komparatistischen Längsschnittanalyse die Frage nach der zeitlichen Stabilität politischer Kulturmuster. Zwar lassen sich durchaus signifikante Veränderungen politischer Kulturen belegen ${ }^{209}$, dennoch überwiegt in der Literatur die Annahme einer Dauerhaftigkeit der subjektiven Orientierungen von Nationen ${ }^{210}$. Während von den drei Bestandteilen politischer Kultur - Meinungen, Einstellungen und Werte Meinungen relativ leicht und in Abhängigkeit von tagespolitischen Einflüssen variieren dürften, handelt es sich bei grundlegenden politischen Einstellungen und Werten um stabilere Elemente des Kultur-Konstruktes: "Wenn auch vieles im Fluß ist, so gibt es doch dauerhafte und stabile Bestandteile, wie grundlegende politische Überzeugungen, Werthaltungen und Primärbindungen, die unser politisches Verhalten und Politik beeinflussen und bedingen.

Wegen der bereits oben festgestellten kulturellen Bedingtheit nationaler Politikstile, kann somit angenommen werden, daß die relative zeitliche Konstanz der politischen Kultur stabilisierend wirkt und eine nennenswerte zeitliche Varianz des nationalen Politikstils verhindert.

Nachdem in den letzten Kapiteln eine hinreichende definitorische Präzisierung und kontextuale Einordnung des Policy-Style-Konzeptes vorgenommen wurde, folgt nun die Diskussion von drei konkreten Ansätzen zur Erklärung und Darstellung nationaler Politikstile. Dabei wurden solche Konzepte ausgesucht, die über eine deskriptive, der Gefahr des reinen "story telling" unterliegenden Heransgehensweise hinaus auch den Versuch der Konstruktion einer konkreten Typologie von Politikstilen unternehmen, und dabei klar definierte Beschreibungsmerkmale von Policy Styles verwenden. Diese Merkmale können dann in der Phase der eigenen

${ }^{209}$ Siehe als Beispiel die von Inglehart beschriebenen Veränderungen der politischen Kultur Deutschlands nach dem zweiten Weltkrieg (Ders. (1988): Politische Kultur . . ., a.a.O., S. 370). Oder die von Almond angefürten Beispiele: Ders. (1987): Zum Konzept . . ., a.a.O., $34 f$.

210 Vgl. Almond, Gabriel A. (1987): Zum Konzept . . ., a.a.O., S. 29; Inglehart, Ronald (1988): Politische Kultur ..., a.a.O., S. 370.

${ }^{211}$ Almond, Gabriel A. (1987): Zum Konzept . . ., a.a.O., S. 36. 
Theoriebildung als Grundlage zur Diskussion und Auswahl der relevanten Politikstildimensionen dienen.

\subsubsection{Ansätze zur Erklärung und Darstellung nationaler Policy Styles}

4.2.3.1 Das Konzept von Richardson (1982): Problemlösungsverhalten und Art der Beziehungen zwischen Regierung und Betroffenen als relevante Politikstildimensionen

Bevor die relevanten Dimensionen zur Identifizierung unterschiedlicher Politikstile explizit festlegt werden, ist grundsätzlich festzustellen, daß mit dem Studium der Politikstile eine Verschiebung des Interesses von den Entscheidungsinhalten hin $\mathrm{zu}$ den unterschiedlichen Entscheidungssystemen und -prozessen stattfindet. Dies bedeutet gleichzeitig, daß die für diese Art von Typologie berücksichtigten Phasen des politischen Prozesses diejenigen der Entscheidung (Policymaking), also der Politikformulierung und der Implementation sind ${ }^{212}$.

Eines der grundlegenden Konzepte zum Thema der Policy Styles stammt von Richardson ${ }^{213}$.

Eine Besonderheit dieses Ansatzes betrifft den Zusammenhang zwischen dem nationalen Wertesystem und dem praktizierten Politikstil. So wird ein Land, in dem die Konsultation Betroffener auch im alltäglichen gesellschaftlichen Umgang einen hohen Stellenwert einnimmt, im politischen Prozeß einen eher langwierigen und breiten Konsultationsmechanismus vorschalten. Dieser Zusammenhang zeigt den bereits in Abschnitt 4.2.2. thematisierten kausalen Nexus zwischen den kulturellen Faktoren (hier: Werteskala) und den politischen Determinanten (hier: Politikstil) des politischen Outputs. ${ }^{214}$

Als Hauptschwierigkeiten bei der Identifizierung unterschiedlicher Arten, in denen Gesellschaften ihre politischen Probleme lösen, sind drei Punkte anzuführen: ${ }^{215}$

${ }^{212}$ Ebenda, S. 2.

${ }^{213}$ Richardson, Jeremy, et al. (1982): The Concept . . , a.a.O.

214 Ebenda.

${ }^{215}$ Ebenda, S. 2 ff. 
- Oft sind es Ausnahmefälle der politischen Entscheidung, welche eine erhöhte Publizität erhalten, und deshalb leicht die eigentliche Regel, d.h. den wahren Politikstil überdecken.

- Ein weiteres Problem kann aus der Tatsache erwachsen, daß sich innerhalb einer Gesellschaft für unterschiedliche Politikbereiche unterschiedliche Politikstile herausgebildet haben, oder daß sogar innerhalb eines Politikfeldes inhaltlich und personell geschlossene „Unter"Bereiche bestehen, in denen ebenfalls verschiedene Policy Styles existieren. Diese Problematik ist insbesondere dann ausgeprägt, wenn in diese Bereiche Entscheidungskompetenz ausgelagert wurde. Diese Schwierigkeiten zwingen den Analytiker dominierender nationaler Politikstile dazu, den Fokus auf eine eher hohe Ebene der politischen Probleme und Entscheidungen zu richten, in der Annahme, daß zumindest die grundsätzlichen Probleme eines Staates auf eine ähnliche, charakteristische Art und Weise gelöst werden.

- Als dritte Einschränkung des Policy Style-Konzeptes ist die These Lowis anzuführen, daß in einer Umkehrung der bisherigen Kausalitätsannahme nicht unterschiedliche Politikstile zu spezifischen Herangehensweisen an ein bestimmtes Problem führen, sondern daß es die Charakteristik des Problems ist, welche die Art und Weise seiner Lösung determiniert $^{216}$. Somit wäre also der Politikstil das jeweils situative $\mathrm{Re}$ sultat einer politischen Problemlage. Diesen Überlegungen ist sicherlich zuzustimmen, doch nur insofern, als daß der Ausprägungsgrad bestimmter Politikstildimensionen im Einzelfall mit der Art und Weise des behandelten Themas korrelieren dürfte, daß jedoch der grundlegende Politikstil eines Staates oder einer Gesellschaft prinzipiell vom Charakter der auf der politischen Agenda stehenden Themen unberührt bleibt.

Laut Richardson sind es vor allem zwei Faktoren, mit denen länderspezifische Ausprägungen des Policymakings hinreichend beschrieben werden können ${ }^{217}$.

1.Der prinzipielle Problemlösungsansatz der Regierung. Das dabei zunächst zugrundegelegte Kontinuum an möglichen Handlungsalternativen reicht von einem als „incrementalist" beschriebenen Ansatz, der

${ }^{216}$ Lowi, Theodore J. (1964): American Business . . ., a.a.O., S. 677-715, sowie Lowi, Theodore, J. (1972): Four Systems of Policy, Politics, and Choice, in: Public Administration Review, 33, S. 298-310.

${ }^{217}$ Richardson, Jeremy, et al. (1982): The Concept . ., a.a.O., S. 12 f. 
als eine Politik der schrittweisen Modifikationen ohne Zwang und durch ausgedehnte Konsultationen charakterisiert werden kann, bis zu einer als "rationalist" bezeichneten Art der Problemlösung. Dieser zweite Idealtyp kann wie folgt definiert werden:

- Existenz einer Zentralinstanz mit weitreichenden Zwangsinstrumenten,

- Vorgabe klarer Handlungsziele,

- Möglichkeit zum radikalen Wandel etc.

Der erste Hauptfaktor bildet also ein Kontinuum, welches von einem sehr frühzeitigen und eigeninitiativen bis hin zu einem reaktiven, erst durch äußeren Anstoß aktivierbaren Problemlösungsverhalten der Regierung reicht.

2. Art der Beziehungen zwischen Regierung und Betroffenen. Die zweite Dimension des Modells beschreibt den Umgang der Regierung mit den anderen Akteuren des politischen Entscheidungs- und Implementationsprozesses. Das hier bestehende Kontinuum weist als seine Extrema zum einen eine eindeutige Konsensfixierung der Regierung und zum anderen das Bestreben, die eigenen Lösungsansätze notfalls unter Inkaufnahme von Konflikten kompromißlos umzusetzen, auf.

Weitere wichtige Dimensionen wie etwa der Zentralisierungsgrad oder die Zugänglichkeit der politischen Arena bleiben unberücksichtigt. Dieses Vorgehen verringert zwar die Exaktheit der Aussagen, erhöht jedoch deren heuristisches Erklärungspotential.

Die Auswahl der beiden Faktoren Problemlösungsverhalten und Art der Beziehungen ermöglicht nun das Aufstellen einer zweidimensionalen Politikstil-Typologie mit vier Grundtypen (Abbildung 8).

Konzertierung. Dieser Politikstil kombiniert ein aktives Problemlösungsverhalten der Regierung mit einer eher konsensualen Beziehung zu den anderen Akteuren des politischen Prozesses.

Konsensualismus. In diesem Fall verhält sich die Regierung ebenfalls kooperativ, sie setzt den Problemlösungsmechanismus jedoch erst im Falle eines äußeren Anstoßes in Gang. Dieser Anstoß kann von der Problematik, die keinen weiteren Aufschub mehr zuläßt, oder von Interessengruppen, die ein bestimmtes Thema auf die politische Agenda setzen, erfolgen.

Dirigismus. Der so genannte Politikstil ist im Gegensatz zu den beiden erstgenannten durch die Bereitschaft der Regierung charakterisiert, ihre Beschlüsse auch gegen das Einverständnis der anderen Akteure des politischen Prozesses durchzusetzen. Dieses konfliktäre Element verbindet sich mit einem aktiven Problemlösungsverhalten. Eine dirigistische Re- 
gierung erkennt Probleme also frühzeitig und oktroyiert ihre Lösungsansätze dann den Betroffenen.

Laissez-faire. Hier sind sowohl das konfliktäre als auch das reaktive Problemlösungsverhalten kombiniert. Eine diesen Politikstil praktizierende Regierung entscheidet Probleme erst im Falle eines äußeren Anstoßes, und dann auch ohne Einbeziehung bzw. Einverständnis der oppositionellen politischen Akteure. Auch eine Politik des Non-Decisionmaking, also der Vermeidung einer Entscheidung (vgl. Abschnitt 4.1.3.3.), gehört in dieses Feld.

\section{Abb. 8: Politikstil-Typologie}

\begin{tabular}{|c|c|c|}
\hline $\begin{array}{c}\text { Art der Beziehungen } \\
\text { Problem- } \\
\text { lösungsverhalten }\end{array}$ & $\begin{array}{c}\text { Konsensuale } \\
\text { Beziehungen }\end{array}$ & $\begin{array}{c}\text { Konfliktäre } \\
\text { Beziehungen }\end{array}$ \\
\hline antizipativ & Konzertierung & Dirigismus \\
\hline reaktiv & Konsensualismus & Laissez-faire \\
\hline
\end{tabular}

Quelle: Richardson (1982), S. 13; Eigene Benennung der Felder.

Für die Staaten Westeuropas kommt Richardson nach der Auswertung verschiedener Länderstudien zu dem Resultat, daß sie erstens einen ähnlichen, zunehmend konvergierenden Politikstil aufweisen, und daß zweitens dieser Politikstil im rechten, oberen Quadranten $\mathrm{zu}$ finden ist ${ }^{218}$. Durch die zunehmende Komplexität der in hochentwickelten Demokratien zu bewältigenden Probleme und der damit inhärenten Tendenz zur Aufteilung politischer Ressourcen und Fähigkeiten auf eine immer größere Vielzahl von Einzelpersonen, Gruppen und Organisationen innerhalb der westeuropäischen Gesellschaften werde ein antizipativer, innovativer Problemlösungsansatz immer schwieriger. Zum anderen hätte die fort-

${ }^{218}$ Richardson, Jeremy (1982): Convergent Policy Styles . . , a.a.O., S. 197. 
schreitende Demokratisierung der Gesellschaften in Verbindung mit einem forcierten Wertewandel zu einer immer größeren Anzahl gesellschaftlicher Interessengruppen geführt, deren Partizipation am politischen Prozeß sogar ein overcrowding ${ }^{219}$ desselben befürchten lassen, also eine Lähmung des Entscheidungsprozesses durch das Aushandeln zwischen zu vielen und zu gegensätzlichen Akteuren.

4.2.3.2 Die Typologie von Kitschelt (1983): Offenheit und Handlungskapazität als relevante Politikstildimensionen

In seiner Studie zu den Energie-Technologiepolitiken westlicher Industriestaaten untersucht Kitschelt unter anderem die Bedeutung bestimmter institutioneller Bedingungen und politischer Regimeformen für umweltpolitische Innovationsfähigkeit ${ }^{220}$.

Aus Zwecken der Vereinfachung ersetzt Kitschelt den mehrphasigen politischen Prozeß durch ein Input-Output-Modell, in welchem als Input die Artikulations-, Aggregations- und Entscheidungsphase und als Output die Implementations-, Evaluations- und Terminationsphase zusammengefaßt werden. Dieses Zwei-Phasen-Modell bildet die Grundlage seiner Politikstil-Typologie ${ }^{221}$.

Die von ihm gewählten Merkmalsdimensionen zur Unterscheidung des nationalen Politikstils sind zum einen die Offenheit für Interessenartikulation und -aggregation (Input) und zum anderen die Organisations- und Ressourcenkapazität der politischen Administration in der Implementationsphase (Output).

Indikatoren für die Offenheit von Artikulation und Aggregation im politischen Prozeß sind die Artikulationsbreite (klein bis groß) und die Aggregationskapazität (gering bis hoch) ${ }^{222}$. Zwischen beiden Faktoren wird da-

${ }^{219}$ Richardson, Jeremy et al. (1982): The Concept . ., a.a.O., S. 6.

${ }^{220}$ Kitschelt, Herbert (1983): Politik und Energie: Energie-Technologiepolitiken in den USA, der Bundesrepublik Deutschland, Frankreich und Schweden, Frankfurt am Main, New York. Die von Kitschelt genannten Politikstil-Phasen entsprechen trotz leicht unterschiedlicher Begrifflichkeit weitgehend den Sequenzen des in Abschnitt 4.1.3. dargestellten PolitikzyklusModells.

${ }^{221}$ Ebenda, S. $70 \mathrm{f}$.

${ }^{222}$ Ebenda, S. 72 f. 
bei eine Beziehung in Form einer konvexen Funktion angenommen, d.h. daß optimale Offenheit nicht in der Nähe der Extrema der beiden Variablen zu finden ist. So führt nämlich eine große Artikulationsbreite bei gleichzeitig geringer Aggregationskapazität zu Immobilismus des politischen Systems, und andererseits wäre eine Folge der Kombination kleine Artikulationsbreite/hohe Aggregationskapazität eine hohe Geschlossenheit des politischen Systems. Die optimale Offenheit ist also im Falle einer Kombination mittlerer Ausprägungen beider Faktoren zu finden.

Auch für die Indikatoren des politischen Output-Prozesses - die Struktur des administrativen Organisationsnetzes (fragmentiert bis konzentriert) sowie die Implementationsressourcen (gering bis groß) - gilt dieser $\mathrm{Zu}$ sammenhang, d.h. eine optimale Implementationskapazität ergibt sich bei eher mittlerer Ausprägung beider Faktoren ${ }^{223}$.

Aufgrund dieser Vorüberlegungen kann ebenfalls ein zweidimensionaes Schema politischer Stile abgebildet werden (Abbildung 9):

Pluralismus. Dieser Politikstil ist auf der Input-Seite durch Offenheit der Interessenartikulation und -aggregation gekennzeichnet. Diese Partizipation wird in der Output-Phase durch mangelnde Implementationskapazitäten jedoch nivelliert. Die Offenheit der politischen Arena wird also praktisch kompensiert durch die Auslagerung der politisch-ökonomischen Ressourcen von der Zentralregierung hin $\mathrm{zu}$ privatwirtschaftlichen Instanzen. Dieser Mangel an externer Handlungskapazität hat dann auch Auswirkungen auf das Innovationsvermögen und den Rationalitätsstil ${ }^{224}$ der jeweiligen Regierung. Das Resultat ist eine Selbstbezogenheit der politischen Organisation, welche zwar die Neuformulierung von Politiken zuläßt, sich aufgrund mangelnder Durchschlagskraft jedoch primär auf eine Absicherung des eigenen Bestandes beschränkt.

${ }^{223}$ Ebenda, S. $73 \mathrm{f}$.

${ }^{224}$ Mit diesem Terminus meint Kitschelt den jeweiligen Erfolgsstandard staatlichen Handelns, d.h. der Rationalitätsstil gibt eine Antwort auf die Frage, ob der Systembestand, Effektivitätsbzw. Effizienzkriterien oder die demokratische Legitimation als Handlungsregulativ dienen soll (Ebd.). 


\begin{tabular}{l|l|l|l|}
\multicolumn{2}{c}{} & \multicolumn{2}{c}{ ARTIKULATIONS- UND } \\
\multicolumn{1}{c|}{ AGGREGATIONSPROZESSE } \\
\cline { 2 - 3 } & \multicolumn{1}{c|}{ offen } & \multicolumn{1}{c|}{ geschlossen } \\
\cline { 2 - 3 } $\begin{array}{l}\text { ORGANISA- } \\
\text { TIONS- UND } \\
\text { RESSOURCEN- } \\
\text { KAPAZITÄT } \\
\text { POLITISCHER } \\
\text { ADMINISTRA- } \\
\text { TION }\end{array}$ & $\begin{array}{l}\text { Pluralismus: } \\
\bullet \text { Policy- } \\
\text { Innovationen } \\
\text { organisatorische } \\
\text { Bestandsrationalität }\end{array}$ & $\begin{array}{l}\text { Segmentierung: } \\
\text { geringe Innovatio- } \\
\text { nen } \\
\text { politische Effizi- } \\
\text { enzrationalität }\end{array}$ \\
\cline { 2 - 3 } & $\begin{array}{l}\text { Polarisierung: } \\
\text { hoch }\end{array}$ & $\begin{array}{l}\text { Struktur- } \\
\text { Innovationen } \\
\text { demokratische Ra- } \\
\text { tionalität }\end{array}$ & $\begin{array}{l}\text { Etatozentrismus: } \\
\text { Policy- } \\
\text { Innovationen } \\
\text { politische Effek- } \\
\text { tivitätsrationalität }\end{array}$ \\
\hline
\end{tabular}

Quelle: Kitschelt (1983), S. 78 ff.; Eigene Zusammenfassung mehrerer Darstellungen

Segmentierung. Geschlossene Artikulations- und Aggregationsprozesse in Verbindung mit einer eher geringen Organisations- und Ressourcenkapazität der politischen Administration führen zu segmentierten Politikzyklen. In den einzelnen Politikbereichen werden die Entscheidungen von den wenigen Zugelassenen getroffen und anschließend von einer sanktionsschwachen Administration im Sinne der jeweiligen Klientel umgesetzt. Das Innovationspotential eines solchen segmentierten politischen Regimes ist daher gering, und es finden meist nur inkrementelle Veränderungen im Sinne einer Effizienzrationalität statt, welche Verbesserungen zu Gunsten der privatwirtschaftlichen Klientel verfolgt.

Polarisierung. Dieser Politikstil ist gekennzeichnet durch Offenheit in der Input-Phase und Stringenz in der Output-Phase des politischen Prozesses.

Die Durchlässigkeit für innovative Problemlösungen führt zu einer Artikulation und Aggregation zahlreicher, gegensätzlicher Handlungsalternativen. Nach der Herbeiführung eines Kompromisses wird die so generierte Entscheidung dann mit hoher Durchsetzungsfähigkeit und unter Einschluß eines Sanktionsinstrumentariums realisiert. Auf diesem Wege sind auch strukturelle Innovationen, welche die aktuellen Vorgehensweisen durch völlig neue Ansätze ersetzen, möglich. Die politische Rationalität 
dieses Regimes dient somit der demokratisch herbeigeführten Entscheidung.

Etatozentrismus. Ein etatozentristischer Politikstil ist charakterisiert durch einen stark limitierten und gefilterten Zugang zur politischen Arena und einem gleichzeitig hohen Implementationspotential der politischen Administration. Eine kleine Elite von politischen Akteuren kann also effektive politische Lösungen im Sinne ihrer Klientel exekutieren. Ähnlich wie im Pluralismus ist auch dieser Politikstil meist nur $\mathrm{zu}$ PolicyInnovationen in der Lage, da strukturändernde Politikalternativen keinen Zugang zur politischen Agenda erhalten.

Beim Vergleich dieses Schemas mit demjenigen Richardsons fallen zwei wesentliche Unterschiede auf.

- Alle Phasen des politischen Prozesses werden berücksichtigt.

- Statt nur nach Handlungspräferenzen der Regierung zu fragen, wird auch die Handlungskapazität in die Analyse einbezogen.

Trotz dieser Unterschiede bleibt jedoch vor allem der Eindruck der Ähnlichkeit zwischen den Entwürfen Richardsons und Kitschelts. Denn zwischen den Merkmalen Offenheit und Konsensorientierung sowie Implemenationskapazität und Antizipationsausmaß bestehen inhaltliche $\mathrm{Zu}$ sammenhänge. Konsens in der Phase des Policymakings setzt notwendigerweise ein gewisses $\mathrm{Ma}$ an Offenheit der Artikulations- und Aggragationsvorgänge des politischen Prozesses voraus. Und aktives, vorausschauendes Regierungshandeln ist ohne entsprechende Handlungskapazitäten der politischen Administration nicht möglich.

4.2.3.3 Die Typologie von Bull (1992): Interessenaggregation nicht-staatlicher Akteure und staatliche Macht als relevante Politikstildimensionen

Ausgehend von seiner Kritik an dem vor allem von Cawson behaupteten Kontinuum zur Beschreibung von Beziehungen zwischen dem Staat und Interessengruppen ${ }^{225}$, welches sich zwischen den beiden Extrema Korporatismus und Pluralismus erstreckt, entwirft Bull durch die Einführung einer zweiten Strukturvariablen eine differenziertere zweidimensionale Typologie ${ }^{226}$. U.a. ist es dadurch möglich, bestimmte Formen der staatlich erzwungenen Konzertierung der gesellschaftlichen Interessengruppen

${ }^{225}$ Cawson, A. (1986): Corporatism and Political Theory, Oxford.

${ }^{226}$ Bull, Martin J. (1992): The Corporatist Ideal-Type . . , a.a.O., S. 264 ff. 
(wie zum Beispiel im italienischen Faschismus) nicht mehr länger der Gruppe des Korporatimus (etwa als state corporatism) zuordnen zu müssen.

Die beiden Dimensionen dieses Modells (Abbildung 10) sind die staatliche Machtfülle (state power) sowie das Ausmaß selbständiger Interessenkonzentration (self-generated concentration of interests).

Der erste Indikator ist ähnlich zu interpretieren wie der von Richardson eingeführte Faktor des staatlichen Antizipationsvermögens oder das bei Kitschelt verwendete Merkmal staatlicher Implementationskapazität. State power liegt offenbar dann vor, wenn der Staat in der Lage ist, Politik aktiv zu gestalten, die Interessen der relevanten politischen Akteure (oder seine eigenen) zu konzertieren und in ausreichender Effektivität zu implementieren.

Der zweite Faktor ist ein Maß zur Beschreibung der Fähigkeit der nichtstaatlichen Akteure, ihre Interessen institutionell und artikulativ $\mathrm{zu}$ formieren. Bull geht davon aus, daß eine solche Interessenkonzentration einen starken Staat trotz seiner Machtkapazitäten zu einem kooperativen Vorgehen veranlaßt bzw. daß die state power ein historischkonstitutionelles Faktum ist, und sich das Ausmaß der Interessenkonzentration der anderen gesellschaftlichen Kräfte je nach Politikstil (demokratisch oder autoritär) daraus ableitet.

Im Falle eines hohen Konzentrationsmaßes der durch die gesellschaftlichen Gruppen selbstformulierten Interessen und einer ausreichenden staatlich-administrativen Durchgriffsmöglichkeit liegt der Politikstil des Korporatismus vor.

Die Kombination von großer staatlicher Machtfülle und eher fragmentierten Interessen der anderen politischen Akteure ergibt den Politikstil des State rule. Allerdings ist auch unter solchen Bedingungen eine Art des Korporatismus denkbar, nämlich dann, wenn die Interessenformierung unter staatlichem Druck erfolgt, die gesellschaftlichen Kräfte also unter staatlicher Aufsicht und Moderation einen dem Gesamtwohl dienenden Interessenausgleich vorzunehmen haben. Dieser "Staats-Korporatimus“ ist die typische Form der Interessenmediation in faschistischen Systemen. 
Abb. 10: Typologie möglicher Beziehungen von Staat und Interessengruppen

\begin{tabular}{|c|c|c|c|}
\hline & \multicolumn{2}{|c|}{$\begin{array}{c}\text { SELBSTÄNDIGE } \\
\text { INTERESSENKONZENTRATION }\end{array}$} \\
\hline & & Niedrig & Hoch \\
\hline \multirow{2}{*}{$\begin{array}{l}\text { STAATLICHE } \\
\text { MACHT }\end{array}$} & Stark & State rule & Korporatismus \\
\hline & Gering & Pluralismus & $\begin{array}{l}\text { Herrschaft von } \\
\text { Privatinteressen }\end{array}$ \\
\hline
\end{tabular}

Quelle: Bull (1992), S. 265; Eigene Übersetzung.

Ein Staat mit eher geringer Macht in Verbindung mit einer hohen Interessenkonzentration der anderen Akteure führt zu monopolistischen Strukturen, in denen die Privatinteressen der am besten formierten gesellschaftlichen Kräfte Politik und Wirtschaft dominieren.

Unter dem Begriff Pluralismus werden schließlich solche Formen des Politikstils zusammengefaßt, in welchen aufgrund eines schwachen Staates und fragmentierter gesellschaftlicher Interessen keine politische oder ökonomische Grundorientierung vorhanden ist.

\subsubsection{Schlußfolgerungen}

Die Skizzierung dieser drei wichtigen Versuche einer Typologie von Politikstilen und daraus resultierenden Regimeformen ergibt für die vorliegenden Arbeit die folgenden Erkenntnisse (siehe auch Abbildung 11):

- In allen Studien wurde ein zweidimensionales Schema gewählt. Diese Vorgehensweise ermöglicht eine ausreichende Differenzierung, bleibt jedoch auf einem anschaulichen Abstraktionsniveau und erlaubt Aussagen mit einem Anspruch auf Allgemeingültigkeit.

- Ebenfalls allen Studien gemeinsam ist als eine Merkmalsdimension ein Indikator zur Messung der politischen Handlungsqualitäten bzw. kapazitäten der Regierung. Gestaltungsmöglichkeiten und -willen der staatlichen Institutionen sind somit aus Sicht der Politikwissenschaft ein grundlegender Faktor zur Beschreibung des Politikstils. 
- Auch die jeweils als zweite Merkmalsdimension gewählten Indikatoren sind sich ähnlich. Stets geht es um die nicht-staatlichen Akteure des politischen Prozesses, deren Zugang zur politischen Arena bzw. deren Partizipationsmöglichkeiten im Umgang mit den staatlichen Entscheidungsträgern. So beschreiben die dargestellten Faktoren die Konsensorientierung der Regierung gegenüber den interest groups (Richardson), die Chancen dieser Gruppen zur Interessenartikulation und aggregation (Kitschelt) sowie das Ausmaß an selbständiger Interessenformierung durch dieselben (Bull). So ist also als zweiter wichtiger Faktor zur Beschreibung des politischen Stils die Rolle der nichtstaatlichen Interessengruppen anzunehmen.

- Als im Sinne der ökonomischen und/oder ökologischen Problemlösungskompetenz günstigster Politikstil wird in allen Untersuchungen (zumindest implizit) ein solcher angesehen, welcher eine aktive, starke Rolle des Staates mit einer weitgehenden Berücksichtigung der Interessen der sonstigen Akteure des politischen Prozesses verbindet.

Im folgenden wird also zu überlegen sein, ob das in der empirischen Untersuchung zu verwendende Schema nationaler Umweltpolitik-Stile (a) ebenfalls zweidimensional konzipiert sein soll und (b) ähnliche Merkmalsdimensionen wie die bereits dargestellten Typologien aufweisen soll.

\section{Abb. 11: Vergleich der vorgestellten Politikstil-Studien}

\begin{tabular}{|c|c|c|c|c|c|c|c|}
\hline \multirow{2}{*}{$\begin{array}{l}\text { Studle } \\
\text { (Jahr) }\end{array}$} & \multicolumn{6}{|c|}{ Policy Cycle-Phasen } & \multirow{2}{*}{$\begin{array}{l}\text { Polidikstil- } \\
\text { dimen- } \\
\text { sionen }\end{array}$} \\
\hline & $\begin{array}{l}\text { Problem- } \\
\text { definition }\end{array}$ & $\begin{array}{l}\text { Agenda } \\
\text { Setting }\end{array}$ & $\begin{array}{l}\text { Politikfor- } \\
\text { mullerung }\end{array}$ & $\begin{array}{l}\text { Implemen- } \\
\text { tation }\end{array}$ & $\begin{array}{c}\text { Evalua- } \\
\text { tion }\end{array}$ & $\begin{array}{l}\text { Terminie- } \\
\text { rung }\end{array}$ & \\
\hline \multirow{2}{*}{$\begin{array}{l}\text { Richardson } \\
\text { (1982) }\end{array}$} & & \multicolumn{5}{|c|}{ Problemlösungsverhalten (reaktiv $v s$ aktiv) } & $\begin{array}{l}\text { 1. Dimen- } \\
\text { slon }\end{array}$ \\
\hline & \multicolumn{4}{|c|}{$\begin{array}{l}\text { Entscheidungsfindung } \\
\text { (Konsens vs Konflikt) }\end{array}$} & & & $\begin{array}{l}\text { 2. Dimen- } \\
\text { slon }\end{array}$ \\
\hline \multirow{2}{*}{$\begin{array}{l}\text { Kitschelt } \\
\text { (1983) }\end{array}$} & & & & \multicolumn{3}{|c|}{$\begin{array}{l}\text { Administrative Kapazitat } \\
\text { (hoch } v s \text { niedrig) }\end{array}$} & $\begin{array}{l}\text { 1. Dimen- } \\
\text { sion }\end{array}$ \\
\hline & \multicolumn{3}{|c|}{$\begin{array}{l}\text { Artikulations- und Aggr.Prozesse } \\
\text { (offen vs geschlossen) }\end{array}$} & & & & $\begin{array}{l}\text { 2. Dimen- } \\
\text { slon }\end{array}$ \\
\hline \multirow{2}{*}{$\begin{array}{l}\text { Bull } \\
\text { (1992) }\end{array}$} & \multicolumn{5}{|c|}{ Staatliche Macht (groB vs gering) } & & $\begin{array}{l}\text { 1. Dimen- } \\
\text { slon }\end{array}$ \\
\hline & \multicolumn{3}{|c|}{$\begin{array}{l}\text { Selbstandige Interessenaggregation } \\
\text { (hoch vs niedrig) }\end{array}$} & ion & & & $\begin{array}{l}\text { 2. Dimen- } \\
\text { slon }\end{array}$ \\
\hline
\end{tabular}




\section{Die Bedeutung des Politikstils als Determinante der Effektivităt nationa- ler Umweltpolitik}

\subsection{Modellentwurf}

Nachfolgend soll ein Modell entworfen werden, welches die für die ökologische Effektivität relevanten Politikstildimensionen und deren Wirkungsmechanismus auf den umweltpolitischen Erfolg darstellt.

Um die Problematik lösen zu können, welche „stilistischen“ Merkmale eine ökologisch effektive Politik aufweisen muß, ist es zunächst notwendig, sich die besonderen Charakteristiken der ökologischen Agenda zu verdeutlichen. Denn wenn die spezifische Art und Beschaffenheit umweltpolitischer Themen klar wird, dann lassen sich daraus auch bestimmte Notwendigkeiten des Policy Styles ableiten.

Umwelthandeln zeichnet sich gegenüber dem Agieren in anderen gesellschaftlichen und politischen Bereichen vor allem durch drei hier besonders ausgeprägte Merkmale aus: (1) Wirkungstiefe, (2) Wirkungsbreite und (3) Komplexität ${ }^{227}$.

(1) Wirkungstiefe bedeutet, daß auf einer bestimmten Ebene getroffene Entscheidungen oder ablaufende Prozesse durch eine geschlossene Kausalkette bis auf weit darunter liegende Ebenen durchgreifen. Der Entschluß zum Bau einer industriellen Großanlage in einem bis dato „unberührten“ Landstrich hat beispielsweise nicht nur Wirkungen auf der direkten Handlungsebene, etwa durch die infrastrukturellen und ökonomischen Veränderungen der Region, er wirkt vielmehr auch deutlich "tiefer". So führen der Verlust der landschaftlichen Unversehrtheit, die Einschränkungen des natürlichen Lebensraumes, die Abgabe von Emissionen, der Ressourcenverbrauch im Produktionsproze $B$ etc. zu einer Minderung der Umweltqualität. Diese Umweltqualitätsminderung wiederum kann auf einer noch tieferen Wirkungsebene einen Rückgang von Fauna und Flora, langfristige Schadstoffablagerungen im Boden oder sogar signifikante Beeinträchtigungen der Gesundheit der ansässigen Bevölkerung bewirken, also zu einer Umweltschädigung führen.

${ }^{227}$ Vgl. Prittwitz, Volker von (1990): Das Katatstrophenparadox . . , a.a.O., S. 54 ff. sowie S. $58 \mathrm{ff}$. 
Ein weiteres Beispiel ist der Entschluß zur Abholzung von Baumbeständen zur Herstellung von Möbeln oder von Papier. Die direkten Effekte die Maßnahme - ein gewisser wirtschaftlicher Nutzen und ein Rückgang der bewaldeten Fläche - sind noch um weitere, indirekte ökologische Folgen zu ergänzen, die sich aus dem Verlust der ökologischen Funktionen des Waldes ergeben: Bodenerosion, Störungen des Wasserkreislaufes, Anstieg des $\mathrm{CO}_{2}$-Gehaltes in der Atmosphäre, Lebensraumverlust für zahlreiche Tier- und Pflanzenarten ${ }^{228}$.

(2) Wirkungsbreite ist in zeitlicher, räumlicher und sachlicher Hinsicht zu verstehen:

- Die zeitliche Wirkungsbreite von Umwelthandeln reicht von nur kurzfristig wirksamen Maßnahmen bis hin zur Produktion von Abfallprodukten beim Betrieb von Atomkraftwerken, deren Lagerung über Tausende von Jahren ein ökologisches Problem bleiben wird. Weitere Beispiele für die Langfristproblematik von Umwelthandeln sind:

$\Rightarrow$ Durch chemische Schadstoffe und ionisierende Strahlung ausgelöste Veränderungen von Keimzellen (genetische Mutationen) übertragen die veränderten genetischen Informationen auf die Körperzellen der nachfolgenden Generationen $^{229}$.

$\Rightarrow$ Der vor allem durch Fluorkohlenwasserstoffe (FCKW) bewirkte Abbau des atmosphärischen Ozons mit seinen Folgen einer Beeinträchtigung von Pflanzenwachstum und maritimen Nahrungsketten sowie einem erhöhten Hautkrebsrisiko aufgrund einer Zunahme der ultravioletten Sonnenstrahlung ist nur sehr langfristig reversibel. Selbst ein sofortiger und sehr weitgehender Rückgang der Produktion von FCKW und anderen problematischen Gasen würde zu keiner schnellen Verbesserung der Lage führen, da die Diffusion dieser Stoffe in die Atmosphäre 20 bis 30 Jahre beansprucht $^{230}$.

\footnotetext{
${ }^{228}$ Vgl. Dieren, Wouter van (1995): Mit der Natur rechnen. Der neue Club-of-Rome-Bericht: Vom Bruttosozialprodukt zum Ökosozialprodukt, Basel u.a., S. 72.

${ }^{229}$ Vgl. Bossel, Hartmut (1990): Umweltwissen, Berlin, S. 122 f.

${ }^{230}$ Ebd. S. 131.
} 
- Die räumliche Wirkungsbreite erstreckt sich über ein Kontinuum, dessen Extrema zum einen regional begrenzte Auswirkungen und zum anderen globale Effekte bilden. Als Beispiele für überregionale und teilweise auch globale Effekte von Umwelthandeln seien erwähnt ${ }^{231}$ :

$\Rightarrow$ Die Belastung der Ostsee durch Schadstoffeinleitungen der Anrainerstaaten und Schadstoffeinträgen über die Luft mit dem Resultat großflächiger Eutrophierung und der Vernichtung großer Meeresregionen als maritime Lebensräume ${ }^{232}$.

$\Rightarrow$ Der sogenannte „Treibhauseffekt", d.h. die Absorbtion der von der Erdoberfläche reflektierten Sonnenstrahlung durch eine Erhöhung von Spurengasen (Kohlendioxyd, FCKW, Halone etc.) und der damit einhergehende Anstieg der bodennahen Temperatur. Die Folgen für die Umwelt liegen in einer polwärtigen Verschiebung der Klima- und Vegetationszonen, einer Veränderung des hydrologischen Kreislaufes mit gravierenden Auswirkungen auf die landwirtschaftlichen Erträge sowie bedeutenden Landverlusten durch ein Ansteigen des Meeresspiegels. Verursacht wird die Zunahme der schädlichen Spurengase vor allem durch die Verbrennung fossiler Energieträger, die Verwendung von FCKW und Halonen bei der Produktion von z.B. Kühlgeräten und Schaumstoffen, durch Abholzung von Waldbeständen sowie durch Nutzviehhaltung und Naßreisanbau ${ }^{233}$.

$\Rightarrow$ Der bereits erwähnte Ozonabbau in der Stratosphäre, der durch die Schädigung des in den obersten Ozonschichten lebenden und einen großen Teil der weltweiten $\mathrm{CO}_{2}$ Emissionen bindenden Phytoplanktons zu einer Beschleunigung des Treibhauseffektes beiträgt.

- Die sachliche Wirkungsbreite schließlich kann sowohl sehr begrenzt sein als auch nahezu alle Bereiche des gesellschaftlichen und staatlichen Lebens erfassen (man denke zum Beispiel an die

${ }^{231}$ Vgl. auch Prittwitz von, Volker (1986): Drei Ansätze . . ., a.a.O., sowie Ders. (1989): Internationale Umweltregime - Ein Fallvergleich, In: Kohler-Koch (Hg.): Regime in den internationalen Beziehungen, Baden-Baden, S. 389-405.

${ }^{232}$ Vgl. auch: Umweltbundesamt (1995): Unweltdaten Deutschland 1995, Berlin, S. 32.

${ }^{233}$ Vgl. Bleischwitz, Raimund (1995): Atmosphäre und Klima, In: Hauchler, Ingomar (Hg.): Globale Trends 95/96. Daten zur Weltentwicklung, Frankfurt am Main, S. $260 \mathrm{ff}$. 
Änderungen des öffentlichen Lebens in den Monaten nach der Tschernobyl-Katastrophe). Prittwitz verdeutlicht die sachliche Wirkungsbreite des umweltpolitischen Handelns wie folgt ${ }^{234}$ : „Stark selektive Schutzmaßnahmen, zum Beispiel eine nur in einem Ort unter bestimmten Bedingungen in Kraft tretende Alarmregelung, und universell wirkende Handlungsformen, zum Beispiel das generelle Verbot der Produktion und Konsumtion eines Schadstoffs, stehen sich gegenüber."

(3) Die hohe Komplexität umweltpolitischer Problematiken ist in den Ausführungen zu Wirkungstiefe und -breite bereits angedeutet worden. Die zahlreichen (Inter-)Dependenzen und Rückkopplungen ökologischer Systeme und Prozesse und deren Vernetzung mit gesellschaftlichen und techno-ökonomischen Rahmenbedingungen läßt sich oft nur mit dem Instrument der Systemanalyse darstellen und untersuchen ${ }^{235}$. Außerdem sei hier auf die Ausführungen Bossels verwiesen, der zur Einleitung seiner besonders anschaulichen Darstellung der komplexen ökologischen Thematik ausführt ${ }^{236}$ :

„Herausragendes Merkmal des Systems Mensch-Technik sind die Rückkopplungen zwischen den Komponenten. Eingriffe des Menschen bleiben also selten auf ihre direkten Wirkungen im ÖkoSystem beschränkt, sondern sie wirken im Zusammenspiel mit den Folgen anderer Eingriffe auf die anderen Komponenten des Systems ein und schließlich auch auf den Menschen zurück. Es kommt auf die Art und Stärke der Verkopplungen zwischen den Komponenten an, ob sich die Effekte in Rückkopplungskreisen abschwächen oder verstärken."

Zusammenfassend läßt sich also formulieren, daß es ein offensichtliches Spezifikum von Umweltpolitik ist, daß die in diesem Politikfeld auftretenden Entwicklungen und die getroffenen Maßnahmen in besonderem Ausmaß über die Eigenschaften der Wirkungstiefe, der kurz- und mittelfristigen Irreversibilität, der überregionalen und globalen Bedeutung, der sachlichen Wirkungsbreite sowie der Komplexität verfügen. Dies vor allem deswegen, weil die Umweltpolitik auf langfristige, nach naturwissen-

\footnotetext{
${ }^{234}$ Prittwitz, Volker von (1990): Das Katatstrophenparadox . . , a.a.O., S. 54 ff. sowie S. 59.

${ }^{235}$ Siehe etwa Abbildung 1 im einleitenden Kapitel dieser Untersuchung.

${ }^{236}$ Bossel, Hartmut (1990): Umweltwissen, Berlin, S.6.
} 
schaftlichen Gesetzmäßigkeiten ablaufende Prozesse reagieren muß bzw. durch ihre Entscheidungen solche in Gang setzen kann, und sich diese dann der nachträglich steuernden politischen Einflußnahme entziehen.

Weil also die Wirkungen auf die Umwelt oft langfristig und irreversibel sind, müssen die umweltpolitischen Maßnahmen in ihrer Art und ihrem Inhalt auch diesen strategischen Implikationen angemessen sein.

Der umweltpolitisch ideale Handlungstypus ist deshalb laut von Prittwitz die strukturelle Ökologisierung von Staat und Gesellschaft ${ }^{237}$. Durch eine langfristig angelegte Politik sollen grundsätzliche Veränderungen der Produktions- und Konsumtionsmechanismen mit einer entsprechend groBen Wirkungsbreite und -tiefe herbeigeführt werden. Grundlage für dieses Vorgehen sind zum einen eine hierfür günstige postmaterielle gesellschaftliche Werteskala und zum anderen die Kooperation aller relevanten Interessengruppen. Diese Betonung der „Allgemeinheit" als Träger einer strukturellen Ökologisierung ist neben der Notwendigkeit einer strategisch-strukturellen Ausrichtung der Politik der zweite wichtige Hinweis für die Beschaffenheit einer effektiven Umweltpolitik.

Ähnliche Ansprüche an eine effektive Umweltpolitik stellt Simonis. Seine Forderung nach einer strategisch-präventiv ausgerichteten Umweltpolitik gegründet er wie folgt ${ }^{238}$ : „Die Strategie des herkömmlichen Umweltschutzes - so wichtig sie zur akuten Gefahrenabwehr und zur Bewältigung von Altlasten sein mag - kann auch deshalb nicht befriedigen, weil bei ansonsten nur wenig veränderter Produkt- und Technologiestruktur das fortgesetzte Wachstum der Produktion überproportional steigende Umweltschutzaufwendungen hervorruft, wenn das Gesamtniveau der Schadstoffemissionen nicht weiter steigen soll. Zukunftsfähigkeit der Industriegesellschaft verlangt daher nach Dynamisierung, nicht nach Festschreiben von Umweltnormen; insbesondere bei rasch wachsendem Produktionsniveau müssen die Input- und Emissionsstandards sukzessive verschärft werden.[...] Es geht [...] um die Betonung und das Einfordern der Präventionskomponente der Umweltpolitik."

Zur argumentativen Unterstützung seines Plädoyers für eine aktivvorsorgende Umweltpolitik führt Simonis in Anlehnung an Scimemi die folgenden Überlegungen an (Abbildung 12) ${ }^{239}$ :

${ }^{237}$ Prittwitz, Volker von (1990): Das Katatstrophenparadox . . , a.a.O., S. 90 ff.

${ }^{238}$ Simonis, Udo E. (1996): Ökologische Umorientierung der Industriegesellschaft, in: Aus Politik und Zeitgeschichte, 7, S. 8 f. 
Im „umweltpolitischen Entscheidungszyklus“ gibt es eine Phasenverschiebung zwischen dem Niveau der Umweltschäden, dem Niveau des technischen und administrativen Wissens zur Problembeseitigung und des öffentlichen Umweltbewußtseins. D. h. die Umweltschädigung tritt zuerst auf, hat bei Erreichen des Schwellenwertes des notwendigen technischen Wissens $(t)$ bereits das Niveau $D_{1}$ erreicht, wird jedoch erst bei Erreichen des Schwellenwertes für das notwendige Umweltbewußtsein $(p)$ auf dem Niveau $\mathrm{D}_{2}$ angegangen.

\section{Abb. 12: Faktoren des Umweltpolitikzyklus}

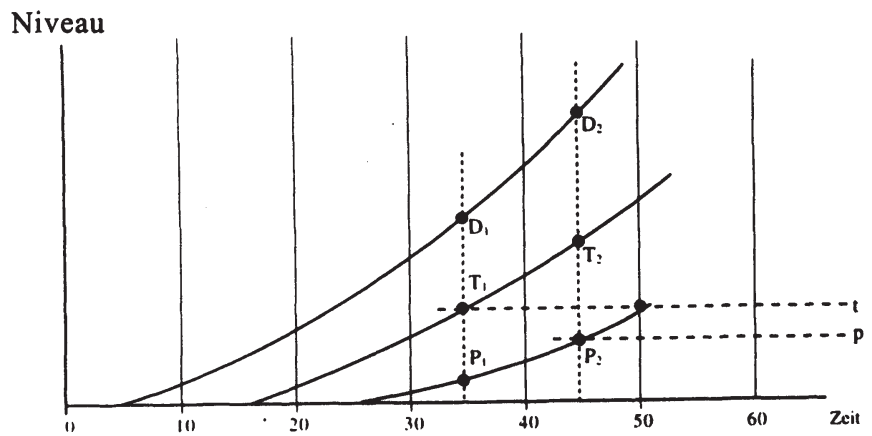

Quelle: Scimemi (Fn. 239)

Anm.: D:=Umweltschäden; $\mathrm{T}:=$ technisches Wissen; $\mathrm{P}:=$ öffentliches Umweltbewußtsein; $t:=$ Schwellenwert des notwendigen technischen Wissens; $p:=$ Schwellenwert des notwendigen Umweltbewußtseins

Eine Verringerung der zeitlichen Lücke zwischen dem Auftreten der Umweltschäden und dem politischen Handeln könne nur durch eine strategische Ausrichtung der Umweltpolitik erreicht werden (Abbildung $13)^{240}$ :

\section{"Im besonderen}

- Reduzierung der „Schwellenwerte“ des [...] notwendigen Umweltbewußtseins (p), konkret: frühzeitige Beteiligung aller Betroffenen, diskursiver Politikdialog;

${ }^{239}$ Scimemi, Gabriele (1988): Environmental Policies and Anticipatory Strategies, In: Simonis, Udo E. (Hg.): Präventive Umweltpolitik, Frankfurt am Main, S. 27-48; zit. nach Simonis, Udo E. (1996): Ökologische Umorientierung ..., a.a.O., S. 9 ff.

${ }^{240}$ Ebd., S. 10. 
- Reduzierung der "Schwellenwerte" des notwendigen technischen Wissens (t), konkret: dynamische Verschärfung der Input- und Emissionsstandards. “

\section{Abb. 13: Strategische Ansătze für eine präventive Umweltpolitik}

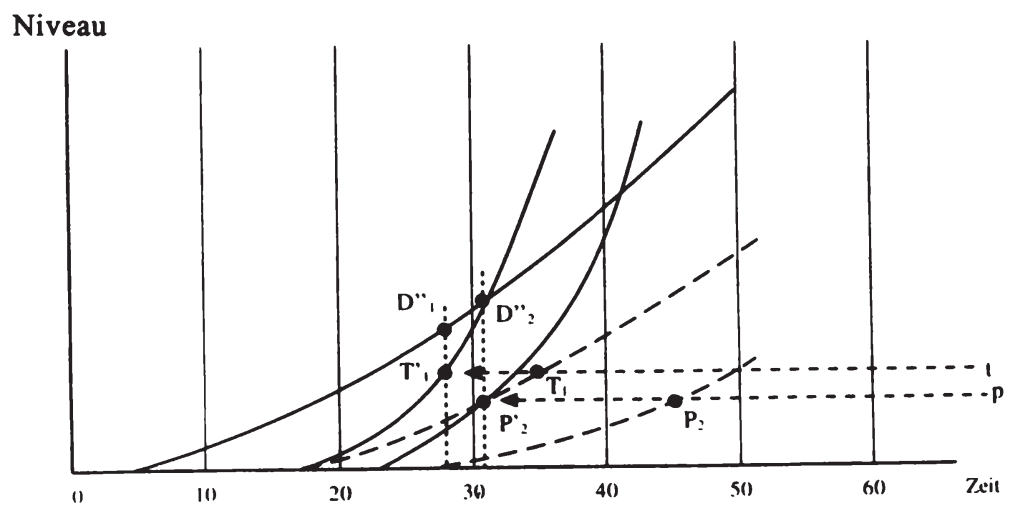

Quelle: Scimemi (Fn. 239)

Anm.: D:=Umweltschäden; $T:=$ technisches Wissen; $P:=0$ ffentliches Umweltbewußtsein; $t:=$ Schwellenwert des notwendigen technischen Wissens; $p:=S c h w e l l e n w e r t ~ d e s$ notwendigen Umweltbewußtseins

Simonis fordert somit eine Flankierung präventiver Umweltpolitik durch die Partzipation der Betroffenen im Rahmen eines ,diskursiven Politikdialoges". Mit der Feststellung dieser beiden Hauptdimensionen erfolgreicher Umweltpolitik ähneln seine Ausführungen den bereits dargestellten Überlegungen von Prittwitz', der eine Umweltpolitik für effektiv erklärt, die sowohl vorausschauend strategische als auch kooperative Elemente enthält.

Auch für Kurz zeichnet sich eine zukunftsfähige Umweltpolitik u.a. durch die Kombination aus Langfristorientierung und dem Praktizieren neuer Formen der Konsensfindung (z.B. Bürgerforen und Mediationsverfahren) aus 241 .

${ }^{241}$ Kurz, Rudi (1996): Innovationen in eine zukunftsfăhige Entwicklung, in: Aus Politik und Zeitgeschichte, 7, S. 20. 
Böhret schließlich schlägt als dem Umweltproblem adäquat ebenfalls eine Mischung aus aktiver, politisch-administrativer Steuerung und partizipativen Elementen vor ${ }^{243}$.

Zusammenfassend läßt sich anhand der obigen Überlegungen also formulieren (Abbildung 14): eine der umweltpolitischen Problematik angemessene Politik muß zum einen strategisch, d.h. vorausschauend und langfristig angelegt sein, und zum anderen einen eher hohen Grad der Partizipation nicht-staatlicher Akteure am politischen Proze $B$ aufweisen. Ist nur jeweils eine der beiden Merkmalsdimensionen in diesem Sinne ausgeprägt, also verbinden sich eine strategische Politikausrichtung mit einem geringen Partizipationsgrad bzw. eine reaktiv-nachsorgende Politik mit einem hohen Partizipationsgad, dann ist mit einer nur mittleren Effektivität der Umweltpolitik zu rechnen. Von einer reaktiven Politik, die außerdem den nicht-stattlichen Akteuren ein geringes Partizipationsausmaß am politischen Entscheidungsprozeß zubilligt, ist schließlich eine eher erfolglose Umweltpolitik zu erwarten.

Zur Plausibilisierung dieser vermuteten Effektivitätsgrade unterschiedlicher Environmental Policy Styles wird nachfolgend eine detaillierte Analyse der Wirkungsmechanismen der beiden Faktoren Strategieorientierung und Partizipationsgrad auf die Effektivität nationaler Umweltpolitik durchgeführt.

${ }^{242}$ Böhret, Carl (1990): Folgen. Entwurf furr eine aktive Politik gegen schleichende Katastrophen, Opladen, S. 14. 
Abb. 14: Merkmalsdimensionen nationaler Umweltpolitik-Stile

\begin{tabular}{|c|c|c|}
\hline $\begin{array}{r}\text { Partizipationsgrad der } \\
\text { nicht-staatlichen } \\
\text { Akteure }\end{array}$ & Hoch & Gering \\
\hline $\begin{array}{c}\text { Problem- } \\
\text { lösungsverhalten }\end{array}$ & Hohe Effektivität & Mittlere Effektivität \\
\hline $\begin{array}{c}\text { Strientiert } \\
\text { Reaktiv }\end{array}$ & Mittlere Effektivität & Geringe Effektivität \\
\hline
\end{tabular}

5.2 Ökologisch relevante Politikstildimensionen

\subsubsection{Strategieorientierung}

Der umweltpolitsche Nutzen einer ausgeprägten Strategieorientierung liegt auf zweierlei Ebenen. Zum einen kommt es darauf an, trendhafte Entwicklungen in ihrer Bedeutung frühzeitig zu erkennen, um präventiv wirken zu können. Zum anderen ist es einem Staat mit einer antizipativ agierenden politischen Elite eher möglich, die eventuellen mittel- und langfristigen Folgen des heutigen Handelns in den Entscheidungsprozeß miteinzubeziehen und notwendige Korrekturen zur Vermeidung von Umweltschäden vorzunehmen.

Um vorausschauend agieren zu können, sind zunächst die folgenden Fähigkeiten notwendig:

- Ein System zur frühzeitigen Identifikation und Prognose von negativen oder chancenreichen mittel- und langfristigen Entwicklungen;

- Staatliche Institutionen zur Formulierung einer langfristigen Politik (institutionell-strukturelle Strategiefähigkeit) ${ }^{243}$;

${ }^{243}$ Vgl. Jänicke, Martin (1990): Erfolgsbedingungen . . , a.a.O., S. 225 ff. 
- Ausreichende ökonomische, technologische und administrative Ressourcen zur Implementierung und Überwachung strategischen Handelns (Handlungskapazität) ${ }^{244}$.

Als Frühwarnsystem zum rechtzeitigen Erkennen langfristiger Entwicklungen soll im Rahmen dieser Untersuchung keine technische Einrichtung mit landesweit dislozierten Stationen zur Messung der Umweltqualität verstanden werden, sondern ein Spezifikum des nationalen Politikstils, also bestimmte Verhaltens- und Verfahrensweisen innerhalb des politischen Prozesses, welche das frühzeitige Erkennen wichtiger Trends erlauben oder sogar fördern. Als in diesem Sinne förderlich ist vor allem eine starke Stellung der subnationalen Einheiten in allen Phasen des Policy Cycles anzuführen.

Die Literatur der Umweltpolitikanaylse liefert zahlreiche Belege für eine umweltpolitische „Überlegenheit dezentraler Strukturen “245.

So bieten gemäß Nelkin und Pollack solche Strukturen den ökologischen Interessengruppen größere Chancen, die Problemdefinition und die Agenda-Gestaltung zu beeinflussen ${ }^{246}$.

Auch für die Implementationsphase gibt es empirische Evidenz für eine positive Wirkung der Partizipation subnationaler Einheiten. So beobachten Knoepfel und Weidner im Rahmen ihrer internationalen Vergleichsstudie der Luftreinhaltepolitik mehrerer OECD-Staaten, daß diese regionalen Exekutiven ihren Handlungsspielraum im Sinne einer effektiveren Umsetzung von Standards nutzen ${ }^{247}$.

${ }^{244}$ Vgl. Prittwitz, Volker von (1989): Internationale Umweltregime - Ein Fallvergleich, In: Kohler-Koch, Beate (Hg.): Technik und internationale Politik, Baden-Baden, S. 241.

${ }^{245}$ Vgl. die Übersicht bei: Kern, Kristine, und Bratzel, Stefan (1994): Erfolgskriterien . . ., a.a.O., S. 56.

${ }^{246}$ Vgl. Nelkin, Dorothy, und Pollak, Michael (1981): The Atom Besieged. Extraparlamentary Dissent in France and Germany, Cambridge und London.

${ }^{247}$ Vgl. Knoepfel, Peter, und Weidner, Helmut (1985): Luftreinhaltepolitik (stationäre Quellen) im internationalen Vergleich, Berlin, sowie Dies. (1986): Explaining Differences in the Performance of Clean Air Policies: An International and Interregional Comparative Study, in: Policy and Politics, 14, S. 71-91. 
Einen eher indirekten Effekt dezentraler stattlicher Strukturen vermuten Jänicke und Mönch, indem sie diese als Teil einer für eine erfolgreiche Umweltpolitik notwendigen Innovationsfähigkeit anführen ${ }^{248}$.

Eine sehr detailierte Beschreibung der umweltpolitisch positiven Wirkungen des Föderalismus liefert schließlich Weidner am Beispiel der inzwischen mehr als 25jährigen Geschichte moderner Umweltpolitik in Deutschland ${ }^{249}$ :

- Die föderalen, subnationalen Politikebenen erlauben den umweltpolitischen Interessengruppen politische „Gehversuche“ (,political training field "), um sich für höhere Verantwortungs- und Handlungsebenen $\mathrm{zu}$ qualifizieren.

- Sie bieten diesen Gruppen außerdem den Zugang (,channels for getting access ") zum politisch-administrativen System.

- Föderale politische Strukturen ermöglichen der betroffenen Öffentlichkeit die Möglichkeit zu direkter Partizipation in konkreten Politikfragen.

- Aufgrund der häufigen Wahlkämpfe (allein 16 Landtagswahlen innerhalb von 4 bis fünf Jahren) können ökologische Streitfragen öfter thematisiert werden.

Deshalb lautet Weidners Fazit ${ }^{250}:$, [...] in the German political context federalism was a necessary [...] condition for the environmental achievements [...] and for the process of political and ecological modernisation $[\ldots]$. “

Allgemein ist es vor allem in zwei Situationstypen denkbar, daß eine föderale Struktur einen positiven Einfluß auf das umweltpolitische Ergebnis hat. Zum einen bei der Entscheidung über primär regional belastende Vorgänge (z.B. Bau und Inbetriebnahme eines Kraftwerkes) und zum anderen im Falle der Möglichkeit einer regionalen Verschärfung zentralstaatlich initiierter Umweltstandards (z.B. Smogwarnstufen). Der erste Fall betrifft insbesondere die ersten drei Phasen des politischen Prozesses. Die frühzeitige Einbeziehung der Regional- und Lokalregierungen in den Entscheidungsproze $ß$ erlaubt die Berücksichtigung sonst nicht be-

${ }^{248}$ Vgl. Jänicke, Martin, und Mönch, Harald (1988): Ökologischer . . ., a.a.O., S. 401.

${ }^{249}$ Vgl. Weidner, Helmut (1995): 25 Years of Modern Environmental Policy in Germany. Treading a Well-Worn Path to the Top of the International Field, Berlin, S. 82.

${ }^{250}$ Ebd., S. 83. 
kannter Argumente, verhindert die einseitige Belastung bestimmter Regionen und erhöht die Legitimität der dann getroffenen Entscheidungen.

Der zweite Fall, nämlich die Nutzbarmachung der subnationalen Einheiten in der Implementationsphase kann sich außer in regional möglichen Verschärfungen nationaler Standards auch in geringeren Kontrollkosten der umweltpolitischen Maßnahmen niederschlagen, indem Überwachung und Sanktionen den bereits bestehenden lokalen Verwaltungen übertragen werden können.

Eine wohlfahrtstheoretische Begründung für die bessere Eignung dezentraler Strukturen zur Allokation des öffentlichen Gutes Umweltqualität liefert das Dezentralisierungs-Theorem Blankerts ${ }^{251}$ :

„Wenn ein öffentliches Gut in abgeschlossenen geographischen Teilräumen angeboten werden kann und wenn dessen Erzeugungsgrenz- und Erzeugungsdurchschnittskosten bei jedem Outputniveau in jedem Teilraum die gleichen sind, unabhängig davon, ob die Leistung zentral oder dezentral erzeugt wird, dann ist es immer effizienter oder wenigstens gleich effizient, wenn lokale Regierungen die an die jeweilige Nachfrage angepaßten Outputs bereitstellen, als wenn die Zentralregierung einen einheitlichen Output (wie groß er auch immer sei) bereitstellt."

Zimmermann und Kahlenborn stellen diesen Zusammenhang graphisch dar (Abbildung 15) ${ }^{252}$. Zur Interpretation dieser Graphik sind aus Gründen der Vereinfachung die folgenden Grundannahmen notwendig:

- die Bevölkerung eines Staates teilt sich in zwei gleich-große regional getrennte Gruppen;

- innerhalb der Gruppen sind die Nachfragekurven der Individuen nach dem öffentlichen Gut Umwelt identisch;

- die Nachfrage ist zwischen beiden Gruppen stark unterschiedlich (Kurve $\mathrm{N}_{1}$ bzw. $\mathrm{N}_{2}$ );

- der Preis für das öffentliche Umweltgut beträgt konstant p* (Grenzkostenpreis).

Unter Berücksichtigung dieser Annahmen ergeben sich jeweils die optimalen Gütermengen $Q_{1}$, bzw. $Q_{2}$, weil die beiden Gruppen jeweils ihren

${ }^{251}$ Blankert, Ch. B. (1991): Öffentliche Finanzen in der Demokratie, München, S. 552; zitiert nach: Zimmermann, Klaus W. und Kahlenborn, Walter (1994): Umweltfoderalismus, a.a.O., S. 35 .

252 Ebenda, S. 36. 
Nettonutzen maximieren (Grenzkosten $=$ Preis $=$ Grenznutzen). Im Falle zentralistischer Regierungsstrukturen würde wahrscheinlich für beide Gruppen das gleiche Niveau, z.B. $Q_{n}$, angeboten ${ }^{253}$. Dieses Niveau würde jedoch für beide Gruppen einen Wohlfahrtsverlust durch Über- bzw. Unterversorgung bedeuten (die Zusatzkosten betrügen pro Individuum die Dreieckflächen $\mathrm{ABC}$ für Gruppe $1 \mathrm{bzw}$. BDE für Gruppe 2).

\section{Abb. 15: Dezentralisierungstheorem}

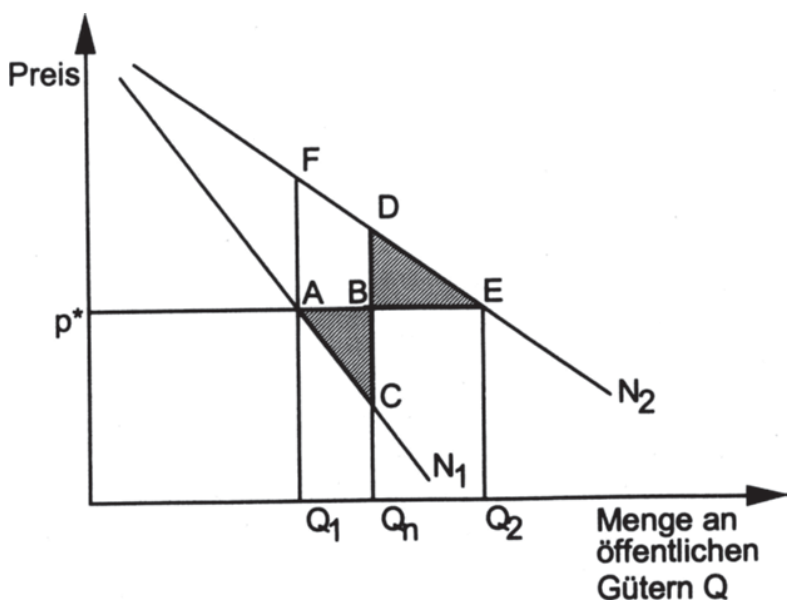

Quelle: Zimmermann und Kahlenborn (1994), S. 36

Auch für den Fall, daß ein Landesteil sein Optimum durchsetzt, ist mit Wohlfahrtsverlusten zu rechnen. Setzt z.B. Gruppe 1 ihr Optimum durch, hat Gruppe 2 den Wohlfahrtsverlust von AFE allein zu tragen. Eine optimale Allokation ökologischer Güter ist also nur im Falle regional differenzierter unterschiedlicher Angebotsniveaus möglich.

${ }^{253}$ Natürlich wäre auch im zentralistischen Staat eine ungleiche Allokation der Gütermengen in Höhe des jeweils optimalen Niveaus denkbar. Dieses Verhalten widerspräche jedoch dem einheitsstaatlichen Ziel der Gleichheit der Lebensbedingungen (vgl. Schultze, Rainer-Olaf (1995): Föderalismus, In: Nohlen, Dieter (Hg.): Wörterbuch . .., a.a.O., S. 156), und soll deshalb nicht weiter behandelt werden. 
Zimmermann und Kahlenborn übertragen diese besondere Eignung föderaler Staatsstrukturen auf nationale (z.B. Verkehr), supranationale (z.B. Fließgewässer) sowie globale Umweltprobleme ${ }^{254}$. In diesen Fällen grenzüberschreitender Umweltschäden (Spillovers) sind nicht die lokale oder regionale Handlungsebene im Sinne einer Kostenminimierung ${ }^{255}$ problemadäquat, sondern die nationale oder supranationale Ebene ${ }^{256}$. Die jeweils problemadäquate Auswahl der Handlungsebene ist aber eher in einem föderalen Staat möglich, während ein zentralistischer Staat primär national oder im internationalen Kontext handeln kann, für lokale oder regionale Umweltprobleme also kaum die kostenoptimale Handlungsebene finden wird.

Im Kontext der Thematik einer institutionell-strukturellen Strategiefähigkeit von Staaten fällt der Blick zuerst auf institutionalisierte Strategiezentren und think tanks einzelner Staaten, allen voran das japanische MITI (Ministerium für Technologie und Außenhandel), aus welchem die sektorale und technologische Langfrist-Steuerung der Volkswirtschaft dieses asiatischen Landes erfolgt ${ }^{257}$. Während es auch in einigen anderen Staaten ähnliche Versuche einer strategischen Wirtschaftsplanung durch die Konzertierung der wichtigsten betroffenen Bereiche gibt ${ }^{258}$, sieht der Institutionalisierungsgrad einer längerfristig angelegten Umweltpolitik in den meisten OECD-Staaten eher dürftig aus.

Ein weiterer Bestandteil der nationalen Fähigkeiten zur frühzeitigen Reaktion auf umweltpolitisch relevante Entwicklungen ist das Vorhandensein ökologischer Instanzen in anderen Politikfeldern. Aufgrund der be-

${ }^{254}$ Ebenda, S. $227 \mathrm{ff}$.

${ }^{255}$ Die $\mathrm{zu}$ minimierenden Kosten sind dabei im einzelnen: Verwaltungs- und Implementierungskosten, Verhandlungskosten, Kosten aus ineffizienter Harmonisierung, Kosten aus internen und externen Effekten, economies of scale, sowie Kosten aus Marktsegmentierung (vgl. jeweils: Ebenda, S. 214 ff.).

${ }^{256}$ Ebd., S. $181 \mathrm{ff}$.

257 Vgl. Johnson, Chalmers (1977): MITI and Japanese International Economic Policy, In: Scalapino, Robert A. (Hg.): The Foreign Policy of Modern Japan, Berkeley, Los Angeles, S. 253-255.

${ }^{258}$ Siehe die Beispiele Schweden und Luxemburg bei Jänicke, Martin (1990): Erfolgsbedingungen ..., a.a.O., S. 226 f. 
stehenden Interdependenzen zwischen Verkehrs-, Wirtschafts- oder Tourismuspolitik auf der einen und der Umweltpolitik auf der anderen Seite wäre es im Sinne einer Berücksichtigung ökologischer Anliegen sinnvoll, wenn die Prüfung auf die Umweltverträglichkeit bestimmter Entscheidungen in Art einer Querschnittsfunktion in allen wichtigen Ressorts bestünde. Je früher im politischen Prozeß diese Einflußnahme geschieht, desto effizienter würde der politische Prozeß verlaufen, da dann die Kosten zur Annulierung getroffener Entscheidungen minimiert werden könnten.

Die Existenz föderaler sowie wirksamer institutionell-intragouvernmentaler Strukturen reicht zum Zweck des strategischen Agierens auf ökologischem Gebiet jedoch nicht aus. Dezentralität und institutionellstrukturelle Strategiefähigkeit sind nur dann wirksam, wenn sie mit einer nennenswerten Handlungskapazität zusammentreffen. D.h. nachdem ein Staat die relevanten Entwicklungen analysiert und eine eigene Handlungsstrategie entworfen hat, müssen die Ressourcen zur Umsetzung und Überwachung dieser langfristigen Absichten vorhanden sein. Vor allem auf drei Feldern sind dazu Mittel notwendig:

- Ökonomie. Eine ökologische Langfriststrategie verlangt ein gewisses Mindestniveau an Wirtschaftskraft. Im Rahmen einer über einen weiten zeitlichen Horizont entworfenen Energiepolitik kann es zum Beispiel notwendig sein, kurzfristig umfangreiche Mittel in alternative, teure Energieformen zu investieren, wissend, daß sich mittel- und langfristig auch die ökonomische Rentabilität dieser Maßnahme herausstellen wird.

- Technologie. Mit dem ersten Punkt eng verknüpft sind die technologischen Ressourcen. Die im Kontext einer präventiven Umwelt- und Energiepolitik meist notwendigen Innovationen im industriellen Produktionsproze $B$ verlangen ein entsprechendes Know-How auf dem Gebiet der Forschung und Entwicklung. Erst der koordinierte Einsatz monetärer und wissenschaftlicher Mittel kann also zum umweltpolitischen Erfolg führen.

- Administration. Ein weiterer wichtiger Punkt ist das Bestehen effizienter bürokratischer Strukturen in geeigneter Größe, welche die notwendigen Maßnahmen initiieren und überwachen. Kitschelt spricht hier von der „Organisations- und Ressourcenkapazität der politischen Administration" ${ }^{\circ 59}$, welche für strukturelle Innovationen mit entsprechender

${ }^{259}$ Vgl. Kitschelt, Herbert (1983): Politik und Energie . . , a.a.O., S. 78 ff. 
Wirkungstiefe und -breite notwendig ist. Da es sich bei umweltpolitischen Grundsatzentscheidungen oft um Fragen mit einer hohen zeitlichen und strukturellen Reichweite handelt, also nicht lediglich oberflächliche und vernachlässigbare Policy-Modifikationen angestrebt werden, ist eine handlungsfähige Administration zur Umsetzung und Kontrolle der gefaßten Maßnahmen unerläßlich.

Erst das Vorliegen ausreichender Mittel auf wirtschaftlichem, technischem und administrativem Gebiet befähigt also einen Staat, eine generierte strategische Entscheidung auch wirksam umzusetzen.

\subsubsection{Partizipationsgrad}

„Gerade in Umweltfragen hat sich gezeigt, daß kompetente Auseinandersetzung nicht den Experten und Politprofis überlassen werden muß, sondern Bürgerbeteiligung möglich und fruchtbar ist. Politik der Nachhaltigkeit verlangt daher eine Stärkung der Beteiligungsmöglichkeiten und rechte der Bürger ...

Mit diesen Ausführungen verdeutlicht Kurz die Bedeutung eines hohen Maßes an Partizipation durch nicht-staatliche Organisationen und Einzelpersonen am umweltpolitischen Prozeß.

In der Policy-Forschung wird vor allem das "Staatsversagen" auf dem Feld der ökologischen Probleme als Grund für die Notwendigkeit partizipativer Problemlösungsverfahren angeführt ${ }^{261}$.

Der spürbaren Zunahme komplexer Umweltprobleme sowie deren Auswirkungen auf Öko-Systeme und Gesellschaften stehen danach inzwischen inadäquate hierarchische und imperative staatliche Steuerungsformen gegenüber. Hinzu kommt die Tatsache, daß die staatlichen Vertreter im traditionellen umweltpolitischen Policy Cycle zunehmend der inhaltlichen und juristischen Kompetenz der betroffenen Unternehmen und Verbände unterlegen sind. Vor diesem Hintergrund wirkt ein Allzuständigkeitsanspruch staatlicher Stellen realitätsfern.

Zur Lösung dieses Dilemmas und zur Verbesserung der Qualität umweltpolitischer Entscheidungsverfahren kann deshalb ein höheres $\mathrm{Maß}$ an

${ }^{260}$ Kurz, Rudi (1996): Innovationen . . ., a.a.O., S. 20.

${ }^{261}$ Vgl. auch im folgenden: Zilleßen, Horst, und Barbian, Thomas (1992): Neue Formen der Konfliktregelung in der Umweltpolitik, in: Aus Politik und Zeitgeschichte, 39-40, S. 14 f., sowie Fietkau, Hans-Joachim, und Weidner, Helmut (1992): Mediationsverfahren in der Umweltpolitik. Erfahrungen in der Bundesrepublik Deutschland, in: Aus Politik und Zeitgeschichte, 39-40, S. 24 f., und die dort gemachten weiteren Literaturhinweise. 
Partizipation beitragen. Diese Partizipation sollte sich jedoch nicht - wie in den traditionellen Beteiligungsverfahren - in politischen und juristischen Abwehrkämpfen gegen nicht gewollte Entscheidungen erschöpfen, sondern gedacht ist vielmehr an eine "Enthierarchisierung der Beziehung zwischen Staat und Gesellschaft ${ }^{\prime 262}$ mit dem Ziel gemeinsamer, konsensorientierter Problemlösungsbemühungen durch alle relevanten Beteiligten und Betroffenen. Zu diesem Zweck wurden vor allem in den USA unter der Bezeichnung "Alternative Dispute Resolution - ADR" zahlreiche Verhandlungs- und Vermittlungsverfahren - auch Mediationsverfahren genannt - erprobt ${ }^{263}$.

Als grundlegende Charakteristika von ADR-Verfahren gelten:

1. Die umweltpolitische Problematik wird von allen Beteiligten und Interessierten in einem gemeinsamen Problemlösungsverfahren behandelt, d.h. es müssen Repräsentanten aller relevanten Institutionen, Organisationen und Gruppierungen einbezogen werden. Dadurch kann das Teilnehmerspektrum neben Vertretern des Staates, der Parteien und der Wirtschaft auch lokale Bürgerinitiativen und überregionale ökologische Pressure Groups wie z.B. Greenpeace umfassen.

2. Es findet ein im Rahmen einer gründlichen Vorbereitung klar strukturierter und in Anlehnung an die Thematik gegliederter Verhandlungsprozeß statt.

3. Ergebnisse dieser Verhandlungen sollen eine konsensfähige Übereinkunft sowie Maßnahmen zu deren Implementation sein.

4. Im Falle besonders konfliktträchtiger Entscheidungen kann das Verfahren durch einen Vermittler (Mediator) geleitet werden.

5. Um eine möglichst effiziente Problemlösung zu erreichen, sollte eine kooperative Atmosphäre vorherrschen.

262 Scharpf, Fritz W. (1991): Die Handlungsfähigkeit des Staates am Ende des 20. Jahrhunderts, in: Politische Vierteljahresschrift, 4, S. 622; zit. nach: Zilleßen, Horst, und Barbian, Thomas (1992): Neue Formen . . ., a.a.O., S. 14.

${ }^{263}$ Vgl. grundlegend: Bingham, Gail (1986): Resolving Environmental Disputes. A Decade of Experience, Washington. 
Abb. 16: Strategieoptionen im bilateralen Verhandlungsprozeß

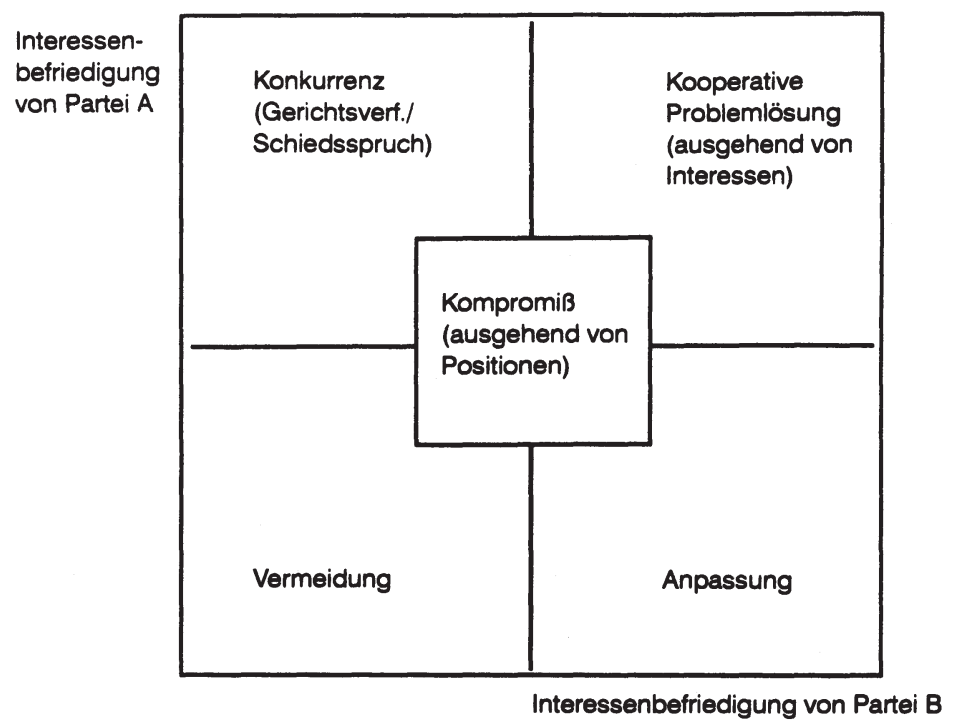

Quelle: Zilleßen und Barbian (1992), S. 18

Zur Verdeutlichung des letzten Punktes sei auf Abbildung 16 verwiesen, welche die möglichen Strategieoptionen der verhandelnden Parteien ${ }^{264}$ darstellt:

- Im Falle der Konkurrenzstrategie wird eine Partei (in diesem Fall Partei A) versuchen, ihre Interessen ohne Rücksichten auf den Verhandlungspartner durchzusetzen. Bedingung für diese Strategie ist ein Machtübergewicht dieser Partei oder die Aussicht, in einem eventuellen Gerichts- bzw. Schiedsverfahren zu obsiegen.

- Die Anpassungsstrategie wird gewählt, wenn eine Partei die Chancen zur Durchsetzung der eigenen Interessen als sehr gering ansieht bzw. es an Motivation und/oder Interesse zur Konfliktaustragung fehlt. Als Resultat ergibt sich wie im Falle der Konkurrenzstrategie die Interessenbefriedigung nur einer Partei bei gleichzeitigem völligen Verlust der anderen Partei.

${ }^{264}$ Zur Vereinfachung der Darstellung sind es hier nur zwei Parteien. 
- Die aus Effizienzgesichtspunkten unsinnigste Strategieoption ist die Vermeidung der Konfliktaustragung. Zu dieser Strategie der NichtEntscheidung kommt es meist aus mangelndem Interesse an der anstehenden Problematik, aus Unsicherheit oder Furcht vor der Konfliktaustragung oder aus der Einschätzung, daß eine Konfliktlösung nicht erreichbar ist.

- Die am häufigsten gewählte Strategieoption ist die Konliktregelung durch einen Kompromiß. Dabei gehen die beiden Parteien von Maximalpositionen aus, die in einem schwierigen Aushandlungsproze $B$, in dem beide Parteien sukzessive auf Teile ihrer Forderungen verzichten, angenähert werden. Als Grundannahmen der Konfliktparteien können im Falle der Kompromißstrategie unterstellt werden:

1. Mißtrauen gegenüber den Absichten der anderen Partei;

2. annähernd gleiche Machtverteilung;

3. der mögliche Nutzenzuwachs bzw. die zur Disposition stehenden Ressourcen sind aus Sicht der Verhandlungspartner begrenzt, und es besteht lediglich die Option einer Aufteilung zwischen beiden Parteien.

- Die in ADR-Verfahren angewandte Strategieoption der kooperativen Problemlösung unterscheidet sich von der Kompromißstrategie vor allem dadurch, daß sie nicht von Positionen sondern von Interessen ausgeht. Wenn es im Verlaufe des Verhandlungsprozesses gelingt, die oft von den Verhandlungspositionen abweichenden tatsächlichen Interessen und Bedürfnisse der Parteien herauszuarbeiten, und sich so herausstellt, daß die Interessen voneinander unabhängig oder sogar komplementär sind, dann kann eine Problemlösung gefunden werden, welche die Bedürfnisse beider Parteien weitgehend erfüllt.

Die Erfolgsbedingungen einer solchen konsensorientierten, kooperativen Problemlösung ergeben sich zum Teil als die sinngemäßen Umkehrungen der Grundannahmen traditioneller Kompromißstrategien.

1. ADR-Verfahren besitzen nur dann Erfolgsaussichten, wenn sich die Verhandlungspartner gegenseitiges Vertrauen entgegenbringen. Gegenteilige Erfahrungen aus früheren Konflikten sowie die aus persönlicher Betroffenheit resultierende Emotionalität einzelner Teilnehmer stehen diesem Punkt häufig entgegen. Auch in diesem Fall kann sich der Einsatz eines neutralen Vermittlers bewähren.

2. Es muß eine grundsätzliche Konsensbereitschaft bestehen, d.h. die Teilnehmer müssen bereit sein, ihre Positionen zu revidieren, wenn ihnen Diskrepanzen zwischen ihren ursprünglichen Forderungen und ihren tatsächlichen Interessen und Bedürfnissen aufgezeigt werden können. 
3. Während sich die beiden ersten Punkte grundsätzlich von den Annahmen im Falle der Kompromißstrategie unterscheiden, gilt die Bedingung annähernd gleicher Machtpositionen für beide Strategien. Ist dies nicht gegeben, besteht die Gefahr eines Strategiewechsels zur Konkurrenzoption durch den übermächtigen Verhandlungspartner. Das oft bestehende Machtungleichgewicht zuungunsten der ökologischen Interessenvertreter kann durch rechtliche Maßnahmen (z.B. Fristen oder Einspruchsrechte) oder durch mediale Aufmerksamkeit ausbalanciert werde.

4. Als weitere Voraussetzung für eine erfolgreiche Anwendung von ADRVerfahren muß schließlich die Bedingung gelten, daß es sich bei der behandelten Streitfrage nicht um eine Grundsatzfrage oder eine bloße Ja-/Nein-Entscheidung handeln darf. Die meisten Fragen des umweltpolitischen Alltags fallen jedoch nicht in diese Kategorien.

Auch in Deutschland gibt es Erfahrungen mit Mediationsverfahren. So diente ein Mediationverfahren des Kreises Neuss zum Abfallwirtschaftskonzept als Untersuchungsobjekt des Wissenschaftszentrums Berlin für Sozialforschung ${ }^{265}$. Von Interesse ist dabei unter anderem der Teilnehmerkreis, zu dem - ähnlich wie in den japanischen, amerikanischen und sonstigen europäischen Mediationsverfahren - u.a. die folgenden Institutionen und Organisationen gehörten:

- Verwaltungen,

- Bürgerinitiativen,

- Gewerkschaften sowie

- Industrieverbände und Firmen.

Diese Teilnehmer (eventuell noch ergänzt durch Parteien, Kirchen etc.) verhandeln also die umweltpolitische Problematik unter neutraler Vermittlung mit dem Ziel einer konsensorientierten, kooperativen Problemlösung. Daraus und aus dem sonst in diesem Abschnitt Angeführten lassen sich für den Partizipationsgrad als Merkmalsdimension eines Environmental Policy Styles noch zwei wichtige, grundlegende Erkenntnisse ableiten:

1. Es gibt eine gewisse strukturelle Ähnlichkeit zwischen dem primär wirtschaftspolitischen Konzept des Korporatismus und den im umweltpolitischen Bereich angewendeten ADR-Verfahren. In beiden Fällen werden Interessengruppen durch wechselseitige Organisationsbezie-

${ }^{265}$ Fietkau, Hans-Joachim, und Weidner, Helmut (1992): Mediationsverfahren . ., a.a.O., S. 31. 
hungen zu staatlichen Stellen sowie untereinander an der Politikformulierung und -implementation beteiligt. Desweiteren geht es sowohl beim Korporatismus als auch bei den ADR-Verfahren um eine Minimierung eventuell entstehender Konfliktkosten durch eine Gesamtverantwortung aller Teilnehmer sowie um eine Orientierung an einem für alle Beteiligten und das Gemeinwohl akzeptablen Konsens. Im Unterschied zum ökonomischen Korporatismus auf dem Gebiet der Tarifproblematik handelt es sich im umweltpolitischen Problemlösungsprozeß bei den konkurrierenden nicht-staatlichen Akteuren jedoch nicht um Vertreter von Unternehmen und Arbeitnehmern, sondern um potentielle Umweltverschmutzer bzw. -verbraucher (Unternehmensverbände/Firmen) sowie um ökologische Interessengruppen (Bürgerinitiativen/Umweltschutzorganisationen), welche weitgehend ohne staatliche Einflußnahme den Versuch einer einvernehmlichen Konfliktregelung unternehmen. Es kann angenommen werden, daß Staaten, welche die ökonomischen Interessengegensätze durch korporatistische Formen der Problemlösung praktizieren, auch der Anwendung von ADR-Verfahren auf umweltpolitischem Gebiet aufgeschlossener gegenüberstehen als nicht-korporatistische Staaten. Diese Annahme kann im empirischen Teil die Operationalisierung der Merkmalsdimension Partizipationsgrad erleichtern.

2. Der im folgenden verwendete Partizipationsbegriff umfaßt neben den reinen Beteiligungsrechten der nicht-staatlichen Akteure auch die $\mathrm{Be}-$ reitschaft der staatlich-administrativen Stellen zu einem kooperativen und konsenorientierten Diskurs. Liegt nämlich lediglich das formale Recht zur Teilnahme am Policy Cycle vor, so können sich daraus dennoch kofliktäre Formen der Problembewältigung ergeben, weil die Parteien Konkurrrenz- oder Kompromißstrategien der Streitaustragung wählen. Allein eine Kombination aus Beteilungsrechten und Kooperation erlaubt hingegen das Erreichen der unter Effizienzgesichtpunkten idealen Konsenslösung mit minimalen Konfliktkosten. Für die spätere Operationalisierung der Merkmalsdimension Partizipationsgrad ist also auch auf das Maß der in den politischen Prozessen eines Staates allgemein üblichen Konsensorientierung der an der Konfliktlösung Beteiligten abzuheben. 


\subsection{Typologie nationaler Umweltpolitikstile}

\subsubsection{Schema}

Aus den in Abschnitt 5.2. angestellten Überlegungen ergibt sich, daß die beiden Merkmalsdimensionen Strategieorientierung und Partizipationsgrad wichtige Variablen zur Identifikation unterschiedlicher ökologisch relevanter Politikstile sind.

Eine Umwandlung des in Abbildung 16 bereits dargestellten Koordinatensystems in ein Schema mit vier idealtypischen umweltpolitischen Regimen erleichtert die Interpretation der vermuteten Zusammenhänge.

Abbildung 17 zeigt die Grundtypen aufgrund der Kombination der beiden Merkmalsdimensionen ${ }^{266}$.

Der planerische Korporatismus ist sowohl strategisch als auch konsensual ausgerichtet und stellt somit den Idealtypus eines umweltpolitischen Regimes mit ausreichender Handlungstiefe und -breite dar.

Ein reaktiver Korporatismus ist zwar ebenfalls durch kooperative Elemente charakterisiert, doch fehlt der planerische Impetus. Vielmehr reagiert der Staat eher passiv auf Anstöße der Interessenvertreter oder nicht mehr aufschiebbaren äußeren Handlungsdruck.

Im Falle des innovativen Pluralismus agiert die Politik aktiv und vorausschauend bei gleichzeitig konfliktären Problemlösungsmechanismen. Die Innovationsfähigkeit eines solchen Regimes ist oft kein Resultat staatlicher Planung, sondern entspringt dem Modernisierungs- und Veränderungsdruck einer durch starken Wettbewerb geprägten Gesellschaft.

${ }^{266}$ Zur Bedeutung der im folgenden verwendeten Korporatismus und Pluralismus-Begriffe sei auf die bereits in Abschnitt 4.1.3.4. erfolgten Ausfuhrungen verwiesen. 
Abb. 17: Umweltpolitikstil-Typologie

\begin{tabular}{|r|r|r|}
$\begin{array}{r}\text { Partizipationsgrad } \\
\text { der nicht-staat- } \\
\text { lichen Ak- } \\
\text { teure }\end{array}$ & Hoch & Gering \\
$\begin{array}{r}\text { Problem- } \\
\text { lösungsverhalten }\end{array}$ & $\begin{array}{c}\text { Planerischer } \\
\text { Korporatismus } \\
\text { orientiert }\end{array}$ & $\begin{array}{c}\text { Innovativer } \\
\text { Pluralismus }\end{array}$ \\
\hline Reaktiv & $\begin{array}{r}\text { Reaktiver } \\
\text { Korporatismus }\end{array}$ & $\begin{array}{c}\text { Laissez } \\
\text { faire }\end{array}$ \\
\hline
\end{tabular}

Als im Sinne einer effektiven Umweltpolitik negativster Politikstil ist der des Laissez faire zu bezeichnen. Sowohl die Strategieorientierung als auch der Partizipationsgrad der nicht-staatlichen Akteure erreichen die geringsten Werte. Kurzfristiges Handeln der politischen Akteure trifft in diesem Fall zusammen mit einer äußerst geringen Bereitschaft, Probleme in einem für alle Betroffenen akzeptablen Konsens zu lösen.

Dieses hier kurz skizzierte Schema, das in den folgenden Abschnitten dieses Kapitels noch ausführlich erläutert wird, bietet trotz seiner Einfachheit und guten Anwendbarkeit jedoch auch Anlaß zur Kritik:

Es ist sicher nicht möglich, die Staaten der untersuchten Grundgesamtheit in jedem Fall einem der vier Idealtypen zweifelsfrei zuzuordnen. Einzelnen Staaten werden möglicherweise eine Art von „Hybrid-Typus“ aufweisen, der sich im Grenzbereich von zwei oder mehr der gerade beschriebenen Regimeformen befindet ${ }^{267}$. Die dann eventuell zu treffende Entschei-

${ }^{267}$ In seiner Typologie politischer Regime ordnet beispielsweise Wildavsky den amerikanischen individualism und die Sozialdemokratie als hybrid regimes ein, welche sich jeweils an den Grenzbereichen zweier politischer Regimeformen ansiedeln (zwischen Individualismus und sectarianism bzw. Kollektivismus und sectarianism) (Wildavsky, Aaron B. (1987): Doing More and Using Less: Utilisation of Research as a Result of Regime, In: Dierkes, Meinolf, et al. (Hg.): Comparative Policy ..., a.a.O., S. 82). 
dung einer Zuordnung zu einem der vier Felder ist somit eher subjektiven Charakters und mit der entsprechenden Vorsicht vorzunehmen.

Desweiteren ist zu bedenken, daß beide Merkmalsdimensionen sicherlich nicht im statistischen Sinne voneinander unabhängig sind. So ist beispielsweise zu vermuten, daß ein eher hoher Partizipationsgrad durch die frühzeitige Einbeziehung von Betroffenen und deren Wissen auch die Strategieorientierung staatlichen Handelns fördert.

Dennoch bildet diese Schema ein starkes heuristische Instrument zur Kategorisierung und Vereinfachung der unterschiedlichen umweltpolitischen Handlungsmuster der noch zu untersuchenden Staatengruppe.

\subsubsection{Planerischer Korporatismus}

Der Politikstil des planerischen Korporatismus vereint zwei Merkmale, die auf den ersten Blick als konkurrierend erscheinen mögen. Zum einen das Bestreben nach einer Einbeziehung aller Betroffenen, also des Schaffens eines allgemeinen Konsenses hinsichtlich der Definition der Ziele und der Implementierung von Maßnahmen, und zum anderen die Fähigkeit zu langfristiger, über die Legislaturperiode hinausgehender Politik. Partizipation und Konsensfindung führen in diesem Falle also nicht zu einer Blockade durch Einzelinteressen oder zu einer Vertagung von Problemen durch Formelkompromisse. Die Bedeutung dieses Politikstiles liegt somit in der Tatsache, daß am Ende eines demokratischen Entscheidungsprozesses „strategiefähige“ Lösungen stehen, der partizipative Pro$z e ß$ der Konsensfindung also Lösungen generiert, die sowohl die einzelnen Teilnehmer des politischen Netzwerkes zufriedenstellen als auch dem Gemeinwohl zuträglich sind.

Der mögliche Nutzen dieses Politikstils liegt damit auf der Hand. Zum einen minimiert ein Kooperationsverhältnis von Staat, Kapital, Arbeit und ökologischen Interessengruppen die Konfliktkosten möglicher Entscheidungen ${ }^{268}$. Auch ist durch die frühzeitige Berücksichtigung umweltrelevanter Argumente ein weniger umweltbelastendes Ergebnis des politischen Prozesses wahrscheinlicher als im Falle der kompromißlosen Verfolgung ökonomischer oder wirtschaftspolitischer Einzelinteressen.

Zum anderen dürfte auch das strategische Element dieses Politikstils positive Effekte auf die Effektivität der Umweltpolitik haben. Denn zwei

${ }^{268}$ Vgl. Jänicke, Martin (1990): Erfolgsbedingungen . . . , a.a.O., S. 223. 
Kernelemente einer langfristig angelegten Politik scheinen zu den Erfolgsbedingungen dieses Politikfeldes zu gehören, nämlich die Innovationsfähigkeit und administrative Kapazität eines Staates.

Ein hohes Innovationsvermögen eines Staates ermöglicht die frühzeitige Adaption politischer, rechtlicher und wirtschaftstechnologischer Neuerungen und schafft somit die politische Offenheit zum Austausch ökonomisch und ökologisch ineffizienter Ziele, Regelungen und Techniken durch Innovationen.

Die administrative Kapazität spiegelt sich wider in einer institutionalisierten, mit ausreichenden ökonomischen Mitteln ausgestatteten Politik, die langfristige Prozesse zum strukturellen Umbau der Volkswirtschaft initiiert. Außer diesem eher zentralistischen Element empfiehlt sich jedoch auch eine Kompetenzverlagerung auf subnationale administrative Einheiten $^{269}$, die sowohl in der Phase des agenda-settings als auch bei der Implementation beschlossener Maßnahmen früher und effektiver als zentralstaatliche Entscheidungsorgane agieren können.

Kern und Bratzel formulieren diesen Zusammenhang so: ${ }^{270}$

„Erfolgreiche (Umwelt-)politik scheint dann am ehesten möglich zu sein, wenn einerseits eine durchsetzungsfähige Behörde auf zentralstaatlicher Ebene existiert, die generelle Mindeststandards setzt. Andererseits müssen auf der dezentralen Ebene genügend Handlungsspielräume vorhanden sein, damit die subnationalen Einheiten, [...], eine Vorreiterposition einnehmen können, indem sie zentralstaatliche Standards verschärfen."

\subsubsection{Reaktiver Korporatismus}

Auch bei diesem Politikstil steht die Suche nach einem Konsens zwischen allen Betroffenen einer umweltpolitischen Maßnahme im Mittelpunkt, d.h. die Ausprägungen der meisten Dimensionen zur Beschreibung der politischen Netzwerke der reaktiv-korporatistischen Staaten entsprechen van Waardens Typen des sektoralen, Makro- oder Staatskorporatismus ${ }^{271}$.

${ }^{269}$ Vgl. Kern, Kristine, und Bratzel, Stefan (1994): Erfolgskriterien . . , a.a.O., S. 18 f.

${ }^{270}$ Ebenda, S. 19.

${ }^{271}$ Waarden, Frans van (1992): Dimensions ..., a.a.O., S. 46 ff. Vgl. auch die Defintion der unterschiedlichen Korporatismus-Typen in Abschnitt 4.1.4.4. 
Bezüglich der Voraussicht und Reichweite der Politik unterscheidet sich diese Gruppe jedoch grundlegend von der zunächst beschriebenen Variante, denn ihr Problemlösungsverhalten ist eher reaktiv als aktiv und antizipativ. Für den Politikstil bedeutet dies im einzelnen:

- Die getroffenen Entscheidungen haben häufig einen nur kurzfristigen, legislaturperioden-weiten Effekt. Es werden kaum solche Problemlösungsansätze umgesetzt, welche erst langfristig positive Effekte auslösen.

- Es gibt kaum politikfeldübergreifende Aktivitäten der einzelnen Ministerien. Eine Berücksichtigung der zahlreichen Interdependenzen politischen Handelns und die daraus entspringende Notwendigkeit der Schaffung von Schnittstellen und der Koordination zwischen den einzelnen Bereichen der Politik erfolgt nur rudimentär. Dies schlägt sich dann auch im Fehlen entsprechender Institutionen der Planung und Koordination nieder.

- Das Verhältnis der zentralstaatlichen Institutionen zu den subnationalregionalen politischen Einheiten ist durch Top-down-Strukturen geprägt, sowohl im Hinblick auf Informationen als auch auf politische Entscheidungen. Umweltpolitische Themen kommen also nicht dann auf die politische Agenda, wenn Probleme dezentral erkannt werden, sondern meist nur dann, wenn die zentralen Stellen diese im Rahmen des agenda-setting für gesamtstaatlich relevant halten. Auch regionale Ausnahmen oder Verschärfungen getroffener politischer Entscheidungen erfolgen eher selten.

- Mangelndes Antizipationvermögen geht oft einher mit fehlendem Interesse am öffentlichen Wohl. Wenn trotz vorhandener Informationen wider besseres Wissen keine langfristige Politik zur Förderung des Gemeinwohls betrieben wird, dann liegt das meist daran, daß sektorale oder individuelle Interessen der Beteiligten, also auch der Parteien, Politiker und Bürokratien den Ausschlag geben.

Das Resultat dieses Politikstils könnte somit eine Blockade des umweltpolitischen Handelns sein. Wenn nämlich einerseits ein Konsens das Ergebnis des politischen Prozesses sein soll, andererseits jedoch keine Eile zur Entscheidung und kein Weitblick bestehen, dürfte es oftmals zu einem langwierigen Prozeß der Verhandlungen und aufschiebenden Kompromisse kommen, und dies solange, bis der Handlungsdruck letztlich doch zu einer wie auch immer gearteten Entscheidung führt. 


\subsubsection{Innovativer Pluralismus}

Der hier als ,innovativer Pluralismus“ bezeichnete Politikstil verbindet ein konfliktäres Problemlösungsverhalten mit eher hohem Antizipationsvermögen.

Innovativ-pluralistische Staaten sind gekennzeichnet durch eine starke Fragmentierung sowohl des gesellschaftlichen Lebens als auch des politischen Prozesses. Gruppen mit eigentlich komplementären oder identischen Interessen finden sich nur zu einem sehr geringen Grade organisatorisch zusammen, der Staatsregierung stehen kaum starke landesweit vertretene Gewerkschaften oder Umweltverbände gegenüber. Vielmehr versuchen einzelne Gruppen durch Lobbyismus oder Klientelismus ihre Interessen durchzusetzen. Für alle Stufen des politischen Prozesses gilt deshalb, daß meist sowohl Sieger als auch Verlierer zurückbleiben.

Dieses Suchen und Austragen des gesellschaftlichen und politischen Konfliktes kombiniert der hier beschriebene Politikstil mit einer eher hoch ausgeprägten Innovations- und Strategiefähigkeit. Innovativpluralistische Staaten sind in dieser Hinsicht durch folgende Charakteristika gekennzeichnet:

- Der stark ausgeprägte innerstaatliche Wettbewerb fördert eine hohe Innovationsbereitschaft, denn nur marktfähige Neuheiten ermöglichen eine ausreichende Performanz.

- Auch die Strategiefähigkeit der innovativ-pluralistisch Staaten entspringt eher den extremen Wettbewerbsbedingungen als staatlicher Planung. In den Abteilungen für strategische Unternehmensentwicklung der Großkonzerne und nicht in einer staatlichen Planungsbehörde werden die notwendigen Investitions- und Technologieentscheidungen mit langfristigem Charakter getroffen.

- Die Staatsorganisation ist eher föderalistisch. Den subnationalen Einheiten wird ihre Funktion als dezentrale Frühwarn- und Implementationsorgane nicht abgesprochen.

\subsubsection{Laissez-faire}

Im Politikstil des Laissez-faire vereinen sich die reaktiven und konfliktären Ausprägungen der beiden wichtigsten Politikstildimensionen. Das dem Pluralismus der Interessen und Zielsetzungen der gesellschaftlichen, politischen und ökonomischen Akteure entspringende Gegeneinander verbindet sich mit einem starren zentralistischen Top-down-Ansatz des 
politischen Prozesses. Die sich aufstauenden Probleme werden nicht im Konsens gelöst, sondern irgendwann - oft nach einer längeren Phase der Eskalation - durch administrative Akte der Zentralregierung entschieden. Auch dieser Entscheidungsakt wird jedoch oft noch angefochten und in Frage gestellt. Stichpunktartig läßt sich der Politikstil des Laissez-faire wie folgt skizzieren:

- Es mangelt an der Fähigkeit zum gesellschaftlichen Diskurs; Konsensfähigkeit gilt als Schwäche.

- Die meist gut organisierten Interessengruppen neigen zur Radikalisierung.

- Konflikte werden meist zu Gunsten einer Partei entschieden.

- Der zentralistische Ansatz verbindet sich nicht mit einem planerischen Impetus, d.h. meist fehlen Elemente einer staatlichen Strukturpolitik.

- Die innerstaatlichen Konflikte führen zu keiner innovationsfördernden Konkurrenzsituation, sondern lediglich zu gegenseitiger Blockade.

- Die zu einem eher großen Prozentsatz verstaatlichten Großunternehmen sehen sich aufgrund von Monopol- oder Oligopolstellungen nicht zu verstärkten Modernisierungs- oder Innovationsanstrengungen gezwungen.

- Eine protektionistische Wirtschaftspolitik fördert diesen Mangel an F\&E-Aktivitäten.

\subsection{Zusammenfassende Bemerkungen}

Das in diesem Kapitel entworfene Modell des Einflusses des Politikstils auf die Effektivität nationaler Umweltpolitik hat unter Bezugnahme auf die spezifische Problematik ökologischen Handelns, welches durch eine hohe Wirkungstiefe und -breite sowie Komplexität gekennzeichnet ist, die Merkmalsdimensionen Strategieorientierung und Partizipationsgrad der nicht-staatlichen Akteure des politischen Prozesses als die relevanten Variablen zur Beschreibung umweltpolitischer Regime identifiziert.

Aus der Kombination dieser beiden Faktoren ergeben sich vier Idealtypen eines umweltpolitischen staatlichen Handelns. Als Idealtypus mit den positivsten Effekten auf die Umweltqualität wurde dabei der planerische Korporatismus identifiziert, welcher sowohl durch ein aktives, antizipatives Agieren des Staates als auch durch eine Einbeziehung der nichtstaatlichen Akteure in alle Phasen des politischen Prozesses charakterisiert werden kann. 
Im folgenden zweiten Teil dieser Analyse sollen die im Rahmen der Theoriebildung aufgestellten Thesen einer empirischen Überprüfung unterzogen werden. 
Teil II: Empirische Untersuchung

\section{Vorhandene empirische Untersuchungen über die Bedeutung des Poli- tikstils für den Erfolg von Umweltpolitik}

\subsection{Die Studien Jänickes}

Im folgenden sollen kurz solche Studien skizziert werden, welche bereits empirische Evidenz für den Zusammenhang zwischen Politikstil und Umweltbelastung geliefert haben. Herauszuheben sind dabei die Überlegungen Jänickes, welche sich vor allem auf die Art der Einbeziehung der nicht-staatlichen Akteure in den politischen Prozeß beziehen ${ }^{272}$. Unter ausdrücklicher Bezugnahme auf die Diskussion über die neokorporatistischen Strukturen in einigen Industrieländern setzt er den Integrationsgrad der Interessenvermittlung in westlichen Industriestaaten mit den in diesen Staaten erreichten Veränderungen der Umweltqualität in Beziehung. Dabei greift er auf eine von Schmidt durchgeführte Einteilung der Staaten in solche mit eher konsensualem bzw. konfliktbetontem Politikstil zurück ${ }^{273}$. Als Indikatoren zur Messung des Erfolges der Umweltpolitik verwendet er die durchschnittlichen Änderungsraten von Luftemissionen, Gewässerreinheit und Kläranlagenbau in den Jahren 1970 bis 1985. Das in Abbildung 18 dargestellte Ergebnis ist eindeutig.

272 Jänicke, Martin (1990): Erfolgsbedingungen . . . , a.a.O., S. 223 f.; Jänicke, Martin; Mönch, Harald; Binder, Manfred et al. (1993): Umweltentlastung durch industriellen Strukturwandel? Eine explorative Studie über 32 Industrieländer (1970 bis 1990), 2. Aufl., Berlin, S. ; Jänicke, Martin (1996): Erfolgsbedingungen von Umweltpolitik; In: Jänicke, Martin (Hg.): Umweltpolitik der Industrieländer. Entwicklung - Bilanz - Erfolgsbedingungen; Berlin, S. 9-28.

${ }^{273}$ Schmidt, Manfred G. (1986): Politische Bedingungen erfolgreicher Wirtschaftspolitik, in: Journal furr Sozialforschung, 3. 


\section{Abb. 18 : Zusammenhang zwischen Konsensorientierung staatlicher Politik und ökologischem Erfolg}

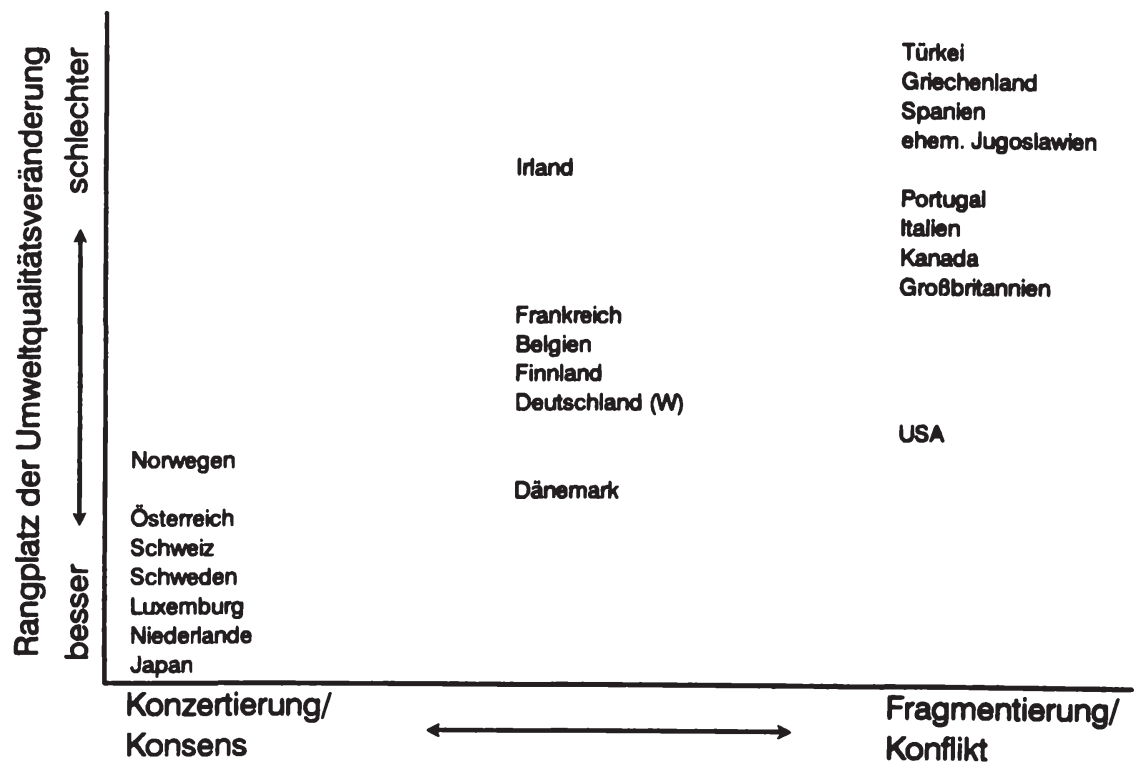

Quelle: Jänicke (1990), S. 224.

Die Erfolgsfälle der Umweltpolitik gehören beinahe ausnahmslos zu den Staaten mit einem eher konsensualen Politikstil, während die konfliktbetonten Problemlösungsstrategien des Laissez faire (z.B. Kanada und Großbritannien) oder des rigiden Zentralismus (z.B. Türkei und Spanien) offenbar mit einer weitgehend erfolglosen Umweltpolitik einhergehen. Zur Begründung dieses Phänomens führt Jänicke zwei Argumente an: 
1. „Offenbar werden in industriepolitischen Aushandlungsprozessen auch die Umweltinteressen früher berücksichtigt.

2. Aus der Tatsache, daß zu den konsensualen Staaten vor allem kleine Staaten gehören, folgert er: „Es könnte [...] sein, daß die ausgeprägt demokratischen Institutionen dieser kleinen Länder einen stärkeren politischen Anpassungsdruck erzeugen und die Fähigkeit zur aktiven Reformpolitik gewissermaßen besser trainieren.

Zwar bezieht sich die von Jänicke beschriebene Konsensfähigkeit ausschließlich auf die Beziehung zwischen Arbeitgebern und -nehmern - und somit nicht auf ökologische Interessenkonflikte -, doch liefern seine Überlegungen einen ersten wichtigen Hinweis in die im Rahmen dieser Untersuchung verfolgte Richtung.

\subsection{Weitere Studien ${ }^{276}$}

Brickman et al. untersuchen die Regelungen zum Umgang mit chemischtoxischen Substanzen in den Vereinigten Staaten, Frankreich, Großbritannien und Deutschland ${ }^{277}$. Dabei finden sie vier Variablen $\mathrm{zu}$ einer sinnvollen Unterscheidung und Charakterisierung der jeweiligen nationalen Vorgehensweisen:

- gesetzlicher und institutioneller Rahmen;

- die strukturellen und politischen Merkmale des Gesetzgebungsprozesses;

- Organisation und Ablauf der Entscheidungsimplementation;

- die Rolle der Gerichte.

274 Jänicke, Martin (1990): Erfolgsbedingungen . . , a.a.O., S. 223.

${ }^{275}$ Ebenda, S. 224; sowie Katzenstein, Peter J. (1985): Small States in World Markets. Industrial Policy in Europe, Ithaca; London.

${ }^{276}$ Die meisten Hinweise sind entnommen aus: Vogel, David (1987): The Comparative Study of Environmental Policy: A Review of the Literature, In: Dierkes, Meinolf, et al. (Hg.): Comparative . . . , a.a.O., S. 167 f.; Jänicke (div.); Kern, Kristine, und Bratzel, Stefan (1994): Erfolgskriterien ..., a.a.O.

${ }^{277}$ Brickman, Ronald; Jasanoff, Sheila, und Ilgen, Thomas (1982): Cancer: A Cross-National Study of Policy and Politics, Cornell. 
Anhand der Ausprägungen dieser Faktoren schlußfolgern die Autoren, daß Unterschiede der nationalen Umweltpolitik vor allem in der Machtverteilung der am politischen Prozeß beteiligten Akteure bestehen. Dabei unterscheidet sich das Vorgehen in den drei westeuropäischen Staaten signifikant von demjenigen in den USA.

Während bei letzteren vor allem die politische Fragmentierung sowie der regulative und detaillierte Charakter der Gesetzgebung zu einem konfliktreichen, oft in gerichtliche Auseinandersetzungen mündenden, politischen Prozeß führen, sind für die westeuropäischen Staaten eher kooperative Politikmuster typisch. Diese führen über konsensual angelegte Konsultationen der Parteien zu einem tendenziell einvernehmlichen Politikergebnis mit einer größeren Stabilität.

Kelman untersucht unterschiedliche Vorgehensweisen schwedischer und US-amerikanischer Behörden bei der Implementation und Kontrolle von Regelungen zum Schutz von durch Umweltbelastungen besonders beeinträchtigten Arbeitsplätzen ${ }^{278}$.

Das hierbei deutlich werdende Muster läßt sich folgendermaßen zusammenfassen $^{279}$ :

- In Schweden verlaufen Implementation und Kontrolle in kooperativen Formen. Die Vorgaben der Regierung werden aktiv umgesetzt und auftretende Probleme zwischen den beteiligten Konfliktparteien einvernehmlich gelöst.

- In den USA versuchen die Akteure aufgrund des individualistischen, auf Selbstverwirklichung des Einzelnen orientierten Wertesystems die jeweils aus Einzelsicht optimale Lösung zu erwirken. Dies führt $\mathrm{zu}$ häufigen Konflikten, welche unter Anwendung des zur Verfügung stehenden Sanktionsinstrumentariums gelöst werden.

Ebenfalls um die Implementation beschlossener Umweltschutzmaßnahmen - in diesem Fall um die Kontrolle von $\mathrm{SO}_{2}$-Emissionen - geht es in den Studien von Knoepfel et al. ${ }^{280}$ Zur untersuchten Grundgesamtheit gehören die Staaten der Europäischen Gemeinschaft und die Schweiz.

${ }^{278}$ Kelman, S. (1981): Regulating America ..., a.a.O.

${ }^{279}$ Ebenda, S. 221.

${ }^{280}$ Knoepfel, Peter; Weidner, H., und Hanf, K. (1979): The Implementation of $\mathrm{SO}_{2}$ Control Policy in the European Community and Switzerland, Air Pollution Control: National und International Perspectives, Aspen; Knoepfel, Peter, und Weidner, H. (1983): Implementing Air 
Der Implementationserfolg wird $\mathrm{zu}$ verschiedenen Einflußfaktoren in Beziehung gesetzt:

- der Art des implementierten Programmes;

- den Chararkteristika der Administration und ihrer eventuellen Einbeziehung in politische Netzwerke;

- den verwendeten Implementationsinstrumenten;

- Aktivitäten und Einfluß nicht-staatlicher Akteure;

- dem Umgang staatlicher Akteure mit Partikularinteressen von Betroffenen oder Verschmutzern (kooperativ oder konfliktär).

Ein signifikanter Einfluß dieser Faktoren auf die SchwefeldioxidVerminderung konnte nicht gemessen werden. Besonders bemerkenswert erscheint im Kontext dieser Studie zudem, daß zwischen der „Offenheit““ des Implementationprozesses, also dem Ausmaß der öffentlichen Partizipation, und den festgelegten Luftqualitätsstandard kein Zusammenhang festgestellt werden konnte.

In einer weiteren international vergleichenden Studie, welche die Unterschiede in der Programmformulierung und -implementation von Luftreinhaltepolitik in der Bundesrepublik Deutschland, Frankreich, Großbritannien, Italien und den Niederlanden zum Gegenstand hat, stellen Knoepfel und Weidner fest, daß insbesondere ein eher großer Handlungsspielraum der regionalen Administration einen günstigen Einfluß auf die Implementation der beschlossenen Regelungen hat ${ }^{281}$.

Die Umweltschutzregime Großbritanniens und der USA werden in der umfangreichen Vergleichsstudie Vogels thematisiert ${ }^{282}$. Obgleich es sich in beiden Fällen um liberale Gesellschaften mit einer eher schwachen Zentralregierung handelt, sind deutlich unterschiedliche Politikstile feststellbar.

Die britische Umweltpolitik setzt eher auf das Aushandeln von Standards und Maßnahmen unter Einbeziehung der Industrie. Das daraus resultierende Regularium ist eher allgemein gefaßt. Eine Einbeziehung von Umweltschutzgruppen in den politischen Prozeß findet nicht statt.

Quality Control Programs in Europe: Some Results of A Comparative Study, In: Downing, P., und Hanf, K . (Hg.): Implementing Pollution Laws: International Comparison, Boston.

${ }^{281}$ Knoepfel, Peter, und Weidner, Helmut (1985): Luftreinhaltepolitik . . ., a.a.O.

282 Vogel, David (1982): Coercion versus Consultation: A Comparison of Environmental Protection in the United States and Great Britain, Denver; Vogel, David (1986): National Styles of Regulations: Environmental Policy in Great Britain and the United States, Ithica. 
Die umweltpolitische Arena in den USA ist dagegen offener, und Interessenaggregation und Politikimplementation sind häufig durch den Streit der Interessengruppen - insbesondere zwischen Regierung und Unternehmen - gekennzeichnet ${ }^{283}$. Die umweltpolitischen Richtlinien sind schließlich eher detailliert und mit einem großen Sanktionsintrumentarium versehen.

Diese Unterschiede der Umweltpolitik, welche ähnlich auch in anderen Feldern der politischen und gesellschaftlichen Auseinandersetzung wiederzufinden sind, haben laut Vogel historische Ursachen. Signifikante Unterschiede im umweltpolitischen Erfolg beider Staaten seien trotz dieser Differenzen nicht feststellbar, doch habe die britische Politik den gleichen umweltentlastenden Effekt mit erheblich geringeren politischen Kosten erreicht ${ }^{284}$. Ähnlich wie Knoepfel und Weidner sieht auch Vogel Vorteile in einer regionalen Kompetenz zur Festlegung und Implementation von Umweltstandards ${ }^{285}$ : „... they are able to tailor regulations to the particular circumstances of the individual firms and industries. "

Zu einem ähnlichen Ergebnis gelangt Badaracco in seinem Vergleich der politischen Problemlösungsmuster von fünf Industrienationen ${ }^{286}$. Zwar stehen am Ende der Bemühungen in allen Ländern ähnliche Reduktionszahlen für Vinylchlorid, doch während die Interaktion der Akteure in Japan, Deutschland, Frankreich und Großbritannien eher kooparativ verläuft, überwiegen in den USA die konfliktären Problembearbeitungsstrategien.

Trotz der Ähnlichkeit des politischen Outcomes hält der Autor die in Japan und Europa praktizierte Vorgehensweise letztlich für überlegen, vor allem im Hinblick auf komplizierte und interdependente Problemati$\operatorname{ken}^{287}$.

Dieser kurze Überblick über fünf Studien der vergleichenden Umweltpolitikforschung zeigt bereits einige wichtige inhaltliche Tendenzen auf:

\footnotetext{
${ }^{283}$ Ebenda, S. 22.

${ }^{284}$ Ebenda, S. 23 f.

${ }^{285}$ Ebenda, S. 24.

${ }^{286}$ Badaracco, Joseph L. (1985): Loading . . ., a.a.O.

${ }^{287}$ Ebenda, S. 161.
} 
- In fast allen Studien werden Faktoren wie die Offenheit des politischen Prozesses, das Partizipationsausmaß der nicht-staatlichen Akteure, die Art der Konfliktlösung etc. als unabhängige Variablen thematisiert.

- Tendenziell erscheinen aus Sicht der Autoren konsensuale Politikmuster in allen Phasen des politischen Prozesses geeigneter zu sein als konfliktäre Vorgehensweisen.

- Alle zitierten Studien stellen signifikante Unterschiede im Politikstil der Vereinigten Staaten sowie der europäischen Staaten fest. Während in den USA viele Fragen durch den Streit der Akteure gelöst werden, überwiegt in Europa (und in Japan) das konsensuale Vorgehen. 


\section{Politikstil und Effektivităt von Umweltpolitik in OECD-Ländern: Unter- suchungsdesign}

Um die bestehende empirische Evidenz bezüglich des Zusammenhanges von Politikstil und Umweltbelastungsniveau zu erweitern, und die im ersten Teil dieser Untersuchung aufgestellten Hypothesen zu überprüfen, wird in den nächsten Kapiteln eine multivariate Datenanalyse durchgeführt.

$\mathrm{Zu}$ diesem Zweck wird zunächst die Frage nach der Größe der zu untersuchenden Grundgesamtheit gestellt. Dann erfolgt die Operationalisierung der abhängigen Größe, also der Effektivität von Umweltpolitik. Das vorliegende Kapitel schließt mit einer detaillierten Beschreibung der Analysestrategie.

\subsection{Die Festlegung der Fallzahl}

Bei der Festlegung der Größe N, also der Grundgesamtheit der zu untersuchenden Staaten, spricht das Streben nach einer hohen Reichweite der gefundenen Resultate für eine möglichst große Fallzahl. Dieses Ziel konkurriert jedoch mit dem Bemühen um ein ebenfalls hohes $\mathrm{MaB}$ an methodischer Verläßlichkeit der Ergebnisse, denn dies ist meist nur im Fall einer homogenen, also eher kleinen Grundgesamtheit gegeben.

Die Festlegung auf eine dieser beiden Prämissen führt zu Problemen:

Im ersten Fall können die kulturellen und ökonomischen Unterschiede zwischen den Erkenntnisobjekten so groß sein, daß die im Rahmen einer Analyse abgeleiteten Erkenntnisse nur wenig gehaltvoll sein dürften.

Wenn nämlich zwischen den Elementen der ausgewählten Staatengruppe große Unterschiede in der historisch-kulturellen Entwicklung und/oder dem wirtschaftlichen Entwicklungsstand bestehen, ist davon auszugehen, $\mathrm{da} B$ die determinierenden Wirkungen dieser Faktoren auf die ökonomischen und ökologischen Prozesse zu groß sind, um in einer quantitativen Analyse empirisch gesicherte Zusammenhänge zwischen anderen erklärenden Faktoren und den Zielgrößen aufzudecken.

Im zweiten Fall besteht dagegen die Gefahr der Erstellung inhaltlich zwar sehr richtiger, komparatistisch jedoch nichtssagender Regional- oder Einzelfallstudien $^{288}$. 
Berg-Schlosser und Stammen beschreiben die Problematik der Festlegung einer Fallzahl wie folgt: „Die „Vergleichbarkeit"verschiedener Fälle ist oft schwer zu bestimmen. Diese sind oft zu heterogen, z.B. in bezug auf die Größenordnung, die geographische Lage, die betrachtete Periode und viele andere jeweils untersuchte Variable, um einen sinnvollen Vergleich zu ermöglichen, "Ähnlichkeit" und „Verschiedenheit" müssen daher in einem sinnvollen Verhältnis zueinander stehen, um wenigstens einige Variable, unter Vernachlässigung anderer als in beiden Fällen „ähnlich" angesehener, näher betrachten zu können.

In diesem Sinne ist in der komparatistischen Praxis meist ein Kompromiß zwischen den Extremen einer möglichst großen Fallzahl mit wenigen Gemeinsamkeiten bzw. einer geringen Fallzahl mit großen Gemeinsamkeiten zu treffen ${ }^{290}$.

Es geht im Rahmen dieser Untersuchung also darum, eine möglichst groBe Staatengruppe mit ähnlichen politischen und ökonomischen Systemen sowie einem ähnlichen Grad der ökologischen Belastung auszuwählen ${ }^{291}$. Unter dieser Prämisse bietet es sich an, die Mitgliedstaaten der OECD zu den Erkenntnisobjekten der nachfolgenden empirischen Analyse zu machen, da hier eine relativ homogene Gruppe demokratisch verfaßter, marktwirtschaftlich orientierter Industrieländer mit ähnlichen sozioökonomischen und politisch-institutionellen Strukturen vorliegt. Mexiko, das sowohl in seiner demokratischen als auch in seiner gesellschaftlichen Entwicklung gegenüber den anderen Staaten eklatant abfällt, wird aus der Analyse ausgeschlossen. Die Türkei stellt in beiderlei Hinsicht aus Sicht des Verfassers einen gerade noch akzeptablen Grenzfall dar.

Die Beschränkung auf 22 Mitgliedstaaten der $\mathrm{OECD}^{292}$ hat noch zwei weitere Gründe:

${ }^{288}$ Vgl. dazu erneut die Anmerkungen Hartmanns aus Fußnote 22.

${ }^{289}$ Berg-Schlosser, Dirk, und Stammen, Dirk (1992): Einführung . ., a.a.O., S. 129 f.

${ }^{290}$ Hartmann, Jürgen (1995): Vergleichende . . ., a.a.O.

${ }^{291}$ Die Methode einer Maximierung der Zahl der homogenen Fälle wird in der Vergleichenden Politikforschung auch als most similar system design bezeichnet (Przeworski, A. und Teune, H. (1970): The Logic of Comparative Social Inquiry, New York, S. 31 ff.; zit. nach: Müller-Rommel, Ferdinand (1993): Grüne Parteien in Westeuropa. Entwicklungsphasen und Erfolgsbedingungen, Opladen, S. 34. 
1. Es gibt für andere Weltregionen (Lateinamerika, Asien, Osteuropa oder Afrika) kein verläßliches Zahlenmaterial im gleichen Umfang wie die Datensammlung „OECD Environmental Data - Donées OCDE sur l'environment ${ }^{\star 293}$.

2. Die Problematik der Umweltverschmutzung ist für hochentwickelte Industrieländer, also die meisten OECD-Staaten, von besonderer Relevanz.

\subsection{Indikatorenauswahl und Index-Bildung}

In Literatur und politischer Praxis finden sich zwei grundsätzliche Ansätze, um die Umweltbelastung durch geeignete Indikatoren zu erfassen. Der eine Ansatz liefert eine Systematik von Indikatoren, welche primär an der Dokumentation ökologischer Schäden orientiert ist, der zweite Ansatz nimmt eine generelle Zweiteilung der Indikatoren in solche zur Messung von Ressourcennutzung und -verbrauch bzw. in solche zur Erfassung der Umweltbelastung durch Schadstoffe vor.

Zur ersten Gruppe der Systematiken gehört das Umweltgutachten des Sachverständigenrates für Umweltfragen ${ }^{294}$. Darin werden unter dem Oberbegriff „Sektoren des Umweltschutzes“ die folgenden Bereiche ökologischer Belastung aufgezählt ${ }^{295}$ :

- Belastung und Schutz der Böden,

- Luftbelastung und Luftreinhaltung,

- Gewässerzustand und Gewässerschutz,

- Verunreinigungen von Lebensmitteln,

- Lärm.

292 Von den 26 OECD-Staaten fehlen Luxemburg, dessen Zahlen aufgrund der Wirtschaftsunion mit denen Belgiens aggregiert wurden, Island sowie die 1994 und 1996 beigetretenen Staaten Mexiko und Tschechien.

${ }^{293}$ Zur Problematik der Vergleichbarkeit von Umweltdaten aus unterschiedlichen Quellen siehe u.a. Bungarten, Harald H. (1978): Umweltpolitik . . ., a.a.O., S. 85 ff. sowie OECD (1994) (Hg.): Environmental Indicators, a.a.O., S.4.

${ }^{294}$ Der Rat von Sachverständigen für Umweltfragen (1988): Umweltgutachten 1987, Stuttgart; Mainz.

${ }^{295}$ Ebenda: das Inhaltsverzeichnis, S. 7 ff. 
Die Messung der Schäden für diese einzelnen Bereiche erfolgt anhand zahlreicher Einzelindikatoren. Dabei werden Aspekte der Nutzung von Ressourcen ohne systematische Trennung als Unterpunkte ${ }^{296}$ oder in einem ganz anderen Kontext ${ }^{297}$ behandelt.

Ähnlich verfährt das Umweltbundesamt in seiner Datensammlung zur Umweltsituation in Deutschland ${ }^{298}$. In einer eher losen Aufzählung werden primär Indikatoren zur Feststellung der Güte von Luft ${ }^{299}$, Wasser ${ }^{300}$ sowie zur Messung des Abfallaufkommens ${ }^{301}$ dargestellt. Auch hier werden Fragen der Ressourcennutzung ohne inhaltliche Trennung $\mathrm{zu}$ anderen Themen subsumiert ${ }^{302}$ oder ohne besondere Berücksichtigung ihrer umweltbelastenden Wirkung in anderen Kapiteln abgehandelt ${ }^{303}$.

Auch Wicke zählt in seiner ökologischen Schadensbilanzierung ausschließlich Umweltlasten auf ${ }^{304}$ :

- Luftverschmutzung,

- Gewässerverschmutzung,

- Bodenbelastung,

- Lärm.

${ }^{296}$ Ebenda, S. 183 ff.: Bodennutzung; S. 319 ff.: Trink- und Abwasserverbrauch.

${ }^{297}$ Die Nutzung fossiler Brennstoffe zur Energiegewinnung wird in einem separaten Kapitel „Umwelt und Energie“ (Ebenda, S. 488 ff.) abgehandelt.

${ }^{298}$ Umweltbundesamt (1995) (Hg.): Umweltdaten Deutschland 1995, Berlin.

${ }^{299}$ Ebenda, S. 16 ff.

${ }^{300}$ Ebenda, S. $28 \mathrm{ff}$.

${ }^{301}$ Ebenda, S. 37 ff.

${ }^{302}$ So wird zum Beispiel die Wasserentnahme im Kapitel „Wasser“ neben zahlreichen Indikatoren der Gewässerverschmutzung aufgeführt (Ebenda, S. 28).

${ }^{303}$ So sind die Flächennutzung im Kapitel „Allgemeine Daten“ (Ebenda, S. 2) und der Energieverbrauch im Kapitel „Umwelt und Energie“ (Ebenda, S. 8 ff.) dargestellt.

${ }^{304}$ Wicke, Lutz (1991): Umweltökonomie, a.a.O., S. 59-88. 
Die Problematik der Ressourcennutzung wird erst in einem späteren Kapitel im Kontext des Zusammenhanges von Umweltschutz und Wirtschaftswachstum thematisiert $^{305}$ :

Die Indikatorsystematik der OECD ist ein sogenanntes PSR-Kausalmodell eingebettet $^{306}$. Danach gibt es drei Kategorien von Umweltindikatoren: Die durch die menschlichen Aktivitäten ausgelösten ökologischen Schäden (Pressures) bedingen einen durch Qualitäten und Quantitäten beschreibbaren Zustand (State) der Umwelt und der natürlichen Ressourcen. Dieser Zustand wiederum löst Reaktionen (Responses) durch ökologische und ökonomische Akteure aus. Zur Messung sind für alle drei Elemente dieses Modells zahlreiche Indikatoren vorhanden. Dabei werden Schadstoffemissionen sowie Ressourcennutzung ohne weitere Differenzierung in der Kategorie der Environmental Pressures subsumiert.

Im Gegensatz zu diesen vier eben geschilderten Ansätzen unterscheidet Bossel in seiner Systematik eindeutig zwischen Umweltbelastungen durch Schadstoffeinträge bzw. durch Nutzung und Verbrauch von Ressourcen ${ }^{307}$. Die erste Kategorie der Schadwirkungen umfaßt dabei im einzelnen ${ }^{308}$ :

- Luftbelastungen,

- Gewässerbelastungen,

- Bodenbelastungen,

- Lebens- und Nahrungsbelastungen sowie

- Radioaktivität.

Die Nutzung erneuerbarer Ressourcen läßt sich vor allem in Land- und Wassernutzung unterscheiden ${ }^{309}$. Beim Verbrauch nicht-erneuerbarer Ressourcen geht es neben Materialressourcen primär um Energieressourcen ${ }^{310}$.

${ }^{305}$ Vgl. insbesondere: ebenda, S. $555 \mathrm{ff}$.

${ }^{306}$ OECD (1994) (Hg.): Environmental Indicators, a.a.O., S. 8 ff.

${ }^{307}$ Bossel, Hartmut (1990): Umweltwissen, a.a.O.; vgl. ebenda: das Inhaltsverzeichnis, S. 7.

${ }^{308}$ Ebenda, S. 119-144.

${ }^{309}$ Ebenda, S. 81-100.

${ }^{310}$ Ebenda, S. 101-118. 
Um zum einen ein möglichst detailliertes Bild der Umweltbelastung der untersuchten Staaten zu gewinnen und zum anderen eventuelle Unterschiede im Ausmaß der Ressourcennutzung bzw. der Schadstoffeinträge eines Staates aufzudecken, sollen in Anlehnung an Bossels Ansatz diese beiden Hauptdimensionen der Umweltbelastung in der vorliegenden Studie getrennt erfaßt werden. Zur Messung werden die folgenden Indikatoren verwendet (in eckigen Klammern sind die im weiteren genutzten Variablen-Kurzbezeichnungen aufgeführt) $)^{311}$ :

\section{Ressourcennutzung:}

Wasserverbrauch [H2O].

Diese Variable mißt den jährlichen Wasserverbrauch eines Staates durch private Haushalte, landwirtschaftliche Bewässerungsmaßnahmen, industrielle Prozesse sowie die Kühlung von Elektrizitätswerken ${ }^{32}$.

$\mathrm{Da}$ alle Lebensvorgänge auf der Erde an das Vorhandensein von Wasser gebunden sind ${ }^{313}$, stellt der Qualität und Quantität dieser Ressource beeinflussende Wasserverbrauch eine wichtige ökologische Variable dar. So kann etwa eine Grundwassernutzung zur Bewässerung landwirtschaftlicher Anbauflächen, welche in ihrer Menge den Wassereintrag durch Niederschläge überschreitet, durch Grundwassersenkung zu schweren Schäden des Öko-Systems führen ${ }^{314}$. Weitere Beispiele für ökologische Schädigungen durch Wasserverbrauch sind Verschmutzungen durch private Haushalte und Industrie ${ }^{315}$ sowie die Reduzierung des Sauerstoffgehaltes durch Erwärmung zu Zwecken der Kraftwerkskühlung $^{316}$.

${ }^{311}$ Die Daten fur die Indikatoren wurden entnommen aus: OECD (div. Jg.): OECD Environmental Data, a.a.O., Tabellen 2.1A-G, 3.1A, 5.1A, 8.1-3A, 12.3A, 15.6A-7A.

312 OECD (1993): OECD Environmental Data, Paris, S. 52; Einzelfallbeispiele für verschiedene Formen des Wasserverbrauches: Jänicke, Martin, und Weidner, Helmut (Hg.) (1995): Successful ..., a.a.O., S. 27-145.

${ }^{313}$ Vgl. Stephan, Petra (1995): Boden, Wasser, Biosphäre, In: Hauchler, Ingomar (Hg.): Globale Trends 95/96 . ., a.a.O., S. $290 \mathrm{ff}$.

${ }^{314}$ Vgl. Bossel, Hartmut (1990): Umweltwissen, a.a.O., S. 95.

${ }^{315}$ Vgl. Meadows, Donella, Meadows, Dennis, und Randers, Jorgen (1992): Die neuen Grenzen des Wachstums, Stuttgart, S. 117 ff.

${ }^{316}$ Vgl. Bossel, Hartmut (1990): Umweltwissen, a.a.O., S. 96; Vgl. Stephan, Petra (1995): Boden ..., a.a.O., S. $290 \mathrm{ff}$. 
Landwirtschaftsfläche [LAND].

Der Flächenverbrauch zu landwirtschaftlichen Zwecken ist die zweite Variable zur Messung der Ressourcennutzung eines Staates. Zwar könnte man einwenden, daß hohe Werte dieses Indikators eher positiv zu werten sind, da landwirtschaftlich genutzte Flächen den Bewohnern eines Staates aufgrund einiger Spezifika - wenig Lärm, keine Bebauung etc. - ein nicht zu vernachlässigendes Erholungspotential bieten. Dennoch überwiegen die negativen Effekte agrarwirtschaftlicher Nutzung. So haben die beiden Hauptformen der in menschlichen Zeiträumen unumkehrbaren Bodenschäden, Degradation und Desertifikation, ihre Ursachen vor allem in Überweidung, Ackerbau sowie Rodung zu Zwecken der Landwirtschaft ${ }^{317}$. Durch Abholzung und Entwaldung sind außerdem zahlreiche Arten gefährdet ${ }^{318}$. Bossel resümmiert deshalb ${ }^{39}:$ „Die Ertragsmaximierung der modernen Landwirtschaft geht auf Kosten der Stabilität des landwirtschaftlichen Ökosystems. " Solange also keine nachhaltige Landwirtschaft betrieben wird ${ }^{320}$, welche die Belastbarkeit der Böden und die volkswirtschaftlichen Kosten der Nutzung dieses Wirtschaftsgutes berücksichtigt, ist der Indikator Flächenverbrauch zu landwirtschaftlichen Zwecken als Umweltbelastungsgröße anzusehen.

\section{Primärenergieverbrauch [ENER].:}

Der Indikator Primärenergieverbrauch ${ }^{321}$, also die gesamte dem Umwandlungsproze $ß$ in Kraftwerken, Raffinerien und Heizwerken zugeführte Energiemenge umfaßt die fossilen Energieträger Kohle, Öl und Gas sowie Kernenergie, Wasserkraft, Biomasse und Sonnenenergie. Während der Verbrauch fossiler, nichtregenerativer Ressourcen ohne weitere Überlegungen als umweltbelastend $\mathrm{zu}$ klassifizieren ist, liegt dies für die anderen Energieträger nicht auf der Hand. Doch da der Anteil fossiler Energieträger am gesamten Energieverbrauch welt-

${ }^{317}$ Vgl. ebenda, S. 284 ff.; OECD (1993): OECD Environmental Data, Paris, S. 256.

${ }^{318}$ Vgl. Bossel, Hartmut (1990): Umweltwissen, a.a.O., S. 86.

${ }^{319}$ Ebd., S. 91.

${ }^{320}$ Zum Thema nachhaltige Landwirtschaft siehe: Ebd., S. 91 ff.; Stephan, Petra (1995): Boden ..., a.a.O., S.288 f.

${ }^{321}$ Dieser Indikator gehört zu den am häufigsten verwendeten Variablen zur Messung der Umweltbelastung (siehe z.B. Jänicke, Martin, et al. (1993): Umweltentlastung . . ., a.a.O., S. 32 ff.; Meadows, Donella, et al. (1992): Die neuen Grenzen . .., a.a.O., S. 94 ff.; Umweltbundesamt (1995) (Hg.): Umweltdaten . ., a.a.O., S. 9 f). 
weit 77 und in den OECD-Staaten sogar 78 Prozent ausmacht, soll dieser Indikator im folgenden ohne weitere Differenzierung bzw. ohne Herausrechnen des ohnehin geringen Anteils der nicht-fossilen Energieträger als Umweltbelastungsgröße gelten.

\section{Schadstoffeinträge:}

Energiebedingte $\mathrm{CO}_{2}$-Emissionen [CO2].

Diese Schadstoffbelastungsvariable eines Staates mißt die jährlichen energiebedingten $\mathrm{CO}_{2}$-Emissionen.

Die bei der Verbrennung fossiler Brennstoffe freiwerdenden Kohlendioxidmengen stellen den größten Anteil der für den Temperaturanstieg der Atmosphäre verantwortlichen Gase ${ }^{322}$. Diese auch „Treibhauseffekt" genannte Entwicklung hat potentiell negative Effekte auf das globale Klima, das Niveau des Meeresspiegels sowie die Landwirtschaft.

\section{Abfallaufkommen [WASTE].}

Dieser Indikator umfaßt die innerhalb eines Staates jährlich anfallenden Abfälle aus privaten Haushalten, Kleingewerbe und Dienstleistungen.

Die negativen Umweltwirkungen der Abfallproduktion liegen außer in der unmittelbaren Verschmutzung von Luft, Wasser und Böden auch im Flächenverbrauch (z.B. für Deponien), Geruchsbelästigungen sowie weiteren ästhetischen Beeinträchtigungen.

Handelsdüngerabsatz [FERT].

Der jährliche Handelsdüngerabsatz eines Staates umfaßt die synthetisch erzeugten Düngerarten aus den Grundstoffen Stickstoff, Phosphat, Kali und Kalk. Die umweltbelastende Wirkung dieser Stoffe beschreibt Bossel wie folgt ${ }^{323}$ : „Hohe Düngergaben in [der] Landwirtschaft [...] führen zu erhöhter Auswaschung von Nitrat im Boden und in die Grundwasserleiter. Nitrat verändert u.a. die Stickstoffbindung im Blut und führt zu Blausucht und Ersticken bei Kleinkindern [...] Eine mögliche Verbindung [...] besteht auch zur Krebsbildung. "

\footnotetext{
${ }^{322} \mathrm{Vgl}$. Meadows, Donella, et al. (1992): Die neuen Grenzen . . ., a.a.O., S. 123 ff.; OECD (1993): OECD Environmental Data, Paris, S. 30; Bossel, Hartmut (1990): Umweltwissen, a.a.O., S. 130.

${ }^{323}$ Ebd., S. 136.
} 
Für die weitere Analyse werden die Werte aller sechs Indikatoren jeweils entweder auf die Einwohnerzahl [POPUL] oder auf das Bruttoinlandsprodukt [BIP] des gleichen Jahres bezogen.

Außerdem werden für beide Dimensionen durch Aggregation der jeweils indexierten Werte der Einzelindikatoren Indexwerte der Ressourcennutzung [RESOU] bzw. der Schadstoffeinträge [PRESS] ermittelt.

Als Basisgrößen gelten dabei die OECD-Durchschnittswerte für alle sechs Indikatorvariablen im Jahr 1980.

OECD-Durchschnitt 1980:

\begin{tabular}{|c|c|c|c|c|c|}
\hline \multicolumn{3}{|c|}{ Wert pro } & \multirow[b]{2}{*}{ Indikator } & \multirow[b]{2}{*}{ POPUL } & \multirow[b]{2}{*}{$B I P$} \\
\hline Indikator & POPUL & $B I P$ & & & \\
\hline$H 2 O^{\mathrm{a}}$ & 720 & 73,1 & $\mathrm{CO}^{\mathrm{d}}$ & 9,8 & 0,92 \\
\hline$L A N D^{\mathrm{b}}$ & 4915 & 578,2 & $W A S T E^{e}$ & 382 & 43,6 \\
\hline$E N E R^{\mathrm{c}}$ & 3,897 & 3,51 & $F E R T^{f}$ & 680 & 7,4 \\
\hline
\end{tabular}
a. $1000 \mathrm{~m}^{3} /$ Einw. bzw. $\mathrm{m}^{3} / 1000$ US-\$
b. $\mathrm{m}^{2} /$ Einw. bzw. $\mathrm{m}^{2} / \mathrm{Mrd}$. US-S
c. Tonnen Erdölăquivalent (TOE)/Einw. bzw. TOE/1000 US-\$
d. t/Einw. bzw. $t / 1000$ US-\$
e. kg/Einw. bzw. kg/1000 US-\$
f. kg/Einw. bzw. kg/1000 US-\$

Zur Erläuterung der weiteren Vorgehensweise wird die Indexberechnung am praktischen Beispiel für die Werte Portugals im Jahre 1975 und die Werte der USA im Jahre 1990 demonstriert.

\begin{tabular}{|c|c|c|c|c|}
\hline \multirow[b]{2}{*}{ Indikator } & \multicolumn{2}{|c|}{$\begin{array}{c}\text { Portugal } \\
\text { Wert (1975) pro }\end{array}$} & \multicolumn{2}{|c|}{$\begin{array}{c}\text { USA } \\
\text { Wert (1990) pro }\end{array}$} \\
\hline & POPUL & $B I P$ & POPUL & $B I P$ \\
\hline $\mathrm{H} 2 \mathrm{O}$ & 181 & 41,4 & 1861 & 102,7 \\
\hline$L A N D$ & 3350 & 764 & 7551 & 417 \\
\hline ENER & 0,859 & 1,96 & 7,633 & 4,22 \\
\hline $\mathrm{CO} 2$ & 2,4 & 0,54 & 20,0 & 1,11 \\
\hline WASTE & 226,4 & 51,3 & 705,7 & 39,0 \\
\hline$F E R T$ & 263,2 & 6,01 & 732,7 & 4,05 \\
\hline
\end{tabular}

Einheiten wie oben. 
Diese Indikatorwerte werden mittels der OECD-Durchschnittswerte für das Jahr 1980 (jeweils bezogen auf die Einwohnerzahl bzw. das Bruttoinlandsprodukt) indexiert.

Wasserverbrauch

Portugal: $\quad(181 / 720) \times 100=25,1$

bzw. $(41,4 / 73,1) \times 100=56,6$

USA:

$(1861 / 720) \times 100=258,5$

bzw. $(102,7 / 73,1) \times 100=140,5$

Landwirtschaftsfläche

Portugal: $\quad(3350 / 4915) \times 100=68,2 \quad$ bzw. $(764 / 578,2) \times 100=132,1$

USA: $\quad(7551 / 4915) \times 100=153,6 \quad$ bzw. $(417 / 578,2) \times 100=71,1$

Primärenergieverbrauch

Portugal: $\quad(0,859 / 3,752) \times 100=22,9 \quad$ bzw. $(1,96 / 3,51) \times 100=55,8$

USA: $\quad(7,633 / 3,752) \times 100=203,4$ bzw. $(4,22 / 3,51) \times 100=120,2$

Energiebedingte $\mathrm{CO}_{2}$-Emissionen

Portugal: $\quad(2,4 / 9,8) \times 100=24,5$

USA: $\quad(20,0 / 9,8) \times 100=204,1$

bzw. $(0,54 / 0,92) \times 100=58,7$

bzw. $(1,11 / 0,92) \times 100=120,7$

Abfallaufkommen

Portugal: $\quad(226,4 / 382) \times 100=59,3 \quad$ bzw. $(51,3 / 43,6) \times 100=117,7$

USA: $\quad(705,7 / 382) \times 100=184,7 \quad$ bzw. $(39,0 / 43,6) \times 100=89,4$

Düngemittelabsatz

Portugal: $\quad(263,2 / 680) \times 100=38,7 \quad$ bzw. $(6,01 / 7,4) \times 100=81,2$

USA: $\quad(732,7 / 680) \times 100=107,8 \quad$ bzw. $(4,05 / 7,4) \times 100=54,7$

Aus diesen indexierten Daten werden die Indexwerte des Ressourcenverbrauches und der Schadstoffeinträge als arithmetische Mittel der drei Einzelindikatoren wie folgt berechnet: 
Ressourcenverbrauchsindex (Basis: Einwohnerzahl)

\begin{tabular}{llllll}
\hline Land & $\begin{array}{l}\text { Indexierter } \\
\text { Wasserver- } \\
\text { brauch }\end{array}$ & $\begin{array}{l}\text { Indexierte } \\
\text { schafts- } \\
\text { fläche }\end{array}$ & $\begin{array}{l}\text { Indexierter } \\
\text { Primär- } \\
\text { energie- } \\
\text { verbrauch }\end{array}$ & $\begin{array}{l}\text { Ressourc.- } \\
\text { verbrauchs } \\
\text {-index }\end{array}$ \\
\hline Portugal & 25,1 & 68,2 & 22,9 & 116,2 & 38,7 \\
USA & 258,5 & 153,5 & 203,4 & 615,4 & 205,1 \\
\hline
\end{tabular}

Schadstoffindex (Basis: Einwohnerzahl)

\begin{tabular}{llllll}
\hline Land & $\begin{array}{l}\text { Indexierte } \\
\mathrm{CO}_{2-} \\
\text { Emissione } \\
\mathrm{n}\end{array}$ & $\begin{array}{l}\text { Indexiertes } \\
\text { Abfallauf- } \\
\text { kommen }\end{array}$ & $\begin{array}{l}\text { Indexierter } \\
\text { Handels- } \\
\text { dünger- } \\
\text { absatz }\end{array}$ & $\begin{array}{l}\text { Schadstoff } \\
\text {-index }\end{array}$ \\
\hline Portugal & 24,5 & 59,3 & 38,7 & 122,5 & 40,8 \\
USA & 204,1 & 184,7 & 107,8 & 496,6 & 165,5 \\
\hline
\end{tabular}

Ressourcenverbrauchsindex (Basis: Bruttoinlandsprodukt)

\begin{tabular}{llllll}
\hline Land & $\begin{array}{l}\text { Indexierter } \\
\text { Wasserver- } \\
\text { brauch }\end{array}$ & $\begin{array}{l}\text { Landwirte } \\
\text { schafts- } \\
\text { fläche }\end{array}$ & $\begin{array}{l}\text { Indexierter } \\
\text { Primär- } \\
\text { energie- } \\
\text { verbrauch }\end{array}$ & $\begin{array}{l}\text { Ressourc.- } \\
\text { verbrauchs } \\
\text {-index }\end{array}$ \\
\hline Portugal & 56,6 & 132,1 & 55,8 & 244,5 & 81,5 \\
USA & 140,5 & 71,1 & 120,2 & 331,8 & 110,6 \\
\hline
\end{tabular}

Schadstoffindex (Basis: Bruttoinlandsprodukt)

\begin{tabular}{llllll}
\hline Land & $\begin{array}{l}\text { Indexierte } \\
\mathrm{CO}_{2-} \\
\text { Emissione } \\
\mathrm{n}\end{array}$ & $\begin{array}{l}\text { Indexiertes } \\
\text { Abfallauf- } \\
\text { kommen }\end{array}$ & $\begin{array}{l}\text { Indexierter } \\
\text { Handels- } \\
\text { dünger- } \\
\text { absatz }\end{array}$ & $\begin{array}{l}\text { Schadstoff } \\
\text {-index }\end{array}$ \\
\hline Portugal & 58,7 & 117,7 & 81,2 & 257,6 & 85,9 \\
USA & 120,7 & 89,4 & 54,7 & 264,8 & 88,3 \\
\hline
\end{tabular}

Beide Indexwerte werden schließlich noch zu einem Gesamt-Index (jeweils pro Einwohnerzahl bzw. Einheit Bruttoinlandsprodukt) zusammengefaßt: 
Umweltbelastungsindex (Basis: Einwohnerzahl)

\begin{tabular}{lllll}
\hline Land & $\begin{array}{l}\text { Ressourcen- } \\
\text { verbrauchs- } \\
\text { index }\end{array}$ & $\begin{array}{l}\text { Schadstoff } \\
\text { index }\end{array}$ & $\Sigma$ & $\begin{array}{l}\text { Umweltbe- } \\
\text { lastungsindex }\end{array}$ \\
\hline Portugal & 38,7 & 40,8 & 79,5 & 39,8 \\
USA & 205,1 & 165,5 & 370,6 & 185,3 \\
\hline
\end{tabular}

Umweltbelastungsindex (Basis: Bruttoinlandsprodukt)

\begin{tabular}{lllll}
\hline Land & $\begin{array}{l}\text { Ressourcen- } \\
\text { verbrauchs- } \\
\text { index }\end{array}$ & $\begin{array}{l}\text { Schadstoff } \\
\text { index }\end{array}$ & $\Sigma$ & $\begin{array}{l}\text { Umweltbe- } \\
\text { lastungsindex }\end{array}$ \\
\hline Portugal & 81,5 & 85,9 & 167,4 & 83,7 \\
USA & 110,6 & 88,3 & 198,9 & 99,5 \\
\hline
\end{tabular}

Diese Art der Indexbildung wirft einige Fragen bezüglich der Methodik auf: Warum werden in unterschiedlichen Einheiten gemessene Indikatoren der Umweltbelastung $\mathrm{zu}$ einem Index zusammengefaßt? Warum werden alle Indikatoren gleich gewichtet? Warum werden pro Dimension der Umweltbelastung nur drei Einzelindikatoren zur Indexbildung genutzt? Werden durch einen einzigen Indexwert pro Land nicht regionale Unterschiede der Umweltbelastung nivelliert?

Die Bildung von Bereichs-Indizes bzw. eines Gesamt-Index ist der Versuch, trotz der Komplexität der Umweltproblematik und der Vielzahl der möglichen Indikatoren ${ }^{324}$, einen heuristischen Maßstab zur Feststellung der ökologischen Belastung eines Staates zu finden. Die Indexierung der Daten für die Einzelindikatoren durch das arithmetische Mittel aller OECD-Staaten im Jahre 1980 erlaubt zum einen die Darstellung der zeitlichen Entwicklung der Umweltbelastung eines Staates und zum anderen einen aussagekräftigen Vergleich der Daten eines Staates mit anderen Ländern auf einer Ratioskala.

Die Gleichgewichtung der Einzelindikatoren mag simplifizierend oder willkürlich erscheinen. Doch solange es kein überzeugendes und praktikables Kriterium zur unterschiedlichen Gewichtung der einzelnen Faktoren gibt, erscheint es

\footnotetext{
${ }^{324}$ So umfassen z.B. die Datenkompendien der OECD Zeitreihen von 103 Indikatoren (Vgl. OECD (1993): OECD Environmental Data, Paris.).
} 
methodisch am redlichsten zu sein, alle mit dem gleichen Gewicht in den Gesamtindex einfließen zu lassen.

Es wäre sicherlich möglich gewesen, eine deutlich höhere Zahl an Einzelindikatoren zu berücksichtigen, doch zahlreiche Gründe lassen eine Beschränkung auf drei Komponenten je Umweltbelastungsbereich (Ressourcennutzung bzw. Schadstoffemissionen) sinnvoll erscheinen:

1. Nicht für alle Indikatoren liegen für den gesamten Beobachtungszeitraum so komplette Zeitreihen vor wie für die sechs ausgewählten Komponenten;

2. Eine Hinzunahme weiterer Indikatoren hätte nicht unbedingt einen weiteren signifikanten Informationsgewinn bedeutet. So machen die $\mathrm{CO}_{2}$-Emissionen unter den gesamten Treibhausgasen nahezu 97 Prozent aus, so daß eine zusätzliche Berücksichtigung der Umweltbelastungsvariable „Emissionen an Treibhausgasen ${ }^{6325}$ zu einer starken Überschneidung zweier Indikatoren führen würde;

3. Außer dem Nachteil geringen Informationsgewinnes bei gleichzeitiger Erhöhung der Aufwandes zur Datengewinnung führt die Berücksichtigung von sich inhaltlich überschneidenden Komponenten auch zu Verzerrungen der Indexwerte, wodurch das Ziel eines globalen Maßstabes zur Messung der Umweltbelastung verfehlt würde.

Dagegen scheinen die sechs ausgewählten Einzelindikatoren relativ heterogen zu sein, denn sie messen sehr unterschiedliche Aspekte der komplexen Umweltbelastungsproblematik. So beziehen sich die drei Variablen zur Messung der Ressourcenausbeutung jeweils auf die Nutzung von Wasser $(\mathrm{H} 2 \mathrm{O})$, Böden ( $L A N D)$ sowie Rohstoffen (ENER), und die Schadstoff-indikatoren auf die Aufnahmemedien Luft (CO2), Böden (WASTE) sowie Wasser (FERT).

Zwar gibt es zwischen einzelnen Regionen innerhalb eines Staates so große Unterschiede im Grad der Umweltbelastung, daß die Berechnung eines einzigen Indexwertes pro Staat als Irreführung erscheinen mag, dennoch werden im folgenden keine regional differenzierten Indizes verwendet. Denn zum einen ist das Datenmaterial über die quantitative Umweltbelastung von geographischen Regionen noch immer sehr lückenhaft, und zum anderen geht es in dieser Studie ja um die politikstilbedingten Unterschiede des Umweltbelastungsgrades von Nationalstaaten. Und so mag es aufgrund historischer, klimatischer und/oder geologischer Spezifika insbesondere in Flächen- und Kontinentalstaaten große Unterschiede der regionalen Umweltbelastung geben, im Kontext dieser Untersuchung wird jedoch unterstellt, daß sich das durchschnittliche Bela- 
stungsniveau innerhalb eines Staates letztlich aus dem nationalen Politikstil ableitet, eine Differenzierung der Indizes nach geographischen Subeinheiten also nicht hilfreich ist.

\subsection{Strategie der Datenanalyse}

Zu Beginn der Datenanalyse erfolgt in Kapitel 8 eine ausführliche Darstellung deskriptiver Statistiken der einzelnen Indikatoren und Indizes. Diese Vorgehensweise soll eine erste Einschätzung der im Rahmen dieser Studie untersuchten Staaten im Hinblick auf ihr Umweltbelastungsniveau ermöglichen. Auch eventuelle Ähnlichkeiten der Datenmuster unterschiedlicher Staaten sollen bereits in diesem frühen Stadium der Analyse aufgedeckt und - soweit möglich erklärt werden.

In einem zweiten Schritt wird in Kapitel 9 das Erklärungspotential ausgewählter struktureller Determinanten des Erfolges nationaler Umweltpolitik untersucht. Von den ökonomischen Faktoren sind dabei die Variablen Wirtschaftskraft (gemessen im Bruttoinlandsprodukt pro Einwohner bzw. dessen Wachstum) sowie -struktur (gemessen anhand der sektoralen Verteilung der Volkswirtschaft) von besonderem Interesse; aus der Gruppe der politischen Faktoren wird der elektorale Erfolg grüner Parteien zum Zwecke einer quantitativen Analyse operationalisiert. Um die Bedeutung dieser drei erklärenden Variablen für das Ausmaß der Umweltbelastungsindikatoren festzustellen, finden dann die statistischen Verfahren der Korrelationsanalyse sowie des t-Testes Anwendung. Zwar wird von diesen Faktoren ein gewisses Erklärungspotential für die Varianz der ökologischen Variablen erwartet, doch die eigentlichen Hypothesentests werden dann im fünften Kapitel des empirischen Teiles der Studie durchgeführt.

Dabei ist es zunächst notwendig, die erklärende Größe, den nationalen Politikstil, hinreichend zu operationalisieren. Die erste der beiden Politikstildimensionen, das staatliche Vermögen, zukünftige Ereignisse zu antizipieren, und die Entwicklung ökologischer Größen mittel- und langfristig strategisch zu beeinflussen, wird dabei anhand der Hauptmerkmale Dezentralisationsgrad, technologische Kapazität und administrative Kapazität gemessen. Während die Staaten anhand von Plausibilitätsüberlegungen in die Gruppe der eher föderalen oder eher zentralistischen Gemeinwesen eingruppiert werden, sind für die beiden anderen Indikatoren der Strategieorientierung Operationalisierungsmöglichkeiten anhand von „harten“ Daten gegeben. Die Messung der technologischen Kapazität erfolgt durch den Anteil der Forschungs- und Entwicklungsausgaben sowie der Spitzenforschungsausgaben eines Staates am BIP und dem 
Export-/Importquotienten im Außenhandel mit Hochtechnologiegütern. Die administrative Kapazität messen wir anhand der Anteile der öffentlichen Ausgaben am BIP sowie der mit der Wahrnehmung staatlicher Aufgaben Beschäftigten an allen Beschäftigten.

Aufgrund der Datenmuster für diese drei Variablenkomplexe kann dann eine Einteilung der Grundgesamtheit in Gruppen mit unterschiedlich ausgeprägter Strategieorientierung erfolgen.

Die zweite grundlegende Politikstildimension, der Grad der Partizipation der durch Entscheidungen Betroffenen am Prozeß der ökologischen Problemlösung, wird durch zwei Variablen operationalisiert. Zum einen ermöglicht eine Sekundäranalyse vorhandener Studien der Politikwissenschaft die Messung des Ausmaßes, in dem Staaten eine korporatistische Politik betreiben, und zum anderen können die Staaten aufgrund ihres empirisch belegbaren Problemlösungsverhaltens in solche unterschieden werden, die Entscheidungen nur unter konsensualer Einbeziehung möglichst aller Betroffenen treffen, bzw. andere, welche eine Lösungstrategie durch die offene Konfliktaustragung der Beteiligten präferieren. Auch die sich hierbei ergebenen Datenmuster lassen eine Einteilung der Grundgesamtheit im Sinne unseres Erklärungsmodells zu, nämlich in Staaten mit einem hohen Partizipationsgrad der nicht-staatlichen Interessengruppen (hoher Korporatismusgrad, konsensuale Konfliktlösung) und in Staaten mit einer Dominanz der staatlichen Akteure im politischen Prozeß (geringer Korporatismusgrad, konfliktäres Problemlösungsverhalten).

Anhand der beiden so gewonnenen Eingruppierungsmöglichkeiten sind die einzelnen Staaten dann in das durch die beiden Hauptmerkmalsdimensionen aufgespannte Koordinatensystem einzuordnen. Die daran anschließende Clusteranalyse soll dreierlei Funktionen erfüllen:

1. Verifizierung bzw. Falsifizierung der Gruppeneinteilung;

2. Bestätigung der weitgehenden statistischen Unabhängigkeit der beiden Hauptmerkmalsdimensionen durch die Feststellung der Orthogonalität der Achsen;

3. Bestätigung der jeweiligen inhaltlichen „Verwandtschaft“ der die Merkmalsdimensionen operationalisierenden Einzelvariablen.

Im Kapitel 11 wird dann als Kern der empirischen Untersuchung das Erklärungspotential des nationalen Politikstils für die Effektivität nationaler Umweltpolitik untersucht.

Dabei sind zunächst die Auswirkungen der einzelnen Politikstildimensionen bzw. deren Einzelelemente auf die Umweltbelastungsindikatoren festzustellen. Hierbei kommt die multivariate Methode der multiplen Regressionsanalyse zur Anwendung. 
Die Bedeutung der kombinatorischen Wirkung beider Hauptmerkmalsdimensionen auf die abhängigen ökologischen Variablen wird dann anhand eines varianzanalytischen Vergleiches der Politikstilgruppen festgestellt. 


\section{Messung der Effektivität nationaler Umweltpolitik}

8.1 Umweltbelastungsindikatoren (Basis: Einwohnerzahl)

\subsubsection{Ressourcenverbrauch}

Unter den Indikatoren zur Messung des Ressourcenverbrauches soll zunächst der Wasserverbrauch pro Einwohner im Jahr 1990 behandelt werden. Dieser Faktor umfaßt den gesamten Wasserverbrauch der einzelnen Volkswirtschaften, also vor allem die Privatnutzung, die zur Bewässerung landwirtschaftlicher Flächen eingesetzten Wassermengen, die industrielle Nutzung sowie das zur Kühlung von Kraftwerken verwendete Wasser ${ }^{326}$.

Den höchsten Wasserverbrauch haben mit einigem Abstand die USA $\left(1861 \mathrm{~m}^{3}\right)$ und Kanada $\left(1761 \mathrm{~m}^{3}\right) \mathrm{zu}$ verzeichnen ${ }^{327}$. Während alle anderen Staaten von Australien bis Norwegen leicht über bzw. unter dem OECD-Mittelwert von 714 Kubikmetern pro Einwohner liegen, weisen Schweden $\left(343 \mathrm{~m}^{3}\right)$, Österreich $\left(275 \mathrm{~m}^{3}\right)$, Großbritannien $\left(246 \mathrm{~m}^{3}\right)$, Dänemark $\left(233 \mathrm{~m}^{3}\right)$, Irland $\left(226 \mathrm{~m}^{3}\right)$ und vor allem die Schweiz $\left(172 \mathrm{~m}^{3}\right)$ signifikant niedrigere Werte auf.

Die hohen Werte der beiden nordamerikanischen Staaten sind dabei zum einen auf eine weniger sparsame Verwendung von Wasser im Privathaushalt zurückzuführen - die USA erreichen hier einen Wert von $217 \mathrm{~m}^{3}$, und Kanada liegt bei $191 \mathrm{~m}^{3}$ (zum Vergleich: Österreich: $69 \mathrm{~m}^{3}$ ) -, und zum anderen auf die großen Wassermengen zur Bewässerung (USA: $774 \mathrm{~m}^{3}$ ) und zur Kraftwerkkühlung (USA: $726 \mathrm{~m}^{3}$; Kanada: $982 \mathrm{~m}^{3}$ )

OECD-weit ist der Wasserverbrauch pro Einwohner seit 1970 leicht angestiegen, und 1990 weisen im Vergleich zum damaligen Wert 10 von 22 Staaten höhere Pro-Kopf-Verbräuche auf ${ }^{329}$. Es gibt jedoch auch sechs Länder, die für alle vier gemessenen Fünf-Jahres-Zeiträume einen konti-

${ }^{326}$ OECD (1993): OECD Environmental Data, a.a.O., S. 47 ff.

${ }^{327}$ Vgl. Tabelle A13.

${ }^{328}$ OECD (1993): OECD Environmental Data, a.a.O., S. 55; eigene Berechnungen aufgrund der dort gemachten Prozentangaben.

329

Vgl. Tabelle A13. 
nuierlichen Rückgang des Wasserverbrauches zu verzeichnen haben: Japan, Australien, Österreich, Belgien, Norwegen und Schweden ${ }^{330}$.

Auch für den zweiten Indikator, die landwirtschaftliche Nutzfläche pro Einwohner, sind zwei statistische "Ausreißer" mit extrem hohen Werten festzustellen: Australien $\left(28,63 \mathrm{~km}^{2}\right)$ und Kanada $\left(15,56 \mathrm{~km}^{2}\right)^{331}$. Während noch weitere Flächenstaaten (USA, Finnland, Türkei) für diesen Indikator überdurchschnittliche Werte aufweisen, sind die Zahlen für einige Industrieländer mit hoher Bevölkerungsdichte (Deutschland, Großbritannien, Belgien, Schweiz, Niederlande und Japan) auffallend niedrig.

Die zeitliche Entwicklung dieses Indikators ist langsam aber stetig rückläufig. Alle Staaten weisen für 1990 niedrigere Werte auf als im Jahr $1970^{332}$. Während jedoch in den europäischen OECD-Staaten und Japan auch die absoluten Werte gesunken sind, haben die Zahlen für die in Australien (1970-1990) und Kanada (1970-1985 und nur leichter Rückgang in 1990) genutzten Landflächen im Zeitverlauf zugenommen ${ }^{333}$.

Eine Analyse der Daten des dritten Indikators zur Messung des Ressourcenverbrauches, dem primären Energieverbrauch pro Einwohner, ergibt mit Kanada (7,915 Tonnen Erdöläquivalent [TOE]) und den Vereinigten Staaten $\left(7,633\right.$ TOE) erneut zwei Staaten mit signifikant hohen Werten ${ }^{334}$. $\mathrm{Da}$ der Energieverbrauch eng mit dem wirtschaftlichen Entwicklungsstand eines Staates korrelieren dürfte ${ }^{335}$, ist es nicht überraschend, daß sich mit den südeuropäischen EU-Mitgliedsstaaten und der Türkei Länder

${ }^{330} \mathrm{Vgl}$. Tabelle A 22.

${ }^{331}$ OECD (1993): OECD Environmental Data, a.a.O., S. 256 f. Auch unter Berücksichtigung der Besonderheit, daß die Daten Australiens etwa 57\% kultiviertes Grasland enthalten, bleibt der Wert fur diesen Staat extrem hoch. Vgl. auch Tabelle A14.

${ }^{332}$ Vgl. ebenda sowie Tabelle A23.

${ }^{333} \mathrm{Vgl}$. Tabellen A2 und A8.

${ }^{334}$ Vgl. Tabelle A15.

${ }^{335}$ Im Falle Kanadas spielen neben dem hohen Wirtschaftsniveau sicherlich auch die klimatischen Verhältnisse eine wichtige Rolle. So betragen z.B. die Durchschnittstemperaturen im Monat Januar selbst im eher suldlich gelegenen Ottawa $-11^{\circ} \mathrm{C}$ (Harenberg (1994): Harenberg Länderlexikon '94/95, Dortmund, S. 229). 
mit am OECD-Standard gemessen eher niedrigen Bruttoinlandsproduktwerten auch als solche mit dem geringsten Pro-Kopf-Energieverbrauch erweisen. Aber auch ökonomisch hochentwickelte Staaten wie Japan $(3,467$ TOE), Österreich $(3,291$ TOE) und Italien (2,684 TOE) gehören zu den Ländern mit unterdurchschnittlichen Indikatorwerten.

Der Mittelwert aller OECD-Staaten ist für diesen Indikator in den letzten 20 Jahren ständig angestiegen, und bis auf Dänemark und Großbritannien weisen alle Elemente der untersuchten Grundgesamtheit 1990 einen höheren Wert auf als 1970. Auch in der letzten Fünf-Jahres-Periode (1985-90), für welche vollständige Datensätze vorliegen, ist der Energieverbrauch pro Einwohner in nahezu allen Staaten angestiegen. Lediglich für Dänemark, Deutschland und Schweden waren sinkende Werte $\mathrm{zu}$ verzeichnen ${ }^{336}$.

Die sich aus den drei geschilderten Indikatorwerten ergebenden IndexWerte für den Ressourcenverbrauch des Jahres 1990 sind in Abbildung 19 dargestellt. Die 22 Staaten lassen sich danach grob in vier Gruppen einteilen. Die drei Kontinentalstaaten USA, Kanada und Australien weisen extrem hohe Index-Werte auf. Finnland und Spanien bilden die zweite Gruppe und liegen in etwa auf dem OECD-Niveau von 1980. Dann folgen 13 weitere Staaten, deren Zahlen alle dicht beisammen liegen. Ausreißer im positiven Sinne sind Irland, Österreich, Großbritannien und die Schweiz mit eher niedrigen Werten zwischen 56 und 46 Index-Punkten.

Der Mittelwert aller OECD-Staaten ist von 1970 bis 1980 leicht angestiegen und liegt seitdem auf diesem Niveau ${ }^{337}$.

In Abbildung 20 ist die zeitliche Entwicklung des Index für sechs ausgewählte Staaten, die insgesamt mehr als $60 \%$ der Bevölkerung und etwa $70 \%$ des Bruttoinlandsproduktes der gesamten OECD repräsentieren, dargestellt. Während die USA und Schweden ihre Werte seit 1980 von einem hohen Niveau ausgehend verbessern konnten, sind die Zahlen für die anderen vier Staaten seitdem zumindest leicht angestiegen, und Frankreich hat Schweden im Hinblick auf diesen Index sogar überholt. Auch die Werte von Deutschland und der Schweiz haben sich in den 20 Jahren des Beobachtungszeitraumes kontinuierlich verschlechtert, während der japanische Pro-Kopf-Ressourcenverbrauch des Jahres 1990 nahezu mit den Zahlen von 1970 identisch ist.

${ }^{336} \mathrm{Vgl}$. Tabelle A24.

${ }^{337} \mathrm{Vgl}$. Tabellen A19 und A28. 
Zusammenfassend läßt sich $\mathrm{zu}$ dieser Indikatorgruppe also folgendes formulieren:

- Der Ressourcenverbrauch pro Einwohner ist in den OECD-Staaten zwischen 1970 und 1980 stark angestiegen und bewegt sich in den folgenden 10 Jahren auf einem etwa konstanten Niveau.

- Die einzelnen Staaten unterscheiden sich deutlich in ihren Verbrauchswerten, und es lassen sich durch grobe Einteilung vier Staatengruppen mit unterschiedlichem Verbrauchsniveau identifizieren.

- Auch die Zeitreihenverläufe des Ressourcenindexes weisen für die einzelnen Staaten teilweise sehr unterschiedliche Entwicklungsmuster auf.

\subsubsection{Schadstoffemissionen}

Den höchsten Wert des Schadstoff-Indikators „Energiebedingte $\mathrm{CO}_{2}$ Emissionen pro Einwohner" weisen die USA mit gut 20 Tonnen auf ${ }^{338}$. Dahinter folgen die Kontinentalstaaten Kanada (16,34 t) und Australien $(15,92 \mathrm{t})$. Die meisten anderen OECD-Staaten liegen mit Werten zwischen 12,54 (Belgien) und 5,83 Tonnen (Spanien) dicht beisammen, während die weniger entwickelten Länder Portugal $(4,36 \mathrm{t})$ und Türkei $(2,46$ t) deutlich niedrigere Daten aufweisen. Der OECD-Mittelwert für 1990 $(9,5 \mathrm{t})$ liegt leicht über der Zahl von 1970 (9,0 t), jedoch ist in den 20 Jahren des Beobachtungszeitraumes kein eindeutiger Trend ablesbar ${ }^{339}$. Während relativ gering industrialisierte Staaten wie die Türkei, Griechenland oder Portugal mit zunehmender okönomischer Modernisierung auch ihren Kohlendioxid-Ausstoß vergrößerten, gibt es unter den Industriestaaten sowohl Erfolgsfälle mit einer sehr starken Reduktion in den 20 Jahren des Beobachtungszeitraumes (Schweden mit einem Rückgang von $43,84 \%$ oder Dänemark mit einer Reduktion um $17,1 \%$ ) als auch Staaten mit einer weiteren Zunahme des Pro-Kopf-Ausstoßes dieses Treibhausgases (Australien: $+26,16 \%)^{340}$.

${ }^{338}$ Vgl. Tabelle A16.

${ }^{339} \mathrm{Vgl}$. Tabelle A25. In absoluten Zahlen ist nach einer kurzzeitigen Trendwende in der ersten Hälfte der 80er Jahre wieder ein kontinuierliches Ansteigen der Werte zu verzeichnen. So liegen die Werte für 1990 für die gesamte OECD ca. 20\% über den Daten von 1970 (Tabellen A4 und A10). Weltweit beträgt dieser Anstieg sogar 47,28\% (siehe: OECD (1993): OECD Environmental Data, a.a.O., S. 31).

${ }^{340} \mathrm{Vgl}$. Tabelle A25. 
Auch für den Indikator „Abfallaufkommen pro Einwohner" sind die drei Kontinentalstaaten unter den Ländern mit den ungünstigsten Zahlenwer$\operatorname{ten}^{341}$. Doch auch Neuseeland und Finnland (mit jeweils ca. $620 \mathrm{~kg} \mathrm{Ab-}$ fallaufkommen pro Einwohner) gehören zu den Staaten mit besonders hohen Indikatorwerten. Bemerkenswert ist der relativ gute Wert der Industriestaaten Deutschland und Frankreich (jeweils ca. $330 \mathrm{~kg}$ ).

Im zeitlichen Ablauf ist eine starke Ausweitung der Müllproduktion in nahezu allen Staaten der OECD zu konstatieren. Von den hochentwickelten Staaten sind lediglich Australien, mit einem - allerdings von einem sehr hohen Niveau ausgehenden - Rückgang zwischen 1975 und 1990 von 19,7 Prozent, die Niederlande $(-3,7 \%)$ und Deutschland $(+1,4 \%)$ als positive Ausnahmen zu nennen ${ }^{342}$.

Die Daten des Indikators „Absatz von Handelsdünger“ ergeben ein sehr heterogenes Bild ${ }^{343}$. Besonders hoch sind die Werte für Irland und Dänemark (1975 bzw. $1231 \mathrm{~kg}$ pro Einwohner). „Schlußlicht“ mit deutlichem Abstand ist Japan $(149 \mathrm{~kg})$, das allerdings wegen seiner relativ geringen landwirtschaftlichen Nutzfläche ${ }^{344}$ einen hohen Düngereinsatz pro Einheit Ackerfläche zu verzeichen hat.

Der OECD-Mittelwert des Düngemittelabsatzes hat sich über die 20 Jahre zwischen 1970 und 1990 nur leicht verändert (von 603,2 $\mathrm{kg}$ auf 615,2 $\mathrm{kg})$. Jedoch haben sich die Zahlen für die einzelnen Staaten sehr unterschiedlich entwickelt. Einen starken Anstieg können wir für die folgenden Staaten feststellen: Kanada $(+107,2 \%)$, Griechenland $(+79,1 \%)$, Portugal $(+89,4 \%)$ und Türkei $(+176,1 \%)^{345}$.

Stark rückläufig war der Düngemitteleinsatz vor allem in Neuseeland ($36,1 \%)$, Deutschland $(-40,8 \%)$ und Schweden $(-38,7 \%)$.

${ }^{341}$ Vgl. Tabelle A17.

${ }^{342}$ Vgl. Tabelle A26; Berechnungen auf Grundlage von Tabelle A17.

${ }^{343}$ Vgl. Tabelle A18.

${ }^{344}$ Vgl. Tabellen A2 und A14.

${ }^{345}$ Vgl. Tabelle A27; Berechnungen auf Grundlage von Tabelle A18. 
Für den zusammenfassenden Schadstoffindex ergibt sich das folgende Bild (Abbildung 21): ${ }^{346}$ Die USA erreichen mit 166 Punkten den ungünstigsten Wert. Im Gegensatz zum Ressourcen-Index gehört Irland (157) zu den Staaten mit den höchsten Werten. Australien (139), Kanada (146) und Finnland (135) sind wie schon beim Ressourcenverbrauch auch im Hinblick auf Schadstoffemissionen eher Fälle ökologischen Mißerfolgs. Unter den hochentwickelten Industrieländern gibt es zwei Staaten, die für beide Indikatoren günstige Werte aufweisen: Österreich (55 bzw. 74) und die Schweiz (52 bzw. 73). Bemerkenswert ist auch der gute Wert des Industriegiganten Japan (72), der wie schon beim Ressourceneinsatz auch im Hinblick auf die Begrenzung des im Rahmen des wirtschaftlichen Transformationsprozesses erzeugten Schadstoff-Outputs relativ positiv abschneidet.

Die zeitliche Entwicklung des Schadstoffindex (siehe für einige ausgesuchte Staaten Abbildung 22$)^{347}$ verläuft äußerst heterogen: Während die USA ihr 70er-Jahre-Niveau ungefähr gehalten haben, lassen sich ansonsten 3 weitere Ländergruppen unterscheiden:

- Zunächst sind solche Industrieländer aufzuführen, welche ihren Schadstoffaussto $ß$ in den letzten 20 Jahren kontinuierlich erhöht haben. Dazu zählen außer Japan (+20 Index-Punkte) und der Schweiz (+24) u.a. auch Staaten wie Kanada (+37), Norwegen $(+16)$ und Italien $(+22)$.

- Die zweite Staatengruppe besteht aus hochentwickelten Staaten, welche ihre ökonomische Entwicklung und die Emission von Schadstoffen entkoppeln konnten. Dies sind z.B. Deutschland (-15), Frankreich (-11) und vor allem Schweden (-24).

- Die dritte Gruppe bilden die ökonomisch nachholenden Staaten der südlichen Peripherie Europas, bei welchen sich ökonomischer Fortschritt und Schadstoffemissionen parallel entwickeln: Griechenland (+44), Portugal (+31), Spanien (+29) und die Türkei (+40).

\footnotetext{
${ }^{346}$ Vgl. auch Tabelle A20.

${ }^{347}$ Vgl. auch Tabelle A29.
} 


\subsubsection{Gesamtbelastung}

Die Aggregation des Ressourcenbelastungs- und des Schadstoffindex zu einem einzigen Umweltbelastungsindex ergibt für das Jahr 1990 das in Abbildung 23 dargestellte Bild ${ }^{348}$.

Eine Dreier-Gruppe aus den beiden nordamerikanischen Staaten und Australien erreicht die höchsten Werte.

Es folgen vier landwirtschaftlich geprägte Staaten, deren hohe Indexwerte zwischen 100 und 124 Punkten zumindest teilweise der überdurchschnittlichen Größe der landwirtschaftlichen Nutzfläche bzw. der Menge des eingesetzten Handelsdüngers entspringen.

Werte zwischen 77 und 96 Punkten sind dann für eine Gruppe von neun weiteren europäischen Staaten - darunter auch Deutschland - zu konstatieren.

Die sechs ökologischen „Erfolgsfälle“ mit einem Index-Score von weniger als 70 Punkten bestehen zum einen aus vier hochentwickelten Industrie- und Dienstleistungsstaaten (Großbritannien, Japan, Österreich und die Schweiz) und zum anderen aus den beiden ökonomisch weniger leistungsfähigen Ländern Portugal und Türkei.

Im zeitlichen Ablauf hat sich der durchschnittliche Umweltbelastungsindex der OECD-Staaten nach einem starken Anstieg zwischen 1970 und 1980 seit 10 Jahren stabilisiert ${ }^{349}$.

An den sechs ausgewählten Ländern der Abbildung 24 lassen sich die folgenden Entwicklungen ablesen:

- Die USA haben ihren Index-Wert zwar um 10 Punkte reduziert, dies ist jedoch unter Berücksichtigung des hohen Ausgangswertes keine bedeutende Veränderung.

- Der japanische Score von 1990 liegt 11 Punkte über dem Wert von 1970. Diese Entwicklung ist trotzdem eher positiv zu bewerten, da zum einen dieser moderate Anstieg einher ging mit einem rasanten Wachstum des Bruttoinlandsproduktes ${ }^{350}$ und zum anderen der aktuelle Wert

${ }^{348}$ Vgl. auch Tabelle A21.

${ }^{349}$ Vgl. Tabelle A30.

${ }^{350}$ Von 779 Mrd. US-\$ (in Preisen und Kaufkraftparitäten von 1985) im Jahre 1970 auf 1817 Mrd. US-\$ in 1990. 
von 69 noch immer den 17. Rangplatz unter allen OECD-Staaten bedeutet $^{351}$.

- Deutschland und Frankreich konnten ihren Index-Wert ungefähr auf dem Niveau der 70er Jahre stabilisieren (+1 bzw. +3 Punkte). Bemerkenswert ist dabei auch, daß Deutschland in der Rangreihe aller OECDStaaten dadurch vom zwölften auf den vierzehnten Platz rückte.

- Eine durchweg positive Entwicklung können wir für Schweden attestieren, denn dem skandinavischen Land gelang es, den Indexwert von 99 auf 81 Punkte zu reduzieren und sich in der OECD-Rangreihe vom siebten auf den 14. Platz zu verändern.

- Die Schweiz erhöhte ihren Score zwar um 16 Punkte, liegt mit einem aktuellen Wert von 59 jedoch an letzter Position der OECD-Rangreihe.

8.2 Umweltbelastungsindikatoren (Basis: BIP)

\subsubsection{Ressourcenverbrauch}

Beziehen wir die Werte der verschiedenen Umweltbelastungsindikatoren statt auf die Einwohnerzahl auf die ökonomische Leistungsfähigkeit - gemessen durch das Bruttoinlandsprodukt -, dann ergeben sich ganz andere Rangreihen der OECD-Staaten.

Für den Wasserverbrauch nimmt nunmehr die Türkei mit einem Wert von 193 Kubikmetern pro 1000 US-Dollar Bruttoinlandsprodukt den Platz als ökologisch ineffizientester Staat ein. Auch die anderen weniger stark entwickelten Staaten Südeuropas finden sich in einer ähnlichen Position. Als erstaunliches Resultat des in Tabelle A31 des Anhangs dargestellten Vergleiches muß jedoch festgehalten werden, daß die beiden Staaten Nordamerikas sowie Australien auch für den Fall, daß wir die Wirtschaftsleistung als Basis heranziehen, zu den Staaten mit eher schlechten Indikatorwerten zählen.

Ein relativ geringer Wasserverbrauch ist feststellbar für die skandinavischen Staaten, die Alpenländer sowie die Inselstaaten Großbritannien und Irland.

Ein Blick auf die zeitliche Entwicklung des Wasserverbrauches pro Einheit BIP in der gesamten OECD zeigt einen kontinuierlichen Rückgang über den kompletten Beobachtungszeitraum (Mittelwert 1970: 85,9 $\mathrm{m}^{3} ; 1990: 63,9 \mathrm{~m}^{3}$ ). Le-

${ }^{351} \mathrm{Da}$ der Index Belastungen mißt, bedeutet ein hinterer Rangplatz einen relativen umweltpolitischen Erfolg. 
diglich Neuseeland und Portugal weisen für 1990 deutlich höhere Werte auf als für 1970, und in den fün Jahren zwischen 1985 und 1990 ist mit Ausnahme von Portugal und der Türkei für alle Staaten ein Rückgang des Wasserverbrauches pro Einheit BIP zu verzeichnen ${ }^{352}$.

Im Hinblick auf den Indikator der landwirtschaftlichen Nutzfläche pro Einheit BIP gelten ähnliche Feststellungen wie für den Wasserverbrauch:

- Die ungünstigsten Werte erreichen Nordamerika und Australien sowie die weniger entwickelten Staaten Südeuropas;

- Entgegen den absoluten Werten ${ }^{353}$ sind die Zahlen pro Einheit BIP in der gesamten OECD wegen der insgesamt positiven ökonomischen Entwicklung stetig gefallen ${ }^{354}$;

- Für alle OECD-Staaten und den gesamten Beobachtungszeitraum gilt somit die Feststellung, daß die moderaten Ausweitungen der landwirtschaftichen Nutzflächen immer geringer ausfielen als das ökonomische Wachstum ${ }^{355}$.

Der dritte Ressourcenverbrauchsindikator, der Energieverbrauch pro Wirtschaftsleistung (gemessen in Tonnen Erdöl-Äquivalant pro 1000 US-\$ BIP), weist für 1990 Kanada mit 4,98 TOE als Staat mit dem ungünstigsten Wert aus $^{356}$. Ebenfalls bemerkenswert sind die hohen Werte der sonst eher positiv vermerkten Staaten Schweden (3,97 TOE) und Norwegen (3,79 TOE). Die geringsten Werte sind für die Schweiz (2,1 TOE) und Italien (2,01 TOE) zu verzeichnen, doch auch die günstigen Werte Spaniens (2,31 TOE) und Portugals (2,5 TOE), die beide deutlich unter dem OECD-Mittelwert (3,20 TOE) liegen, sind aufgrund der eigentlich zu vermuteten Abhängigkeit der Effizienz der Energienutzung vom ökonomischen Entwicklungsstand hervorzuheben.

Wie die beiden anderen Indikatoren so ist auch der Energieverbrauch pro Einheit BIP während des Beobachtungszeitraumes stark gesunken (OECDMittelwert 1970: 3,73 TOE; 1990: 3,20 TOE) ${ }^{357}$. Mit Ausnahme der vier südeu-

${ }^{352}$ Vgl. Tabelle A40.

${ }^{353}$ Vgl. Tabelle A2.

${ }^{354}$ Vgl. Tabelle A41.

355 Einzige Ausnahme: Neuseeland im Zeitraum 1975-80, in welchem der Indikatorwert um 10,83 Prozent anstieg (vgl. Tabelle A41).

${ }^{356}$ Vgl. Tabelle A33. 
ropäischen Staaten, Neuseelands und der Schweiz weisen alle Staaten für 1990 niedrigere Werte auf als für 1970.

Die für die drei Einzelindikatoren festgestellte Tendenz des beinahe stetigen Rückganges spiegelt sich somit auch im zusammenfassenden Ressourcenverbrauchsindex wider (Abbildung 25) ${ }^{358}$ :

- Die höchsten Werte sind für die Staaten der südlichen Peripherie Europas sowie die Flächen- bzw. Kontinentalstaaten Australien, Kanada, USA und Finnland zu notieren).

- Die günstigsten Werte (unter 55 Punkten) weisen die Alpenländer (inklusive Deutschland), Dänemark, Großbritannien und Japan auf.

- Bis auf Neuseeland (+8 Punkte) und Portugal (+8) ist der Indexwert in den 20 datenmäßig erfaßten Jahren für alle OECD-Staaten gesunken ${ }^{359}$.

- Während die Werte der meisten in Abbildung 26 dargestellten ausgewählten Staaten stetig um moderate Werte von durchschnittlich etwa 5 Prozent pro Fünf-Jahres-Zeitraum gesunken bzw. im Fall der Schweiz ungefähr konstant geblieben sind, stellt die Entwicklung des japanischen Indexwertes eine Ausnahme dar. Der Rückgang des Indikatorswertes beträgt ca. 3 Prozent pro Jahr, und in der OECD-Rangreihe veränderte sich der Rang des asiatischen Staates vom 15. auf den 19. Platz.

\subsubsection{Schadstoffemissionen}

In Tabelle A34 des Anhangs ist das Ausmaß der durch Energieverbrauch induzierten Kohlendioxid-Emissionen pro Einheit BIP dargestellt. Außer den drei englischsprachigen Kontinentalstaaten liegen auch die Werte für Griechenland $(1,29 \mathrm{t}$ pro 1000 US-\$) und Irland $(1,08 \mathrm{t})$ über der 1-t-Marke. Besonders positive Werte sind dagegen für Frankreich $(0,47 \mathrm{t})$, Schweden $(0,47 \mathrm{t})$ und die Schweiz $(0,37 \mathrm{t}) \mathrm{zu}$ verzeichnen.

In Hinblick auf die zeitliche Entwicklung dieses Indikatorswertes ist die gleiche Tendenz wie bei den Ressourcenverbrauchsindikatoren feststellbar, nämlich ein stetiger Rückgang des OECD-Mittelwerts von 1,07 t im Jahre 1970 bis 0,77 t für 1990. Im 20-Jahres-Vergleich erhöhten sich die Werte lediglich in Neuseeland, Griechenland, Portugal, Spanien und der Türkei, und im 5-Jahres-

\footnotetext{
${ }^{357}$ Vgl. Tabelle A42.

${ }^{358} \mathrm{Vgl}$. Tabelle A37.

${ }^{359} \mathrm{Vgl}$. Tabelle A46.
} 
Vergleich (85-90) in Australien, Neuseeland, Griechenland, Portugal und der Türkei $^{360}$.

Auch die Müllproduktion pro Einheit BIP ist im Gegensatz zum absoluten Wert kontinuierlich rückläufig ${ }^{361}$.

Den positivsten Wert im Jahre 1990 weist Deutschland mit knapp $22 \mathrm{~kg}$ pro 1000 US-\$ auf. Die direkten deutschen Nachbarstaaten Frankreich, Österreich, Belgien und die Schweiz folgen mit Werten unter $26 \mathrm{~kg}$. Bemerkenswert hoch ist die Zahl von $123,2 \mathrm{~kg}$ für die Türkei, welche trotz eines mehr als fünfundzwanzig-prozentigen Rückganges zwischen 1985 und 1990 damit den eklatant höchsten Wert innerhalb der gesamten OECD aufweist ${ }^{362}$.

Zwar zeigt die allgemeine Entwicklung dieses Indikators nach unten, doch gab es im Beobachtungszeitraum 85-90 insgesamt neun Staaten mit ansteigenden Werten, darunter die USA $(+4,98 \%)$, Österreich $(+24,85)$, Schweden $(+8,93)$ und die Schweiz $(+4,61)^{363}$.

Den höchsten Einsatz von Handelsdünger pro Einheit BIP gab es 1990 in Irland mit 22,04 kg pro 1000 US-\$ BIP. Ökologische Spitzenwerte sind dagegen für Japan $(1,01 \mathrm{~kg})$, die Schweiz $(1,41 \mathrm{~kg})$ und Deutschland $(1,67 \mathrm{~kg})$ zu notieren $^{364}$.

Im Zeitraum 1985-90 ist der Düngemitteleinsatz pro BIP in allen OECDStaaten gesunken, und im Vergleich zu 1970 weisen 1990 lediglich Kanada, Griechenland, Portugal und die Türkei höhere Werte auf ${ }^{365}$.

Für den zusammenfassenden Schadstoffindex gelten die folgenden Aussagen (Abbildungen 27 und 28) ${ }^{366}$ :

${ }^{360}$ Vgl. Tabelle A43.

${ }^{361} \mathrm{Vgl}$. Tabellen A5 sowie A35.

${ }^{362} \mathrm{Vgl}$. Tabelle A44.

${ }^{363}$ Ebenda.

${ }^{364} \mathrm{Vgl}$. Tabelle A36.

${ }^{365} \mathrm{Vgl}$. Tabelle A45.

${ }^{366}$ Vgl. auch Tabelle A38. 
- Der mit deutlichem Abstand geringste Wert für das Jahr 1990 ist derjenige der Schweiz (39 Punkte).

- Es folgt eine Gruppe von fünf hochentwickelten Staaten - darunter Deutschland und Japan mit Werten zwischen 47 und 51 Punkten.

- Unter den neun Staaten mit den höchsten Punktewerten (>87 Punkte) befinden sich fünf angelsächsisch geprägte Länder (einzige Ausnahme: Großbritannien).

- Die mit Abstand höchsten Scores der OECD-Rangreihe sind für die Türkei (179) und Irland (165) zu verzeichnen.

- 18 von 22 OECD-Staaten weisen für 1990 niedrigere Werte als im Jahre $1970 \mathrm{auf}^{367}$.

- Im Fünf-Jahres-Zeitraum 1985-90 sind mit Ausnahme von Griechenland in allen untersuchten Staaten die Werte des Schadstoffindexes gefallen ${ }^{368}$.

- Auffallend ist die Verbesserung Deutschlands von 97 (1970) auf 51 (1990) Punkte bzw. vom 15. auf den 17. Platz der OECD-Rangreihe, eine positive Entwicklung die jedoch noch von Schweden übertroffen wird (107/49 Punkte bzw. 10./20. Rang) $)^{369}$.

\subsubsection{Gesamtbelastung}

Für den Umweltbelastungsindex gelten ähnliche Beobachtungen wie für den Schadstoffindex ${ }^{370}$. Die ungünstigsten Werte erreichen zum einen die ökonomisch nachholenden Staaten der südeuropäischen Peripherie und zum anderen die angelsächsisch geprägten Staaten mit Ausnahme von Großbritannien. Die skandinavischen und Benelux-Länder liegen im Mittelfeld, während die Alpenländer sowie die vier „klassischen“ Industrienationen Japan, Großbritannien, Deutschland und Italien die niedrigsten Score-Werte erzielen (Abbildung 29).

Im zeitlichen Ablauf können wir einen allgemeinen Rückgang konstatieren ${ }^{371}$. Die in Abbildung 30 erfaßten sechs ausgewählten Staaten weisen die folgenden Charakteristika ihrer Zeitreihen auf:

\footnotetext{
${ }^{367} \mathrm{Vgl}$. ebenda.

${ }^{368}$ Vgl. Tabelle A47.

${ }^{369} \mathrm{Vgl}$. Tabelle A37.

${ }^{370} \mathrm{Vgl}$. Tabelle A39.

${ }^{371}$ Vgl. Tabelle A48.
} 
- Die USA können zwar auf einen stetigen Rückgang ihres Index-Wertes verweisen, ihr aktueller Wert von 99 Punkten ist jedoch noch immer der sechstschlechteste aller OECD-Staaten.

- Japan, Deutschland, Frankreich und Schweden verringerten die von ihnen induzierte Umweltbelastung ebenfalls kontinuierlich, und insbesondere die Beinahehalbierung (von 98 auf 56 Punkte) des schwedischen sowie der deutliche, mit einem sehr starken Wirtschaftswachstum einhergehende Rückgang des japanischen Wertes (von 79 auf 47 Punkte) sind hervorzuheben.

- Die Schweiz ist zwar einer von drei Staaten, deren 90er Wert über den Zahlen von 1970 liegt, dennoch ist die von ihr verursachte Umweltbelastung pro Einheit BIP mit Abstand die niedrigste aller OECD-Staaten.

Ein Vergleich dieser Resultate mit den Index-Werten bezogen auf die Einwohnerzahl läßt die folgenden Schlußfolgerungen zu:

- Sowohl mit der Einwohnerzahl als auch mit dem Bruttoinlandsprodukt als Basis ergeben sich für die angelsächsisch geprägten Staaten (Ausnahme: Großbritannien) die ungünstigsten Werte. Dies gilt insbesondere für die Staaten Nordamerikas und Australien.

- Während die Staaten des südlichen Randes Europas für die Indexwerte bezogen auf die Einwohnerzahl relativ gut abschneiden, sind für die Umweltbelastung-BIP-Relationen deutlich negativere Werte zu attestieren, da die ökologische Effizienz des Wirtschaftskreislaufes anscheinend mit der Wirtschaftsleistung zunimmt.

- Die Benelux-Länder, die skandinavischen Staaten mit Ausnahme Schwedens sowie Frankreich liegen für beide Indizes im mittleren Wertebereich.

- Die Alpenländer, Schweden sowie vier klassische Industrieländer (Japan, Deutschland, Großbritannien und Italien) erreichen jeweils relativ gute Werte.

Während die Umweltbelastung pro Einwohner OECD-weit im günstigsten Fall zu stagnieren scheint, nimmt die Umweltlast pro Einheit BIP stetig ab, d.h. es scheint den hochindustrialisierten Ländern in Westeuropa, Nordamerika und Japan weitgehend gelungen zu sein, das Wirtschaftswachstum und den Anstieg der Umweltbelastung zu entkoppeln. 
Abb. 19: Ressourcenverbrauchsindex pro Einwohner 1990

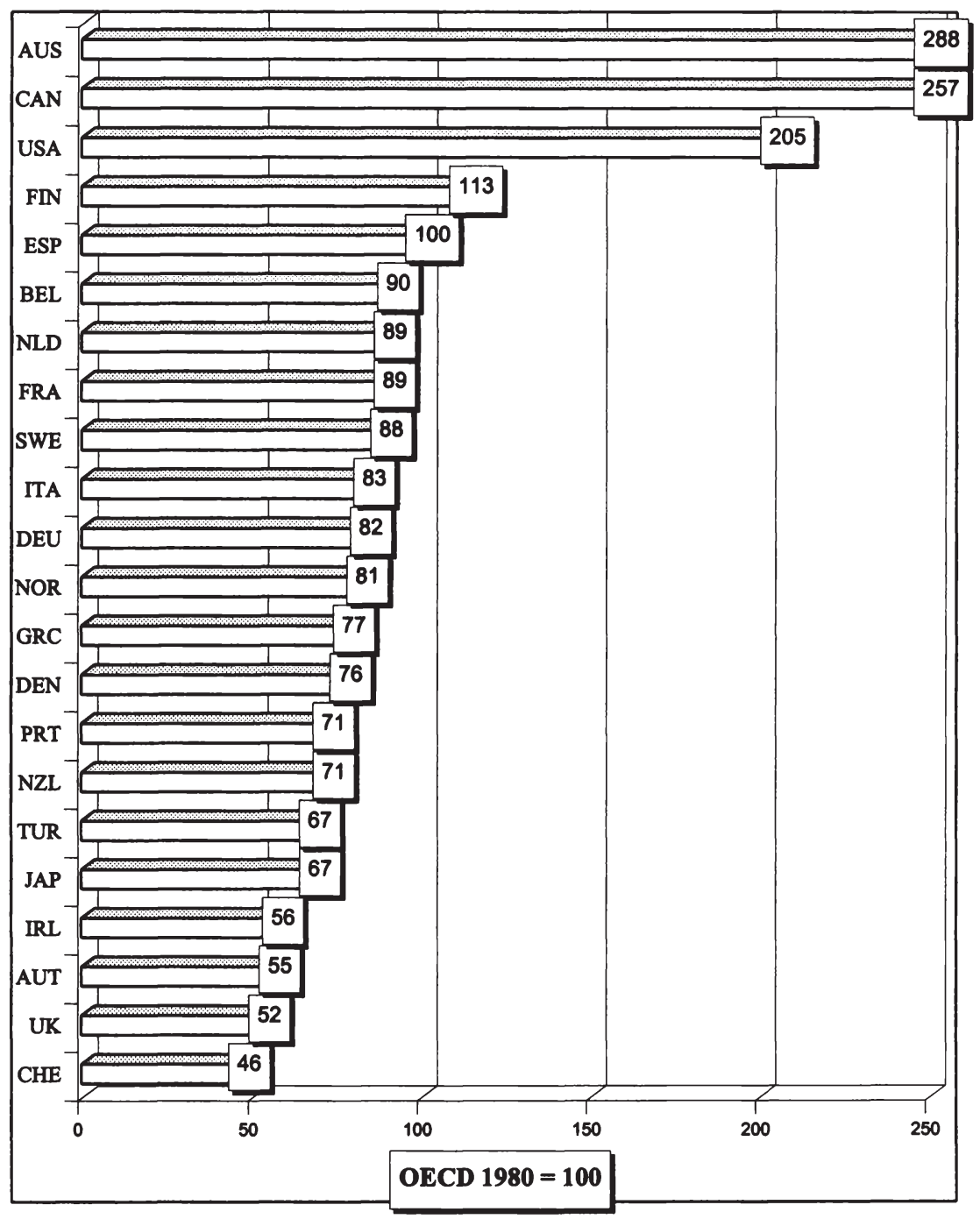


Abb. 20: Ressourcenverbrauchsindex pro Einwohner ausgewählter Staaten 1970 bis 1990

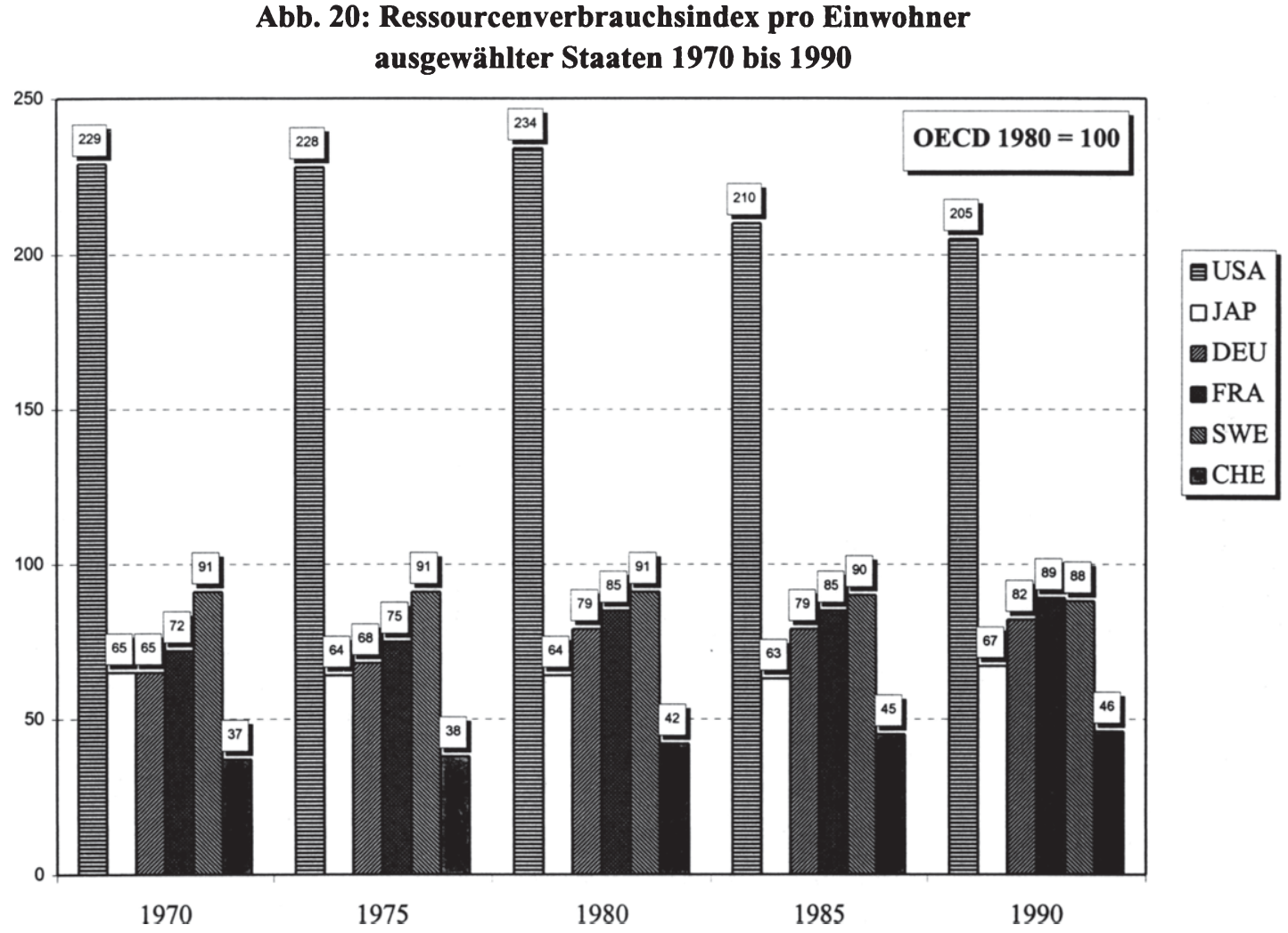




\section{Abb. 21: Schadstoffindex pro Einwohner 1990}

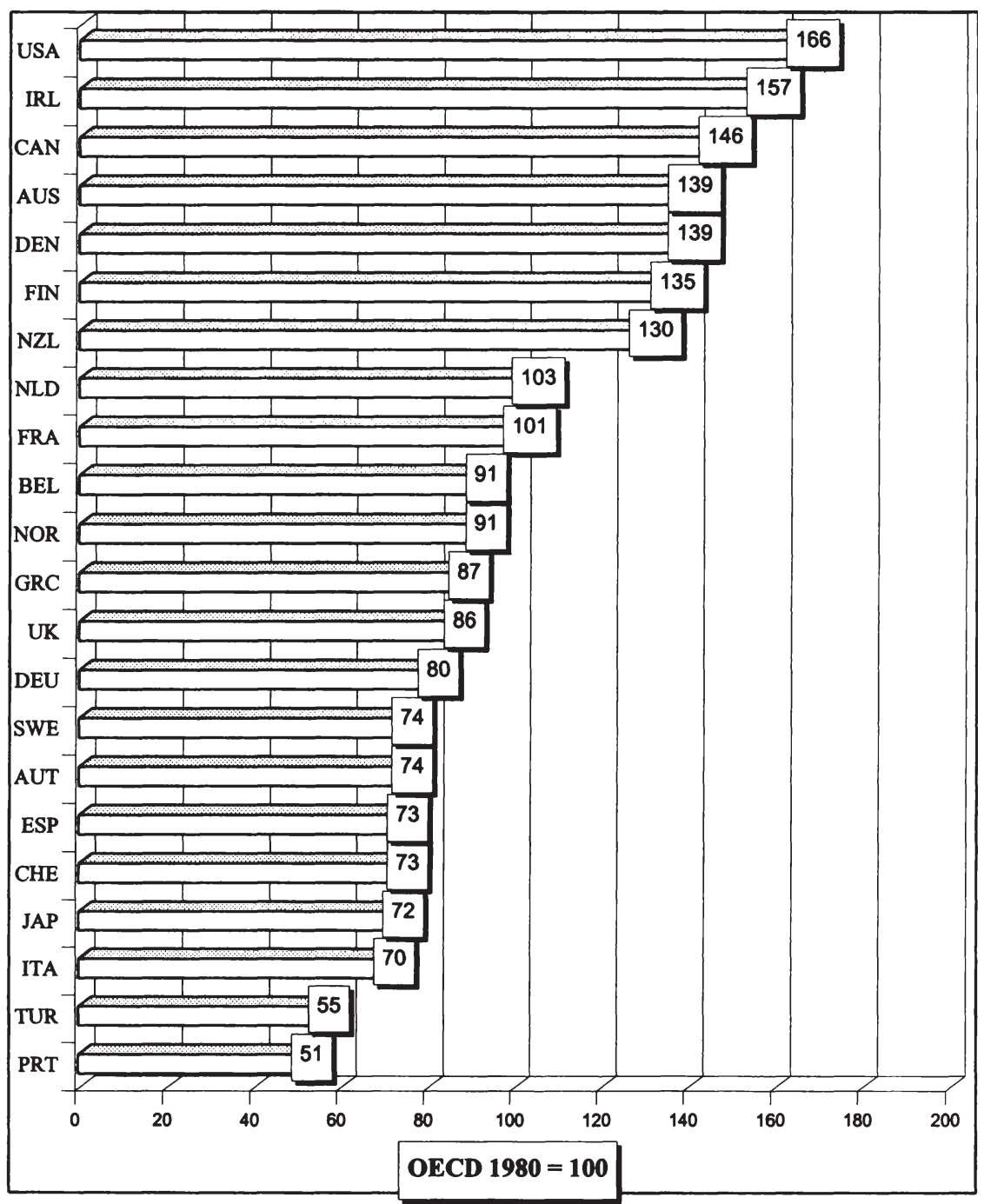


क

Abb. 22: Schadstoffindex pro Einwohner ausgewählter Staaten 1970 bis 1990

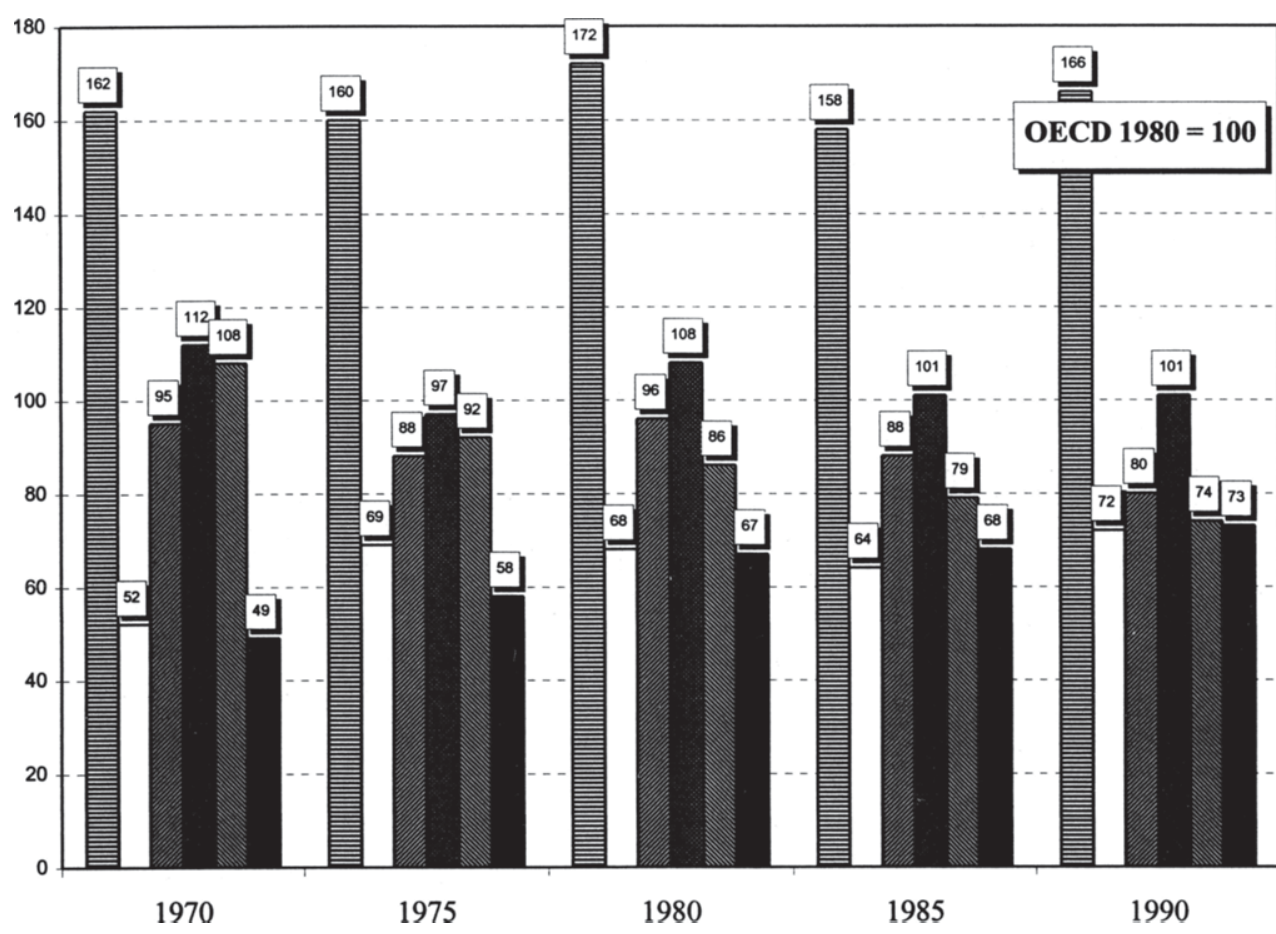

目USA 口JAP DEU -FRA $\triangle \mathrm{SWE}$ -CHE 


\section{Abb. 23: Umweltbelastungsindex pro Einwohner 1990}

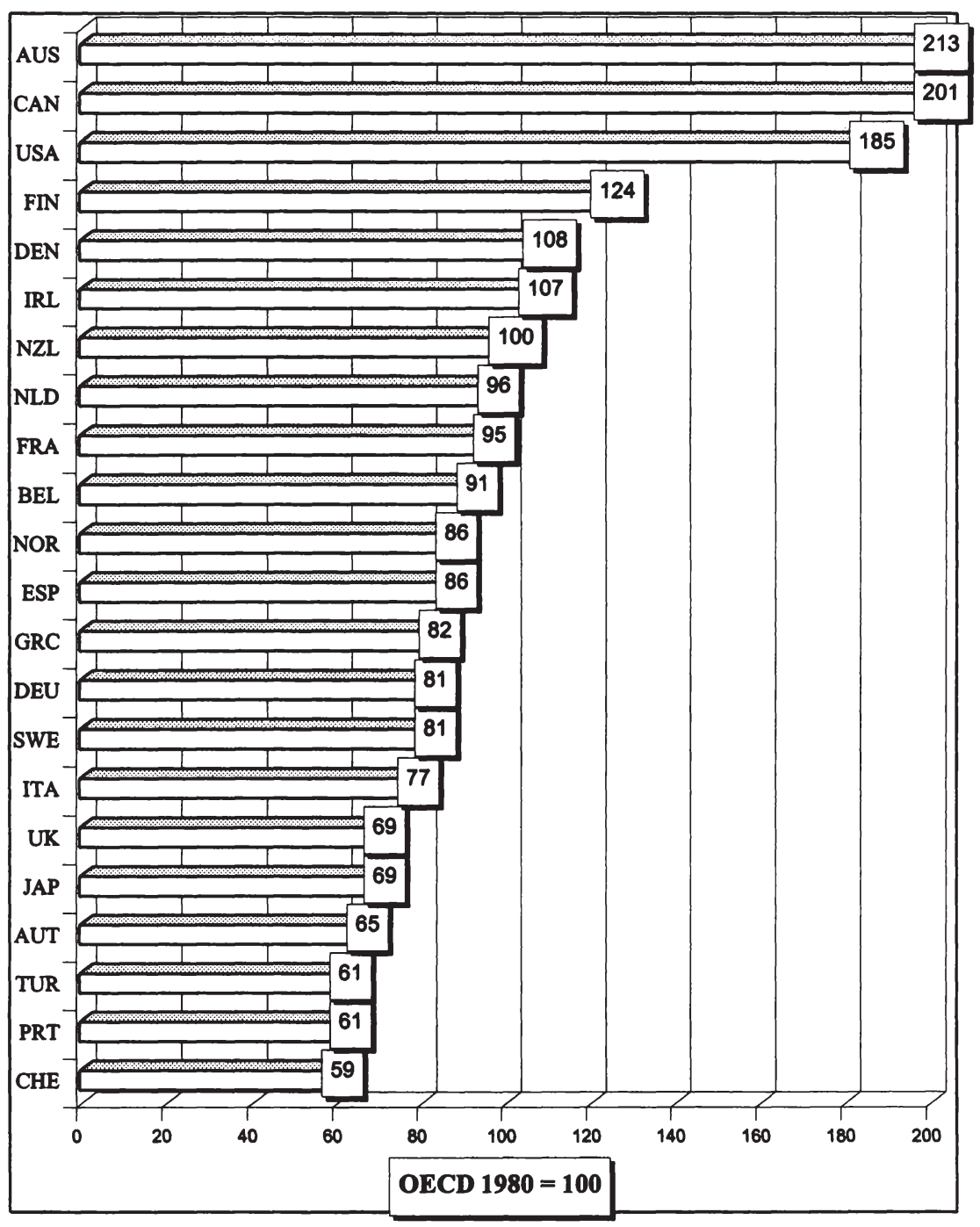


Abb. 24: Umweltbelastungsindex pro Einwohner

ausgewählter Staaten 1970 bis 1990
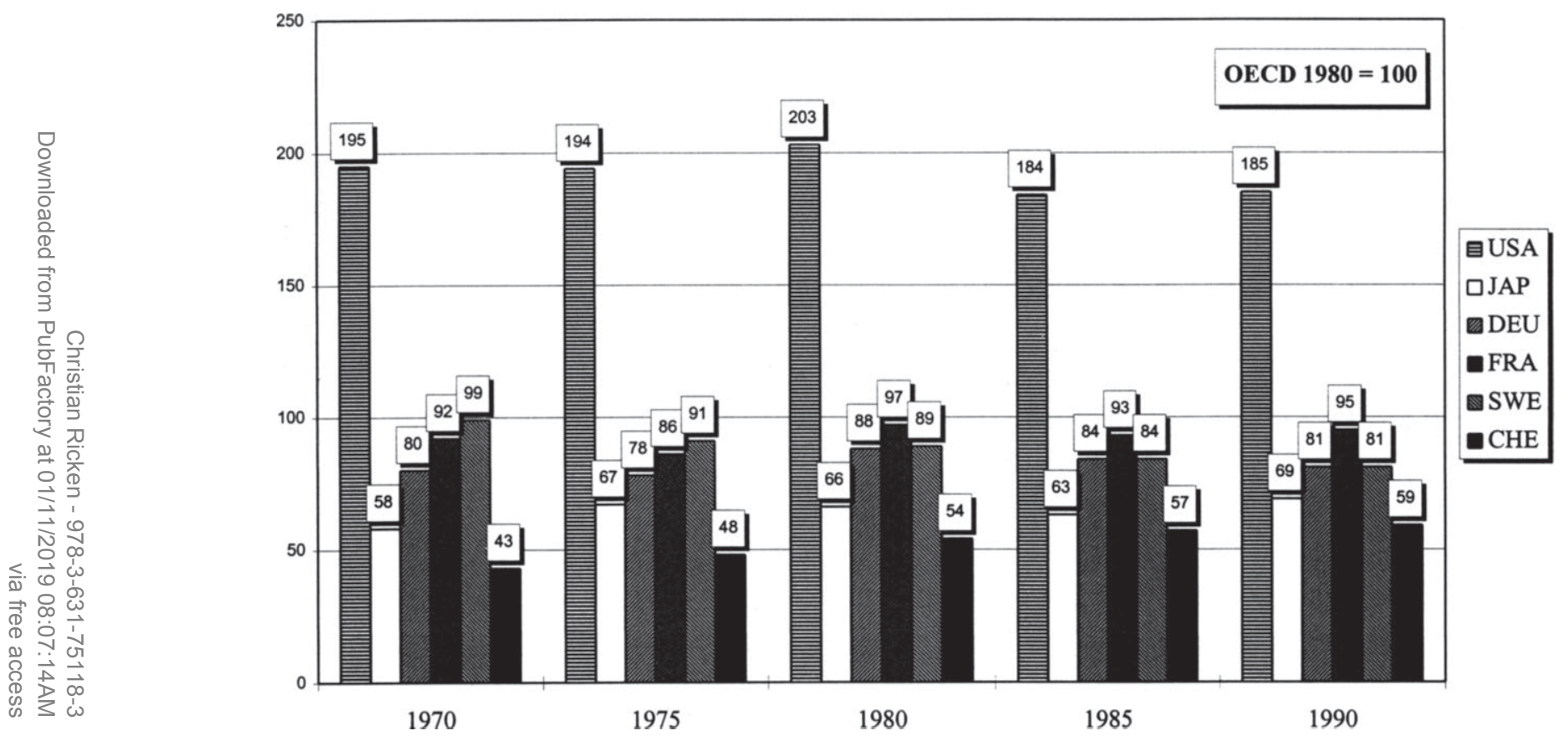
Abb. 25: Ressourcenverbrauchsindex pro BIP 1990

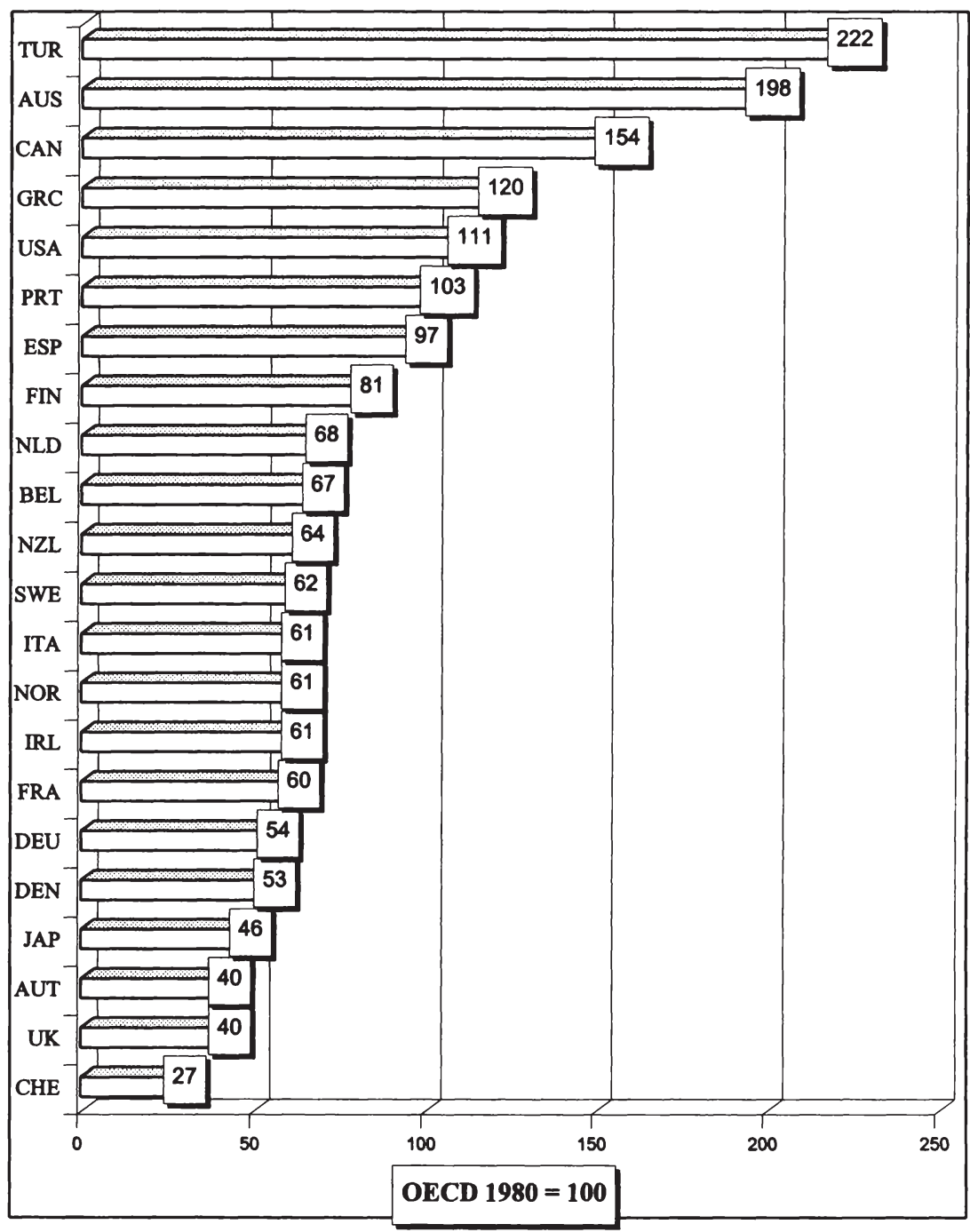


Abb. 26: Ressourcenverbrauchsindex pro Einheit BIP ausgewählter Staaten 1970 bis 1990
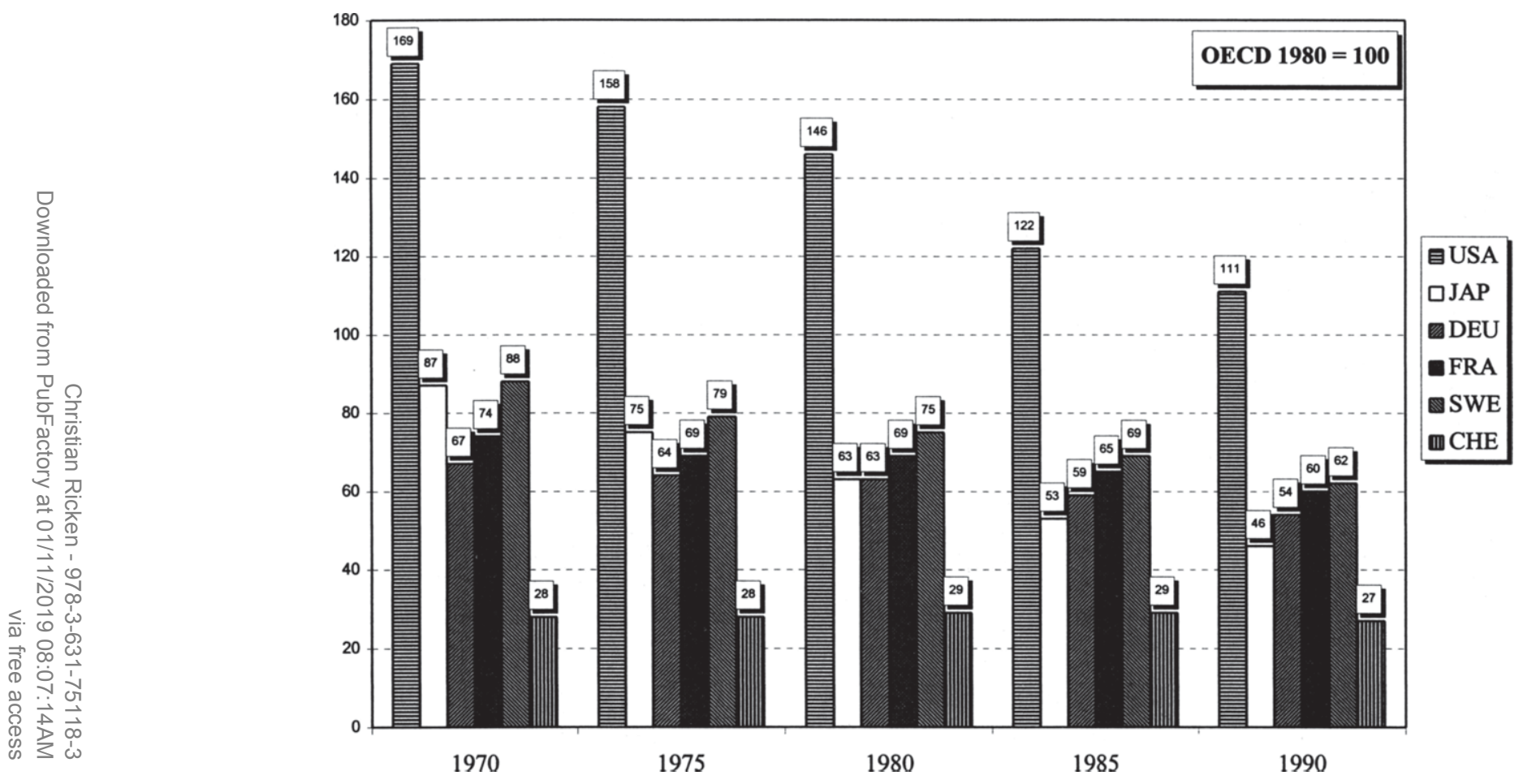


\section{Abb. 27: Schadstoffindex pro Einheit BIP 1990}

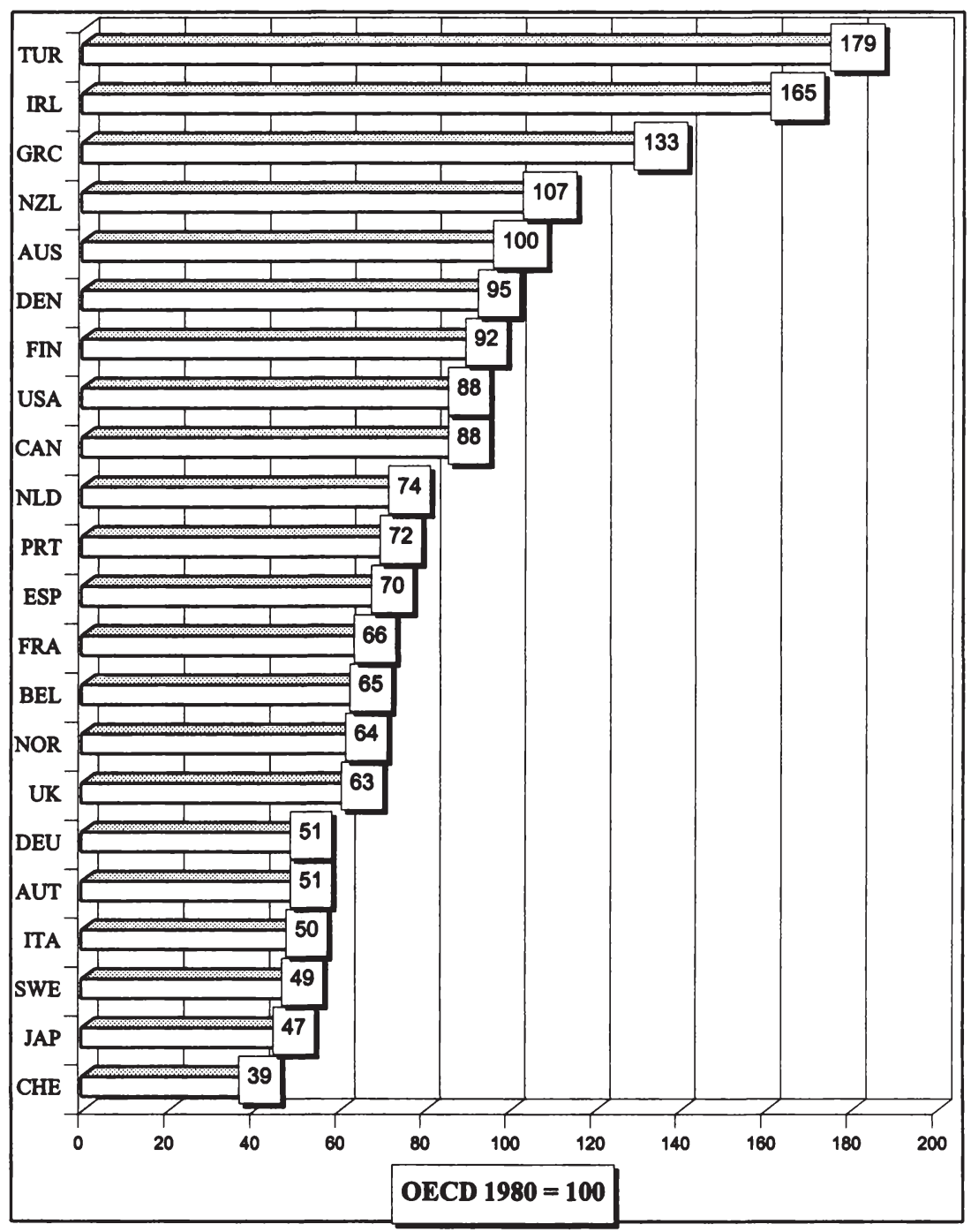


깅

Abb. 28: Schadstoffindex pro Einheit BIP ausgewählter Staaten 1970 bis 1990

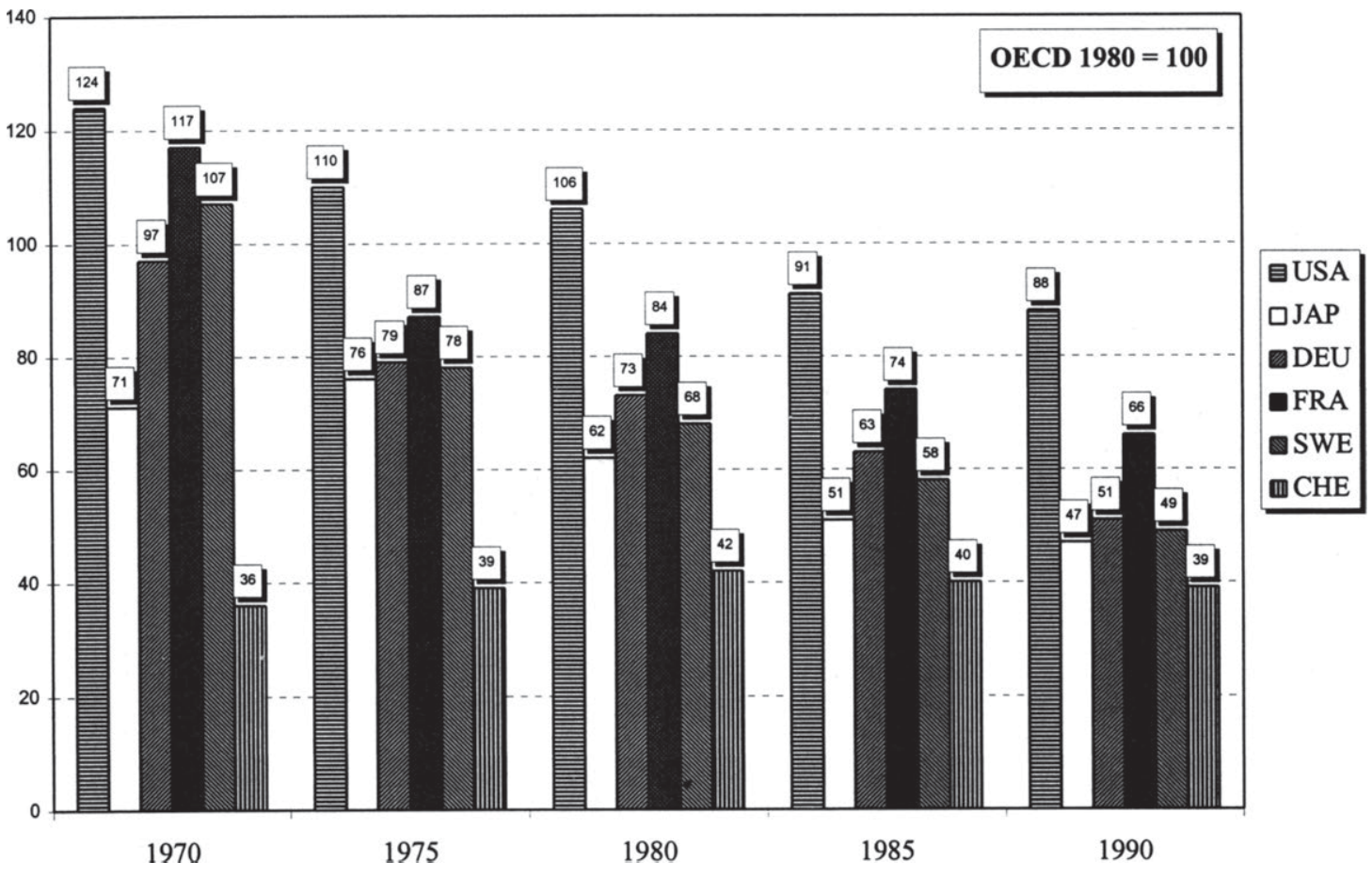




\section{Abb. 29: Umweltbelastungsindex pro Einheit BIP 1990}

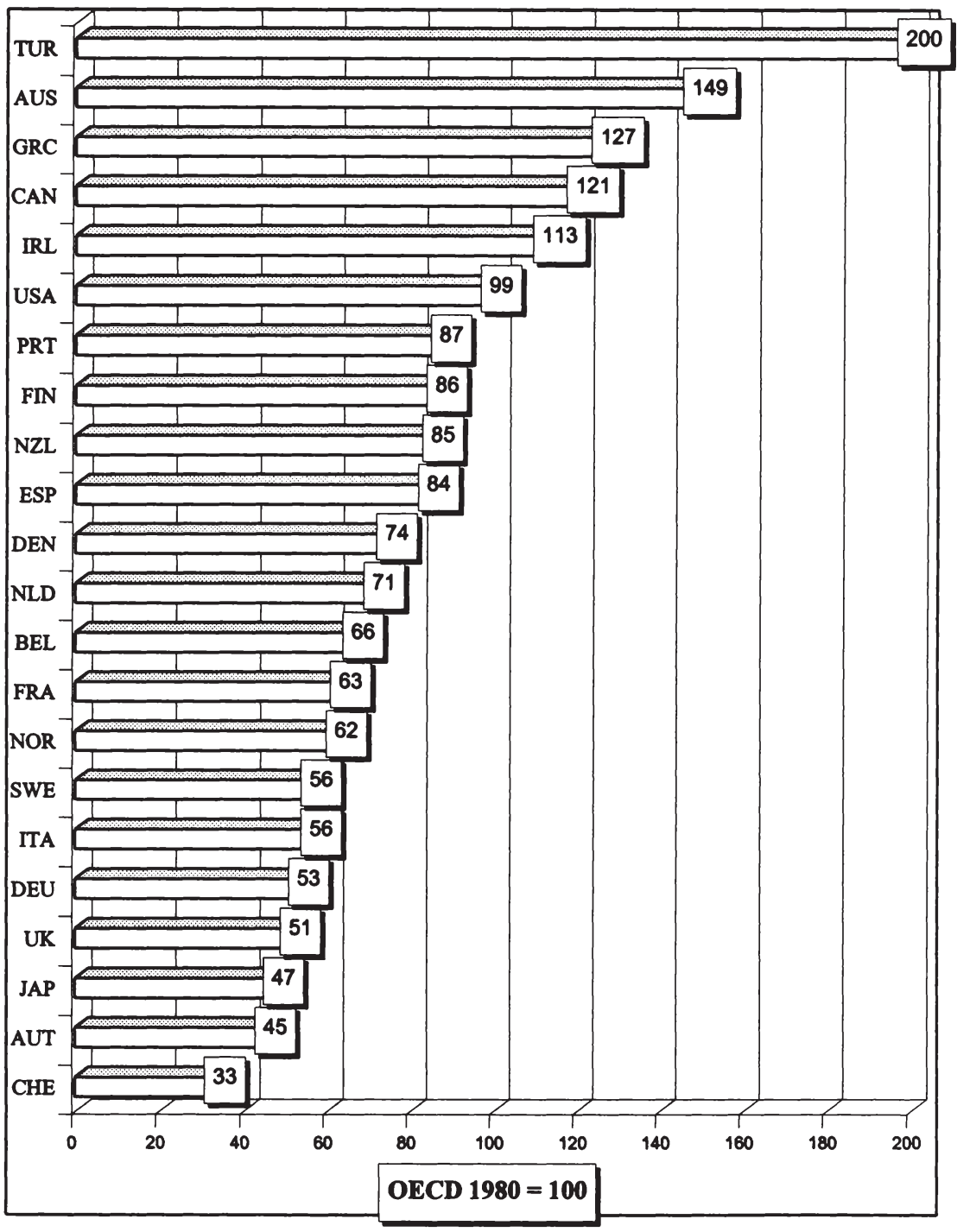


ล

Abb. 30: Umweltbelastungsindex pro Einheit BIP

ausgewählter Staaten 1970 bis 1990

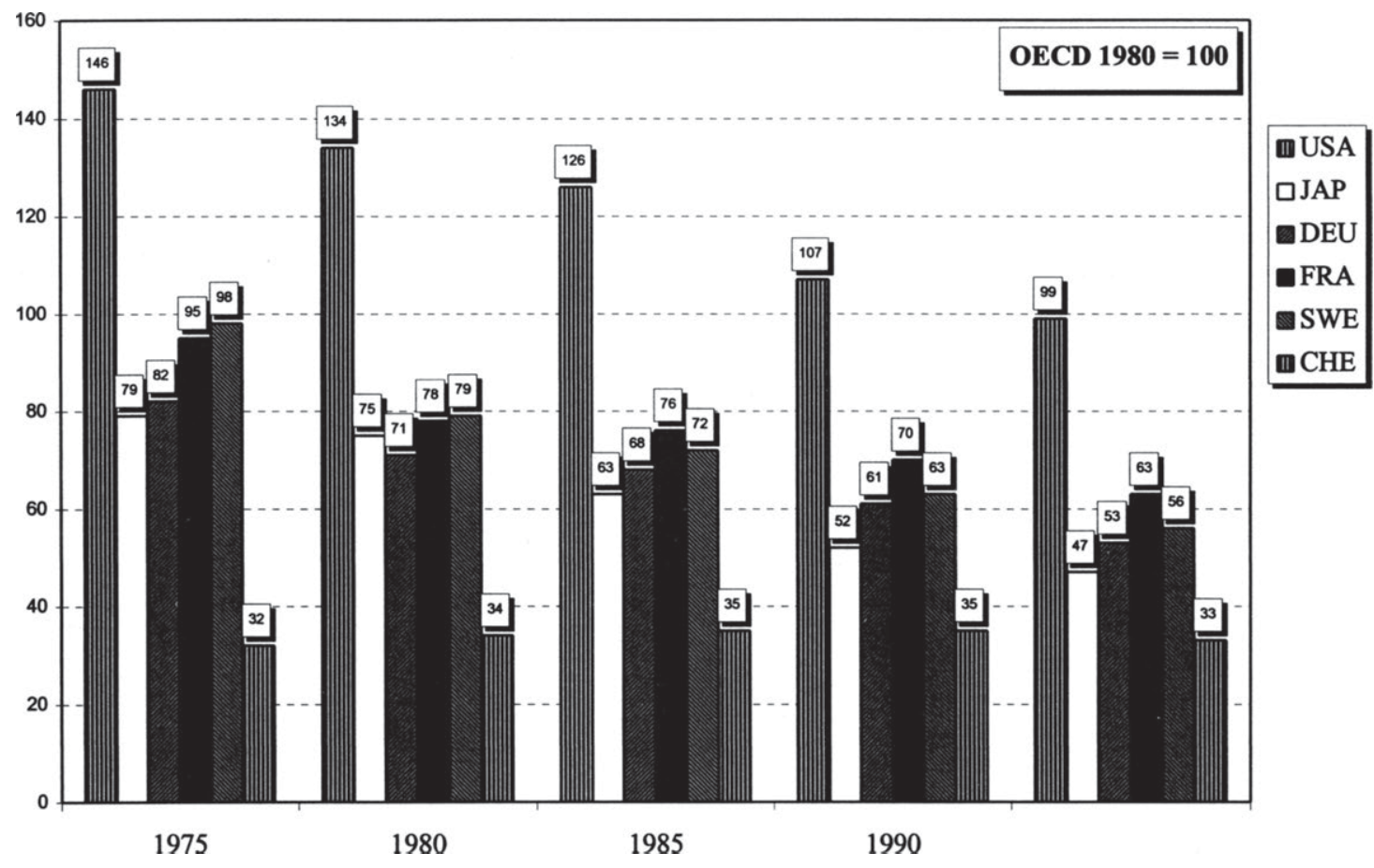




\section{Determinanten der Effektivităt nationaler Umweltpolitik}

Bevor in den Kapiteln 10 und 11 die Dimensionen des nationalen Politikstils operationalisiert und gemäß der Grundthese als Determinanten der Umweltbelastungsindikatoren getestet werden, sollen in diesem Kapitel einige wichtige ökonomische und politische Faktoren im Hinblick auf ihr Erklärungspotential als Bestimmungsgrößen des Ausmaßes von Ressourcenverbrauch und Schadstoffemissionen betrachtet werden. Sollten sich einzelne Faktoren in diesem Zusammenhang als besonders relevant erweisen, sind diese in den folgenden Analysen statistisch zu kontrollieren um Fehlinterpretationen von aufgrund von Kollinearitäten der unabhängigen Variablen erzeugten Ergebnissen zu vermeiden.

Die nachfolgend betrachteten Faktoren sind im einzelnen:

- Ökonomisch:

- Das Wirtschaftsniveau (BIP pro Einwohner) und dessen Wachstum als Variablen der Performanz und Dynamik von Volkswirtschaften;

- Die sektorale Verteilung der Volkswirtschaft als Indikator einer eher agrarwirtschaftlichen, industriellen oder DienstleistungsAusrichtung;

- Politisch:

- Der elektorale Erfolg grüner Parteien als Variable zur Beschreibung der Wirkung des Parteiensystems auf die umweltpolitische Effektivität von Staaten.

\section{1 Ökonomische Determinanten}

\subsection{1 Ökonomisches Niveau}

Eine Korrelationsanalyse des Bruttoinlandsproduktes pro Einwohner mit den jeweiligen Umweltbelastungsindikatoren des gleichen Zeitraumes ergibt zahlreiche signifikante Zusammenhänge zwischen beiden Variablenkomplexen (Tabelle 1).

Sowohl für die Gruppe der Indikatoren zur Messung des Ressourcenverbrauches als auch für diejenige der Schadstoffindikatoren ist eine positive Beziehung zum Ausmaß des wirtschaftlichen Entwicklungsstandes festzustellen. Von den Einzelindikatoren des Ressourcenverbrauchsindexes 
sind vor allem die signifikanten Korrelationen von Wasser- und Energieverbrauch mit dem Wohlstandsniveau hervorzuheben. Der quadrierte Korrelationskoeffizient als das Maß der erklärten Varianz zwischen ProKopf-Primärenergieverbrauch und -Bruttoinlandsprodukt beträgt z.B. für das Jahr 1985 0.64, d.h. 64 Prozent der Varianz der Energieverbrauchsdaten der untersuchten Staaten lassen sich durch die unterschiedlichen Ausprägungen des Bruttoinlandsproduktes erklären.

Die positiven Korrelationskoeffizienten des Schadstoffemissionsindexes [PRESS/POPUL] sind vor allem auf die Werte des energiebedingten Kohlendioxidausstoßes sowie des Abfallaufkommens zurückzuführen. Diese Zusammenhänge wandeln sich jedoch anscheinend im Laufe der Zeit. Während die Varianz des $\mathrm{CO}_{2}$-Ausstoßes immer weniger vom Wohlstandsniveau abhängt (1970: 50\%; 1990: 35\%), gilt für das $\mathrm{Ab}$ fallaufkommen das Gegenteil (1975: 7\%; 1990: 24\%).

Insgesamt ist festzustellen, daß der Korrelationskoeffizient des Umweltbelastungsindexes pro Einwohner [ENVIR/POPUL] im Verlauf des Untersuchungszeitraumes stetig abgenommen hat und für die Daten des Jahres 1990 erstmals kein signifikanter Zusammenhang mit der Wirtschaftsleistung mehr feststellbar ist. Oder anders formuliert: der relativ geringe, durch das Bruttoinlandsprodukt pro Einwohner erklärte Anteil der Varianz der Umweltbelastungsindexwerte des Jahres 1970, nämlich ca. 21 Prozent, ist im Jahr 1990 auf etwas mehr als 12 Prozent abgesunken. Somit scheint zwar das Ausmaß der Umweltbelastung pro Einwohner positiv mit dem Pro-Kopf-Einkommen zu korrelieren, dieser Zusammenhang ist jedoch relativ schwach, und hat im Verlauf des Untersuchungszeitraumes zudem stetig abgenommen.

Dennoch gibt es ausreichende empirische Evidenz für die These, daß mit einer Zunahme des individuellen Wohlstandsniveaus auch ein Anstieg der auf jeden einzelnen Einwohner umgerechneten Umweltbelastungen einher geht. Für die Indikatoren Primärenergieverbrauch und energiebedingte $\mathrm{CO}_{2}$-Emissionen gilt dieser Zusammenhang von Wohlstand und Umweltstreß im besonderen Maße, da hier die Korrelationskoeffizienten auch für die Werte des Jahres 1990 noch auf dem 0.01\%-Niveau signifikant sind und das Maß der durch das BIP pro Einwohner erklärten Varianz 56,25 bzw. $34,81 \%$ beträgt. 
Tabelle 1: Korrelationsanalyse zwischen den Umweltbelastungsindikatoren pro Einwohner und der Wirtschaftsleistung

BIP/POPUL

\begin{tabular}{llllll}
\cline { 2 - 6 } & 1970 & 1975 & 1980 & 1985 & 1990 \\
\hline H2O/POPUL & $0.38^{*}$ & $0.39^{*}$ & $0.42^{*}$ & 0.36 & 0.25 \\
LAND/POPUL & 0.05 & $0.09^{*}$ & 0.08 & 0.10 & 0.06 \\
ENER/POPUL & $0.74^{* *}$ & $0.77^{* *}$ & $0.77^{* *}$ & $0.80^{* *}$ & $0.75^{* *}$ \\
RESOU/POPUL & 0.31 & 0.35 & $0.37^{*}$ & $0.37^{*}$ & 0.30 \\
\hline CO2/POPUL & $0.71^{* *}$ & $0.72^{* *}$ & $0.71^{* *}$ & $0.69^{* *}$ & $0.59^{* *}$ \\
WASTE/POPUL & $k . A$. & 0.27 & 0.33 & $0.40^{*}$ & $0.49^{*}$ \\
FERT/POPUL & 0.17 & 0.15 & 0.06 & 0.05 & -0.09 \\
PRESS/POPUL & $0.53^{* *}$ & $0.52^{* *}$ & $0.46^{*}$ & $0.43^{*}$ & 0.35 \\
\hline ENVIR/POPUL & $0.46^{*}$ & $0.45^{*}$ & $0.44^{*}$ & $0.43^{*}$ & 0.35 \\
\hline
\end{tabular}

Anm.: Für die Umweltbelastungsindikatoren wurde jeweils mit den Werten des gleichen Jahres gerechnet, in dem auch der Bruttoinlandsproduktwert erhoben wurde. $\mathrm{N}=22 ;{ }^{*}: \mathrm{p}<0.05 ;^{* *}: \mathrm{p}<0.01$

Da das Erklärungspotential der absoluten Höhe des Pro-Kopf-BIP für die Varianz der Umweltbelastungswerte innerhalb der Grundgesamtheit der OECD insgesamt jedoch begrenzt erscheint, soll nachfolgend untersucht werden, ob vom Wachstum des Pro-Kopf-BIP, also von der ökonomischen Expansionsdynamik, stärkere Einflüsse auf das Ausmaß des Umweltstresses ausgehen. Hinter dieser Fragestellung steht also die Vermutung, daß sich ein hohes $\mathrm{Ma} B$ an Wirtschaftswachstum in eher hohen Zahlen der Belastungsindikatoren niederschlägt, da die Fokussierung der ökonomischen und politischen Anstrengungen auf das Ziel der Wohlstandsmehrung die umweltpolitischen Probleme auf die hinteren Ränge der Agenda rückt (These 1). Eine entgegengesetzte These könnte lauten, $\mathrm{da} ß$ erst ein hinreichend hohes BIP-Wachstum die Bildung ausreichender finanzieller und technischer Ressourcen für die ökologisch notwendige Entkopplung von ökonomischem Output und dadurch verursachtem Umweltstreß ermöglicht (These 2). 
Tabelle 2: Korrelationsanalyse zwischen den Umweltbelastungsindikatoren pro Einwohner und dem Wachstum der Wirtschaftsleistung

Durchschnittliche jährliche Wachstumsrate von BIP/POPUL

$$
1970-75 \quad 1975-80 \quad 1980-85 \quad 1985-90
$$

\begin{tabular}{lllll}
\hline H2O/POPUL & 0.01 & -0.03 & -0.12 & -0.15 \\
LAND/POPUL & 0.09 & -0.19 & 0.08 & -0.21 \\
ENER/POPUL & -0.33 & 0.01 & 0.23 & $-0.47^{*}$ \\
RESOU/POPUL & -0.09 & -0.13 & 0.07 & -0.30 \\
\hline CO2/POPUL & $-0.37^{*}$ & 0.00 & 0.13 & -0.32 \\
WASTE/POPUL & -0.03 & $-0.44^{*}$ & $-0.42^{*}$ & $-0.55^{*}$ \\
FERT/POPUL & -0.11 & -0.21 & 0.29 & 0.04 \\
PRESS/POPUL & -0.25 & -0.27 & 0.34 & -0.29 \\
\hline ENVIR/POPUL & -0.10 & -0.19 & 0.18 & -0.32 \\
\hline
\end{tabular}

Anm.i Für die Umweltbelastungsindikatoren wurde jeweils mit den Werten des letzten Jahres des Fünf-Jahres-Zeitraumes gerechnet, in welchem das BIP-Wachstum errechnet wurde.

Lesebeispiel: Für den Umweltbelastungsindex pro Einwohner [ENVIR/POPUL] des Jahres 1975 und dem durchschnittlichen Pro-Kopf-Einkommen-Wachstum des Zeitraumes 1970 bis 1975 [BIP/POPUL1970-75] ergibt sich ein Korrelationskoeffizient von -0.10 .

$\mathrm{N}=22 ;^{*}: \mathrm{p}<0.05 ;^{* *}: \mathrm{p}<0.01$

Tabelle 2 gibt die Zusammenhänge zwischen dem Wachstum des Bruttoinlandsproduktes pro Einwohner sowie den Umweltbelastungsindikatoren wieder. Das Erklärungspotential des Wohlstandswachstums für die Änderungen der ökologischen Indikatoren ist anscheinend sehr gering. Lediglich Energieverbrauch und Müllaufkommen scheinen im Sinne von These 2 mit zunehmendem ökonomischen Wachstumsniveau zu sinken.

1990 erreicht der Korrelationskoeffizient zwischen der unabhängigen und der wichtigsten der abhängigen Variablen, dem Umweltbelastungsindex, zwar mit -0.32 den höchsten Wert, das Maß der erklärten Varianz ist dabei mit lediglich $10 \%$ jedoch nur gering und nicht im statistischen Sinne signifikant. 
Während also das absolute BIP-pro-Einwohner-Niveau insofern als Determinante der Effektivität nationaler Umweltpolitik taugt, als daß zwischen ihm und den Umweltbelastungsindikatoren positive, wenn auch nur teilweise statistisch signifikante Korrelationen meßbar sind, und das BIPWachstum als erklärende Variable für das absolute Niveau der Umweltbelastung anscheinend ausfällt, gibt Tabelle 3 einen Überblick über den $\mathrm{Zu}$ sammenhang zwischen dem jeweiligen Anstieg von Wirtschaftsleistung bzw. Umweltbelastungsindikatoren.

Auch für diesen Zusammenhang sind wieder zwei entgegengesetzte Vermutungen möglich. Zum einen ist eine stark positive Korrelation denkbar, nämlich im Falle eines Gleichklanges von Veränderung des volkswirtschaftlichen Produktionsvolumens und dadurch induzierter Umweltbelastung, oder zum anderen eher negative oder nicht-signifikante Koeffizienten, falls durch ein hohes $\mathrm{Maß}$ an BIP-Wachstum eine Entkopplung der produktionsbedingten Umweltbelastung gelingt.

Bei der Analyse der in Tabelle 3 dargestellten Daten fallen einige interessante Zusammenhänge auf: Während für die Perioden 1970-75 sowie 1985-90 zahlreiche statistisch signifikante, positive Korrelationen zwischen beiden Variablenkomplexen meßbar sind, gilt dies für die $10 \mathrm{Jahre}$ von 1975 bis 1985 nicht.

Für den Zeitraum von 1975 bis 1980 besteht lediglich für die Einzelindikatoren Energieverbrauch und $\mathrm{CO}_{2}$-Emissionen ein signifikant positiver Zusammenhang zwischen dem Wachstum des Indikators sowie dem der Wirtschaftsleistung.

Die Periode 1980-85 weist überhaupt keine nennenswerten Korrelationen zwischen den ökonomischen und ökologischen Variablen auf.

Eine Begründung für dieses Phänomen könnte in der Tatsache liegen, daß das durchschnittliche Wachstum der OECD-Volkswirtschaften im Zeitraum 1975-80 im Vergleich zur vorausgegangenen 5-Jahres-Periode um jährlich knapp ein Prozent niedriger ausfiel und zwischen 1980 und 1985 noch einmal um durchschnittlich ca. ein Prozent jährlich zurückging, während die zweite Hälfte der 80er Jahre wieder eine Periode stärkeren Wachstums war ${ }^{372}$.

372 Vgl. Tabelle A51 sowie die Darstellung bei Cusack, Thomas R. (1994): Economic Problems ..., a.a.O., S. 4 ff. 
Tabelle 3: Korrelationsanalyse zwischen dem Wachstum der Umweltbelastungsindikatoren pro Einwohner und dem Wachstum der Wirtschaftsleistung

\section{Durchschnittliche jährliche Wachstumsrate von BIP/POPUL}
$\Delta 70-75$
$\Delta 75-80$
$\Delta 80-85$
$\Delta 85-90$

\begin{tabular}{lllll}
\hline$\triangle H 2 O / P O P U L$ & 0.15 & -0.01 & 0.03 & 0.24 \\
$\Delta$ LAND/POPUL & -0.05 & -0.22 & -0.01 & 0.29 \\
$\Delta$ ENER/POPUL & $0.65^{* *}$ & $0.56^{* *}$ & 0.13 & $0.45^{*}$ \\
$\Delta$ RESOU/POPUL & $0.38^{*}$ & 0.24 & 0.10 & 0.29 \\
\hline$\triangle$ CO2/POPUL & $0.67^{* *}$ & $0.38^{*}$ & 0.09 & $0.44^{*}$ \\
$\Delta$ WASTE/POPUL & $k . A$. & $-0.48^{*}$ & 0.06 & 0.20 \\
$\Delta$ FERT/POPUL & $0.43^{*}$ & 0.09 & 0.02 & $0.52^{* *}$ \\
$\Delta$ PRESS/POPUL & $0.45^{*}$ & 0.04 & -0.04 & $0.70^{* *}$ \\
\hline$\Delta E N V I R / P O P U L$ & $0.49^{*}$ & 0.19 & 0.01 & $0.51^{* *}$ \\
\hline
\end{tabular}

Anm.i Für die Umweltbelastungsindikatoren wurde jeweils mit den Werten des gleichen Fünf-Jahres-Zeitraumes gerechnet, in welchem auch das BIP-Wachstum errechnet wurde.

Lesebeispiel: Für das Wachstum des Umweltindikators pro Einwohner [ENVIR/POPUL] im Zeitraum 1970 bis 1975 und das BIP-Wachstum des gleichen Zeitraumes ergibt sich ein Korrelationskoeffizient von 0.49 .

$\mathrm{N}=22 ;^{*}: \mathrm{p}<0.05 ;^{* *}: \mathrm{p}<0.01$;

Somit scheint in ökonomischen Wachstumsphasen zu gelten: je höher das Wirtschaftswachstum, desto größer ist auch der Anstieg der Umweltbelastung pro Einwohner. In Zeiten einer Rezession ist dagegen kein Zusammenhang zwischen beiden Variablenkomplexen meßbar.

Dies widerspricht also der Plausibilitätsüberlegung, daß gerade in Zeiten des stetigen Wachstums ein Teil des möglichen ökonomischen Zuwachses für eine Reduktion der Umweltbelastung investiert wird. Im Gegensatz zu dieser Annahme ist es anscheinend vielmehr so, daß die Dynamik starken wirtschaftlichen Wachstums weniger Platz für die Implementation umweltschonender Produktions-und Konsumtionsverfahren läßt, während sich der Rückgang bzw. die Verlangsamung des Anstieges der Industrieproduktion in wirtschaftlich schwachen Perioden als Gratiseffekt in einer 
Stagnation bzw. einer Reduktion der Umweltbelastungsindikatoren pro Einwohner niederschlagen.

Nachdem in der bisherigen Analyse Ressourcenverzehr und Schadstoffemissionen als $\mathrm{Ma} B$ für den individuellen Umweltstreß, den jedes Subjekt einer Volkswirtschaft im Durchschnitt verursacht, auf die Einwohnerzahl bezogen wurden, sollen in einem weiteren Analyseschritt nun die Zusammenhänge zwischen dem ökonomischen Wohlstandsniveau und den Belastungsindikatoren pro Einheit Bruttoinlandsprodukt untersucht werden. Als Maßzahlen des Umweltstresses dienen also im Sinne einer ökologisch-ökonomischen Effizienz auf den wirtschaftlichen Output bezogene Belastungswerte.

In Tabelle 4 sind die entsprechenden Korrelationskoeffizienten dargestellt.

Die gemessenen signifikanten Zusammenhänge weisen beinahe ausnahmslos ein negatives Vorzeichen auf. Auch die zunächst positiven Korrelationen zwischen Pro-Kopf-Einkommen sowie Primärenergieverbrauch und energiebedingtem Kohlendioxidaussto $B$ (jeweils gemessen pro Einheit BIP) schwächen sich im Verlauf des Untersuchungszeitraumes ab bzw. ändern das Vorzeichen.

Besonders deutlich ist der Zusammenhang zwischen dem BIP pro Einwohner und dem Umweltbelastungsindex [ENVIR/BIP] im Jahre 1990. Knapp $40 \%$ der Varianz der Umweltbelastung wird durch das ökonomische Wohlstandsniveau erklärt. Somit spricht ein hohes Maß an empirischer Evidenz für die folgende Aussage: Je höher das Pro-KopfEinkommen, desto geringer ist das Ausmaß der pro Einheit des ökonomischen Outputs verursachten Umweltbelastungen.

Diese Beobachtung stützt also die Annahme, daß die mit dem ökonomischen Niveau ebenfalls ansteigende technologische Kompetenz eine weitere Steigerung der wirtschaftlichen Wohlfahrt bei einem relativ abnehmenden ökologischen Faktoreinsatz zuläßt, d.h. die Umweltgrenzbelastung pro Einheit BIP ist dann mit Ansteigen des ökonomischen Entwicklungsstandes negativ. 
Tabelle 4: Korrelationsanalyse zwischen den Umweltbelastungsindikatoren pro Einheit BIP und der Wirtschaftsleistung

BIP/POPUL

\begin{tabular}{llllll}
\cline { 2 - 6 } & 1970 & 1975 & 1980 & 1985 & 1990 \\
\hline$H 2 O / B I P$ & -0.34 & -0.33 & -0.28 & $-0.38^{*}$ & $-0.59^{* *}$ \\
LAND/BIP & $-0.47^{*}$ & $-0.43^{*}$ & $-0.45^{*}$ & $-0.41^{*}$ & $-0.41^{*}$ \\
ENER/BIP & $0.39^{*}$ & 0.36 & 0.33 & 0.29 & 0.08 \\
RESOU/BIP & $-0.41^{*}$ & $-0.37^{*}$ & $-0.36^{*}$ & $-0.38^{*}$ & $-0.48^{*}$ \\
\hline CO2/BIP & 0.32 & 0.28 & 0.20 & -0.02 & -0.25 \\
WASTE/BIP & $k . A$. & $-0.60^{* *}$ & $-0.60^{* *}$ & $-0.66^{* *}$ & $-0.68^{* *}$ \\
FERT/BIP & -0.28 & $-0.40^{*}$ & -0.51 & $-0.51^{* *}$ & $-0.58^{* *}$ \\
PRESS/BIP & -0.11 & $-0.58^{*}$ & $-0.60^{* *}$ & $-0.64^{* *}$ & $-0.69^{* *}$ \\
\hline ENVIR/BIP & $-0.39^{*}$ & $-0.49^{*}$ & $-0.50^{* *}$ & $-0.54^{* *}$ & $-0.63^{* *}$ \\
\hline
\end{tabular}

Anm.i Für die Umweltbelastungsindikatoren wurde jeweils mit den Werten des gleichen Jahres gerechnet, in dem auch der entsprechende Bruttoinlandsproduktwert gemessen wurde.

$\mathrm{N}=22 ; *: \mathrm{p}<0.05 ; * *: \mathrm{p}<0.01$

Wie schon im Hinblick auf die Umweltbelastung pro Einwohner, so scheint jedoch auch für die Belastungsindikatoren pro Einheit BIP einerseits und dem BIP-Wachstum andererseits kein linearer Zusammenhang zu bestehen (Tabelle 5). Das Ausmaß des ökonomischen Wachstums hat also mit Ausnahme des Fünf-Jahres-Zeitraumes von 1970 bis 1975 kaum meßbare Einflüsse auf die absoluten Werte der Umweltbelastungsindikatoren.

Ein anderes Bild ergibt sich für die Zusammenhänge zwischen dem Wachstum der Umweltbelastungsindikatoren pro Einheit BIP und dem Wachstum der Wirtschaftsleistung (Tabelle 6).

Im Unterschied zu den Indikatorwerten pro Einwohner (siehe Tabelle 3) gibt es hier signifikante Beziehungen gerade für die Perioden ökonomisch geringen Wachstums (1975-85). 
Tabelle 5: Korrelationsanalyse zwischen den Umweltbelastungsindikatoren pro Einheit BIP und dem Wachstum der Wirtschaftsleistung

$$
\begin{aligned}
& \text { Durchschnittliche jährliche Wachstumsrate von } \\
& \text { BIP/POPUL }
\end{aligned}
$$

\begin{tabular}{lllll}
\cline { 2 - 5 } & $1970-75$ & $1975-80$ & $1980-85$ & $1985-90$ \\
\hline H2O/BIP & $0.52^{* *}$ & -0.19 & -0.21 & 0.17 \\
LAND/BIP & $0.39^{*}$ & -0.30 & 0.04 & 0.02 \\
ENER/BIP & 0.06 & -0.04 & 0.26 & $-0.36^{*}$ \\
RESOU/BIP & $0.45^{*}$ & -0.28 & -0.01 & 0.03 \\
\hline CO2/BIP & -0.03 & -0.02 & 0.03 & -0.06 \\
WASTE/BIP & $0.45^{*}$ & $-0.37^{*}$ & 0.12 & 0.09 \\
FERT/BIP & 0.23 & -0.20 & 0.13 & 0.28 \\
PRESS/BIP & $0.44^{*}$ & -0.34 & 0.14 & 0.19 \\
\hline ENVIR/BIP & $0.49^{*}$ & -0.34 & 0.06 & 0.10 \\
\hline
\end{tabular}

Anm:i Für die Umweltbelastungsindikatoren wurde jeweils mit den Werten des letzten Jahres des Fünf-Jahres-Zeitraumes gerechnet, in welchem das BIP-Wachstum errechnet wurde.

Lesebeispiel: Für den Umweltindikator pro Einheit BIP [ENVIR/BIP] des Jahres 1975 und dem BIP-Wachstum des Zeitraumes 1970 bis 1975 ergibt sich ein Korrelationskoeffizient von 0.49 .

$\mathrm{N}=22 ;{ }^{*}: \mathrm{p}<0.05 ;{ }^{* *}: \mathrm{p}<0.01$

Die negative Korrelation weist auf eine gegenläufige Entwicklung von Bruttoinlandsprodukt und Umweltbelastung hin: je mehr der Wert des BIP in einer Rezession sinkt, desto mehr steigt der Wert der Umweltbelastung pro Einheit BIP an.

Dieser Effekt könnte dadurch zu erklären sein, daß in wirtschaftlichen Stagnationszeiten das geringe ökonomische Wachstum mit den vorhandenen Produktionsmitteln bewältigt werden kann, und Investitionen in moderne, auf einem höheren Niveau der Schadstoffvermeidung - oder verminderung befindliche Technologien unterbleiben. 
Tabelle 6: Korrelationsanalyse zwischen dem Wachstum der Umweltbelastungsindikatoren pro Einheit BIP und dem Wachstum der Wirtschaftsleistung

Durchschnittliche jährliche Wachstumsrate von BIP/POPUL
$\Delta 70-75$
$\Delta 75-80$
$\Delta 80-85$
$\Delta 85-90$

\begin{tabular}{lllll}
\hline$\triangle$ H2O/POPUL & -0.20 & $-0.47^{*}$ & -0.22 & 0.14 \\
$\triangle$ LAND/POPUL & $-0.56^{* *}$ & $-0.80^{* *}$ & $-0.61^{* *}$ & $-0.64^{* *}$ \\
$\triangle$ ENER/POPUL & $0.44^{*}$ & -0.42 & $-0.39^{*}$ & -0.19 \\
$\Delta$ RESOU/POPUL & $-0.47^{*}$ & $-0.63^{* *}$ & -0.35 & -0.12 \\
\hline$\triangle$ CO2/POPUL & $0.42^{*}$ & -0.35 & -0.23 & -0.03 \\
$\Delta$ WASTE/POPUL & $k . A$. & $-0.66^{* *}$ & -0.18 & -0.28 \\
$\Delta$ FERT/POPUL & 0.23 & -0.25 & -0.29 & 0.12 \\
$\Delta$ PRESS/POPUL & $0.38^{*}$ & $-0.63^{* *}$ & $-0.46^{*}$ & -0.14 \\
\hline$\triangle$ ENVIR/POPUL & 0.19 & $-0.68^{* *}$ & $-0.56^{* *}$ & -0.12 \\
\hline
\end{tabular}

Anm.: Für die Umweltbelastungsindikatoren wurde jeweils mit den Werten des gleichen Fünf-Jahres-Zeitraumes gerechnet, in welchem auch das BIP-Wachstum errechnet wurde.

Lesebeispiel: Für das Wachstum des Umweltindikators pro Einheit BIP [ENVIR/BIP] im Zeitraum 1970 bis 1975 und das BIP-Wachstum des gleichen Zeitraumes ergibt sich ein Korrelationskoeffizient von 0.19 .

$\mathrm{N}=22 ;{ }^{*}: \mathrm{p}<0.05 ;^{* *}: \mathrm{p}<0.01$

In einer Rezessionsphase führt ein Rückgang des Bruttoinlandproduktes bei gleichzeitiger Konstanz bzw. geringer Zunahme der absoluten Umweltbelastung somit zu einer Vergrößerung des Quotienten aus Umweltbelastung und Wirtschaftsleistung und so zu einer negativen Korrelation zwischen dem Anstieg der Belastungsindikatoren und dem Wachstum des Pro-Kopf-Einkommens.

Betrachtet man die bisher dargestellten Analyseresultate im Zusammenhang, muß man feststellen, daß zwar die Umweltbelastung pro Einheit der erstellten Wirtschaftsleistung mit zunehmendem ökonomischem Niveau abnimmt, daß aber für den Indikator Umweltbelastung pro Einwohner diese Entkopplung vom Wirtschaftswachstum nicht eintritt. Dies würde 
bedeuten, daß Staaten mit einem hohen wirtschaftlichen und technologischen Niveau zwar in der Lage sind, zusätzlichen Output mit einem geringeren ökologisch Faktoreinsatz (Umwelt als Ressource und Medium zur Aufnahme von Schadstoffen) zu erzielen, daß jedoch der Faktoreinsatz und somit auch die Umweltbelastungen absolut und pro Einwohner weiter ansteigen.

Zur weiteren Prüfung der Entkopplungsthese wurde die Grundgesamtheit deshalb in zwei gleich große Staatengruppen unterschieden, deren BIP pro Einwohner des Jahres 1990 jeweils über- bzw. unterhalb von 13500 US-\$ in Preisen von 1985 liegt. Für diese Gruppen wurde erwartet, daß die Wirtschaftsleistung der ökonomisch schwächeren Staaten mit der Umweltbelastung pro Einwohner positiv korrelieren würde, während im Falle der „reichen" Staaten ein negativer Zusammenhang oder keine Korrelation zwischen den Variablen zu attestieren wäre. Diese Erwartungen werden nur teilweise erfüllt, d.h. die Umweltbelastungsindizes der Staaten mit einem Pro-Kopf-Einkommen von weniger als 13500 US-Dollar weisen wie die Grundgesamtheit einen positiven Zusammenhang mit der Wirtschaftsleistung auf (Tabelle 7). Die Korrelationskoeffizienten für die zweite Staatengruppe sind entgegen der These jedoch ebenfalls positiv und in einem Fall sogar auf dem 0.1-Niveau signifikant.

Eine genauere Analyse der einzelnen Datenpaare ergibt, daß die Werte der USA und Kanadas erhebliche Ausreißer darstellen, da sich in diesen Fällen sehr hohe Pro-Kopf-Einkommen mit ebenfalls weit überdurchschnittlichen Umweltbelastungsindikatorwerten verbinden.

Werden diese beiden Staaten aus der Gruppe der „reichen“ Staaten entfernt und eine erneute Korrelationsanalyse durchgeführt, dann erhält man die in der mittleren Datenspalte von Tabelle 7 dargestellten Werte.

Die auf dem 0.1-Niveau (bzw. in einem Fall sogar trotz der geringen Anzahl von 9 Datenpaaren auf dem 0.05-Niveau) signifikanten Zusammenhänge sind linear-negativ. Für die um Kanada und die USA bereinigte Gruppe der Staaten mit überdurchschnittlichem ökonomischem Leistungsniveau gilt also die Aussage: je höher das wirtschaftliche Niveau, desto geringer die Umweltbelastung pro Einwohner.

Somit scheint es tatsächlich ein typisches Muster des Zusammenhanges zwischen Bruttoinlandsprodukt und der Höhe der Indikatorwerte pro Einwohner zu geben: während die Belastungsindikatorwerte zunächst mit 
steigendem BIP ebenfalls ansteigen, kehrt sich diese Beziehung ab einem bestimmten ökonomischen und damit auch technologischen Niveau um. ${ }^{373}$

Tabelle 7: Korrelationen zwischen den Umweltbelastungsindizes und der Wirtschaftsleistung von Staaten mit hohem bzw. niedrigem BIP pro Einwohner

\begin{tabular}{|l|c|c|c|}
\hline \multirow{4}{*}{} & \multicolumn{3}{|c|}{ BIP/POPUL (1990 in US-\$) } \\
\cline { 2 - 4 } & \multicolumn{2}{|c|}{$>13500$} & $<13500$ \\
\cline { 2 - 4 } Umwelt-Index (1990) & $(n=11)$ & $(n=9)^{1)}$ & $(n=11)$ \\
\cline { 2 - 4 } & $0.42^{*}$ & $-0.60^{* *}$ & 0.31 \\
RESOU/POPUL & 0.29 & $-0.44^{*}$ & 0.39 \\
ENESS/POPUL & 0.39 & $-0.58^{*}$ & 0.40 \\
\hline
\end{tabular}

Anm.: ${ }^{*}: p<0.1 ; * *: p<0.05 .{ }^{1)}$ Ohne USA und Kanada.

Als die wichtigsten Ergebnisse der Analyse der Bedeutung des ökonomischen Niveaus als Determinante der Effektivität nationaler Umweltpolitik sind somit festzuhalten:

- Eine Zunahme der Wirtschaftsleistung scheint auf die Umweltbelastung pro Einwohner zunächst einen verstärkenden Einfluß zu haben; ab einem bestimmten Wohlstandsniveau findet jedoch eine Entkopplung bzw. Umkehr des Zusammenhanges zwischen ökonomischer Entwicklung und dem Anstieg von Ressourcenverbrauch bzw. Schadstoffemissionen statt, und die zunehmende ökonomische und technologische $\mathrm{Ka}$ pazität wird vielmehr zu einer Reduktion der Umweltbelastung genutzt.

${ }^{373}$ Dieses Niveau liegt bei einem Bruttoinlandsprodukt pro Einwohner von ca. 13500 US-\$ (in Preisen von 1985 unter Berücksichtigung von Kaufkraftparităten) und wird von den untersuchten Staaten am ehesten von Belgien ausgewiesen (BIP/Einw. in 1990: 13625 US-\$ [siehe Tabelle A52]). 
- Mit zunehmender Wirtschaftsleistung sinkt die Umweltbelastung pro Einheit der erbrachten Wirtschaftsleistung, d.h. eine Ausweitung der Wirtschaftstätigkeit geht generell mit einer ökonomisch effizienteren Nutzung der Umwelt einher.

- Das durchschnittliche Wachstum der Wirtschaftstätigkeit während einer Periode hat keinen meßbaren Einfluß auf die Ausmaße der Umweltbelastung am Ende dieser Periode, d.h. das absolute Maß des ökonomischen Wohlstandes, jedoch nicht das relative Tempo seines Wachstums, wirkt sich auf das Niveau von Ressourcenverbrauch und Schadstoffemissionen aus.

- In weltwirtschaftlichen Wachstumsphasen bedeutet ein starkes Wachstum eine ebenfalls starke Zunahme der Umweltbelastung pro Einwohner.

- In Rezessionsphasen geht ein Rückgang des BIP tendenziell mit einem Anstieg der Umweltbelastung pro Einheit BIP, also einer Minderung der ökologischen Effizienz, einher.

- Insgesamt gibt es also meßbare Zusammenhänge zwischen grundlegenden ökonomischen Leistungsindikatoren einerseits sowie den Größen zur Erfassung der ökologischen Belastung andererseits. Der durch die Wirtschaftsleistung erklärte Anteil der Varianz der Umweltbelastungsindikatoren ist insgesamt jedoch eher gering. Deshalb ist die vielfach postulierte Dominanz des ökonomischen Faktors aus der Analyse der bisher dargestellten Zusammenhänge nicht ableitbar.

\subsubsection{Sektorale Aufteilung der Volkswirtschaft}

Für den vermuteten Zusammenhang zwischen der sektoralen Aufteilung von Volkswirtschaften sowie der Ausprägung von Indikatoren, welche umweltrelevante Größen messen, sollen zunächst die folgende Thesen gelten:

- Für den primären Sektor, also die Landwirtschaft, ist zu erwarten, daß Staaten mit einem eher hohen Anteil dieses Sektors an der volkswirtschaftlichen Wertschöpfung aufgrund tendenziell größerer Flächennutzung und höherem Düngemitteleinsatz auch eher höhere Indexwerte für Ressourcennutzung und Emissionen aufweisen.

- Je höher der Anteil des sekundären Sektors an der volkswirtschaftlichen Wertschöpfung, desto größer ist aufgrund der umweltbelastenden Produktionsprozesse auch der Umweltstreß. 
- Oder anders formuliert: je höher der Anteil des tertiären Sektors (Dienstleistungen), desto geringer ist eben wegen des teilweisen Wegfalls der industriellen Produktion die Umweltbelastung ${ }^{374}$.

Eine Überprüfung dieser Annahmen ergibt die in Tabelle 8 dargestellten einfachen und partiellen Korrelationskoeffizienten.

Während der Anteil des primären Sektors am Bruttoinlandsprodukt mit den auf die Einwohnerzahl bezogenen Umweltbelastungsvariablen meist negativ korreliert - eine Ausnahme bildet lediglich der Düngemittelabsatz -, besteht mit den Indikator- und Indexwerten pro Einheit BIP ein positiver, in vielen Fällen sogar hochsignifikanter Zusammenhang. Da für den Landwirtschaftsanteil und das allgemeine Wohlstandsniveau (gemessen im Pro-Kopf-Einkommen) jedoch ein einfacher Korrelationskoeffizient von -0.85 gemessen werden kann, Staaten mit einem hohen Agraranteil also ein eher geringes Pro-Kopf-Einkommen aufweisen, und im obigen Abschnitt ein Zusammenhang zwischen Wirtschaftsleistung und Umweltbelastung festgestellt wurde, lag es nahe, in einem weiteren Analyseschritt die Einflüsse der Variable Pro-Kopf-Einkommen durch die Bestimmung der partiellen Korrelationskoeffizienten herauszurechnen ${ }^{375}$. Wie aus Tabelle $8 \mathrm{zu}$ entnehmen ist, ergeben sich dann für die Pro-KopfUmweltbelastung zwar positive Koeffizienten, diese Zusammenhänge sind jedoch nicht signifikant. Für die auf das Bruttoinlandsprodukt bezogenen Indizes gilt folgendes: zwischen Agraranteil und Ressourcennutzung scheint es keinen Zusammenhang zu geben, während die Emissionsmenge pro Einheit BIP mit der Größe des primären Sektors signifikant ansteigt (das Maß der erklärten Varianz beträgt hier knapp 50 Prozent).

Entgegen der oben aufgestellten These bestehen zwischen dem Anteil des sekundären volkswirtschaftlichen Sektors, also der Industrie, und den Umweltbelastungsvariablen vornehmlich negative Korrelationen, dies gilt insbesondere für die Pro-Kopf-Indikator- und -Indexwerte. Auch in die-

${ }^{374}$ Der Effekt ökonomischer Strukturen auf die Umweltbelastung ist auch Thema einer Studie von Jänicke, Martin; Mönch, Harald; Binder, Manfred et al. (1993): Umweltentlastung . . . , a.a.O. Allerdings betrachten sie ausschließlich den industriellen Sektor. Dabei konstatieren sie die besondere Bedeutung des intrasektoralen Wandels, d.h. durch branchen- oder sogar unternehmensinterne Maßnahmen werden ökologische Belastungen reduziert (S. 144 ff.).

${ }^{375}$ Um die Einflüsse der anderen Variablen auszuschalten, wird im Verfahren der partiellen Korrelationsanalyse die ,tatsächliche“ Korrelation zwischen zwei Variablen anhand eines partiellen Korrelationskoeffizienten errechnet, d.h. die Effekte der anderen Variablen werden durch geeignete Rekursionsformeln konstant gehalten. Der partielle Korrelationskoeffizient liegt wie der einfache Korrelationskoeffizient zwischen -1 und 1. 
sem Fall kann das Analyseergebnis durch das Herausrechnen des vom Niveau des Pro-Kopf-Einkommens ausgeübten Einflusses präzisiert werden.

Während die Ressourcennutzung vom Anteil des sekundären Sektors am BIP weitgehend unabhängig ist, gilt für den anderen Bereichsindex: je höher der Industrieanteil, desto geringer ist das Ausmaß der Schadstoffbelastung pro Einwohner bzw. pro Einheit BIP. Ein eher hoher Anteil der industriellen Produktion am BIP scheint also mit spezifischen, im Hinblick auf die Freisetzung von Schadstoffen eher restriktiven Produktionsund Konsumtionsmustern einherzugehen.

Auch der zwischen dem Anteil des tertiären Sektors am BIP sowie den Umweltbelastungsvariablen gemessene Zusammenhang entspricht nicht der Ausgangsthese. Von den im Rahmen einer einfachen Korrelationsanalyse gemessenen signifikanten Beziehungen verbleiben nach Eliminierung des Einflusses der ökonomischen Variable keine nicht-zufälligen Korrelationen.

Ein sparsamer Umgang mit Ressourcen und ein eher geringes Niveau der freigesetzten Schadstoffmenge sind also nicht - wie zunächst angenommen - Kennzeichen von ausgeprägten Dienstleistungsgesellschaften. 
Tabelle 8: Einfache und partielle Korrelationsanalyse zwischen den Umweltbelastungsindikatoren und der sektoralen Verteilung der Volkswirtschaften

Anteil des jeweiligen Sektors am BIP

Umweltbelast- Landwirtschaft Industrie Dienstleistung ungsvariablen

\begin{tabular}{|c|c|c|c|}
\hline \multicolumn{4}{|c|}{ Einfache Korrelationskoeffizienten } \\
\hline H20/POPUL & -.21 & -.11 & .20 \\
\hline LAND/POPUL & -.02 & -.21 & .17 \\
\hline ENER/POPUL & $-.56 * *$ & -.33 & $.62 * *$ \\
\hline RESOU/POPUL & -.21 & -.25 & .33 \\
\hline CO2/POPUL & $-.43 *$ & $-.36^{*}$ & $.57 * *$ \\
\hline WASTE/POPUL & -.19 & -.36 & $.40^{*}$ \\
\hline FERT/POPUL & .29 & $-.52 * *$ & .24 \\
\hline PRESS/POPUL & -.07 & $-.58 * *$ & $.51 * *$ \\
\hline ENVIR/POPUL & -.18 & $-.41^{*}$ & $.44 *$ \\
\hline $\mathrm{H} 20 / \mathrm{BIP}$ & $.54 * *$ & .09 & -.45 \\
\hline LAND/BIP & $.43 *$ & -.05 & -.25 \\
\hline ENER/BIP & .10 & $-.44^{*}$ & .27 \\
\hline RESOU/BIP & $.50 * *$ & -.06 & -.30 \\
\hline CO2/BIP & $.38^{*}$ & $-.42 *$ & .09 \\
\hline WASTE/BIP & $.78 * *$ & .03 & $-.54 * *$ \\
\hline FERT/BIP & $.73 * *$ & -.30 & -.23 \\
\hline PRESS/BIP & $.85 * *$ & -.27 & -.35 \\
\hline ENVIR/BIP & $.72 * *$ & -.17 & -.35 \\
\hline Partielle Korrela & koeffizic & & \\
\hline RESOU/POPUL & .36 & .10 & -.03 \\
\hline PRESS/POPUL & .37 & $-.56 * *$ & .37 \\
\hline BIP/POPUL & $-.90 * *$ & .15 & $.55^{* *}$ \\
\hline RESOU/BIP & -.17 & .11 & -.08 \\
\hline PRESS/BIP & $.69 * *$ & $-.44^{*}$ & .16 \\
\hline BIP/POPUL & $-.69 * *$ & -.36 & $.56 * *$ \\
\hline
\end{tabular}

Anm.: Alle Werte von 1990. Die Werte für die Anteile der einzelnen Sektoren sind entnommen aus: OECD (1993): OECD Environmental Data, Paris, S. 317. 


\subsection{Politische Determinanten}

Die Bedeutung der politischen Stärke oder Dominanz unterschiedlicher Ideologien und der sich daraus ergebenden volkswirtschaftlichen Prioritäten für den Erfolg nationaler Wirtschaftspolitik ist eine empirisch inzwischen gut erschlossene Fragestellung ${ }^{376}$. Dabei steht insbesondere der Effekt entweder sozial-demokratisch oder liberal-konservativ geprägter Politik auf den makroökonomischen Outcome im Zentrum der Analyse ${ }^{377}$. Empirisch-quantitative Studien über den bivariaten Zusammenhang zwischen „grüner“ Politik und ökonomischen oder ökologischen Erfolgsgrößen sind dagegen kaum auffindbar ${ }^{378}$. Dies liegt wahrscheinlich zum einen an dem bisher geringen Maß politisch überregionaler oder sogar nationaler Verantwortung grüner Parteien, und zum anderen an der a priori gemachten Annahme einer negativen Korrelation zwischen ökologisch orientierter Politik und traditionellen ökonomischen Erfolgsgrößen wie Wachstum, Beschäftigung oder Preisstabilität, welche einer empirischen Überprüfung nicht zu bedürfen scheint. Statt als Determinante gesellschaftlicher Entwicklungen wird der Erfolg grüner Parteien in der politikund wirtschaftswissenschaftlichen Literatur deshalb eher als zu erklärende Variable thematisiert ${ }^{379}$. Aber auch in diesen Studien findet keine Kausalanalyse zwischen Wahlerfolgen bzw. parlamentarischer Präsenz ökologisch orientierter Gruppierungen einerseits und Umweltbelastungsoder -qualitätsindikatoren andererseits statt.

Da wir im Rahmen des Modellentwurfes unter den strukturellökonomischen Faktoren, welche den Erfolg nationaler Umweltpolitik beeinflussen, die politische Präsenz grüner Parteien als relevant erachtet haben, soll nachfolgend eine empirische Überprüfung dieser Annahme

${ }^{376}$ Vgl. u.a. Cusack; Thomas R. (1994): Economic Problems . ., a.a.O.

${ }^{377}$ Vgl. Ebenda, S. 52 ff.

${ }^{378}$ Dagegen gibt es zumindest qualitative Einzellfallstudien, die dem parlamentarischen Erfolg grüner Parteien einen Einfluß auf das umweltpolitische Handeln der jeweiligen Exekutive zuschreiben (vgl. etwa Mez, Lutz (1995): Reduction of Exhaust Gases at Large Combustion Plants in the Federal Republic of Germany, In: Jänicke, Martin, und Weidner, Helmut (Hg.): Succesful . . ., a.a.O., oder Holzinger, Katharina (1995): A Surprising Success in EC Environmental Policy: The Small Car Exhaust Emission Directive of 1989, In: Jänicke, Martin, und Weidner, Helmut (Hg.): Succesful . . ., a.a.O.).

${ }^{379}$ Vgl. Müller-Rommel, Ferdinand (1993): Grüne Parteien . . ., a.a.O., S. 190. 
erfolgen. Als zu falsifizierende Hypothese soll dabei die folgende Überlegung gelten:

Wahlerfolge und parlamentarische Präsenz grüner Parteien wirken sich entweder durch direkte Regierungsbeteiligung oder inhaltliche Beeinflussung der dominierenden politischen Formationen in einem Rückgang ökologischer Belastungsindikatoren aus.

Zur Messung des (wahl-)politischen Erfolges und des Ausmaßes der parlamentarischen Beteiligung grüner Parteien wurden die von MüllerRommel zusammengestellten Daten für die Jahre 1979 bis 1989 übernommen.

Tabelle 9: Elektoral erfolgreiche und erfolglose grüne Parteien in Westeuropa (1979-1989)

\begin{tabular}{lccc}
\hline Länder & $\begin{array}{c}\text { Beteiligung } \\
\text { an Wahlen } \\
(N)\end{array}$ & $\begin{array}{c}\text { Durchschnitt- } \\
\text { liches } \\
\text { Wahlergebnis } \\
\text { (in Prozent) }\end{array}$ & $\begin{array}{c}\text { Konzentra- } \\
\text { tionsgrad im } \\
\text { nationalen } \\
\text { Parlament }\end{array}$ \\
\hline erfolgreich & 7 & 6.3 & 4.24 \\
Belgien & 6 & 5.7 & 8.46 \\
Deutschland (W) & 3 & 5.6 & 4.00 \\
Niederlande & 3 & 5.2 & 3.50 \\
Schweiz & 3 & 4.0 & 4.37 \\
Österreich & & & \\
erfolglos & 7 & 4.3 & 0.00 \\
Frankreich & 3 & 2.9 & 8.03 \\
Schweden & 6 & 2.8 & 0.00 \\
Großbritannien & 3 & 1.9 & 2.00 \\
Finnland & 2 & 4.4 & 2.06 \\
Italien & 4 & 1.4 & 0.60 \\
Irland & 2 & 1.3 & 0.00 \\
Dänemark & 2 & 1.2 & 0.00 \\
Spanien & 1 & 1.1 & 0.33 \\
Griechenland & 3.9 & 3.4 & 2.85 \\
\hline Mittelwert & & &
\end{tabular}

Anm.: Der Konzentrationsgrad definiert sich als Anteil der Sitze Grüner Parteien an der Gesamtzahl der Sitze im Parlament.

Quelle: Müller-Rommel (1993), S. 190. 
Der elektorale Erfolg wird dabei anhand von drei Kriterien definiert. Für eine erfolgreiche Partei gelten die folgenden Bedingungen ${ }^{380}$ :

1. Nominaler Wahlerfolg: Der durchschnittliche Stimmenanteil bei den nationalen Wahlen des Zeitraumes 1979-89 muß mindestens 4 Prozent betragen.

2. Parlamentarische Präsenz: Der Konzentrationsgrad im nationalen Parlament, d.h. der Anteil der Sitze grüner Parteien an der Gesamtzahl der Sitze im Parlament, muß mindestens 3,5 Prozent betragen.

3. Beständigkeit im nationalen Parteiensystem, d.h. Beteiligung an mindestens drei nationalen Wahlen.

Bei gleichzeitiger Berücksichtigung dieser drei Kriterien sind fünf grüne Parteien als elektoral erfolgreich anzusehen, nämlich diejenigen in Belgien, Deutschland, den Niederlanden, der Schweiz und Österreich ${ }^{381}$. Die weiteren neun Parteien sind definitionsgemäß erfolglos. Hierzu zählen die Grünen in Frankreich, Schweden, Großbritannien, Finnland, Italien, Irland, Dänemark, Spanien und Griechenland.

In Abbildung 31 sind die prozentualen Änderungsraten der Umweltbelastungsindizes im Zeitraum 1970-1990 für die beiden Staatengruppen mit elektoral erfolgreichen bzw. erfolglosen grünen Parteien dargestellt.

$\mathrm{Da}$ die meisten europäischen grünen Parteien die sogenannte Mobilisierungshürde, definiert durch die Gründung einer nationalen Parteiorganisation und die Erstellung von Kandidatenlisten für nationale Parlamentswahlen ${ }^{382}$, erst gegen Ende der 70er Jahre überwinden konnten, kann die umweltentlastende Wirkung des elektoralen Erfolges der grünen erst für die Zeiträume 1980-85 bzw. 1985-90 angenommen werden.

Die Entwicklung der Umweltbelastungsindizes in diesen Perioden liefert jedoch keine empirische Evidenz für die Gültigkeit der Ausgangsthese. Zwar sind die Schadstoffindexwerte in den beiden Zeiträume 80-85 sowie 85-90 in den Staaten mit elektoral erfolgreichen grünen Parteien stärker zurückgegangen bzw. weniger stark angestiegen als in den Staaten mit erfolglosen Umweltparteien, doch zum einen sind diese Unterschiede nur

${ }^{380}$ Ebd., S. 189 ff.

${ }^{381}$ Nicht berücksichtigt wird erneut Luxemburg, daß bei Anwendung der Kriterien auch zu den Ländern mit erfolgreicher grüner Partei zählen würde.

${ }^{382}$ Vgl. Müller-Rommel, Ferdinand (1993): Grüne Parteien . ., a.a.O., S. 31 ff. 
in einem Fall statistisch signifikant ${ }^{383}$, und zum anderen weist die Staatengruppe mit erfolglosen grünen Parteien für die Änderungsraten der Ressourcenverbrauchsindizes günstigere Werte auf.

$\mathrm{Da}$ in Kapitel 9.1. ein Einfluß des Wachstums der Wirtschaftsleistung auf das Wachstum der Umweltbelastungsindikatoren, insbesondere derjenigen pro Einwohner, festgestellt wurde ${ }^{384}$, und außerdem eine Abhängigkeit des elektoralen Erfolges grüner Parteien von der ökonomischen Entwicklung möglich wäre ${ }^{385}$, ist ein weiterer Analyseschritt, in dem die Variable Wirtschaftswachstum kontrolliert wird, naheliegend.

Wie Müller-Rommel im Rahmen seiner umfangreichen empirischen Untersuchung über die Erfolgsbedingungen grüner Parteien in Westeuropa jedoch statistisch nachweisen konnte, ,deuten die empirischen Befunde auf keinen nennenswerten Zusammenhang zwischen [...] dem Wirtschaftswachstum einerseits und der Höhe der Wahlergebnisse Grüner Parteien andererseits hin.

Deshalb können die in Abbildung 31 dargestellten Ergebnisse ohne weitere Analyse als eine weitgehende Bestätigung der Nullhypothese gelten: Es besteht kein kontinuierlicher Zusammenhang zwischen dem elektoralen Erfolg grüner Parteien und der Entwicklung der Umweltbelastungsindizes.

${ }^{383}$ Der Rückgang des Schadstoffindex pro Einwohner betrug im Zeitraum 1980-85 in den Staaten mit elektoral erfolgreichen grünen Parteien 5,58 Prozent. In derselben Periode stieg der Wert für diesen Index in der Staatengrupppe mit erfolglosen Parteien um 2,25 Prozent. Dieser Unterschied zwischen beiden Gruppen erweist sich im t-Test auf einem Signifikanzniveau von $\mathrm{p}=0.05$ als nicht zufällig.

${ }^{384} \mathrm{Vgl}$. Tabelle 3.

${ }^{385}$ Vgl. Müller-Rommel, Ferdinand (1993): Grüne Parteien . ., a.a.O., S. 107.

${ }^{386}$ Ebd., S. 110. Der gemessene Korrelationskoeffizient zwischen beiden Variablen beträgt $\mathrm{r}=-.10$. 


\section{Abb. 31: Entwicklung der Umweltbelastungsindizes in Abhängigkeit vom elektoralen Erfolg grüner Parteien}
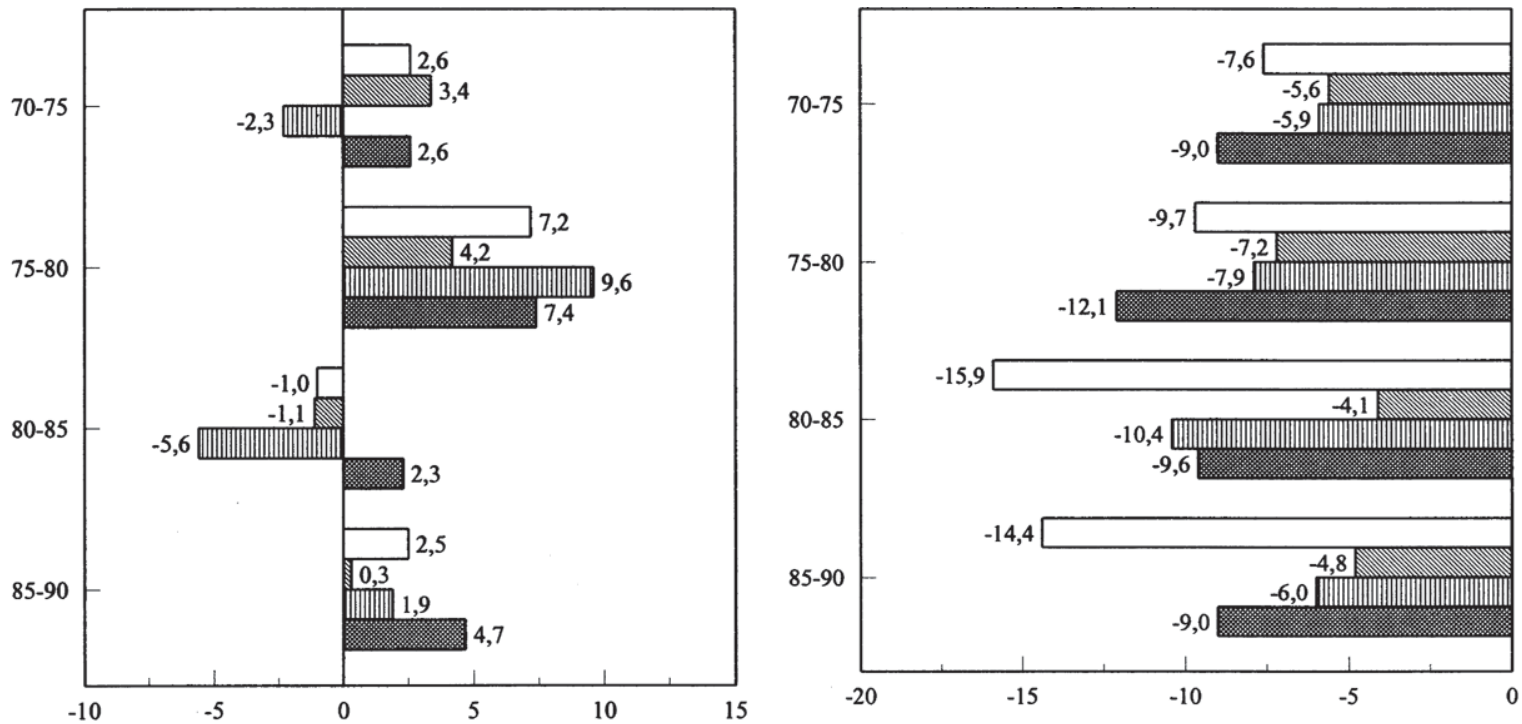

$\square$ RESOU/POPUL(Grün+) \$RESOU/POPUL(Grün-) 四PRESS/POPUL(Grün+) 1 PRESS/POPUL(Grün-)

\section{$\square$ RESOU/BIP(Grün+) $\mathbb{\mathbb { Q }}$ RESOU/BIP(Grün-)} 四PRESS/BIP(Grün+) 1 PRESS/BIP(Grün-)

Anm: Veränderungen im jeweiligen Zeitraum in Prozent. (Grün+):=Staaten mit elektoral erfolgreichen grünen Parteien; (Grün-):=Staaten mit elektoral erfolglosen grünen Parteien. Lesebeispiel: Die Veränderungsrate der Ressourcennutzung pro Einwohner betrug im Zeitraum 1970 bis 1975 in den Ländern mit elektoral erfolgreichen grünen Parteien 2,5\%. 


\section{Messung des nationalen Politikstils}

\subsection{Strategieorientierung}

\subsection{1 (De-)Zentralisationsgrad}

Gemäß der in Kapitel 7 des zweiten Teils dieser Studie dargelegten Analysestrategie soll die Operationalisierung der Merkmalsdimension Strategieorientierung zunächst anhand der Variable Dezentralisationsgrad erfolgen.

Ein dezentraler Staatsaufbau spiegelt sich in föderalen Strukturen wieder, in welchen sich die Regelungskompetenzen der zentralen Staatsmacht weitgehend auf die für das Funktionieren des Gesamtstaates notwendigen Kernbereiche staalichen Handelns (militärische Sicherheit, Finanz- und Währungspolitik, AuBenbeziehungen) beschränken, während die anderen Politikbereiche im Rahmen vertikaler Gewaltenteilung von demokratisch legitimierten Legislativen und Exekutiven subnationaler, anhand geographischer Kriterien definierter Einheiten gestaltet werden ${ }^{387}$.

Ein weiteres Merkmal eines föderalen Staatsaufbaus ist die Einbeziehung dieser subnationalen, regionalen Einheiten in gesamtstaatlich dominierte Problemlösungsprozesse, falls deren Interessen durch die auf der Agenda stehende Thematik berührt werden. Ein Beispiel hierfür ist die Einbeziehung von Instanzen der zweiten oder einer noch niedrigeren Systemebene im Falle einer Nutzung von im Besitz des Gesamtstaates befindlichen Grundstücken oder Liegenschaften zu militärischen Übungszwecken, oder im Falle von bilateralen Regelungen des Grenzregimes mit Staaten, welche direkt an das Territorium einer subnationalen Einheit angrenzen.

Diese meist konstitutionell geregelten Mechanismen der Kooperation zwischen Zentralmacht und regionalen Einheiten haben für die gesamtstaatlichen Staatsorgane trotz der objektiv gegebenen Beschneidung ihrer Machtfülle zwei wichtige Vorteile:

1. Die regionale Umsetzung und Kontrolle zentral verfügter Maßnahmen kann unter der Voraussetzung der Existenz ausreichender Kompetenzen und Ressourcen dezentral viel effektiver erfolgen, da die Installation schwerfälliger

${ }^{387}$ Vgl. im folgenden auch die Ausfuhrungen bei: Schultze, Rainer-Olaf (1983): Föderalismus, In: Schmidt, Manfred G. (Hg.): Westliche Industriegesellschaften. Wirtschaft - Gesellschaft - Politik, München, S. 93-105, sowie: Schultze, Rainer-Olaf (1995): Föderalismus, In: Nohlen, Dieter (Hg.): Wörterbuch Staat und Politik, Bonn, S. 155-164. 
Implementations- und Überwachungsbürokratie weitgehend entfällt bzw. durch die subnationalen Einheiten erfolgt. Neben dieser Entlastung ist es auBerdem möglich, daß national festgelegte Standards aufgrund regionaler Spezifika noch übertroffen werden können.

2. Die regionalen Systemebenen können in gesamtstaatlich relevanten Fragen als „Frühwarnsysteme“ fungieren, indem sie krisenhafte bzw. chancenreiche Entwicklungen weitergeben und damit rechtzeitiges und vorausschauendes Handeln der Zentralmacht initiieren. Vor allem dieser zweite Vorteil eines föderalen Staatsaufbaus läßt dieses Merkmal als wertvollen Indikator zur Messung staatlicher Strategieorientierung erscheinen.

Von den hier untersuchten 22 OECD-Staaten sind nach eingehender Analyse lediglich sieben Staaten als föderalistisch strukturiert zu bezeichnen: Kanada, die Vereinigten Staaten, Australien, Österreich, Belgien, Deutschland und die Schweiz ${ }^{388}$.

Der kanadische Staat gliedert sich in 10 Provinzen und 2 Bundesterritorien. Diese regeln Streitfragen mit der Bundesregierung in konfliktorientierten Mechanismen des vertikalen Aushandelns. Der Pluralismus der Provinzen und ihr stetes Spannungsverhältnis zum Bund wird noch überlagert durch die These der deux nations der frankophonen Gesellschaft Quebecs. Dennoch hat der Staatsaufbau Kanadas einen eindeutig föderalen Charakter, und wichtige Fragen der Wirtschafts- und Umweltpolitik fallen in den Regelungsbereich der Provinzregierungen $^{389}$.

Die USA setzen sich aus 50 Einzelstaaten, dem District of Columbia sowie zahlreichen überseeischen Gebieten mit unterschiedlichem rechtlichen Status zusammen. Ein besonderes Kennzeichen des amerikanischen Föderalismus ist das hohe $\mathrm{Ma} ß$ an Entscheidungsautomonie der direkt gewählten Exekutiven der Einzelstaaten. Während die Bundesregierung auf den von ihr dominierten Politikfeldern meist ohne Abstimmung mit den betroffenen subnationalen Einheiten agiert, so gilt dies umgekehrt auch für diese Staaten und die von ihnen bestimmten Politikbereiche ${ }^{390}$. Insbesondere die Umweltpolitik ist in den USA eine Domäne der einzelnen Bundesstaaten, was etwa auf dem Gebiet der Luft- und Gewässerreinhalteregime zu großen, durch die regionalen, sozio-strukturellen

${ }^{388}$ Ebenda.

${ }^{389}$ Vgl. ebenda, S. 97 ff.

390 Zum Regierungssystem der USA vgl. ebenda sowie Ranney, Austin (1992): Politics . . ., a.a.O. 
Entwicklungsdisparitäten bedingten Heterogenitäten des Belastungsniveaus geführt hat ${ }^{391}$.

Die Kompetenzverteilung zwischen Bund und Gliedstaaten erfolgt in Australien (mit 7 Einzelstaaten und 2 Bundesterritorien) ähnlich wie in Kanada. Während der Bund die in der Verfassung niedergelegten Kernkompetenzen besitzt, fallen die Residualkompetenzen an die Provinzen. Die ebenfalls zu konstatierende Konfliktorientierung wird jedoch durch die Dominanz der britischprotestantischen Kultur sowie die Präponderanz des Bundes in Streitfragen abgemildert $^{392}$.

Im Gegensatz zu den Ausprägungen des Dezentralismus der drei bisher aufgeführten englischsprachigen konföderalen Bundesstaaten ist der österreichische Föderalismus unitaristisch und konsensorientiert. Sowohl vertikal zwischen dem Bund und den 9 Bundesländern als auch horizontal zwischen den einzelnen Gliedstaaten hat sich eine Kultur des geduldigen Aushandelns etabliert, an deren Ende eine für alle Beteiligten akzeptable Problemlösung zu stehen hat ${ }^{393}$.

Einen ganz besonderen Fall des Föderalismus bildet Belgien. Der im Laufe der 80er Jahre von einem dezentralen Einheitsstaat in einen föderalen Bundesstaat transformierte Staat gliedert sich in drei Regionen (Flandern, Wallonien und Brüssel), wobei der Dualismus zwischen der flämischen und der wallonischen Bevölkerungsgruppe die grundlegende Konstante der belgischen Politik bildet. Der Kampf um eine Ausweitung der Kompetenzen der Regionen ist somit primär ein Ausdruck der Segmentierung der Gesellschaft und des Wettbewerbs der beiden Volksgruppen. Dennoch hat die 1994 vollzogene Verfassungsreform, welche insbesondere direkt gewählte Regionalparlamente der einzelnen Landesteile vorsieht, den letzten Schritt der Umwandlung des einstmals zentralistischen belgischen Staates in ein funktionierendes föderales Gemeinwesen bedeutet $^{394}$.

Auch die Bundesrepublik Deutschland wird zu den föderalistischen Staaten gezählt. Die Kompetenzen der 16 Gliedstaaten sind jedoch von ganz spezieller Natur. Während die Gesetzgebungskompetenz in den meisten Politikfeldern beim Bund liegt, und den Ländern primär die Administration bundesstaatlicher Entscheidungen zukommt, besteht über den Bundesrat dennoch eine starke

\footnotetext{
${ }^{391}$ Vgl. etwa Ringquist, Evan J. (1993): Environmental Protection . . ., a.a.O.

${ }^{392}$ Vgl. Schultze, Rainer-Olaf (1983): Foderalismus, a.a.O., S. 96 ff.

${ }^{393}$ Ebenda, S. 97 ff.

${ }^{394}$ Ebenda.
} 
bundespolitische Einflußmöglichkeit der einzelnen Gliedstaaten ${ }^{395}$. Kitschelt führt im Zusammenhang mit der Zustimmungspflichtigkeit zahlreicher Gesetze durch den Bundesrat und somit einem de-facto-Vetorecht der Länder in wichtigen Fragen sogar aus: "[...] der föderale Charakter des westdeutschen Regierungssystems, welcher in vielen Politikbereichen eine Verflechtung politischer Entscheidungskompetenzen zwischen Bund, Ländern und Gemeinden mit sich bringt, [restringiert] den Spielraum implementierbarer Politiken $[\ldots]^{396}$. Dieser Zustand macht eine möglichst konfliktarme Zusammenarbeit von Zentralregierung und regionalen Einheiten (z.B. in den Bund-Länder-Konferenzen) zu einer wichtigen Bedingung für das Funktionieren des Gesamtstaates. Neben dieser Verzahnung von Bund und Ländern besteht außerdem ein hohes $\mathrm{Maß}$ an interstaatlicher Kooperation zwischen den einzelnen Gliedstaaten (z.B. Finanzausgleich) $)^{397}$.

Ein weiterer Sonderfall des europäischen Föderalismus ist derjenige der Schweiz. Die möglichen Konfliktpotentiale der Bikonfessionalität und der Mehrsprachigkeit des Staates werden durch eine strikte Dezentralisierung in 23 Kantone aufgefangen. Innerhalb und zwischen den einzelnen Systemebenen gilt dabei das konsenorientierte System des konkordanzdemokratischen Aushandelns ${ }^{398}$. Fragen von besonderer politischer oder gesellschaftlicher Relevanz werden zudem durch Referenda entschieden ${ }^{399}$.

Die Heterogenität der hier geschilderten sieben Fälle föderaler Systeme zeigt die Schwierigkeit einer Kategorisierung der untersuchten Staaten im Hinblick auf das Ausmaß der Eigenständigkeit ihrer subnationalen Einheiten.

${ }^{395}$ Zum Föderalismus in der Bundesrepublik Deutschland vgl. auch Scharpf, F. W. (1976): Politikverflechtung. Theorie und Empirie des kooperativen Föderalismus in der Bundesrepublik, Kronberg/Ts.; Scharpf, Fritz W. (1985): Die Politikverflechtungsfalle: Europäische Integration und deutscher Főderalismus im Vergleich, in: Politische Vierteljehresschrift, 26, S. 323-356; Scharpf, F. W. (1994): Optionen des Föderalismus in Deutschland und Europa, Frankfurt am Main.

${ }^{396}$ Kitschelt, Herbert (1983): Politik und Energie . ., a.a.O., S. 88.

397 Zum Regierungssystem der Bundesrepublik Deutschlands vgl. außerdem: Hartmann, Jürgen (1995): Vergleichende Politikwissenschaft, a.a.O., S. 67 ff.; Hartmann, Jürgen (1983): Politik . ., a.a.O., S. 168 ff.; Dalton, Russel, J. (1992): Politics . ., a.a.O.

${ }^{398}$ Zum Thema Konkordanzdemokratie vgl. Lehmbruch, Gerhard (1995): Konkordanzdemokratie, In: Nohlen, Dieter (Hg.): Wörterbuch . . ., a.a.O., S. 350-354.

${ }^{399}$ Vgl. Schultze, Rainer-Olaf (1983): Föderalismus, a.a.O., S. 97 ff. 
Auch die fünf unter dem Begriff des dezentralen Einheitsstaates zu subsumierenden Fälle - Frankreich, Italien, Spanien, Japan und die Niederlande (Tabelle 10) - weisen eklatante Unterschiede im Staatsaufbau auf, dennoch sind in allen diesen Ländern der Eigenständigkeit und Autonomie der vorhandenen Regionen enge Grenzen gesetzt, so daß die entscheidenden politischen Strukturelemente primär auf der gesamtstaatlichen Entscheidungsebene konzentriert sind. Die verbleibenden zehn Staaten sind weitgehende Einheitsstaaten, in welchen es zwar geographisch definierte Subeinheiten staatlicher Gewalt gibt, aufgrund weitgehender sozio-kultureller Homogenität der Territiorien oder des politischen Credos einer Gleichheit der Lebensbedingungen die Entscheidungen über die Vergabe von Ressourcen jedoch nahezu ausschließlich durch die Staatsregierung getroffen werden.

\section{Tabelle 10: Föderalismusgrad der untersuchten Staaten}

$\begin{array}{lll}\text { konföderaler/ } & \text { dezentraler } & \text { Einheitsstaat } \\ \text { unitaristischer } & \text { Einheitsstaat } & \\ \text { Bundesstat } & & \end{array}$

Bundesstaat

Kanada

USA

Australien

Deutschland

Österreich

Schweiz

Belgien
Niederlande

Japan

Frankreich

Italien

Spanien

Dänemark
Finnland
Norwegen
Schweden
Großbritannien
Irland
Neuseeland
Portugal
Griechenland
Türkei

Dänemark

Norwegen

Schweden

Irland

Neuseeland

Portugal

Türkei

Quelle: Schultze (1983); S. 93 ff. sowie eigene Einschätzungen 


\subsubsection{Technologische Kapazitäten}

Zur Fähigkeit eines Staates, strategisch agieren zu können, gehört, wie im Modellentwurf festgestellt, auch eine entsprechende ökonomisch-technologische Handlungskapazität. Zur Messung dieser Größe wurden aus dem vorhandenen Datenmaterial drei Indikatoren ausgewählt: der Anteil der Forschungs- und Entwicklungs-(F\&E-)Ausgaben (staatlich und privat) als Prozentsatz des gesamten BIP eines Staates, das Export-/Import-Verhältnis im Handel mit Hochtechnologieprodukten sowie der Anteil der staatlichen Ausgaben für Spitzenausbildungsförderung im F\&E-Bereich (Higher Education Sector Expenditure on $R \& D$ ) am BIP.

Die relative Höhe der F\&E-Ausgaben stellt dabei ein Maß für die technologische Innovationsbereitschaft und -fähigkeit eines Staates dar. Der gesamtstaatlich von Wirtschaft und Industrie zu diesem Zweck zur Verfügung gestellte Anteil der volkswirtschaftlichen Wertschöpfung ist somit ein guter Indikator für die in einem Staat herrschende Zukunftsorientierung bzw. sein Vermögen, sich den mittel- und langfristig absehbaren ökonomischen und ökologischen Herausforderungen zu stellen. Außerdem sind nur bei Vorhandensein ausreichender F\&E-Ressourcen die technologischen Innovationen in neue Produktionsformen möglich, welche die Voraussetzung für den Prozeß der Entkopplung der Umweltbelastung vom ökonomischen Wachstum sind.

Wie Tabelle 11 zeigt, sind die Staaten mit den besten Rangplätzen bezüglich dieses Indikators klassische, hochentwickelte Industriestaaten, welche ihre gegenwärtig gute weltwirtschaftliche Position insbesondere dem Fahrzeug- und Maschinenbau verdanken. Die hohen F\&E-Ausgaben dieser Länder sind somit Ausdruck des Versuches, die gute technologische Position in diesen Industriesektoren zu halten und sich außerdem auch in anderen strategisch wichtigen Bereichen der Hochtechnologiegüter zu positionieren.

Bemerkenswert sind die schlechten Rangplätze der G7-Industriestaaten Italien (16.) und Kanada (14.). Hier scheint im Rahmen der innerstaatlichen Ressourcenallokation ein wichtiger Bereich zur Bewahrung ausreichender technologischer Kapazitäten benachteiligt zu werden.

Ein ähnliches Bild ergibt sich auch für den Quotienten aus den Export- und Importumsätzen von Gütern aus F\&E-intensiven Branchen. Diese Zahl ist ein guter Indikator für das $\mathrm{Maß}$, in welchem sich die F\&E-Ausgaben auch tatsächlich in der Fähigkeit zu Produktion und Export von Hochtechnologiegütern niederschlagen. Negative Beispiele sind in diesem Kontext Finnland und Norwegen, die für den High-Tech-Quotienten einen wesentlich schwächeren Rangplatz aufweisen als für ihre F\&E-Ausgaben. Hier scheinen Forschungsgelder wenig strategiegerecht in eher anwendungsferne Bereiche zu fließen. 
Tabelle 11: Technologische Kapazitäten

\begin{tabular}{|c|c|c|c|c|c|c|c|}
\hline \multirow[t]{2}{*}{ Land } & \multicolumn{6}{|c|}{ Indikatoren der technologischen Kapazität } & \multirow[b]{2}{*}{$\begin{array}{l}\text { Durch- } \\
\text { schnittl. } \\
\text { Rang- } \\
\text { platz }\end{array}$} \\
\hline & $\begin{array}{l}\text { F\&E- } \\
\text { Ausg. } \\
\text { in \% des } \\
\text { BIP }\end{array}$ & $\begin{array}{l}\text { Rang- } \\
\text { platz }\end{array}$ & $\begin{array}{l}\text { Exporte/ } \\
\text { Importe v. } \\
\text { HighTech } \\
\text {-gütern }^{\text {b }}\end{array}$ & $\begin{array}{l}\text { Rang- } \\
\text { platz }\end{array}$ & $\begin{array}{l}\text { F\&E-Bil- } \\
\text { dungsausg } \\
\text { in } \% \text { des } \\
\text { BIP }^{c}\end{array}$ & $\begin{array}{l}\text { Rang- } \\
\text { platz }\end{array}$ & \\
\hline Japan & 3,00 & 1 & 3,67 & 1 & 0,56 & 3 & 1,67 \\
\hline Schweiz & 2,68 & 4 & 1,60 & 3 & 0,67 & 2 & 3,00 \\
\hline Schweden & 2,86 & 2 & 1,00 & 8 & 0,79 & 1 & 3,67 \\
\hline Deutschland & 2,50 & 5 & 1,03 & 5 & 0,41 & 9 & 6,33 \\
\hline USA & 2,81 & 3 & 1,02 & 7 & 0,40 & 11 & 7,00 \\
\hline Frankreich & 2,40 & 6 & 1,04 & 4 & 0,37 & 13 & 7,67 \\
\hline Niederlande & 1,86 & 9 & 0,89 & 10 & 0,48 & 5 & 8,00 \\
\hline Finnland & 2,18 & 8 & 0,74 & 13 & 0,48 & 5 & 8,67 \\
\hline Dänemark & 1,70 & 11 & 1,03 & 5 & 0,38 & 12 & 9,33 \\
\hline Großbritannien & 2,22 & 7 & 0,99 & 9 & 0,36 & 14 & 10,00 \\
\hline Belgien & 1,67 & 12 & 0,77 & 12 & 0,44 & 7 & 10,33 \\
\hline Norwegen & 1,84 & 10 & 0,34 & 18 & 0,49 & 4 & 10,67 \\
\hline Österreich & 1,53 & 13 & 0,74 & 13 & 0,44 & 7 & 11,00 \\
\hline Irland & 1,08 & 17 & 1,63 & 2 & 0,25 & 18 & 12,33 \\
\hline Kanada & 1,51 & 14 & 0,48 & 15 & 0,40 & 10 & 13,00 \\
\hline Italien & 1,31 & 16 & 0,82 & 11 & 0,27 & 17 & 14,67 \\
\hline Australien & 1,35 & 15 & 0,15 & 20 & 0,35 & 15 & 16,67 \\
\hline Portugal & 0,61 & 20 & 0,44 & 16 & 0,22 & 19 & 18,33 \\
\hline Spanien & 0,85 & 19 & 0,43 & 17 & 0,21 & 20 & 18,67 \\
\hline Türkei & 0,50 & 21 & 0,19 & 19 & 0,34 & 16 & 18,67 \\
\hline Neuseeland & 0,88 & 18 & 0,14 & 21 & 0,17 & 21 & 20,00 \\
\hline Griechenland & 0,46 & 22 & 0,11 & 22 & 0,16 & 22 & 22,00 \\
\hline
\end{tabular}

Quelle: OECD (1995): OECD in Figures, in: The OECD Observer, Nr. 194, S. 56-61. Siehe auch die dortigen Anmerkungen.

Anm.:

a. Inländische Bruttoausgaben für Forschung und Entwicklung als Prozentsatz des Bruttoinlandsproduktes (in Kaufkraftparitäten). Daten soweit verfügbar aus 1992.

b. Quotient aus Export und Import von Gütern aus F\&E-intensiven Industriesektoren: Luftund Raumfahrt, Büromaschinen, Computer, elektronische Bauteile, Arzneimittel, Elektrogeräte. Daten soweit verfügbar aus 1992.

c. Öffentliche Bildungsausgaben zur F\&E-Spitzenförderung als Prozentsatz des Bruttoinlands-produktes. Daten aus 1991-92. 
Erstaunlicherweise ganz anders ist der Fall bei Irland gelagert, das für diesen Indikator den zweiten Rangplatz (!) aufweist, während sich seine F\&EAusgaben auf den hinteren Positionen befinden.

Wie schon für den ersten Faktor, so ist auch für das Export-/Importverhältnis von Hochtechnologieprodukten der japanische Wert der beste. Der extrem hohe Quotient von 3,67 spiegelt das ausgeprägte Vermögen Japans zu einer strategisch angelegten, auf ökonomische und technologische Zukunftsfelder ausgerichteten Wirtschaftspolitik wider. Bemerkenswert in diesem Kontext ist deshalb auch, daß drei G7-Staaten über einen eher mäßigen Mittelplatz nicht hinaus kommen (Großbritannien: 9.; Italien: 11.; Kanada: 15.).

Der dritte Indikator mißt die öffentlichen Bildungsausgaben eines Staates auf dem Gebiet der F\&E-Spitzenförderung, also die Bereitschaft eines Landes, einen Teil seiner monetären Ressourcen in den Erhalt und die Erweiterung des Humankapitals zu investieren. Ein geringer Wert für diese Variable bedeutet den Verzicht auf einen für die Innovationsfähigkeit eines Staates notwendigen Bildungsgrad, da auf die Rekrutierung neuer wissenschaftlicher Eliten weitgehend verzichtet wird.

Den besten Wert für diesen Indikator erreicht Schweden (0,79\%). Außer diesem Staat befinden sich noch zwei weitere skandinavische Staaten, nämlich Norwegen (4. Rangplatz) und Finnland (5.) auf den vorderen Plätzen.

Unter den großen G7-Staaten liegen die Werte Deutschlands $(0,41 \%, 9$.$) , Kana-$ das $(0,40 \%, 10$. ) sowie vor allem Japans $(0,56 \%, 3$.) auf einem eher hohen $\mathrm{Ni}$ veau, während die vier anderen Nationen einen erstaunlich niedrigen Indikatorwert aufweisen. Zwar gelingt es den USA und Frankreich, durch ein System elitärer privater Bildungs- und Forschungseinrichtungen ein ausreichend großes Humankapital sicherzustellen, doch für Großbritannien $(0,36 \%, 14$.) und Italien $(0,27 \%, 17$. $)$ ist $z u$ erwarten, daß sich die geringen Investitionen in Spitzenforschung und -ausbildung mittel- oder langfristig in einem die Konkurrenz- und Innovationsfähigkeit irreparabel schädigenden Verlust an Know-How niederschlagen werden.

Wie auch bei den anderen beiden Indikatoren, bilden die südeuropäischen Staaten sowie Australien und Neuseeland die Schlußlichter der Rangreihe.

In der letzten Spalte von Tabelle 11 ist der durchschnittliche Rangplatz der einzelnen Staaten für die drei ausgewählten Indikatoren technologischer Handlungskapazität aufgeführt.

Die besten Werte für diesen Teilaspekt staatlicher Strategieorientierung weisen Japan, die Schweiz und Schweden auf. Hier scheinen die drei wichtigsten Elemente einer zukunftsfähigen Technologiepolitik zusammenzutreffen: die Bereitstellung von Ressourcen zu F\&E-Zwecken, die Lenkung dieser Aktivitäten in Richtung zukunftsträchtiger Hochtechnologiebereiche sowie die langfristige Unterstützung dieser Maßnahmen durch die Heranbildung und ständige Ergän- 
zung einer gut ausgebildeten techno-ökonomischen Elite. In diesem Sinne beweisen diese Staaten ihre Fähigkeit zu strategieorientierter Politik.

Es bleibt jedoch anzumerken, daß die zur Messung der technologischen Kapazität verwendeten Indikatoren teilweise relative Zahlen messen, also die Fertigkeiten auf technischem Gebiet gemessen an der Größe der jeweiligen Volkswirtschaften. Da bestimmte technologische Innovationen jedoch ein so hohes absolutes Investitionskapital benötigen, daß selbst multinationale Großkonzerne diese Vorhaben nur noch in strategischen Allianzen mit anderen Partnern bewältigen können, konzentrieren sich die entsprechenden F\&E-Aktivitäten auch auf die größeren Industriestaaten. Diese verfügen zwar über geringere relative, dafür jedoch absolut ausreichende technologische Kapazitäten für solche Projekte. In diesem Sinne bedeuten die relativ guten Rangplätze der großen Industriestaaten Japan, Frankreich, Deutschland und USA daß die eigentliche Fähigkeit zu strategischer Technologiepolitik bei diesen Ländern liegt. Und diese Fähigkeit zu strategischen, investitionsintensiven Innovationen ist wiederum notwendig, damit zum einen durch additive Maßnahmen die ökologische und ökologisch-ökonomische Effizienz bestehender Produktionsverfahren verbessert und zum anderen vollkommen neue, ökologisch entlastende Fertigungsund Energiegewinnungsmethoden entwickelt werden können.

Bedenklich erscheint deshalb der relative Mangel an technologischen Kapazitäten in den wichtigen Industrieländern Großbritannien, Kanada und Italien.

Die südeuropäischen EU-Staaten sowie die Türkei bilden die Schlußlichter der OECD-Rangfolge und verfügen demnach über nur sehr geringe Möglichkeiten einer mittel- oder langfristigen F\&E-Politik auf dem Gebiet von Hochtechnologieprodukten.

\subsubsection{Administrative Kapazitäten}

Das Bestehen effizienter administrativer Strukturen zur Implementation und Kontrolle von getroffenen Maßnahmen ist ein unerläßlicher Bestandteil einer strategische Politik ermöglichenden Handlungskapazität. Fehlen die notwendigen monetären und humanen Ressourcen im Bereich der Administration, können weitreichend intendierte politische Entscheidungen zu bloßen Policy- Modifikationen ohne praktische Relevanz degenerieren.

Zur Messung der administrativen Kapazitäten eines Staates werden im folgenden die beiden Indikatoren Ausgaben für staatliche Dienstleistungen (inklusive Verwaltung) als Prozentsatz des BIP sowie der Anteil der im öffentlichen Sektor Beschäftigten an der Gesamtzahl aller Beschäftigten verwendet.

Zwar bedeuten das Ausmaß der Ausgaben für staatliche Dienstleistungen sowie die schiere Größe der öffentlichen Verwaltung noch keine automatische Effizi- 
enz administrativen Handelns, dennoch macht die Verwendung dieser Indikatoren aus Sicht des Verfassers Sinn:

- Der Rückgriff auf verläßliches Zahlenmaterial zur quantitativen Erfassung der Effizienz und Handlungskapazität von öffentlichen Verwaltungen ist nicht möglich.

- Die Ausgabenhöhe für Dienstleistungen des Staates gibt insofern einen guten Hinweis auf die Handlungskapazität eines Landes, als daß alleine durch die Möglichkeit der Zuweisung von finanziellen Ressourcen die Fähigkeit zum zielgerichteten administrativen Handeln gegeben ist, oder negativ formuliert: ohne eine ausreichende Finanzausstattung der öffentlichen Verwaltung ist eine effektive Administration ausgeschlossen.

Zwar bergen große Verwaltungen immer die Gefahr der Ineffizienz und Selbstvermehrung in sich, dennoch ist die Bewältigung komplexer und regional gebundener Implementations- und Kontrollaufgaben als Konsequenz staatlicher Gesetzgebung (zum Beispiel auch auf dem Feld des Umweltschutzes) nur möglich, wenn eine Administration personell hinreichend bestückt ist.

Somit können die in Tabelle 12 dargestellten Resultate mit der gebotenen Sorgfalt interpretiert werden.

Auffällig ist das hohe Maß an Korrelation zwischen beiden Variablen. Für kaum einen Staat weichen die Rangplätze bedeutend voneinander ab.

Wie im Falle der Ausgaben für F\&E-Spitzenförderung, so nehmen die skandinavischen Staaten auch im Hinblick auf die Bereitstellung öffentlicher Mittel zur Schaffung von administrativen Ressourcen die vorderen Plätze ein. Lediglich Frankreich erreicht hier ähnlich hohe Werte.

Die über hohe relative und absolute technologische Kapazitäten verfügenden Industriestaaten nehmen demgegenüber entweder nur schwache mittlere (USA, Deutschland und die Schweiz) oder absolut hintere Ränge (Niederlande und Japan) ein. 


\begin{tabular}{|c|c|c|c|c|c|}
\hline \multirow[b]{2}{*}{ Land } & \multicolumn{4}{|c|}{ Indikatoren der administrativen Kapazität } & \multirow[b]{2}{*}{$\begin{array}{l}\text { Durchschnittl } \\
\text { Rangplatz }\end{array}$} \\
\hline & $\begin{array}{l}\text { Öfentliche } \\
\text { Ausgaben in } \\
\% \text { des BIP }\end{array}$ & Rangplatz & $\begin{array}{l}\text { Anteil der } \\
\text { durch den } \\
\text { Staat Be- } \\
\text { schäftigten an } \\
\text { allen Be- } \\
\text { schäffigten }^{b} \\
\end{array}$ & Rangplatz & \\
\hline Schweden & 21,2 & 1 & 32,4 & 1 & 1 \\
\hline Dänemark & 19,4 & 2 & 31,1 & 2 & 2 \\
\hline Finnland & 19,1 & 3 & 24,8 & 4 & 3,5 \\
\hline Norwegen & 16,3 & 5 & 28,7 & 3 & 4 \\
\hline Frankreich & 17,0 & 4 & 24,3 & 5 & 4,5 \\
\hline Kanada & 14,2 & 7 & 20,7 & 8 & 7,5 \\
\hline Österreich & 14,0 & 8 & 21,0 & 7 & 7,5 \\
\hline Irland & 14,3 & 6 & 18,5 & 11 & 8,5 \\
\hline Großbritannien & 12,7 & 10 & 19,3 & 9 & 9,5 \\
\hline Neuseeland & 11,7 & 16 & 22,1 & 6 & 11 \\
\hline Italien & 12,8 & 9 & 16,1 & 13 & 11 \\
\hline Belgien & 11,9 & 15 & 19,3 & 9 & 12 \\
\hline Spanien & 12,1 & 13 & 15,0 & 14 & 13,5 \\
\hline Portugal & 12,7 & 10 & 13,6 & 17 & 13,5 \\
\hline USA & 12,5 & 12 & 14,5 & 16 & 14 \\
\hline Deutschland & 10,3 & 17 & 14,9 & 15 & 16 \\
\hline Schweiz & 12,1 & 13 & 12 & 19 & 16 \\
\hline Australien & 4,0 & 22 & 17,3 & 12 & 17 \\
\hline Türkei & 10,7 & 18 & k.A. & & 18 \\
\hline Niederlande & 9,9 & 19 & 12,8 & 18 & 18,5 \\
\hline Griechenland & 9,7 & 20 & k.A. & & 20 \\
\hline Japan & 7,7 & 21 & 5,9 & 20 & 20,5 \\
\hline
\end{tabular}

Quelle: OECD (1995): OECD in Figures, in: The OECD Observer, Nr. 194, S. 38 f. und 40 f. Siehe auch die dortigen Anmerkungen.

Anm:

a. Ausgaben für öffentliche Aufgaben (government services)

b. Anteil der mit der Erfüllung öffentlicher Aufgaben Beschäftigter 


\subsubsection{Gesamteinschätzung}

Eine Integration aller drei Teilaspekte staatlicher Strategieorientierung ergibt die in Tabelle 13 dargestellte Gesamteinschätzung der untersuchten Staaten. Danach erfüllen 8 der 22 OECD-Staaten das hier untersuchte Kriterium. Insbesondere in Kanada (föderalistischer Staat mit hoher administrativer Kapazität), Frankreich (dezentraler Einheitsstaat mit hoher technologischer und administrativer Kapazität), Deutschland (föderalistischer Staat mit hoher technologischer Kapazität) und den USA (wie Deutschland) scheinen die politischen Eliten über ein hohes $\mathrm{Maß}$ an Strategieorientierung zu verfügen. Auch in den Alpenländern sowie Belgien und Schweden überwiegen hinsichtlich der Einzelindikatoren die positiven Einschätzungen. Während in Österreich und Belgien die nur durchschnittlichen Handlungskapazitäten auf den Gebieten der Technologie und Verwaltung durch den föderalen Staatsaufbau kompensiert werden, gleicht die Schweiz mangelnde administrative Ressourcen durch Technologieorientierung und den besonders ausgeprägten Dezentralismus seines Kantonalsystems aus. Einen Sonderfall bildet auch der zentralistische Einheitsstaat Schweden. Zwar fehlen hier die Vorteile eines föderalen Staatsaufbaus, doch der dritte Rangplatz bei den Indikatoren zur Messung der technologischen Kapazitäten und der erste Rangplatz für die Verwaltungskapazität führen zur Einstufung Schwedens als strategieorientiertem Staat.

$\mathrm{Zu}$ den reaktiven Staaten zählen die vier sozio-kulturell angelsächsisch geprägten Länder Australien, Neuseeland, Irland und Großbritannien sowie Italien und die vier Staaten der südlichen Peripherie Europas. Mit Griechenland und der Türkei gibt es sogar zwei Staaten, die in allen drei Einzelindikatoren negative Bewertungen erhalten. Der politische Prozeß scheint von den politischen Eliten dieser Staaten mit einem äußerst geringen $\mathrm{Ma}$ an Vorausssicht und präventiver Aktion vollzogen $\mathrm{zu}$ werden.

\subsection{Partizipationsgrad}

\subsubsection{Korporatismusgrad}

Die in Tabelle 14 dargestellten Korporatismusgrade der einzelnen OECDStaaten stellen die Durchschnittswerte zahlreicher Quantifizierungsversuche der politikwissenschaftlichen Literatur dar ${ }^{400}$. Während in einigen Studien die Ein- 
stufungen das Resultat von Plausibilitätsüberlegungen sind, wurde in den meisten Analysen eine Operationalisierung dieser Variable anhand der Aggregierung von objektiv meßbaren Indikatoren durchgeführt.

Da der Korporatismusbegriff zur Beschreibung kooperativer, nongovernmentaler Aushandlungsmechanismen zwischen Wirtschafts- und Arbeitnehmervertretern, in welchen die Regierung höchstens die Rolle eines Initiators oder Moderators übernimmt, verwendet wird, sind die genutzten Indikatoren folglich auch Größen zur Messung der Konflikthäufigkeit und -intensität zwischen diesen beiden Interessengruppen sowie des Grades der Formierung und Organisation ihrer jeweiligen Verbände (insbesondere der Gewerkschaften).Die so gewonnenen durchschnittlichen Indikatorwerte werden im Zusammenhang dieser Studie zur Messung des Partizipationsgrades der nicht-staatlichen Interessengruppen am politischen Entscheidungsprozeß genutzt. Die Übertragung dieser eigentlich den wirtschaftspolitischen Prozeß betreffenden Variable auf das Gebiet der Ökologie erscheint deshalb sinnvoll, weil es sich bei den korporatistischen Strukturen nicht um bewußt geschaffene, zweckbezogene Instrumente staatlicher Wirtschaftspolitik handelt, sondern um den Ausdruck allgemeiner, kulturell und historisch gewachsener Konfliktlösungsmuster einzelner Gesellschaften. Diese verinnerlichten Konfliktlösungsmuster werden sich folglich auch in anderen Feldern staatlichen Handelns in ähnlicher Form wiederfinden lassen. Mit anderen Worten: ein Staat, dessen Beziehungen zwischen Arbeit und Kapital eher korporatistisch organisiert sind, wird auch im ökologischen Politikfeld korporatistisch agieren. Dies meint nicht unbedingt, da $\beta$ zwischen Emittenten und Ressourcennutzern einerseits sowie Umweltschutzverbänden und ökologischen Parteien andererseits institutionalisierte Verhandlungsrunden über ein akzeptables $\mathrm{Ma} \beta$ an Umweltbelastung abgehalten werden, sondern daß die politischen Eliten ihre Aufgabe auch auf dem Feld der Umweltpolitik als das Initiieren und Steuern von gesellschaftlichen Prozessen des Ausgleichs von ökonomischen und ökologischen Interessen, sowie solchen der Konsumenten, verstehen.

Ein Blick auf den degree of corporatism der einzelnen OECD-Staaten zeigt deutliche Unterschiede. Sehr hohe Werte von mehr als 1 erreichen Österreich, Norwegen und Schweden. Ebenfalls positive Werte zwischen 0.258 und 0.518 sind für die beiden anderen skandinavischen Staaten, Deutschland und die Schweiz zu verzeichnen. Dieses Resultat belegt die Einschätzung, daß korpora-

${ }^{400}$ Vgl. Crepaz, Markus M. L. (1992): Corporatism . . ., a.a.O., S. 148 f. sowie die dort gemachten Literaturhinweise auf insgesamt 12 quantitative Korporatismus-Studien aus den Jahren 1981 bis 1985 . Deren Werte wurden so standardisiert, daß der Mittelwert 0 und die Standardabweichung 1 beträgt. 
tistische Strukturen eher für bevölkerungsschwache Staaten typisch sind (Ausnahme: Deutschland).

Tabelle 13: Strategieorientierung der OECD-Staaten

\begin{tabular}{lcccc}
\hline & \multicolumn{3}{c}{ Kriterien der Strategieorientierung } & \\
\cline { 2 - 4 } Land & Föderalismus & $\begin{array}{c}\text { Technologische } \\
\text { Kapazităt }\end{array}$ & $\begin{array}{c}\text { Administrative } \\
\text { Kapaziăta }\end{array}$ & $\begin{array}{c}\text { Maß der } \\
\text { Strategieorient. }\end{array}$ \\
\hline Australien & + & - & - & niedrig \\
Österreich & + & 0 & + & hoch \\
Belgien & + & 0 & 0 & hoch \\
Kanada & + & 0 & + & hoch \\
Dänemark & - & 0 & + & mittel \\
Finnland & - & 0 & + & mittel \\
Frankreich & 0 & + & + & hoch \\
Deutschland & + & + & - & hoch \\
Griechenland & - & - & - & niedrig \\
Irland & - & 0 & 0 & niedrig \\
Italien & 0 & - & 0 & niedrig \\
Japan & 0 & + & - & mittel \\
Niederlande & 0 & + & - & mittel \\
Neuseeland & - & - & 0 & niedrig \\
Norwegen & - & 0 & + & mittel \\
Portugal & - & - & 0 & niedrig \\
Spanien & 0 & - & 0 & niedrig \\
Schweden & - & + & + & hoch \\
Schweiz & + & + & - & hoch \\
Türkei & - & - & - & niedrig \\
Großbritannien & - & 0 & 0 & niedrig \\
USA & + & + & 0 & hoch \\
\hline & & & &
\end{tabular}

Anm.:

a) Bewertung gemäß Tabelle 10; + := konföderaler/unitaristischer Einheitsstaat ; 0 := dezentraler Einheitsstaat; - := Einheitsstaat

b) Bewertung gemäß Tabelle $11 ;+$ := erster bis siebter durchschnittlicher Rangplatz; 0 := achter bis fünfzehnter durchschnittlicher Rangplatz; - := sechzehnter bis zweiundzwanzigster durchschnittlicher Rangplatz

c) Bewertung gemäß Tabelle 12; Bedeutung der Zeichen: siehe Anm. b)

d) hoch := „+“ überwiegt; mittel := „+“ und „““ halten sich die Waage; niedrig := „““ überwiegt 
Einen Mittelplatz nimmt Japan ein. An diesem Land scheiden sich offenbar die Geister der Korporatismusforscher, da die Konsensbeziehungen zwischen Kapital und Arbeit im Falle Nippons unter der stark akzentuierten Aufsicht der Regierung bei gleichzeitig eher geringer Formierung des Arbeitnehmerlagers organisiert sind. Wissenschaftler, die in ihrer Definiton des Korporatismusbegriffes vor allem auf die Freiwilligkeit der Kooperationsbemühungen abheben, sehen in japanischen Modell statt Korporatismus eher einer Form von "concertation without labour" " ${ }^{401}$ Für die anderen Autoren, welche den Konsensgedanken zwischen den ökonomischen Interessengruppen als das entscheidende Merkmal verstehen, sind die ökonomischen Strukturen des ostasiatischen Landes als im hohen Maße korporatistisch zu bezeichnen ${ }^{402}$.

Negative Korporatismusgrade lassen sich zum einen für die Gruppe der sechs angelsächsisch geprägten Staaten, deren ökonomischen Binnenbeziehung durch die liberalen Grundsätze des unbedingten Wettbewerbs sowie des Konfliktes der gesellschaftlichen Schichten geprägt sind, sowie die Mittelmeeranrainer Frankreich und Italien feststellen. Für die drei südeuropäischen Länder und die Türkei finden sich in den vorliegenden Studien zwar keine Angaben, die durch den ökonomischen Transformationsprozeß erst teilweise erfaßten, durch eine lange Periode autoritärer Dominanz von Regierung und Großkapital geprägten politischen und ökonomischen Strukturen lassen eine Subsumierung dieser Staaten unter die nicht-korporatistischen Staaten jedoch sinnvoll erscheinen.

\subsubsection{Konfliktlösungsverhalten}

Mit der Variable Konfliktlösungsverhalten soll gemessen werden, inwieweit ein Staat kooperative, konsensbetonte Mechanismen der Konfliktlösung kultiviert hat. Es geht anders als im Fall des Korporatismus also nicht darum, die Problemlösung den machtpolitisch mehr oder weniger gleichstarken Betroffenen alleine zu überlassen, sondern ein konsensuales Konfliktlösungsverhalten liegt dann vor, wenn die Regierung ihre legislative und exekutive Macht nicht zu einer die gesellschaftliche Fragmentierung fördernden, rigorosen Durchsetzung

\footnotetext{
${ }^{401}$ Vgl. Lehmbruch, Gerhard (1984): Concertation ..., a.a.O.

${ }^{402}$ Vgl. Schmidt, Manfred G. (1982): Does Corporatism Matter?, a.a.O.
} 
souverän beschlossener Maßnahmen nutzt, sondern die Betroffenen in den Pro$z e ß$ der Entscheidungsfindung und -implementation integriert.

Die sich anhand dieser Vorüberlegungen ergebende Skala reicht also von einem aktiv-kooperativen Politikstil bis hin zu einem konfliktären Vorgehen des Laissez-faire. Gemäß Katzenstein sind es vor allem die kleineren Staaten, die sich aufgrund des ökonomischen Anpassungsdrucks des Weltmarktes eine aktive Reformpolitik antrainiert haben ${ }^{403}$.

Wie ein Blick auf die in Tabelle 14 angegebenen, aufgrund von Plausibilitätsüberlegungen vergebenen Werte für diese Variable zeigt, sind es jedoch anscheinend auch historisch-kulturelle Bestimmungsgrößen, die zu unterschiedlichen Ausprägungen des nationalen Konfliktlösungsverhaltens führen.

So zählen zu den konfliktären Staaten außer Großbritannien noch drei weitere am Westminster-Demokratiemodell orientierte Staaten (Kanada, Australien und Neuseeland). Auch die drei romanischen Mittelmeerländer Spanien, Portugal und Italien können als kulturell-historisch homogen Gruppen angesehen werden. Die Gruppe der konfliktären Staaten wird komplettiert durch die beiden erst vor kurzem demokratisierten ehemaligen Diktaturen Griechenland und Türkei sowie die Vereinigten Staaten.

Irland, Finnland sowie die vier unmittelbar aneinander angrenzenden Staaten Deutschland, Frankreich, Belgien und Dänemark bilden die Gruppe der Länder mit einem weder besonders kooperativ noch konfliktär ausgeprägten Konfliktlösungsverhalten.

Die kosensualen Staaten sind die konkordanzdemokratischen Länder Schweiz und Österreich sowie Norwegen, Schweden und die Niederlande. Während diese fünf Staaten tatsächlich dem Katzenbergschen Typus der kleinen, anpassungsfähigen „Reformstaaten“ entsprechen, bildet Japan als großer Industriestaat eine Ausnahme in dieser Gruppe der konsensorientierten Staaten.

${ }^{403}$ Katzenstein, Peter (1985): Small States in World Markets. Industrial Policy in Europe, Ithaca; London, zit. nach: Jänicke, Martin (1990): Umweltpolitik . . ., a.a.O. S. 224. 
Tabelle 14: Partizipationsgrad der OECD-Staaten

\begin{tabular}{lccc}
\hline & \multicolumn{2}{c}{ Kriterien des Partizipationssgrades } & \\
\cline { 2 - 3 } Land & Korporatismusgrad & $\begin{array}{c}\text { Konfliktlosung- }^{\text {verhalten }}{ }^{\mathrm{b}} \\
\text { Partizipationssgrad }\end{array}$ \\
\hline Australien & -1.025 & $-1^{*}$ & niedrig \\
Österreich & 1.6 & 1 & hoch \\
Belgien & 0.258 & 0 & mittel \\
Kanada & -1.335 & -1 & niedrig \\
Dänemark & 0.518 & 0 & mittel \\
Finnland & 0.427 & 0 & mittel \\
Frankreich & -0.725 & 0 & mittel \\
Deutschland & 0.480 & 0 & mittel \\
Griechenland & $-1^{*}$ & -1 & niedrig \\
Irland & -0.528 & 0 & mittel \\
Italien & -0.851 & -1 & niedrig \\
Japan & 0.053 & 1 & hoch \\
Niederlande & 1.006 & 1 & hoch \\
Neuseeland & -1.106 & $-1^{*}$ & niedrig \\
Norwegen & 1.531 & 1 & hoch \\
Portugal & $-1^{*}$ & -1 & niedrig \\
Spanien & $-1^{*}$ & -1 & niedrig \\
Schweden & 1.396 & 1 & hoch \\
Schweiz & 0.505 & 1 & hoch \\
Türkei & $-1^{*}$ & -1 & niedrig \\
Großbritannien & -0.862 & -1 & niedrig \\
USA & -1.341 & -1 & niedrig \\
\hline
\end{tabular}

\section{Anm.:}

a) Die Zahl gibt den durchschnittlichen Korporatismusgrad einzelner Staaten gemäß der zusammenfassenden Studie von Crepaz (1992) an. Ein positiver Wert bedeutet dabei einen zunehmend hohen Grad an korporatistischen Politikstrukturen und -prozessen, ein negativer Wert das Gegenteil.

b) Die Zahl gibt das Ausmaß Konsensorientierung der Beteiligten im politischen Prozeß gemäß einer Studie von Schmidt (1986) an. Dabei bedeutet der Wert „1“ ein hohes, der Wert „0" ein mittleres und der Wert ,-1" ein geringes $\mathrm{Ma} ß$ an Konsensorientierung.

c) Die Einschätzung ,hoch“ ergibt sich, wenn beide Indikatorwerte größer als Null sind, die Einschätzung ,niedrig“, wenn beide Werte kleiner als Null sind. In allen anderen Fällen ergibt sich die Einschätzung ,mittel“".

* := Eigene Einschätzung 


\subsubsection{Gesamteinschätzung der Staaten}

Da sich die Ausprägungen der beiden Indikatorwerte für die meisten Staaten kaum signifikant voneinander unterscheiden, kommen wir hinsichtlich der Gesamteinschätzung zu ähnlichen Staatengruppen wie in den beiden vorhergehenden Abschnitten.

$\mathrm{Zu}$ den partizipativen Staaten zählen somit:

- die beiden skandinavischen Länder Schweden und Norwegen,

- die Alpenländer Österreich und Schweiz,

- die Niederlande sowie

- Japan.

Eine mittlere Gesamteinschätzung ergibt sich für:

- die beiden anderen skandinavischen Staaten: Dänemark und Finnland,

- die Nachbarstaaten Deutschland, Belgien und Frankreich sowie

- Irland.

Die Gruppe der konfliktären Staaten bilden hingegen:

- die übrigen fünf angelsächsisch geprägten Länder und

- die fünf Mittelmeeranrainerstaaten.

\subsection{Positionierung der Staaten}

Ausgehend von den Gesamteinschätzungen der OECD-Staaten bezüglich des Grades ihrer jeweiligen Strategieorientierung und ihres Partizipationsgrades lassen sich die 22 Staaten in einer zweidimensionalen 9-Felder-Matrix positionieren (Abbildung 32).

Daraus ergibt sich, daß mit Österreich, Schweden und der Schweiz drei Staaten für beide Politikstildimensionen einen hohen Ausprägungsgrad aufweisen. Drei weitere Staaten - Japan, die Niederlande und Norwegen - verbinden einen hohen Kooperationsgrad mit einem mittleren $\mathrm{Maß}$ an Strategieorientierung. Eine weitere Staatengruppe bilden Deutschland, Frankreich und Belgien. 


\begin{tabular}{|c|c|c|c|c|}
\hline & & & $\begin{array}{l}\text { egieorientier } \\
\text { litischen Akt } \\
\text { gem. Tabelle }\end{array}$ & der \\
\hline & & hoch & mittel & niedrig \\
\hline & hoch & $\begin{array}{l}\text { Österreich } \\
\text { Schweden } \\
\text { Schweiz }\end{array}$ & $\begin{array}{l}\text { Japan } \\
\text { Niederlande } \\
\text { Norwegen }\end{array}$ & \\
\hline $\begin{array}{l}\text { Partizipations- } \\
\text { grad der } \\
\text { nicht-staat- } \\
\text { lichen Akteure } \\
\text { (gem. Tabelle } \\
\text { 14) }\end{array}$ & mittel & $\begin{array}{l}\text { Belgien } \\
\text { Frankreich } \\
\text { Deutschland }\end{array}$ & $\begin{array}{l}\text { Dänemark } \\
\text { Finnland }\end{array}$ & Irland \\
\hline & niedrig & $\begin{array}{l}\text { Kanada } \\
\text { USA }\end{array}$ & & $\begin{array}{l}\text { Australien } \\
\text { Griechenland } \\
\text { Italien } \\
\text { Neuseeland } \\
\text { Portugal } \\
\text { Spanien } \\
\text { Türkei } \\
\text { Großbritan- } \\
\text { nien } \\
\end{array}$ \\
\hline
\end{tabular}

Deren Kennzeichen ist eine Kombination aus einem hohen $\mathrm{Ma} \beta$ an strategischen Fähigkeiten und einer eher durchschnittlich ausgeprägten Kooperationsfähigkeit der Teilnehmer am politischen Prozeß. Für beide Merkmalsdimensionen mittlere Werte weisen Dänemark und Finnland auf. In einem der neun Matrix-Felder finden wir nur einen Staat: Irland verfügt über ein nur geringes $\mathrm{Ma} ß$ 
an Strategieorientierung bei gleichzeitig mittlerem Kooperationsgrad. Während das Feld mit der Merkmalskombination ,partizipativ/gering strategieorientiert" leer bleibt, finden sich für das entgegengesetzte Feld „konfliktär/strategieorientiert" zwei Staaten: Kanada und die USA. Die meisten Objekte weist das Feld des konfliktären und nur geringfügig strategieorientierten Politikstils aus. Hier sind sowohl die ökonomisch nachholenden Staaten Südeuropas (Spanien, Portugal und Türkei), Italien als auch drei historisch-kulturell angelsächsisch geprägte Staaten (Neuseeland, Australien und Großbritannien) positioniert.

Eine Analyse dieser noch recht groben Methode einer Positionierung anhand von ordinalskalierten Ausprägungen der Politikstilindikatoren ergibt drei wesentliche Erkenntnisse:

- Es scheint zwar eine gewisse Korrelation zwischen beiden Dimensionen zur Messung des nationalen Politikstils zu geben (in den drei diagonalen Feldern sind 13 der 22 OECD-Staaten positioniert), dennoch erlauben diese ein hohes $\mathrm{Ma} ß$ an Differenzierung ( 7 der 9 Felder sind besetzt);

- Die aufgrund „harter“ Indikatoren vorgenommene Positionierung ergibt eine erstaunliche Homogenität der so gewonnenen Ländergruppen hinsichtlich geographischer (USA/Kanada oder Belgien/Frankreich/Deutschland) oder historisch-kultureller (Spanien/Portugal) Merkmale;

- Eine Kombination von hohem Kooperationsgrad sowie nur gering strategieorientiertem Problemlösungsverhalten kommt als praktischer Politikstil nicht vor. Die im Modellentwurf gewonnenen Idealtypen sind aus diesem Grunde zu modifizieren.

Um eine aussagekräftigere Positionierung der untersuchten Staaten zu gewährleisten, wurden diese mittels einer Clusteranalyse anhand der acht Einzelindiaktoren beider Dimensionen zu Gruppen zusammengefaßt. Dieses multivariate Statistikverfahren separiert die Grundgesamtheit auf Grundlage ihrer Variablenwerte in verschiedene Cluster, die zwei Hauptkriterien erfüllen:

- Zum einen sollen die Varianzen innerhalb der einzelnen Cluster möglichst gering sein, und

- zum anderen soll die Distanz zwischen den Gruppen einen möglichst großen Wert einnehmen ${ }^{404}$.

${ }^{404}$ Backhaus, K. et al. (1980): Multivariate Analysemethoden - Eine anwendungsorientierte Einführung, Berlin; Heidelberg; New York, S. 105 ff. 


\section{Tabelle 15: Clusteranalyse der OECD-Staaten aufgrund ihrer Politikstil- merkmale (Gruppenzentroide)}

\begin{tabular}{|c|l|r|r|r|r|r|r|r|}
\hline $\begin{array}{l}\text { Cluster- } \\
\text { nummer }\end{array}$ & $\begin{array}{l}\text { Dezentr. } \\
\text { grad }\end{array}$ & $\begin{array}{l}\text { F\&E- } \\
\text { Ausgb. }\end{array}$ & $\begin{array}{l}\text { Ex/Imp. } \\
\text { HiTech }\end{array}$ & $\begin{array}{l}\text { Bildg.- } \\
\text { ausgb. }\end{array}$ & $\begin{array}{l}\text { Öffentl. } \\
\text { Ausgb. }\end{array}$ & $\begin{array}{l}\text { Staats- } \\
\text { Beschaf. }\end{array}$ & $\begin{array}{l}\text { Korpora- } \\
\text { timus }\end{array}$ & $\begin{array}{l}\text { Konflikt- } \\
\text { losung }\end{array}$ \\
\hline 1 & .541 & 1.026 & 1.593 & 1.175 & -.618 & -1.357 & .730 & 1.418 \\
\hline 2 & -.336 & -1.120 & -.743 & -.974 & -.823 & -.400 & -.850 & -.982 \\
\hline 3 & -.534 & .394 & -.142 & .817 & 1.055 & 1.384 & 1.329 & .938 \\
\hline 4 & .486 & .398 & .161 & -.113 & .334 & -.007 & -.413 & -.296 \\
\hline
\end{tabular}

Anm.: Die Werte sind die z-transformierten Mittelwerte der einzelnen Gruppen. Es wurde folgendes partionierendes Clusterverfahren ausgewăhlt: Iteratives Minimaldistanzverfahren und Gruppenzentroide

Tabelle 16: Clustervarianzen und -zugehörigkeiten

\begin{tabular}{|l|l|l|l|l|}
\hline Cluster-Nr. & 1 & \multicolumn{1}{|c|}{2} & \multicolumn{1}{c|}{4} \\
\hline $\begin{array}{l}\text { Cluster- } \\
\text { varianz }\end{array}$ & 12.0 & 13.8 & 20.6 & 22.2 \\
\hline $\begin{array}{l}\text { Cluster- } \\
\text { bezeichnung }\end{array}$ & $\begin{array}{l}\text { Technologisch- } \\
\text { dezentraler } \\
\text { Korporatismus }\end{array}$ & $\begin{array}{c}\text { Laissez- } \\
\text { faire }\end{array}$ & $\begin{array}{l}\text { Administrativ- } \\
\text { zentralistischer } \\
\text { Korporatismus }\end{array}$ & $\begin{array}{l}\text { Innovativer } \\
\text { Pluralismus }\end{array}$ \\
\hline Elemente & $\begin{array}{l}\text { Japan } \\
\text { Niederlande } \\
\text { Schweiz }\end{array}$ & $\begin{array}{l}\text { Australien } \\
\text { Neuseeland } \\
\text { Griechenland } \\
\text { Italien } \\
\text { Portugal } \\
\text { Spanien } \\
\text { Türkei }\end{array}$ & $\begin{array}{l}\text { Österreich } \\
\text { Dänemark } \\
\text { Finnland } \\
\text { Norwegen } \\
\text { Schweden }\end{array}$ & $\begin{array}{l}\text { Kanada } \\
\text { USA } \\
\text { Belgien } \\
\text { Frankreich } \\
\text { Deutschland } \\
\text { Irland } \\
\text { G.-Britannien }\end{array}$ \\
\hline
\end{tabular}

Das Ergebnis der Analyse ist in den Tabellen 15 und 16 dargestellt. Die vier Cluster lassen sich wie folgt beschreiben:

- Gruppe 1: Dieses Cluster, aufgrund der geringen Fallzahl das homogenste unter allen Gruppen (niedrigste Clustervarianz), ist charakterisiert durch ein hohes $\mathrm{Ma} \beta$ an Dezentralität, sehr hohe technologische Kapazitäten bei gleichzeitig geringen administrativen Ressourcen sowie einem hohen Partizipationsgrad während des politischen Prozesses. D.h. diese Gruppe weicht insofern vom „idealen“ Politikstil des antizipativen Korporatismus ab, als daß 
ein Element der Strategieorientierung, nämlich ein hohes $\mathrm{Ma} ß$ an administrativer Kapazität, fehlt. Den durch Gruppe 1 repräsentierten Politikstil wollen wir deshalb im folgenden als „technologisch-dezentralen Korporatismus“ bezeichnen.

- Gruppe 2: Die Merkmalsausprägungen dieses Clusters, nämlich ein eher geringer Föderalismusgrad, niedrige technologische und administrative Kapazitäten sowie ein sehr konfliktäres Politikverhalten entsprechen exakt dem im Modellentwurf als „Laissez faire“ bezeichneten Politikstil. Bemerkenswert ist auch die für eine große Homogenität sprechende, trotz der relativ hohen Fallzahl von sieben Clusterelementen eher geringe Varianz innerhalb der Gruppe.

- Gruppe 3: Wie Gruppe 1 weist auch dieses Cluster neben einem hohen Partizipationsgrad nur für zwei der drei Strategieorientierung ausmachenden Elemente hohe Werte auf. Die Position des Gruppenzentroids belegt zum einen ein eher hohes $\mathrm{Ma} ß$ an technologischer Kapazität und zum anderen die Existenz sehr ausgeprägter monetärer und personeller Ressourcen zur administrativen Implementation strategisch gewollter Politikinhalte. Der Unterschied dieses Clusters im Vergleich mit dem idealen Politikstil liegt also im zentralistischen Staatsaufbau. Die Bezeichnung dieser Gruppe soll deshalb „administrativ-zentralistischer Korporatismus“ lauten.

- Gruppe 4: Anhand des Gruppenzentroids läßt sich dieses Cluster wie folgt beschreiben: eher dezentraler Staatsaufbau, leicht überdurchschnittliche technologische und administrative Kapazitäten sowie ein eher konfliktäres Verhalten im politischen Prozeß. Als Bezeichnung für dieses mit Abstand heterogenste Cluster soll die im Modellentwurf für einen strategieorientierten und konfliktären Politikstil eingeführte Benennung als ,innovativer Pluralismus“ beibehalten werden, auch wenn die Werte des Gruppenzentroids nur leicht über dem einen mittleren Merkmalsausprägungsgrad repräsentierenden Nullpunkt beider Hauptdimensionen liegen.

Insgesamt ist also festzustellen, daß zwei der mittels Clusteranalyse gefundenen Gruppen (2 und 4) einen Politikstil aufweisen, der mit den im Modellentwurf beschriebenen Idealtypen weitgehende Ähnlichkeiten aufweist. Während das Feld des reaktiven Korporatismus leer bleibt, ergeben sich für die Kombination von hoher Strategieorientierung und ausgeprägtem Kooperationsgrad zwei Cluster, die im Falle einer zweidimensionalen Darstellung ihrer Zentroide nahe beieinander liegen (Abbildung 33). Anscheinend ist ein Politikstil. der sowohl partizipativ ausgerichtet ist, als auch für alle drei Kriterien der Strategieorientierung überdurchschnittliche Werte aufweist, praktisch nicht möglich. Dies mag vor allem daran liegen, daß Zentralismus und administrative Kapazitäten positiv korrelieren, und Kombinationen wie Österreichs oder Kanadas, die einen föderalen Staatsaufbau mit einer gleichzeitig hohen Ressourcenbereitstellung für 
administrative Zwecke verbinden, die Ausnahme bilden. Ein im Vergleich zur 9-Felder-Matrix weiterer auffälliger Aspekt des Resultates der Clusteranalyse ist die mit Ausnahme von Gruppe 1 noch weitgehende regionale und historischkulturelle Homogenität der Cluster bzw. Gruppen innerhalb der Cluster. So finden sich alle Staaten Südeuropas in Gruppe 2, sämtliche skandinavischen Staaten in Gruppe 3 und die beiden nordamerikanischen Staaten, die beiden englischsprachigen Inselstaaten sowie die Staatengruppe Deutschland-FrankreichBelgien in Gruppe 4.

Diese Tatsache ist ein guter Indikator für die Richtigkeit der Vermutung, daß der sich in quantitativen Indikatorwerten der Staaten ausdrückende Politikstil im wesentlichen kulturell determiniert ist. Mit der Einführung weiterer Politikstildimensionen ließe sich womöglich eine in diesem Sinne noch homogenere Clusterbildung erreichen.

In den nachfolgenden Analyseschritten sollen die mittels der Clusteranalyse gewonnenen Ländergruppen auf die jeweilige Effektivität ihrer Umweltpolitik untersucht werden. Dabei soll in Modifikation der Ausgangsthese davon ausgegangen werden, daß sowohl der technologisch-dezentrale als auch der administrativ-zentralistische Korporatismus signifikant bessere Werte für die Umweltbelastungsindikatoren aufweisen werden als die beiden anderen Politikstiltypen, insbesondere besser als der Laissez-faire-Stil. Wie sich die beiden Korporatismustypen hinsichtlich ihrer ökologischen Performanz unterscheiden, ist ebenfalls zu untersuchen. 


\section{Abb. 33: Positionierung der Clusterzentroide}

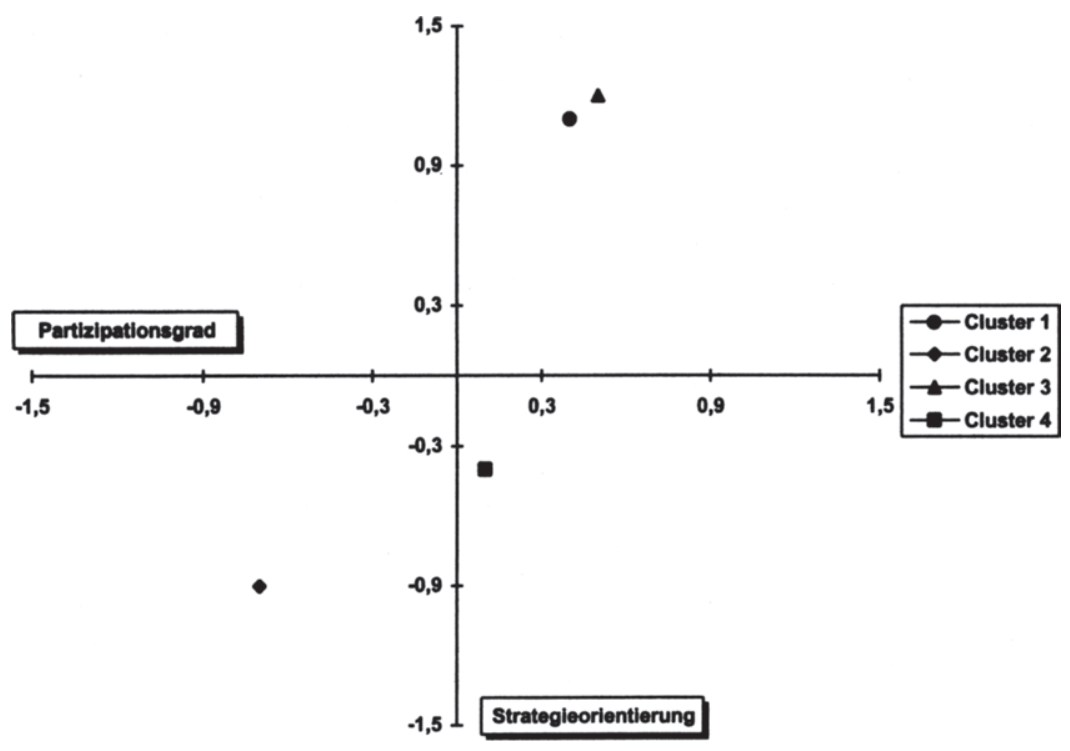

Anm:-Die Abbildung zeigt die Position der Cluster anhand der für beide Hauptmerkmalsdimensionen ermittelten Durchschnittswerte der z-transformierten Zentroidwerte ihrer jeweiligen Politikstilvariablen. 


\section{Politikstil als Determinante der Effektivităt nationaler Umweltpolitik}

\subsection{Politikstildimensionen als Korrelate der Umweltbelastung}

In den folgenden Abschnitten wird die Bedeutung der beiden Politikstildimensionen Strategieorientierung sowie Partizipationsgrad auf die Höhe der Umweltbelastungsindikatoren gemessen. Dies geschieht anhand der einfachen bzw. multiplen Korrelationskoeffizienten der Indikatoren dieser Poltikstildimensionen mit den Variablen zur Messung von Ressourcenverbrauch und Schadstoffemissionen. Dabei wird damit gerechnet, daß sich hohe Werte der Indikatoren staatlicher Strategieorientierung und der Partizipation der durch umweltpolitische Entscheidungen Betroffenen in eher niedrigen Werten der Belastungsindikatoren niederschlagen.

\subsubsection{Strategieorientierung}

Tabelle 17 zeigt die Korrelationskoeffizienten zwischen den Indikatoren einer der beiden Hauptdimensionen nationalen Politikstils, der Strategieorientierung, und den verschiedenen Umweltbelastungesindikatoren und -indizes pro Kopf. Es ist festzustellen, daß es insgesamt keine einzige hochsignifikante Korrelation auf dem 0.01-Niveau gibt.

Auffällig ist auch, daß der Föderalismusgrad mit einigen Indikatoren positiv korreliert, nämlich mit dem Pro-Kopf-Wasserverbrauch, den energiebedingten $\mathrm{CO}_{2}$-Emissionen sowie dem Ressourcenverbrauchsindex. Das Maß der erklärten Varianz beträgt jedoch maximal 27 Prozent. Zwar weisen die Vorzeichen der anderen Koeffizienten überwiegend die gemäß dem Erklärungsmodell zu erwartende Richtung auf (d.h. die meisten Belastungsindikatoren korrelieren mit den Indikatoren staatlicher Strategieorientierung negativ), doch insgesamt bestehen nur sechs (und davon sogar vier positive) auf dem $0.05 \%$-Niveau signifikante Korrelationen. So ist zusammenfassend zu formulieren: die Politikstildimension Strategieorientierung ist keine wichtige Determinante der Pro-KopfUmweltbelastung. 


\section{Tabelle 17: Indikatoren der Strategieorientierung als Korrelate der Um- weltbelastung (Basis: Einwohnerzahl)}

\begin{tabular}{|l|c|c|c|c|c|c|}
\cline { 2 - 7 } \multicolumn{1}{c|}{} & \multicolumn{7}{c|}{ Indikatoren staatlicher Strategieorientierung } \\
\hline $\begin{array}{l}\text { Belastungs- } \\
\text { indikator }\end{array}$ & 1 & 2 & 3 & 4 & 5 & 6 \\
\hline H2O & $.52^{*}$ & -.02 & -.19 & -.20 & -.33 & -.32 \\
\hline LAND & .33 & -.20 & -.35 & -.13 & -.34 & .05 \\
\hline ENER & .33 & $.42^{*}$ & -.16 & .37 & .13 & .25 \\
\hline CO2 & $.48^{*}$ & .21 & -.09 & .01 & -.28 & -.09 \\
\hline WASTE & .13 & .16 & -.20 & .07 & -.07 & .10 \\
\hline FERT & -.35 & -.30 & -.10 & $-.39^{*}$ & .31 & $.38^{*}$ \\
\hline RESOU & $.46^{*}$ & -.05 & -.32 & -.08 & -.31 & -.02 \\
\hline PRESS & .01 & -.05 & -.16 & -.22 & .06 & .24 \\
\hline ENVIR & .34 & -.06 & -.31 & -.14 & -.20 & .08 \\
\hline
\end{tabular}

Anmi:

$\begin{array}{llll}1= & \text { Föderalismusgrad } & 2= & \text { F\&E-Ausgaben } \\ 3= & \text { Exp/Imp. HighTech } & 4= & \text { Ausgaben für Spitzenforschung } \\ 5= & \text { Öffentliche Ausgaben } & 6= & \text { Staatsbeschäftigte }\end{array}$

(Siehe zur Bedeutung der Variablen auch Kapitel 10.1.)

Für die Belastungsindikatoren wurde jeweils mit den Werten des Jahres 1990 bezogen auf die Einwohnerzahl gerechnet. Für die Variablen der Strategieorientierung gingen jeweils die neuesten verfügbaren Werte (1990-93) in die Berechnung ein.

*: $=\mathrm{p}<0.05 \quad \mathrm{~N}=22$

Ganz andere Ergebnisse sind aus Tabelle 18 abzulesen, in welcher die Resultate einer Korrelationsanalyse derselben Politikstilindikatoren mit den Variablen zur Messung der Umweltbelastung pro Einheit BIP dargestellt sind. Sämtliche auf dem 0,05\%-Niveau signifikanten Korrelationen - insgesamt sind es 14 - stützen die Ausgangsthese einer negativen Beziehung zwischen den Indikatoren staatlicher Strategieorientierung einerseits und den Umweltbelastungsdimensionen Ressourcenverbrauch bzw. Schadstoffemissionen andererseits. 


\section{Tabelle 18: Indikatoren der Strategieorientierung als Korrelate der Um- weltbelastung (Basis: Einheit BIP)}

\begin{tabular}{|l|c|c|c|c|c|c|}
\cline { 2 - 7 } \multicolumn{1}{c|}{} & \multicolumn{7}{c|}{ Indikatoren staatlicher Strategieorientierung } \\
\hline $\begin{array}{l}\text { Belastungs- } \\
\text { indikator }\end{array}$ & 1 & 2 & 3 & 4 & 5 & 6 \\
\hline H2O & .30 & -.36 & -.31 & $-.43^{*}$ & -.37 & $-.39^{*}$ \\
\hline LAND & .26 & -.30 & -.38 & -.21 & -.36 & .02 \\
\hline ENER & .03 & -.06 & -.35 & .13 & .15 & .36 \\
\hline CO2 & .21 & -.21 & -.19 & -.31 & -.35 & -.11 \\
\hline WASTE & -.31 & $-.44^{*}$ & $-.41^{*}$ & $-.40^{*}$ & -.11 & .09 \\
\hline FERT & $-.42^{*}$ & $-.42^{*}$ & -.04 & $-.45^{*}$ & .22 & .24 \\
\hline RESOU & .29 & -.33 & $-.44^{*}$ & -.27 & -.36 & -.06 \\
\hline PRESS & -.31 & $-.47^{*}$ & -.18 & $-.51^{*}$ & .03 & .16 \\
\hline ENVIR & .05 & $-.47^{*}$ & $-.40^{*}$ & $-.45^{*}$ & -.24 & .04 \\
\hline
\end{tabular}

\section{Anmi:}

$\begin{array}{llll}1= & \text { Föderalismusgrad } & 2= & \text { F\&E-Ausgaben } \\ 3= & \text { Exp/Imp. HighTech } & 4= & \text { Ausgaben für Spitzenforschung } \\ 5= & \text { Öffentliche Ausgaben } & 6= & \text { Staatsbeschäftigte }\end{array}$

(Siehe zur Bedeutung der Variablen auch Kapitel 10.1.)

Für die Belastungsindikatoren wurde jeweils mit den Werten des Jahres 1990 bezogen auf das Bruttoinlandsprodukt gerechnet. Für die Variablen der Strategieorientierung gingen jeweils die neuesten verfügbaren Werte (1990-93) in die Berechnung ein.

$*:=\mathrm{p}<0.05 \quad \mathrm{~N}=22$

Das Ausmaß des Wasserverbrauches korreliert dabei signifikant negativ mit dem F\&E-Bildungsanteil am BIP sowie dem Anteil der administrativ Tätigen an der Gesamtbevölkerung. Ebenfalls negative Korrelationen sind zwischen dem Abfallaufkommen und allen drei Indikatoren zur Messung der technologischen Kapazität zu attestieren. Auch der Einsatz künstlicher Düngemittel scheint mit dem Ausmaß technologischer Kapazität und dezentraler Strukturen zu sinken. Für die drei Indizes gilt vor allem ein negativer Zusammenhang mit den Technologieindikatoren. Im Sinne unseres Erklärungsmodells kann also festgestellt werden, daß die Umweltbelastung eines Staates (pro Einheit BIP) durch Ressourcenverbrauch und Schadstoffemissionen mit einer zunehmend starken Ausprägung seiner Strategieorientierung - insbesondere seiner technologischen Kapazitäten - sinkt. Oder anders formuliert: die Effektivität nationaler 
Umweltpolitik steigt mit dem Ausmaß, in welchem Strategieorientierung Merkmal des nationalen Politikstils ist.

\subsubsection{Partizipationsgrad}

Sowohl der Korporatismusgrad als auch das Ausmaß des konsualen Konfliktlösungsverhaltens korrelieren negativ mit allen Unweltbelastungsindikatoren und -indizes pro Einwohner (Tabelle 19).

Den höchsten Wert mit -.53, d.h. 28 Prozent erklärter Varianz, erreicht der Zusammenhang zwischen Korporatismusgrad und Wasserverbrauch (hochsignifikant auf dem 0,01\%-Niveau). Alle anderen signifikanten Korrelationen erreichen lediglich das 0,05\%-Niveau. Während die Beziehungen zwischen den Indikatoren des Partizipationsgrades und den Variablen des Ressourcenverbauches mit Ausnahme des Primärenergieverbrauches ein statistisch relevantes Signifikanzniveau erreichen, gilt für die Variablen der Schadstoffemission nur ein deutlich schwächerer Zusammenhang.

Obwohl insgesamt nur eine einzige Korrelation in Tabelle 19 hochsignifikant ist, kann aufgrund der Datenlage insgesamt von einer umweltentlastenden Wirkung der Determinante Partizipationsgrad ausgegangen werden.

Dieses Ergebnis wird durch eine Korrelationsanalyse dieses Merkmals mit den auf die Höhe der Wirtschaftsleistung bezogenen Umweltbelastungswerten noch verstärkt (Tabelle 20).

Auch die Schadstoffemissionsindikatoren weisen in diesem Fall signifikante Korrelationen mit den Politikstilmerkmalen auf. Die höchsten Koeffizienten ergeben sich für die Korrelation von Korporatismusgrad und Konsensorientierung mit dem Wasserverbrauch. Das $\mathrm{Maß}$ der erklärten Varianz beträgt in diesen Fällen jeweils mehr als 36 Prozent. 
Tabelle 19: Indikatoren des Partizipationsgrades als Korrelate der Umweltbelastung (Basis: Einwohnerzahl)

\begin{tabular}{|l|c|c|}
\cline { 2 - 3 } \multicolumn{1}{c|}{} & \multicolumn{2}{c|}{ Indikatoren des Partizipationsgrades } \\
\hline $\begin{array}{l}\text { Belastungs- } \\
\text { indikator }\end{array}$ & Korporatismusgrad & Konfliktlösungsverhalten \\
\hline H2O & $-.53^{* *}$ & $-.49^{*}$ \\
\hline LAND & $-.40^{*}$ & $-.43^{*}$ \\
\hline ENER & -.03 & -.04 \\
\hline CO2 & -.28 & -.29 \\
\hline WASTE & -.19 & -.19 \\
\hline FERT & -.26 & -.20 \\
\hline RESOU & $-.45^{*}$ & $-.46^{*}$ \\
\hline PRESS & -.33 & -.30 \\
\hline ENVIR & $-.45^{*}$ & $-.45^{*}$ \\
\hline
\end{tabular}

\section{Anme:}

Siehe zur Bedeutung der Variablen auch Kapitel 10.2.

Für die Belastungsindikatoren wurde jeweils mit den Werten des Jahres 1990 bezogen auf die Einwohnerzahl gerechnet. Für die Variablen des Partizipationsgrades gingen die Werte aus Kapitel 10.2. in die Berechnung ein.

$*:=\mathrm{p}<0.05 \quad * *:=\mathrm{p}<0.01 \quad \mathrm{~N}=22$

Auch für die Ressourcenverbrauchs- und Umweltbelastungsindizes gelten hochsignifikante Zusammenhänge mit den beiden Politikstilindikatoren. Dies bedeutet insgesamt, daß ein eher partizipativer Politikstil zu tendenziell deutlich günstigeren Werten für die Umweltbelastungsindikatoren und somit zu einer effektiveren nationalen Umweltpolitik zu führen scheint. 
Tabelle 20: Indikatoren des Partizipationsgrades als Korrelate der Umweltbelastung (Basis: Einheit BIP)

\begin{tabular}{|l|c|c|}
\cline { 2 - 3 } \multicolumn{1}{c|}{} & \multicolumn{2}{c|}{ Indikatoren des Partizipationsgrades } \\
\hline $\begin{array}{l}\text { Belastungs- } \\
\text { indikator }\end{array}$ & Korporatismusgrad & Konfliktlösungsverhalten \\
\hline H2O & $-.61^{* *}$ & $-.61^{* *}$ \\
\hline LAND & $-.42^{*}$ & $-.46^{*}$ \\
\hline ENER & -.10 & -.15 \\
\hline CO2 & $-.40^{*}$ & $-.43^{*}$ \\
\hline WASTE & -.36 & $-.41^{*}$ \\
\hline FERT & -.25 & -.18 \\
\hline RESOU & $-.53^{* *}$ & $-.57^{* *}$ \\
\hline PRESS & -.38 & -.35 \\
\hline ENVIR & $-.56^{* *}$ & $-.58^{* *}$ \\
\hline
\end{tabular}

Anm.:

Siehe zur Bedeutung der Variablen auch Kapitel 10.2.

Für die Belastungsindikatoren wurde jeweils mit den Werten des Jahres 1990 bezogen auf das Bruttoinlandsprodukt gerechnet. Für die Variablen des Partizipationsgrades gingen die Werte aus Kapitel 10.2. in die Berechnung ein.

*:= $\mathrm{p}<0.05 \quad * *:=\mathrm{p}<0.01 \quad \mathrm{~N}=22$

11.2 Multiple Regressionsanalysen aller Politikstilvariablen zur Erklärung der Varianz der Umweltbelastung

Da die einfache Korrelationsanalyse aufgrund von Kollinearitäten über das tatsächliche Ausmaß des Zusammenhanges zwischen zwei Variablen täuschen könnte, und da die multiple Regression vieler Einflußgrößen zu einem noch höheren Bestimmtheitsmaß der abhängigen Variable führen kann, wurde das Verfahren der multiplen Regressionsanalyse angewendet, d.h. der gleichzeitige Effekt aller acht Politikstilvariablen auf die Umweltbelastungsindizes (jeweils bezogen auf die Einwohnerzahl bzw. das BIP) wurde unter Ausschaltung der Kollinearitäten gemessen.

Die Tabellen 21 bis 23 zeigen die Ergebnisse dieser Vorgehensweise zunächst für die Indexwerte pro Einwohner.

Tabelle 21 listet die Prädiktoren des Kriteriums Ressourcenverbrauchsindex pro Einwohner auf. Während vier Variablen keinen signifikanten Beitrag zur 
Erklärung der abhängigen Größe leisten, erweisen sich die vier anderen Prädiktoren als signifikant und erklären insgesamt 38,4 Prozent der Kriteriumsvarianz. Diese drei Variablen - Föderalismusgrad, öffentliche Ausgaben sowie Korporatismusgrad - entsprechen hinsichtlich der Vorzeichen ihrer standardisierten Regressionsgewichte Beta den einfachen Korrelationen der Tabellen 17 und 19. Im Gegensatz dazu widerspricht der Prädiktor Anteil der Staatsbeschäftigten mit einem Beta-Gewicht von .77 dem Ergebnis der einfachen Korrelationsanalyse und der Hypothese, daß sich eine hohe personelle Kapazität im administrativen Bereich einen umweltentlastenden Effekt hat ${ }^{405}$.

\section{Tabelle 21: Beta-Gewichte und t-Werte signifikanter Prädiktoren der Höhe des Ressourcenverbrauchsindexes pro Einwohner (multiple Regressionsa- nalyse)}

\begin{tabular}{|l|c|c|c|}
\hline \multicolumn{1}{|c|}{ Prädiktor } & Beta & t-Wert & Sign.Niv. \\
\hline Föderalismusgrad & .54 & 3.38 & 0.004 \\
\hline Öffentliche Ausgaben in \% des BIP & -.46 & -1.83 & 0.082 \\
\hline Anteil der Staatsbeschäftigten & .77 & 2.93 & 0.009 \\
\hline Korporatismusgrad & -.52 & -3.23 & 0.005 \\
\hline
\end{tabular}

Anm.: $\mathrm{r}=.62 \quad \mathrm{~F}=6.89 \quad$ Sign.Niv. $=0.002 \quad \mathrm{n}=22$

Die Gesamtregressionsgleichung findet sich in Tabelle A55.1 des Anhanges. Die dortigen Beta-Gewichte unterscheiden sich von denjenigen der obigen Tabelle, da die Werte im Anhang die Ausgangssituation der multiplen Regressionsanalyse vor der stufenweise Variablenreduktion widerspiegeln.

Anscheinend wirkt sich eine nur personelle Ausweitung der Administration auf dem Gebiet der Umweltpolitik eher innovations- und effektivitätsmindernd aus. Das Beta-Gewicht der erklärenden Variable Föderalismusgrad entspricht mit seinem positiven Vorzeichen zwar der einfachen Korrelation, entgegen der ur-

\footnotetext{
${ }^{405}$ Statistisch bedeutet ein solcher Vorzeichenwechsel das folgende: der in der einfachen Korrelationanalyse gemessene negative Zusammenhang ist das Resultat einer Prämissenverletzung des linearen Regressionsmodells, nämlich der Unabhängigkeit der Regressoren untereinander. Im Gegensatz zum einfachen Korrelationskoeffizienten gibt der standardisierte multiple Korrelationskoeffizient Beta nur den ,Nettoeffekt" jedes einzelnen Regressors auf die abhängige Variable wider (vgl. Backhaus, K. et al. (1980): Multivariate . .., a.a.O., S. 74 f.).
} 
sprünglichen Annahme des Erklärungsmodells scheint es jedoch zumindest für das auf die Einwohnerzahl bezogene Umweltkriterium so zu sein, daß zentrale Strukturen günstigere Wirkungen auf die Effektivität nationaler Umweltpolitik haben als eine föderale Staatsorganisation.

Desweiteren ist der Tabelle $21 \mathrm{zu}$ entnehmen, daß hohe monetäre Ressourcen der Administration sowie korporatistische Wege der Interessenvermittlung eine umweltentlastende Wirkung ausüben. Insgesamt ist es also anscheinend so, daß niedrige Kriteriumswerte für Staaten mit den folgenden Ausprägungen der relevanten Politikstilvariablen zu erwarten sind: personell „schlanke“, finanziell gut ausgestattete Einheitsstaaten mit korporatistischen Netzwerkstrukturen.

Diese Einschätzung wird durch die multiplen Regressionsanalysen, in welchen der Schadstoff- bzw. Umweltbelastungsindex als Kriterium fungieren, weitgehend bestätigt.

Schadstoffbelastung eines Staates korreliert - wie aus Tabelle 22 ersichtlich positiv mit den Föderalismusgrad sowie dem Anteil der Staatsbeschäftigten an der Gesamtzahl der Beschäftigten. Gleichzeitig bildet der Korporatismusgrad auch für diesen Indikator der Umweltbelastung ein wichtiges Kriterium in dem Sinne, daß eher korporatistisch verfaßte Staaten günstigere Werte des Schadstoffindexes ausweisen.

Tabelle 22: Beta-Gewichte und t-Werte signifikanter Prädiktoren der Höhe des Schadstoffindexes pro Einwohner (multiple Regressionsanalyse)

\begin{tabular}{|l|c|c|c|}
\hline \multicolumn{1}{|c|}{ Prädiktor } & Beta & t-Wert & Sign.Niv. \\
\hline Föderalismusgrad & .29 & 3.65 & 0.002 \\
\hline Anteil der Staatsbeschäftigten & .57 & 2.41 & 0.025 \\
\hline Korporatismusgrad & -.46 & -3.24 & 0.004 \\
\hline
\end{tabular}

Anm. $r=.53 \quad F=6.84 \quad$ Sign.Niv. $=0.003 \quad n=22$

Die Gesamtregressionsgleichung findet sich in Tabelle A55.2 des Anhanges. Die dortigen Beta-Gewichte unterscheiden sich von denjenigen der obigen Tabelle, da die Werte im Anhang die Ausgangssituation der multiplen Regressionsanalyse vor der stufenweise Variablenreduktion widerspiegeln.

Für den Umweltbelastungsindex pro Einwohner ergibt die multiple Regressionsanalyse nur noch zwei relevante Prädiktoren, nämlich den Staatsbeschäftigtenanteil sowie den Korporatismusgrad. Aus den Vorzeichen der Beta-Gewichte kann insgesamt die folgende Schlußfolgerung gezogen werden: sie Umweltbelastung pro Einwohner ist in einem Staat um so kleiner, je geringer der Anteil 
des staatlichen Sektors der Beschäftigtenzahl ist, und je korporatistischer die Interessenvermittlung innerhalb dieses Staates abläuft.

Tabelle 23: Beta-Gewichte und t-Werte signifikanter Prädiktoren der Höhe des Umweltbelastungsindexes pro Einwohner (multiple Regressionsanalyse)

\begin{tabular}{|l|c|c|c|}
\hline \multicolumn{1}{|c|}{ Prädiktor } & Beta & t-Wert & Sign.Niv. \\
\hline Anteil der Staatsbeschäftigten & .33 & 2.08 & 0.049 \\
\hline Korporatismusgrad & -.50 & -1.88 & 0.072 \\
\hline
\end{tabular}

Anm.: $r=.23 \quad F=2.82 \quad$ Sign.Niv. $=0.083 \quad n=22$

Die Gesamtregressionsgleichung findet sich in Tabelle A55.3 des Anhanges. Die dortigen Beta-Gewichte unterscheiden sich von denjenigen der obigen Tabelle, da die Werte im Anhang die Ausgangssituation der multiplen Regressionsanalyse vor der stufenweise Variablenreduktion widerspiegeln.

Als Begründung für die dem Erklärungsmodell bisher weitgehend widersprechende Rolle des Prädiktors Föderalismusgrad mag die Tatsache dienen, daß gerade die föderalen Bundesstaaten USA und Kanada sehr hohe Werte für die Umweltbelastungsindizes aufweisen ${ }^{406}$, während die Werte der zentralistischen Einheitsstaaten Skandinaviens für diese Variablen eher niedrig ausfallen. Da die Clusteranalyse in Kapitel 10.3 die OECD-Staaten jedoch in jeweils zwei Gruppen mit hohem bzw. niedrigem Föderalismusgrad geteilt hat (Tabelle 16), ist noch genauer zu untersuchen, ob nicht ein ausgeprägter Föderalismus wie z.B. bei den Staaten der Cluster-Gruppe 1 (Japan, Niederlande, Schweiz) nicht in Kombination mit anderen Variablenausprägungen doch umweltentlastend wirkt.

Weitere multiple Regressionsanalysen, diesmal mit den Umweltindizes bezogen auf das Bruttoinlandsprodukt, führen $\mathrm{zu}$ anderen Resultaten (Tabellen 24 bis 26).

${ }^{406}$ Vgl. noch einmal ausführlich Kapitel 8.1. des empirischen Teils. 
Tabelle 24: Beta-Gewichte und t-Werte signifikanter Prädiktoren der Höhe des Ressourcenverbrauchsindexes pro Einheit BIP (multiple Regressionsanalyse)

\begin{tabular}{|l|c|c|c|}
\hline \multicolumn{1}{|c|}{ Prädiktor } & Beta & t-Wert & Sign.Niv. \\
\hline Föderalismusgrad & -.06 & -3.22 & 0.005 \\
\hline Anteil der Staatsbeschäftigten & .04 & 2.87 & 0.010 \\
\hline Korporatismusgrad & -.56 & -3.28 & 0.004 \\
\hline
\end{tabular}

Anm.i $r=.51 \quad F=6.28 \quad$ Sign.Niv. $=0.005 \quad n=22$

Die Gesamtregressionsgleichung findet sich in Tabelle A55.4 des Anhanges. Die dortigen Beta-Gewichte unterscheiden sich von denjenigen der obigen Tabelle, da die Werte im Anhang die Ausgangssituation der multiplen Regressionsanalyse vor der stufenweise Variablenreduktion widerspiegeln.

Zwar leistet auch hier der Prädiktor Korporatismusgrad mit durchgehend negativen Beta-Werten für alle drei Indizes einen signifikanten Beitrag zur Erklärung der Kriteriumsvarianz, doch im Gegensatz zum ersten Analysekomplex spielen in diesem Fall Föderalismus und administrative Kapazitäten eine deutlich geringere Rolle.

Dafür liefern die Variablen zur Erfassung der technologischen Kapazität einen deutlich höheren Beitrag zur Erklärung der Kriterien Schadstoff- und Umweltbelastungsindex (jeweils pro Einheit BIP).

So sinkt - wie Tabelle 25 zeigt - die Schadstoffbelastung mit der Zunahme der F\&E-Ausgaben deutlich (Beta-Gewicht -.79).

Auch zwei weitere der insgesamt fünf Prädiktoren, die einen signifikanten Beitrag zur Erklärung der Kriteriumsvarianz leisten (der durch die multiple Regressionsanalyse bestimmte Varianzanteil beträgt insgesamt knapp 50 Prozent) gehören zur Gruppe der Indikatoren technologischer Handlungskapazität. So ist die Belastung durch Schadstoffemissionen in solchen Staaten tendenziell niedriger, deren Ex-/Importrelation technologieintensiver Waren einen eher hohen Wert erreicht. Auch für die Variable Bildungsausgaben zur F\&ESpitzenförderung in Prozent des BIP ist im Einklang mit dem Modellentwurf und der einfachen Korrelationsanalyse (Tabelle 18) ein negatives Vorzeichen des standardisierten Regressionskoeffizienten Beta (-.25) abzulesen. 
Tabelle 25: Beta-Gewichte und t-Werte signifikanter Prädiktoren der Höhe des Schadstoffindexes pro Einheit BIP (multiple Regressionsanalyse)

\begin{tabular}{|l|c|c|c|}
\hline \multicolumn{1}{|c|}{ Prädiktor } & Beta & t-Wert & Sign.Niv. \\
\hline F\&E-Ausgaben in \% des BIP & -.79 & -2.39 & 0.028 \\
\hline HighTech-Export-Import-Ratio & -.15 & -2.04 & 0.056 \\
\hline $\begin{array}{l}\text { Ausgaben zur F\&E-Spitzenförderung \% d. } \\
\text { BIP }\end{array}$ & -.25 & -3.34 & 0.004 \\
\hline Öffentliche Ausgaben in \% des BIP & -.13 & -1.76 & 0.095 \\
\hline Korporatismusgrad & -.36 & -3.67 & 0.002 \\
\hline
\end{tabular}

Anm.: $\mathrm{r}=.71 \quad \mathrm{~F}=7.66 \quad$ Sign.Niv. $=0.001 \quad \mathrm{n}=22$

Die Gesamtregressionsgleichung findet sich in Tabelle A55.5 des Anhanges. Die dortigen Beta-Gewichte unterscheiden sich von denjenigen der obigen Tabelle, da die Werte im Anhang die Ausgangssituation der multiplen Regressionsanalyse vor der stufenweise Variablenreduktion widerspiegeln.

Wie bei den allgemeinen F\&E-Ausgaben und der Technologiequote des Warenhandels scheint es bei diesem Prädiktor so zu sein, daß sich ein eher hoher finanzieller Input des Staates positiv, also mindernd auf das Niveau der Schadstoffbelastung auswirkt.

Ein Blick auf Tabelle 26, in welcher die Resultate einer multiplen Regressionsanalyse bezogen auf das Kriterium Umweltbelastung pro Einheit BIP dargestellt sind, bestätigt diese Überlegungen. 36 Prozent der Kriteriumsvarianz werden durch die drei, einen signifikanten Beitrag leistenden Prädiktoren F\&EAusgaben, Bildungsausgaben sowie Korporatismusgrad erklärt.

Zusammenfassend läßt sich für den zweiten Analysekomplex - also die multiplen Regressionsanalysen mit den Belastungsindizes pro Einheit BIP als abhängigen Variablen - formulieren, daß eine eher stark ausgegrägte Technologieorientierung, die sich in tendenziell hohen Werten der F\&E-Ausgaben und einem hohen Ex-/Importquotienten von Hochtechnologiegütern ausdrückt, sowie korporatistische Muster der Interessenvermitttlung für auf das BIP bezogene Umweltbelastungsindizes entlastend wirken. Die Pradikatoren administrative Kapazität und Föderalismusgrad spielen im Gegensatz dazu nur eine untergeordnete Rolle. 
Tabelle 26: Beta-Gewichte und t-Werte signifikanter Prädiktoren der Höhe des Umweltbelastungsindexes pro Einheit BIP (multiple Regressionsanalyse)

\begin{tabular}{|l|c|c|c|}
\hline \multicolumn{1}{|c|}{ Prädiktor } & Beta & t-Wert & Sign.Niv. \\
\hline F\&E-Ausgaben in \% des BIP & -.90 & -3.62 & 0.002 \\
\hline $\begin{array}{l}\text { Ausgaben zur F\&E-Spitzenförderung \% d. } \\
\text { BIP }\end{array}$ & -.74 & -2.38 & 0.027 \\
\hline Korporatismusgrad & -.68 & -3.18 & 0.005 \\
\hline
\end{tabular}

Anm.i $\quad r=.60 \quad F=9.09 \quad$ Sign.Niv. $=0.001 \quad n=22$

Die Gesamtregressionsgleichung findet sich in Tabelle A55.6 des Anhanges. Die dortigen Beta-Gewichte unterscheiden sich von denjenigen der obigen Tabelle, da die Werte im Anhang die Ausgangssituation der multiplen Regressionsanalyse vor der stufenweise Variablenreduktion widerspiegeln.

11.3 Varianzanalysen der Politikstilcluster anhand der Umweltbelastungsindizes

Wie oben bereits festgestellt, eignen sich die einfache bzw. multiple Regressionsanalyse zur Ermittlung des Einflusses, den einzelne bzw. mehrere Variablen gleichzeitig auf die abhängige Größe besitzen. Da die jeweiligen Ausprägungen der Prädiktoren bekannt sind, wäre es durch simples Einsetzen der tatsächlichen Datenkombination (Prädiktorwerte) eines Staates in die multiple Regressionsgleichung also möglich, eine weitgehend zutreffende Aussage über die für diesen Staat zu erwartenden Umweltbelastungsdaten (Kriteriumswerte) zu machen. Weil es in dieser Studie jedoch darum geht, die Wirkung typischer Kombinationen von Politikstilstilvariablen - sprich: Politikstile - auf das Umweltbelastungsausmaß zu untersuchen, soll nachfolgend im Rahmen von Varianzanaly$\operatorname{sen}^{407}$ untersucht werden, ob sich die ökologischen Belastungsdaten der einzelnen Politikstilcluster signifikant voneinander unterscheiden.

Tabelle 27 stellt die Analyseresultate für die vier in Kapitel 10.3. generierten Staatengruppen bezogen auf die Umweltbelastungswerte pro Einwohner dar. Für das Kriterium des Ressourcenverbrauches ergeben sich wegen der geringen Fallzahl zwar sowohl durch eine Varianzanalyse als auch für alle paarweisen

${ }^{407}$ Vgl. Backhaus, K. et al. (1980): Multivariate Analysemethoden . ., a.a.O., S. 9 ff. 
Mittelwertvergleiche keine statistisch signifikanten Unterschiede, dennoch weisen die Daten der einzelnen Cluster deutlich voneinander abweichende Werte auf. So ist der Mittelwert der Staaten, welche einen technologieorientierten, föderalen Korporatismus als Politikstil praktizieren, der mit Abstand niedrigste (M: 67.3). Ebenfalls recht niedrige Werte hat der administrativ-zentralistische Korporatismus der skandinavischen Staaten und Österreichs zu verzeichnen (M: 82.6).

Mit deutlichem Abstand folgt der Politikstil des Laissez-faire (M: 108.1), und die ungünstigsten Werte sind für die Staatengruppe des innovativen Pluralismus abzulesen (M: 118.7).

Eine andere Reihenfolge ergibt sich für den Schadstoffindex [PRESS/POPUL]. Zwar weist auch für diese Variable die Clustergruppe A die niedrigsten Werte auf (M: 82.7), doch knapp dahinter liegen bereits die Laissez-faire-Staaten (M: 86.4). Während der administrative Korporatismus mit einem Mittelwert von 102.6 Indexpunkten diesmal eher ungünstige Werte aufweist, ist für die innovativ-pluralistischen Staaten auch im Hinblick auf diesen Indikator der mit Abstand schlechteste Wert abzulesen (M: 118.1). Damit ist der Mittelwert der Clustergruppe C signifikant höher als derjenige von Gruppe D (Signifikanzniveau: 0.05).

Der Umweltindex [ENVIR/POPUL] spiegelt als Durchschnittswert der beiden anderen Indizes die allgemeine Tendenz des eben Ausgeführten wider: der technologieorientierte, dezentrale Korporatismus der aus Japan, der Schweiz und den Niederlanden bestehenden Clustergruppe A ist im Hinblick auf die Umweltbelastungsdaten pro Einwohner der deutlich effektivste Politikstil.

Mit deutlichem Abstand folgen der zentralistisch-administrative Korporatismus der skandinavischen Staaten und Österreichs einerseits sowie der Laissez-fairePolitikstil der ökonomisch nachholenden Staaten Südeuropas und der pazifischen Staaten Australien und Neuseeland andererseits. 
Tabelle 27: Varianzanalyse der Staatengruppen anhand ihrer Umweltbelastungswerte (Basis: Einwohnerzahl) I

Statistiken:

Gruppen

A: Techn.- dezentr. Korporatismus

B: Adm.-zentr. Korporatismus

C: Innovativer Pluralismus

D: Laissez faire

Varianzanalyse:

Mittelwert-Vergleiche:

\begin{tabular}{|c|c|c|c|c|}
\hline & $A$ & $B$ & $C$ & $D$ \\
\hline$A$ & & 1) & 1) & 1) \\
\hline$B$ & 1) & & n.s. & n.s. \\
\hline$C$ & 1) & n.s. & & n.s. \\
\hline$D$ & 1) & n.s. & n.s. & \\
\hline
\end{tabular}

PRESSYPOPUL

\begin{tabular}{|r|r|r|}
\hline \multicolumn{1}{|c|}{$M$} & \multicolumn{1}{|c|}{ SD } & N \\
\hline 82.7 & 17.6 & 3 \\
\hline 102.6 & 32.2 & 5 \\
\hline 118.1 & 36.7 & 7 \\
\hline 86.4 & 35.0 & 7 \\
\hline
\end{tabular}

TG $1.44 \mathrm{p}=0.27(2.16$ d.f.)

(Vi: 1224.7

Vz: 1760.3)

\begin{tabular}{|c|c|c|c|c|}
\hline & $A$ & $B$ & $C$ & $D$ \\
\hline$A$ & & $1)$ & $1)$ & $1)$ \\
\hline$B$ & $1)$ & & n.s. & n.s. \\
\hline$C$ & $1)$ & n.s. & & $*$ \\
\hline$D$ & $1)$ & n.s. & $*$ & \\
\hline
\end{tabular}

\section{ENVIRPOPUL}

\begin{tabular}{|r|r|r|}
\hline \multicolumn{1}{|c|}{$M$} & \multicolumn{1}{c|}{ SD } & N \\
\hline 74.7 & 19.1 & 3 \\
\hline 92.8 & 23.3 & 5 \\
\hline 118.4 & 52.5 & 7 \\
\hline 97.1 & 52.9 & 7 \\
\hline
\end{tabular}

TG $0.54 \mathrm{p}=0.59$ (2.16 d.f.)

(Vi: 2218.2

Vz: 1206.6)

\begin{tabular}{|c|c|c|c|c|}
\hline & $A$ & $B$ & $C$ & $D$ \\
\hline$A$ & & 1) & 1) & 1) \\
\hline$B$ & $1)$ & & n.s. & n.s. \\
\hline$C$ & 1) & n.s. & & n.s. \\
\hline$D$ & 1) & n.s. & n.s. & \\
\hline
\end{tabular}

Anmi

M = Mittelwert

SD $=$ Standardabweichung

$\mathrm{TG}=\mathrm{TestgroBe}$

d.f. $=$ Freiheitsgrade

$V i=$ Varianz innerhalb der Gruppen
$\mathrm{Vz}=$ Varianz zwischen den Gruppen n.s. $=$ nicht signifikant

1) Stichproben mit $N<4$ nicht im Test
Mittelwert-Vergleiche mittels Wilcoxon-Test

$*:=p<0.05$

Alle Daten aus 1990 
Statistiken:

Gruppen

A: Techn.- dezentr. Korporatismus

B: Adm.-zentr. Korporatismus

C: Innovativer Pluralismus

D: Laissez faire

Varianzanalyse:

Anm.i

$M=$ Mittelwert

$\mathrm{SD}=$ Standardabweichung

TG $=$ Testgroße

d.f. $=$ Freiheitsgrade

$\mathrm{Vi}=$ Varianz innerhalb der Gruppen
RESOU/BIP

\begin{tabular}{|r|r|r|}
\hline \multicolumn{1}{|c|}{$\mathrm{M}$} & \multicolumn{1}{c|}{$\mathrm{SD}$} & $\mathrm{N}$ \\
\hline 46.9 & 20.5 & 3 \\
\hline 59.4 & 14.9 & 5 \\
\hline 78.3 & 40.1 & 7 \\
\hline 123.4 & 62.8 & 7 \\
\hline
\end{tabular}

TG $3.15 p=0.07$ (2.16 d.f.)

(Vi: 2135.7

Vz: 6734..6)

\begin{tabular}{|c|c|c|c|c|}
\hline & $A$ & $B$ & $C$ & $D$ \\
\hline$A$ & & 1) & 1) & 1) \\
\hline$B$ & 1) & & n.s. & $* *$ \\
\hline$C$ & 1) & n.s. & & $*$ \\
\hline$D$ & 1) & $* *$ & $*$ & \\
\hline
\end{tabular}

PRESS/BIP

\begin{tabular}{|r|r|l|}
\hline \multicolumn{1}{|c|}{$\mathrm{M}$} & \multicolumn{1}{c|}{ SD } & $\mathrm{N}$ \\
\hline 53.3 & 18.7 & 3 \\
\hline 70.1 & 22.1 & 5 \\
\hline 83.6 & 38.4 & 7 \\
\hline 101.6 & 43.9 & 7 \\
\hline
\end{tabular}

TG $1.07 p=0.37$ (2.16 d.f.)

(Vi: 1398.6

Vz: 1498.5)

\begin{tabular}{|c|c|c|c|c|}
\hline & $A$ & $B$ & $C$ & $D$ \\
\hline$A$ & & 1) & 1) & 1) \\
\hline$B$ & $1)$ & & n.s. & n.s. \\
\hline$C$ & $1)$ & n.s. & & n.s. \\
\hline$D$ & 1) & n.s. & n.s. & \\
\hline
\end{tabular}

ENVIR/BIP

\begin{tabular}{|r|r|r|}
\hline \multicolumn{1}{|c|}{$\mathrm{M}$} & \multicolumn{1}{c|}{$\mathrm{SD}$} & $\mathrm{N}$ \\
\hline 50.1 & 19.4 & 3 \\
\hline 64.8 & 16.0 & 5 \\
\hline 80.9 & 29.5 & 7 \\
\hline 112.5 & 49.4 & 7 \\
\hline
\end{tabular}

TG $2.78 \mathrm{p}=0.09$ (2.16 d.f.)

(Vi: 1306.5

Vz: 3629.6)

\begin{tabular}{|c|c|c|c|c|}
\hline & $A$ & $B$ & $C$ & $D$ \\
\hline$A$ & & $1)$ & $1)$ & $1)$ \\
\hline$B$ & $1)$ & & n.s. & $*$ \\
\hline$C$ & $1)$ & n.s. & & n.s. \\
\hline$D$ & $1)$ & $*$ & n.s. & \\
\hline
\end{tabular}

$\mathrm{z}=$ Varianz zwischen den Gruppen n.s. $=$ nicht signifikant

1) Stichproben mit $N<4$ nicht im Test

Alle Daten aus 1990
Mittelwert-Vergleiche mittels Wilcoxon-Test

$:=p<0.05$

$*:=p<0.01$ 
Tabelle 29: Varianzanalyse der Staatengruppen anhand ihrer Umweltbelastungswerte (Basis: Einwohnerzahl) II

\section{RESOU/POPUL}

Gruppen-Statistiken:

A/B: Korporatismus

C: Innovativer Pluralismus

D: Laissez faire

Varianzanalyse:

Mittelwert-Vergleiche:

\begin{tabular}{|c|c|c|c|}
\hline & $A / B$ & $C$ & $D$ \\
\hline$A / B$ & & n.s. & n.s. \\
\hline$C$ & n.s. & & n.s. \\
\hline$D$ & n.s. & n.s. & \\
\hline
\end{tabular}

TG $0.86 p=0.44$ ( 2.16 d.f.)

(Vi: 4189.4

Vz: 3596.7)

Anmi

$M=$ Mittelwert

SD $=$ Standardabweichung

$\mathrm{TG}=$ Testgroße

d.f. $=$ Freiheitsgrade

$V i=$ Varianz innerhalb der Gruppen
$\mathbf{V z}=$ Varianz zwischen den Gruppen

n.s. $=$ nicht signifikant

Alle Daten aus 1990
PRESSYPOPUL

\begin{tabular}{|r|r|r|}
\hline \multicolumn{1}{|c|}{ M } & SD & N \\
\hline 95.1 & 28.1 & 8 \\
\hline 118.1 & 36.7 & 7 \\
\hline 86.4 & 35.0 & 7 \\
\hline
\end{tabular}

ENVIR/POPUL

\begin{tabular}{|r|r|r|}
\hline \multicolumn{1}{|c|}{$\mathrm{M}$} & \multicolumn{1}{|c|}{ SD } & N \\
\hline 86.0 & 22.4 & 8 \\
\hline 118.4 & 52.5 & 7 \\
\hline 97.1 & 52.9 & 7 \\
\hline
\end{tabular}

TG $1.71 \mathrm{p}=0.21$ ( 2.16 d.f.)

(Vi: 1103.2

Vz: 1890.7)

TG $1.03 \mathrm{p}=0.38$ (2.16 d.f.)

(Vi: 1939.0

Vz: 2001.0)

\begin{tabular}{|c|c|c|c|}
\hline & $A / B$ & $C$ & $D$ \\
\hline$A / B$ & & n.s. & n.s. \\
\hline$C$ & n.s. & & $*$ \\
\hline$D$ & n.s. & $*$ & \\
\hline
\end{tabular}

Mittelwert-Vergleiche mittels Wilcoxon-Test : $:=0.05$ 


\section{RESOU/BIP}

Gruppen-Statistiken:

A/B: Korporatismus

C: Innovativer Pluralismus

D: Laissez faire

Varianzanalyse:

\begin{tabular}{|c|c|c|c|c|}
\hline \multirow[b]{2}{*}{ Mittelwert-Vergleiche: } & & \multicolumn{3}{|c|}{$\begin{array}{l}\text { (Vi: } 1857.9 \\
\text { Vz: } 8988.8)\end{array}$} \\
\hline & & $A / B$ & $C$ & $D$ \\
\hline & $A / B$ & & n.s. & $* *$ \\
\hline & $C$ & n.s. & & $\bullet$ \\
\hline & $D$ & ** & $*$ & \\
\hline
\end{tabular}

\section{PRESSBBIP}

\begin{tabular}{|r|r|r|}
\hline \multicolumn{1}{|c|}{$M$} & SD & N \\
\hline 63.8 & 21.3 & 8 \\
\hline 83.6 & 38.4 & 7 \\
\hline 101.6 & 43.9 & 7 \\
\hline
\end{tabular}

$T G=2.15 \quad p=0.14$ (2.16 d.f.)

(Vi: 1242.3

Vz: 2674.8)

\begin{tabular}{|c|c|c|c|}
\hline & $A / B$ & $C$ & $D$ \\
\hline$A / B$ & & n.s. & $*$ \\
\hline$C$ & n.s. & & n.s. \\
\hline$D$ & $*$ & n.s. & \\
\hline
\end{tabular}

ENVIR/BIP

\begin{tabular}{|r|r|r|}
\hline \multicolumn{1}{|c|}{ M } & \multicolumn{1}{|c|}{ SD } & N \\
\hline 59.3 & 17.6 & 8 \\
\hline 80.9 & 29.5 & 7 \\
\hline 112.5 & 49.4 & 7 \\
\hline
\end{tabular}

$T G=4.58 p=0.02(2.16$ d.f. $)$

(Vi: 1160.9

Vz: 5319.20)

\begin{tabular}{|c|c|c|c|}
\hline & $A / B$ & $C$ & $D$ \\
\hline$A / B$ & & n.s. & $*$ \\
\hline$C$ & n.s. & & n.s. \\
\hline$D$ & $*$ & n.s. & \\
\hline
\end{tabular}

Anmi:

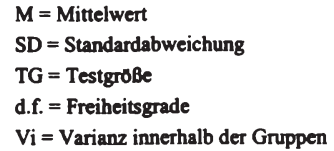

$\mathrm{Vz}=$ Varianz zwischen den Gruppen n.s. $=$ nicht signifikant

Daten aus 1990
Mittelwert-Vergleiche mittels Wilcoxon-Test

$:=\mathrm{p}<0.05$

$*:=p<0.01$ 
Am ökologisch uneffektivsten erscheint der innovative Pluralismus der Clustergruppe $\mathrm{C}$. Es ist jedoch zu bedenken, daß in dieser Gruppe außer Belgien und Irland fünf der hochindustrialisierten G7-Staaten vertreten sind. Da alle diese Staaten ein ausgesprochen hohes ökonomisches Niveau aufweisen, und in Kapitel 9.1.1. der Zusammenhang zwischen dem eben dieses Niveau repräsentierenden BIP-pro-Kopf-Wert und der Umweltbelastung nachgewiesen wurde (Tabelle 1), bleibt jedoch abzuwarten, ob die aus Tabelle 27 abzulesende Reihenfolge der Politikstile auch dann Bestand behält, wenn die Belastungsdaten auf das BIP der untersuchten Staaten bezogen wird.

Dieser Analyseschritt erfolgt in der in Tabelle 28 dargestellten Varianzanalyse.

Für den Ressourcenverbrauchsindex [RESSOU/BIP] ergibt dieses multivariate Analyseverfahren, daß die Varianz zwischen den Gruppen signifikant höher ist als die interne Gruppenvarianz (Signifikanzniveau: 0.07).

Wie in der vorausgegangenen Analyse ist auch diesmal für Clustergruppe A der günstigste Wert abzulesen (M: 46.9). Einen ebenfalls recht niedrigen Mittelwert weist die Gruppe der zentralistisch-administrativen Korporatismus-Staaten mit 59.4 Indexpunkten auf. Dieser Wert ist sogar hoch signifikant (Niveau: 0.01) kleiner als derjenige der Laissez-faire-Staaten (M: 123.4). Die Tatsache, daß Clustergruppe $\mathrm{C}$ mit einem durchschnittlichen Indexwert von 78.3 für diese Variable ebenfalls signifikant besser liegt als Gruppe D, bestätigt die oben hinsichtlich der durch das BIP-Niveau bedingten Verzerrungen der Umweltbelastungsdaten gemachten Überlegungen. Zur Erreichung des Zieles möglichst niedriger Umweltbelastungsdaten pro Einheit ökonomischen Outputs scheint der technologieorientierte, dezentrale Politikstil der Gruppe $\mathrm{C}$ deutlich effektiver zu sein als der auf die Abstützung durch technologische und administrative Kapazitäten verzichtende Politikstil der Clustergruppe D.

Diese Einschätzung bestätigt sich auch mit Blick auf die beiden anderen Indexwerte [PRESS/BIP] sowie [ENVIR/BIP]. Allerdings sind für den Schadstoffindex trotz identischer Reihenfolge der Clustergruppen keine signifikanten Varianz- und Mittelwertunterschiede zwischen den Gruppen zu attestieren.

Um die Unterschiede zwischen den Gruppen noch stärker herauszuarbeiten, und um für alle Gruppen eine varianzanalytisch ausreichende Fallzahl zu erzeugen, wurden die beiden Korporatismus-Cluster für die weiteren Analyseschritte zu einer einzigen, acht Staaten umfassenden Gruppe (A/B) zusammengefaßt (Tabellen 29 und 30).

Zwar gelingt es auch durch diese Vorgehensweise nicht, signifikante Mittelwertunterschiede zwischen der Gruppe der korporatistischen Staaten einerseits und der jeweils zweitbesten Clustergruppe andererseits zu erzeugen, die Analyseergebnisse sind jedoch um einiges deutlicher, und zumindest für die Varianzanalysen anhand der Umweltbelastungswerte pro Einheit BIP (Tabelle 30) er- 
geben sich teilweise sogar hochsignifikante Varianzunterschiede zwischen den Gruppen.

Als Resümee der varianzanalytischen Untersuchungen der Tabellen 27 bis 30 lassen sich die folgenden Erkenntnisse ableiten:

- Die anhand des Politikstils generierten Staatengruppen weisen erhebliche Unterschiede hinsichtlich ihrer Umweltbelastungsdaten auf.

- Als umweltpolitisch am effektivsten erweisen sich die beiden Varianten des Korporatismus.

- Ein technologieorientierter, in föderale Strukturen eingebundener Korporatismus ist der mit Abstand günstigste Politikstil, sowohl im Hinblick auf die Umweltbelastungsdaten pro Einwohner als auch pro Einheit BIP.

- Ebenfalls überdurchschnittlich gute Umweltbelastungsindexwerte ergeben sich für die Staaten mit einem „traditionell“ korporatistischen Politikstil, der konsensuale Politikformen mit einer starken zentralen Implementierungskapazität verbindet.

- Unterschiedliche Resultate gibt es für die Politikstile des Laissez-faire und des innovativen Pluralismus. Während der eine (Laissez-faire) für die auf die Einwohnerzahl bezogenen Belastungswerte besser abschneidet, erweist sich der andere für die Werte pro Einheit BIP als der effektivere Politikstil. Unter Berücksichtigung des ökonomischen Niveaus ist eine Kombination aus tendenziell konkurrenzorientierten und föderalen Elementen somit günstiger als ein stark zentralistischer und konfliktärer Politikstil. 


\section{Teil III: Schlußfolgerungen und Ausblick}

In diesem abschließenden Kapitel werden zwei Zwecke verfolgt. Erstens sollen die in den vergangenen Kapiteln gewonnenen Resultate der quantitativen Analyse noch einmal dahingehend hinterfragt werden, ob sie die Ausgangsthesen dieser Untersuchung und den daraus abgeleiteten Modellentwurf stützen oder ob sie diese Grundannahmen in weiten Teilen falsifizieren. Zweitens sollen Methodik und Strategie der Datenanalyse noch einmal kritisch hinterfragt werden. Dies geschieht u.a. mit dem Ziel, für an diese Arbeit anknüpfende Falsifizierungsbemühungen eventuell noch reliablere Vorgehensweisen insbesondere in Hinblick auf Quantität und Operationalisierung der Merkmalsdimensionen von abhängiger (Effektivität nationaler Umweltpolitik) und unabhängiger Variable (Politikstil) zu finden.

\section{Zusammenfassung und Interpretation der empirischen Untersuchungs- ergebnisse}

Als erste Erkenntnis aus den in den Kapiteln 8 bis 11 gewonnenen Analyseresultaten kann festgehalten werden, daß mittels der verwendeten Politikstildimensionen eine Clusterung der Grundgesamtheit in Gruppen mit signifikanten Varianzunterschieden möglich ist, d.h. es lassen sich anhand der Variablenausprägungen weitgehend homogene Staatengruppen gewinnen, und gleichzeitig kann zwischen diesen Gruppen gut differenziert werden. Reduzieren wir den in diesem Fall achtdimensionalen Variablenraum auf die zwei Grunddimensionen Partizipationsgrad und Strategieorientierung, lassen sich die vier Politikstiltypen an unterschiedlichen Punkten positionieren. Ein Cluster faßt Staaten mit einer typischen Kombination aus Strategieorientierung und eher geringem Partizipationgrad zusammen (Innovativer Pluralismus), eine weitere Gruppe wird aus Staaten mit nur geringfügig strategisch und partizipativ orientierten Verhaltensmustern in den Politikformulierungs- und -implementationsphasen des $\mathrm{Po}$ licy Cycles gebildet, und zwei weitere Cluster schließlich sind im Feld desjenigen Politikstiltypus positioniert, der partizipative und aktive, strategieorientierte Elemente kombiniert (Planerischer Korporatismus; auf eine Differenzierung zwischen diesen beiden Clustern wird anschließend noch einmal näher eingegangen). Ein Politikstil der partizipative und reaktive Elemente verbindet, konnte nicht identifiziert werden.

Die zweite wichtige Erkenntnis aus der empirischen Untersuchung ist die Tatsache, daß die zentrale These dieser Untersuchung, daß nämlich diese unterschiedlichen Typen der Formulierung und Implementierung von Politik, sprich: 
Politikstile, einen signifikanten Beitrag zur Erklärung unterschiedlicher Niveaus der Effektivität nationaler Umweltpolitik leisten, weitgehend verifiziert werden konnte. Die Effektivität nationaler Umweltpolitik, operationalisiert durch die beiden auf das okonomische Niveau und die Einwohnerzahl bezogenen Umweltbelastungsfaktoren Schadstoffemissionen und Ressourcenverbrauch, variiert stark in Abhängigkeit von der konkreten Ausgestaltung des jeweiligen nationalen Politikstils.

Dabei ist zunächst der jeweils isolierte Effekt der beiden einzelnen Politikstildimensionen zu betrachten. Zwischen dem Partizipationsgrad der von umweltpolitischen Entscheidungen Betroffenen und den Indikatoren zur Messung der Umweltbelastung besteht eine durchgehend starke und negative Korrelation, d.h. je höher der Partizipationsgrad, desto geringer ist das Ausmaß der Umweltbelastung. Die Vermutung, daß durch die Einbeziehung der Betroffenen und der sie vertretenden privaten Interessengruppen (Bürgerinitiativen, Umweltschutzorganisationen) in den umweltpolitischen Entscheidungsprozeß ökologisch weniger belastende Lösungen gefunden werden, hat sich weitgehend bestätigt. Weniger eindeutig ist die Beziehung zwischen der zweiten Politikstildimension, der Strategieorientierung, und dem Ausmaß der Umweltbelastung. Tendenziell ist zwar auch für den Zusammenhang dieser beiden Variablenkomplexe eine negative Korrelation feststellbar, doch während dies für die Technologische Kapazität als einer Variable zur Operationalisierung der Konstruktes Strategieorientierung im starken Maße gültig ist, sind die Korrelationen zwischen den beiden anderen Variablen, der Bereitstellung Administativer Kapazitäten und dem Agieren in föderalen Strukturen (Föderalismusgrad), sowie den Umweltbelastungindikatoren weniger eindeutig. Diese Feststellung mag als erster Hinweis darauf dienen, daß es notwendig sein könnte, die zweidimensionale Betrachtungsweise nationaler Politikstile aufzugeben, und insbesondere den Faktor Strategieorientierung zum Zwecke einer stärkeren Differenzierung durch mindestens zwei andere Dimensionen zu ersetzen.

Ein jedoch zunächst erfolgender abschließender Blick auf die zweidimensionale Politikstiltypologie führt zu folgendem Resümee:

1. Das höchste Maß an umweltpolitischer Effektivität erreichen die Staaten des Planerischen Korporatismus, d.h. eine Politik, die einen starken aktivplanerischen Impetus mit einer konsensualen Einbeziehung privater Interessengruppen kombiniert, führt zu den aus ökologischer Sicht positivsten Resultaten.

2. Der Politikstil des Laissez-faire, d.h. eine Kombination aus geringfügiger Strategieorientierung und einem Mangel an Partizipation der nicht-staatlichen Akteure, verursacht eher hohe Werte der Umweltbelastungsindikatoren, insbesondere wenn diese in Relation zum jeweiligen ökonomischen Niveau gesetzt werden. 
3. Eine mittlere Position hinsichtlich der umweltpolitischen Effektivität nimmt der Innovative Pluralismus ein, d.h. eine Strategieorientierung und ein nur geringes $\mathrm{Ma} \beta$ an partizipativen Mustern der Interessenvermittlung kombinierende Politik. Während die Umweltbelastungsdaten bezogen auf die Einwohnerzahl eher schlecht sind, fallen die Werte pro Einheit BIP im Vergleich zum Laissez-faire-Stil deutlich besser aus.

Da bereits die Clusteranalyse gezeigt hat, daß es sich beim Planerischen Korporatismus um keinen homogenen Politikstil handelt, sondern daß sich zwei Cluster innerhalb dieses Typus gewinnen lassen, die für die Variablen zur Messung der Strategieorientierung deutlich unterschiedliche Datenmuster aufweisen, ergibt sich die folgende inhaltliche Differenzierung dieser beiden Staatengruppen: Die Staaten des als Technolgisch-dezentraler Korporatismus bezeichneten Clusters weisen bei einem eher durchschnittlichen Niveau der administrativen Kapazität eine typische Merkmalskombination aus föderalem oder zumindest dezentralem Staatsaufbau und einer hohen technologischen Kapazität auf. Diese technologische Kapazität ist andererseits bei den Staaten des Administrativzentralistischen Korporatismus weniger stark ausgeprägt. Im Gegensatz dazu verfügen diese Staaten über eine vergleichsweise hohe administrative Kapazität bei gleichzeitig eher zentralem Staatsaufbau.

Die empirischen Analysen haben aufgezeigt, daß das korporatistische, partizipative Politikstilelement stets einen direkten positiven Effekt auf die Resultate der Umweltpolitik hat. Für die Elemente der Strategieorientierung gilt jedoch, $\mathrm{daß}$ sie nur in spezifischen Kombinationen (Föderalismus/Technologische Kapazität bzw. Zentralismus/Administrative Kapazität) umweltentlastend wirken. Als wichtigste Erkenntnis der vorliegenden Untersuchung hat somit die Verifizierung des folgenden Zusammenhanges zu gelten:

Ein Politikstil, der zum einen partizipativ angelegt ist, und zum anderen über das strategische Moment entweder einer in dezentralen Strukturen wirkenden technologischen Kapazität oder einer zentralstaatlich verankerten administrativen Kapazität verfügt, führt zu einer vergleichsweise hohen Effektivität der nationalen Umweltpolitik.

Die für die zweite Politikstildimension, die Strategieorientierung, etwas umständliche Formulierung des obigen Satzes verweist auf einen ersten Ansatzpunkt für die nachfolgend dargelegte Methodenkritik. 


\section{Kritik an Methodik und Strategie der Datenanalyse}

Die praktische Bewährungsprobe der angewendeten Methodik und Analysestrategie gibt aus der Ex-post-Perspektive Anlaß zu einigen Anmerkungen:

- Das zweidimensionale Politikstil-Modell scheint zur Erklärung der umweltpolitischen Realitäten ergänzungsbedürftig zu sein. Die als Merkmale staatlicher Strategieorientierung angenommenen Einzelindikatoren - dezentrale Entscheidungs- und -implementationsstrukturen, technologisches Know-How sowie administrative Handlungskapazitäten - erweisen sich in der quantitativen Analyse nicht als Elemente eines Faktors, sondern machen aufgrund der heterogenen Datenmuster eine mehrdimensionale Interpretation der Ergebnisse notwendig. Für weitere Untersuchungen dürfte es also günstig sein, bereits ex ante zwischen einer eher technologie- bzw. einer eher administrativ orientierten Ausrichtung staatlichen Handelns als zwei unterschiedlichen Dimensionen des Politikstils zu unterscheiden, und den Dezentralisationsgrad weniger als Beschreibungsmerkmal staatlichen Handelns, sondern eher als indirekt wirkende Moderatorvariable der politischen Struktur zu behandeln.

- Während für die Indikatorvariablen der Umweltbelastung und der technologischen und administrativen Kapazitäten aufgrund des quantitativen Datenmaterials und der detaillierten, sich über lange Zeitreihen erstreckenden Datenquellen von einer hohen Reliabilität ausgegangen werden kann, erfolgte die Messung von Partizipations- und Dezentralisationsgrad aufgrund weniger Beobachtungen und primär anhand von subjektiven Einschätzungen, also eher „weichem“ Datenmaterial. Eine weitere Überlegung zur Absicherung der Untersuchungsergebnisse müßte es also sein, die Operationalisierung dieser beiden Faktoren durch eine größere Zahl von „objektiveren“, d.h. quantitativ leichter meßbaren Einzelindikatoren zu verbessern.

- Der dritte Kritikpunkt schließlich bezieht sich auf die Grundgesamtheit der vorliegenden Untersuchung. Die Begründung für den gewählten Kompromiß zwischen der Homogenität der Untersuchungsobjekte und der Verallgemeinbarkeit der Ergebnisse wurde bereits in Kapitel 7.1. geliefert. Dennoch wäre es sicherlich wünschenswert, eine Aussage über den Zusammenhang zwischen Politikstil und Effektivität nationaler Umweltpolitik auch für die sogenannten „emerging markets“, also die ökonomisch aufstrebenden Staaten Asiens und Lateinamerikas sowie die erfolgreichen Reformstaaten Mittelund Osteuropas, machen zu können, vor allem vor den Hintergrund der Frage, ob die mit dem enormen ökonomischen Wachstumstempo einhergehenden Umweltbelastungen durch den jeweilgen nationalen Politikstil noch verstärkt werden oder nicht. Zwar scheitert der Versuch einer Erweiterung der untersuchten Grundgesamtheit zunächst ohnehin noch am fehlenden Daten- 
material für die betreffenden Staaten, dennoch ist es denkbar, im Falle des Vorliegens der benötigten Daten (etwa durch Beitritt dieser Staaten zur OECD wie bei Mexiko, Südkorea, der Tschechischen Republik und Ungarn kürzlich geschehen) die Anzahl der Untersuchungsobjekte vorsichtig zu erweitern. 


\section{Literatur:}

Alemann, Ulrich von (1995): Politikbegriffe, In: Nohlen, Dieter (Hg.): Wörterbuch Staat und Politik, Bonn, S. 542-545.

Almond, Gabriel A., und Verba, Sidney (1963): The Civic Culture, Boston.

Almond, Gabriel A. und Powell, Jr., Bingham G. (1978): Comparative Politics. System, Process, and Policy, Boston;Toronto.

Almond, Gabriel A. (1987): Zum Konzept der politischen Kultur. Politische Kultur-Forschung - Rückblick und Ausblick, In: Berg-Schlosser, Dirk, und Schissler, Jakob (Hg.): Politische Kultur in Deutschland. Bilanz und Perspektiven der Forschung, Politische Vierteljahresschrift, Sonderheft 18, Opladen, S. 27-38.

Almond, Gabriel A., und Powell, Jr., Bingham G. (1992) (Hg.): Comparative Politics Today: A World View, 5. Aufl., New York.

Anderson, James E. (1979): Public Policy-Making, Chicago; San Franzisko.

Anton, Thomas J. (1969): Policy Making and Political Culture in Sweden, in: Scandinavian Political Studies, 4, S. 88-102.

Backhaus, K., Erichson, B., Plinke, W. und Weiber, R. (1980): Multivariate Analysemethoden - Eine anwendungsorientierte Einführung, Berlin; Heidelberg; New York.

Badaracco, Joseph L. (1985): Loading the Dice. A Five-Country Study of Vinyl Chloride Regulation, Boston.

Berg-Schlosser, Dirk (1987): Politische Kultur, In: In: Nohlen, Dieter, und Schultze, Rainer-Olaf (Hg.): Politikwissenschaft. Theorien - Methoden - Begriffe, München; Zürich, S. 746-751.

Berg-Schlosser, Dirk, und Schissler, Jakob (1987) (Hg.): Politische Kultur in Deutschland. Bilanz und Perspektiven der Forschung, Politische Vierteljahresschrift, Sonderheft 18, Opladen. 
Berg-Schlosser, Dirk, und Stammen, Theo (1992): Einführung in die Politikwissenschaft, München.

Bingham, Gail (1986): Resolving Environmental Disputes. A Decade of Experience, Washington.

Blankart, Ch. B. (1991): Öffentliche Finanzen in der Demokratie, München.

Blankenburg, Erhard; Schmid, Günther, und Treiber, Hubert (1974): Von der reaktiven zur aktiven Politik? Darstellung und Kritik des Policy Sciences-Ansatzes, In: Grottian, Peter, und Murswieck, Axel (Hg.): Handlungsspielräume der Staatsadministration, Hamburg, S. 35-51.

Böhret, Carl (1990): Folgen. Entwurf für eine aktive Politik gegen schleichende Katastrophen, Opladen.

Bleischwitz, Raimund (1995): Atmosphäre und Klima, In: Hauchler, Ingomar (Hg.): Globale Trends 1996. Daten zur Weltentwicklung, Frankfurt am Main, S. 259-280.

Bossel, Hartmut (1990): Umweltwissen, Berlin.

Brewer, Garry D. (1978): Termination: Hard Choices - Harder Questions, in: Public Administration Review, 4, S. 338-344.

Brewer, Garry D., und deLeon, Peter (1983): The Foundations of Policy Analysis, Homewodd.

Brickman, Ronald; Jasanoff, Sheila, und Ilgen, Thomas (1982): Cancer: A Cross-National Study of Policy and Politics, Cornell.

Bull, Martin J. (1992): The Corporatist Ideal-Type and Political Exchange, in: Political Studies, 40, S. 255-272.

Bungarten, Harald H. (1978): Umweltpolitik in Westeuropa, Bonn.

Cameron, David R. (1984): Social Democracy, Corporatism, Labour Quiescence and the Representation of Economic Interest in Advanced Capitalist Society, In: Goldthorpe, J.H. (Hg.): Order and Conflict in 
Contemporary Capitalism: Studies in the Political Economy of Western European Nations, Oxford, S. 143-178.

Cawson, A. (1986): Corporatism and Political Theory, Oxford.

Cobb, Roger W., und Elder, Charles D. (1983): Participation in American Politics: The Dynamics of Agenda-Building, Boston.

Crepaz, Markus M. L. (1992): Corporatism in Decline? An Empirical Analysis of the Impact of Corporatism on Macroeconomics Performance and Industrial Disputes in 18 Industrialized Democracies, in: Comparative Political Sciences, 2, S. 139-168.

Cusack, Thomas R. (1994): Economic Problems in the OECD Countries during the Period 1950 through 1990, unveröffentlichter Entwurf, Berlin.

Czada, Roland (1983): Konsensbedingungen und Auswirkungen neokorporatistischer Politikentwicklung, in: Journal für Sozialforschung, 4, S. 421-441.

Czada, Roland (1983): Korporatismus, In: Schmidt, Manfred G. (Hg.): Westliche Industriegesellschaften. Wirtschaft - Gesellschaft - Politik, München, S. 209-217.

Czada, Roland, und Windhoff-Héretier, Adrienne (1990) (Hg.): Political Choice, Institutions, Rules and the Limits of Rationality, Frankfurt a.M

Czada, Roland (1995): Korporatismus/Neo-Korporatismus, In: Nohlen, Dieter (Hg.): Wörterbuch Staat und Politik, Bonn, S. 365-370.

Dalton, Russel, J. (1992): Politics in Germany, In: Almond, Gabriel A., und Powell, Jr., Bingham G. (Hg.): Comparative Politics Today: A World View, 5. Aufl., New York, S. 239-296.

Decker, Frank (1994): Ökologie und Verteilung. Eine Analyse der sozialen Folgen des Umweltschutzes, in: Aus Politik und Zeitgeschichte, 49, S. 22-32. 
Dieren, Wouter van (1995): Mit der Natur rechnen. Der neue Club-ofRome-Bericht: Vom Bruttosozialprodukt zum Ökosozialprodukt, Basel; Boston; Berlin.

Der Rat von Sachverständigen für Umweltfragen (1988): Umweltgutachten 1987, Stuttgart; Mainz.

Dierkes, Meinolf, et al. (1987) (Hg.): Comparative Policy Research, Aldershot.

Dogan, Mattei, und Pelassy, Dominique (1990): How to Compare Nations. Strategies in Comparative Politics, Chatham.

Downing, Paul, und Hanf, Kenneth (1983): Implementing Pollution Laws: International Comparison, Boston.

Downs, Anthony (1972): Up and Down with Ecology - The IssueAttention-Cycle, in: Public Interest, 28, S. 38-50.

Dror, Yehezkel (1971): Ventures in Policy Sciences: Concept and Applications, New York.

Dye, Thomas R. (1976): Policy Analysis: What Governments Do, Why They Do it, ans What Difference it Makes, Alabama.

Dyson, Kenneth (1982): West-Germany: The Search for a Rationalist Consensus, In: Richardson, Jeremy, et al. (Hg.): Policy Styles in Western Europe, London, S. 17-46.

Easton, David (1965): A Framework for Political Analysis, Englewood Cliffs.

Easton, David (1967): A Systems Analysis of Political Life, New York

Ehrmann, Henry W., und Shain, Martin A. (1992): Politics in France, In: Almond, Gabriel A., und Powell, Jr., Bingham G. (Hg.): Comparative Politics Today: A World View, 5. Aufl., New York, S. 189-238.

Eisfeld, Rainer (1995): Pluralismus/Pluralismustheorie, In: Nohlen, Dieter (Hg.): Wörterbuch Staat und Politik, Bonn, S. 537-542. 
Enloe, Cynthia H. (1975): The Politics of Pollution in a Comparative Perspective. Ecology and Power in Four Nations, New York.

Feick, Jürgen, und Jann, Werner (1988): „Nations matter“ - Vom Elektizismus zur Integration in der vergleichenden Policy-Forschung?, In: Schmidt, Manfred G. (Hg.): Staatstätigkeit: International und historisch vergleichende Analysen, Politische Vierteljahresschrift, Sonderheft 19, S. 196-220.

Fenner, Christian (1995): Politische Kultur, In: Nohlen, Dieter (Hg.): Wörterbuch Staat und Politik, Bonn, S. 565-571.

Flanagan, Scott C., und Richardson, Bradley M. (1992): Politics in Japan, In: Almond, Gabriel A., und Powell, Jr., Bingham G. (Hg.): Comparative Politics Today: A World View, 5. Aufl., New York, S. 297-350.

Freeman, Gary P. (1985): National Styles and Policy Sectors: Explaining Structured Variation, in: Journal of Public Policy, 5, S. 467-496.

Fietkau, Hans-Joachim, und Weidner, Helmut (1992): Mediationsverfahren in der Umweltpolitik. Erfahrungen in der Bundesrepublik Deutschland, in: Aus Politik und Zeitgeschichte, 39-40, S. 24-34.

Goldthorpe, J.H. (1984) (Hg.): Order and Conflict in Contemporary Capitalism: Studies in the Political Economy of Western European Nations, Oxford.

Grant, W. (1985) (Hg.): The Political Economy of Corporatism, New York

Grottian, Peter, und Murswieck, Axel (1974): Handlungsspielräume der Staatsadministration, Hamburg.

Harenberg (1994): Harenberg Länderlexikon '94/95, Dortmund.

Hartmann, Jürgen (1980) (Hg.): Vergleichende politische Systemforschung. Konzepte und Analysen, Köln; Wien.

Hartmann, Jürgen (1980): Vergleichende politische Systemforschung und komparative Methoden, In: Hartmann, Jürgen (Hg.): Vergleichende politische Systemforschung. Konzepte und Analysen, Köln; Wien, S. 1959. 
Hartmann, Jürgen (1980): Das amerikanische Regierungssystem: eine Skizze aus Sicht der Vergleichenden Regierungslehre, In: Hartmann, Jürgen (Hg.): Vergleichende politische Systemforschung. Konzepte und Analysen, Köln; Wien, S. 63-81.

Hartmann, Jürgen (1983): Politik und Gesellschaft in Japan, USA, Westeuropa. Ein einführender Vergleich, Frankfurt/Main; New York.

Hartmann, Jürgen (1995): Vergleichende Politikwissenschaft, Frankfurt/Main; New York.

Hartwich, Hans-Hermann (1985) (Hg.): Policy-Forschung in der Bundesrepublik Deutschland. Ihr Selbstverständnis und ihr Verhältnis zu den Grundfragen der Politikwissenschaft, Opladen.

Hashimoto, Michio (1989): The Environmental Monitoring and Reporting System: An Outline, In: Tsuru, Shigeto, und Weidner, Helmut (Hg): Environmental Policy in Japan, Berlin, S. 453-460.

Hauchler, Ingomar (1993) (Hg.): Globale Trends 93/94. Daten zur Weltentwicklung, Frankfurt am Main.

Hauchler, Ingomar (1995) (Hg.): Globale Trends 1996. Daten zur Weltentwicklung, Frankfurt am Main.

Hayward, Jack (1982): Mobilizing Private Interests in the Service of Public Ambitions: The Salient Element in the Dual French Policy Style?, In: Richardson, Jeremy, et al. (Hg.): Policy Styles in Western Europe, Boston; Sydney, S. 111-140.

Heidenheimer, Arnold J., und Johansen, Lars (1985): Organized Medicine and Scandinavian Professional Unionism: Hospital Policies and Exit Options in Denmark and Sweden, in: Journal of Health Politics, Policy, and Law, 2, S. 347-370.

Henn, Heike, et al. (1993): Weltökologie, In: Hauchler, Ingomar (Hg.): Globale Trends 93/94. Daten zur Weltentwicklung, Frankfurt am Main, S. 277-330.

Héritier, Adrienne (1993) (Hg.): Policy-Analyse, Kritik und Neuorientierung, Politische Vierteljahresschrift, Sonderheft 24, Opladen. 
Hird, John A. (1993): Environmental Policy and Equity: The Case of Superfund, in: Journal of Policy Analysis and Management, 12, S. 323343.

Holzinger, Katharina (1995): A Surprising Success in EC Environmental Policy: The Small Car Exhaust Emission Directive of 1989, In: Jänikke, Martin, und Weidner, Helmut (Hg.): Successful Environmental Policy. A Critical Evaluation of 24 Cases, Berlin, S. 187-202.

Imamura, Tsunao (1989): Environmental Responsibilities at the National Level, In: Tsuru, Shigeto, und Weidner, Helmut (Hg.): Environmental Policy in Japan, Berlin, S. 43-53.

Imura, Hidefumi (1989): Administration of Pollution Control at Local Level, In: Tsuru, Shigeto, und Weidner, Helmut (Hg): Environmental Policy in Japan, Berlin, S. 54-96.

Inglehart, Ronald (1977): The Silent Reution in Europe: Changing Values and Political Styles among Western Publics, Princeton.

Inglehart, Ronald (1988): Politische Kultur und stabile Demokratie, in: Politische Vierteljahresschrift, 3, S. 369-387.

Jänicke, Martin, und Mönch, Harald (1988): Ökologischer und wirtschaftlicher Wandel im Industrieländervergleich, in: Schmidt, Manfred G. (Hg.): Staatstätigkeit. International und historisch vergleichende Analysen, Politische Vierteljahresschrift, Sonderheft 19, Opladen, S. 389-405.

Jänicke, Martin (1990): Erfolgsbedingungen von Umweltpolitik im internationalen Vergleich, in: Zeitschrift für Umweltpolitik und Umweltrecht, 3, S. 213-232.

Jänicke, Martin; Mönch, Harald; Binder, Manfred et al. (1993): Umweltentlastung durch industriellen Strukturwandel? Eine explorative Studie über 32 Industrieländer (1970 bis 1990), 2. Aufl., Berlin.

Jänicke, Martin; Bratzel, Stefan; Carius, Alexander; Kern, Kristine; Mönch, Harald (1995): Umweltpolitik im internationalen Vergleich, FFU-report 95-5, Berlin. 
Jănicke, Martin, und Weidner, Helmut (1995) (Hg.): Successful Environmental Policy. A Critical Evaluation of 24 Cases, Berlin.

Jănicke, Martin, und Weidner, Helmut (1995): Successful Environmental Policy: An Introduction, In: Jănicke, Martin, und Weidner, Helmut (Hg.): Successful Environmental Policy. A Critical Evaluation of $24 \mathrm{Ca}$ ses, Berlin, S. 10-26.

Jănicke, Martin (1996) (Hg.): Umweltpolitik der Industrieländer. Entwicklung - Bilanz - Erfolgsbedingungen; Berlin.

Jănicke, Martin (1996): Erfolgsbedingungen von Umweltpolitik; In: Jänicke, Martin (Hg.): Umweltpolitik der Industrielănder. Entwicklung Bilanz - Erfolgsbedingungen; Berlin, S. 9-28.

Jann, Werner (1981): Kategorien der Policy-Forschung, Speyer.

Jann, Werner (1987): Policy, In: Nohlen, Dieter, und Schultze, RainerOlaf (Hg.): Politikwissenschaft. Theorien - Methoden - Begriffe, München; Zürich, S. 702.

Jann, Werner (1987): Politics, In: Nohlen, Dieter, und Schultze, RainerOlaf (Hg.): Politikwissenschaft. Theorien - Methoden - Begriffe, München; Zürich, S. 704.

Jann, Werner (1995): Politikfeldanalyse, In: Nohlen, Dieter (Hg.): Wörterbuch Staat und Politik, Bonn, S. 550-555.

Jarre, Jan (1976): Die verteilungspolitische Bedeutung von Umweltschäden, Göttingen.

Johnson, Chalmers (1977): MITI and Japanese International Economic Policy, In: Scalapino, Robert A. (Hg.): The Foreign Policy of Modern Japan, Berkeley; Los Angeles, S. 253-255.

Joppke, Christian (1993): Mobilizing against Nuclear Energy: A Comparison of Germany and the United States, Berkeley; Los Angeles.

Junne, Gerd (1993): Neue Technologien, In: Hauchler, Ingomar (Hg.): Globale Trends 93/94. Daten zur Weltentwicklung, Frankfurt am Main, S. 369-384. 
Kaase, M., und Klingemann (1983) (Hg.): Wahlen und System, Opladen

Kaase, M. (1983): Sinn und Unsinn des Konzeptes politischer Kultur für die vergleichende Politikforschung, In: Kaase, M., und Klingemann (Hg.): Wahlen und System, Opladen, S. 144-172.

Katzenstein, Peter J. (1978): Between Power and Plenty. Foreign Economic Policies of Advanced Industrial States, Madison.

Katzenstein, Peter J. (1985): Small States in World Markets. Industrial Policy in Europe, Ithaca; London.

Kelman, Steven (1981): Regulating America, Regulating Sweden: A Comparative Study of Occupational Safety and Health Policy, Cambridge.

Kern, Kristine, und Bratzel, Stefan (1994): Erfolgskriterien und Erfolgsbedingungen von (Umwelt-)politik im internationalen Vergleich: Eine Literaturstudie, FFU-report 94-3, Berlin.

Kern, Kristine, und Bratzel, Stefan (1994): Umweltpolitischer Erfolg im internationalen Vergleich, unveröffentlichtes Manuskript, Berlin.

Kitschelt, Herbert (1983): Politik und Energie: EnergieTechnologiepolitiken in den USA, der Bundesrepublik Deutschland, Frankreich und Schweden, Frankfurt/Main; New York.

Knoepfel, Peter; Weidner, Helmut, und Hanf, Kenneth (1979): The Implementation of $\mathrm{SO}_{2}$ Control Policy in the European Community and Switzerland, Air Pollution Control: National und International Perspectives, Aspen.

Knoepfel, Peter, und Weidner, Helmut (1985): Luftreinhaltepolitik (stationäre Quellen) im internationalen Vergleich, Berlin.

Knoepfel, Peter, und Weidner, Helmut (1986): Explaining Differences in the Performance of Clean Air Policies: An International and Interregional Comparative Study, in: Policy and Politics, 14, S. 71-91. 
Knoepfel, Peter, et al. (1987): Comparing Environmental Policies: Different Styles, Similar Content, In: Dierkes, Meinolf, et al. (Hg.): Comparative Policy Research, Aldershot, S. 171-186.

Knoepfel, Peter (1993): Bedingungen einer wirksamen Umsetzung umweltpolitischer Programme - Erfahrungen aus Westeuropäischen Staaten, Lausanne.

Kohler-Koch, Beate (1986) (Hg.): Technik und internationale Politik, Baden-Baden.

Kohler-Koch, Beate (1989) (Hg.): Regime in den internationalen Beziehungen, Baden-Baden.

Kreiner, Josef (1994): Religionen heute, In: Mayer, Hans Jürgen, und Pohl, Manfred (Hg.): Länderbericht Japan, Bonn.

Kurz, Rudi (1996): Innovationen in eine zukunftsfähige Entwicklung, in: Aus Politik und Zeitgeschichte, 7, S. 14-22.

Lasswell, Harold D. (1956): The Decision Process, Maryland.

Lasswell, Harold D. (1971): A Pre-View of Policy Sciences, New York.

Lehmbruch, Gerhard (1977): Liberal Corporatism and Party Government, in: Comparative Political Studies, 10, S. 91-126.

Lehmbruch, Gerhard, und Schmitter, Phillipe C. (1982) (Hg.): Patterns of Corporatist Policy Making, Beverly Hills.

Lehmbruch, Gerhard (1984): Concertation and the Structure of Corporatist Networks, In: Goldthorpe, J.H. (Hg.): Order and Conflict in Contemporary Capitalism: Studies in the Political Economy of Western European Nations, Oxford.

Lehmbruch, Gerhard (1990): Public Bureaucracies, Strategic Choice, and the Development of Political Networks, In: Czada, Roland, und Windhoff-Héretier, Adrienne (Hg.): Political Choice, Institutions, Rules and the Limits of Rationality, Frankfurt a.M., S. 121-158. 
Lehmbruch, Gerhard (1995): Konkordanzdemokratie, In: Nohlen, Dieter (Hg.): Wörterbuch Staat und Politik, Bonn, S. 350-354.

deLeon, Peter (1983): Policy Evaluation and Program Termination, In: May, Judith V., und Wildavsky, Aaron B. (Hg.): The Policy Cycle, London u.a., S. 279-300.

Lerner, Daniel, und Lasswell, Harold D. (1951) (Hg.): The Policy Sciences. Recent Development in Scope and Method, Stanford.

Lowi, Theodore, J. (1964): American Business, Public Policy, Case Studies, and Political Theory, in: World Politics, Jg. 16, 4, S. 677-715.

Lowi, Theodore, J. (1969): The End of Liberalism, New York.

Lowi, Theodore, J. (1972): Four Systems of Policy, Politics, and Choice, in: Public Administration Review, 33, S. 298-310.

Lundquist, Lennart J. (1974): Do Political Structures Matter in Environmental Politics? The Case of Air Pollution Control in Canada, Sweden, and the United States, in: Canadian Public Administration, 17, S. 119-141.

Lundquist, Lennart J. (1980): The Hare and the Tortoise: Clean Air Policies in the United States and Sweden, Ann Arbor.

Lundquist, Lennart J. (1995): Municipal Sewage Treatment in Sweden. From Bans on Bathing to Schools of Salmon, In: Jänicke, Martin, und Weidner, Helmut (Hg.): Successful Environmental Policy. A Critical Evaluation of 24 Cases, Berlin, S. 43-60.

Mazmanian, Daniel (1995): Electric Energy Conservation Policy and Environmental Protection in California, In: (Hg.): Jänicke, Martin, und Weidner, Helmut (Hg.): Successful Environmental Policy. A Critical Evaluation of 24 Cases, Berlin, S.272-287.

Malunat, Bernd M. (1994): Die Umweltpolitik der Bundesrepublik Deutschland, in: Aus Politik und Zeitgeschichte, 49, S. 3-12.

May, Judith V., und Wildavsky, Aaron B. (1983) (Hg.): The Policy Cycle, London u.a. 
Mayer, Hans Jürgen, und Pohl, Manfred (1994) (Hg.): Länderbericht Japan, Bonn.

Mayer, Hans Jürgen (1994): Umweltpolitik auf internationaler Ebene: Anpassungsdruck als „Motor des Fortschritts“, In: Mayer, Hans Jürgen, und Pohl, Manfred (Hg.): Länderbericht Japan, Bonn, S. 179-182.

Mayntz, R., et al. (1982): Regulative Politik und politisch-administrative Kultur. Ein Vergleich von fünf Ländern und vier Interventionsprogrammen (Projektbericht des Instituts für angewandte Sozialforschung), Köln.

Meadows, Donella; Meadows, Dennis, und Randers, Jorgen (1992): Die neuen Grenzen des Wachstums, Stuttgart.

Meter, Donald S. van, und Horn, Carl E. van (1975): The Policy Implementation Process. A Conceptual Framework, in: Administration and Society, S. 445-488.

Mez, Lutz (1995): Reduction of Exhaust Gases at Large Combustion Plants in the Federal Republic of Germany, In: Jänicke, Martin, und Weidner, Helmut (Hg.): Successful Environmental Policy. A Critical Evaluation of 24 Cases, Berlin, S. 173-186.

Müller-Rommel, Ferdinand (1992): Erfolgsbedingungen Grüner Parteien in Westeuropa, in: Politische Vierteljahresschrift, 2, S. 189-218.

Müller-Rommel, Ferdinand (1993): Grüne Parteien in Westeuropa. Entwicklungsphasen und Erfolgsbedingungen, Opladen.

Nagel, Stuart S. (1991): Public Policy. Goals, Means, and Methods, Lanham.

Nelkin, Dorothy, und Pollack, Michael (1981): The Atom Besieged. Extraparlamentary Dissent in France and Germany, Cambridge; London.

Nohlen, Dieter, und Schultze, Rainer-Olaf (1987): Politikwissenschaft. Theorien - Methoden - Begriffe, München; Zürich.

Nohlen, Dieter, und Thibaut, Bernhard (1995): Politisches System, In: Nohlen, Dieter (Hg.): Wörterbuch Staat und Politik, Bonn; S. 610-613 
Nohlen, Dieter (1995) (Hg.): Wörterbuch Staat und Politik, Bonn.

OECD (div. Jg.) (Hg.): OECD Environmental Data - Données OCDE sur l'environnement, Paris.

OECD (1994) (Hg.): Environmental Indicators, Paris.

OECD (1995) (Hg.): OECD in Figures. Statistics on the Member Countries, Paris.

Pearce, D.W. (1982): The Distribution of the Costs and Benefits of Environmental Policy, Environment Committee OECD, ENV/ECO, 82.3, vervielf. Manuskript, Paris.

Pihan, Andreas (1995): Der Policy Cycle der Pflegesicherung - Zur Bedeutung von Politiknetzwerken, Dissertation, Hamburg.

Popper, Karl (1976): Logik der Forschung, 6. Aufl.,Tübingen.

Pressman, Jeffrey, und Wildavsky, Aaron B. (1973): Implementation, Berkeley.

Prittwitz von, Volker (1986): Drei Ansätze zur Analyse internationaler Umweltpolitik, In: Kohler-Koch, Beate (Hg.): Technik und internationale Politik, Baden-Baden, S. 489-505.

Prittwitz von, Volker (1989): Internationale Umweltregime - Ein Fallvergleich, In: Kohler-Koch, Beate (Hg.): Regime in den internationalen Beziehungen, Baden-Baden, S. 389-405.

Prittwitz von, Volker (1990): Das Katastrophenparadox - Elemente einer Theorie der Umweltpolitik, Opladen.

Prittwitz von, Volker (1993): Katastrophenparadox und Handlungskapazität. Theoretische Orientierungen der Politikanalyse, in: Héritier, Adrienne (Hg.): Policy-Analyse, Kritik und Neuorientierung, Politische Vierteljahresschrift, Sonderheft 24, Opladen, S. 372-394.

Prittwitz von, Volker (1993): Politikfeldanalyse, Opladen. 
Prokop, H. (1987): Koordinierung und Abschottung. Muster staatlichen Problemlösungsverhaltens in Frankreich und Italien, Dissertation, Speyer.

Pryor, Frederic L. (1988): Corporatism as an Economic System: A Review Essay, in: Journal Of Comparative Economics, 12, S. 317-344.

Putnam, Robert D. (1973): The Beliefs of Politicians, New Haven.

Przeworski, A., und Teune, H. (1970): The Logic of Comparative Social Inquiry, New York.

Ranney, Austin (1992): Politics in the United States, In: Almond, Gabriel A. und Powell, Jr., Bingham G. (Hg.): Comparative Politics Today: A World View, 5. Aufl., New York, S. 563-601.

Reich, Michael R. (1984): Mobilizing for Environmental Policy in Italy and Japan, in: Comparative Politics, 16, S. 379-402.

Richardson, Jeremy, et al. (1982) (Hg.): Policy Styles in Western Europe, Boston; Sydney.

Richardson, Jeremy; Gustafsson, Gunnel, und Jordan, Grant (1982): The Concept of Policy Style, In: Richardson, Jeremy, et al. (Hg.): Policy Styles in Western Europe, Boston; Sydney, S. 1-16.

Richardson, Jeremy (1982): Convergent Policy Styles in Europe?, In: Richardson, Jeremy, et al. (Hg.): Policy Styles in Western Europe, Boston; Sydney, S. 197-209.

Ringquist, Evan J. (1993): Environmental Protection at the State Level. Politics and Progress in Controlling Pollution, Armonk; London.

Rohe, K. (1987): Politische Kultur und der kulturelle Aspekt von politischer Wirklichkeit - Konzeptionelle und typologische Überlegungen zu Gegenstand und Fragestellung Politischer Kulturforschung, In: BergSchlosser, Dirk, und Schissler, Jakob (Hg.): Politische Kultur in Deutschland. Bilanz und Perspektiven der Forschung, Politische Vierteljahresschrift, Sonderheft 18, Opladen, S. 39-48. 
Rose, Richard (1992): Politics in England, In: Almond, Gabriel A., und Powell, Jr., Bingham G. (Hg.): Comparative Politics Today: A World View, 5. Aufl., New York, S.131-188.

Rothstein, Bo (1991): State Structure and Variations in Corporatism: The Swedish Case, in: Scandinavian Political Studies, 2, S. 149-170.

Ruin, Olof (1982): Sweden in the 1970s: Policy Making Becomes More Difficult, In: Richardson, Jeremy, et al. (Hg.): Policy Styles in Western Europe, Boston; Sydney, S. 141-167.

Sagami, Yoshikazu (1989): The Environmental Pollution Dispute Settlement System, In: Tsuru, Shigeto, und Weidner, Helmut (Hg.): Environmental Policy in Japan, Berlin, S. 196-206..

Scalapino, Robert A. (1977) (Hg): The Foreign Policy of Modern Japan, Berkeley; Los Angeles.

Scharpf, Fritz W. (1976): Politikverflechtung. Theorie und Empirie des kooperativen Föderalismus in der Bundesrepublik, Kronberg/Ts.

Scharpf, Fritz W. (1985): Die Politikverflechtungsfalle: Europäische Integration und deutscher Föderalismus im Vergleich, in: Politische Vierteljehresschrift, 26, S. 323-356.

Scharpf, Fritz W. (1987): Sozialdemokratische Krisenpolitik in Europa, Frankfurt am Main; New York.

Scharpf, Fritz W. (1987): Die Handlungsfähigkeit des Staates am Ende des 20. Jahrhunderts, in: Politische Vierteljahresschrift, 4, S. 621-634.

Scharpf, Fritz W. (1994): Optionen des Föderalismus in Deutschland und Europa, Frankfurt am Main.

Schmidt, Manfred G. (1982): Does Corporatism Matter? Economic Crisis, Politics, and Rates of Unemployment in Capitalist Democracies in the 1970's, In: Lehmbruch, G., und Schmitter, P. (Hg.): Patterns of Corporatist Policy Making, Beverly Hills, S. 237-258. 
Schmidt, Manfred, G. (1982): Wohlfahrtstaatliche Politik unter bürgerlichen und sozialdemokratischen Regierungen. Ein internationaler Vergleich, Frankfurt/Main; New York.

Schmidt, Manfred G. (1983) (Hg.): Westliche Industriegesellschaften. Wirtschaft - Gesellschaft - Politik, München.

Schmidt, Manfred G. (1986): Politische Bedingungen erfolgreicher Wirtschaftspolitik, in: Journal für Sozialforschung, 3.

Schmidt, Manfred G. (1988) (Hg.): Staatstätigkeit. International und historisch vergleichende Analysen, Politische Vierteljahresschrift, Sonderheft 19, Opladen.

Schmidt, Manfred G. (1990): Die Politik des mittleren Weges. Besonderheiten der Staatstätigkeit in der Bundesrepublik Deutschland, in: Aus Politik und Zeitgeschichte, 9-10, S. 23-31.

Schmidt, Manfred G. (1993): Theorien in der international vergleichenden Staatstätigkeitsforschung, in: Héritier, Adrienne (Hg.): PolicyAnalyse, Kritik und Neuorientierung, Politische Vierteljahresschrift, Sonderheft 24, Opladen, S. 372-394.

Schmitter, Phillipe C. (1981): Interest Intermediation and Regime Governability in Western Europe and North America, In: Berger, S. (Hg.): Organizing Interests in Western Europe, Cambridge, S. 287-330.

Schmitter, Phillipe C. (1985): Neo-corporatism and the State, In: Grant, W. (Hg.): The Political Economy of Corporatism, New York, S. 32-63.

Schubert, Klaus (1991): Politikfeldanalyse, Opladen.

Schultze, Rainer-Olaf (1983): Föderalismus, In: Schmidt, Manfred G. (Hg.): Westliche Industriegesellschaften. Wirtschaft - Gesellschaft - Politik, München, S. 93-105.

Schultze, Rainer-Olaf, und Sturm, Roland (1983): Regionalismus, In: Schmidt, Manfred G. (Hg.): Westliche Industriegesellschaften. Wirtschaft - Gesellschaft - Politik, München, S. 380-390. 
Schultze, Rainer-Olaf (1995): Föderalismus, In: Nohlen, Dieter (Hg.): Wörterbuch Staat und Politik, Bonn, S. 155-164.

Scimemi, Gabriele (1988): Environmental Policies and Anticipatory Strategies, in: Simonis, Udo E. (Hg.): Präventive Umweltpolitik, Frankfurt am Main; New York, S. 27-48.

Schulz, Werner (1985): Der monetäre Wert besserer Luft: Eine empirische Analyse individueller Zahlungsbereitschaften und ihrer Determinanten auf der Basis von Repräsentativumfragen, Frankfurt/Main; Bern; New York.

Schumann, Jochen (1987): Grundzüge der mikroökonomischen Theorie, 5. Aufl., Berlin; Heidelberg; New York.

Simon, Harald; Bauer, Brigitte; Jägeler, Franz (1993): Auf der Suche nach Europas Stärken. Managementkulturen und Erfolgsfaktoren, Landsberg/Lech.

Simonis, Udo Ernst (1988) (Hg.): Präventive Umweltpolitik, Frankfurt/Main; New York.

Simonis, Udo Ernst (1996): Ökologische Umorientierung der Industriegesellschaft, in: Aus Politik und Zeitgeschichte, 7, S. 3-13.

Smith, Zachary A. (1992): The Environmental Policy Paradox, Englewood Cliffs.

Solesbury, William (1976): Issues and Innovation in Environmental Policy in Britain, West Germany, and California, in: Policy Analysis, 2, S. $1-38$.

Spinner, Helmut F. (1995): Kritischer Rationalismus bzw. Pluralismus, In: Nohlen, Dieter (Hg.): Wörterbuch Staat und Politik, Bonn, S. 377-382.

Stahmer, Carsten (1996): Aufstand der Erbsenzähler, in: DIE ZEIT (Sonderheftdruck ZEIT-Punkte): Wie teuer ist uns die Natur? Ökonomie und Ökologie an der Schwelle zum 21. Jahrhundert, 6, S. 12-16. 
Stephan, Petra (1995): Boden, Wasser, Biosphäre, In: Hauchler, Ingomar (Hg.): Globale Trends 1996. Daten zur Weltentwicklung, Frankfurt am Main, S. 281-308.

Streeck, W., und Schmitter, Phillipe C. (1991): From National Corporatism to Transnational Pluralism: Organized Interests in the Single European Market, in: Politics and Society, 19, 133-164.

Sturm, R. (1985): Die Politikstilanalyse. Zur Konkretisierung des Konzeptes der Politischen Kultur in der Policy-Analyse, in: Hartwich, HansHermann (Hg.): Policy-Forschung in der Bundesrepublik Deutschland. Ihr Selbstverständnis und ihr Verhältnis zu den Grundlagen der Politikwissenschaft, Opladen, S. 111-116.

Tsuru, Shigeto, und Weidner, Helmut (1989) (Hg.): Environmental Policy in Japan, Berlin.

Ui, Jun (1989): Anti-Pollution Movements and Other Grass-Roots Organizations, In: Tsuru, Shigeto, und Weidner, Helmut (Hg): Environmental Policy in Japan, Berlin, S. 109-120.

Umweltbundesamt (1995) (Hg.): Umweltdaten Deutschland 1995, Berlin.

Vogel, David (1982): Coercion versus Consultation: A Comparison of Environmental Protection in the United States and Great Britain, Denver.

Vogel, David (1986): National Styles of Regulation: Environmental Policy in Great Britain and the United States, Ithaca.

Vogel, David (1987): The Comparative Study of Environmental Policy: A Review of the Literature, In: Dierkes, Meinolf, et al. (Hg.): Comparative Policy Research, Aldershot, S. 99-170.

Waarden, Frans van (1992): Dimensions and Types of Policy Networks, in: European Journal of Political Research, 21, S. 29-52.

Weidner, Helmut (1988): Bausteine einer präventiven Umweltpolitik. Anregungen aus Japan, In: Simonis, Udo Ernst (Hg.): Präventive Umweltpolitik, Frankfurt/Main; New York, S. 143-165. 
Weidner, Helmut (1989): Environmental Monitoring and Reporting by Local Government, In: Tsuru, Shigeto, und Weidner, Helmut (Hg): Environmental Policy in Japan, Berlin, S. 461-476.

Weidner, Helmut (1989): Japanese Environmental Policy in an International Perspective: Lessons for a Preventive Approach, In: Tsuru, Shigeto, und Weidner, Helmut (Hg): Environmental Policy in Japan, Berlin, S. 479-552.

Weidner, Helmut (1994): Entwicklungslinie und Merkmale der Umweltpolitik, In: Mayer, Hans Jürgen, und Pohl, Manfred (Hg.): Länderbericht Japan, Bonn, S. 125-133.

Weidner, Helmut (1995): Reduction in $\mathrm{SO}_{2}$ and $\mathrm{NO}_{2}$ Emissions from Stationary Sources in Japan, In: Jänicke, Martin, und Weidner, Helmut (Hg.): Successful Environmental Policy. A Critical Evaluation of $24 \mathrm{Ca}-$ ses, Berlin, S. 146-172.

Weidner, Helmut (1995): 25 Years of Modern Environmental Policy in Germany. Treading a Well-Worn Path to the Top of the International Field, Arbeitspapier FS II 95-301 des Wissenschaftszentrums Berlin für Sozialforschung (WZB), Berlin.

Wicke, Lutz (1991): Umweltökonomie. Eine praxisorientierte Einführung, München.

Wilensky, Harold L. (1976): The „New Corporatism“: Centralization and the Welfare State, Beverly Hills.

Wildavsky, Aaron B. (1987): Doing More and Using Less: Utilisation of Research as a Result of Regime, In: Dierkes, Meinolf et al. (Hg.): Comparative Policy Research, Aldershot.

Williamson, Peter J. (1989): Corporatism in Perspective - An Introducery Guide to Corporatist Theory, London.

Windhoff-Héritier, Adrienne (1987): Policy-Analyse. Eine Einführung, Frankfurt/Main; New York.

Ziemer, Klaus (1995): Klientelismus, In: Nohlen, Dieter (Hg.): Wörterbuch Staat und Politik, Bonn, S. 315-317. 
Zilleßen, Horst, und Barbian, Thomas (1992): Neue Formen der Konfliktregelung in der Umweltpolitik, in: Aus Politik und Zeitgeschichte, 39-40, S. 14-23.

Zimmermann, Klaus W. (1983): Ansatzpunkte einer verteilungsorientierten Umweltpolitik, in: Kyklos, 3, S. 420-449.

Zimmermann, Klaus W. (1985): Umweltpolitik und Verteilung. Eine Analyse der Verteilungswirkungen des öffentlichen Gutes Umwelt, Berlin.

Zimmermann, Klaus W. (1992): Umweltpolitische Perspektiven in den neuen Ländern, in: Aus Politik und Zeitgeschichte, 39-40, S. 3-13.

Zimmermann, Klaus W., und Kahlenborn, Walter (1994): Umweltföderalismus. Einheit und Einheitlichkeit in Deutschland und Europa, Berlin. 
Christian Ricken - 978-3-631-75118-3

Downloaded from PubFactory at 01/11/2019 08:07:14AM

via free access 


\section{Anhang}

Tabelle A1: Wasserverbrauch (Millionen $\left.\mathrm{m}^{3}\right)^{\mathrm{a}, \mathrm{b}}$

\begin{tabular}{|c|c|c|c|c|c|}
\hline & 1970 & 1975 & 1980 & 1985 & 1990 \\
\hline Kanada & 24057 & 28128 & 37864 & 42383 & 46878 \\
\hline USA & 440000 & 472500 & 525000 & 467000 & 468000 \\
\hline Japan & k.A. & 87600 & 88200 & 89200 & 89290 \\
\hline Australien $^{c}$ & k.A. & 17800 & k.A. & k.A. & k.A. \\
\hline Neuseeland & 990 & 1045 & 1200 & 1900 & k.A. \\
\hline Österreich & k.A. & 2620 & 2190 & 2120 & 2120 \\
\hline Belgien $^{c}$ & 9481 & k.A. & 9030 & k.A. & k.A. \\
\hline Dänemark & 720 & 1205 & k.A. & k.A. & 1200 \\
\hline Finnland & 3300 & 3550 & 3700 & 4000 & 3001 \\
\hline Frankreich & 23500 & 27000 & 35104 & 34887 & 37730 \\
\hline Deutschland $^{d}$ & 29488 & 33572 & 42206 & 41216 & 47507 \\
\hline Griechenland & 4254 & 5847 & 6945 & k.A. & k.A. \\
\hline Irland $^{c}$ & k.A. & k.A. & 793 & k.A. & k.A. \\
\hline Italien & 41900 & k.A. & 56200 & 52000 & 56200 \\
\hline Niederlande & 13270 & 13734 & 14794 & 14481 & k.A. \\
\hline Norwegen & k.A. & 2380 & k.A. & 2025 & k.A. \\
\hline Portugal & k.A. & k.A. & 1687 & 2003 & 7288 \\
\hline Spanien & 24600 & 36080 & 39920 & 46250 & 36900 \\
\hline Schweden & 4073 & 4135 & 4106 & 2901 & 2932 \\
\hline Schweiz & 1140 & 1129 & 1103 & 1143 & 1166 \\
\hline Türkei & 11760 & 16041 & 16200 & 19400 & 30600 \\
\hline Großbritannien & k.A. & k.A. & 13912 & 12947 & 14237 \\
\hline Nordamerika & 464057 & 500628 & 562864 & 509383 & 514878 \\
\hline OECD-Europa ${ }^{e}$ & 193100 & 224900 & 255700 & 258700 & 275400 \\
\hline $\mathrm{EU}^{\mathrm{e}, \mathrm{f}}$ & 168100 & 195000 & 226300 & 227100 & 233200 \\
\hline $\mathrm{OECD}^{\mathrm{e}}$ & 756900 & 832000 & 927000 & 879700 & 900800 \\
\hline
\end{tabular}

Anm.:

a. Privater Verbrauch, Bewalsserung und industrieller Verbrauch

b. Alle Daten soweit nicht anders vermerkt aus: OECD (1993): OECD Environmental Data, Paris, S. 53.

c. Daten aus: OECD (1989): OECD Environmental Data, Paris, S. 51

d. Alte Bundeslănder

e. Schătzung, falls fur ein Land keine Angaben (k.A.) vorliegen

f. 11 Staaten (ohne Österreich, Finnland, Schweden und Luxemburg) 
Tabelle A2: Landwirtschaftsflache $\left(\mathrm{km}^{2}\right)^{\mathrm{a}, \mathrm{b}}$

\begin{tabular}{lrrrrr}
\hline & 1970 & 1975 & 1980 & 1985 & 1990 \\
\hline Kanada & 386500 & 392640 & 406680 & 416800 & 414290 \\
USA & 1905000 & 1882180 & 1906240 & 1899150 & 1889150 \\
Japan & 55100 & 50880 & 48810 & 47580 & 45960 \\
Australien $^{c}$ & 417870 & 423870 & 441860 & 473100 & 489190 \\
Neuseeland & 5790 & 4060 & 4530 & 5110 & 4120 \\
Osterreich & 16810 & 16090 & 16350 & 15250 & 15050 \\
Belgien & 8500 & 8360 & 7730 & 7570 & 7620 \\
Dänemark & 26760 & 26600 & 26530 & 26140 & 25670 \\
Finnland & 26670 & 26410 & 25630 & 24100 & 25440 \\
Frankreich & 184960 & 189540 & 188940 & 192420 & 192090 \\
Deutschland & 75740 & 75730 & 74960 & 74530 & 74920 \\
Griechenland & 39100 & 38670 & 39250 & 39400 & 39340 \\
Irland & 13800 & 12360 & 11100 & 10320 & 9430 \\
Italien & 149300 & 123130 & 124360 & 121140 & 119730 \\
Niederlande & 8670 & 8410 & 8600 & 8920 & 9440 \\
Norwegen & 8140 & 7920 & 8170 & 8580 & 8640 \\
Portugal & 30910 & 31180 & 31410 & 31560 & 31730 \\
Spanien & 211690 & 208330 & 204990 & 204160 & 205440 \\
Schweden & 30530 & 30060 & 29790 & 29220 & 28450 \\
Schweiz & 4620 & 4740 & 4930 & 4940 & 4940 \\
Türkei & 273390 & 276620 & 281750 & 275300 & 278570 \\
Großbritannien & 71990 & 69540 & 69960 & 70610 & 66570 \\
\hline Nordamerika & 2291500 & 2274820 & 2312920 & 2315950 & 2313440 \\
OECD-Europa & 1183430 & 1155570 & 1156370 & 1146080 & 1145040 \\
EU ${ }^{e}$ & 822070 & 792460 & 788420 & 787330 & 782550 \\
\hline OECD & 3953690 & 3909190 & 3964490 & 3987820 & 3997750 \\
\hline & & & & &
\end{tabular}

Anm.:

a. Stłndiges und temporăres Ackerland

b. Alle Daten soweit nicht anders vermerkt aus: OECD (1993): OECD Environmental Data, Paris, S. 257

c. Angabe enthalt über $270000 \mathrm{~km}^{2}$ kultivierte Grasflalchen

d. Alte Bundeslander

e. 11 Staten (ohne Österreich, Finnland, Schweden und Luxemburg) 
Tabelle A3: Primårenergieverbrauch (MTOE) $)^{a, b, c}$

\begin{tabular}{lrrrrr}
\hline & 1970 & 1975 & 1980 & 1985 & 1990 \\
\hline Kanada & 132,1 & 161,1 & 192,1 & 193,3 & 210,7 \\
USA & 1545,9 & 1646,7 & 1801,0 & 1771,9 & 1919,9 \\
Japan & 256,4 & 307,4 & 345,6 & 359,6 & 428,3 \\
Australien & 51,3 & 61,0 & 70,4 & 73,9 & 88,1 \\
Neuseeland & 7,0 & 8,5 & 8,9 & 11,2 & 14,0 \\
Osterreich & 18,3 & 20,4 & 23,5 & 23,2 & 25,4 \\
Belgien & 40,3 & 42,6 & 46,1 & 44,0 & 48,3 \\
Dannemark & 20,2 & 18,0 & 19,5 & 19,6 & 18,3 \\
Finnland & 18,1 & 20,0 & 25,0 & 26,2 & 28,5 \\
Frankreich & 147,3 & 162,2 & 190,7 & 200,7 & 221,2 \\
Deutschland & 234,4 & 239,1 & 272,9 & 270,0 & 278,2 \\
Griechenland & 8,1 & 12,2 & 16,0 & 18,6 & 22,1 \\
Irland & 6,3 & 6,9 & 8,5 & 8,9 & 10,6 \\
Italien & 110,7 & 124,4 & 139,2 & 136,6 & 154,7 \\
Niederlande & 49,9 & 59,9 & 65,5 & 61,6 & 66,4 \\
Norwegen & 13,9 & 15,2 & 18,9 & 20,4 & 21,5 \\
Portugal & 6,0 & 8,0 & 10,3 & 11,4 & 16,4 \\
Spanien & 38,4 & 58,5 & 68,7 & 71,9 & 88,0 \\
Schweden & 38,0 & 39,5 & 41,0 & 47,6 & 47,8 \\
Schweiz & 16,5 & 18,0 & 21,0 & 23,1 & 25,0 \\
Türkei & 12,1 & 26,8 & 31,8 & 39,0 & 53,1 \\
Großbritannien & 207,7 & 201,9 & 201,2 & 203,0 & 21,8 \\
\hline Nordamerika & 1677,9 & 1807,8 & 1993,1 & 1965,1 & 2130,5 \\
OECD-Europa & 990,9 & 1078,0 & 1204,2 & 1230,1 & 1342,1 \\
EU & 873,3 & 937,3 & 1042,2 & 1049,5 & 1139,5 \\
\hline OECD & 2983,6 & 3262,7 & 3622,1 & 3629,9 & 4002,9 \\
\hline & & & & &
\end{tabular}

Anm.:

a. Inlandische Produktion + Importe - Exporte

b. Alle Daten soweit nicht anders vermerkt aus: OECD (1993): OECD Environmental Data, Paris, S. 193

c. MTOE $:=$ Millionen Tonnen Roholaqivalent

d. Alte Bundeslander

e. 11 Staaten (ohne Osterreich, Finnland, Schweden und Luxemburg) 
Tabelle A4: Energiebedingte $\mathrm{CO}_{2}$-Emissionen (Mio. $\left.\mathrm{t}\right)^{\mathrm{a}, \mathrm{D}}$

\begin{tabular}{lrrrrr}
\hline & 1970 & 1975 & 1980 & 1985 & 1990 \\
\hline Kanada & 342 & 402 & 439 & 406 & 437 \\
USA & 4267 & 4444 & 4913 & 4732 & 5038 \\
Japan & 781 & 912 & 937 & 912 & 1060 \\
Australien & 163 & 191 & 221 & 229 & 272 \\
Neuseeland & 16 & 19 & 19 & 23 & 26 \\
Osterreich & 49 & 53 & 59 & 55 & 59 \\
Belgien & 134 & 130 & 136 & 111 & 125 \\
Dänemark & 64 & 56 & 64 & 64 & 56 \\
Finnland & 41 & 47 & 60 & 53 & 55 \\
Frankreich & 443 & 462 & 499 & 395 & 385 \\
Deutschland & 749 & 716 & 791 & 721 & 713 \\
Griechenland & 25 & 39 & 51 & 62 & 81 \\
Irland & 21 & 22 & 27 & 28 & 34 \\
Italien & 307 & 342 & 382 & 369 & 411 \\
Niederlande & 161 & 175 & 184 & 167 & 183 \\
Norwegen & 28 & 28 & 32 & 30 & 32 \\
Portugal & 16 & 22 & 27 & 28 & 43 \\
Spanien & 119 & 170 & 203 & 198 & 227 \\
Schweden & 98 & 85 & 75 & 65 & 56 \\
Schweiz & 39 & 39 & 42 & 41 & 44 \\
Türkei & 39 & 63 & 75 & 101 & 139 \\
Großbritannien & 662 & 614 & 601 & 574 & 598 \\
\hline Nordamerika & 4609 & 4845 & 5352 & 5138 & 5475 \\
OECD-Europa & 3279 & 3354 & 3622 & 3392 & 3529 \\
EUd & 2983 & 3038 & 3277 & 3045 & 3141 \\
\hline OECD $^{\text {d }}$ & 8848 & 9321 & 10150 & 9694 & 10361 \\
\hline & & & & &
\end{tabular}

Anm: :
a. Inklusive Hochseebunkerung
b. Alle Daten soweit nicht anders vermerkt aus: OECD (1993): OECD Environmental Data, Paris, S. 31
c. Alte Bundeslănder
d. 11 Staaten (ohne Ôsterreich, Finnland, Schweden und Luxemburg) 
Tabelle A5: Abfallaufkommen (1 $000 \mathrm{t})^{\mathrm{a}, \mathrm{b}}$

\begin{tabular}{|c|c|c|c|c|}
\hline & 1975 & 1980 & 1985 & 1990 \\
\hline Kanada & k.A. & 12600 & k.A. & 16000 \\
\hline USA & 116200 & 137400 & 149100 & 177500 \\
\hline Japan & 42180 & 43950 & 41530 & 50441 \\
\hline Australien & k.A. & k.A. & k.A. & 10000 \\
\hline Neuseeland & 1150 & 2106 & k.A. & k.A. \\
\hline Osterreich & 1407 & 1673 & 1727 & 2506 \\
\hline Belgien & 2900 & 3082 & k.A. & 3410 \\
\hline Dänemark & k.A. & 2046 & 2430 & k.A. \\
\hline Finnland & k.A. & k.A. & 2500 & 3100 \\
\hline Frankreich & 14330 & 15570 & 16220 & 18510 \\
\hline Deutschland $^{c}$ & 20423 & 21417 & 19387 & 21172 \\
\hline Griechenland & k.A. & 2500 & 3023 & 3000 \\
\hline Irland & 555 & 640 & 1100 & k.A. \\
\hline Italien & 14095 & 14041 & 15000 & 20033 \\
\hline Niederlande & k.A. & 7050 & 6307 & 7430 \\
\hline Norwegen & 1700 & 1700 & 1970 & 2000 \\
\hline Portugal & k.A. & 2091 & 2448 & 2538 \\
\hline Spanien & 8028 & 10100 & 10014 & 12546 \\
\hline Schweden & 2400 & 2510 & 2650 & 3200 \\
\hline Schweiz & 1900 & 2240 & 2500 & 3000 \\
\hline Türkei & k.A. & k.A. & k.A. & 19500 \\
\hline Großbritannien & 16000 & 15500 & 17000 & 20000 \\
\hline Nordamerika $^{d}$ & 128000 & 150000 & 165000 & 194000 \\
\hline OECD-Europa $^{d}$ & 118000 & 125000 & 130000 & 150000 \\
\hline EU d,e & 89000 & 94000 & 98000 & 115000 \\
\hline $\mathrm{OECD}^{d}$ & 299000 & 331000 & 350000 & 408000 \\
\hline
\end{tabular}

Anm.:

a. Abfalle aus privaten Haushalten, Kleingewerbe und Dienstleistungen

b. Alle Daten soweit nicht anders vermerkt aus: OECD (1993): OECD Environmental Data, Paris, S.

c. Alte Bundeslander

d. Schătzung falls für ein Land keine Angaben (k.A.) vorliegen

e. 11 Staaten (ohne Osterreich, Finnland, Schweden und Luxemburg) 
Tabelle A6: Handelsdüngerabsatz $(1000 t)^{a, b}$

\begin{tabular}{lrrrrr}
\hline & 1970 & 1975 & 1980 & 1985 & 1990 \\
\hline Kanada & 802 & 1308 & 1939 & 2325 & 2074 \\
USA & 15535 & 18914 & 21480 & 17831 & 18429 \\
Japan & 1955 & 1801 & 1816 & 2034 & 1838 \\
Australien & 971 & 722 & 1162 & 1155 & 1164 \\
Neuseeland & 448 & 516 & 464 & 427 & 343 \\
Osterreich & 408 & 313 & 407 & 388 & 304 \\
Belgien & 517 & 451 & 447 & 421 & 384 \\
Dänemark & 598 & 639 & 627 & 634 & 633 \\
Finnland & 486 & 517 & 489 & 507 & 443 \\
Frankreich & 4651 & 4686 & 5609 & 5695 & 5684 \\
Deutschland & 2609 & 2557 & 2733 & 2439 & 1609 \\
Griechenland & 337 & 463 & 527 & 685 & 696 \\
Irland & 423 & 432 & 601 & 620 & 692 \\
Italien & 1338 & 1490 & 2111 & 2102 & 1832 \\
Niederlande & 650 & 635 & 679 & 701 & 561 \\
Norwegen & 199 & 230 & 259 & 238 & 210 \\
Portugal & 129 & 245 & 259 & 241 & 278 \\
Spanien & 1216 & 1402 & 1662 & 1734 & 1977 \\
Schweden & 503 & 525 & 484 & 419 & 328 \\
Schweiz & 148 & 143 & 181 & 180 & 168 \\
Türkei & 431 & 891 & 1456 & 1427 & 1888 \\
Großbritannien & 1894 & 1820 & 2054 & 2524 & 2370 \\
\hline Nordamerika & 16338 & 20221 & 23418 & 20155 & 20502 \\
OECD-Europa & 16559 & 17465 & 20616 & 20981 & 20080 \\
EU & 14362 & 14818 & 17310 & 17796 & 16716 \\
\hline OECD & 36271 & 40725 & 47477 & 44752 & 43927 \\
\hline & & & & &
\end{tabular}

Anm.:

a. Stickstoff, Phosphat, Kali und Kalk

b. Alle Daten soweit nicht anders vermerkt aus: OECD (1993): OECD Environmental Data, Paris, S. 273

c. Alte Bundesländer

d. 11 Staaten (ohne Osterreich, Finnland, Schweden und Luxemburg) 
Tabelle A7: Verănderung des Wasserverbrauches (in \%)

\begin{tabular}{|c|c|c|c|c|}
\hline & $1970-1975$ & $1975-1980$ & $1980-1985$ & $1985-1990$ \\
\hline Kanada & 16,9 & 34,6 & 11,9 & 10,6 \\
\hline USA & 7,4 & 11,1 & $-11,0$ & 0,2 \\
\hline Japan & k.A. & 0,7 & 1,1 & 0,1 \\
\hline Australien & k.A. & k.A. & k.A. & k.A. \\
\hline Neuseeland & 5,6 & 14,8 & 58,3 & k.A. \\
\hline Osterreich & k.A. & $-16,4$ & $-3,2$ & k.A. \\
\hline Belgien & k.A. & $-1,9$ & k.A. & k.A. \\
\hline Dänemark & 67,4 & k.A. & k.A. & $-0,4$ \\
\hline Finnland & 7,6 & 4,2 & 8,1 & $-25,0$ \\
\hline Frankreich & 14,9 & 30,0 & $-0,6$ & 8,1 \\
\hline Deutschland $^{c}$ & 13,9 & 25,7 & $-2,4$ & 15,3 \\
\hline Griechenland & 37,4 & 18,8 & k.A & k.A. \\
\hline Irland & k.A. & k.A. & k.A. & k.A. \\
\hline Italien & 15,8 & 15,9 & $-7,5$ & 8,1 \\
\hline Niederlande & 3,5 & 7,7 & $-2,1$ & k.A. \\
\hline Norwegen & k.A. & $-6,9$ & $-8,6$ & k.A. \\
\hline Portugal & k.A. & k.A. & 18,7 & 263,9 \\
\hline Spanien & 46,7 & 10,6 & 15,9 & $-20,2$ \\
\hline Schweden & 1,5 & $-0,7$ & $-29,3$ & 1,1 \\
\hline Schweiz & $-1,0$ & $-2,3$ & 3,6 & 2,0 \\
\hline Türkei & 36,4 & 1,0 & 19,8 & 57,7 \\
\hline Großbritannien & k.A. & k.A. & $-6,9$ & 10,0 \\
\hline Nordamerika $^{d}$ & 12,2 & 22,9 & 0,5 & $\overline{5,4}$ \\
\hline OECD-Europa ${ }^{d}$ & 20,8 & 7,1 & $-1,2$ & $-0,1$ \\
\hline$E U^{d, e}$ & 28,5 & 15,3 & 2,2 & 3,5 \\
\hline $\mathrm{OECD}^{d}$ & 19,6 & 8,6 & 3,9 & $\overline{5,2}$ \\
\hline
\end{tabular}

Anm.:

a. Privater Verbrauch, Bewlssenung und industrieller Verbrauch

b. Eigene Berechnungen auf Grundlage von Tabelle Al

c. Alte Bundeslander

d. Mittelwert

e. 11 Staaten (ohne Osterreich, Finnland, Schweden und Luxemburg)

Mittelwerte 1985-1990 ohne Portugal 
Tabelle A8: Verănderung der Landwirtschaftsfläche (in \%)"

\begin{tabular}{|c|c|c|c|c|}
\hline & $1970-1975$ & $1975-1980$ & $1980-1985$ & $1985-1990$ \\
\hline Kanada & 1,6 & 3,6 & 2,5 & $-0,6$ \\
\hline USA & $-1,2$ & 1,3 & $-0,4$ & 0,0 \\
\hline Japan & $-7,7$ & $-4,1$ & $-2,5$ & $-3,4$ \\
\hline Australien & 1,4 & 4,2 & 7,1 & 3,4 \\
\hline Neuseeland & $-29,9$ & 11,6 & 12,8 & $-19,4$ \\
\hline Österreich & $-4,3$ & 1,6 & $-6,7$ & $-1,3$ \\
\hline Belgien & $-1,7$ & $-7,5$ & $-2,1$ & 0,7 \\
\hline Dänemark & $-0,6$ & $-0,3$ & $-1,5$ & $-1,8$ \\
\hline Finnland & $-1,0$ & $-3,0$ & $-6,0$ & 5,6 \\
\hline Frankreich & 2,5 & $-0,3$ & 1,8 & $-0,2$ \\
\hline Deutschland $^{c}$ & 0,0 & $-1,0$ & $-0,6$ & 0,5 \\
\hline Griechenland & $-1,1$ & 1,5 & 0,4 & $-0,2$ \\
\hline Irland & $-10,4$ & $-10,2$ & $-7,0$ & $-8,6$ \\
\hline Italien & $-17,5$ & 1,0 & $-2,6$ & $-1,2$ \\
\hline Niederlande & $-3,0$ & 2,3 & 3,7 & 5,8 \\
\hline Norwegen & $-2,7$ & 3,2 & 5,0 & 0,7 \\
\hline Portugal & 0,9 & 0,7 & 0,5 & 0,5 \\
\hline Spanien & $-1,6$ & $-1,6$ & $-0,4$ & 0,6 \\
\hline Schweden & $-1,5$ & $-0,9$ & $-1,9$ & $-2,6$ \\
\hline Schweiz & 2,6 & 4,0 & 0,2 & 0,0 \\
\hline Türkei & 1,2 & 1,9 & $-2,3$ & 1,2 \\
\hline Großbritannien & $-3,4$ & 0,6 & 0,9 & $-5,7$ \\
\hline Nordamerika $^{d}$ & 0,2 & 2,5 & 1,1 & $-0,3$ \\
\hline OECD-Europa $^{d}$ & $-2,7$ & $-0,6$ & $-1,0$ & $-0,5$ \\
\hline $\mathrm{EU}^{\mathrm{d}, \mathrm{e}}$ & $-3,3$ & $-1,3$ & $-0,6$ & $-0,9$ \\
\hline $\mathrm{OECD}^{d}$ & $-3,5$ & 0,4 & 0,0 & $-1,2$ \\
\hline
\end{tabular}

Anm: :
a. Standiges und temporäres Ackerland
b. Eigene Berechnungen auf Grundlage von Tabelle A2
c. Alte Bundeslănder
d. Mittelwert
c. 11 Staaten (ohne Ősterreich, Finnland, Schweden und Luxemburg) 
Tabelle A9: Verănderung des Primărenergieverbrauches (in

\begin{tabular}{|c|c|c|c|c|}
\hline & $1970-1975$ & $1975-1980$ & $1980-1985$ & $1985-1990$ \\
\hline Kanada & 22,0 & 19,2 & 0,6 & 9,0 \\
\hline USA & 6,5 & 9,4 & $-1,6$ & 8,3 \\
\hline Japan & 19,9 & 12,4 & 4,1 & 19,1 \\
\hline Australien & 18,9 & 15,4 & 5,0 & 19,2 \\
\hline Neuseeland & 21,4 & 4,7 & 25,8 & 25,0 \\
\hline Österreich & 11,5 & 15,2 & $-1,3$ & 9,5 \\
\hline Belgien & 5,7 & 8,2 & $-4,6$ & 9,8 \\
\hline Dänemark & $-10,9$ & 8,3 & 0,5 & $-6,6$ \\
\hline Finnland & 10,5 & 25,0 & 4,8 & 8,8 \\
\hline Frankreich & 10,1 & 17,6 & 5,2 & 10,2 \\
\hline Deutschland $^{c}$ & 2,0 & 14,1 & $-1,1$ & 3,0 \\
\hline Griechenland & 50,6 & 31,1 & 16,3 & 18,8 \\
\hline Irland & 9,5 & 23,2 & 4,7 & 19,1 \\
\hline Italien & 12,4 & 11,9 & $-1,9$ & 13,3 \\
\hline Niederlande & 20,0 & 9,4 & $-6,0$ & 7,8 \\
\hline Norwegen & 9,4 & 24,3 & 7,9 & 5,4 \\
\hline Portugal & 33,3 & 28,8 & 10,7 & 43,9 \\
\hline Spanien & 52,3 & 17,4 & 4,7 & 22,4 \\
\hline Schweden & 3,9 & 3,8 & 16,1 & 0,4 \\
\hline Schweiz & 9,1 & 16,7 & 10,0 & 8,2 \\
\hline Türkei & 119,7 & 18,7 & 22,6 & 36,1 \\
\hline Großbritannien & $-2,8$ & $-0,4$ & 0,9 & 4,3 \\
\hline Nordamerika ${ }^{d}$ & 14,3 & 14,3 & $-0,5$ & 8,7 \\
\hline OECD-Europa $^{d}$ & 14,2 & 15,9 & 4,2 & 11,1 \\
\hline$E U^{d, e}$ & 16,6 & 15,4 & 2,7 & 13,3 \\
\hline OECD $^{d}$ & 15,0 & 15,2 & 5,6 & 13,4 \\
\hline
\end{tabular}

Anm:

a. Inlandische Produktion + Importe - Exporte

b. Eigene Berechnungen auf Grundlage von Tabelle A3

c. Alte Bundeslander

d. Mittelwert (1970-1975 ohne Turkei)

e. 11 Stasten (ohne Österreich, Finnland, Schweden und Luxemburg) 
Tabelle A10: Verănderung der $\mathrm{CO}_{2}$-Emissionen (in \%),

\begin{tabular}{|c|c|c|c|c|}
\hline & $1970-1975$ & $1975-1980$ & $1980-1985$ & $1985-1990$ \\
\hline Kanada & 17,5 & 9,2 & $-7,5$ & 7,1 \\
\hline USA & 4,1 & 10,6 & $-3,7$ & 6,5 \\
\hline Japan & 16,8 & 2,7 & $-2,7$ & 16,2 \\
\hline Australien & 17,2 & 15,7 & 3,6 & 18,8 \\
\hline Neuseeland & 18,8 & 0,0 & 21,1 & 13,0 \\
\hline Osterreich & 8,2 & 11,3 & $-6,8$ & 7,3 \\
\hline Belgien & $-3,0$ & 4,6 & $-18,4$ & 12,6 \\
\hline Dänemark & $-12,5$ & 14,3 & 0,0 & $-12,5$ \\
\hline Finnland & 14,6 & 27,7 & $-11,7$ & 3,8 \\
\hline Frankreich & 4,3 & 8,0 & $-20,8$ & $-2,5$ \\
\hline Deutschland ${ }^{c}$ & $-4,4$ & 10,5 & $-8,9$ & $-1,1$ \\
\hline Griechenland & 56,0 & 30,8 & 21,6 & 30,7 \\
\hline Irland & 4,8 & 22,7 & 3,7 & 21,4 \\
\hline Italien & 11,4 & 11,7 & $-3,4$ & 11,4 \\
\hline Niederlande & 8,7 & 5,1 & $-9,2$ & 9,6 \\
\hline Norwegen & 0,0 & 14,3 & $-6,3$ & 6,7 \\
\hline Portugal & 37,5 & 22,7 & 3,7 & 53,6 \\
\hline Spanien & 42,9 & 19,4 & $-2,5$ & 14,7 \\
\hline Schweden & $-13,3$ & $-11,8$ & $-13,3$ & $-13,8$ \\
\hline Schweiz & 0,0 & 7,7 & $-2,4$ & 7,3 \\
\hline Türkei & 61,5 & 19,1 & 34,7 & 37,6 \\
\hline Großbritannien & $-7,3$ & $-2,1$ & $-4,5$ & 4,2 \\
\hline Nordamerika & 10,8 & 9,9 & $-5,6$ & $\overline{6,8}$ \\
\hline OECD-Europa ${ }^{d}$ & 9,2 & 12,3 & $-5,0$ & 9,6 \\
\hline $\mathrm{EU}^{\mathrm{d}, \mathrm{e}}$ & 12,6 & 13,4 & $-3,5$ & 12,9 \\
\hline $\mathrm{OECD}^{d}$ & 12,9 & 11,6 & $-1,5$ & 11,5 \\
\hline
\end{tabular}

Anm.:

a. Inklusive Hochseebunkerung

b. Eigene Berechnungen auf Grundlage von Tabelle A4

c. Alte Bundesländer

d. Mittelwert

e. 11 Staaten (ohne Österreich, Finnland, Schweden und Luxemburg) 
Tabelle A11: Verănderung des Abfallaufkommen

\begin{tabular}{|c|c|c|c|}
\hline & $1975-1980$ & $1980-1985$ & $1985-1990$ \\
\hline Kanada & k.A. & k.A. & 11,9 \\
\hline USA & 18,2 & 8,5 & 19,1 \\
\hline Japan & 4,2 & $-5,5$ & 21,4 \\
\hline Australien & k.A. & k.A. & k.A. \\
\hline Neuseeland & 83,1 & k.A. & k.A. \\
\hline Österreich & 18,9 & 3,2 & 45,1 \\
\hline Belgien & 6,3 & 5,1 & 5,2 \\
\hline Dänemark & k.A. & 18,8 & k.A. \\
\hline Finnland & k.A. & k.A. & k.A. \\
\hline Frankreich & 8,7 & 4,2 & 14,1 \\
\hline Deutschland $^{c}$ & 4,9 & $-9,4$ & 9,2 \\
\hline Griechenland & k.A. & 20,9 & $-0,8$ \\
\hline Irland & 15,3 & 71,9 & k.A. \\
\hline Italien & $-0,4$ & 6,8 & 33,6 \\
\hline Niederlande & k.A. & $-10,5$ & 17,8 \\
\hline Norwegen & k.A. & 15,9 & 1,5 \\
\hline Portugal & k.A. & 17,1 & 3,7 \\
\hline Spanien & 25,8 & $-0,9$ & 25,3 \\
\hline Schweden & 4,9 & 5,6 & 20,8 \\
\hline Schweiz & 17,9 & 11,6 & 20,0 \\
\hline Türkei & k.A. & k.A. & k.A. \\
\hline Großbritannien & $-3,1$ & 9,7 & 17,7 \\
\hline Nordamerika $^{d}$ & 18,2 & 8,5 & 15,5 \\
\hline OECD-Europa ${ }^{d}$ & 9,9 & 11,3 & 16,4 \\
\hline $\mathrm{EU}^{\mathrm{d}, \mathrm{e}}$ & 8,2 & 12,2 & 14,0 \\
\hline $\mathrm{OECD}^{d}$ & 15,7 & 10,2 & 16,6 \\
\hline
\end{tabular}

Anm.:

a. Abfalle aus privaten Haushalten, Kleingewerbe und Dienstleistungen

b. Eigene Berechnungen auf Grundlage von Tabelle A5

c. Alte Bundeslander

d. Mittelwert

e. 11 Staaten (ohne Osterreich, Finnland, Schweden und Luxemburg) 
Tabelle A12: Verănderung des Handelsdüngerabsatzes (in \%)

\begin{tabular}{|c|c|c|c|c|}
\hline & $1970-1975$ & $1975-1980$ & $1980-1985$ & $1985-1990$ \\
\hline Kanada & 63,1 & 48,2 & 19,9 & $-10,8$ \\
\hline USA & 21,8 & 13,6 & $-17,0$ & 3,4 \\
\hline Japan & $-7,9$ & 0,8 & 15,9 & $-12,7$ \\
\hline Australien & $-25,6$ & 60,9 & $-0,6$ & 0,8 \\
\hline Neuseeland & 15,2 & $-10,1$ & $-8,0$ & $-19,7$ \\
\hline Österreich & $-23,3$ & 30,0 & $-4,7$ & $-21,6$ \\
\hline Belgien & $-12,8$ & $-0,9$ & $-5,2$ & $-8,8$ \\
\hline Dänemark & 6,9 & $-1,9$ & 1,1 & $-0,2$ \\
\hline Finnland & 6,4 & $-5,4$ & 3,7 & $-12,6$ \\
\hline Frankreich & 0,8 & 19,7 & 1,5 & $-0,2$ \\
\hline Deutschland $^{c}$ & $-2,0$ & 6,9 & $-10,8$ & $-34,0$ \\
\hline Griechenland & 37,4 & 13,8 & 30,0 & 1,6 \\
\hline Irland & 2,1 & 39,1 & 3,2 & 11,6 \\
\hline Italien & 11,4 & 41,7 & $-0,4$ & $-12,8$ \\
\hline Niederlande & $-2,3$ & 6,9 & 3,2 & $-20,0$ \\
\hline Norwegen & 15,6 & 12,6 & $-8,1$ & $-11,8$ \\
\hline Portugal & 89,9 & 5,7 & $-7,0$ & 15,4 \\
\hline Spanien & 15,3 & 18,5 & 4,3 & 14,0 \\
\hline Schweden & 4,4 & $-7,8$ & $-13,4$ & $-21,7$ \\
\hline Schweiz & $-3,4$ & 26,6 & $-0,6$ & $-6,7$ \\
\hline Türkei & 106,7 & 63,4 & $-2,0$ & 32,3 \\
\hline Großbritannien & $-3,9$ & 12,9 & 22,9 & $-6,1$ \\
\hline Nordamerika $^{d}$ & 42,5 & 30,9 & 1,5 & $-3,7$ \\
\hline OECD-Europa $^{d}$ & 8,9 & 13,7 & 1,2 & $-7,1$ \\
\hline $\mathrm{EU}^{\mathrm{d}, \mathrm{e}}$ & 13,0 & 14,8 & 3,9 & $-3,6$ \\
\hline $\mathrm{OECD}^{d}$ & 14,4 & 18,0 & 1,3 & $-\overline{-5,5}$ \\
\hline
\end{tabular}

Anm.:
a. Stickstoff, Phosphat, Kali und Kalk
b. Eigene Berechnungen auf Grundlage von Tabelle A6
c. Alte Bundesländer
d. Mittelwert
e. 11 Staaten (ohne Österreich, Finnland, Schweden und Luxemburg) 
Tabelle A13: Wasserverbrauch pro Einwohner $\left(1000 \mathrm{~m}^{3}\right)^{\mathrm{anb}}$

\begin{tabular}{|c|c|c|c|c|c|c|c|c|c|c|}
\hline & \multicolumn{2}{|c|}{1970} & \multicolumn{2}{|c|}{1975} & \multicolumn{2}{|c|}{1980} & \multicolumn{2}{|c|}{1985} & \multicolumn{2}{|c|}{1990} \\
\hline & Menge & Rang & Menge & Rang & Menge & Rang & Menge & Rang & Menge & Rang \\
\hline Kanada & 1128 & 3 & 1238 & 3 & 1573 & 2 & 1683 & 2 & 1761 & 2 \\
\hline USA & 2146 & 1 & 2188 & 1 & 2305 & 1 & 1952 & 1 & 1861 & 1 \\
\hline Japan & 845 & 6 & 786 & 8 & 755 & 9 & 739 & 9 & 723 & 10 \\
\hline Australien & 1389 & 2 & 1281 & 2 & 1211 & 3 & 1127 & 4 & 1042 & 3 \\
\hline Neuseeland & 351 & 15 & 339 & 17 & 382 & 15 & 581 & 13 & 562 & 14 \\
\hline Österreich & 351 & 15 & 346 & 16 & 290 & 17 & 280 & 17 & 275 & 18 \\
\hline Belgien & 982 & 5 & 939 & 6 & 917 & 7 & 916 & 6 & 906 & 7 \\
\hline Dänemark & 146 & 22 & 238 & 20 & 235 & 19 & 236 & 18 & 233 & 20 \\
\hline Finnland & 716 & 9 & 753 & 9 & 774 & 8 & 816 & 8 & 602 & 13 \\
\hline Frankreich & 463 & 14 & 512 & 13 & 652 & 12 & 632 & 12 & 669 & 12 \\
\hline Deutschland $^{c}$ & 486 & 12 & 543 & 12 & 686 & 11 & 675 & 11 & 751 & 8 \\
\hline Griechenland & 484 & 13 & 646 & 10 & 720 & 10 & 699 & 10 & 685 & 11 \\
\hline Irland & 269 & 18 & 250 & 18 & 233 & 20 & 224 & 20 & 226 & 21 \\
\hline Italien & 781 & 7 & 875 & 7 & 996 & 6 & 910 & 7 & 975 & 4 \\
\hline Niederlande & 1018 & 4 & 1005 & 5 & 1046 & 5 & 999 & 5 & 969 & 5 \\
\hline Norwegen & 614 & 10 & 594 & 11 & 542 & 13 & 488 & 14 & 477 & 16 \\
\hline Portugal & 195 & 20 & 181 & 21 & 172 & 22 & 202 & 21 & 739 & 9 \\
\hline Spanien & 726 & 8 & 1016 & 4 & 1068 & 4 & 1201 & 3 & 947 & 6 \\
\hline Schweden & 506 & 11 & 505 & 14 & 494 & 14 & 347 & 16 & 343 & 17 \\
\hline Schweiz & 182 & 21 & 176 & 22 & 173 & 21 & 175 & 22 & 172 & 22 \\
\hline Türkei & 330 & 17 & 398 & 15 & 362 & 16 & 383 & 15 & 542 & 15 \\
\hline Großbritannien & 250 & 19 & 247 & 19 & 247 & 18 & 229 & 19 & 248 & 19 \\
\hline Nordamerika $^{\mathrm{d}}$ & 1637 & & 1713 & & 1939 & & 1818 & & 1811 & \\
\hline OECD-Europa ${ }^{d}$ & 511 & & 552 & & 578 & & 564 & & 576 & \\
\hline$\underline{E U^{\text {d,e }}}$ & 527 & & 587 & & 634 & & 629 & & 668 & \\
\hline $\mathrm{OECD}^{d}$ & 653 & & 684 & & 720 & & 704 & & 714 & \\
\hline
\end{tabular}

Anm:

a. Privater Verbrauch, Bewasserung und industrieller Verbrauch

b. Eigene Berechnungen auf Grundlage von Tabelle A1

c. Alte Bundeslander

d. Mittelwert

e. 11 Staaten (ohne Osterreich, Finnland, Schweden und Luxemburg) 
Tabelle A14: Landwirtschaftsflăche pro Einwohner $\left(m^{2}\right)^{a, b}$

\begin{tabular}{|c|c|c|c|c|c|c|c|c|c|c|}
\hline & \multicolumn{2}{|c|}{1970} & \multicolumn{2}{|c|}{1975} & \multicolumn{2}{|c|}{1980} & \multicolumn{2}{|c|}{1985} & \multicolumn{2}{|c|}{1990} \\
\hline & Fläche & Rang & Fläche & Rang & Fläche & Rang & Fläche & Rang & Fläche & Rang \\
\hline Kanada & 18130 & 2 & 17280 & 2 & 16900 & 2 & 16550 & 2 & 15560 & 2 \\
\hline USA & 9290 & 3 & 8716 & 3 & 8370 & 3 & 7937 & 3 & 7551 & 3 \\
\hline Japan & 531 & 22 & 456 & 22 & 418 & 22 & 394 & 22 & 372 & 22 \\
\hline Australien & 32600 & 1 & 30510 & 1 & 30070 & 1 & 29970 & 1 & 28630 & 1 \\
\hline Neuseeland & 2053 & 16 & 1315 & 16 & 1441 & 16 & 1562 & 16 & 1219 & 16 \\
\hline Osterreich & 2251 & 14 & 2123 & 14 & 2166 & 14 & 2018 & 15 & 1950 & 15 \\
\hline Belgien & 881 & 19 & 853 & 19 & 785 & 19 & 768 & 19 & 765 & 19 \\
\hline Dänemark & 5429 & 7 & 5257 & 7 & 5177 & 7 & 5111 & 6 & 4993 & 6 \\
\hline Finnland & 5790 & 6 & 5605 & 6 & 5362 & 6 & 4916 & 7 & 5102 & 5 \\
\hline Frankreich & 3643 & 11 & 3597 & 11 & 3507 & 10 & 3488 & 10 & 3405 & 9 \\
\hline Deutschland $^{c}$ & 1249 & 18 & 1225 & 18 & 1218 & 18 & 1221 & 18 & 1185 & 17 \\
\hline Griechenland & 4447 & 9 & 4275 & 8 & 4071 & 8 & 3966 & 8 & 3880 & 8 \\
\hline Irland & 4678 & 8 & 3890 & 9 & 3264 & 11 & 2915 & 13 & 2692 & 12 \\
\hline Italien & 2782 & 13 & 2221 & 13 & 2204 & 13 & 2121 & 14 & 2077 & 13 \\
\hline Niederlande & 665 & 21 & 615 & 21 & 608 & 21 & 616 & 21 & 631 & 21 \\
\hline Norwegen & 2098 & 15 & 1977 & 15 & 1999 & 15 & 2966 & 12 & 2037 & 14 \\
\hline Portugal & 3568 & 12 & 3350 & 12 & 3199 & 12 & 3186 & 11 & 3218 & 11 \\
\hline Spanien & 6249 & 5 & 5866 & 5 & 5483 & 5 & 5302 & 5 & 5273 & 4 \\
\hline Schweden & 3769 & 10 & 3669 & 10 & 3584 & 9 & 3499 & 9 & 3324 & 10 \\
\hline Schweiz & 737 & 20 & 740 & 20 & 772 & 20 & 756 & 20 & 727 & 20 \\
\hline Türkei & 7678 & 4 & 6856 & 4 & 6298 & 4 & 5434 & 4 & 4933 & 7 \\
\hline Großbritannien & 1294 & 17 & 1237 & 17 & 1242 & 17 & 1247 & 17 & 1160 & 18 \\
\hline Nordamerika & 13710 & & 12998 & & 12635 & & 12244 & & 11556 & \\
\hline OECD-Europa ${ }^{d}$ & 3096 & & 2906 & & 2790 & & 2756 & & 2651 & \\
\hline$E U^{d, e}$ & 3171 & & 2944 & & 2796 & & 2722 & & 2662 & \\
\hline $\mathrm{OECD}^{d}$ & 5446 & & 5074 & & 4915 & & 4816 & & 4577 & \\
\hline
\end{tabular}

Anm.:

a. Ständige und temporäre Ackerflächen

b. Eigene Berechnungen auf Grundlage von Tabelle A2

c. Alte Bundeslander

d. Mittelwert

c. 11 Staaten (ohne Österreich, Finnland, Schweden und Luxemburg) 
Tabelle A15: Primårenergieverbrauch pro Einwohner (TOE) a,b,c

\begin{tabular}{|c|c|c|c|c|c|c|c|c|c|c|}
\hline & \multicolumn{2}{|c|}{1970} & \multicolumn{2}{|c|}{1975} & \multicolumn{2}{|c|}{1980} & \multicolumn{2}{|c|}{1985} & \multicolumn{2}{|c|}{1990} \\
\hline & Menge & Rang & Menge & Rang & Menge & Rang & Menge & Rang & Menge & Rang \\
\hline Kanada & 6.195 & 2 & 7.088 & 2 & 7.981 & 1 & 7.676 & 1 & 7.915 & 1 \\
\hline USA & 7.539 & 1 & 7.626 & 1 & 7.908 & 2 & 7.405 & 2 & 7.633 & 2 \\
\hline Japan & 2.472 & 15 & 2.756 & 14 & 2.959 & 15 & 2.978 & 16 & 3.467 & 15 \\
\hline Australien & 4.002 & 6 & 4.391 & 4 & 4.791 & 5 & 4.681 & 6 & 5.157 & 5 \\
\hline Neuseeland & 2.482 & 14 & 2.753 & 15 & 2.831 & 16 & 3.423 & 14 & 4.143 & 10 \\
\hline Osterreich & 2.451 & 16 & 2.692 & 16 & 3.113 & 14 & 3.070 & 15 & 3.291 & 16 \\
\hline Belgien & 4.176 & 4 & 4.349 & 6 & 4.682 & 6 & 4.463 & 7 & 4.846 & 7 \\
\hline Dånemark & 4.098 & 5 & 3.557 & 11 & 3.805 & 10 & 3.833 & 10 & 3.560 & 14 \\
\hline Finnland & 3.930 & 7 & 4.244 & 7 & 5.230 & 3 & 5.345 & 4 & 5.716 & 3 \\
\hline Frankreich & 2.901 & 12 & 3.078 & 12 & 3.539 & 12 & 3.638 & 11 & 3.921 & 11 \\
\hline Deutschland $^{d}$ & 3.865 & 8 & 3.867 & 8 & 4.433 & 9 & 4.424 & 8 & 4.400 & 9 \\
\hline Griechenland & 0.921 & 20 & 1.349 & 20 & 1.659 & 20 & 1.872 & 19 & 2.179 & 20 \\
\hline Irland & 2.136 & 17 & 2.172 & 18 & 2.499 & 17 & 2.514 & 17 & 3.026 & 17 \\
\hline Italien & 2.063 & 18 & 2.244 & 17 & 2.467 & 18 & 2.391 & 18 & 2.684 & 18 \\
\hline Niederlande & 3.827 & 9 & 4.383 & 5 & 4.629 & 7 & 4.251 & 9 & 4.441 & 8 \\
\hline Norwegen & 3.583 & 11 & 3.793 & 9 & 4.624 & 8 & 4.912 & 5 & 5.070 & 6 \\
\hline Portugal & 0.693 & 21 & 0.859 & 21 & 1.049 & 21 & 1.151 & 21 & 1.663 & 21 \\
\hline Spanien & 1.134 & 19 & 1.647 & 19 & 1.838 & 19 & 1.867 & 20 & 2.259 & 19 \\
\hline Schweden & 4.725 & 3 & 4.822 & 3 & 4.933 & 4 & 5.701 & 3 & 5.585 & 4 \\
\hline Schweiz & 2.633 & 13 & 2.811 & 13 & 3.289 & 13 & 3.536 & 13 & 3.679 & 13 \\
\hline Türkei & 0.343 & 22 & 0.664 & 22 & 0.711 & 22 & 0.770 & 22 & 0.940 & 22 \\
\hline Großbritannien & 3.733 & 10 & 3.592 & 10 & 3.573 & 11 & 3.585 & 12 & 3.689 & 12 \\
\hline Nordamerika $^{e}$ & 6.867 & & 7.357 & & 7.945 & & 7.541 & & 7.774 & \\
\hline OECD-Europa ${ }^{\circ}$ & 3.233 & & 3.240 & & 3.460 & & 3.535 & & 3.751 & \\
\hline EU & 3.104 & & 3.024 & & 3.107 & & 3.090 & & 3.333 & \\
\hline $\mathrm{OECD}^{\mathrm{e}}$ & 3.576 & & 3.661 & & 3.897 & & 3.939 & & 4.206 & \\
\hline
\end{tabular}

Anm.:

a. Inlandische Produktion + Importe - Exporte

b. Eigene Berechnungen auf Grundlage von Tabelle A3

c. TOE := Tonne Endolaquivalent

d. Alte Bundeslander

e. Mittelwert

f. 11 Staaten (ohne Osterreich, Finnland, Schweden und Luxemburg) 
Tabelle A16: Energiebedingte $\mathrm{CO}_{2}$-Emissionen pro Einwohner $(\mathrm{t})^{\mathrm{a}, \mathrm{b}}$

\begin{tabular}{|c|c|c|c|c|c|c|c|c|c|c|}
\hline & \multicolumn{2}{|c|}{1970} & \multicolumn{2}{|c|}{1975} & \multicolumn{2}{|c|}{1980} & \multicolumn{2}{|c|}{1985} & \multicolumn{2}{|c|}{1990} \\
\hline & Menge & Rang & Menge & Rang & Menge & Rang & Menge & Rang & Menge & Rang \\
\hline Kanada & 16,0 & 2 & 17,7 & 2 & 18,2 & 2 & 16,1 & 2 & 16,3 & 2 \\
\hline USA & 20,8 & 1 & 20,6 & 1 & 21,6 & 1 & 19,8 & 1 & 20,0 & 1 \\
\hline Japan & 7,5 & 12 & 8,2 & 12 & 8,0 & 12 & 7,6 & 12 & 8,6 & 11 \\
\hline Australien & 12,7 & 5 & 13,8 & 3 & 15,0 & 3 & 14,5 & 3 & 15,9 & 3 \\
\hline Neuseeland & 5,7 & 17 & 6,2 & 16 & 6,0 & 18 & 7,0 & 16 & 7,7 & 13 \\
\hline Österreich & 6,6 & 15 & 7,0 & 13 & 7,8 & 14 & 7,3 & 13 & 7,6 & 14 \\
\hline Belgien & 13,9 & 3 & 13,3 & 4 & 13,8 & 4 & 11,3 & 7 & 12,5 & 4 \\
\hline Dänemark & 13,0 & 4 & 11,1 & 7 & 12,5 & 8 & 12,5 & 4 & 10,9 & 8 \\
\hline Finnland & 8,9 & 10 & 10,0 & 10 & 12,6 & 7 & 10,8 & 8 & 11,0 & 7 \\
\hline Frankreich & 8,7 & 11 & 8,8 & 11 & 9,3 & 10 & 7,2 & 14 & 6,8 & 17 \\
\hline Deutschland $^{c}$ & 12,4 & 6 & 11,6 & 6 & 12,9 & 6 & 11,8 & 5 & 11,3 & 6 \\
\hline Griechenland & 2,8 & 20 & 4,3 & 20 & 5,3 & 20 & 6,2 & 19 & 8,0 & 12 \\
\hline Irland & 7,1 & 14 & 6,9 & 15 & 7,9 & 13 & 7,9 & 10 & 9,7 & 10 \\
\hline Italien & 5,7 & 17 & 6,2 & 16 & 6,8 & 16 & 6,5 & 17 & 7,1 & 16 \\
\hline Niederlande & 12,4 & 6 & 12,8 & 5 & 13,0 & 5 & 11,5 & 6 & 12,2 & 5 \\
\hline Norwegen & 7,2 & 13 & 7,0 & 13 & 7,8 & 14 & 7,2 & 14 & 7,6 & 14 \\
\hline Portugal & 1,9 & 21 & 2,4 & 21 & 2,8 & 21 & 2,8 & 21 & 4,4 & 21 \\
\hline Spanien & 3,5 & 19 & 4,8 & 19 & 5,4 & 19 & 5,1 & 20 & 5,8 & 20 \\
\hline Schweden & 12,2 & 8 & 10,4 & 9 & 9,0 & 11 & 7,8 & 11 & 6,5 & 18 \\
\hline Schweiz & 6,2 & 16 & 6,1 & 18 & 6,6 & 17 & 6,3 & 18 & 6,5 & 18 \\
\hline Türkei & 1,1 & 22 & 1,6 & 22 & 1,7 & 22 & 2,0 & 22 & 2,5 & 22 \\
\hline Großbritannien & 11,9 & 9 & 10,9 & 8 & 10,7 & 9 & 10,1 & 9 & 10,4 & 9 \\
\hline Nordamerika $^{d}$ & 18,4 & & 19,2 & & 19,9 & & 18,0 & & 18,2 & \\
\hline OECD-Europa ${ }^{d}$ & 8,4 & & 8,4 & & 9,0 & & 8,3 & & 8,6 & \\
\hline $\mathrm{EU}^{\mathrm{d}, \mathrm{e}}$ & 8,5 & & 8,5 & & 9,1 & & 8,4 & & 9,0 & \\
\hline $\mathrm{OECD}^{\mathrm{d}}$ & 9,0 & & 9,2 & & 9,8 & & 9,1 & & 9,5 & \\
\hline
\end{tabular}

Anm:

a. Inklusive Hochseeebunkerung

b. Eigene Berechnungen auf Grundlage von Tabelle A4

c. Alte Bundeslander

d. Mittelwert

e. 11 Staaten (ohne Ǒsterreich, Finnland, Schweden und Luxemburg) 
Tabelle A17: Abfallaufkommen pro Einwohner (kg)

\begin{tabular}{|c|c|c|c|c|c|c|c|c|}
\hline & \multicolumn{2}{|l|}{1975} & \multicolumn{2}{|c|}{1980} & \multicolumn{2}{|l|}{1985} & \multicolumn{2}{|c|}{1990} \\
\hline & Menge & Rang & Menge & Rang & Menge & Rang & Menge & Rang \\
\hline Kanada & 554,4 & 2 & 523,5 & 4 & 567,9 & 4 & 601,1 & 4 \\
\hline USA & 538,1 & 3 & 603,3 & 3 & 623,1 & 3 & 705,7 & 1 \\
\hline Japan & 378,2 & 9 & 376,3 & 10 & 343,9 & 11 & 408,3 & 10 \\
\hline Australien & 719,8 & 1 & 680,5 & 1 & 633,4 & 2 & 585,3 & 5 \\
\hline Neuseeland & 372,5 & 10 & 669,8 & 2 & 643,6 & 1 & 623,3 & 2 \\
\hline Österreich & 185,6 & 21 & 221,6 & 20 & 228,5 & 22 & 324,7 & 18 \\
\hline Belgien & 296,1 & 13 & 313,0 & 13 & 328,7 & 12 & 342,1 & 15 \\
\hline Dănemark & 404,3 & 8 & 399,2 & 9 & 475,2 & 6 & 472,2 & 7 \\
\hline Finnland & 530,6 & 4 & 523,0 & 5 & 510,0 & 5 & 621,7 & 3 \\
\hline Frankreich & 271,9 & 17 & 289,0 & 15 & 294,0 & 18 & 328,1 & 17 \\
\hline Deutschland $^{c}$ & 330,3 & 11 & 347,9 & 12 & 317,7 & 13 & 334,8 & 16 \\
\hline Griechenland & 276,4 & 16 & 259,3 & 18 & 304,3 & 16 & 295,9 & 21 \\
\hline Irland & 174,7 & 22 & 188,2 & 22 & 310,7 & 15 & 314,0 & 20 \\
\hline Italien & 254,2 & 18 & 248,9 & 19 & 262,6 & 19 & 347,5 & 13 \\
\hline Niederlande & 515,9 & 5 & 498,2 & 6 & 435,2 & 8 & 497,0 & 6 \\
\hline Norwegen & 424,3 & 7 & 416,0 & 8 & 474,4 & 7 & 471,6 & 8 \\
\hline Portugal & 226,4 & 19 & 213,0 & 21 & 247,1 & 21 & 257,4 & 22 \\
\hline Spanien & 226,0 & 20 & 270,2 & 17 & 260,1 & 20 & 322,0 & 19 \\
\hline Schweden & 293,0 & 14 & 302,0 & 14 & 317,4 & 14 & 373,9 & 11 \\
\hline Schweiz & 296,7 & 12 & 350,8 & 11 & 382,7 & 10 & 441,4 & 9 \\
\hline Türkei & 483,3 & 6 & 435,9 & 7 & 384,9 & 9 & 345,3 & 14 \\
\hline Großbritannien & 284,6 & 15 & 275,2 & 16 & 300,3 & 17 & 348,4 & 12 \\
\hline Nordamerika $^{\mathrm{d}}$ & 546,3 & & 563,4 & & 595,5 & & 653,4 & \\
\hline OECD-Europa ${ }^{d}$ & 311,9 & & 319,7 & & 340,6 & & 380,8 & \\
\hline $\mathrm{EU}^{\mathrm{d}, \mathrm{e}}$ & 296,4 & & 300,2 & & 321,4 & & 350,9 & \\
\hline $\mathrm{OECD}^{d}$ & 365,3 & & 382,0 & & 393,0 & & 425,5 & \\
\hline
\end{tabular}

Anm.:

a. Abfalle aus privaten Haushalten, Kleingewerbe und Dienstleistungen

b. Eigene Berechnungen auf Grundlage von Tabelle A5

c. Alte Bundeslander

d. Mittelwert

e. 11 Staaten (ohne Osterreich, Finnland, Schweden und Luxemburg) 
Tabelle A18: Handelsdüngerabsatz pro Einwohner (kg)

\begin{tabular}{|c|c|c|c|c|c|c|c|c|c|c|}
\hline & \multicolumn{2}{|c|}{1970} & \multicolumn{2}{|l|}{1975} & \multicolumn{2}{|c|}{1980} & \multicolumn{2}{|l|}{1985} & \multicolumn{2}{|c|}{1990} \\
\hline & Menge & Rang & Menge & Rang & Menge & Rang & Menge & Rang & Menge & Rang \\
\hline Kanada & 371,6 & 15 & 575,5 & 8 & 805,6 & 7 & 923,3 & 6 & 779,1 & 6 \\
\hline USA & 757,6 & 6 & 875,9 & 6 & 943,1 & 6 & 745,2 & 7 & 732,7 & 7 \\
\hline Japan & 188,5 & 20 & 161,5 & 22 & 155,5 & 22 & 174,3 & 22 & 148,8 & 22 \\
\hline Australien & 757,6 & 6 & 519,7 & 10 & 790,7 & 8 & 731,6 & 8 & 681,3 & 9 \\
\hline Neuseeland & 1589,0 & 1 & 1672,0 & 1 & 1476,0 & 2 & 1305,0 & 2 & 1015,0 & 3 \\
\hline Österreich & 546,4 & 9 & 413,0 & 15 & 539,1 & 12 & 513,4 & 11 & 393,9 & 13 \\
\hline Belgien & 535,7 & 10 & 460,4 & 13 & 453,9 & 14 & 427,1 & 16 & 385,3 & 14 \\
\hline Dänemark & 1213,0 & 3 & 1263,0 & 3 & 1223,0 & 3 & 1240,0 & 3 & 1231,0 & 2 \\
\hline Finnland & 1055,0 & 4 & 1097,0 & 4 & 1023,0 & 5 & 1034,0 & 4 & 888,5 & 5 \\
\hline Frankreich & 916,1 & 5 & 889,2 & 5 & 1041,0 & 4 & 1032,0 & 5 & 1007,0 & 4 \\
\hline Deutschland $^{c}$ & 430,2 & 13 & 413,6 & 14 & 443,9 & 16 & 399,7 & 17 & 254,5 & 20 \\
\hline Griechenland & 383,3 & 14 & 511,8 & 11 & 546,6 & 11 & 689,6 & 9 & 686,4 & 8 \\
\hline Irland & 1434,0 & 2 & 1360,0 & 2 & 1767,0 & 1 & 1751,0 & 1 & 1975,0 & 1 \\
\hline Italien & 249,3 & 18 & 268,8 & 18 & 374,2 & 17 & 367,9 & 18 & 317,8 & 18 \\
\hline Niederlande & 498,5 & 12 & 464,7 & 12 & 479,9 & 13 & 483,7 & 13 & 375,2 & 16 \\
\hline Norwegen & 513,0 & 11 & 574,0 & 9 & 633,7 & 9 & 573,1 & 10 & 495,2 & 11 \\
\hline Portugal & 148,9 & 21 & 263,2 & 19 & 263,8 & 21 & 243,3 & 21 & 282,0 & 19 \\
\hline Spanien & 359,0 & 16 & 394,8 & 16 & 444,6 & 15 & 450,3 & 14 & 507,5 & 10 \\
\hline Schweden & 625,4 & 8 & 640,9 & 7 & 582,4 & 10 & 501,8 & 12 & 383,2 & 15 \\
\hline Schweiz & 236,2 & 19 & 223,3 & 20 & 283,5 & 20 & 275,5 & 20 & 247,2 & 21 \\
\hline Türkei & 121,1 & 22 & 220,8 & 21 & 325,5 & 19 & 281,7 & 19 & 334,3 & 17 \\
\hline Großbritannien & 340,5 & 17 & 323,8 & 17 & 364,7 & 18 & 445,8 & 15 & 412,8 & 12 \\
\hline Nordamerika $^{\mathrm{d}}$ & 564,6 & & 725,7 & & 874,4 & & 834,3 & & 755,9 & \\
\hline OECD-Europa ${ }^{d}$ & 592,8 & & 597,6 & & 654,0 & & 651,8 & & 615,2 & \\
\hline$E U^{\mathrm{d}, e}$ & 591,7 & & 601,2 & & 673,0 & & 684,6 & & 675,9 & \\
\hline $\mathrm{OECD}^{\mathrm{d}}$ & 603,2 & & 617,6 & & 680,0 & & 663,2 & & 615,2 & \\
\hline
\end{tabular}

Anm.:

a. Stickstoff, Phosphat, Kali und Kalk

b. Eigene Berechnungen auf Grundlage von Tabelle A6

c. Alte Bundesländer

d. Mittelwert

e. 11 Staaten (ohne Österreich, Finnland, Schweden und Luxemburg) 
Tabelle A19: Ressourcenverbrauchsindex (Basis: Einwohnerzahl)

\begin{tabular}{|c|c|c|c|c|c|c|c|c|c|c|}
\hline & \multicolumn{2}{|c|}{1970} & \multicolumn{2}{|c|}{1975} & \multicolumn{2}{|c|}{1980} & \multicolumn{2}{|c|}{1985} & \multicolumn{2}{|c|}{1990} \\
\hline & Wert & Rang & Wert & Rang & Wert & Rang & Wert & Rang & Wert & Rang \\
\hline Kanada & 230 & 2 & 237 & 2 & 258 & 2 & 258 & 2 & 257 & 2 \\
\hline USA & 229 & 3 & 228 & 3 & 234 & 3 & 210 & 3 & 205 & 3 \\
\hline Japan & 65 & 14 & 64 & 16 & 64 & 16 & 63 & 16 & 67 & 17 \\
\hline Australien & 321 & 1 & 305 & 1 & 302 & 1 & 297 & 1 & 288 & 1 \\
\hline Neuseeland & 52 & 19 & 49 & 20 & 53 & 19 & 68 & 15 & 71 & 15 \\
\hline Osterreich & 53 & 18 & 54 & 18 & 56 & 17 & 54 & 18 & 55 & 20 \\
\hline Belgien & 89 & 6 & 88 & 8 & 89 & 8 & 87 & 8 & 90 & 6 \\
\hline Dănemark & 80 & 9 & 78 & 9 & 80 & 11 & 80 & 10 & 76 & 14 \\
\hline Finnland & 107 & 4 & 111 & 4 & 119 & 4 & 119 & 4 & 113 & 4 \\
\hline Frankreich & 72 & 12 & 75 & 11 & 85 & 9 & 85 & 9 & 89 & 7 \\
\hline Deutschland ${ }^{b}$ & 65 & 14 & 68 & 15 & 79 & 13 & 79 & 12 & 82 & 11 \\
\hline Griechenland & 61 & 17 & 71 & 13 & 76 & 14 & 76 & 14 & 77 & 13 \\
\hline Irland & 63 & 16 & 57 & 17 & 55 & 18 & 52 & 19 & 56 & 19 \\
\hline Italien & 73 & 11 & 76 & 10 & 83 & 10 & 78 & 13 & 83 & 10 \\
\hline Niederlande & 86 & 7 & 90 & 7 & 94 & 6 & 88 & 7 & 89 & 7 \\
\hline Norwegen & 74 & 10 & 75 & 11 & 80 & 11 & 80 & 10 & 81 & 12 \\
\hline Portugal & 39 & 21 & 39 & 21 & 39 & 22 & 41 & 22 & 71 & 15 \\
\hline Spanien & 86 & 7 & 101 & 5 & 103 & 5 & 108 & 5 & 100 & 5 \\
\hline Schweden & 91 & 5 & 91 & 6 & 91 & 7 & 90 & 6 & 88 & 9 \\
\hline Schweiz & 37 & 22 & 38 & 22 & 42 & 21 & 45 & 21 & 46 & 22 \\
\hline Türkei & 70 & 13 & 71 & 13 & 66 & 15 & 61 & 17 & 67 & 17 \\
\hline Großbritannien & 43 & 20 & 52 & 19 & 52 & 20 & 51 & 20 & 52 & 21 \\
\hline Nordamerika $^{c}$ & 230 & & 233 & & 246 & & 234 & & 231 & \\
\hline OECD-Europa ${ }^{c}$ & 70 & & 73 & & 76 & & 76 & & 78 & \\
\hline EU & 69 & & 72 & & 76 & & 75 & & 79 & \\
\hline $\mathrm{OECD}^{c}$ & 95 & & 96 & & 100 & & 99 & & 100 & \\
\hline
\end{tabular}

Anm.:

a. OECD $1980=100$. Zur Berechnung des Index siehe Kapitel II.2.2

b. Alte Bundeslander

c. Mittelwert

d. 11 Staaten (ohne Osterreich, Finnland, Schweden und Luxemburg) 
Tabelle A20: Schadstofinndex (Basis: Einwohnerzahl)"

\begin{tabular}{|c|c|c|c|c|c|c|c|c|c|c|}
\hline & \multicolumn{2}{|c|}{1970} & \multicolumn{2}{|c|}{1975} & \multicolumn{2}{|c|}{1980} & \multicolumn{2}{|c|}{1985} & \multicolumn{2}{|c|}{1990} \\
\hline & Wert & Rang & Wert & Rang & Wert & Rang & Wert & Rang & Wert & Rang \\
\hline Kanada & 109 & 9 & 137 & 2 & 147 & 4 & 150 & 2 & 146 & 3 \\
\hline USA & 162 & 1 & 160 & 1 & 172 & 1 & 158 & 1 & 166 & 1 \\
\hline Japan & 52 & 16 & 69 & 15 & 68 & 16 & 64 & 18 & 72 & 19 \\
\hline Australien & 121 & 6 & 135 & 3 & 149 & 3 & 140 & 5 & 139 & 4 \\
\hline Neuseeland & 146 & 3 & 135 & 3 & 151 & 2 & 144 & 4 & 130 & 7 \\
\hline Osterreich & 74 & 15 & 60 & 17 & 72 & 15 & 70 & 16 & 74 & 15 \\
\hline Belgien & 110 & 8 & 94 & 10 & 97 & 10 & 88 & 11 & 91 & 10 \\
\hline Dänemark & 155 & 2 & 135 & 3 & 137 & 6 & 145 & 3 & 139 & 4 \\
\hline Finnland & 123 & 5 & 134 & 6 & 138 & 5 & 132 & 7 & 135 & 6 \\
\hline Frankreich & 112 & 7 & 97 & 9 & 108 & 9 & 101 & 8 & 101 & 9 \\
\hline Deutschland ${ }^{b}$ & 95 & 12 & 88 & 13 & 96 & 11 & 88 & 11 & 80 & 14 \\
\hline Griechenland & 43 & 20 & 64 & 16 & 67 & 17 & 82 & 14 & 87 & 12 \\
\hline Irland & 142 & 4 & 105 & 8 & 130 & 7 & 140 & 5 & 157 & 2 \\
\hline Italien & 48 & 18 & 56 & 20 & 63 & 20 & 63 & 19 & 70 & 20 \\
\hline Niederlande & 100 & 11 & 111 & 7 & 111 & 8 & 101 & 8 & 103 & 8 \\
\hline Norwegen & 75 & 14 & 89 & 12 & 94 & 12 & 94 & 10 & 91 & 10 \\
\hline Portugal & 20 & 21 & 41 & 22 & 41 & 22 & 43 & 22 & 51 & 22 \\
\hline Spanien & 44 & 19 & 55 & 21 & 64 & 19 & 62 & 20 & 73 & 17 \\
\hline Schweden & 108 & 10 & 92 & 11 & 86 & 13 & 79 & 15 & 74 & 15 \\
\hline Schweiz & 49 & 17 & 58 & 18 & 67 & 17 & 68 & 17 & 73 & 17 \\
\hline Türkei & 15 & 22 & 58 & 18 & 60 & 21 & 54 & 21 & 55 & 21 \\
\hline Großbritannien & 86 & 13 & 78 & 14 & 78 & 14 & 83 & 13 & 86 & 13 \\
\hline Nordamerika $^{c}$ & 136 & & 149 & & 160 & & 154 & & 156 & \\
\hline OECD-Europa ${ }^{c}$ & 87 & & 85 & & 91 & & 90 & & 93 & \\
\hline$E U^{c, d}$ & 87 & & 84 & & 90 & & 91 & & 94 & \\
\hline $\mathrm{OECD}^{c}$ & 90 & & 93 & & 100 & & 98 & & 100 & \\
\hline
\end{tabular}

Anm.:

a. OECD $1980=100$. Zur Berechnung des Index siehe Kapitel II.2.2

b. Alte Bundesiander

c. Mittelwert

d. 11 Staaten (ohne Österreich, Finnland, Schweden und Luxemburg) 
Tabelle A21: Umweltbelastungsindex (Basis: Einwohnerzahl)

\begin{tabular}{|c|c|c|c|c|c|c|c|c|c|c|}
\hline & \multicolumn{2}{|c|}{1970} & \multicolumn{2}{|c|}{1975} & \multicolumn{2}{|l|}{1980} & \multicolumn{2}{|c|}{1985} & \multicolumn{2}{|c|}{1990} \\
\hline & Wert & Rang & Wert & Rang & Wert & Rang & Wert & Rang & Wert & Rang \\
\hline Kanada & 169 & 3 & 187 & 3 & 203 & 2 & 204 & 2 & 201 & 2 \\
\hline USA & 195 & 2 & 194 & 2 & 203 & 2 & 184 & 3 & 185 & 3 \\
\hline Japan & 58 & 18 & 67 & 16 & 66 & 17 & 63 & 18 & 69 & 17 \\
\hline Australien & 221 & 1 & 220 & 1 & 226 & 1 & 219 & 1 & 213 & 1 \\
\hline Neuseeland & 99 & 7 & 92 & 7 & 102 & 6 & 106 & 6 & 100 & 7 \\
\hline Ósterreich & 63 & 16 & 57 & 20 & 64 & 19 & 62 & 19 & 65 & 19 \\
\hline Belgien & 99 & 7 & 91 & 8 & 93 & 9 & 88 & 10 & 91 & 10 \\
\hline Dănemark & 117 & 4 & 107 & 5 & 108 & 5 & 113 & 5 & 108 & 5 \\
\hline Finnland & 115 & 5 & 123 & 4 & 128 & 4 & 125 & 4 & 124 & 4 \\
\hline Frankreich & 92 & 11 & 86 & 10 & 97 & 8 & 93 & 9 & 95 & 9 \\
\hline Deutschland $^{b}$ & 80 & 12 & 78 & 13 & 88 & 12 & 84 & 13 & 81 & 14 \\
\hline Griechenland & 52 & 19 & 68 & 15 & 72 & 16 & 79 & 15 & 82 & 13 \\
\hline Irland & 102 & 6 & 81 & 12 & 93 & 9 & 96 & 7 & 107 & 6 \\
\hline Italien & 61 & 17 & 66 & 17 & 73 & 15 & 71 & 16 & 77 & 16 \\
\hline Niederlande & 93 & 10 & 100 & 6 & 102 & 6 & 94 & 8 & 96 & 8 \\
\hline Norwegen & 74 & 13 & 82 & 11 & 87 & 13 & 87 & 11 & 86 & 11 \\
\hline Portugal & 30 & 22 & 40 & 22 & 40 & 22 & 42 & 22 & 61 & 20 \\
\hline Spanien & 65 & 14 & 78 & 13 & 84 & 14 & 85 & 12 & 86 & 11 \\
\hline Schweden & 99 & 7 & 91 & 8 & 89 & 11 & 84 & 13 & 81 & 14 \\
\hline Schweiz & 43 & 20 & 48 & 21 & 54 & 21 & 57 & 21 & 59 & 22 \\
\hline Türkei & 43 & 20 & 65 & 18 & 63 & 20 & 58 & 20 & 61 & 20 \\
\hline Großbritannien & 65 & 14 & 65 & 18 & 65 & 18 & 67 & 17 & 69 & 17 \\
\hline Nordamerika $^{c}$ & 182 & & 191 & & 203 & & 194 & & 193 & \\
\hline OECD-Europa ${ }^{c}$ & 78 & & 79 & & 84 & & 83 & & 86 & \\
\hline$E U^{e, d}$ & 78 & & 78 & & 83 & & 83 & & 87 & \\
\hline $\mathrm{OECD}^{\mathrm{c}}$ & 93 & & 95 & & 100 & & 98 & & 100 & \\
\hline
\end{tabular}

Anm.:

a. OECD $1980=100$. Zur Berechnung des Index siehe Kapitel II.2.2

b. Alte Bundeslander

c. Mittelwert

d. 11 Staaten (ohne Osterreich, Finnland, Schweden und Luxemburg) 
Tabelle A22: Verănderung des Wasserverbrauches pro Einwohner (in \%)"

\begin{tabular}{|c|c|c|c|c|}
\hline & $1970-1975$ & $1975-1980$ & $1980-1985$ & $1985-1990$ \\
\hline Kanada & 9,8 & 27,1 & 7,0 & 4,6 \\
\hline USA & 2,0 & 5,3 & $-15,3$ & $-4,7$ \\
\hline Japan & $-7,0$ & $-3,9$ & $-2,1$ & $-2,2$ \\
\hline Australien & $-7,8$ & $-5,5$ & $-6,9$ & $-7,5$ \\
\hline Neuseeland & $-3,4$ & 12,7 & 52,1 & $-3,3$ \\
\hline Österreich & $-1,4$ & $-16,2$ & $-3,5$ & $-1,8$ \\
\hline Belgien & $-4,4$ & $-2,3$ & $-0,1$ & $-1,1$ \\
\hline Dänemark & 63,0 & $-1,3$ & 0,4 & $-1,3$ \\
\hline Finnland & 5,2 & 2,8 & 5,4 & $-26,2$ \\
\hline Frankreich & 10,6 & 27,3 & $-3,1$ & 5,9 \\
\hline Deutschland $^{b}$ & 11,7 & 26,3 & $-1,6$ & 11,3 \\
\hline Griechenland & 33,5 & 11,5 & $-2,9$ & $-2,0$ \\
\hline Irland & $-7,1$ & $-6,8$ & $-3,9$ & 0,9 \\
\hline Italien & 12,0 & 13,8 & $-8,6$ & 7,1 \\
\hline Niederlande & $-1,3$ & 4,1 & $-4,5$ & $-3,0$ \\
\hline Norwegen & $-3,3$ & $-8,8$ & $-10,0$ & $-2,3$ \\
\hline Portugal & $-7,2$ & $-5,0$ & 17,4 & 265,8 \\
\hline Spanien & 40,0 & 5,1 & 12,5 & $-21,1$ \\
\hline Schweden & $-0,2$ & $-2,2$ & $-29,8$ & $-1,2$ \\
\hline Schweiz & $-3,3$ & $-1,7$ & 1,2 & $-1,7$ \\
\hline Türkei & 20,6 & $-9,1$ & 5,8 & 41,5 \\
\hline Großbritannien & $-1,2$ & 0,0 & $-7,3$ & 8,3 \\
\hline Nordamerika $^{c}$ & 5,9 & 16,2 & $-4,2$ & $-0,1$ \\
\hline OECD-Europa ${ }^{c}$ & 9,2 & 2,9 & $-2,4$ & $-1,9$ \\
\hline$E^{c, d}$ & 13,6 & 6,6 & $-0,2$ & 0,5 \\
\hline $\mathrm{OECD}^{\mathrm{c}}$ & 7,3 & 3,3 & 0,1 & 0,0 \\
\hline
\end{tabular}

Anm.:

a. Eigene Berechnungen auf Grundlage von Tabelle A13

b. Alte Bundesländer

c. Mittelwert

d. 11 Staaten (ohne Osterreich, Finnland, Schweden und Luxemburg)

Mittelwerte 1985-1990 ohne Portugal 
Tabelle A23: Verănderung der Landwirtschaftsfiăche pro Einwohner (in \%)

\begin{tabular}{|c|c|c|c|c|}
\hline & $1970-1975$ & $1975-1980$ & $1980-1985$ & $1985-1990$ \\
\hline Kanada & $-4,7$ & $-2,2$ & $-2,1$ & $-6,0$ \\
\hline USA & $-6,2$ & $-4,0$ & $-5,2$ & $-4,9$ \\
\hline Japan & $-14,1$ & $-8,3$ & $-5,7$ & $-5,6$ \\
\hline Australien & $-6,4$ & $-1,4$ & $-0,3$ & $-4,5$ \\
\hline Neuseeland & $-35,9$ & 9,6 & 8,4 & $-22,0$ \\
\hline Österreich & $-5,7$ & 2,0 & $-6,8$ & $-3,4$ \\
\hline Belgien & $-3,2$ & $-8,0$ & $-2,2$ & $-0,4$ \\
\hline Dănemark & $-3,2$ & $-1,5$ & $-1,3$ & $-2,3$ \\
\hline Finnland & $-1,3$ & $-4,3$ & $-8,3$ & 3,8 \\
\hline Frankreich & $-1,9$ & $-2,5$ & $-0,5$ & $-2,4$ \\
\hline Deutschland ${ }^{\mathbf{b}}$ & $-3,9$ & $-0,6$ & 0,2 & $-3,0$ \\
\hline Griechenland & $-16,8$ & $-4,8$ & $-2,6$ & $-2,2$ \\
\hline Irland & $-20,2$ & $-16,1$ & $-10,7$ & $-7,7$ \\
\hline Italien & $-7,5$ & $-0,8$ & $-3,8$ & $-2,1$ \\
\hline Niederlande & $-5,8$ & $-1,1$ & 1,3 & 2,4 \\
\hline Norwegen & $-6,1$ & 1,1 & 3,4 & $-1,4$ \\
\hline Portugal & $-6,1$ & $-4,5$ & $-0,4$ & 1,0 \\
\hline Spanien & $-3,4$ & $-6,5$ & $-3,3$ & $-0,6$ \\
\hline Schweden & 0,4 & $-2,3$ & $-2,4$ & $-5,0$ \\
\hline Schweiz & $-10,7$ & 4,3 & $-2,1$ & $-3,8$ \\
\hline Türkei & $-4,4$ & $-8,1$ & $-13,7$ & $-9,2$ \\
\hline GroBbritannien & $-1,2$ & 0,4 & 0,4 & $-7,0$ \\
\hline Nordamerika $^{c}$ & $-5,5$ & $-3,1$ & $-3,7$ & $-5,5$ \\
\hline OECD-Europa ${ }^{c}$ & $-6,0$ & $-2,8$ & $-2,4$ & $-2,1$ \\
\hline EU & $-6,7$ & $-4,2$ & $-2,1$ & $-2,2$ \\
\hline $\mathrm{OECD}^{\mathrm{c}}$ & $-7,7$ & $-2,7$ & $-2,6$ & $-3,9$ \\
\hline
\end{tabular}

Anm.:

a. Eigene Berechnungen auf Grundlage von Tabelle A14

b. Alte Bundeslănder

c. Mittelwert

d. 11 Staaten (ohne Ősterreich, Finnland, Schweden und Luxemburg) 
Tabelle A24: Veränderang des Primlrrenergieverbrauches pro Einwohner (in \%)"

\begin{tabular}{|c|c|c|c|c|}
\hline & $1970-1975$ & $1975-1980$ & $1980-1985$ & $1985-1990$ \\
\hline Kanada & 14,4 & 12,6 & $-3,8$ & 3,1 \\
\hline USA & 1,2 & 3,7 & $-6,4$ & 3,1 \\
\hline Japan & 11,5 & 7,4 & 0,6 & 16,4 \\
\hline Australien & 9,7 & 9,1 & $-2,3$ & 10,2 \\
\hline Neuseeland & 10,9 & 2,8 & 20,9 & 21,0 \\
\hline Österreich & 9,8 & 15,6 & $-1,4$ & 7,2 \\
\hline Belgien & 4,1 & 7,7 & $-4,7$ & 8,6 \\
\hline Dänemark & $-13,2$ & 7,0 & 0,7 & $-7,1$ \\
\hline Finnland & 8,0 & 23,2 & 2,2 & 6,9 \\
\hline Frankreich & 6,1 & 15,0 & 2,8 & 7,8 \\
\hline Deutschland $^{b}$ & 0,1 & 14,6 & $-0,2$ & $-0,5$ \\
\hline Griechenland & 46,5 & 23,0 & 12,8 & 16,4 \\
\hline Irland & 1,7 & 15,1 & 0,6 & 20,3 \\
\hline Italien & 8,8 & 9,9 & $-3,1$ & 12,2 \\
\hline Niederlande & 14,5 & 5,6 & $-8,2$ & 4,5 \\
\hline Norwegen & 5,9 & 21,9 & 6,2 & 3,2 \\
\hline Portugal & 24,0 & 22,1 & 9,7 & 44,5 \\
\hline Spanien & 45,2 & 11,6 & 1,6 & 21,0 \\
\hline Schweden & 2,1 & 2,3 & 15,6 & $-2,0$ \\
\hline Schweiz & 6,8 & 17,0 & 7,5 & 4,0 \\
\hline Türkei & 93,6 & 7,1 & 8,3 & 22,1 \\
\hline Großbritannien & $-3,8$ & $-0,5$ & 0,3 & 2,9 \\
\hline Nordamerika $^{c}$ & 7,8 & 8,2 & $-5,1$ & 3,1 \\
\hline OECD-Europa ${ }^{c}$ & 10,4 & 13,2 & 2,7 & 9,4 \\
\hline$E U^{c, d}$ & 12,2 & 11,9 & 1,1 & 11,9 \\
\hline $\mathrm{OECD}^{c}$ & 14,0 & 11,5 & 2,7 & 10,3 \\
\hline
\end{tabular}

Anm.:

a. Eigene Berechnungen auf Grundlage von Tabelle A15

b. Alte Bundeslănder

c. Mittelwert

d. 11 Staaten (ohne Osterreich, Finnland, Schweden und Luxemburg)

Mittelwerte 1970-1975 ohne Turkei 
Tabelle A25: Verănderung der energiebedingten $\mathrm{CO}_{2}$-Emissio pro Einwohner (in \%)"

\begin{tabular}{|c|c|c|c|c|}
\hline & $1970-1975$ & $1975-1980$ & $1980-1985$ & $1985-1990$ \\
\hline Kanada & 10,3 & 3,1 & $-11,6$ & 1,4 \\
\hline USA & $-1,1$ & 4,8 & $-8,3$ & 1,3 \\
\hline Japan & 8,6 & $-2,0$ & $-5,9$ & 13,6 \\
\hline Australien & 8,1 & 9,4 & $-3,6$ & 9,8 \\
\hline Neuseeland & 8,5 & $-1,8$ & 16,4 & 9,4 \\
\hline Österreich & 6,6 & 11,9 & $-6,9$ & 4,9 \\
\hline Belgien & $-4,4$ & 4,1 & $-18,5$ & 11,4 \\
\hline Dănemark & $-14,7$ & 12,8 & 0,2 & $-12,9$ \\
\hline Finnland & 12,0 & 25,9 & $-13,9$ & 2,0 \\
\hline Frankreich & 0,5 & 5,6 & $-22,7$ & $-4,8$ \\
\hline Deutschland ${ }^{b}$ & $-6,2$ & 11,0 & $-8,0$ & $-4,6$ \\
\hline Griechenland & 51,8 & 22,7 & 18,0 & 28,0 \\
\hline Irland & $-2,8$ & 14,7 & $-0,4$ & 22,8 \\
\hline Italien & 7,9 & 9,7 & $-4,6$ & 10,4 \\
\hline Niederlande & 3,7 & 1,5 & $-11,4$ & 6,3 \\
\hline Norwegen & $-3,2$ & 12,0 & $-7,8$ & 4,6 \\
\hline Portugal & 27,6 & 16,5 & 2,9 & 54,1 \\
\hline Spanien & 36,5 & 13,3 & $-5,3$ & 13,4 \\
\hline Schweden & $-14,8$ & $-13,1$ & $-13,7$ & $-15,9$ \\
\hline Schweiz & $-2,1$ & 8,0 & $-4,6$ & 3,0 \\
\hline Türkei & 41,8 & 7,7 & 18,5 & 23,6 \\
\hline GroBbritannien & $-8,2$ & $-2,3$ & $-5,0$ & 2,8 \\
\hline Nordamerika $^{c}$ & 4,6 & 4,0 & $-10,0$ & 1,4 \\
\hline OECD-Europa ${ }^{c}$ & 5,6 & 9,6 & $-6,4$ & 7,8 \\
\hline$\underline{E U^{c, d}}$ & 8,3 & 10,0 & $-5,0$ & 11,5 \\
\hline $\mathrm{OECD}^{c}$ & 7,6 & 8,0 & $-4,4$ & 8,4 \\
\hline
\end{tabular}

Anm.:

a. Eigene Berechnungen auf Grundlage von Tabelle A16

b. Alte Bundeslander

c. Mittelwert

d. 11 Stasten (ohne Osterreich, Finnland, Schweden und Luxemburg) 
Tabelle A26: Verænderung des Abfallaufikommen pro Einwohner (in \%)

\begin{tabular}{|c|c|c|c|}
\hline & $1975-1980$ & $1980-1985$ & $1985-1990$ \\
\hline Kanada & $-5,6$ & 8,5 & 5,8 \\
\hline USA & 12,1 & 3,3 & 13,3 \\
\hline Japan & $-0,5$ & $-8,6$ & 18,7 \\
\hline Australien & $-5,5$ & $-6,9$ & $-7,6$ \\
\hline Neuseeland & 79,8 & $-3,9$ & $-3,1$ \\
\hline Österreich & 19,4 & 3,1 & 42,1 \\
\hline Belgien & 5,7 & 5,0 & 4,1 \\
\hline Dänemark & $-1,3$ & 19,0 & $-0,5$ \\
\hline Finnland & $-1,4$ & $-2,5$ & 21,9 \\
\hline Frankreich & 6,3 & 1,7 & 11,6 \\
\hline Deutschland $^{b}$ & 5,3 & $-8,7$ & 5,4 \\
\hline Griechenland & $-6,2$ & 17,4 & $-2,8$ \\
\hline Irland & 7,7 & 65,1 & 1,1 \\
\hline Italien & $-2,1$ & 5,5 & 32,3 \\
\hline Niederlande & $-3,4$ & $-12,6$ & 14,2 \\
\hline Norwegen & $-2,0$ & 14,0 & $-0,6$ \\
\hline Portugal & $-5,2$ & 16,0 & 4,2 \\
\hline Spanien & 19,6 & $-3,7$ & 23,8 \\
\hline Schweden & 3,1 & 5,1 & 17,8 \\
\hline Schweiz & 18,2 & 9,1 & 15,3 \\
\hline Türkei & $-9,8$ & $-11,7$ & $-10,3$ \\
\hline Großbritannien & $-3,3$ & 9,1 & 16,0 \\
\hline Nordamerika $^{c}$ & 3,3 & 5,9 & 9,6 \\
\hline OECD-Europa ${ }^{c}$ & 3,8 & 8,9 & 12,9 \\
\hline$E U^{c, d}$ & 2,1 & 10,3 & 9,9 \\
\hline $\mathrm{OECD}^{c}$ & 6,0 & 5,6 & 10,1 \\
\hline
\end{tabular}

Anm:

a. Eigene Berechnungen auf Grundlage von Tabelle A17

b. Alte Bundeslander

c. Mittelwert

d. 11 Stasten (ohne Osterreich, Finnland, Schweden und Luxemburg) 
Tabelle A27: Veræ̌nderung des Handelsdüngerabsatzes pro Einwohner (in \%)

\begin{tabular}{|c|c|c|c|c|}
\hline & $1970-1975$ & $1975-1980$ & $1980-1985$ & $1985-1990$ \\
\hline Kanada & 53,0 & 40,0 & 14,6 & $-15,6$ \\
\hline USA & 15,6 & 7,7 & $-21,0$ & $-1,7$ \\
\hline Japan & $-14,3$ & $-3,7$ & 12,1 & $-14,6$ \\
\hline Australien & $-31,4$ & 52,2 & $-7,5$ & $-6,9$ \\
\hline Neuseeland & 5,2 & $-11,7$ & $-11,6$ & $-22,2$ \\
\hline Osterreich & $-24,4$ & 30,5 & $-4,8$ & $-23,3$ \\
\hline Belgien & $-14,1$ & $-1,4$ & $-5,9$ & $-9,8$ \\
\hline Danemark & 4,1 & $-3,2$ & 1,4 & $-0,7$ \\
\hline Finnland & 4,0 & $-6,8$ & 1,1 & $-14,1$ \\
\hline Frankreich & $-2,9$ & 17,1 & $-0,9$ & $-2,4$ \\
\hline Deutschland ${ }^{b}$ & $-3,9$ & 7,3 & $-10,0$ & $-36,3$ \\
\hline Griechenland & 33,5 & 6,8 & 26,2 & $-0,5$ \\
\hline Irland & $-5,2$ & 29,9 & $-0,9$ & 12,8 \\
\hline Italien & 7,8 & 39,2 & $-1,7$ & $-13,6$ \\
\hline Niederlande & $-6,8$ & 3,3 & 0,8 & $-22,4$ \\
\hline Norwegen & 11,9 & 10,4 & $-9,6$ & $-13,6$ \\
\hline Portugal & 76,8 & 0,2 & $-7,8$ & 15,9 \\
\hline Spanien & 10,0 & 12,6 & 1,3 & 12,7 \\
\hline Schweden & 2,5 & $-9,1$ & $-13,8$ & $-23,6$ \\
\hline Schweiz & $-5,5$ & 27,0 & $-2,8$ & $-10,3$ \\
\hline Türkei & 82,3 & 47,4 & $-13,5$ & 18,7 \\
\hline GroBbritannien & $-4,9$ & 12,6 & 22,2 & $-7,4$ \\
\hline Nordamerika & 34,3 & 23,9 & $-3,2$ & $-8,6$ \\
\hline OECD-Europa ${ }^{c}$ & 5,2 & 11,0 & $-0,3$ & $-8,5$ \\
\hline EU & 8,6 & 11,3 & 2,2 & $-4,7$ \\
\hline $\mathrm{OECD}^{\mathrm{c}}$ & 8,8 & 14,0 & $-1,5$ & $-8,1$ \\
\hline
\end{tabular}

Anm:

a. Eigene Berechnungen auf Grundlage von Tabelle A18

b. Alte Bundeslander

c. Mittelwert

d. 11 Staaten (ohne Osterreich, Finnland, Schweden und Luxemburg) 
Tabelle A28: Verånderung des Ressourcenverbrauchsindex (Basis: Einwohnerzahl; in \%)"

\begin{tabular}{|c|c|c|c|c|}
\hline & $1970-1975$ & $1975-1980$ & $1980-1985$ & $1985-1990$ \\
\hline Kanada & 3,0 & 8,9 & 0,0 & $-0,4$ \\
\hline USA & $-0,4$ & 2,6 & $-10,3$ & $-2,4$ \\
\hline Japan & $-1,5$ & 0,0 & $-1,6$ & 6,3 \\
\hline Australien & $-5,0$ & $-1,0$ & $-1,7$ & $-3,0$ \\
\hline Neuseeland & $-5,8$ & 8,2 & 28,3 & 4,4 \\
\hline Osterreich & 1,9 & 3,7 & $-3,6$ & 1,9 \\
\hline Belgien & $-1,1$ & 1,1 & $-2,3$ & 3,4 \\
\hline Dănemark & $-2,5$ & 2,6 & 0,0 & $-5,0$ \\
\hline Finnland & 3,7 & 7,2 & 0,0 & $-5,0$ \\
\hline Frankreich & 4,2 & 13,3 & 0,0 & 4,7 \\
\hline Deutschland $^{b}$ & 4,6 & 16,2 & 0,0 & 3,8 \\
\hline Griechenland & 16,4 & 7,0 & 0,0 & 1,3 \\
\hline Irland & $-9,5$ & $-3,5$ & $-5,5$ & 7,7 \\
\hline Italien & 4,1 & 9,2 & $-6,0$ & 6,4 \\
\hline Niederlande & 4,7 & 4,4 & $-6,4$ & 1,1 \\
\hline Norwegen & 1,4 & 6,7 & 0,0 & 1,3 \\
\hline Portugal & 0,0 & 0,0 & 5,1 & 73,2 \\
\hline Spanien & 17,4 & 2,0 & 4,9 & $-7,4$ \\
\hline Schweden & 0,0 & 0,0 & $-1,1$ & $-2,2$ \\
\hline Schweiz & 2,7 & 10,5 & 7,1 & 2,2 \\
\hline Türkei & 1,4 & $-7,0$ & $-7,6$ & 9,8 \\
\hline Großbritannien & $-3,7$ & 0,0 & $-1,9$ & 2,0 \\
\hline Nordamerika $^{c}$ & 1,3 & 5,8 & $-5,2$ & $-1,4$ \\
\hline OECD-Europa ${ }^{c}$ & 2,8 & 5,0 & $-0,6$ & 5,6 \\
\hline$E U^{c, d}$ & 3,1 & 4,8 & $-1,1$ & 8,3 \\
\hline $\mathrm{OECD}^{\mathrm{c}}$ & 1,6 & 4,2 & $-0,1$ & 4,7 \\
\hline
\end{tabular}

Anm.:
a. Eigene Berechnungen auf Grundlage von Tabelle A19
b. Alte Bundeslander
c. Mittelwert
d. 11 Staaten (ohne Osterreich, Finnland, Schweden und Luxemburg) 
Tabelle A29: Verănderung des Schadstofinindex

(Basis: Einwohnerzahl; in \%)

\begin{tabular}{|c|c|c|c|c|}
\hline & $1970-1975$ & $1975-1980$ & $1980-1985$ & $1985-1990$ \\
\hline Kanada & 25,7 & 7,3 & 2,0 & $-2,7$ \\
\hline USA & $-1,2$ & 7,5 & $-8,1$ & 5,1 \\
\hline Japan & 32,7 & $-1,5$ & $-5,9$ & 12,5 \\
\hline Australien & 11,6 & 10,4 & $-6,0$ & $-0,7$ \\
\hline Neuseeland & $-7,5$ & 11,9 & $-4,6$ & $-9,7$ \\
\hline Österreich & $-18,9$ & 20,0 & $-2,8$ & 5,7 \\
\hline Belgien & $-14,5$ & 3,2 & $-9,3$ & 3,4 \\
\hline Dänemark & $-12,9$ & 1,5 & 5,8 & $-4,1$ \\
\hline Finnland & 8,9 & 3,0 & $-4,4$ & 2,3 \\
\hline Frankreich & $-13,4$ & 11,3 & $-6,5$ & 0,0 \\
\hline Deutschland ${ }^{b}$ & $-7,4$ & 9,1 & $-8,3$ & $-9,1$ \\
\hline Griechenland & 48,8 & 4,7 & 22,4 & 6,1 \\
\hline Irland & $-26,1$ & 23,8 & 7,7 & 12,1 \\
\hline Italien & 16,7 & 12,5 & 0,0 & 11,1 \\
\hline Niederlande & 11,0 & 0,0 & $-9,0$ & 2,0 \\
\hline Norwegen & 18,7 & 5,6 & 0,0 & $-3,2$ \\
\hline Portugal & 105,0 & 0,0 & 4,9 & 18,6 \\
\hline Spanien & 25,0 & 16,4 & $-3,1$ & 17,7 \\
\hline Schweden & $-14,8$ & $-6,5$ & $-8,1$ & $-6,3$ \\
\hline Schweiz & 18,4 & 15,5 & 1,5 & 7,4 \\
\hline Türkei & 286,7 & 3,4 & $-10,0$ & 1,9 \\
\hline Großbritannien & $-9,3$ & 0,0 & 6,4 & 3,6 \\
\hline Nordamerika $^{c}$ & 12,3 & 7,4 & $-3,1$ & 1,2 \\
\hline OECD-Europa ${ }^{c}$ & 8,5 & 7,5 & $-0,2$ & 4,2 \\
\hline $\mathrm{EU}^{\mathrm{c}, \mathrm{d}}$ & 11,2 & 7,5 & 1,0 & 5,6 \\
\hline $\mathrm{OECD}^{\mathrm{c}, \mathrm{e}}$ & 9,4 & 7,2 & $-1,6$ & 3,4 \\
\hline
\end{tabular}

Anm:
a. Eigene Berechnungen auf Grundlage von Tabelle A20
b. Alte Bundesländer
c. Mittelwert
d. 11 Staaten (ohne Österreich, Finnland, Schweden und Luxemburg)
e. Wert 70-75 ohne die Türkei 
Tabelle A30: Verd̆nderung des Umweltbelastungsindex (Basis: Einwohnerzahl; in \%)

\begin{tabular}{|c|c|c|c|c|}
\hline & $1970-1975$ & $1975-1980$ & $1980-1985$ & $1985-1990$ \\
\hline Kanada & 10,7 & 8,6 & 0,5 & $-1,5$ \\
\hline USA & $-0,5$ & 4,6 & $-9,4$ & 0,5 \\
\hline Japan & 15,5 & $-1,5$ & $-4,6$ & 9,5 \\
\hline Australien & $-0,5$ & 2,7 & $-3,1$ & $-2,7$ \\
\hline Neuseeland & $-7,1$ & 10,9 & 3,9 & $-5,7$ \\
\hline Österreich & $-9,5$ & 12,3 & $-3,1$ & 4,8 \\
\hline Belgien & $-8,1$ & 2,2 & $-5,4$ & 3,4 \\
\hline Dänemark & $-8,6$ & 0,9 & 4,6 & $-4,4$ \\
\hline Finnland & 7,0 & 4,1 & $-2,3$ & $-0,8$ \\
\hline Frankreich & $-6,5$ & 12,8 & $-4,1$ & 2,2 \\
\hline Deutschland $^{b}$ & $-2,5$ & 12,8 & $-4,6$ & $-3,6$ \\
\hline Griechenland & 30,8 & 5,9 & 9,7 & 3,8 \\
\hline Irland & $-20,6$ & 14,8 & 3,2 & 11,5 \\
\hline Italien & 8,2 & 10,6 & $-2,7$ & 8,5 \\
\hline Niederlande & 7,5 & 2,0 & $-7,8$ & 2,1 \\
\hline Norwegen & 10,8 & 6,1 & 0,0 & $-1,2$ \\
\hline Portugal & 33,3 & 0,0 & 5,0 & 45,2 \\
\hline Spanien & 20,0 & 7,7 & 1,1 & 1,2 \\
\hline Schweden & $-8,1$ & $-2,2$ & $-5,6$ & $-3,6$ \\
\hline Schweiz & 11,6 & 12,5 & 5,6 & 3,5 \\
\hline Türkei & 51,2 & $-3,1$ & $-7,9$ & 5,2 \\
\hline Großbritannien & $-7,1$ & 0,0 & 3,1 & 3,0 \\
\hline Nordamerika $^{c}$ & 5,1 & 6,6 & $-4,5$ & $-0,5$ \\
\hline OECD-Europa ${ }^{c}$ & 3,6 & 6,4 & $-0,2$ & 4,7 \\
\hline$E U^{c, d}$ & 4,2 & 6,3 & 0,2 & 6,6 \\
\hline $\mathrm{OECD}^{\mathrm{ces}}$ & 3,6 & 5,7 & $-1,1$ & 3,7 \\
\hline
\end{tabular}

Anm.:

a. Eigene Berechnungen auf Grundlage von Tabelle A21

b. Alte Bundeslander

c. Mittelwert

d. 11 Staaten (ohne Osterreich, Finnland, Schweden und Luxemburg)

e. Wert 70-75 ohne die Turkei 
Tabelle A31: Wasserverbrauch pro Einheit BIP $\left(\mathrm{m}^{3} / 1000 \text { US- } \$\right)^{2, b}$

\begin{tabular}{|c|c|c|c|c|c|c|c|c|c|c|}
\hline & \multicolumn{2}{|c|}{1970} & \multicolumn{2}{|c|}{1975} & \multicolumn{2}{|c|}{1980} & \multicolumn{2}{|c|}{1985} & \multicolumn{2}{|c|}{1990} \\
\hline & Menge & Rang & Menge & Rang & Menge & Rang & Menge & Rang & Menge & Rang \\
\hline $\begin{array}{l}\text { Kanada } \\
\text {. }\end{array}$ & 117,1 & 7 & 106,8 & 7 & 118,5 & 6 & 115,0 & 6 & 110,8 & 4 \\
\hline USA & 162,1 & 2 & 155,2 & 2 & 147,4 & 2 & 116,3 & 4 & 102,7 & 5 \\
\hline Japan & 112,5 & 8 & 90,7 & 10 & 73,0 & 11 & 61,4 & 11 & 49,2 & 12 \\
\hline Australien & 149,2 & 3 & 123,4 & 5 & 107,1 & 6 & 91,5 & 6 & 78,7 & 7 \\
\hline Neuseeland & 37,9 & 19 & 32,6 & 19 & 37,2 & 16 & 50,5 & 12 & 49,7 & 11 \\
\hline Osterreich & 43,5 & 18 & 35,9 & 18 & 25,4 & 19 & 23,1 & 19 & 19,9 & 19 \\
\hline Belgien & 116,6 & 7 & 95,4 & 8 & 80,6 & 9 & 77,6 & 9 & 66,5 & 10 \\
\hline Dänemark & 15,6 & 21 & 23,6 & 21 & 20,9 & 21 & 18,3 & 21 & 16,9 & 21 \\
\hline Finnland & 93,0 & 11 & 81,8 & 11 & 73,6 & 10 & 69,0 & 10 & 43,7 & 15 \\
\hline Frankreich & 49,3 & 16 & 48,1 & 14 & 53,5 & 13 & 49,4 & 14 & 46,1 & 14 \\
\hline Deutschland $^{c}$ & 49,2 & 17 & 50,4 & 13 & 53,9 & 12 & 49,9 & 13 & 49,2 & 12 \\
\hline Griechenland & 125,1 & 4 & 134,4 & 4 & 128,8 & 4 & 120,6 & 3 & 110,9 & 3 \\
\hline Irland & 57,5 & 13 & 45,3 & 15 & 36,2 & 17 & 32,0 & 17 & 25,3 & 17 \\
\hline Italien & 98,3 & 10 & 99,2 & 7 & 91,0 & 7 & 78,6 & 8 & 73,1 & 8 \\
\hline Niederlande & 107,3 & 9 & 94,9 & 9 & 89,8 & 8 & 83,6 & 7 & 72,8 & 9 \\
\hline Norwegen & 85,0 & 12 & 67,8 & 12 & 49,9 & 14 & 38,7 & 15 & 35,7 & 16 \\
\hline Portugal & 51,3 & 14 & 41,4 & 17 & 32,3 & 18 & 36,6 & 16 & 111,3 & 2 \\
\hline Spanien & 120,8 & 5 & 137,7 & 3 & 139,6 & 3 & 151,0 & 2 & 96,8 & 6 \\
\hline Schweden & 49,8 & 15 & 44,6 & 16 & 41,4 & 15 & 26,7 & 18 & 24,4 & 18 \\
\hline Schweiz & 13,3 & 22 & 12,7 & 22 & 11,4 & 22 & 11,0 & 22 & 9,8 & 22 \\
\hline Turkei & 206,3 & 1 & 196,1 & 1 & 172,5 & 1 & 163,6 & 1 & 193,3 & 1 \\
\hline GroBbritannien & 28,7 & 20 & 25,9 & 20 & 23,7 & 20 & 19,9 & 20 & 18,8 & 20 \\
\hline Nordamerika ${ }^{d}$ & 139,6 & & 131,0 & & 133,0 & & 115,7 & & 106,8 & \\
\hline OECD-Europa $^{d}$ & 69,0 & & 64,9 & & 59,5 & & 55,4 & & 51,3 & \\
\hline$E U^{d, e}$ & 74,5 & & 72,4 & & 68,2 & & 65,2 & & 62,5 & \\
\hline $\mathrm{OECD}^{d}$ & 85,9 & & 79,3 & & 73,1 & & 67,5 & & 63,9 & \\
\hline
\end{tabular}

Anm.:

a. Privater Verbrauch, Bewlsserung und industrieller Verbrauch

b. Eigene Berechnungen auf Grundlage von Tabelle Al

c. Alte Bundeslander

d. Mittelwert

e. 11 Staaten (ohne Osterreich, Finnland, Schweden und Luxemburg) 
Tabelle A32: Landwirtschaftsflăche pro Einheit BIP $\left(\mathrm{m}^{2} / \mathrm{Mrd}\right.$. US-S) ${ }^{a, b}$

\begin{tabular}{|c|c|c|c|c|c|c|c|c|c|c|}
\hline & \multicolumn{2}{|c|}{1970} & \multicolumn{2}{|c|}{1975} & \multicolumn{2}{|c|}{1980} & \multicolumn{2}{|c|}{1985} & \multicolumn{2}{|c|}{1990} \\
\hline & Flache & Rang & Flache & Rang & Flache & Rang & Flyche & Rang & Fläche & Rang \\
\hline Kanada & 1881 & 3 & 1491 & 3 & 1273 & 3 & 1131 & 3 & 980 & 3 \\
\hline USA & 702 & 9 & 618 & 8 & 535 & 7 & 473 & 7 & 417 & 7 \\
\hline Japan & 71 & 20 & 53 & 21 & 40 & 22 & 33 & 22 & 25 & 22 \\
\hline Australien & 3503 & 2 & 2939 & 2 & 2659 & 2 & 2431 & 1 & 2162 & 1 \\
\hline Neuseeland & 222 & 16 & 127 & 17 & 140 & 16 & 136 & 16 & 108 & 16 \\
\hline Osterreich & 279 & 15 & 220 & 15 & 190 & 14 & 166 & 14 & 141 & 15 \\
\hline Belgien & 105 & 19 & 87 & 19 & 69 & 19 & 65 & 19 & 56 & 19 \\
\hline Dänemark & 578 & 10 & 522 & 10 & 459 & 10 & 397 & 10 & 362 & 9 \\
\hline Finnland & 751 & 8 & 609 & 9 & 510 & 8 & 416 & 8 & 370 & 8 \\
\hline Frankreich & 388 & 11 & 338 & 11 & 288 & 12 & 272 & 11 & 235 & 12 \\
\hline Deutschland $^{c}$ & 127 & 18 & 114 & 18 & 96 & 18 & 90 & 18 & 78 & 18 \\
\hline Griechenland & 1150 & 4 & 889 & 4 & 728 & 4 & 684 & 4 & 628 & 4 \\
\hline Irland & 1000 & 6 & 706 & 7 & 507 & 9 & 416 & 8 & 300 & 10 \\
\hline Italien & 350 & 13 & 252 & 13 & 201 & 13 & 183 & 13 & 156 & 13 \\
\hline Niederlande & 70 & 21 & 58 & 20 & 52 & 20 & 52 & 20 & 47 & 20 \\
\hline Norwegen & 291 & 14 & 226 & 14 & 184 & 15 & 164 & 15 & 152 & 14 \\
\hline Portugal & 940 & 7 & 764 & 6 & 601 & 6 & 577 & 6 & 484 & 6 \\
\hline Spanien & 1040 & 5 & 795 & 5 & 717 & 5 & 667 & 5 & 539 & 5 \\
\hline Schweden & 373 & 12 & 324 & 12 & 300 & 11 & 269 & 12 & 236 & 11 \\
\hline Schweiz & 54 & 22 & 53 & 21 & 51 & 21 & 48 & 21 & 41 & 21 \\
\hline Türkei & 4796 & 1 & 3382 & 1 & 3001 & 1 & 2321 & 2 & 1760 & 2 \\
\hline Großbritannien & 148 & 17 & 130 & 16 & 119 & 17 & 109 & 17 & 88 & 17 \\
\hline Nordamerika $^{d}$ & 1291,50 & & 1054,50 & & 904,00 & & 802,00 & & 698,50 & \\
\hline OECD-Europa $^{d}$ & 477,75 & & 380,44 & & 317,00 & & 285,94 & & 244,56 & \\
\hline $\mathrm{EU}^{\mathrm{d}, \mathrm{e}}$ & 536,00 & & 423,18 & & 348,82 & & 319,27 & & 270,27 & \\
\hline $\mathrm{OECD}^{d}$ & 855,41 & & 668,05 & & 578,18 & & 504,55 & & 425,68 & \\
\hline
\end{tabular}

Anm.:

a. Standige und temporäre Ackerflächen

b. Eigene Berechnungen auf Gundlage von Tabelle A2

c. Alte Bundeslănder

d. Mittelwert

e. 11 Staaten (ohne Osterreich, Finnland, Schweden und Luxemburg) 
Tabelle A33: Primårenergieverbrauch pro Einheit BIP (TOE/1000 US-\$) a,b,c

\begin{tabular}{|c|c|c|c|c|c|c|c|c|c|c|}
\hline & \multicolumn{2}{|c|}{1970} & \multicolumn{2}{|c|}{1975} & \multicolumn{2}{|c|}{1980} & \multicolumn{2}{|c|}{1985} & \multicolumn{2}{|c|}{1990} \\
\hline & Menge & Rang & Menge & Rang & Menge & Rang & Menge & Rang & Menge & Rang \\
\hline Kanada & 6,43 & 1 & 6,12 & 1 & 6,01 & 1 & 5,25 & 1 & 4,98 & 1 \\
\hline USA & 5,69 & 2 & 5,41 & 2 & 5,06 & 2 & 4,41 & 3 & 4,22 & 2 \\
\hline Japan & 3,29 & 13 & 3,18 & 14 & 2,86 & 16 & 2,48 & 18 & 2,36 & 19 \\
\hline Australien & 4,30 & 9 & 4,23 & 7 & 4,24 & 5 & 3,80 & 6 & 3,89 & 5 \\
\hline Neuseeland & 2,68 & 16 & 2,65 & 18 & 2,76 & 17 & 2,98 & 14 & 3,67 & 7 \\
\hline Österreich & 3,04 & 15 & 2,80 & 17 & 2,73 & 18 & 2,52 & 17 & 2,38 & 18 \\
\hline Belgien & 4,96 & 4 & 4,42 & 4 & 4,12 & 7 & 3,78 & 7 & 3,56 & 8 \\
\hline Dănemark & 4,36 & 8 & 3,53 & 12 & 3,37 & 13 & 2,98 & 14 & 2,58 & 16 \\
\hline Finnland & 5,10 & 3 & 4,61 & 3 & 4,97 & 3 & 4,52 & 2 & 4,15 & 3 \\
\hline Frankreich & 3,09 & 14 & 2,89 & 15 & 2,91 & 15 & 2,84 & 16 & 2,71 & 15 \\
\hline Deutschland $^{d}$ & 3,91 & 12 & 3,59 & 11 & 3,49 & 10 & 3,27 & 11 & 2,88 & 13 \\
\hline Griechenland & 2,38 & 18 & 2,81 & 16 & 2,97 & 14 & 3,23 & 12 & 3,53 & 9 \\
\hline Irland & 4,57 & 7 & 3,94 & 9 & 3,88 & 9 & 3,59 & 8 & 3,38 & 10 \\
\hline Italien & 2,60 & 17 & 2,54 & 19 & 2,26 & 20 & 2,07 & 22 & 2,01 & 22 \\
\hline Niederlande & 4,03 & 11 & 4,14 & 8 & 3,98 & 8 & 3,56 & 9 & 3,34 & 12 \\
\hline Norwegen & 4,96 & 4 & 4,33 & 5 & 4,26 & 4 & 3,89 & 5 & 3,79 & 6 \\
\hline Portugal & 1,82 & 22 & 1,96 & 22 & 1,97 & 22 & 2,08 & 21 & 2,50 & 17 \\
\hline Spanien & 1,87 & 21 & 2,23 & 20 & 2,40 & 19 & 2,35 & 19 & 2,31 & 20 \\
\hline Schweden & 4,65 & 6 & 4,26 & 6 & 4,13 & 6 & 4,38 & 4 & 3,97 & 4 \\
\hline Schweiz & 1,93 & 20 & 2,02 & 21 & 2,17 & 21 & 2,23 & 20 & 2,10 & 21 \\
\hline Türkei & 2,14 & 19 & 3,28 & 13 & 3,39 & 12 & 3,29 & 10 & 3,35 & 11 \\
\hline Großbritannien & 4,28 & 10 & 3,76 & 10 & 3,42 & 11 & 3,13 & 13 & 2,79 & 14 \\
\hline Nordamerika $^{e}$ & 6,06 & & 5,77 & & 5,54 & & 4,83 & & 4,60 & \\
\hline OECD-Europa ${ }^{e}$ & 3,60 & & 3,36 & & 3,31 & & 3,15 & & 3,00 & \\
\hline$E U^{e, f}$ & 3,44 & & 3,26 & & 3,16 & & 2,99 & & 2,87 & \\
\hline $\mathrm{OECD}^{\mathrm{e}}$ & 3,73 & & 3,58 & & 3,52 & & 3,30 & & 3,20 & \\
\hline
\end{tabular}

Anm:

a. Inlăndische Produktion + Importe - Exporte

b. Eigene Berechnungen auf Grundlage von Tabelle A3

c. TOE := Tonne Erdolaquivalent

d. Alte Bundesländer

e. Mittelwert

f. 11 Staaten (ohne Österreich, Finnland, Schweden und Luxemburg) 
Tabelle A34: Energiebedingte $\mathrm{CO}_{2}$-Emissionen pro Einheit BIP (t/1000 US-\$) ${ }^{\text {a,b }}$

\begin{tabular}{|c|c|c|c|c|c|c|c|c|c|c|}
\hline & \multicolumn{2}{|c|}{1970} & \multicolumn{2}{|c|}{1975} & \multicolumn{2}{|c|}{1980} & \multicolumn{2}{|c|}{1985} & \multicolumn{2}{|c|}{1990} \\
\hline & Menge & Rang & Menge & Rang & Menge & Rang & Menge & Rang & Menge & Rang \\
\hline Kanada & 1,66 & 1 & 1,53 & 1 & 1,37 & 2 & 1,10 & 4 & 1,03 & 5 \\
\hline USA & 1,57 & 3 & 1,46 & 2 & 1,38 & 1 & 1,18 & 1 & 1,11 & 3 \\
\hline Japan & 1,00 & 12 & 0,94 & 11 & 0,78 & 13 & 0,63 & 14 & 0,58 & 16 \\
\hline Australien & 1,36 & 6 & 1,33 & 4 & 1,33 & 3 & 1,18 & 1 & 1,20 & 2 \\
\hline Neuseeland & 0,61 & 19 & 0,59 & 20 & 0,59 & 20 & 0,61 & 15 & 0,68 & 13 \\
\hline Osterreich & 0,81 & 15 & 0,73 & 17 & 0,69 & 18 & 0,60 & 16 & 0,55 & 18 \\
\hline Belgien & 1,65 & 2 & 1,35 & 3 & 1,21 & 5 & 0,95 & 8 & 0,92 & 6 \\
\hline Dänemark & 1,38 & 5 & 1,10 & 8 & 1,11 & 8 & 0,97 & 6 & 0,79 & 10 \\
\hline Finnland & 1,16 & 11 & 1,08 & 9 & 1,19 & 6 & 0,91 & 9 & 0,80 & 9 \\
\hline Frankreich & 0,93 & 14 & 0,82 & 14 & 0,76 & 14 & 0,56 & 19 & 0,47 & 20 \\
\hline Deutschland $^{c}$ & 1,25 & 9 & 1,07 & 10 & 1,01 & 10 & 0,87 & 11 & 0,74 & 12 \\
\hline Griechenland & 0,74 & 16 & 0,90 & 13 & 0,95 & 11 & 1,08 & 5 & 1,29 & 1 \\
\hline Irland & 1,52 & 4 & 1,26 & 5 & 1,23 & 4 & 1,13 & 3 & 1,08 & 4 \\
\hline Italien & 0,72 & 17 & 0,70 & 18 & 0,62 & 19 & 0,56 & 19 & 0,53 & 19 \\
\hline Niederlande & 1,30 & 8 & 1,21 & 6 & 1,12 & 7 & 0,96 & 7 & 0,92 & 6 \\
\hline Norwegen & 1,00 & 12 & 0,80 & 15 & 0,72 & 16 & 0,57 & 18 & 0,56 & 17 \\
\hline Portugal & 0,49 & 21 & 0,54 & 21 & 0,52 & 21 & 0,51 & 21 & 0,66 & 14 \\
\hline Spanien & 0,58 & 20 & 0,65 & 19 & 0,71 & 17 & 0,65 & 13 & 0,60 & 15 \\
\hline Schweden & 1,20 & 10 & 0,92 & 12 & 0,76 & 14 & 0,60 & 16 & 0,47 & 20 \\
\hline Schweiz & 0,46 & 22 & 0,44 & 22 & 0,43 & 22 & 0,40 & 22 & 0,37 & 22 \\
\hline Türkei & 0,68 & 18 & 0,77 & 16 & 0,80 & 12 & 0,85 & 12 & 0,88 & 8 \\
\hline Großbritannien & 1,36 & 6 & 1,14 & 7 & 1,02 & 9 & 0,88 & 10 & 0,79 & 10 \\
\hline Nordamerika $^{d}$ & 1,62 & & 1,50 & & 1,38 & & 1,14 & & 1,07 & \\
\hline OECD-Europa ${ }^{d}$ & 1,03 & & 0,92 & & 0,88 & & 0,76 & & 0,72 & \\
\hline$E U^{d, e}$ & 1,08 & & 0,98 & & 0,93 & & 0,83 & & 0,80 & \\
\hline $\mathrm{OECD}^{d}$ & 1,07 & & 0,97 & & 0,92 & & 0,81 & & 0,77 & \\
\hline
\end{tabular}

Anm:

a. Inklusive Hochseeebunkerung

b. Eigene Berechnungen auf Grundlage von Tabelle A4

c. Alte Bundesländer

d. Mittelwert

e. 11 Staaten (ohne Osterreich, Finnland, Schweden und Luxemburg) 
Tabelle A35: Abfallaufkommen pro Einheit BIP (kg/1000 US-\$) $)^{\text {a,b }}$

\begin{tabular}{|c|c|c|c|c|c|c|c|c|}
\hline & \multicolumn{2}{|l|}{1975} & \multicolumn{2}{|l|}{1980} & \multicolumn{2}{|l|}{1985} & \multicolumn{2}{|l|}{1990} \\
\hline & Menge & Rang & Menge & Rang & Menge & Rang & Menge & Rang \\
\hline Kanada & 47,8 & 8 & 39,5 & 8 & 38,8 & 8 & 37,8 & 8 \\
\hline USA & 38,2 & 11 & 38,6 & 9 & 37,1 & 10 & 39,0 & 6 \\
\hline Japan & 43,7 & 9 & 36,4 & 11 & 28,6 & 14 & 27,8 & 14 \\
\hline Australien & 69,4 & 2 & 60,2 & 3 & 51,4 & 4 & 44,2 & 5 \\
\hline Neuseeland & 35,8 & 12 & 65,2 & 2 & 56,0 & 2 & 55,1 & 2 \\
\hline Österreich & 19,3 & 22 & 19,4 & 22 & 18,8 & 22 & 23,5 & 20 \\
\hline Belgien & 30,1 & 16 & 27,5 & 15 & 27,8 & 15 & 25,1 & 19 \\
\hline Danemark & 40,1 & 10 & 35,4 & 12 & 36,9 & 11 & 34,2 & 12 \\
\hline Finnland & 57,6 & 3 & 49,7 & 4 & 43,1 & 7 & 45,1 & 4 \\
\hline Frankreich & 25,5 & 20 & 23,7 & 19 & 23,0 & 20 & 22,6 & 21 \\
\hline Deutschland $^{\mathfrak{C}}$ & 30,6 & 14 & 27,4 & 16 & 23,5 & 19 & 21,9 & 22 \\
\hline Griechenland & 57,5 & 4 & 46,4 & 5 & 52,5 & 3 & 47,9 & 3 \\
\hline Irland & 31,7 & 13 & 29,2 & 14 & 44,4 & 6 & 35,0 & 11 \\
\hline Italien & 28,8 & 18 & 22,7 & 21 & 22,7 & 21 & 26,1 & 17 \\
\hline Niederlande & 48,7 & 6 & 42,8 & 6 & 36,4 & 12 & 37,4 & 9 \\
\hline Norwegen & 48,4 & 7 & 38,3 & 10 & 37,6 & 9 & 35,3 & 10 \\
\hline Portugal & 51,3 & 5 & 40,0 & 7 & 44,8 & 5 & 38,8 & 7 \\
\hline Spanien & 30,6 & 14 & 35,3 & 13 & 32,7 & 13 & 32,9 & 13 \\
\hline Schweden & 25,9 & 19 & 25,3 & 18 & 24,4 & 17 & 26,6 & 15 \\
\hline Schweiz & 21,4 & 21 & 23,1 & 20 & 24,1 & 18 & 25,2 & 18 \\
\hline Türkei & 238,4 & 1 & 207,7 & 1 & 164,4 & 1 & 123,2 & 1 \\
\hline Großbritannien & 29,8 & 17 & 26,4 & 17 & 26,2 & 16 & 26,3 & 16 \\
\hline Nordamerika $^{d}$. & 43,0 & & 39,1 & & 38,0 & & 38,4 & \\
\hline OECD-Europa ${ }^{d}$ & 36,1 & & 32,0 & & 32,4 & & 31,5 & \\
\hline $\mathrm{EU}^{\mathrm{d}, \mathrm{e}}$ & 36,8 & & 32,4 & & 33,7 & & 31,7 & \\
\hline $\mathrm{OECD}^{d}$ & 47,8 & & 43,6 & & 40,7 & & 37,8 & \\
\hline
\end{tabular}

Anm.:

a. Abfalle aus privaten Haushalten, Kleingewerbe und Dienstleistungen

b. Eigene Berechnungen auf Grundlage von Tabelle A5

c. Alte Bundeslander

d. Mittelwert

e. 11 Staaten (ohne Osterreich, Finnland, Schweden und Luxemburg) 
Tabelle A36: Handelsdungerabsatz pro Einheit BIP (kg/1000 US-\$) ${ }^{a, b}$

\begin{tabular}{|c|c|c|c|c|c|c|c|c|c|c|}
\hline & \multicolumn{2}{|c|}{1970} & \multicolumn{2}{|c|}{1975} & \multicolumn{2}{|c|}{1980} & \multicolumn{2}{|c|}{1985} & \multicolumn{2}{|c|}{1990} \\
\hline & Menge & Rang & Menge & Rang & Menge & Rang & Menge & Rang & Menge & Rang \\
\hline Kanada & 3,90 & 18 & 4,97 & 14 & 6,07 & 9 & 6,31 & 8 & 4,90 & 10 \\
\hline USA & 5,72 & 14 & 6,21 & 9 & 6,03 & 10 & 4,44 & 12 & 4,05 & 12 \\
\hline Japan & 2,51 & 21 & 1,86 & 21 & 1,50 & 22 & 1,45 & 22 & 1,01 & 22 \\
\hline Australien & 8,14 & 7 & 5,01 & 13 & 6,99 & 8 & 5,94 & 9 & 5,14 & 9 \\
\hline Neuseeland & 17,16 & 2 & 16,07 & 2 & 14,37 & 3 & 11,36 & 4 & 8,98 & 4 \\
\hline Österreich & 6,78 & 10 & 4,29 & 17 & 4,73 & 15 & 4,22 & 14 & 2,85 & 15 \\
\hline Belgien & 6,36 & 11 & 4,68 & 15 & 3,99 & 17 & 3,62 & 18 & 2,83 & 16 \\
\hline Dănemark & 12,92 & 4 & 12,53 & 3 & 10,85 & 4 & 9,64 & 5 & 8,92 & 5 \\
\hline Finnland & 13,69 & 3 & 11,91 & 4 & 9,72 & 6 & 8,74 & 6 & 6,45 & 7 \\
\hline Frankreich & 9,76 & 6 & 8,35 & 7 & 8,55 & 7 & 8,06 & 7 & 6,95 & 6 \\
\hline Deutschland $^{c}$ & 4,36 & 16 & 3,84 & 18 & 3,49 & 18 & 2,95 & 20 & 1,67 & 20 \\
\hline Griechenland & 9,91 & 5 & 10,64 & 6 & 9,78 & 5 & 11,89 & 3 & 11,12 & 3 \\
\hline Irland & 30,65 & 1 & 24,69 & 1 & 27,44 & 1 & 25,00 & 1 & 22,04 & 1 \\
\hline Italien & 3,14 & 20 & 3,05 & 20 & 3,42 & 20 & 3,18 & 19 & 2,38 & 19 \\
\hline Niederlande & 5,26 & 15 & 4,39 & 16 & 4,12 & 16 & 4,05 & 15 & 2,82 & 17 \\
\hline Norwegen & 7,11 & 9 & 6,55 & 8 & 5,83 & 11 & 4,54 & 11 & 3,70 & 13 \\
\hline Portugal & 3,92 & 17 & 6,01 & 10 & 4,95 & 13 & 4,41 & 13 & 4,24 & 11 \\
\hline Spanien & 5,97 & 13 & 5,35 & 12 & 5,81 & 12 & 5,66 & 10 & 5,19 & 8 \\
\hline Schweden & 6,15 & 12 & 5,66 & 11 & 4,87 & 14 & 3,86 & 17 & 2,72 & 18 \\
\hline Schweiz & 1,73 & 22 & 1,61 & 22 & 1,87 & 21 & 1,73 & 21 & 1,41 & 21 \\
\hline Türkei & 7,56 & 8 & 10,89 & 5 & 15,51 & 2 & 12,03 & 2 & 11,93 & 2 \\
\hline Großbritannien & 3,90 & 18 & 3,39 & 19 & 3,49 & 18 & 3,89 & 16 & 3,12 & 14 \\
\hline Nordamerika $^{d}$ & 4,81 & & 5,59 & & 6,05 & & 5,38 & & 4,48 & \\
\hline OECD-Europa ${ }^{d}$ & 8,23 & & 7,31 & & 7,06 & & 6,59 & & 5,53 & \\
\hline $\mathrm{EU}^{\mathrm{d}, \mathrm{e}}$ & 8,74 & & 7,90 & & 7,81 & & 7,49 & & 6,48 & \\
\hline $\mathrm{OECD}^{\mathrm{d}}$ & 8,03 & & 7,36 & & 7,43 & & 6,68 & & 5,66 & \\
\hline
\end{tabular}

Anm.:

a. Stickstoff, Phosphat, Kali und Kalk

b. Eigene Berechnungen auf Grundlage von Tabelle A6

c. Alte Bundesländer

d. Mittelwert

e. 11 Staaten (ohne Ôsterreich, Finnland, Schweden und Luxemburg) 
Tabelle A37: Ressourcenverbrauchsindex (Basis: BIP)"

\begin{tabular}{|c|c|c|c|c|c|c|c|c|c|c|}
\hline & \multicolumn{2}{|c|}{1970} & \multicolumn{2}{|c|}{1975} & \multicolumn{2}{|c|}{1980} & \multicolumn{2}{|c|}{1985} & \multicolumn{2}{|c|}{1990} \\
\hline & Wert & Rang & Wert & Rang & Wert & Rang & Wert & Rang & Wert & Rang \\
\hline Kanada & 223 & 3 & 193 & 3 & 185 & 3 & 168 & 3 & 154 & 3 \\
\hline USA & 169 & 4 & 158 & 4 & 146 & 4 & 122 & 6 & 111 & 5 \\
\hline Japan & 87 & 15 & 75 & 15 & 63 & 17 & 53 & 19 & 46 & 19 \\
\hline Australien & 311 & 2 & 266 & 2 & 242 & 2 & 218 & 2 & 198 & 2 \\
\hline Neuseeland & 56 & 20 & 47 & 21 & 51 & 19 & 59 & 17 & 64 & 11 \\
\hline Österreich & 65 & 19 & 56 & 19 & 48 & 21 & 44 & 21 & 40 & 20 \\
\hline Belgien & 106 & 9 & 90 & 9 & 80 & 10 & 75 & 8 & 67 & 10 \\
\hline Dănemark & 82 & 16 & 74 & 16 & 68 & 15 & 60 & 16 & 53 & 18 \\
\hline Finnland & 134 & 6 & 116 & 7 & 110 & 7 & 98 & 7 & 81 & 8 \\
\hline Frankreich & 74 & 17 & 69 & 17 & 69 & 14 & 65 & 14 & 60 & 16 \\
\hline Deutschland $^{b}$ & 67 & 18 & 64 & 18 & 63 & 17 & 59 & 17 & 54 & 17 \\
\hline Griechenland & 146 & 5 & 139 & 5 & 129 & 5 & 125 & 5 & 120 & 4 \\
\hline Irland & 127 & 8 & 99 & 8 & 83 & 8 & 73 & 10 & 61 & 13 \\
\hline Italien & 90 & 13 & 84 & 12 & 75 & 11 & 66 & 13 & 61 & 13 \\
\hline Niederlande & 91 & 12 & 86 & 10 & 82 & 9 & 75 & 8 & 68 & 9 \\
\hline Norwegen & 103 & 10 & 85 & 11 & 74 & 13 & 64 & 15 & 61 & 13 \\
\hline Portugal & 95 & 11 & 82 & 13 & 68 & 15 & 70 & 11 & 103 & 6 \\
\hline Spanien & 133 & 7 & 130 & 6 & 128 & 6 & 130 & 4 & 97 & 7 \\
\hline Schweden & 88 & 14 & 79 & 14 & 75 & 11 & 69 & 12 & 62 & 12 \\
\hline Schweiz & 28 & 22 & 28 & 22 & 29 & 22 & 29 & 22 & 27 & 22 \\
\hline Türkei & 391 & 1 & 316 & 1 & 284 & 1 & 240 & 1 & 222 & 1 \\
\hline Großbritannien & 50 & 21 & 55 & 20 & 50 & 20 & 45 & 20 & 40 & 20 \\
\hline Nordamerika $^{c}$ & 196 & & 176 & & 166 & & 145 & & 133 & \\
\hline OECD-Europa ${ }^{c}$ & 92 & & 84 & & 77 & & 72 & & 66 & \\
\hline$E U^{c, d}$ & 96 & & 88 & & 81 & & 77 & & 71 & \\
\hline $\mathrm{OECD}^{c}$ & 123 & & 109 & & 100 & & 91 & & 84 & \\
\hline
\end{tabular}

Anm.:

a. OECD $1980=100$. Zur Berechnung des Index siehe Kapitel II.2.2

b. Alte Bundeslander

c. Mittelwert

d. 11 Stasten (ohne Osterreich, Finnland, Schweden und Luxemburg) 
Tabelle A38: Schadstofinndex (Basis: BIP)

\begin{tabular}{|c|c|c|c|c|c|c|c|c|c|c|}
\hline & \multicolumn{2}{|c|}{1970} & \multicolumn{2}{|c|}{1975} & \multicolumn{2}{|c|}{1980} & \multicolumn{2}{|c|}{1985} & \multicolumn{2}{|c|}{1990} \\
\hline & Wert & Rang & Wert & Rang & Wert & Rang & Wert & Rang & Wert & Rang \\
\hline Kanada & 117 & 8 & 114 & 8 & 107 & 8 & 98 & 8 & 88 & 8 \\
\hline USA & 124 & 7 & 110 & 9 & 106 & 9 & 91 & 9 & 88 & 8 \\
\hline Japan & 71 & 19 & 76 & 18 & 62 & 19 & 51 & 21 & 47 & 21 \\
\hline Australien & 129 & 6 & 123 & 6 & 125 & 4 & 108 & 5 & 100 & 5 \\
\hline Neuseeland & 149 & 4 & 121 & 7 & 135 & 3 & 116 & 4 & 107 & 4 \\
\hline Osterreich & 90 & 16 & 60 & 21 & 61 & 20 & 55 & 19 & 51 & 17 \\
\hline Belgien & 133 & 5 & 93 & 12 & 83 & 12 & 72 & 13 & 65 & 14 \\
\hline Dănemark & 162 & 2 & 127 & 4 & 116 & 6 & 107 & 6 & 95 & 6 \\
\hline Finnland & 155 & 3 & 137 & 3 & 125 & 4 & 105 & 7 & 92 & 7 \\
\hline Frankreich & 117 & 8 & 87 & 13 & 84 & 11 & 74 & 11 & 66 & 13 \\
\hline Deutschland $^{b}$ & 97 & 15 & 79 & 15 & 73 & 15 & 63 & 17 & 51 & 17 \\
\hline Griechenland & 107 & 10 & 124 & 5 & 114 & 7 & 132 & 3 & 133 & 3 \\
\hline Irland & 290 & 1 & 181 & 2 & 190 & 2 & 187 & 2 & 165 & 2 \\
\hline Italien & 60 & 20 & 61 & 20 & 55 & 21 & 52 & 20 & 50 & 19 \\
\hline Niederlande & 106 & 12 & 100 & 10 & 91 & 10 & 81 & 10 & 74 & 10 \\
\hline Norwegen & 102 & 13 & 95 & 11 & 81 & 13 & 70 & 15 & 64 & 15 \\
\hline Portugal & 53 & 21 & 85 & 14 & 71 & 17 & 72 & 13 & 72 & 11 \\
\hline Spanien & 72 & 18 & 71 & 19 & 79 & 14 & 74 & 11 & 70 & 12 \\
\hline Schweden & 107 & 10 & 78 & 17 & 68 & 18 & 58 & 18 & 49 & 20 \\
\hline Schweiz & 36 & 22 & 39 & 22 & 42 & 22 & 40 & 22 & 39 & 22 \\
\hline Türkei & 88 & 17 & 258 & 1 & 256 & 1 & 210 & 1 & 179 & 1 \\
\hline Großbritannien & 100 & 14 & 79 & 15 & 73 & 15 & 69 & 16 & 63 & 16 \\
\hline Nordamerika $^{c}$ & 121 & & 112 & & 107 & & 95 & & 88 & \\
\hline OECD-Europa ${ }^{c}$ & 112 & & 94 & & 88 & & 82 & & 75 & \\
\hline $\mathrm{EU}^{\mathrm{c}, \mathrm{d}}$ & 118 & & 99 & & 94 & & 89 & & 82 & \\
\hline $\mathrm{OECD}^{c}$ & 112 & & 104 & & 100 & & 90 & & 82 & \\
\hline
\end{tabular}

Anm.:

a. OECD $1980=100$. Zur Berechnung des Index siche Kapitel II.2.2

b. Alte Bundeslander

c. Mittelwert

d. II Staaten (ohne Osterreich, Finnland, Schweden und Luxemburg) 
Tabelle A39: Umweltbelastungsindex (Basis: BIP)"

\begin{tabular}{|c|c|c|c|c|c|c|c|c|c|c|}
\hline & \multicolumn{2}{|c|}{1970} & \multicolumn{2}{|c|}{1975} & \multicolumn{2}{|c|}{1980} & \multicolumn{2}{|c|}{1985} & \multicolumn{2}{|c|}{1990} \\
\hline & Wert & Rang & Wert & Rang & Wert & Rang & Wert & Rang & Wert & $\overline{\text { Rang }}$ \\
\hline Kanada & 170 & 4 & 153 & 3 & 146 & 3 & 133 & 3 & 121 & 4 \\
\hline USA & 146 & 5 & 134 & 5 & 126 & 5 & 107 & 6 & 99 & 6 \\
\hline Japan & 79 & 17 & 75 & 17 & 63 & 19 & 52 & 20 & 47 & 20 \\
\hline Australien & 220 & 2 & 195 & 2 & 184 & 2 & 163 & 2 & 149 & 2 \\
\hline Neuseeland & 102 & 12 & 84 & 13 & 93 & 9 & 87 & 9 & 85 & 9 \\
\hline Osterreich & 77 & 18 & 58 & 21 & 55 & 21 & 49 & 21 & 45 & 21 \\
\hline Belgien & 119 & 9 & 92 & 11 & 81 & 12 & 73 & 12 & 66 & 13 \\
\hline Dănemark & 122 & 8 & 101 & 8 & 92 & 10 & 83 & 10 & 74 & 11 \\
\hline Finnland & 145 & 6 & 126 & 7 & 117 & 7 & 102 & 7 & 86 & 8 \\
\hline Frankreich & 95 & 15 & 78 & 16 & 76 & 14 & 70 & 14 & 63 & 14 \\
\hline Deutschland $^{b}$ & 82 & 16 & 71 & 19 & 68 & 17 & 61 & 17 & 53 & 18 \\
\hline Griechenland & 127 & 7 & 132 & 6 & 121 & 6 & 129 & 5 & 127 & 3 \\
\hline Irland & 209 & 3 & 140 & 4 & 137 & 4 & 130 & 4 & 113 & 5 \\
\hline Italien & 75 & 19 & 72 & 18 & 65 & 18 & 59 & 18 & 56 & 16 \\
\hline Niederlande & 99 & 13 & 93 & 10 & 87 & 11 & 78 & 11 & 71 & 12 \\
\hline Norwegen & 103 & 10 & 90 & 12 & 78 & 13 & 67 & 15 & 62 & 15 \\
\hline Portugal & 74 & 21 & 83 & 14 & 70 & 16 & 71 & 13 & 87 & 7 \\
\hline Spanien & 103 & 10 & 100 & 9 & 103 & 8 & 102 & 7 & 84 & 10 \\
\hline Schweden & 98 & 14 & 79 & 15 & 72 & 15 & 63 & 16 & 56 & 16 \\
\hline Schweiz & 32 & 22 & 34 & 22 & 35 & 22 & 35 & 22 & 33 & 22 \\
\hline Türkei & 240 & 1 & 287 & 1 & 270 & 1 & 225 & 1 & 200 & 1 \\
\hline GroBbritannien & 75 & 19 & 67 & 20 & 61 & 20 & 57 & 19 & 51 & 19 \\
\hline Nordamerika $^{\mathrm{C}}$ & 158 & & 144 & & 136 & & 120 & & 110 & \\
\hline OECD-Europa $^{c}$ & 102 & & 89 & & 82 & & 77 & & 70 & \\
\hline $\mathrm{EU}^{\mathrm{ced}}$ & 107 & & 94 & & 87 & & 83 & & 77 & \\
\hline $\mathrm{OECD}^{\mathrm{c}}$ & 118 & & 107 & & 100 & & 91 & & 83 & \\
\hline
\end{tabular}

Anm.:

a. OECD $1980=100$. Zur Berechnung des Index siehe Kapitel II.2.2

b. Alte Bundeslander

c. Mittelwert

d. 11 Staaten (ohne Osterreich, Finnland, Schweden und Luxemburg) 
Tabelle A40: Verănderung des Wasserverbrauches pro Einheit BIP (in \%)"

\begin{tabular}{|c|c|c|c|c|}
\hline & $1970-1975$ & $1975-1980$ & $1980-1985$ & $1985-1990$ \\
\hline Kanada & $-8,8$ & 11,0 & $-3,0$ & $-3,7$ \\
\hline USA & $-4,3$ & $-5,0$ & $-21,1$ & $-11,7$ \\
\hline Japan & $-19,4$ & $-20,0$ & $-15,9$ & $-19,9$ \\
\hline Australien & $-17,3$ & $-13,2$ & $-14,6$ & $-14,0$ \\
\hline Neuseeland & $-14,2$ & 14,1 & 36,0 & $-1,6$ \\
\hline Österreich & $-17,5$ & $-29,1$ & $-9,3$ & -14 \\
\hline Belgien & $-18,1$ & $-15,5$ & $-3,8$ & $-14,3$ \\
\hline Dănemark & 52,0 & $-11,8$ & $-12,2$ & $-7,7$ \\
\hline Finnland & $-12,0$ & $-10,1$ & $-6,2$ & $-36,7$ \\
\hline Frankreich & $-2,5$ & 11,3 & $-7,8$ & $-6,5$ \\
\hline Deutschland $^{b}$ & 2,3 & 7,0 & $-7,4$ & $-1,4$ \\
\hline Griechenland & 7,4 & $-4,2$ & $-6,4$ & $-8,0$ \\
\hline Irland & $-21,1$ & $-20,1$ & $-11,7$ & $-21,0$ \\
\hline Italien & 0,9 & $-8,2$ & $-13,7$ & $-7,0$ \\
\hline Niederlande & $-11,6$ & $-5,3$ & $-6,9$ & $-12,9$ \\
\hline Norwegen & $-20,2$ & $-26,4$ & $-22,5$ & $-7,6$ \\
\hline Portugal & $-19,4$ & $-22,0$ & 13,5 & 203,9 \\
\hline Spanien & 14,0 & 1,4 & 8,2 & $-35,9$ \\
\hline Schweden & $-10,5$ & $-7,2$ & $-35,4$ & $-8,8$ \\
\hline Schweiz & $-4,7$ & $-10,2$ & $-3,3$ & $-11,1$ \\
\hline Türkei & $-4,9$ & $-12,0$ & $-5,2$ & 18,2 \\
\hline Großbritannien & $-9,4$ & $-8,8$ & $-15,8$ & $-5,9$ \\
\hline Nordamerika $^{c}$ & $-6,6$ & 3,0 & $-12,1$ & $-7,7$ \\
\hline OECD-Europa ${ }^{c, e}$ & $-4,4$ & $-10,0$ & $-8,8$ & $-13,3$ \\
\hline EU, c,e,e & $-0,5$ & $-6,9$ & $-5,8$ & $-12,1$ \\
\hline $\mathrm{OECD}^{\mathrm{c}, \mathrm{e}}$ & $-6,3$ & $-8,4$ & $-7,5$ & $-11,0$ \\
\hline
\end{tabular}

Anm.:

a. Eigene Berechnungen auf Grundlage von Tabelle A31

b. Alte Bundeslander

c. Mittelwert

d. 11 Staaten (ohne Österreich, Finnland, Schweden und Luxemburg)

e. Wert $85-90$ ohne Portugal 
Tabelle A41: Veræ̊nderung der Landwirtschaftsnäche pro Einheit BIP (in \%)

\begin{tabular}{|c|c|c|c|c|}
\hline & $1970-1975$ & $1975-1980$ & $1980-1985$ & $1985-1990$ \\
\hline Kanada & $-20,7$ & $-14,6$ & $-11,2$ & $-13,4$ \\
\hline USA & $-11,9$ & $-13,4$ & $-11,6$ & $-11,8$ \\
\hline Japan & $-25,6$ & $-23,3$ & $-18,9$ & $-22,7$ \\
\hline Australien & $-16,1$ & $-9,5$ & $-8,6$ & $-11,1$ \\
\hline Neuseeland & $-43,0$ & 10,8 & $-3,1$ & $-20,6$ \\
\hline Osterreich & $-21,1$ & $-13,8$ & $-12,6$ & $-15,1$ \\
\hline Belgien & $-17,1$ & $-20,4$ & $-5,8$ & $-13,7$ \\
\hline Dănemark & $-9,8$ & $-12,0$ & $-13,4$ & $-9,0$ \\
\hline Finnland & $-19,0$ & $-16,3$ & $-18,4$ & $-10,9$ \\
\hline Frankreich & $-13,0$ & $-14,7$ & $-5,5$ & $-13,7$ \\
\hline Deutschland $^{b}$ & $-10,2$ & $-15,7$ & $-5,8$ & $-14,0$ \\
\hline Griechenland & $-22,7$ & $-18,1$ & $-6,1$ & $-8,1$ \\
\hline Irland & $-29,4$ & $-28,2$ & $-17,9$ & $-27,8$ \\
\hline Italien & $-28,1$ & $-20,0$ & $-9,1$ & $-14,9$ \\
\hline Niederlande & $-17,1$ & $-10,1$ & $-1,4$ & $-7,8$ \\
\hline Norwegen & $-22,4$ & $-18,4$ & $-11,0$ & $-6,9$ \\
\hline Portugal & $-18,7$ & $-21,4$ & $-3,9$ & $-16,0$ \\
\hline Spanien & $-23,6$ & $-9,8$ & $-7,0$ & $-19,2$ \\
\hline Schweden & $-13,2$ & $-7,4$ & $-10,3$ & $-12,2$ \\
\hline Schweiz & $-1,3$ & $-4,4$ & $-6,6$ & $-12,8$ \\
\hline Türkei & $-29,5$ & $-11,3$ & $-22,7$ & $-24,2$ \\
\hline Großbritannien & $-12,6$ & $-8,2$ & $-8,7$ & $-19,4$ \\
\hline Nordamerika $^{c}$ & $-16,3$ & $-14,0$ & $-11,4$ & $-12,6$ \\
\hline OECD-Europa ${ }^{c}$ & $-17,5$ & $-14,9$ & $-9,0$ & $-13,8$ \\
\hline$E U^{c, d}$ & $-18,4$ & $-16,2$ & $-7,7$ & $-14,9$ \\
\hline $\mathrm{OECD}^{\mathrm{c}}$ & $-19,4$ & $-13,6$ & $-10,0$ & $-14,8$ \\
\hline
\end{tabular}

Anm:

a. Eigene Berechnungen auf Grundlage von Tabelle A32

b. Alte Bundesiander

c. Mittelwert

d. 11 Staten (ohne Osterreich, Finnland, Schweden und Luxemburg) 
Tabelle A42: Verănderung des Primărenergieverbrauches pro Einheit BIP (in \%)

\begin{tabular}{|c|c|c|c|c|}
\hline & $1970-1975$ & $1975-1980$ & $1980-1985$ & $1985-1990$ \\
\hline Kanada & $-4,9$ & $-1,7$ & $-12,8$ & $-5,1$ \\
\hline USA & $-5,0$ & $-6,5$ & $-12,7$ & $-4,4$ \\
\hline Japan & $-3,4$ & $-10,2$ & $-13,4$ & $-4,7$ \\
\hline Australien & $-1,6$ & 0,1 & $-10,3$ & 2,5 \\
\hline Neuseeland & $-1,3$ & 4,0 & 8,1 & 23,0 \\
\hline Osterreich & $-8,1$ & $-2,4$ & $-7,5$ & $-5,8$ \\
\hline Belgien & $-10,9$ & $-6,9$ & $-8,2$ & $-5,9$ \\
\hline Dănemark & $-19,1$ & $-4,4$ & $-11,7$ & $-13,5$ \\
\hline Finnland & $-9,6$ & 7,9 & $-9,1$ & $-8,2$ \\
\hline Frankreich & $-6,5$ & 0,6 & $-2,3$ & $-4,8$ \\
\hline Deutschland $^{b}$ & $-8,4$ & $-2,8$ & $-6,2$ & $-11,9$ \\
\hline Griechenland & 17,8 & 5,8 & 8,8 & 9,3 \\
\hline Irland & $-13,6$ & $-1,6$ & $-7,5$ & $-5,9$ \\
\hline Italien & $-2,1$ & $-11,4$ & $-8,4$ & $-2,5$ \\
\hline Niederlande & 2,6 & $-3,9$ & $-10,6$ & $-6,1$ \\
\hline Norwegen & $-12,8$ & $-1,7$ & $-8,6$ & $-2,6$ \\
\hline Portugal & 7,5 & 0,4 & 5,8 & 20,2 \\
\hline Spanien & 18,4 & 7,6 & $-2,3$ & $-1,7$ \\
\hline Schweden & $-8,4$ & $-3,0$ & 6,2 & $-9,4$ \\
\hline Schweiz & 4,9 & 7,3 & 2,6 & $-5,7$ \\
\hline Türkei & 53,1 & 3,4 & $-2,9$ & 2,0 \\
\hline Großbritannien & $-12,0$ & $-9,1$ & $-8,7$ & $-10,8$ \\
\hline Nordamerika $^{c}$ & $-5,0$ & $-4,1$ & $-12,8$ & $-4,8$ \\
\hline OECD-Europa ${ }^{c}$ & $-3,8$ & $-1,1$ & $-4,2$ & $-4,1$ \\
\hline$E U^{c, d}$ & $-2,4$ & $-2,3$ & $-4,7$ & $-3,1$ \\
\hline $\mathrm{OECD}^{c}$ & $-1,1$ & $-1,3$ & $-5,1$ & $-2,4$ \\
\hline
\end{tabular}

Anm.:

a. Eigene Berechnungen auf Grundlage von Tabelle A33

b. Alte Bundesländer

c. Mittelwert

d. 11 Staaten (ohne Osterreich, Finnland, Schweden und Luxemburg) 
Tabelle A43: Verănderung der energiebedingten $\mathrm{CO}_{2}$-Emissio pro Einheit BIP(in \%)"

\begin{tabular}{|c|c|c|c|c|}
\hline & $1970-1975$ & $1975-1980$ & $1980-1985$ & $1985-1990$ \\
\hline Kanada & $-8,3$ & $-10,0$ & $-19,8$ & $-6,6$ \\
\hline USA & $-7,1$ & $-5,6$ & $-14,6$ & $-6,1$ \\
\hline Japan & $-5,9$ & $-17,9$ & $-19,0$ & $-7,0$ \\
\hline Australien & $-3,0$ & 0,4 & $-11,5$ & 2,1 \\
\hline Neuseeland & $-3,4$ & $-0,7$ & 4,1 & 11,2 \\
\hline Osterreich & $-10,8$ & $-5,7$ & $-12,7$ & $-7,7$ \\
\hline Belgien & $-18,1$ & $-10,0$ & $-21,4$ & $-3,6$ \\
\hline Dänemark & $-20,5$ & 0,8 & $-12,1$ & $-18,9$ \\
\hline Finnland & $-6,2$ & 10,2 & $-23,4$ & $-12,4$ \\
\hline Frankreich & $-11,5$ & $-7,5$ & $-26,5$ & $-15,7$ \\
\hline Deutschland ${ }^{b}$ & $-14,1$ & $-6,0$ & $-13,6$ & $-15,5$ \\
\hline Griechenland & 22,0 & 5,5 & 13,7 & 20,3 \\
\hline Irland & $-17,4$ & $-1,9$ & $-8,4$ & $-4,1$ \\
\hline Italien & $-2,9$ & $-11,4$ & $-9,9$ & $-4,3$ \\
\hline Niederlande & $-7,1$ & $-7,6$ & $-13,7$ & $-4,5$ \\
\hline Norwegen & $-20,2$ & $-9,7$ & $-20,5$ & $-1,6$ \\
\hline Portugal & 10,9 & $-4,3$ & $-0,8$ & 28,1 \\
\hline Spanien & 11,1 & 9,4 & $-8,9$ & $-8,0$ \\
\hline Schweden & $-23,5$ & $-17,6$ & $-20,7$ & $-22,4$ \\
\hline Schweiz & $-4,0$ & $-0,9$ & $-9,0$ & $-6,6$ \\
\hline Turkei & 12,6 & 3,8 & 6,6 & 3,1 \\
\hline GroBbritannien & $-16,0$ & $-10,7$ & $-13,5$ & $-11,0$ \\
\hline Nordamerika $^{c}$ & $-7,7$ & $-7,8$ & $-17,2$ & $-6,4$ \\
\hline OECD-Europa ${ }^{c}$ & $-8,0$ & $-4,2$ & $-12,6$ & $-5,5$ \\
\hline EU & $-5,8$ & $-4,0$ & $-10,5$ & $-3,4$ \\
\hline $\mathrm{OECD}^{c}$ & $-6,5$ & $-4,4$ & $-11,6$ & $-4,1$ \\
\hline
\end{tabular}

Anm.:

a. Eigene Berechnungen auf Grundlage von Tabelle A34

b. Alte Bundesiander

c. Mittelwert

d. 11 Stanten (ohne Osterreich, Finnland, Schweden und Luxemburg) 
Tabelle A44: Veræ̌nderung des Abfallaufkommen pro Einheit BIP (in \%)

\begin{tabular}{|c|c|c|c|}
\hline & $1975-1980$ & $1980-1985$ & $1985-1990$ \\
\hline Kanada & $-17,5$ & $-1,6$ & $-2,6$ \\
\hline USA & 1,0 & $-3,8$ & 5,0 \\
\hline Japan & $-16,7$ & $-21,4$ & $-2,8$ \\
\hline Australien & $-13,2$ & $-13,6$ & $-14,0$ \\
\hline Neuseeland & 82,0 & $-14,1$ & $-1,6$ \\
\hline Osterreich & 0,8 & $-3,3$ & 24,9 \\
\hline Belgien & $-8,5$ & 1,2 & $-9,8$ \\
\hline Dănemark & $-11,8$ & 4,3 & $-7,3$ \\
\hline Finnland & $-13,7$ & $-13,3$ & 4,7 \\
\hline Frankreich & $-7,0$ & $-3,3$ & $-1,4$ \\
\hline Deutschland ${ }^{b}$ & $-10,7$ & $-14,2$ & $-6,6$ \\
\hline Griechenland & $-19,3$ & 13,2 & $-8,7$ \\
\hline Irland & $-7,9$ & 51,8 & $-21,0$ \\
\hline Italien & $-21,1$ & $-0,3$ & 14,9 \\
\hline Niederlande & $-12,1$ & $-14,9$ & 2,6 \\
\hline Norwegen & $-20,9$ & $-1,8$ & $-6,2$ \\
\hline Portugal & $-22,0$ & 11,9 & $-13,4$ \\
\hline Spanien & 15,3 & $-7,4$ & 0,6 \\
\hline Schweden & $-2,2$ & $-3,5$ & 8,9 \\
\hline Schweiz & 8,4 & 4,1 & 4,6 \\
\hline Türkei & $-12,9$ & $-20,8$ & $-25,1$ \\
\hline Großbritannien & $-11,6$ & $-0,8$ & 0,7 \\
\hline Nordamerika $^{c}$ & $-8,3$ & $-2,7$ & $\overline{1,2}$ \\
\hline OECD-Europa ${ }^{c}$ & $-9,0$ & 1,5 & $-0,8$ \\
\hline $\mathrm{EU}^{\mathrm{c}, \mathrm{d}}$ & $-10,6$ & 3,8 & $-4,5$ \\
\hline $\mathrm{OECD}^{c}$ & $-5,5$ & $-2,3$ & $-2,4$ \\
\hline
\end{tabular}

Anm:

a. Eigene Berechnungen auf Grundlage von Tabelle A35

b. Alte Bundeslander

c. Mittelwert

d. 11 Staaten (ohne Osterreich, Finnland, Schweden und Luxemburg) 
Tabelle A45: Verănderung des Handelsdüngerabsatzes pro Einheit BIP (in \%)"

\begin{tabular}{|c|c|c|c|c|}
\hline & $1970-1975$ & $1975-1980$ & $1980-1985$ & $1985-1990$ \\
\hline Kanada & 27,2 & 22,3 & 4,0 & $-22,3$ \\
\hline USA & 8,6 & $-3,0$ & $-26,4$ & $-8,9$ \\
\hline Japan & $-25,7$ & $-19,4$ & $-3,5$ & $-30,2$ \\
\hline Australien & $-38,5$ & 39,6 & $-15,1$ & $-13,3$ \\
\hline Neuseeland & $-6,4$ & $-10,6$ & $-20,9$ & $-21,0$ \\
\hline Österreich & $-36,7$ & 10,2 & $-10,7$ & $-32,6$ \\
\hline Belgien & $-26,4$ & $-14,7$ & $-9,4$ & $-21,8$ \\
\hline Dănemark & $-3,0$ & $-13,4$ & $-11,2$ & $-7,5$ \\
\hline Finnland & $-13,0$ & $-18,4$ & $-10,1$ & $-26,2$ \\
\hline Frankreich & $-14,5$ & 2,4 & $-5,8$ & $-13,7$ \\
\hline Deutschland $^{b}$ & $-11,9$ & $-9,0$ & $-15,4$ & $-43,6$ \\
\hline Griechenland & 7,3 & $-8,1$ & 21,6 & $-6,5$ \\
\hline Irland & $-19,4$ & 11,1 & $-8,9$ & $-11,8$ \\
\hline Italien & $-3,0$ & 12,3 & $-7,1$ & $-25,0$ \\
\hline Niederlande & $-16,6$ & $-6,0$ & $-1,8$ & $-30,3$ \\
\hline Norwegen & $-7,8$ & $-11,0$ & $-22,1$ & $-18,5$ \\
\hline Portugal & 53,2 & $-17,5$ & $-11,0$ & $-3,7$ \\
\hline Spanien & $-10,4$ & 8,6 & $-2,6$ & $-8,4$ \\
\hline Schweden & $-8,0$ & $-13,8$ & $-20,8$ & $-29,4$ \\
\hline Schweiz & $-7,1$ & 16,3 & $-7,3$ & $-18,6$ \\
\hline Türkei & 44,0 & 42,4 & $-22,4$ & $-0,8$ \\
\hline Großbritannien & $-13,0$ & 3,0 & 11,2 & $-19,7$ \\
\hline Nordamerika $^{c}$ & 17,9 & 9,7 & $-11,2$ & $-15,6$ \\
\hline OECD-Europa $^{c}$ & $-8,1$ & $-3,0$ & $-7,0$ & $-19,8$ \\
\hline$E U^{c, d}$ & $-5,2$ & $-2,8$ & $-3,7$ & $-17,5$ \\
\hline $\mathrm{OECD}^{\mathrm{c}}$ & $-5,5$ & 1,1 & $-8,9$ & $-18,8$ \\
\hline
\end{tabular}

Anm:

a. Eigene Berechnungen auf Grundlage von Tabelle A36

b. Alte Bundeslander

c. Mittelwert

d. 11 Staaten (ohne Osterreich, Finnland, Schweden und Luxemburg) 
Tabelle A46: Verănderung des Ressourcenverbrauchsindex (Basis: BIP; in \%)

\begin{tabular}{|c|c|c|c|c|}
\hline & $1970 \cdot 1975$ & $1975-1980$ & $1980-1985$ & $1985-1990$ \\
\hline Kanada & $-13,5$ & $-4,3$ & $-9,3$ & $-7,9$ \\
\hline USA & $-6,4$ & $-7,4$ & $-16,4$ & $-9,3$ \\
\hline Japan & $-13,9$ & $-15,9$ & $-14,9$ & $-13,3$ \\
\hline Australien & $-14,5$ & $-8,8$ & $-10,1$ & $-9,4$ \\
\hline Neuseeland & $-14,9$ & 8,2 & 15,6 & 7,7 \\
\hline Osterreich & $-14,2$ & $-12,9$ & $-9,1$ & $-9,8$ \\
\hline Belgien & $-14,9$ & $-11,8$ & $-6,0$ & $-10,3$ \\
\hline Dănemark & $-9,2$ & $-8,5$ & $-12,5$ & $-10,9$ \\
\hline Finnland & $-13,3$ & $-5,3$ & $-10,7$ & $-17,9$ \\
\hline Frankreich & $-7,3$ & $-0,3$ & $-5,0$ & $-7,5$ \\
\hline Deutschland $^{b}$ & $-5,0$ & $-0,6$ & $-6,6$ & $-8,0$ \\
\hline Griechenland & $-4,7$ & $-7,4$ & $-3,0$ & $-3,8$ \\
\hline Irland & $-22,3$ & $-16,4$ & $-12,0$ & $-16,2$ \\
\hline Italien & $-6,5$ & $-11,2$ & $-11,5$ & $-6,9$ \\
\hline Niederlande & $-5,9$ & $-4,8$ & $-8,4$ & $-9,6$ \\
\hline Norwegen & $-17,2$ & $-13,2$ & $-13,2$ & $-4,6$ \\
\hline Portugal & $-14,1$ & $-16,6$ & 2,5 & 46,9 \\
\hline Spanien & $-2,4$ & $-1,5$ & 1,4 & $-25,0$ \\
\hline Schweden & $-10,1$ & $-5,1$ & $-8,0$ & $-9,9$ \\
\hline Schweiz & 2,0 & 2,4 & 0,6 & $-7,3$ \\
\hline Türkei & $-19,3$ & $-10,0$ & $-15,6$ & $-7,6$ \\
\hline Großbritannien & $-11,5$ & $-8,9$ & $-10,2$ & $-11,0$ \\
\hline Nordamerika $^{c}$ & $-10,0$ & $-5,9$ & $-12,9$ & $-8,6$ \\
\hline OECD-Europa ${ }^{c}$ & $-9,8$ & $-7,6$ & $-7,0$ & $-7,0$ \\
\hline$E U^{c, d}$ & $-9,4$ & $-8,0$ & $-6,5$ & $-5,7$ \\
\hline $\mathrm{OECD}^{\mathrm{c}}$ & $-10,9$ & $-7,3$ & $-7,4$ & $-6,9$ \\
\hline
\end{tabular}

Anm.:

a. Eigene Berechnungen auf Grundlage von Tabelle A37

b. Alte Bundesiânder

c. Mittelwert

d. 11 Staaten (ohne Osterreich, Finnland, Schweden und Luxemburg) 
Tabelle A47: Verănderung des Schadstofinndex

(Basis: BIP; in \%)"

\begin{tabular}{|c|c|c|c|c|}
\hline & $1970-1975$ & $1975-1980$ & $1980-1985$ & $1985-1990$ \\
\hline Kanada & $-2,5$ & $-6,1$ & $-8,6$ & $-10,0$ \\
\hline USA & $-11,5$ & $-3,2$ & $-14,6$ & $-3,3$ \\
\hline Japan & 5,9 & $-17,6$ & $-18,4$ & $-8,2$ \\
\hline Australien & $-4,7$ & 1,8 & $-13,6$ & $-7,5$ \\
\hline Neuseeland & $-19,0$ & 11,9 & $-14,5$ & $-7,7$ \\
\hline Österreich & $-33,1$ & 1,0 & $-9,7$ & $-7,9$ \\
\hline Belgien & $-30,0$ & $-10,7$ & $-13,1$ & $-9,5$ \\
\hline Dănemark & $-22,0$ & $-8,5$ & $-7,9$ & $-11,2$ \\
\hline Finnland & $-12,1$ & $-8,6$ & $-15,7$ & $-12,2$ \\
\hline Frankreich & $-25,5$ & $-3,1$ & $-12,1$ & $-11,4$ \\
\hline Deutschland ${ }^{b}$ & $-18,5$ & $-8,0$ & $-14,1$ & $-18,9$ \\
\hline Griechenland & 16,0 & $-8,5$ & 16,6 & 0,8 \\
\hline Irland & $-37,6$ & 5,3 & $-1,7$ & $-11,8$ \\
\hline Italien & 0,9 & $-9,6$ & $-6,1$ & $-3,7$ \\
\hline Niederlande & $-5,6$ & $-8,9$ & $-11,7$ & $-7,9$ \\
\hline Norwegen & $-7,1$ & $-14,4$ & $-14,4$ & $-8,4$ \\
\hline Portugal & 61,4 & $-16,5$ & 1,4 & $-0,1$ \\
\hline Spanien & $-1,8$ & 11,1 & $-6,3$ & $-5,3$ \\
\hline Schweden & $-26,7$ & $-12,5$ & $-15,9$ & $-14,5$ \\
\hline Schweiz & 7,7 & 6,1 & $-3,2$ & $-3,8$ \\
\hline Türkei & 191,9 & $-0,5$ & $-18,2$ & $-14,6$ \\
\hline Großbritannien & $-21,0$ & $-8,3$ & $-4,7$ & $-9,8$ \\
\hline Nordamerika ${ }^{c}$ & $-7,0$ & $-4,7$ & $-11,6$ & $-6,7$ \\
\hline OECD-Europa ${ }^{c}$ & $-9,7$ & $-5,9$ & $-7,4$ & $-8,5$ \\
\hline$E U^{c, d}$ & $-7,6$ & $-6,0$ & $-5,4$ & $-8,1$ \\
\hline $\mathrm{OECD}^{\mathrm{c}, 0}$ & $-8,9$ & $-4,9$ & $-9,4$ & $-8,5$ \\
\hline
\end{tabular}

Anm:

a. Eigene Berechnungen auf Grundlage von Tabelle A38

b. Alte Bundesländer

c. Mittelwert

d. 11 Stanten (ohne Osterreich, Finnland, Schweden und Luxemburg)

e. Wert 70-75 ohne die Turkei 
Tabelle A48: Verlnderung des Umweltbelastungsindex (Basis: BIP; in \%)"

\begin{tabular}{|c|c|c|c|c|}
\hline & $1970-1975$ & $1975-1980$ & $1980-1985$ & $1985-1990$ \\
\hline Kanada & $-9,7$ & $-5,0$ & $-9,0$ & $-8,6$ \\
\hline USA & $-8,5$ & $-5,7$ & $-15,6$ & $-6,7$ \\
\hline Japan & $-5,0$ & $-16,7$ & $-16,6$ & $-10,8$ \\
\hline Australien & $-11,6$ & $-5,5$ & $-11,3$ & $-8,8$ \\
\hline Neuseeland & $-17,8$ & 10,9 & $-6,2$ & $-2,5$ \\
\hline Österreich & $-25,2$ & $-5,7$ & $-9,4$ & $-8,7$ \\
\hline Belgien & $-23,3$ & $-11,2$ & $-9,6$ & $-9,9$ \\
\hline Dänemark & $-17,7$ & $-8,6$ & $-9,6$ & $-11,1$ \\
\hline Finnland & $-12,7$ & $-7,1$ & $-13,4$ & $-15,0$ \\
\hline Frankreich & $-18,4$ & $-1,9$ & $-8,9$ & $-9,6$ \\
\hline Deutschland $^{b}$ & $-13,0$ & $-4,7$ & $-10,6$ & $-13,6$ \\
\hline Griechenland & 4,0 & $-7,9$ & 6,2 & $-1,5$ \\
\hline Irland & $-32,9$ & $-2,4$ & $-4,8$ & $-13,0$ \\
\hline Italien & $-3,5$ & $-10,5$ & $-9,2$ & $-5,5$ \\
\hline Niederlande & $-5,7$ & $-7,0$ & $-10,2$ & $-8,7$ \\
\hline Norwegen & $-12,1$ & $-13,9$ & $-13,8$ & $-6,6$ \\
\hline Portugal & 13,0 & $-16,5$ & 2,0 & 23,0 \\
\hline Spanien & $-2,2$ & 2,9 & $-1,5$ & $-17,9$ \\
\hline Schweden & $-19,2$ & $-8,8$ & $-11,8$ & $-12,0$ \\
\hline Schweiz & 5,3 & 4,6 & $-1,7$ & $-5,3$ \\
\hline Türkei & 19,6 & $-5,8$ & $-16,8$ & $-10,9$ \\
\hline Großbritannien & $-17,4$ & $-8,5$ & $-6,9$ & $-10,3$ \\
\hline Nordamerika $^{c}$ & $-9,1$ & $-5,4$ & $-12,3$ & $-7,7$ \\
\hline OECD-Europa $^{c}$ & $-11,3$ & $-6,7$ & $-7,1$ & $-7,9$ \\
\hline $\mathrm{EU}^{\mathrm{c}, \mathrm{d}}$ & $-10,6$ & $-6,9$ & $-5,7$ & $-7,1$ \\
\hline $\mathrm{OECD}^{c}$ & $-9,7$ & $-6,1$ & $-8,6$ & $-7,9$ \\
\hline
\end{tabular}

Anm:

a. Eigene Berechnungen auf Grundlage von Tabelle A39

b. Alte Bundesländer

c. Mittelwert

d. 11 Staaten (ohne Österreich, Finnland, Schweden und Luxemburg) 
Tabelle A49: Einwohnerzahl (in Tausend)

\begin{tabular}{lrrrrr}
\hline & 1970 & 1975 & 1980 & 1985 & 1990 \\
\hline Kanada & 21324 & 22727 & 24070 & 25181 & 26620 \\
USA & 205052 & 215937 & 227757 & 239279 & 251523 \\
Japan & 103720 & 111520 & 116800 & 120750 & 123540 \\
Australien & 12817 & 13893 & 14695 & 15788 & 17085 \\
Neuseeland & 2820 & 3087 & 3144 & 3272 & 3379 \\
Osterreich & 7467 & 7579 & 7549 & 7558 & 7718 \\
Belgien & 9651 & 9795 & 9847 & 9858 & 9967 \\
Dänemark & 4929 & 5060 & 5125 & 5114 & 5141 \\
Finnland & 4606 & 4712 & 4780 & 4902 & 4986 \\
Frankreich & 50772 & 52699 & 53880 & 55170 & 56420 \\
Deutschland & 60651 & 61829 & 61566 & 61024 & 63232 \\
Griechenland & 8793 & 9046 & 9642 & 9934 & 10140 \\
Irland & 2950 & 3177 & 3401 & 3540 & 3503 \\
Italien & 53661 & 55441 & 56416 & 57128 & 57647 \\
Niederlande & 13039 & 13666 & 14150 & 14491 & 14951 \\
Norwegen & 3879 & 4007 & 4087 & 4153 & 4241 \\
Portugal & 8663 & 9308 & 9819 & 9905 & 9859 \\
Spanien & 33876 & 35515 & 37386 & 38505 & 38959 \\
Schweden & 8043 & 8192 & 8311 & 8350 & 8559 \\
Schweiz & 6267 & 6404 & 6385 & 6533 & 6796 \\
Türkei & 35605 & 40348 & 44737 & 50664 & 56473 \\
Großbritannien & 55632 & 56215 & 56314 & 56618 & 57411 \\
\hline Nordamerika & 226376 & 238664 & 251827 & 264460 & 278143 \\
OECD-Europa & 332879 & 342645 & 348658 & 352783 & 359530 \\
EU ${ }^{b}$ & 302617 & 311751 & 317546 & 321287 & 327230 \\
\hline OECD & 714217 & 750157 & 779861 & 807717 & 838150 \\
\hline & & & & &
\end{tabular}

Quelle: OECD (div. Jahrgange): OECD Environmental Data, a.a.O.

Anm:

a. Alte Bundesilander

b. 11 Staaten (ohne Osterreich, Finnland, Schweden und Luxemburg) 
Tabelle A50: Bruttoinlandsprodukt (in Mrd. US-\$)

\begin{tabular}{lrrrrr}
\hline & 1970 & 1975 & 1980 & 1985 & 1990 \\
\hline Kanada & 206 & 263 & 319 & 368 & 423 \\
USA & 2715 & 3045 & 3563 & 4017 & 4555 \\
Japan & 779 & 966 & 1209 & 1453 & 1817 \\
Australien & 119 & 144 & 166 & 195 & 226 \\
Neuseeland & 26 & 32 & 32 & 38 & 38 \\
Osterreich & 60 & 73 & 86 & 92 & 107 \\
Belgien & 81 & 96 & 112 & 116 & 136 \\
Dänemark & 46 & 51 & 58 & 66 & 71 \\
Finnland & 36 & 43 & 50 & 58 & 69 \\
Frankreich & 476 & 561 & 656 & 707 & 818 \\
Deutschland & 599 & 667 & 783 & 826 & 966 \\
Griechenland & 34 & 44 & 54 & 58 & 63 \\
Irland & 14 & 18 & 22 & 25 & 31 \\
Italien & 426 & 489 & 618 & 662 & 769 \\
Niederlande & 124 & 145 & 165 & 173 & 199 \\
Norwegen & 28 & 35 & 44 & 52 & 57 \\
Portugal & 33 & 41 & 52 & 55 & 66 \\
Spanien & 204 & 262 & 286 & 306 & 381 \\
Schweden & 81 & 93 & 99 & 109 & 120 \\
Schweiz & 86 & 89 & 97 & 104 & 119 \\
Türkei & 57 & 82 & 94 & 119 & 158 \\
Großbritannien & 486 & 537 & 588 & 650 & 759 \\
\hline Nordamerika & 2921 & 3308 & 3882 & 4385 & 4978 \\
OECD-Europa & 2814 & 3244 & 3770 & 4059 & 4731 \\
EUc & 2523 & 2911 & 3394 & 3644 & 4259 \\
\hline OECD $^{6}$ & 6716 & 7776 & 9153 & 10249 & 11948 \\
\hline & & & & &
\end{tabular}

Quelle: OECD (div. Jahrgănge): OECD Environmental Data, a.a.O.

Anm.:

a. In Preisen von 1985 und Kaufkraftparitaten

b. Alte Bundesländer

c. 11 Staaten (ohne Österreich, Finnland, Schweden und Luxemburg) 
Tabelle A51: Wachstum des Bruttoinlandsproduktes (in Proze

\begin{tabular}{|c|c|c|c|c|}
\hline & $1970-75$ & $1975-80$ & $1980-85$ & $1985-90$ \\
\hline Kanada & 27,67 & 21,29 & 15,36 & 14,95 \\
\hline USA & 12,15 & 17,01 & 12,74 & 13,39 \\
\hline Japan & 24,01 & 25,16 & 20,18 & 25,05 \\
\hline Australien & 21,01 & 15,28 & 17,47 & 15,90 \\
\hline Neuseeland & 23,08 & 0,00 & 18,75 & 0,00 \\
\hline Osterreich & 21,67 & 17,81 & 6,98 & 16,30 \\
\hline Belgien & 18,52 & 16,67 & 3,57 & 17,24 \\
\hline Dänemark & 10,87 & 13,73 & 13,79 & 7,58 \\
\hline Finnland & 19,44 & 16,28 & 16,00 & 18,97 \\
\hline Frankreich & 17,86 & 16,93 & 7,77 & 15,70 \\
\hline Deutschland ${ }^{b}$ & 11,35 & 17,39 & 5,49 & 16,95 \\
\hline Griechenland & 29,41 & 22,73 & 7,41 & 8,62 \\
\hline Irland & 28,57 & 22,22 & 13,64 & 24,00 \\
\hline Italien & 14,79 & 26,38 & 7,12 & 16,16 \\
\hline Niederlande & 16,94 & 13,79 & 4,85 & 15,03 \\
\hline Norwegen & 25,00 & 25,71 & 18,18 & 9,62 \\
\hline Portugal & 24,24 & 26,83 & 5,77 & 20,00 \\
\hline Spanien & 28,43 & 9,16 & 6,99 & 24,51 \\
\hline Schweden & 14,81 & 6,45 & 10,10 & 10,09 \\
\hline Schweiz & 3,49 & 8,99 & 7,22 & 14,42 \\
\hline Turkei & 43,86 & 14,63 & 26,60 & 32,77 \\
\hline GroBbritannien & 10,49 & 9,50 & 10,54 & 16,77 \\
\hline Nordamerika $^{c}$ & 19,91 & 19,15 & 14,05 & 14,17 \\
\hline OECD-Europe ${ }^{c}$ & 18,49 & 16,91 & 9,09 & 15,75 \\
\hline EU & 19,22 & 17,76 & 7,90 & 16,60 \\
\hline $\mathrm{OECD}^{c}$ & 20,35 & 16,54 & 11,66 & 16,09 \\
\hline
\end{tabular}

Quelle: OECD (div. Jahroulnge): OECD Environmental Data, a.2.O.; eigene Berechnungen Anm:

a. In Preisen von 1985 und Kaufkrafparitaten

b. Alte Bundeslander

c. Mittelwert

d. 11 Staaten (ohne Osterreich, Finnland, Schweden und Luxemburg) 
Tabelle A52: Bruttoinlandsprodukt pro Einwohner (in US-S)

\begin{tabular}{|c|c|c|c|c|c|}
\hline & 1970 & 1975 & 1980 & 1985 & 1990 \\
\hline Kanada & 9637 & 11590 & 13270 & 14630 & 15887 \\
\hline USA & 13242 & 14099 & 15643 & 16786 & 18109 \\
\hline Japan & 7510 & 8666 & 10352 & 12035 & 14704 \\
\hline Australien & 9308 & 10379 & 11310 & 12326 & 13246 \\
\hline Neuseeland & 9255 & 10398 & 10274 & 11491 & 11305 \\
\hline Österreich & 8062 & 9632 & 11405 & 12159 & 13838 \\
\hline Belgien & 8424 & 9842 & 11374 & 11808 & 13625 \\
\hline Dănemark & 9393 & 10079 & 11278 & 12867 & 13811 \\
\hline Finnland & 7707 & 9211 & 10523 & 11832 & 13779 \\
\hline Frankreich & 9383 & 10649 & 12173 & 12811 & 14495 \\
\hline Deutschland $^{b}$ & 9875 & 10781 & 12718 & 13536 & 15271 \\
\hline Griechenland & 3867 & 4809 & 5590 & 5798 & 6173 \\
\hline Irland & 4678 & 5508 & 6439 & 7006 & 8964 \\
\hline Italien & 7943 & 8822 & 10945 & 11585 & 13340 \\
\hline Niederlande & 9487 & 10596 & 11640 & 11952 & 13297 \\
\hline Norwegen & 7218 & 8758 & 10864 & 12617 & 13369 \\
\hline Portugal & 3798 & 4383 & 5326 & 5523 & 6644 \\
\hline Spanien & 6010 & 7380 & 7650 & 7952 & 9785 \\
\hline Schweden & 10170 & 11328 & 11948 & 13006 & 14067 \\
\hline Schweiz & 13659 & 13898 & 15161 & 15889 & 17525 \\
\hline Türkei & 1601 & 2027 & 2099 & 2341 & 2803 \\
\hline Großbritannien & 8734 & 9546 & 10440 & 11473 & 13227 \\
\hline Nordamerika $^{c}$ & 11440 & 12845 & 14457 & 15708 & 16998 \\
\hline OECD-Europa ${ }^{c}$ & 8026 & 9076 & 10342 & 11113 & 12576 \\
\hline$E U^{c, d}$ & 7417 & 8400 & 9598 & 10210 & 11694 \\
\hline $\mathrm{OECD}^{c}$ & 8135 & 9199 & 10383 & 11247 & 12603 \\
\hline
\end{tabular}

Quelle: OECD (div. Jahrgange): OECD Environmental Data, a.a.o.

Anm.:
a. In Preisen von 1985 und Kaufkraftparitaten
b. Alte Bundeslander
c. Mittelwert
d. 11 Staaten (ohne Ôsterreich, Finnland, Schweden und Luxemburg) 
Tabelle A53: Wachstum des BIP pro Einwohner (in Prozent)"

\begin{tabular}{|c|c|c|c|c|}
\hline & $1970-75$ & $1975-80$ & $1980-85$ & $1985-90$ \\
\hline Kanada & 20,3 & 14,5 & 10,3 & 8,6 \\
\hline USA & 6,5 & 11 & 7,3 & 7,9 \\
\hline Japan & 15,4 & 19,5 & 16,3 & 22,2 \\
\hline Australien & 11,5 & 9 & 9 & 7,5 \\
\hline Neuseeland & 12,4 & $-1,2$ & 11,9 & $-1,6$ \\
\hline Österreich & 19,5 & 18,4 & 6,6 & 13,8 \\
\hline Belgien & 16,8 & 15,6 & 3,8 & 15,4 \\
\hline Dänemark & 7,3 & 11,9 & 14,1 & 7,3 \\
\hline Finnland & 19,5 & 14,3 & 12,4 & 16,5 \\
\hline Frankreich & 13,5 & 14,3 & 5,2 & 13,1 \\
\hline Deutschland $^{b}$ & 9,2 & 18 & 6,4 & 12,8 \\
\hline Griechenland & 24,4 & 16,3 & 3,7 & 6,5 \\
\hline Irland & 17,8 & 16,9 & 8,8 & 28 \\
\hline Italien & 11,1 & 24,1 & 5,8 & 15,2 \\
\hline Niederlande & 11,7 & 9,9 & 2,7 & 11,3 \\
\hline Norwegen & 21,4 & 24 & 16,1 & 6 \\
\hline Portugal & 15,4 & 21,5 & 3,7 & 20,3 \\
\hline Spanien & 22,8 & 3,7 & 4 & 23 \\
\hline Schweden & 11,4 & 5,5 & 8,9 & 8,2 \\
\hline Schweiz & 1,8 & 9,1 & 4,8 & 10,3 \\
\hline Turkei & 26,6 & 3,5 & 11,5 & 19,7 \\
\hline GroBbritannien & 9,3 & 9,4 & 9,9 & 15,3 \\
\hline Nordamerika $^{c}$ & 13,4 & 12,8 & 8,8 & 8,3 \\
\hline OECD-Europa $^{c}$ & 14,6 & 14,6 & 7,3 & 13,9 \\
\hline$E U^{c, d}$ & 14,5 & 14,7 & 6,2 & 15,3 \\
\hline $\mathrm{OECD}^{c}$ & 14,8 & 13,1 & 8,3 & 13,1 \\
\hline
\end{tabular}

Quelle: OECD (div. Jahrginge): OECD Environmental Data, a.a.O.; eigene Berechnungen Anm:
a. In Preisen von 1985 und Kaufkraftparititen
b. Alte Bundeslinder
c. Mittelwert
d. 11 Staaten (ohne Osterreich, Finnland, Schweden und Luxemburg) 
Tabelle A54: Sektorale Aufteilung der Volkswirtschaft 1990 (in \%)

\begin{tabular}{|c|c|c|c|}
\hline & Landwirtschaft & Industrie & Dienstleistung \\
\hline Kanada & 2,4 & 29,8 & 67,8 \\
\hline USA & 2,0 & 29,2 & 68,8 \\
\hline Japan & 2,5 & 41,8 & 55,7 \\
\hline Australien & 4,1 & 31,0 & 65,0 \\
\hline Neuseeland & 8,6 & 26,7 & 64,7 \\
\hline Österreich & 3,2 & 36,1 & 60,8 \\
\hline Belgien & 1,8 & 30,1 & 68,1 \\
\hline Dănemark & 3,9 & 24,4 & 71,7 \\
\hline Finnland & 5,4 & 30,0 & 64,7 \\
\hline Frankreich & 3,4 & 29,0 & 68,0 \\
\hline Deutschland $^{b}$ & 1,5 & 38,7 & 59,2 \\
\hline Griechenland & 13,8 & 24,8 & 62,1 \\
\hline Irland & 9,6 & 32,6 & 57,8 \\
\hline Italien & 3,1 & 33,0 & 63,8 \\
\hline Niederlande & 4,2 & 31,5 & 64,2 \\
\hline Norwegen & 2,9 & 35,5 & 60,8 \\
\hline Portugal & 5,8 & 37,8 & 55,7 \\
\hline Spanien & 4,5 & 34,5 & 61,0 \\
\hline Schweden & 2,6 & 29,6 & 67,9 \\
\hline Schweiz & 3,6 & 35,5 & 60,9 \\
\hline Türkei & 15,4 & 36,9 & 47,7 \\
\hline Großbritannien & 1,3 & 29,5 & 69,2 \\
\hline Nordamerika ${ }^{c}$ & 2,2 & 29,5 & 68,3 \\
\hline OECD-Europa ${ }^{c}$ & 4,4 & 32,0 & 63,5 \\
\hline$E^{c, d}$ & 4,8 & 31,4 & 63,7 \\
\hline $\mathrm{OECD}^{c}$ & 4,8 & 32,2 & 63,0 \\
\hline
\end{tabular}

Quelle: OECD (1993): OECD Environmental Data, a.a.O.

Anm:
a. Anteil der einzelnen Sektoren am Bruttoinlandsprodukt
b. Alte Bundeslander
c. Mittelwert
d. 11 Staaten (ohne Osterreich, Finnland, Schweden und Luxemburg) 
Tab. A55: Regressionsgleichungen der multiplen

\section{Regressionsanalysen}

A55.1

[PRESS/C_90] $=61.852^{*}$

$$
\begin{aligned}
& +8.2981^{*} \text { [FÖDERAL] } \\
& -31.252^{*} \text { [KORPORAT] } \\
& +26.734^{*}[\text { PARTIZIP] } \\
& +15.438^{*}[\text { F\&E01] } \\
& +0.7163^{*}[\text { F\&E02] } \\
& -109.54^{*}[\text { F\&E03] } \\
& -2.2574^{*}[\text { ADM01] } \\
& +4.4817^{*} \text { [ADM02] }
\end{aligned}
$$

Beta-Gewicht

$-0.215$

$-0.894$

0.664

0.356

0.016

$-0.493$

$-0.058$

0.870

$\mathrm{N}=22 \quad \mathrm{SE}=\mathbf{3 3 . 0 3 3}$

A55.2

[RESOU/C_90] $=11.171^{*}$

$$
\begin{aligned}
& +31.248^{*} \text { [FÖDERAL] } \\
& -46.318^{*} \text { [KORPORAT] } \\
& -5.6162^{*} \text { [PARTIZIP] } \\
& -4.8147^{*}[\text { F\&E01] } \\
& -10.369^{*}[\text { F\&E02] } \\
& +213.8^{*}[\text { F\&E03] } \\
& -8.7481^{*}[\text { ADM01] } \\
& +7.0404^{*} \text { [ADM02] }
\end{aligned}
$$

Beta-Gewicht

$-0.429$

$-0.708$

$-0.075$

$-0.059$

$-0.122$

0.514

$-0.534$

0.730

$\mathrm{N}=\mathbf{2 2}$

$\mathrm{SE}=46.292$ 
A55.3

[ENVIR/C_90] $=36.426^{*}$

$$
\begin{aligned}
& +19.745^{*} \text { [FÖDERAL] } \\
& -38.248^{*} \text { [KORPORAT] } \\
& +10.14^{*} \text { [PARTIZIP] } \\
& +5.0676^{*}[\text { F\&E01] } \\
& -4.5091^{*}[\text { F\&E02] } \\
& +51.146 * \text { F\&E03] } \\
& -5.4605^{*}[\text { ADM01] } \\
& +5.7637^{*}[\text { ADM02] }
\end{aligned}
$$

Beta-Gewicht

$$
\mathrm{N}=22 \quad \mathrm{SE}=35.792
$$

A55.4

[PRESS/BIP_90] $=109.19^{*}$

$$
\begin{aligned}
& -12.805^{*} \text { [FODERAL] } \\
& -27.814^{*} \text { [KORPORAT] } \\
& +19.624^{*} \text { [PARTIZIP] } \\
& -27.93^{*} \text { [F\&E01] } \\
& -1.5951^{*}[\text { [F\&E2] } \\
& -51.011^{*} \text { [F\&E03] } \\
& -1.4137^{*}[\text { ADM01] } \\
& +0.9386^{*} \text { [ADM02] }
\end{aligned}
$$$$
-0.398
$$$$
-0.852
$$$$
0.196
$$$$
0.091
$$$$
-0.077
$$$$
0.179
$$$$
-0.486
$$$$
0.871
$$

Beta-Gewicht

$\mathrm{N}=\mathbf{2 2} \quad \mathrm{SE}=\mathbf{3 2 . 2 6 9}$

A55.5

[RESOU/BIP_90] $=76.822^{*}$

$$
\begin{aligned}
& -0.7861^{*} \text { [FODERAL] } \\
& -30.001^{*} \text { [KORPORAT] } \\
& -14.535^{*} \text { [PARTIZIP] } \\
& -43.64^{*} \text { [F\&E01] } \\
& -14.99^{*}[\text { F\&E02] } \\
& -331.92^{*} \text { [F\&E03] } \\
& -5.1218^{*} \text { [ADM01] } \\
& +1.2618^{*} \text { [ADM02] }
\end{aligned}
$$$$
0.307
$$$$
-0.736
$$$$
0.451
$$$$
-0.596
$$$$
-0.033
$$$$
0.212
$$$$
-0.149
$$$$
0.168
$$

Beta-Gewicht

$\mathrm{N}=\mathbf{2 2}$

$\mathrm{SE}=\mathbf{3 4 . 0 4 6}$ 
A55.6

[ENVIR/BIP_90] $=93.001^{*}$

- 6.7893* [FÖDERAL]

Beta-Gewicht

- 28.913* [KORPORAT]

0.153

$+2.5414 *$ [PARTIZIP]

$-0.720$

- 35.796* [F\&E01] 0.055

- 8.2873* [F\&E02]

$-0.719$

$-191.52 *$ [F\&E03]

$-0.159$

- 3.2709* [ADM01]

0.750

$+1.1024 *$ [ADM02]

$-0.325$

0.186

$\mathrm{N}=\mathbf{2 2} \quad \mathrm{SE}=\mathbf{2 9 . 8 4 9}$

(Die Bedeutung der Variablenkurzbezeichnungen kann dem Ablärzungsverzeichnis entnommen werden.) 
Christian Ricken - 978-3-631-75118-3

Downloaded from PubFactory at 01/11/2019 08:07:14AM

via free access 


\section{SCHRIFTEN ZUR WIRTSCHAFTSTHEORIE UND WIRTSCHAFTSPOLITIK}

Herausgegeben von Rolf Hasse, Wolf Schäfer, Thomas Straubhaar, Klaus W. Zimmermann

Band 1 Lars Būnning: Die Konvergenzkriterien des Maastricht-Vertrages unter besonderer Berücksichtigung ihrer Konsistenz. 1997.

Band 2 Andreas Henning: Beveridge-Kurve, Lohnsetzung und Langzeitarbeitslosigkeit. Eine theoretische Untersuchung unter Berücksichtigung des Insider-Outsider-Ansatzes und der Entwertung des Humankapitals. 1997.

Band 3 Iris Henning: Die Reputation einer Zentralbank. Eine theoretische Untersuchung unter besonderer Berücksichtigung der Europaischen Zentralbank. 1997.

Band 4 Rüdiger Hermann: Ein gemeinsamer Markt für Elektrizttät in Europa. Optionen einer Wettbewerbsordnung zwischen Anspruch und Wintlichkeit. 1997.

Band 5 Alexander Tiedtke: Japan und der Vorwurf des Trittbrettfahrenverhaltens in der US-amerikanisch-japanischen Allianz. 1997.

Band 6 Wolfgang Grimme: Ordnungspolitisches Konzept der Regionalpolitik. Darstellung der Defizite und des Reformbedarts der Regionalpolitik am Beispiel Mecklenburg-Vorpommerns. 1997.

Band 7 Christian Ricken: Determinanten der Effektlvität der Umweltpolitik. Der nationale Politikstil im Spannungsfeld von Ökonomie, Pollitik und Kultur. 1997. 
Christian Ricken - 978-3-631-75118-3

Downloaded from PubFactory at 01/11/2019 08:07:14AM

via free access 